\title{
Der elastisch aufgehängte starre Körper
}

\author{
Dissertation \\ zur Erlangung des Doktorgrades \\ der Mathematisch-Naturwissenschaftlichen Fachbereiche \\ der Georg-August-Universität zu Göttingen
}

vorgelegt von

Henning Dathe

aus Braunschweig

Göttingen 2001 
D7

Referent: Prof. Dr. H. Nägerl

Korreferent: Prof. Dr. G. Hegerfeldt

Tag der mündlichen Prüfung: 2. Mai 2001 
Die Elastizität der Körper (und es wird wohl keine völlig harten oder völlig weichen geben) ist gleichsam das Leben derselben, wir bekommen dadurch ein Gefühl ihrer Gegenwart durch das Gehör, Gesicht und öfters das Gefühl, ein Körper, welcher dieses Lebens beraubt ist, würde, unkenntlich und unbrauchbar, seine Lücke ausfüllen. Die elastischen Kräfte der Körper sind die Dolmetscher wodurch sie so zu sagen mit uns sprechen.

Georg Christoph Lichtenberg, Sudelbücher, Heft A, 8 (1765) 


\section{Inhaltsverzeichnis}

\begin{tabular}{lll}
\hline 1 & Einleitung & 1
\end{tabular}

1.1 Überblick und Vorgehensweise . . . . . . . . . . . . . . . . . 5

\begin{tabular}{lll}
\hline 2 & Statik elastischer Aufhängungen & 19
\end{tabular}

2.1 Grundbegriffe . . . . . . . . . . . . . . . . . . . . . 20

$2.1 .1 \quad$ Vektoren und Symmetrieoperationen . . . . . . . . . . 20

2.1 .2 Verallgemeinerte Koordinaten und Schrauben . . . . . . . 25

2.2 Die elastischen Matrizen . . . . . . . . . . . . . . . . . . . . . . . . 32

$2.2 .1 \quad$ Die Flexibilitätsmatrix . . . . . . . . . . . . . . . . . 33

2.2 .2 Die Steifheitsmatrix $\ldots \ldots \ldots$. . . . . . . . 37

2.2 .3 Das elastische Potential . . . . . . . . . . . . . . 40

$2.2 .4 \quad$ Das Widerstandszentrum . . . . . . . . . . . . . . . . . . . . 41

$2.2 .5 \quad$ Das elastische Zentrum . . . . . . . . . . . . . . . . . . . 45

2.3 Die Gesamtheit der Schraubachsen . . . . . . . . . . . . . . . . . . 53

$2.3 .1 \quad$ Die Aufpunktfläche . . . . . . . . . . . . . . . . . . 53

2.3 .2 Die Abstandsfläche . . . . . . . . . . . . . 56

2.3 .3 Die Steigungsfläche . . . . . . . . . . . . . . . . . . 58

$2.3 .4 \quad$ Die Kongruenz der Schraubachsen . . . . . . . . . . . . . . 59

$2.4 \quad$ Funktionelle Achsen und andere ausgezeichnete Richtungen . . . . . 61

$2.4 .1 \quad$ Funktionelle Achspaare und Richtungen $\ldots . . . . . . .663$ 
2.5 Standardformen der elastischen Matrizen . . . . . . . . . . . . . . . 64

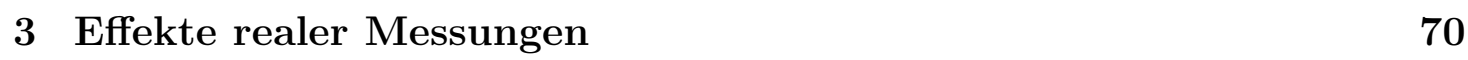

3.1 Zustandsgleichungen und Dynamik . . . . . . . . . . . . . . . . 70

$3.1 .1 \quad$ Kinetische Energie $\ldots \ldots . \ldots . \ldots 71$

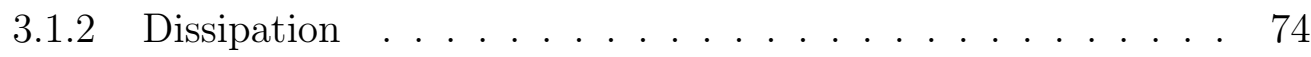

$3.1 .3 \quad$ Zeitabhängigkeit und Superpositionsprinzip . . . . . . . . 75

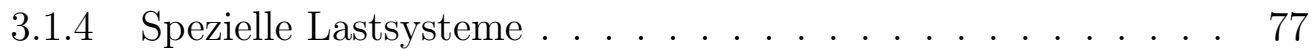

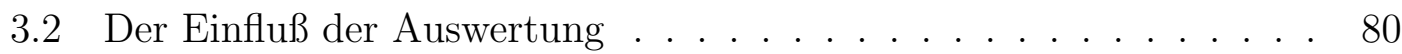

$3.2 .1 \quad$ Lineare Regression und Fehlerfortpflanzung . . . . . . . . . 81

3.2 .2 Herausrechnen mittels Zeitentwicklung . . . . . . . . . . . . 82

$3.2 .3 \quad$ Ausgleichsrechnung mit sechs Freiheitsgraden . . . . . . . . 83

$3.3 \quad$ Der Einfluß der Meßapparatur . . . . . . . . . . . . . . . . . . . . . 85

3.3.1 Der Einfluß der Elastizität der Meßapparatur . . . . . . . . 86

3.3 .2 Inkompatible Einheitensysteme . . . . . . . . . . . . . 86

3.3 .3 Verdrehung der Referenzsysteme. . . . . . . . . . . . . . . 88

3.3 .4 Der Einfluß der Vorlast . . . . . . . . . . . . . . . . . . . . . 91

3.4 Nicht symmetrisierbare elastische Matrizen . . . . . . . . . . . . . . 100

4 Das elastische Potential einer dünnen Schicht 102

4.1 Federn im Raum . . . . . . . . . . . . . . . . . . . . . . . 103

$4.1 .1 \quad$ Die Einzelfeder . . . . . . . . . . . . . . . . . 103

$4.1 .2 \quad$ Systeme von Federn mit gleichen Endpunkten . . . . . . . . 105

4.2 Die dünne elastische Schicht zwischen zwei starren Berandungen als lokal ebenes Problem . . . . . . . . . . . . . . . 106

$4.2 .1 \quad$ Die Federmatrix eines isotropen Materials . . . . . . . . . . 106

$4.2 .2 \quad$ Die Federmatrix eines faserigen Materials . . . . . . . . . . . 107

4.2 .3 Das Federmodell . . . . . . . . . . . . . . . . . . . . . . 108 
$4.3 \quad$ Schichtgrößen, Materialkonstanten und Abmessungen . . . . . . . . 110

$4.3 .1 \quad$ Dimensionsbetrachtungen . . . . . . . . . . . . . . . . 111

4.3 .2 Ähnlichkeitsbetrachtungen . . . . . . . . . . . . . . . . 112

4.4 Stab- und Federmodell in der Kontinuumsmechanik . . . . . . . . . 115

$4.4 .1 \quad$ Die Flexibilitätsmatrix eines Stabes . . . . . . . . . . . . . . 115

$4.4 .2 \quad$ Die allgemeine Form der elastischen Energie . . . . . . . . . 119

$4.4 .3 \quad$ Identifikation der Schichtgrößen . . . . . . . . . . . . . 120

4.5 Zusammengesetzte Aufhängungen . . . . . . . . . . . . . . . . . . . 129

4.5.1 Die Analogie zum Schwerpunktsatz und ihre Grenzen . . . . 130

4.5.2 Superposition von Widerstandszentren . . . . . . . . . . . 131

5 Verschiedene Aufhängungen $\quad 135$

5.1 Einfache Aufhängungen und elementare Rechenmethoden . . . . . . 136

$5.1 .1 \quad$ Die rechteckige Scheibe . . . . . . . . . . . . . 136

5.1 .2 Die Kreisscheibe $\ldots \ldots \ldots$. . . . . . . . . . . . . 137

$5.1 .3 \quad$ Vergleich von Quadrat und Kreis . . . . . . . . . . . . . . . 139

$5.1 .4 \quad$ Eine Aufhängung ohne Widerstandszentrum . . . . . . . . . 139

5.1 .5 Die rechteckige Scheibe mit variabler Schichtdicke . . . . . . 142

5.1.6 Ebene Aufhängungen mit konstanten Schichtgrößen . . . . . 146

5.2 Aufhängungen mit hoher Symmetrie . . . . . . . . . . . . . . . . 151

$5.2 .1 \quad$ Voll- und Halbkugel $\ldots \ldots \ldots$. . . . . . . . . . . 152

$5.2 .2 \quad$ Der Kreiszylinder $\ldots \ldots \ldots$. . . . . . . . . . . 153

$5.2 .3 \quad$ Der Kreiskegel . . . . . . . . . . . . . . . . . . . . . 154

5.3 Komplexere Aufhängungen . . . . . . . . . . . . . . . . . . . 156

$5.3 .1 \quad$ Der zweiseitige Keil . . . . . . . . . . . . . . . . 156

$5.3 .2 \quad$ Der Kreiszylinder mit Boden . . . . . . . . . . . . . . . . 158

$5.3 .3 \quad$ Das Rotationsparaboloid . . . . . . . . . . . . . . . . 159 
5.3.4 Die allgemeine rotationssymmetrische Aufhängung. . . . . . . 161

5.3 .5 Der Schaft einer Hüftendoprothese . . . . . . . . . . . . . . 164

5.4 Diagonalform, Transversalkraft und Reziprozitätstheorem . . . . . . 167

$\begin{array}{|ll|}6 & \text { Anwendung auf biomechanische Messungen }\end{array}$

$6.1 \quad$ Die Auswertung im Überblick . . . . . . . . . . . . . . . . . . . . . 169

$6.1 .1 \quad$ In jedem Fall durchzuführende Schritte . . . . . . . . . . . . 169

6.1 .2 Der Translationsanteil . . . . . . . . . . . . . . . . . . . 170

6.1 .3 Der Rotationsanteil . . . . . . . . . . . . . . . . . . . 171

6.1 .4 Optionale Schritte . . . . . . . . . . . . . . . 172

6.2 Das Wirbelsäulensegment ． . . . . . . . . . . . . . . . . 172

6.3 Ein zylindrischer Probekörper . . . . . . . . . . . . . . . . . . . 178

6.4 Initiale Zahnbeweglichkeit . . . . . . . . . . . . . . . . . . . 183

6.4.1 Elastische Faser vs. inkompressible Flüssigkeit? . . . . . . . 183

6.4 .2 In vivo . . . . . . . . . . . . . . . . . . . . . 195

6.4 .3 In vitro . . . . . . . . . . . . . . . . . 207

6.5 Statik eines Hüftendoprothesenschaftes . . . . . . . . . . . . . . . . 210

6.6 Schraubenfedern . . . . . . . . . . . . . . . . . 213

\begin{tabular}{lll}
\hline 7 & Zusammenfassung & 217 \\
\hline
\end{tabular}

7.1 Thesen . . . . . . . . . . . . . . . . . . . . . . 217

$7.2 \quad$ Was zu tun bleibt . . . . . . . . . . . . . . . . . . . . . . . . . 219

7.3 Danksagung . . . . . . . . . . . . . . . . . . . . . . 220

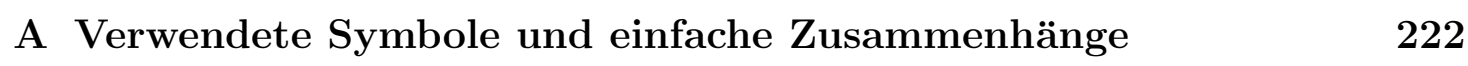

A.1 Notation . . . . . . . . . . . . . . . . . . . . . . . . . . 222

A.1.1 Allgemeine Symbole . . . . . . . . . . . . . . . . 222

A.1.2 Sub- und Superskripte . . . . . . . . . . . . . . . . . 223 
A.1.3 Kernbuchstaben . . . . . . . . . . . . . . . . . . . 224

A.2 Einfache Zusammenhänge . . . . . . . . . . . . . . . . . . . . . . . 228

A.2.1 Projektoren . . . . . . . . . . . . . . . . . . . . . 228

A.2.2 Der $\varepsilon$-Tensor, Kreuzprodukte und antisymmetrische Matrizen 229

A.2.3 Die Güte einer Matrix . . . . . . . . . . . . . . . . 234

A.3 Rechnungen mit Mathematica . . . . . . . . . . . . . . . . . . 235

A.3.1 Prozeduren zur Vektor- und Tensorrechnung und für Block-

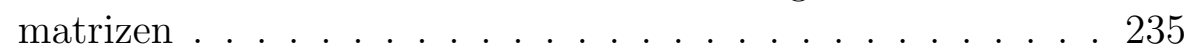

A.3.2 $\quad$ Prozeduren zur Differentialgeometrie . . . . . . . . . . . . . 241

A.3.3 Prozeduren zur direkten Auswertung . . . . . . . . . . . . . 242

\begin{tabular}{ll}
\hline B Miscellana & 246
\end{tabular}

B.1 Widerstandsachsen . . . . . . . . . . . . . . . . . . . 246

B.1.1 Die Achsfläche . . . . . . . . . . . . . . . . . . 247

B.2 Das ebene Widerstandszentrum . . . . . . . . . . . . . . 252

B.2.1 Definition des ebenen Widerstandszentrums . . . . . . . . . 252

B.2.2 Eine allgemeine ebene Aufhängung . . . . . . . . . . . . . 253

B.2.3 Vergleich mit dem Schwerpunkt der Aufhängung . . . . . . . 255

B.2.4 Im räumlichen Formalismus . . . . . . . . . . . . . . 255

B.3 Der lineare Zwangskontakt . . . . . . . . . . . . . . . . . . . . 257

B.3.1 Angreifende und tatsächlich wirkende Lasten . . . . . . . . . 258

B.3.2 Tatsächlich mögliche Lagen . . . . . . . . . . . . . . . . . . 259

B.3.3 Die Flexibilitätsmatrix eines linear gefesselten Systems . . . 260

B.3.4 Steifheitsmatrix und Potential eines linear gefesselten Systems 262

B.3.5 Zwei lineare Fesselungen . . . . . . . . . . . . . . . . 262 


\section{Kapitel 1}

\section{Einleitung}

Synarthrosen sind spezielle Gelenke ohne Gelenkhöhle, bei denen zwei Knochen haftend und kontinuierlich durch Gewebe miteinander verbunden sind, so zum Beispiel der Zahnhalteapparat und die Bandscheibe des menschlichen Körpers. Ziel dieser Arbeit ist es, eine mathematische Beschreibung des mechanischen Verhaltens von Synarthrosen zu finden.

In der Medizin gilt das Augenmerk dem beteiligten Gewebe, entsprechend werden spezielle Begriffe verwendet. Handelt es sich um eine bandhafte Verbindung durch kollagenes Bindegewebe, so spricht man von einer Syndesmose; enthält das Gewebe Faserknorpel, so nennt man das Gelenk eine Symphyse; besteht es dagegen aus hyalinem Knorpel, dann heißt es Synchondrose. Eine Ausnahme bilden durch Wachstum des Knochens geschlossene Knorpelfugen, welche man Synostosen nennt. Beispiele von Syndesmosen sind die Verbindung zwischen Elle und Speiche oder Schien- und Wadenbein, die Nähte zwischen den Schädelknochen und die Zahnwurzelhaut. Bei der Verbindung der Beckenhälften handelt es sich um eine Symphyse. Die Verbindung von Rippe und Brustbein und die Bandscheibe ist eine Synchondrose. Da in dieser Arbeit nach einer gemeinsamen mathematischen Beschreibung solcher Gelenke gesucht werden soll, dürfen derartige anatomische Unterschiede nicht in die grundlegenden Ansätze eingehen. Erst in den zahlenmäßig durchgerechneten Beispielen wird auf die jeweilige Anatomie wieder genauer eingegangen.

Mit Ausnahme der Synostosen, welche hier nicht weiter von Interesse sind, können die beteiligten Knochen im Vergleich zum übrigen Gewebe in guter Näherung als starr betrachtet werden. Ihre Verbindung ist die nicht näher spezifizierte elastische Aufhängung. Wählt man die Lage, also Position und Orientierung, des einen Knochen als raumfest, so genügen zur Beschreibung der Lage des anderen die sechs Freiheitsgrade eines starren Körpers im Raum. Zunächst muß daher eine mathematische Zuordnung von statisch angreifenden Lasten, also Kräften und Drehmomenten, zur Lage des bewegten Körpers gefunden werden. Ferner soll diese Zuordnung 
mit einer Auswertevorschrift verbunden werden, die reproduzierbare Aussagen über die Stärke und Struktur der Aufhängung erlaubt.

Messungen zur initialen Zahnbeweglichkeit [3, 4 $]^{1}$ in unserer Arbeitsgruppe waren die ursprüngliche Motivation zur Beschäftigung mit diesem Thema. Später folgten noch Messungen zur initialen Kinematik der Wirbelsäule [59] und zur Statik von Hüftendoprothesen [72] sowie deren Dynamik [21]. An Messungen mangelte es also nicht, eher an der Interpretierbarkeit der Ergebnisse. Meine Aufgabe sollte es demnach sein, eine passende und genügend einfache Theorie für derartige Messungen zusammenzutragen oder notfalls selbst zu entwickeln.

Das vordringlich zu lösende Problem ist die Abhängigkeit der die Lasten und Lagen beschreibenden Zahlen vom gewählten Koordinatenursprung. Diese überträgt sich auf die Zuordnung von angreifenden Lasten zu den Lageänderungen und damit auf die Meßwerte selbst. Hängen nämlich Lasten und Lagen vom Ursprung der Koordinatensystems ab, und ist kein ausgezeichneter Ursprung definiert, so wird auch die Abhängigkeit der Lagen von den Lasten eine Funktion des Ortes sein. Gerade weil in unserer Arbeitsgruppe Messungen an Synarthrosen durchgeführt wurden, war eine solche Vergleichbarkeit, etwa mit Meßergebnissen anderer Arbeitsgruppen wie [53], unbedingt erstrebenswert.

Durch einen vor allen anderen Punkten ausgezeichneten Bezugspunkt zusammen mit einer ebenso ausgezeichneten Orientierung der Koordinatenachsen wäre diese Mehrdeutigkeit behoben.

Mit dem von H. NÄGERL [49] in seiner Habilitationsschrift in direkter Analogie zum Schwerpunkt in zwei Dimensionen verwendeten Begriff des Widerstandszentrums als ausgezeichneter Bezugspunkt zur Beschreibung ebener Lasten und Lagen mochte ich mich aber aus formalen Gründen nicht recht anfreunden: In der räumlichen Elastostatik hat man es mit Tensoren zweiter Stufe zu tun; bei der Definition des Schwerpunktes wird aber mit der Massendichte ein Skalar verwandt. Aus der Gesamtmasse im Nenner wird hier eine inverse Matrix. Wegen der Nichtkommutativität des Matrixproduktes und der noch unklaren Berechnung eines Ortsvektors aus diesen Matrizen vermutete ich Schwierigkeiten. Der Begriff ließ sich zudem nicht bis auf seinen historischen Ursprung zurückverfolgen: Weder in einem Abriß der Geschichte der Elastizitätstheorie [70] noch in der grundlegenden Arbeit zur Statik des Zahnhalteapparates [64] ist die Rede von einem vergleichbaren Objekt. In [34], fand ich schließlich (S. 144) eine kleingedruckte Bemerkung über die Existenz eines „Schwerpunktes der Federkräfte“ für ebene Aufhängungen mit den gewünschten Eigenschaften - leider ohne Beweis oder Referenz. ${ }^{2}$ Die früheste mir bekannte Verwendung dieses Begriffes in der kieferorthopädischen Literatur stammt aus dem Jahre 1917 [17]. Der Begriff „Widerstandszentrum“ ist demnach

\footnotetext{
${ }^{1}$ Mit Nummern in eckigen Klammern sollen auch in Zukunft Zitate gekennzeichnet werden. Die Zuordnung zur jeweiligen Quelle ergibt sich aus dem Literaturverzeichnis auf Seite 268.

${ }^{2} \mathrm{Im}$ zweiten Teil der Einleitung wird dieser Beweis geführt.
} 
als Übersetzung des der ebenen Elastostatik entlehnten und mittlerweile in der Zahnmedizin üblichen Terminus "center of resistance" inklusive seiner nicht hinterfragten Übertragung auf den räumlichen Fall zu verstehen. Im Widerstandszentrum ([49], Kap. 4) fand ich dennoch eine Vorstellung, die mir zwar nur unter stark vereinfachenden Annahmen (skalare Federstärke, ebene Aufhängung) korrekt erschien, ansonsten aber die notwendige Einfachheit besaß, um als Leitbild einer allgemeineren Definition zu dienen.

Obwohl eine elastische Aufhängung zwischen zwei starren Körpern neben der medizinischen Bedeutung auch in vielen technischen Bereichen eine Rolle spielt (z. B. in einer Autofederung), gibt es keine Quellen, die als Referenz bezeichnet werden könnten. Nur wenig fündig wurde ich in der schon eben erwähnten mathematischen Arbeit über den Zahnhalteapparat [64, 65], die mir teils wegen der gemachten Annahmen (das Periodont als dünne inkompressible Membran) und anderen teils wegen der sehr anspruchsvollen mathematischen Methoden (RIEMANnsche Normalkoordinaten), als zu kompliziert erschien. Mit diesem Formalismus waren zu wenige exakte Lösungen zu erwarten. Vor allen Dingen war das Augenmerk auf die Vorgänge innerhalb des Periodonts zu detailliert, wobei dennoch die faserigen gegenüber den flüssigen Anteile vernachlässigt wurden. In Abhandlungen des Maschinenbaues, speziell über Maschinenfundamente, und der Architektur, dort insbesondere über erdbebensichere Fundamente von Gebäuden, findet das in Lehrbüchern der Elastizitätstheorie, etwa [40, standardmäßig durchgerechnete Beispiel eines elastischen Halbraumes zwar vielfältige Anwendung, aber auf die Aufgabenstellung hier ist es so nicht anwendbar. Modifikationen dieses Ansatzes im Rahmen der strengen Elastizitätstheorie erschienen mir zu komplex. Die Dynamik von gekoppelten Mehrkörpersystemen war da ergiebiger: Im Raketenbau [58, bei der Robotik [2] und in der technischen Numerik geht es insbesondere um das Schwingungsverhalten komplexer mechanischer Systeme, speziell um Simulationsprogramme [43, 58, 29]. Dort, wie auch in allgemeinen Lehrbüchern, etwa [73] $\S 12.2-12.5$ oder [1], konnte ich viele Anregungen zum mathematischen Formalismus finden - allerdings ohne eine Anwendung auf das vorliegende Problem. Meistens tauchen die von mir für relevant befundenen Phänomene durch Untersuchen von symmetrischen oder zu stark vereinfachten Objekten gar nicht erst nicht auf, so etwa in einem aktuellen Handbuch [33], Part II, Chap. 4.

Meines Wissens also wurde das einfache Modell eines starr berandeten elastischen Mediums als elastisch aufgehängter starrer Körper, speziell für eine dünne Schicht, noch nicht adäquat untersucht.

Zuerst sind die nächstliegenden vereinfachenden Annahmen zu untersuchen. In den verwendeten Variablen, wahlweise den Lagen oder den Lasten, wird daher linearisiert. Insbesondere erlaubt das die Verwendung des Drehvektors für die Freiheitsgrade der Rotation. Hookesches Verhalten eines isotropen Mediums dient als Leitfaden für Vorstellungen über ideale Meßwerte und später als Anhaltspunkt zum Vorhersagen der Ergebnisse und damit zum Beurteilen realer Messungen. In den 
Koeffizienten dieser Linearisierung läßt sich dann unter Benutzung ihres Transformationsverhaltens die Information der ausgezeichneten Bezugssysteme ausfindig machen. Diese Information besteht aus einem Bezugspunkt der Lasten und einem der Lagen sowie je einer Orientierung der zugehörigen Koordinatenachsen. Damit ist die unter EuKLIDischen Transformationen der Bezugssysteme invariante Form der Meßwerte bestimmt.

Als Nächstes muß der Gültigkeitsbereich dieser idealisierten Vorstellung bestimmt und eingegrenzt werden. Dabei kommen die Einflüsse dynamischer, thermodynamischer, statistischer und experimenteller Effekte zur Sprache.

Für Vorhersagen gibt es ausgereifte numerische Verfahren, wie etwa die Methode der Finiten Elemente. Diese erschienen mir aber hier nicht brauchbar, da mit ihnen weder Aussagen über Klassen von Meßobjekten mit stetig veränderlicher Form oder andere Abhängigkeiten von einzelnen Parametern des Modells möglich sind. Es galt demnach, eine mit möglichst wenig einschränkenden Vereinfachungen auskommende Methode der Vorhersage von Meßwerten zu entwickeln. Im Gegensatz zur SynGEschen Theorie werden hier den festen oder faserigen Anteilen der Gewebe die Haupteffekte zugeschrieben, da bei einer quasistatischen Messung die durch die Gewebsflüssigkeit hervorgerufene Dämpfung keine Rolle mehr spielen sollte.

Nähert man die Form der Aufhängung durch ein einfaches geometrisches Modell an, so bietet die Differentialgeometrie von Flächen im Raum, vgl. etwa [22], die passende Beschreibung der räumlichen Situation. Die Materialkonstanten dünner isotroper Schichten lassen sich mit Hilfe der Darstellungstheorie auf zwei reduzieren: Eine modelliert das Verhalten für Bewegungen senkrecht zur Schicht, und die andere solche innerhalb der Schicht. Unter Annahme einer lokal homogenen Deformation können diese Schichtgrößen mit Hilfe der Elastizitätstheorie näher spezifiziert werden.

Mit diesen beiden Grundzutaten, einer standardisierten Form der Meßwerte und einem möglichst analytischen Wunschergebnis, werden dann schließlich die Auswertungen durchgeführt.

Diese Arbeit ist notwendigerweise interdisziplinär: Obwohl die formale Sprache der Mathematik bzw. der Theoretischen Physik das Hauptinstrument darstellt, sind die Anwendungen eher im medizinischen oder ingenieurswissenschaftlichen Kontext zu finden. Der Versuch, natürliche Objekte durch Anwendung von Physik besser zu verstehen, war bislang eine Domäne der Bionik. Da es hier zunächst nur um die Anwendung von Lasten auf biologische Strukturen und ihre Effekte geht, ist die Disziplin, zu der die Zielsetzung dieser Arbeit gehört, die Biomechanik. 


\section{1 Überblick und Vorgehensweise}

In dieser Arbeit geht es um eine mathematische Beschreibung der Veränderung der Lage eines elastisch aufgehängten starren Körpers unter Laständerungen. Die Problematik soll an einem, auch im Folgenden eingerückten, zweidimensionalen Beispiel erläutert werden. Feinheiten werden dabei zunächst bewußt übergangen, da diese im Hauptteil dieser Arbeit, dem dreidimensionalen Fall, genügend ausführlich besprochen werden. Die Notation in dieser Einleitung ist entsprechend als Propädeutik für den dreidimensionalen Fall gewählt.

Eine allgemeine Last in zwei Dimensionen, bestehend aus einer Kraft in der $x y$-Ebene und einem Drehmoment senkrecht zu dieser Ebene, ruft an einer elastischen Aufhängung, etwa dem Periodont eines vorderen Schneidezahnes, eine Lageänderung, bestehend aus einer Translation in der $x y$-Ebene und einer Drehung senkrecht dazu, hervor.

Last und Lageänderung können nach Einführung von Koordinaten und einem Maßsystem zu Zahlentripeln

$$
f=\left(\begin{array}{c}
F_{x} \\
F_{y} \\
T_{z}
\end{array}\right)=\left(\begin{array}{c}
\boldsymbol{F} \\
T
\end{array}\right) \quad \text { und } \quad q=\left(\begin{array}{c}
d_{x} \\
d_{y} \\
\theta_{z}
\end{array}\right)=\left(\begin{array}{c}
\boldsymbol{d} \\
\theta
\end{array}\right)
$$

oder, aus der Sicht des Theoretikers, zu verallgemeinerten Koordinaten zusammengefaßt werden. Dabei müssen weder die Koordinatensysteme der Lasten und Lageänderungen übereinstimmen, noch deren Einheiten zueinander passen. Das ist oft eine experimentelle Gegebenheit.

Diese mathematische Beschreibung besteht aus zwei voneinander getrennten Teilen: aus einer Vorschrift, wie Meßwerte zu handhaben sind, und einer Vorhersage über die Struktur der zu erwartenden Ergebnisse. Gesucht ist zunächst eine Beschreibung der Änderungen der Lage bei angelegten Lasten.

Der einfachste und gleichzeitig wichtigste Zusammenhang zwischen kleinen Größen $q$ und $f$ wird beschrieben durch die lineare Näherung

$$
q(f)=\mathcal{F} f
$$

mit der Flexibilitätsmatrix $\mathcal{F}$. Ohne weitere Annahmen ist diese Matrix als voll besetzt anzunehmen. Sie habe die Komponenten

$$
\mathcal{F}=\left(\begin{array}{ccc}
D_{x x} & D_{x y} & s_{x z} \\
D_{y x} & D_{y y} & s_{y z} \\
s_{z x} & s_{z y} & m_{z z}
\end{array}\right):=\left(\begin{array}{cc}
\boldsymbol{D} & \boldsymbol{s}_{2} \\
\boldsymbol{s}_{1}^{T} & m
\end{array}\right)
$$


welche die das System charakterisierenden elastischen Konstanten sind. Die einfachste Meßvorschrift zum Bestimmen von $\mathcal{F}$ besteht im Anlegen dreier Lasten in den verschiedenen Koordinatenrichtungen $F_{1}$ in $x-, F_{2}$ in $y$ - und $T_{3}$ in $z$-Richtung des Koordinatensystems der Lasten bei gleichzeitigem Messen der Lageänderungen $q_{1}, q_{2}$ und $q_{3}$ im Koordinatensystem der Lagen. Die Flexibilitätsmatrix besteht dann aus den durch die Last dividierten Lageänderungen

$$
\begin{aligned}
\mathcal{F} & =\left(\begin{array}{lll}
d_{1 x} / F_{1} & d_{2 x} / F_{2} & d_{3 x} / T_{3} \\
d_{1 y} / F_{1} & d_{2 y} / F_{2} & d_{3 y} / T_{3} \\
\theta_{1 z} / F_{1} & \theta_{2 z} / F_{2} & \theta_{3 z} / T_{3}
\end{array}\right) \\
& =\left(\begin{array}{lll}
d_{1 x} & d_{2 x} & d_{3 x} \\
d_{1 y} & d_{2 y} & d_{3 y} \\
\theta_{1 z} & \theta_{2 z} & \theta_{3 z}
\end{array}\right)\left(\begin{array}{ccc}
F_{1} & 0 & 0 \\
0 & F_{2} & 0 \\
0 & 0 & T_{3}
\end{array}\right)^{-1},
\end{aligned}
$$

und hängt mit diesen von der Wahl der Koordinatensysteme der Lasten und Lagen ab. Die einfachste Theorie zur Berechnung einer Flexibilitätsmatrix beruht auf dem Hookeschen Gesetz. Durch Angabe von $\mathcal{F}$ ist eine elastische Aufhängung vollständig charakterisiert, da nun mit dem Superpositionsprinzip Lageänderungen zu beliebig angelegten Lasten vorhergesagt werden können.

Da der starre Körper sechs Freiheitsgrade besitzt, läßt sich der Zusammenhang zwischen kleinen Lasten und Lagen ohne Kenntnis der inneren Struktur der elastischen Aufhängung in linearer Näherung als $6 \times 6$-Matrix beschreiben. Deren 36 Einträge, einheitenbehaftete reelle Zahlen, sind die Meßwerte, die es im Rahmen einer noch zu klärenden Theorie handzuhaben gilt.

Genauer war es die in verschiedenen Quellen [52, 53, 42] immer wieder betonte aber nur unzureichend begründete Symmetrie der Flexibilitätsmatrix, die nicht mit den Ergebnissen unserer Arbeitsgruppe [3, 4, 72, 59] übereinstimmte. Trotz verschiedener Apparaturen zeigten unsere Messungen alle ähnliche Phänomene. Daher versuche ich in dieser Arbeit, für diese und verwandte Aufgaben einen brauchbaren mathematischen Hintergrund zu liefern. Warum also sollte diese gemessene Matrix symmetrisch sein?

Die durch eine Lageänderung verrichtete Arbeit hat einen Translationsanteil und einen Rotationsanteil. Sind nun die Lasten und Lagen im selben Koordinatensystem bekannt, so lauten diese Anteile $\int \boldsymbol{F}^{T} d \boldsymbol{d}$ und $\int T d \theta$. Dazu müssen etwa die Lasten in das Koordinatensystem der Lagen transformiert werden. Das geschieht durch eine Translation des Koordinatenursprungs und eine Drehung der Koordinatenachsen. Da das die Translation beschreibende Hebelgesetz (vgl. dieser Abschnitt später) und die Drehung lineare Operationen sind, werden diese Trans- 
formationen mit einer $3 \times 3$-Matrix $\mathcal{T}_{q f}$ beschrieben, d. h.

$$
f_{q}=\mathcal{T}_{q f} f
$$

Führt man nun die Steifheitsmatrix $\mathcal{S}=\mathcal{F}^{-1}$ über $f=\mathcal{S} q$ ein, so schreibt sich die Formänderungsarbeit $\Phi$ allgemeiner

$$
\Phi_{12}=\int_{q_{1}}^{q_{2}} f_{q}^{T} d q=\int_{q_{1}}^{q_{2}} f^{T} \mathcal{T}_{q f}^{T} d q=\int_{q_{1}}^{q_{2}} q^{T} \mathcal{S}^{T} \mathcal{T}_{q f}^{T} d q
$$

Ist nun die Matrix $\mathcal{S}_{q q}:=\mathcal{T}_{q f} \mathcal{S}$ symmetrisch, so ist der Integrand genau die Ableitung von

$$
\Phi=\frac{1}{2} q^{T} \mathcal{S}_{q q} q
$$

und die Lasten verrichten als Potentialkräfte unabhängig vom Weg der Zustandsänderung immer dieselbe Arbeit. Antisymmetrische Anteile von $\mathcal{S}_{q q}$ trügen wegen

$$
q^{T} \mathcal{S} q=q^{T}\left(\mathcal{S}^{S}+\mathcal{S}^{A}\right) q=q^{T} \mathcal{S}^{S} q+q^{T} \mathcal{S}^{A} q
$$

und

$$
q^{T} \mathcal{S}^{A} q=\left(q^{T} \mathcal{S}^{A} q\right)^{T}=q^{T}\left(\mathcal{S}^{A}\right)^{T} q=-q^{T} \mathcal{S}^{A} q \quad \Rightarrow \quad q^{T} \mathcal{S}^{A} q=0
$$

in dieser quadratischen Form nicht zur Formänderungsarbeit bei und zerstörten die Wegunabhängigkeit der Formänderungsarbeit. Die Symmetrie der elastischen Matrizen $\mathcal{S}$ und $\mathcal{F}$ liegt also nur dann vor, wenn

- $f$ und $q$ im selben Koordinatensystem beschrieben sind, und

- die elastische Aufhängung durch ein Potential beschrieben wird.

Eine weitere Möglichkeit, antisymmetrische Terme vorzutäuschen, liegt im verwendeten Maßsystem begründet: Die Dimensionen von

$$
\boldsymbol{s}_{2}=\frac{\boldsymbol{d}_{3}}{T_{3}} \quad \text { und } \quad\left(\boldsymbol{s}_{1}\right)_{1,2}=\frac{\theta_{1,2}}{F_{1,2}}
$$

sind sicherlich gleich, nicht notwendigerweise aber auch die Einheiten und damit die Zahlenwerte. Werden etwa die Längen in $m m$ gemessen, so sind die Drehmomente in $N m m$ anzugeben, um die Symmetrie $\boldsymbol{s}_{2}^{T}=$ $s_{1}$ zu erhalten. Zusätzlich hat man also zu beachten, daß

- die Einheiten und Vorsätze passend gewählt sind.

Insbesondere sind die Winkel im Bogenmaß rad zu messen, was auch in den folgenden Überlegungen zum Transformationsverhalten der beteiligten Größen deutlich werden wird. Im SI-System ohne Verwendung von Vorsätzen hat man sicherlich keine Probleme. 
Aus Gründen der Einfachheit soll die Existenz eines Potentials als Arbeitshypothese angenommen werden. Dann sind die Lasten der Gradient des Potentials nach den Lageänderungen - insbesondere sind dadurch Lasten und Lagen im selben Koordinatensystem beschrieben. Die Steifheitsmatrix im Koordinatensystem der Lageänderungen ist dann die zweite Ableitung dieses Potentials. Das Hauptproblem liegt demnach in der Abhängigkeit der Lasten und Lagen vom gewählten Koordinatensystem. Diese Wahl der Koordinatensysteme läßt eine Translation der Ursprünge und eine Rotation der Koordinatenachsen zu. Zuerst soll der Translationsanteil allein näher untersucht werden.

Das Experiment muß nicht so aufgebaut sein, daß Kräfte im selben Bezugssystem angreifen werden, in dem die Lageänderung gemessen wird; das verbietet sich oft aus Platzgründen. Es muß daher eine rechnerische Transformation zwischen zwei Koordinatenursprüngen $O$ und $O^{\prime}$ zweier beliebiger Laborsysteme gefunden werden. Mit $\overrightarrow{O^{\prime} O}=\left(\begin{array}{l}a \\ b\end{array}\right)$ erhält man für die Lasten das Hebelgeset $2^{3}$

$$
\begin{aligned}
\left.\boldsymbol{F}\right|_{O^{\prime}} & =\left.\boldsymbol{F}\right|_{O} \\
\left.T\right|_{O^{\prime}} & =\left.T\right|_{O}+a F_{y}-b F_{x},
\end{aligned}
$$

das mit einer Transformationsmatrix $\mathcal{T}$

$$
f^{\prime}=\underbrace{\left(\begin{array}{ccc}
1 & 0 & 0 \\
0 & 1 & 0 \\
-b & a & 1
\end{array}\right)}_{=: \mathcal{T}_{f}} f
$$

zusammengefaßt geschrieben werden kann. Dieses Gesetz für Transformationen der Koordinatenursprünge für die Drehmomente läßt sich leicht anhand der Abbildung 1.1 auf Seite 9 einsehen!

Für kleine Lageänderungen erhält man durch Entwickeln der Drehmatrix

$$
\begin{aligned}
\left.\theta\right|_{O^{\prime}} & =\left.\theta\right|_{O} \\
\left.\boldsymbol{d}\right|_{O^{\prime}} & =\left.\boldsymbol{d}\right|_{O}+\left[\left(\begin{array}{cc}
\cos \theta & -\sin \theta \\
\sin \theta & \cos \theta
\end{array}\right)-\left(\begin{array}{ll}
1 & 0 \\
0 & 1
\end{array}\right)\right]\left(\begin{array}{l}
a \\
b
\end{array}\right) \\
& =\left.\boldsymbol{d}\right|_{O}+\theta\left(\begin{array}{c}
-b \\
a
\end{array}\right)+\mathcal{O}\left(\theta^{2}\right)
\end{aligned}
$$

\footnotetext{
$\left.{ }^{3} *\right|_{O}$ lies: $*$ bezüglich $O$.

${ }^{4}$ Falls eine Abbildung dieser Arbeit nicht mehr auf die Seite paßt, in der sie benötigt wird, befindet sie sich an der nächsten sinnvollen Stelle, meist auf der folgenden Seite oben.
} 


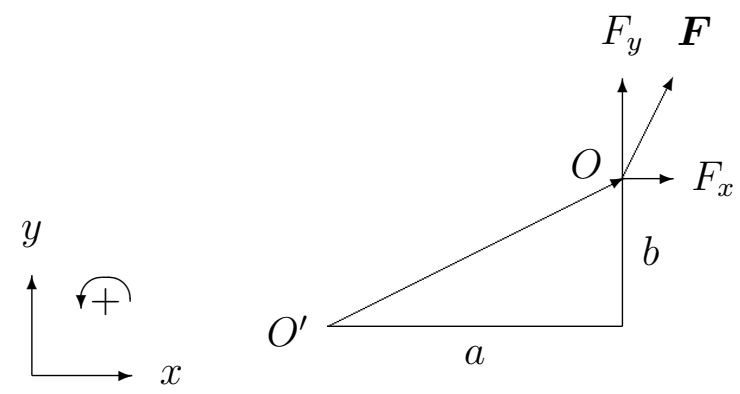

Abbildung 1.1: Das Hebelgesetz in zwei Dimensionen.

wieder zusammenfaßbar zu

$$
q^{\prime}=\underbrace{\left(\begin{array}{ccc}
1 & 0 & -b \\
0 & 1 & a \\
0 & 0 & 1
\end{array}\right)}_{=: \mathcal{T}_{q}} q
$$

Anhand der Zeichnung 1.2 läßt sich auch dieses Transformationsgesetz mit elementaren Mitteln ${ }^{5}$ herleiten.

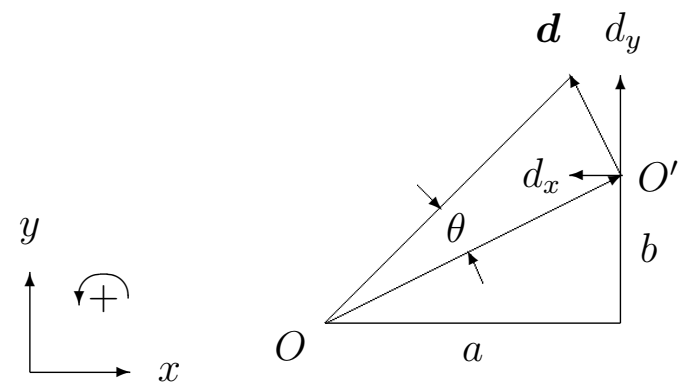

Abbildung 1.2: Die Transformation der Translation.

Eine infinitesimale Drehung läßt sich also in zwei Dimensionen als Wirbelfeld

$$
\boldsymbol{d}(x, y)=\left(\begin{array}{c}
-y \\
x
\end{array}\right) \theta
$$

beschreiben, das linear mit dem Abstand vom Ursprung wächst. In einem anderen Ursprung $O^{\prime}$ mit $x=x^{\prime}+a$ und $y=y^{\prime}+b$ muß dessen

${ }^{5}$ Für kleine Winkel $\theta$ kann die Sekante zwischen Anfangs- und Endpunkt der Bewegung durch die Tangente am Anfang ersetzt werden. Das entspricht der in Abb. 1.2 gezeigten Situation. Das Ausmaß der Bewegung ist $d=d_{x}^{2}+d_{y}^{2}=\sqrt{a^{2}+b^{2}} \theta$, wobei $\theta$ im Bogenmaß zu messen ist. Über ähnliche Dreiecke erhält man die einzelnen Beträge $\left|d_{x}\right| / d=|b| / \sqrt{a^{2}+b^{2}}$ und $\left|d_{y}\right| / d=$ $|a| / \sqrt{a^{2}+b^{2}}$, die zusammen mit den Vorzeichen aus der Zeichnung nach Kürzen der Wurzel das Ergebnis $d_{x}=-b \theta$ und $d_{y}=a \theta$ liefern. 
eigene Verrückung abgezogen werden, und es ergibt sich

$$
\begin{aligned}
\boldsymbol{d}^{\prime}\left(x^{\prime}, y^{\prime}\right) & =\boldsymbol{d}\left(x^{\prime}+a, y^{\prime}+b\right)-\boldsymbol{d}(a, b)=\left(\begin{array}{c}
-y^{\prime}-b \\
x^{\prime}+a
\end{array}\right) \theta-\left(\begin{array}{c}
-b \\
a
\end{array}\right) \theta \\
& =\left(\begin{array}{c}
-y^{\prime} \\
x^{\prime}
\end{array}\right) \theta=\boldsymbol{d}\left(x^{\prime}, y^{\prime}\right) .
\end{aligned}
$$

Die durch ein Wirbelfeld beschreibbare Drehung sieht daher in jedem Punkt gleich aus, d. h. $\theta^{\prime}=\theta$.

Es gilt $\mathcal{T}_{q}=\mathcal{T}_{f}^{T}$, die Transformationsmatrizen beschreiben auch die Symmetrie der Gleichungen.

In drei Dimensionen lassen sich die Transformationsgleichungen für Drehmoment und Translation formäquivalent durch Kreuzprodukte mit dem Differenzvektor der Koordinatenursprünge formulieren. In Matrizenschreibweise führt das auf $6 \times 6$ Matrizen. Wegen dieser formalen Übereinstimmung im Transformationsverhalten gehören Drehmoment und Translation beide zur Klasse der freien Vektoren.

Die Transformationen der elastischen Matrizen folgen aus denen der Lasten und Lagen, sie lauten

$$
\tilde{\mathcal{S}}=\mathcal{T}_{f} \mathcal{S} \mathcal{T}_{q} \quad \text { und } \quad \tilde{\mathcal{F}}=\mathcal{T}_{q} \mathcal{S} \mathcal{T}_{f}
$$

Zum Beschreiben ein- und derselben elastischen Aufhängung steht nun eine Klasse von äquivalenten Matrizen zur Verfügung.

Die Abhängigkeit der Lasten und der Lagen von dem gewählten Koordinatenursprung überträgt sich auf die sie miteinander verknüpfende Matrix. Selbst Messungen an derselben Aufhängung können daher zu unterschiedlichen Ergebnissen führen. In diesem Fall ist nicht einmal die Vergleichbarkeit von Meßergebnissen gewährleistet. Es ist also eine $\ddot{A}$ quivalenzklasse von Steifheitsmatrizen zur Beschreibung einer elastischen Aufhängung zulässig.

Erst eine von experimentellen Bedingungen bis auf unvermeidliche Meßfehler unabhängige Form der Meßwerte aber ermöglicht den Vergleich von Messungen. Nur diese läßt dann tiefere Einsichten über den Gegenstand des Interesses zu.

Vor allem schien mir der Versuch, eine solche Aufhängung unabhängig von der Wahl des Koordinatensystems zu beschreiben, nicht unternommen worden zu sein. Dabei bieten sich zwei prinzipiell unterschiedliche Vorgehensweisen an:

- Eine tatsächliche Unabhängigkeit vom gewählten Koordinatensystem, wie etwa in der Differentialgeometrie, oder 
- Ein reproduzierbar aus der Äquivalenzklasse auswählbarer Repräsentant, also ein durch die Meßwerte selbst eindeutig festgelegtes Koordinatensystem.

Hier soll der letzte Weg beschritten werden.

Nachdem ihr Transformationsverhalten nun bekannt ist, können die freien Vektoren $T$ und $\boldsymbol{d}$ in geeigneten Koordinatensystemen wegtransformiert werden. Das Verschwinden des Drehmomentes $\left.T\right|_{O^{\prime}}$ ist gleichbedeutend mit

$$
\left(\begin{array}{ll}
F_{y} & -F_{x}
\end{array}\right)\left(\begin{array}{c}
a \\
b
\end{array}\right)+\left.T\right|_{O}=0
$$

was die Gleichung einer Geraden in der Ebene der Ortsvektoren ist, der Wirkungslinie der Gesamtkraft. Ein Ort, an dem $\left.\boldsymbol{d}\right|_{O^{\prime}}$ verschwindet, ist der Drehpol

$$
\left(\begin{array}{l}
a \\
b
\end{array}\right)=\frac{1}{\theta}\left(\begin{array}{c}
-d_{y} \\
d_{x}
\end{array}\right)
$$

der, als Aufpunkt zusammengenommen mit der Ebenennormale, wieder eine Gerade oder Linie im Raum festlegt, den Drehvektor. Auch zwischen zwei beliebigen Lagen in der Ebene kann eine endliche Drehung mit zugehörigem Pol angegeben werden, im Raum also wieder ein solcher Drehvektor.

Kraft und Drehvektor gehören im räumlichen Fall der Klasse der linienflüchtigen Vektoren an. Durch das Transformationsverhalten der freien Vektoren können bestimmte Bezugssysteme innerhalb der äquivalenten Lasten und Lagen ausgezeichnet werden.

Durch das Studium des Transformationsverhaltens der beteiligten Größen, insbesondere unter Wechsel des Koordinatensystems am starren Körper, kann also ein Matrixformalismus für $6 \times 6$-Matrizen zum Transformieren der Meßwerte gefunden werden. Dieser wird auch von anderen Autoren ${ }^{6}$ in ähnlicher Form verwendet. Damit war zwar noch nicht das Problem der mehrdeutigen Meßwerte gelöst, aber immerhin war nun eine Transformation auf jedes beliebige Koordinatensystem möglich.

Bei der Formänderungsarbeit hatte man translatorische und rotatorische Anteile. Auch bei den Matrixeinträgen hätte man gern eine Trennung in $\boldsymbol{F}$ und $\boldsymbol{d}$ auf der einen Seite, und $T$ und $\theta$ auf der anderen. Das unterscheidet polare und axiale Vektoren. Da insgesamt vier freie Parameter bei der Wahl der Bezugssysteme zur Verfügung stehen, können

\footnotetext{
${ }^{6}$ Vgl. etwa [58] oder [43].
} 
nun zwei Koordinatensysteme $\boldsymbol{w}_{f}$ und $\boldsymbol{w}_{q}$ bestimmt werden, in denen die abseits der Diagonalen stehenden Matrizen verschwinden. Für die Steifheitsmatrix mit den Komponenten

$$
\mathcal{S}=\left(\begin{array}{cc}
\boldsymbol{A} & \boldsymbol{b}_{2} \\
\boldsymbol{b}_{1}^{T} & c
\end{array}\right)
$$

gilt beispielsweise

$$
\begin{aligned}
\tilde{\mathcal{S}} & =\left(\begin{array}{cc}
\boldsymbol{I} & \mathbf{0} \\
\boldsymbol{w}_{f}^{T} & 1
\end{array}\right)\left(\begin{array}{cc}
\boldsymbol{A} & \boldsymbol{b}_{2} \\
\boldsymbol{b}_{1}^{T} & c
\end{array}\right)\left(\begin{array}{cc}
\boldsymbol{I} & \boldsymbol{w}_{q} \\
\mathbf{0}^{T} & 1
\end{array}\right) \\
& =\left(\begin{array}{cc}
\boldsymbol{I} & \mathbf{0} \\
\boldsymbol{w}_{f}^{T} & 1
\end{array}\right)\left(\begin{array}{cc}
\boldsymbol{A} & \boldsymbol{b}_{2}+\boldsymbol{A} \boldsymbol{w}_{q} \\
\boldsymbol{b}_{1}^{T} & c+\boldsymbol{b}_{1}^{T} \boldsymbol{w}_{q}
\end{array}\right) \\
& =\left(\begin{array}{cc}
\boldsymbol{A} & \boldsymbol{b}_{2}+\boldsymbol{A} \boldsymbol{w}_{q} \\
\boldsymbol{b}_{1}^{T}+\boldsymbol{w}_{f}^{T} \boldsymbol{A} & c+\boldsymbol{b}_{1}^{T} \boldsymbol{w}_{q}+\boldsymbol{w}_{f}^{T} \boldsymbol{b}_{2}+\boldsymbol{w}_{f}^{T} \boldsymbol{A} \boldsymbol{w}_{q}
\end{array}\right)
\end{aligned}
$$

Daher hat nach einer Transformation von $\mathcal{S}$ auf die Lösungen von $\boldsymbol{b}_{2}+$ $\boldsymbol{A} \boldsymbol{w}_{q}=\mathbf{0}$ und $\boldsymbol{b}_{1}+\boldsymbol{A}^{T} \boldsymbol{w}_{f}=\mathbf{0}$ die Steifheitsmatrix die gewünschte Gestalt

$$
\tilde{\mathcal{S}}=\left(\begin{array}{cc}
\boldsymbol{A} & \mathbf{0} \\
\mathbf{0}^{T} & c-\boldsymbol{b}_{1}^{T} \boldsymbol{A}^{-1} \boldsymbol{b}_{2}
\end{array}\right) \quad .
$$

Für eine symmetrische Ausgangsmatrix gilt $\boldsymbol{w}_{f}=\boldsymbol{w}_{q}$, und man spricht vom Widerstandszentrum $W$. Die elastischen Matrizen haben in diesem Widerstandszentrum Blockdiagonalgestalt.

Die Auswertevorschrift gründet sich auf einer Transformation, die mit Hilfe eines durch die Meßwerte selbst festgelegten Koordinatensystems Messungen an gleichen Meßobjekten überhaupt erst vergleichbar macht.

Den Ursprung dieses dem Schwerpunkt einer Massenverteilung analogen Koordinatensystems möchte ich das elastische Zentrum nennen. Seine im ersten Teil dieser Arbeit begründete Definition ist gleichzeitig ihre Hauptaussage. Dieses Objekt ist für alle durch eine symmetrische elastische Matrix beschreibbaren Aufhängungen wohldefiniert, also auch unabhängig von den Vorhersageversuchen für Steifheitsmatrizen des zweiten Teils dieser Arbeit.

Sind die zu untersuchenden elastischen Matrizen nicht symmetrisch, so werden durch die Meßwerte zwei Koordinatensysteme festgelegt. Eines ist der Bezugspunkt der Lasten und das andere das der Lagen. Das Bezugssystem der Lagen erhält man, indem reine Drehmomente angelegt werden, ich möchte es daher Drehmomentszentrum nennen. Im Bezugssystem der Lasten sucht man nach reinen Translationen, weshalb ich es Translationszentrum nennen möchte.

Der Versuch, die Abweichungen vom idealen Verhalten näher zu untersuchen, führt zurück auf ausgezeichnete Last- und Lagesysteme des Raumes, die Schraubachsen. 
Ihre geometrische Bedeutung erlaubt Visualisierungen: die Aufpunktfläche, die $A b$ standsfläche und die Steigungsfläche. Die abstrakte Menge aller der Schraubachsen, die für die Definition des elastischen Zentrum von Bedeutung waren, ist die Kongruenz der Schraubachsen. Bei elastischen Matrizen, die nicht durch eine Verschiebung ihrer Bezugspunkte symmetrisiert werden, verdoppelt sich teilweise die Anzahl der eben genannten Objekte, es existieren dann zwei ausgezeichnete Bezugssysteme.

Bei der dürftigen Quellenlage war es notwendig, eigene Begriffe für mathematisch sinnvolle und physikalisch brauchbare Objekte zu erfinden. Das elastische Zentrum, die Aufpunkt- und Abstandsfläche sowie die funktionellen Achsen und die Kongruenz der Schraubachsen sind solche Begriffe. Da ich viele Objekte aus den Randbereichen der Physik oder Mathematik, wie etwa der Kinematik, als für meine Zwecke nützlich befand, tauchen außer den eigenen Wortschöpfungen in dieser Arbeit noch weitere für Physiker ungebräuchliche Begriffe auf. Ich habe mich bemüht, an der Stelle ihres ersten Auftauchens eine Quelle zu nennen, der dann alle nötigen Informationen für ein tieferes Verständnis zu entnehmen sind - oder eben klarzustellen, daß es sich um einen von mir eingeführten Begriff handelt.

Ein Koordinatensystem als Referenz besteht aus einem Ursprung und einer Orientierung. Die Untersuchung weiterer Eigenschaften des elastischen Zentrums führt, wieder analog zum starren Körper, zu einer Definition der funktionellen Achsen. Hierbei sind die vorangegangenen Erkenntnisse über die Schraubachsen hilfreich.

Durch Drehungen der Koordinatensysteme der Lasten und Lagen mit den Drehmatrizen $\boldsymbol{R}_{f}$ und $\boldsymbol{R}_{q}$ um die $z$-Achse läßt sich die Matrix $\boldsymbol{A}$ auf Diagonalgestalt $\boldsymbol{R}_{f}^{T} \boldsymbol{A} \boldsymbol{R}_{q}=\operatorname{diag}\left(A_{i}\right)$ transformieren. $A_{1}$ und $A_{2}$ sind dann die singulären Werte von $\boldsymbol{A}$. Diese Werte können der Größe nach angeordnet werden, ohne die Händigkeit des Koordinatensystems zu ändern. Damit sind die elastischen Matrizen auf ihre Normalform gebracht, etwa

$$
\mathcal{S}_{N}=\left(\begin{array}{ccc}
A_{1} & 0 & 0 \\
0 & A_{2} & 0 \\
0 & 0 & \tilde{c}
\end{array}\right)
$$

Erst in dieser Normalform sind die elastischen Matrizen miteinander vergleichbar. Da die Formänderungsarbeit immer positiv ist, sind die Koeffizienten $A_{1}, A_{2}$ und $\tilde{c}$ immer $\geq 0$. Für symmetrische Ausgangsmatrizen ist die Zerlegung in die singulären Werte genau die Hauptachsentransformation auf die Eigenwerte.

Nach einer Drehung der Koordinatenachsen auf diese funktionellen Achsen werden die 36 Zahlen auf eine Normalform transformiert, die dann erstmalig die Vergleichbarkeit von Meßwerten erlaubt. Diese Normalform eignet sich auch für theoretische Zwecke zum Studium der Eigenschaften der elastischen Aufhängung. 
Für das zweidimensionale Problem ist die allgemeine Last einer einzelnen Kraft äquivalent. Sie kann beschrieben werden durch das Lot $\boldsymbol{d}$ vom Widerstandszentrum zur Kraftwirkungslinie mit Länge $d$ und Richtung $\boldsymbol{r}$ sowie der Stärke $F$ der Kraft, vgl. Abb. 1.3. Liegt $\mathcal{S}$ in Normalform vor, können $d, \boldsymbol{r}=\left(\begin{array}{l}r_{1} \\ r_{2}\end{array}\right)$ und der Drehpol $\boldsymbol{p}$ in Beziehung gesetzt werden.

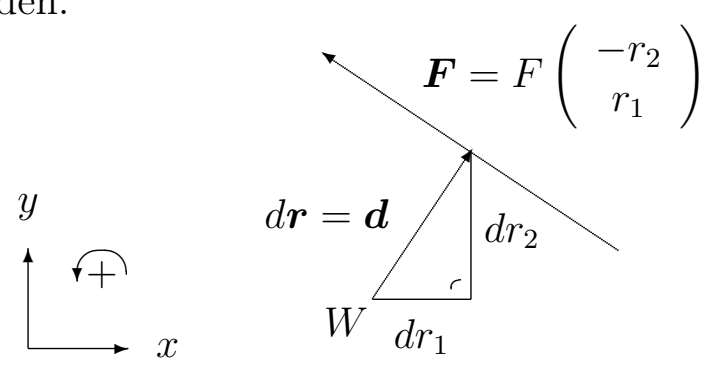

Abbildung 1.3: Die allgemeine ebene Last.

Die Last

$$
f=F\left(\begin{array}{c}
-r_{2} \\
r_{1} \\
d
\end{array}\right)
$$

ruft die Lageänderung $\mathcal{S}_{N} f$ mit den Komponenten

$$
q=F\left(\begin{array}{c}
-A_{1} r_{2} \\
A_{2} r_{1} \\
\tilde{c} d
\end{array}\right)
$$

hervor. Für den Pol $\boldsymbol{p}$ folgt der Ausdruck

$$
\boldsymbol{p}=-\frac{1}{\tilde{c} d}\left(\begin{array}{c}
A_{2} r_{1} \\
A_{1} r_{2}
\end{array}\right)
$$

welcher nicht mehr von der Stärke der Kraft abhängt. Weist $\boldsymbol{r}$ nun in eine Eigenrichtung von $\boldsymbol{A}$, so gilt entweder $r_{1}=0$ oder $r_{2}=0$, und dann liegen sich $\boldsymbol{d}$ und $\boldsymbol{p}$ bezüglich $W$ genau gegenüber. Die Lage des Pols ist dann durch eine skalare Funktion $p=|\boldsymbol{p}|$ beschreibbar. Diese Funktion $p(d)$ ist dann eine Hyperbel mit der Gleichung

$$
p_{1,2} \cdot d_{1,2}=-\frac{A_{2,1}}{\tilde{c}} .
$$

Dieser Zusammenhang ist das Reziprozitätstheorem der Synarthrosen [48]. Für eine allgemeine symmetrische Ausgangsmatrix $\boldsymbol{A}$ gilt es nur für deren Eigenrichtungen, da nur für diese $r_{1}$ oder $r_{2}$ verschwindet. Abseits der Eigenrichtungen muß wieder vektoriell gerechnet werden. Man erhält das Skalarprodukt

$$
\boldsymbol{d} \cdot \boldsymbol{p}=\left(\begin{array}{ll}
r_{1} & r_{2}
\end{array}\right)\left(\begin{array}{cc}
-A_{2} / \tilde{c} & 0 \\
0 & -A_{1} / \tilde{c}
\end{array}\right)\left(\begin{array}{l}
r_{1} \\
r_{2}
\end{array}\right),
$$


welches nicht mehr von dem Abstand der Kraftwirkungslinie zum Ursprung sondern nur von dessen Richtung abhängt. Setzt man entsprechend $r_{1}=\cos (\alpha)$ und $r_{2}=\sin (\alpha)$, so folgt

$$
\boldsymbol{d} \cdot \boldsymbol{p}=\frac{1}{\tilde{c}}\left(A_{2} \cos ^{2} \alpha+A_{1} \sin ^{2} \alpha\right),
$$

also ist $\boldsymbol{d} \cdot \boldsymbol{p}=$ const die Gleichung einer Schar konform ähnlicher Ellipsen.

Das Reziprozitätstheorem beispielsweise, eine der ursprünglichen Aufgabestellungen, ist in drei Dimensionen nur noch unter sehr speziellen Umständen gültig.

Im nächsten Kapitel 3 dieser Arbeit wird auf die Effekte realer Messungen eingegangen, die mit einer einzelnen elastischen Matrix nicht beschrieben werden können. Die störenden Einflüsse werden dabei nur im Hinblick auf eine weitgehende Ausschaltung diskutiert. Da Messungen immer die Zeit beinhalten, geht es zunächst um Grundzüge der Dynamik einer elastischen Aufhängung. Die Gleichungen des bis dahin entwickelten Formalismus lassen sich in den Kontext der linearen Viskoelastizität einbinden. Nach der Festlegung auf eine für das Problem angemessene Zustandsgleichung werden statistische Verfahren erörtert. Als Nächstes werden apparative Effekte diskutiert - einige lassen sich prinzipiell nachträglich herausrechnen. Einen besonders interessanten systematischen Effekt fand ich in [43]: Es handelt sich um den Einfluß einer raumfesten Vorlast, die bei kleinen Auslenkungen, die ja auch Transformationen des Koordinatensystems sind, in linearer Näherung wie eine Laständerung wirkt. Dieser Effekt hat den Nachteil, daß er in der linearen Näherung nicht von anderen unterschieden und damit nicht herausgerechnet werden kann.

Erst Vorhersagen über Meßergebnisse erlauben ihr Verständnis. Ziel des nun folgenden Kapitels 4 und zweiten Hauptteiles dieser Arbeit ist eine Theorie der Struktur der Steifheitsmatrix von Aufhängungen aus dünnen elastischen Schichten. Für Synarthrosen ist eine solche Beschreibung angemessen.

Eine zweidimensionale Synarthrose ist eine Parallelschaltung von kleinen Federn, die zwischen zwei Linien angeordnet sind. Anfangs- und Endpunkt der Feder definieren ein lokales Koordinatensystem, in dem die i-te elastische Matrix $\hat{\mathcal{S}}_{i}$ Diagonalgestalt hat. Alle Matrizen werden im globalen Koordinatensystem ausgedrückt oder auf dieses gedreht

$$
\tilde{\mathcal{S}}_{i}=\left(\begin{array}{cc}
\boldsymbol{R}_{i}^{T} & \mathbf{0} \\
\mathbf{0}^{T} & 1
\end{array}\right) \hat{\mathcal{S}}_{i}\left(\begin{array}{cc}
\boldsymbol{R}_{i} & \mathbf{0} \\
\mathbf{0}^{T} & 1
\end{array}\right)
$$

und auf dessen Ursprung transformiert

$$
\mathcal{S}_{i}=\left(\mathcal{T}_{f}\right)_{i} \tilde{\mathcal{S}}_{i}\left(\mathcal{T}_{q}\right)_{i}
$$


für komplexere Anordnungen sind sie dann wieder voll besetzt. Da sich die Federkräfte addieren, können die einzelnen Steifheitsmatrizen zur Gesamtsteifheitsmatrix summiert werden

$$
\mathcal{S}=\sum_{i} \mathcal{S}_{i}
$$

Bei einer kontinuierlichen Anordnung aus Federn geht diese Summe in ein Linienintegral über.

Wie in der Technischen Mechanik üblich, werden dabei einige vereinfachende aber erprobte und plausible Annahmen verwendet; zusätzlich werden Elemente der Darstellungstheorie [14] aus der Kontinuumsmechanik eingesetzt. Insbesondere erlaubt die dünne elastische Schicht grundlegende Vereinfachungen. Diese Theorie möchte ich das Federmodell nennen.

Es ist sinnvoll, die die Synarthrose begrenzende Kurve zu parametrisieren, etwa $(x(t), y(t))$. Besonders einfach werden die Verhältnisse durch eine Parametrisierung in der Bogenlänge $s$. Der Tangentenvektor $\boldsymbol{t}(s)$ und der Normalenvektor $\boldsymbol{n}(s)$ lauten nun

$$
\boldsymbol{t}=(\dot{\boldsymbol{x}}, \dot{\boldsymbol{y}}) \quad \text { und } \quad \boldsymbol{n}=(-\dot{\boldsymbol{y}}, \dot{\boldsymbol{x}})
$$

Durch die Eigenschaft der Synarthrose, dünn zu sein, wird für ein isotropes Medium eine Richtung ausgezeichnet, wahlweise durch $\boldsymbol{t}$ oder durch $\boldsymbol{n}$.

Eine dünne elastische Schicht wird als Fläche mit der Oberflächennormale als ausgezeichneter Richtung beschrieben. Dadurch lassen sich die elastischen Matrizen der einzelnen Flächenelemente im globalen Koordinatensystem ausdrücken.

Die Teilmatrix $\tilde{\mathcal{S}}_{i}$ bzw. $\tilde{\mathcal{S}}(s)$ habe die Struktur $\tilde{\mathcal{S}}=\left(\begin{array}{cc}\boldsymbol{A} & \mathbf{0} \\ \mathbf{0} & c\end{array}\right)$. Ist nun der Normalenvektor die einzige ausgezeichnete Richtung, so läßt sich $\boldsymbol{A}$ in der Form

$$
\boldsymbol{A}=a_{1} \boldsymbol{n n ^ { T }}+a_{2} \boldsymbol{I}
$$

mit den zwei von der Linie und den elastischen Konstanten des Mediums abhängenden Größen $a_{1}$ und $a_{2}$ ansetzen.

Aufgrund der differentialgeometrischen Beschreibung ist das Problem lokal eben, die einzige ausgezeichnete Richtung einer isotropen Schicht ist folglich die Ebenennormale. Im Raum kann daher ein kleines Flächenelement durch zwei Schichtgrößen und den Normalenvektor der Schicht beschrieben werden. Diese Schichtgrößen modellieren das statische Verhalten senkrecht zur und innerhalb der Schicht. 
Mit dem Vektor $\boldsymbol{n}$ ist die Matrix $\boldsymbol{A}(s)$ nun gegeben durch

$$
\boldsymbol{A}(s)=a_{1}(s)\left(\begin{array}{cc}
\dot{y}^{2} & \dot{y} \dot{x} \\
\dot{y} \dot{x} & \dot{x}^{2}
\end{array}\right)+a_{2}(s)\left(\begin{array}{cc}
1 & 0 \\
0 & 1
\end{array}\right),
$$

der Skalar $c(s)$, auch eine Funktion der Kurvenform und der elastischen Konstanten, bleibt von dieser Näherung unberührt. Zusammen mit den Transformationsmatrizen

$$
\mathcal{T}_{f}(s)=\left(\begin{array}{ccc}
1 & 0 & 0 \\
0 & 1 & 0 \\
-y & x & 1
\end{array}\right) \quad \text { und } \quad \mathcal{T}_{q}(s)=\left(\begin{array}{ccc}
1 & 0 & -y \\
0 & 1 & x \\
0 & 0 & 1
\end{array}\right)
$$

ergibt sich die gesamte Steifheitsmatrix zu

$$
\mathcal{S}=\int \mathcal{T}_{f}(s) \mathcal{S}(s) \mathcal{T}_{q}(s) d s
$$

mit den Komponenten

$$
\begin{aligned}
\mathcal{S}= & \int a_{1}(s)\left(\begin{array}{ccc}
\dot{y}^{2} & -\dot{y} \dot{x} & -\dot{y}(y \dot{y}+x \dot{x}) \\
-\dot{y} \dot{x} & \dot{x}^{2} & \dot{x}(y \dot{y}+x \dot{x}) \\
-\dot{y}(y \dot{y}+x \dot{x}) & \dot{x}(y \dot{y}+x \dot{x}) & (y \dot{y}+x \dot{x})^{2}
\end{array}\right) d s \\
& +\int a_{2}(s)\left(\begin{array}{ccc}
1 & 0 & -y \\
0 & 1 & x \\
-y & x & 1
\end{array}\right) d s+\int c(s)\left(\begin{array}{ccc}
0 & 0 & 0 \\
0 & 0 & 0 \\
0 & 0 & 1
\end{array}\right) d s
\end{aligned}
$$

Für genügend einfache Aufhängungen lassen sich diese Integrationen geschlossen ausführen, womit die zweidimensionale Synarthrose analytisch beschrieben ist.

Mit einfachen Annahmen über das Verhalten eines kleinen Elementes der Berandung kann schließlich die Struktur der Steifheitsmatrix einer beliebigen dünnen elastischen Berandung als Flächenintegral abgeleitet werden. Diese Struktur reicht aus für allgemeine qualitative Aussagen über die Gesamtaufhängung, etwa über die Existenz eines Widerstandszentrums.

Mit Hilfe von Ähnlichkeitsbetrachtungen werden die Schichtkonstanten auf die elastischen Konstanten des Materials und die Schichtdicke reduziert; noch sind sie aber quantitativ unbestimmt. Mit einem plausiblen Ansatz über die lokale Deformation im Inneren der dünnen elastischen Schicht kann diese Unbestimmtheit mit den Mitteln der Elastizitätstheorie beseitigt werden. Die elastischen Konstanten werden dadurch auf die elastischen Konstanten des Mediums und die Schichtdicke zurückgeführt.

Im darauf folgenden Kapitel 5 wird an einigen Beispielen vorgeführt, wie und mit welchen Methoden solche Aufhängungen konkret zu berechnen sind. Entweder 
analytisch, unter Zuhilfenahme eines Computeralgebrasystems oder numerisch lassen sich dann diverse Aufhängungen berechnen. Bei einer Hintereinanderschaltung von solchen Aufhängungen addieren sich deren Lageänderungen unter Last. Die Flexibilitätsmatrix der gesamten Aufhängung ist daher die Summe der einzelnen Flexibilitätsmatrizen. Damit lassen sich auch zusammengesetzte Aufhängungen, wie etwa die Wirbelsäule oder ein mehrwurzeliger Zahn, beschreiben.

Im letzten Teil dieser Arbeit werden die Erkenntnisse aus den vorherigen Hauptteilen auf konkrete Meßergebnisse oder konkurrierende Theorien angewendet. An diesen Beispielen, die für Praktiker von vorrangiger Bedeutung sind, wird die Leistungsfähigkeit des Formalismus demonstriert. Dabei kommen zwei Auswertungen zum Einsatz: Eine direkt ohne Informationsverlust durch reine Anwendung der Transformationen auf das elastische Zentrum und auf die funktionellen Achsen. Dadurch entsteht eine auch auf reale Meßwerte anwendbare Normalform der Steifheits- bzw. Flexibilitätsmatrix. Die andere Auswertung kann nur unter Zuhilfenahme von plausiblen Annahmen über die Haupteffekte durchgeführt werden: über Abspaltung von Vorlasten, Drehungen bzw. Polarzerlegungen bis schließlich hin zu gewöhnlichen Mittelungen. Dabei geht zwar Information verloren, aber die Matrizen bekommen so erst ihre theoriekonforme symmetrische oder gar diagonale Gestalt, die für den Vergleich mit den Vorhersagen nötig ist. Diese Art der Auswertung soll nur für schon zu Anfang bereits gut zur Theorie passende Matrizen durchgeführt werden.

Im Anhang finden sich neben der Notation einige Ergebnisse die abseits der Hauptlinie dieser Arbeit liegen aber dennoch interessant sind. Sie sollen das Gesamtbild durch Aufzeigen von möglichen Erweiterungen dieser Theorie abrunden. 


\section{Kapitel 2}

\section{Statik elastischer Aufhängungen}

In diesem Teil der Arbeit geht es um die Einführung der Grundbegriffe. Die Vektoren von Kraft und Drehmoment werden zur Last zusammengefaßt und die von Translation und Rotation zur Lage. Danach wird das Transformationsverhalten dieser Objekte studiert, was auf die Begriffe der äquivalenten Last- bzw. Lagesysteme führt. Diese Mehrdeutigkeit bei der Wahl von Koordinatensystemen kann durch die Einführung von Schraubachsen beseitigt werden. Diese haben den Vorteil einer gewissen Anschaulichkeit und den Nachteil, nichtlinear zu sein. Da gleiche Messungen aber mit gleichen Zahlen beschrieben werden sollten, wird diese Abhängigkeit genauer untersucht.

Die interessierenden Meßgrößen sind die Koeffizienten der linearisierten Abhängigkeit zwischen Lasten und Lagen, Flexibilitäts- und Steifheitsmatrix. Diese sind, ebenso wie die Lasten und Lagen selber, abhängig von dem im Experiment gewählten Koordinatensystem. Für den konkreten Vergleich von Meßwerten muß also eine Vorschrift gefunden werden, welche die Meßwerte zum gleichen Objekt ineinander überführt - unabhängig von der vorherigen Durchführung des Experimentes. Für den Ursprung gelingt das mit der Einführung von zwei aus den Meßwerten selbst heraus festgelegten Orten, dem Drehmoments- und Translationszentrum. Streng genommen sind diese Definitionen nur korrekt für HookEsche Verhältnisse, also bei Existenz eines elastischen Potentials. Da aber Hookesche Anteile etwa im Sinne der linearen Viskoelastizität [19] klar definiert sind, sind die Resultate durchaus von Belang.

Dabei zeigt sich, daß die ursprünglich angenommene Existenz eines Widerstandszentrums, ein Ort an dem sich die elastische Aufhängung wie eine Punktmasse benimmt, in drei Dimensionen nicht allgemein gegeben ist.

Der Versuch, einzelne Eigenschaften dieser Wunschvorstellung für die neuen Definitionen zu präzisieren, führt über eine abermalige Benutzung der Schraubachsen zu den Begriffen der Aufpunktfläche, der Abstandsfläche und der Steigungsfläche. 
Die Gesamtheit der Schraubachsen läßt sich als Kongruenz beschreiben.

Nach der Translation auf das Widerstandszentrum können die verbleibenden Freiheitsgrade der Rotation dazu benutzt werden, analog zu den Hauptträgheitsachsen eines starren Körpers, Richtungen bzw. Achsen im Raum auszuzeichnen. Das führt zu einer Normalform der Meßwerte, wieder in Analogie zum Trägheitstensor in Diagonalgestalt, die erstmals eine Vergleichbarkeit von Messungen an verschiedenen Apparaturen oder Meßobjekten erlaubt.

\subsection{Grundbegriffe}

Es wird ein Zusammenhang gesucht zwischen Kraft $\boldsymbol{F}$ und Drehmoment $\boldsymbol{T}$ auf der einen Seite, und Translation $\boldsymbol{d}$ und Rotation $\boldsymbol{\theta}$ auf der anderen Seite. Diese vier Vektoren möchte ich im Folgenden die grundlegenden Vektoren nennen. Obwohl sie im Experiment teilweise aus der Messung zu bestimmen sind, haben sie in dieser Theorie den Charakter von Variablen. Im Gegensatz zu den aus der linearen Algebra bekannten Vektoren hängen diese Größen unterschiedlich von der Wahl des Ursprungs und der Händigkeit des verwendeten Koordinatensystems ab. Das führt zu den Begriffen der axialen und polaren sowie der linienflüchtigen und freien Vektoren.

\subsubsection{Vektoren und Symmetrieoperationen}

Als Erstes soll das Transformationsverhalten der grundlegenden Vektoren studiert werden. Bei der Betrachtung des starren Körpers kommt zu der üblicherweise untersuchten Punktspiegelung als zusätzliche Transformation der Wechsel des Bezugspunktes hinzu.

\subsubsection{Punktspiegelungen, polare und axiale Vektoren}

Bei einer Punktspiegelung am Ursprung ändert sich die Händigkeit des Koordinatensystems. Man unterscheidet polare und axiale Vektoren, je nachdem, ob das einen Einfluß auf das Vorzeichen des Vektors hat. Die grundlegenden Vektoren lassen sich nach ihrem Verhalten unter Punktspiegelungen wie folgt einordnen:

\begin{tabular}{l|l|l} 
Typ des Vektors & Vorzeichen & Beispiele \\
\hline \hline polar & ändert sich & Kraft $\boldsymbol{F}$, Translation $\boldsymbol{d}$ \\
\hline axial & ändert sich nicht & Drehmoment $\boldsymbol{T}$, Rotation $\boldsymbol{\theta}$
\end{tabular}


Durch die Darstellung von polaren Vektoren als Pfeile und axialen Vektoren als, hier perspektivisch dargestellte, Kreisbögen mit Umlaufsinn ergibt sich bezüglich des hier als Punkt dargestellten Symmetriezentrums die Visualisierung

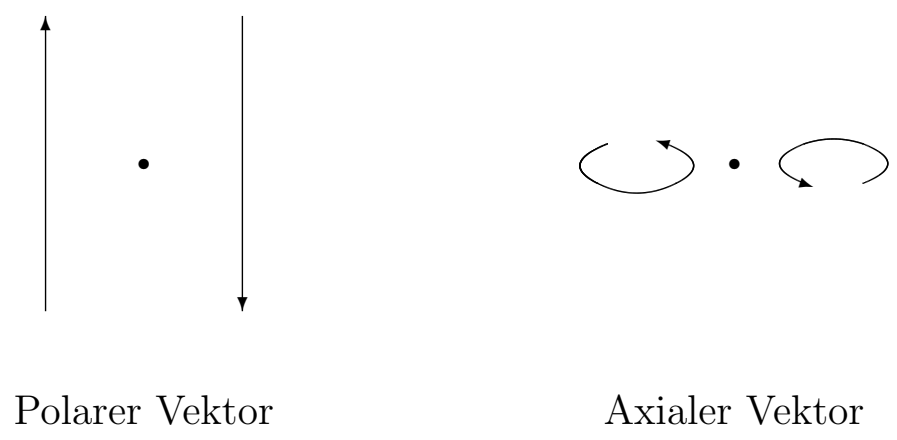

Da axiale Vektoren praktisch nur gemeinsam mit Kreuzprodukten auftauchen, lassen sie sich gut in Form von antisymmetrischen Matrizen handhaben. Dadurch wird aus dem Vektorprodukt eine lineare Abbildung des $\mathbb{R}^{3}$. So ist diese zum Vektor $\boldsymbol{x}$ gehörende Abbildung $\boldsymbol{\Omega}_{x}:=(\boldsymbol{x} \times)$ in Komponenten gegeben durch ${ }^{2}$

$$
\left(\boldsymbol{\Omega}_{x}\right)_{i j}:=\sum_{k=1}^{3} \boldsymbol{\varepsilon}_{i k j}(\boldsymbol{x})_{k},
$$

bzw. als Matrix mit $\boldsymbol{x}=(x, y, z)^{T}$

$$
\boldsymbol{\Omega}_{x}=\left(\begin{array}{ccc}
0 & -z & y \\
z & 0 & -x \\
-y & x & 0
\end{array}\right)
$$

Mehr über dieses Thema findet sich im Anhang A.2.2.

\subsubsection{Wechsel des Bezugspunktes, freie und linienflüchtige Vektoren}

Der starre Körper läßt jeden seiner körpereigenen Punkte und darüber hinaus jeden als starr verbunden gedachten Punkt als Ursprung des Koordinatensystems gleichberechtigt zu. Ein solcher Wechsel beeinflußt die Komponenten der Vektoren unterschiedlich. Man unterscheidet ferner die passive Transformation, bei der nur der Koordinatenursprung verschoben wird, und die aktive Transformation, bei der tatsächlich der Vektor verschoben wird. Es ergeben sich folgende Typen von Vektoren:

\footnotetext{
${ }^{1}$ Beispielsweise wird das Kreuzprodukt zweier polarer Vektoren durch eine Punktspiegelung abgebildet auf den Vektor „(-polar $) \times($-polar $) “=$ „polar $\times$ polar"; er ist also immer ein axialer Vektor. Als Faustregel kann in der Mechanik gelten, daß alle Vektoren, die mit Drehungen zu tun haben, axial sind.

${ }^{2}$ Ich möchte, von dieser Formel abgesehen, im Folgenden die Summationskonvention verwenden, bei der über doppelt vorkommende Indizes summiert wird.
} 
Ortsfest: Ein ortsfester Vektor $\boldsymbol{o}$ ist eine Zuordnung von einem Punkt $P$ bezüglich des Ursprungs $O$ zu einem Vektor $\overrightarrow{O P}$. Dabei nennt man $O$ auch den Anfangspunkt, und $P$ den Endpunkt des Vektors. Ein Wechsel des Koordinatenursprungs $O$ nach $O^{\prime}$ ändert die Koordinaten eines Aufpunktes $P$ dann von $\overrightarrow{O A}$ nach

$$
\overrightarrow{O^{\prime} P}=\overrightarrow{O^{\prime} O}+\overrightarrow{O P}
$$

er transformiert sich additiv mit der Differenz der Koordinatenursprünge.

Linienflüchtig: Längs einer Geraden im Raum kann der linienflüchtige Vektor $\boldsymbol{l}$ aktiv verschoben werden, ohne daß sich an der Gleichgewichtssituation etwas ändert. Beispielsweise beschreiben Drehvektoren $\boldsymbol{\theta}$, die auf einer gemeinsamen Geraden im Raum liegen und denselben Betrag und Richtung haben, dieselbe Drehung. Kräfte $\boldsymbol{F}$ dürfen längs ihrer Wirkungslinie verschoben werden ${ }^{3}$ Hierauf basiert die bekannte graphische Methode der Addition von ebenen Kraftsystemen, bei der erst die Kräfte in den gemeinsamen Schnittpunkt der Kraftwirkungslinien verschoben werden, um dann mit der Parallelogrammkonstruktion zur Gesamtkraft mit entsprechender Wirkungslinie addiert zu werden. Unter einer passiven Verschiebung des Koordinatenursprunges hängen die Komponenten eines linienflüchtigen Vektors nicht von der Wahl des Bezugspunktes ab; etwa lautet die Bedingung für das Gleichgewicht von Kräften in allen Bezugssystemen $\sum \boldsymbol{F}=\mathbf{0}$. Eine Drehung um den Ursprung, beschrieben durch die Drehmatrix $\boldsymbol{R}$ mit $\overrightarrow{O Q}=\boldsymbol{R} \overrightarrow{O P}$, ändert bei einem Wechsel von $O$ nach $O^{\prime}$ wegen $\overrightarrow{O O^{\prime}}+\overrightarrow{O^{\prime} Q}=\boldsymbol{R}\left(\overrightarrow{O O^{\prime}}+\overrightarrow{O^{\prime} P}\right)$ nicht den Drehanteil, denn es kommt nur eine zusätzliche Translation hinzu: $\overrightarrow{O^{\prime} Q}=\boldsymbol{R} \overrightarrow{O^{\prime} P}+(\boldsymbol{R}-\boldsymbol{I}) \overrightarrow{O O^{\prime}} ; \boldsymbol{I}$ ist hierbei die Einheitsmatrix. Die Transformationsgleichung

$$
\begin{aligned}
\boldsymbol{l}(O) & =\boldsymbol{l}\left(O^{\prime}\right) \\
\text { „linienflüchtig }(O) & =\operatorname{linienflüchtig}\left(O^{\prime}\right)^{“},
\end{aligned}
$$

bei der die Koordinaten Funktionen der Koordinatenursprünge sind, faßt also $\boldsymbol{F}_{O}=\boldsymbol{F}_{O^{\prime}}$ und $\boldsymbol{\theta}_{O}=\boldsymbol{\theta}_{O^{\prime}}$ zusammen. Die Indizes können demnach bei linienflüchtigen Vektoren weggelassen werden. Die Änderung der Situation unter aktiver Transformation der zugehörigen Linie, also einer Geraden im Raum, kann daher nicht an den linienflüchtigen Vektoren selber liegen. Die

\footnotetext{
${ }^{3}$ Die Verschiebung einer Kraft längs ihrer Wirkungslinie kann bei einem zwangsgeführten starren Körper die Stabilität verändern, und zwar, etwa bei einer ebenen Bewegung, abhängig von der Lage des Kraftangriffspunktes zum momentanen Drehpol und zum Krümmkreismittelpunkt der Polbahn. Selbst bei einem freien starren Körper im Raum macht es einen Unterschied, ob die Kraft vor oder hinter dem Schwerpunkt (gemessen in Richtung der Bewegung) angreift. Denkt man sich diesen Angriffspunkt am Körper befestigt, so erzeugt beim Kraftangriff vor dem Schwerpunkt eine Störung zusammen mit den Trägheitskräften im Schwerpunkt ein rücktreibendes Drehmoment. Erfolgt der Kraftangriff hinter dem Schwerpunkt, verstärkt das Drehmoment die Störung, und das Gleichgewicht wird instabil.
} 
die Situation verändernden Vektoren sind die im Folgenden definierten freien Vektoren.

Frei: Eine aktive Verschiebung eines freien Vektors $\boldsymbol{f}$ ändert die Situation nicht 4 Anderenfalls hängt er von seinem linienflüchtigen Partner ab, insbesondere von dessen ortsfesten Bezugspunkt; ein freier Vektor ist also im Grunde ein Vektorfeld. Der einfachste freie Vektor ist eine Translation $\overrightarrow{P Q}$, die als Differenzvektor wegen $\overrightarrow{P Q}=\overrightarrow{O Q}-\overrightarrow{O P}$ in allen Koordinatensystemen gleicher Orientierung dieselben Komponenten hat. Das im Punkt $O$ zu einer festen Kraft $\boldsymbol{F}_{O^{\prime}}$ gehörende Drehmoment im Punkt $O^{\prime}$ ist nach dem Hebelgesetz gegeben durch $\boldsymbol{T}_{O^{\prime}}=\boldsymbol{T}_{O}+\overrightarrow{O^{\prime} O} \times \boldsymbol{F}_{O}$ bzw. $\boldsymbol{T}_{O^{\prime}}=\boldsymbol{T}_{O}+\overrightarrow{O^{\prime} O} \times \boldsymbol{F}$, da der Index an der Kraft weggelassen werden kann. Die mit einer kleinen Drehung $\delta \boldsymbol{\theta}$ (auch hier kann der Index wegen $\boldsymbol{\theta}_{O}=\boldsymbol{\theta}_{O^{\prime}}$ weggelassen werden) verbundene Translation im Ort $O^{\prime}$ ist gegeben durch $\delta \boldsymbol{\theta} \times \overrightarrow{O O^{\prime}}$, also gilt insgesamt $\delta \boldsymbol{d}_{O^{\prime}}=\delta \boldsymbol{d}_{O}+\delta \boldsymbol{\theta} \times \overrightarrow{O O^{\prime}}=\delta \boldsymbol{d}_{O}+\overrightarrow{O O^{\prime}} \times \delta \boldsymbol{\theta}$. Die Transformationsgleichung der freien und linienflüchtigen Vektoren zu verschiedenen Aufpunkten $O$ und $O^{\prime}$ lautet, da $\overrightarrow{O O^{\prime}}$ ein ortsfester Vektor ist

$$
\begin{aligned}
\boldsymbol{l}(O) & =\boldsymbol{l}\left(O^{\prime}\right) \\
\text { „linienflüchtig }(O) & =\text { linienflüchtig }\left(O^{\prime}\right)^{“} \\
\boldsymbol{f}(O) & =\boldsymbol{f}\left(O^{\prime}\right)+\overrightarrow{O^{\prime}} \times \boldsymbol{l} \\
& =\boldsymbol{f}\left(O^{\prime}\right)+\boldsymbol{\Omega}_{O O^{\prime}} \boldsymbol{l} \\
\text { „frei }(O) & =\text { frei }\left(O^{\prime}\right)+\text { ortsfest } \times \text { linienflüchtig“ },
\end{aligned}
$$

und verknüpft alle drei in diesem Abschnitt besprochenen Vektoren. Dieses Transformationsverhalten kann als Definition der freien und linienflüchtigen Partner betrachtet werden: Ein Vektor und dessen Partner gehören dann und nur dann zusammen, wenn sie sich nach obigen Gesetzen transformieren.

\begin{tabular}{lr|l|l|l}
\multicolumn{2}{l|}{ Typ des Vektors } & aktive Trsf. & passive Trsf. & Beispiele \\
\hline \hline ortsfest & $\boldsymbol{o}$ & Ä. d. Endpkt. & Ä. d. Anfangspkt. & Ortsvektor $\overrightarrow{O P}$ \\
\hline linienflüchtig & $\boldsymbol{l}$ & k. ̈̈. (Linie) & k. Änderung & $\boldsymbol{F}, \boldsymbol{\theta}$ \\
\hline frei & $\boldsymbol{f}$ & k. ̈̈. (Raum) & $\boldsymbol{f}^{\prime}=\boldsymbol{f}+\boldsymbol{o} \times \boldsymbol{l}$ & $\boldsymbol{T}, \boldsymbol{d}$
\end{tabular}

\footnotetext{
${ }^{4}$ Für Translationen ist das klar. Für Drehmomente gibt es dazu ein bekanntes Experiment, bei dem eine auf einem Luftkissen gelagerte Scheibe mit zirkular polarisiertem Licht bestrahlt wird: Die resultierende Bewegung der Scheibe hängt nicht von dem Auftreffpunkt der Strahlung ab. Ein anderes Beispiel aus dem Alltag ist das Anfahren von Autos: Sowohl front-, heck- oder allradgetriebene Fahrzeuge gehen dabei gleichermaßen hinten „in die Federn“ und kommen vorne hoch.
} 


\subsubsection{Vergleich der Symmetrieoperationen}

Ortsvektoren sind immer polar. Durch Angabe eines Eigenschaftspaares läßt sich jeder Grundvektor eindeutig festlegen:

\begin{tabular}{c||c|c} 
& linienflüchtig & frei \\
\hline \hline polar & Kraft & Translation \\
\hline axial & Rotation & Drehmoment
\end{tabular}

$\mathrm{Zu}$ einem Paar von Grundvektoren, jeweils einer aus den Lasten und der andere aus den Lagen, läßt sich genau eine gemeinsame charakteristische Eigenschaft finden:

\begin{tabular}{c||c|c} 
& Translation & Rotation \\
\hline \hline Kraft & polar & linienflüchtig \\
\hline Drehmoment & frei & axial
\end{tabular}

Dies entspricht genau der von A. Sommerfeld in seinem Lehrbuch ([60], §23 4) konstatierten „kreuzweisen Reziprozität zwischen Statik und Kinematik“. Allerdings taucht dort das Problem der endlichen Drehungen nicht auf, da von vornherein nur Geschwindigkeiten diskutiert werden.

Entgegengesetzt gleich große linienflüchtige Vektoren mit unterschiedlichen Aufpunkten nennt man Paare. Sie ergeben reine freie Vektoren, d. h. der linienflüchtige Partner verschwindet. In der Statik benutzt man den Begriff des Kräftepaares zur Definition eines reinen Drehmomentes, da sich die Kräfte genau aufheben. Ebenso kann man, wie wieder in [60] bemerkt, in der Kinematik den Begriff des Drehpaares zur Definition einer reinen infinitesimalen Translation verwenden, in Analogie zur Vorgehensweise beim reinen Drehmoment.

Das reine Drehmoment ist zwar ein freier Vektor und kann irgendwo am starren Körper angreifen ohne die Wirkung auf den Körper zu verändern. Dabei ist aber zu vermeiden, daß durch die Art der Aufbringung dieser Last dem Meßobjekt eine Drehachse, also ein linienflüchtiger Vektor, aufgezwungen wird. Im Experiment haben sich zur Vermeidung dieses Problems durch Fäden übertragene Paare von Zugkräften bewährt [3, 4].

Die Abhängigkeit der freien Vektoren vom gewählten Bezugspunkt wird im Folgenden dazu verwandt, um spezielle Punkte auszuzeichnen. 


\subsubsection{Verallgemeinerte Koordinaten und Schrauben}

In diesem Abschnitt sollen die die Lage des starren Körpers beschreibenden Koordinaten genauer untersucht werden. Dabei werden zum einen die verallgemeinerten Koordinaten aus der theoretischen Mechanik, und zum anderen der mathematische Formalismus der räumlichen Kinematik, hier auf infinitesimale Bewegungen eingeschränkt, verwendet.

\subsubsection{1 Äquivalente Last- und Lagesysteme}

Im Folgenden sei ein Koordinatensystem vorgegeben, die Vektoren sind nun Tripel reeller Zahlen. Im Sinne verallgemeinerter Kräfte lassen sich zu jedem Punkt $A$ Kraft $\boldsymbol{F}$ und Drehmoment $\boldsymbol{T}_{A}$ an einem Punkt zur Last $f_{A}$ mit

$$
f_{A}:=\left(\begin{array}{c}
\boldsymbol{F} \\
\boldsymbol{T}_{A}
\end{array}\right)
$$

zusammenfassen. Man nennt Lasten zu verschiedenen Punkten $A$ und $B$ äquivalent, wenn sie dem Hebelgesetz $\boldsymbol{T}_{A}=\boldsymbol{T}_{B}+\overrightarrow{A B} \times \boldsymbol{F}$, also insgesamt

$$
f_{A}=\underbrace{\left(\begin{array}{cc}
\boldsymbol{I} & \mathbf{0} \\
\boldsymbol{\Omega}_{A B} & \boldsymbol{I}
\end{array}\right)}_{=: \mathcal{T}_{A B}} f_{B}=\mathcal{T}_{A B} f_{B}
$$

genügen. 5 Die Transformationsmatrizen

$$
\mathcal{T}_{A B}=\left(\begin{array}{cc}
\boldsymbol{I} & \mathbf{0} \\
\boldsymbol{\Omega}_{A B} & \boldsymbol{I}
\end{array}\right)
$$

die eine Last vom Bezugssystem $B$ ins Bezugssystem $A$ transformiert, hat die Inverse

$$
\mathcal{T}_{A B}^{-1}=\left(\begin{array}{cc}
\boldsymbol{I} & \mathbf{0} \\
-\boldsymbol{\Omega}_{A B} & \boldsymbol{I}
\end{array}\right)=\left(\begin{array}{cc}
\boldsymbol{I} & \mathbf{0} \\
\boldsymbol{\Omega}_{B A} & \boldsymbol{I}
\end{array}\right)=\mathcal{T}_{B A}
$$

da Ortsvektoren $\overrightarrow{A B}$ beim Vorzeichenwechsel Anfangs- und Endpunkt vertauschen. Wegen $\operatorname{det} \mathcal{T}=1$ gehören die Transformationsmatrizen zur Gruppe der unimodularen Matrizen.

Die Grundgleichungen der Statik lassen sich nun besonders prägnant formulieren: Für ein System aus in den Punkten $P$ angreifenden Kräften und Drehmomenten gilt im Ursprung $O$ einfach

$$
\sum \mathcal{T}_{O P} f_{P}=0
$$

\footnotetext{
${ }^{5}$ Geschweifte Klammern unterhalb von Teilen einer Formel möchte ich auch im Folgenden für Definitionen, Abkürzungen oder Zwischenergebnisse verwenden.
} 
Im Sinne verallgemeinerter Koordinaten können Translation $\boldsymbol{d}_{A}$ und Drehung $\boldsymbol{\theta}$ an einem Punkt $A$ zur Lage $q_{A}$ mit

$$
q_{A}:=\left(\begin{array}{c}
\boldsymbol{d}_{A} \\
\boldsymbol{\theta}
\end{array}\right)
$$

zusammengefaßt werden. Der Drehvektor $\boldsymbol{\theta}$ ist aber nicht immer die günstigste Wahl zum Beschreiben eines Objektes der Drehgruppe, da die gleiche Drehung durch verschiedene Drehvektoren beschrieben werden kann. Am leichtesten ist das einzusehen über den Zusammenhang mit der zum Drehvektor $\boldsymbol{\theta}$ gehörenden Drehmatrix $\boldsymbol{R}_{\theta}$

$$
\boldsymbol{R}_{\theta}=\exp \left(\boldsymbol{\Omega}_{\theta}\right),
$$

die bei einer zusätzlichen vollen Drehung unverändert bleibt, während die Norm des Drehvektors um $2 \pi$ zunimmt. In der Tat ist es nicht möglich, für die Drehgruppe des $\mathbb{R}^{3}$ eine globale 1-zu-1-Parametrisierung mit weniger als 5 Parametern zu finden, die keine singulären Punkte hat, vgl. auch im Folgenden [62]. Redundante Parameter erlauben aber keine einfache Analysis mehr, es muß dann mit Nebenbedingungen gearbeitet werden. Solche redundanten Parametersätze mit zusammen 12 Komponenten sind die Drehmatrix $\boldsymbol{R}$ und der Verschiebevektor $\boldsymbol{d}$, oder gleich die Transformationsmatrix $\left(\begin{array}{cc}\boldsymbol{R} & \boldsymbol{d} \\ \mathbf{0}^{T} & 1\end{array}\right)$ mit insgesamt 16 und offensichtlich mehr Einträgen als notwendig. Diese letzte Form ist für homogen geschriebene Vektoren $\left(\begin{array}{c}\boldsymbol{x} \\ 1\end{array}\right)$ günstig, da sie eine Transformation zwischen Rast- und Gangsystem mit einer einzigen Matrixmultiplikation beschreibt. Neunparametrige Darstellungen sind die Koordinaten dreier Punkte oder die Angabe der ersten beiden Spalten der Drehmatrix samt dem Verschiebevektor. EulERparameter [1, 2, 30] bilden zusammen mit dem Translationsvektor eine 7-parametrige 1-zu-2-Parametrisierung und stellen einen Kompromiß dar. Da hier nur kleine Lageänderungen von Interesse sind, reicht der Drehvektor als Darstellung kleiner Drehungen bzw. als lokale Karte der Drehgruppe aus. Trotzdem sind die durch endliche Drehungen verursachten Effekte als mögliche Fehlerquellen immer in Betracht zu ziehen. Eine theoretische Betrachtung derart verursachter sog. geometrischer Nichtlinearitäten, insbesondere mit ihren Auswirkungen auf die Steifheitsmatrix (vgl. Abschnitt 2.2.2), findet sich in [57].

Eine endliche Drehung von Ortsvektoren um den Winkel $\theta:=|\boldsymbol{\theta}|$ bei gegebener Drehrichtung $\boldsymbol{\alpha}:=\boldsymbol{\theta} / \theta$ mit $|\boldsymbol{\alpha}|=1$ wird beschrieben durch die Drehmatrix $\boldsymbol{R}$ mit den Komponenten

$$
R_{i j}=\underbrace{\delta_{i j}}_{(=: \boldsymbol{I})_{i j}}+\sin \theta \cdot \underbrace{\boldsymbol{\varepsilon}_{i k j} \alpha_{k}}_{=\left(\boldsymbol{\Omega}_{\alpha}\right)_{i j}}+(\cos \theta-1) \cdot \underbrace{\left(\delta_{i j}-\alpha_{i} \alpha_{j}\right)}_{=:\left(\boldsymbol{P}_{\alpha}^{\perp}\right)_{i j}} .
$$

Diese Gleichung ist die vom Transformationsverhalten korrekte und in beliebigen Koordinatensystemen gültige am Beispiel einer Drehmatrix $\boldsymbol{R}_{z}$ in $z$-Richtung wohl- 
bekannte Gleichung

$$
\begin{aligned}
\boldsymbol{R}_{z} & =\left(\begin{array}{ccc}
\cos \theta & -\sin \theta & 0 \\
\sin \theta & \cos \theta & 0 \\
0 & 0 & 1
\end{array}\right) \\
& =\left(\begin{array}{lll}
1 & 0 & 0 \\
0 & 1 & 0 \\
0 & 0 & 1
\end{array}\right)+\sin \theta\left(\begin{array}{ccc}
0 & -1 & 0 \\
1 & 0 & 0 \\
0 & 0 & 0
\end{array}\right)+(\cos \theta-1)\left(\begin{array}{lll}
1 & 0 & 0 \\
0 & 1 & 0 \\
0 & 0 & 0
\end{array}\right)
\end{aligned}
$$

Eine Drehung erzeugt von einem beliebigen Punkt $B$ der Drehachse aus gesehen im Punkt $A$ eine zusätzliche Auslenkung $\boldsymbol{R} \overrightarrow{B A}-\overrightarrow{B A}=(\boldsymbol{R}-\boldsymbol{I}) \overrightarrow{B A}$. Für eine kleine Lageänderung, bestehend aus Translation $\delta \boldsymbol{d}$ und Drehung $\delta \boldsymbol{\theta}=\boldsymbol{\alpha} \delta \theta$, gilt also

$$
\begin{aligned}
\delta \boldsymbol{d}_{A} & =\delta \boldsymbol{d}_{B}+(\boldsymbol{R}-\boldsymbol{I}) \overrightarrow{B A} \\
& =\delta \boldsymbol{d}_{B}+\left(\sin \delta \theta \cdot \boldsymbol{\Omega}_{\alpha}+(\cos \delta \theta-1) \cdot \boldsymbol{P}_{\alpha}^{\perp}\right) \overrightarrow{B A} \\
& =\delta \boldsymbol{d}_{B}+\left(\delta \theta \boldsymbol{\Omega}_{\alpha}-\frac{1}{2} \delta \theta^{2} \boldsymbol{P}_{\alpha}^{\perp}\right) \overrightarrow{B A}+\mathcal{O}\left(\delta \theta^{3}\right) \\
& =\delta \boldsymbol{d}_{B}+\delta \theta \boldsymbol{\alpha} \times \overrightarrow{B A}+\mathcal{O}\left(\delta \theta^{2}\right) \\
& =\delta \boldsymbol{d}_{B}+\delta \boldsymbol{\theta} \times \overrightarrow{B A}+\mathcal{O}\left(\delta \theta^{2}\right) \\
& =\delta \boldsymbol{d}_{B}-\overrightarrow{B A} \times \delta \boldsymbol{\theta}+\mathcal{O}\left(\delta \theta^{2}\right) \\
& =\delta \boldsymbol{d}_{B}+\overrightarrow{A B} \times \delta \boldsymbol{\theta}+\mathcal{O}\left(\delta \theta^{2}\right) \\
& =\delta \boldsymbol{d}_{B}+\Omega_{A B} \cdot \delta \boldsymbol{\theta}+\mathcal{O}\left(\delta \theta^{2}\right) .
\end{aligned}
$$

Entsprechend dieser Sichtweise wird die Transformation der Translation $\delta \boldsymbol{d}$ üblicherweise geschrieben als $\delta \boldsymbol{d}_{A}=\delta \boldsymbol{d}_{B}+\overrightarrow{A B} \times \delta \boldsymbol{\theta}$ und manchmal auch als "Hebelgesetz der Auslenkungen" bezeichnet.

Es werden also im Sinne verallgemeinerter Koordinaten infinitesimale Translation $\delta \boldsymbol{d}_{A}$ und infinitesimale Drehung $\delta \boldsymbol{\theta}$ an einem Punkt zur Lageänderung $\delta q_{A}$ mit

$$
\delta q_{A}:=\left(\begin{array}{c}
\delta \boldsymbol{d}_{A} \\
\delta \boldsymbol{\theta}
\end{array}\right)
$$

zusammengefaßt. Analog zu dem Hebelgesetz (2.1 $)^{6}$ lautet das Transformationsverhalten für die kleinen Lageänderungen $\delta q$ zu verschiedenen Aufpunkten

$$
\delta q_{A}=\left(\begin{array}{cc}
\boldsymbol{I} & \boldsymbol{\Omega}_{A B} \\
\mathbf{0} & \boldsymbol{I}
\end{array}\right) \delta q_{B}=\mathcal{T}_{B A}^{T} \delta q_{B}
$$

Im Gegensatz zum Hebelgesetz gilt diese Formel nur für kleine Lageänderungen, weshalb eine Definition von äquivalenten Lagesystemen mit dieser Formel daher

\footnotetext{
${ }^{6}$ Mit Zahlen in runden Klammern sollen Verweise auf Formeln bezeichnet werden. Deren Nummern befinden sich jeweils rechts der Formel am Rand der Textbreite.
} 
zunächst nur genähert möglich zu sein scheint. Im Kapitel 2.1.2.2 über Schraubachsen zeigt sich, daß die Analogie aber dennoch möglich ist.

Ein weiteres Problem liegt darin, daß die Lagen gleichzeitig eine Doppelrolle als Referenzsystem und Observable einnehmen; die Größen $\overrightarrow{A B}$ und $\boldsymbol{\theta}$ stellen gleichzeitig eine Transformation des Koordinatensystems und die kleinen Lageänderungen selbst dar. Dies wird aber erst im Kapitel 3.3.4 über Vorlasten von Bedeutung werden, da sich nur für große Vorlasten Effekte erster Ordnung ergeben.

Die Existenz von äquivalenten Last- bzw. Lagesystemen ist aus der Sicht des Messenden unbefriedigend: Gleichen Erscheinungen werden in verschiedenen Bezugssystemen verschiedene Zahlen zugeordnet. Diese fehlende Eindeutigkeit wird sich entsprechend auch auf einen Zusammenhang zwischen Lasten und Lagen übertragen. Eine eindeutige Beschreibung von äquivalenten Lasten und Lagen sind die im Folgenden zu besprechenden Schrauben. Da für den Zusammenhang zwischen Lasten und Lagen diese nur noch die Rolle von Variablen spielen, muß das Problem dort anders angegangen werden.

\subsubsection{Kraft- und Drehschrauben}

Äquivalente Last- und Lagesysteme lassen sich auf eine eindeutige Form bringen, die sog. Kraft- und Drehschrauben. Diese sind Geraden im Raum, also geometrische Gebilde, versehen mit zwei weiteren physikalischen Größen, einem Skalar und einem Pseudoskalar. Für diese Eindeutigkeit muß die Linearität preisgegeben werden: Schraubachsen sind nicht superponierbar.

Über das Transformationsverhalten der freien Vektoren lassen sich bestimmte Koordinatensysteme definieren: Wird als Aufpunkt der Ursprung des Koordinatensystems so gewählt, daß der freie Vektor parallel zu seinem linienflüchtigen Partner ist, so liegt dieser Aufpunkt auf einer Geraden im Raum, der Schraubachse. Auf $\operatorname{der}$ Achse der $\left\{\begin{array}{c}\text { Kraftschraube } \\ \text { Drehschraube }\end{array}\right\}$ sind $\left\{\begin{array}{c}\text { Kraft } \\ \text { Drehung }\end{array}\right\}$ und $\left\{\begin{array}{c}\text { Drehmoment } \\ \text { Translation }\end{array}\right\}$ parallel.

Die Transformationsformeln (2.1) und (2.3) der freien Vektoren liefern nur zu dieser Achse senkrechte Anteile. Daher wächst die Norm des freien Vektors über den Satz des Pythagoras mit dem Abstand zur Schraubachse. Insbesondere ist die Norm des freien Vektors auf der Schraubachse minimal. Dies sieht man rechnerisch ein, indem man den freien Vektor in Anteile senkrecht und parallel zu seinem linienflüchtigen Partner zerlegt, etwa $\boldsymbol{T}=\boldsymbol{T}^{\|}+\boldsymbol{T}^{\perp}$. Der Vektor $\overrightarrow{O P} \times \boldsymbol{F}$ steht für alle $P$ wie $\boldsymbol{T}^{\perp}$ senkrecht auf $\boldsymbol{F}$. Daher ist die Gleichung $\boldsymbol{T}^{\perp}+\overrightarrow{O P} \times \boldsymbol{F}=\mathbf{0}$ wie im Fall eines ebenen Lastsystems mit der Formel für doppelte Kreuzprodukte lösbar, wobei $P$ dann der Punkt $K$ der Kraftschraube mit kleinstem Abstand zum Ursprung $O$ 
ist. Dieselbe Lösung ergibt sich aus den analytischen Minimierungsproblemen

$$
\begin{aligned}
|\boldsymbol{T}(\overrightarrow{O K}, \boldsymbol{F})| & =\underset{\overrightarrow{O K}}{\min !} \\
|\delta \boldsymbol{d}(\overrightarrow{O D}, \delta \boldsymbol{\theta})| & =\min _{\overrightarrow{O D}} !
\end{aligned}
$$

für die freien Vektoren als abhängige Variable. Man erhält damit die Formeln

$$
\begin{aligned}
\overrightarrow{O K} & =\frac{1}{|\boldsymbol{F}|^{2}}(\boldsymbol{F} \times \boldsymbol{T}) \\
\overrightarrow{O D} & =\frac{1}{|\delta \boldsymbol{\theta}|^{2}}(\delta \boldsymbol{\theta} \times \delta \boldsymbol{d}),
\end{aligned}
$$

für die Aufpunkte $K$ der Kraftschraube und $D$ der Drehschraube oder, wieder zusammengefaßt mit dem Aufpunktvektor $\boldsymbol{a}$

$$
\begin{aligned}
\boldsymbol{a} & =\frac{1}{\boldsymbol{l}^{2}}(\boldsymbol{l} \times \boldsymbol{f}) \\
\text { Schraubachsaufpunkt } & =\frac{1}{\mid \text { linienflüchtig }\left.\right|^{2}} \cdot(\text { linienflüchtig } \times \text { frei }) .
\end{aligned}
$$

Diese zu den Aufpunkten gehörenden Ortsvektoren stehen senkrecht auf der Richtung der Schraubachse und haben daher im Vergleich zu allen anderen Aufpunkten minimalen Abstand zum Ursprung. Damit ist eine Schraube als Gerade im Raum in der Punkt-Richtungs-Form eindeutig gegeben.

Warnung: Auch diese Formel gilt nur für infinitesimale Verschiebungen! Unter Benutzung von (2.2), am einfachsten in z-Richtung einzusehen, lautet sie für Drehschrauben korrekt

$$
\overrightarrow{O D}=\frac{1}{2}\left(\boldsymbol{d}^{\perp}+\cot \frac{\theta}{2}(\boldsymbol{\alpha} \times \boldsymbol{d})\right),
$$

wobei $\boldsymbol{d}^{\perp}:=\boldsymbol{P}_{\alpha}^{\perp} \boldsymbol{d}$ der zu $\boldsymbol{\alpha}$ senkrechte Anteil von $\boldsymbol{d}$ ist. Diese Formel erlaubt eine hier nicht weiter relevante geometrische Deutung [69].

Daher kann auch einer endlichen Bewegung eines starren Körpers im Raum, die etwa durch Anfangs- und Endposition festgelegt ist, eindeutig eine Schraubung zugeordnet werden. Das ist genau der Inhalt des Satzes von CHASLES, siehe etwa [1] Ch. 2.5.

Diese Beschreibung liefert bislang insgesamt nur vier der sechs insgesamt nötigen Freiheitsgrade. Die Richtung der Schraubachse legt zwei Größen bis auf ein Vorzeichen fest, die dritte Komponente entfällt wegen der Normierung des Richtungsvektors. Der Schraubachsaufpunkt entspricht auch nur zwei weiteren Größen, da sein Ortsvektor nach den Formeln (2.4) und (2.5) senkrecht auf dieser Richtung steht. Um auf den vollen Informationsgehalt der sechs verallgemeinerten Koordinaten bzw. Kräfte zu kommen, fehlen also noch zwei Größen. Das können entweder 
die Beträge der beiden beteiligten Vektoren sein oder etwa der Betrag des linienflüchtigen Vektors und die Schraubsteigung $\tau$

$$
\begin{aligned}
\tau_{K} & =\frac{1}{|\boldsymbol{F}|^{2}}(\boldsymbol{F} \cdot \boldsymbol{T}) \\
\tau_{D} & =\frac{1}{|\delta \boldsymbol{\theta}|^{2}}(\delta \boldsymbol{\theta} \cdot \delta \boldsymbol{d}) .
\end{aligned}
$$

Für Drehschrauben etwa entspricht sie der Ganghöhe einer materiellen Schraube, was den Vorteil der Anschaulichkeit hat. In beiden Fällen hat die Schraubsteigung die Dimension einer Länge. Die Verbalisierung lautet diesmal

$$
\begin{aligned}
& \tau=\frac{1}{|\boldsymbol{l}|^{2}}(\boldsymbol{l} \cdot \boldsymbol{f}) \\
& \text { Schraubsteigung }=\frac{1}{\mid \text { linienflüchtig| }\left.\right|^{2}} \cdot(\text { linienflüchtig } \cdot \text { frei }) \\
& .
\end{aligned}
$$

Die Formeln (2.4), (2.5), (2.6) und (2.7) lassen sich mit dem Operator o, welcher wahlweise das Vektor- oder das Skalarprodukt beschreibt, symbolisch zusammenfassen $\mathrm{zu}$

$$
\frac{1}{|\boldsymbol{l}|^{2}}(\boldsymbol{l} \circ \boldsymbol{f})
$$

Das ist der allgemeine Ausdruck für die Schraubparameter.

Damit ist die Analogie zwischen Kraft- und Drehschrauben noch nicht erschöpft. Es kann außerdem gezeigt werden, daß die sich durch Superposition zweier Kraftschrauben ergebende dritte Kraftschraube mit den beiden Ausgangsschrauben ein Gemeinlot, das ist eine gemeinsame Senkrechte, hat. Die entsprechende Aussage der Kinematik ist, daß die zu drei gegeneinander bewegten räumlichen Systemen gehörenden drei momentanen Schraubachsen ein Gemeinlot haben ${ }^{7}$. Die Schraubung vom ersten aufs dritte Bezugssystem ist dabei die Ausführung der ersten beiden nacheinander.

Wie auch bei den Formeln für den Schraubachsaufpunkt entstehen numerische Schwierigkeiten für einen reinen freien Vektor, da der Nenner quadratisch gegen Null geht und dann die Ausdrücke divergieren. Für diese Fälle könnte man analytisch den Durchgang durchs Unendliche zulassen, was aber für numerische Zwecke unangemessen ist. Für infinitesimale Bewegungen oder Lasten kann man auf eine andere Beschreibungsform ausweichen. Dann nämlich geht auch der Zähler gegen Null, so daß sich mit dem Satz von DE L'HôpITAL endliche Ausdrücke für Aufpunkt und Steigung der Schraube ergeben. Für die Drehschraube etwa ergibt sich mit $\boldsymbol{\theta}=\theta \boldsymbol{\alpha}$ für $\theta \rightarrow 0$ und $\boldsymbol{d} \rightarrow 0$ die Beziehung

$$
\frac{1}{|\boldsymbol{\theta}|^{2}}(\boldsymbol{\theta} \circ \boldsymbol{d})=\boldsymbol{\alpha} \circ \frac{\boldsymbol{d}}{\theta}=\boldsymbol{\alpha} \circ \frac{\dot{\boldsymbol{d}}}{\dot{\theta}}
$$

\footnotetext{
${ }^{7} \mathrm{Vgl}$. etwa das auch weitere interessante Beschreibungsmöglichkeiten für Lagen enthaltende Buch [30], Abschnitt 2.2.11.
} 
für die Schraubparameter $\overrightarrow{O D}$ bzw. $\tau_{D}$ und entsprechende Ausdrücke für die Kraftschraube.

\subsubsection{Die Invarianzeigenschaft der Schraubparameter}

Die Schraubparameter beschreiben eine Last- bzw. eine Lageänderung fast eindeutig, nur der Aufpunkt der Schraubachse kann auf dieser frei gewählt werden. Es ist daher zu erwarten, daß sich diese Eigenschaft in einem besonderen Transformationsverhalten unter der Wahl des Bezugspunktes äußert.

2.1.2.3.1 Die Schraubsteigung. Nur der zum linienflüchtigen Vektor parallele Anteil des freien Vektors geht in die Formel für die Schraubsteigung ein. Es gilt zu verschiedenen Bezugspunkten $A$ und $B$

$$
\begin{aligned}
\tau_{A} & =\frac{1}{\boldsymbol{l}_{A}^{2}}\left(\boldsymbol{l}_{A} \cdot \boldsymbol{f}_{A}\right)=\frac{1}{\boldsymbol{l}^{2}}\left(\boldsymbol{l} \cdot \boldsymbol{f}_{A}\right)=\frac{1}{\boldsymbol{l}^{2}}\left(\boldsymbol{l} \cdot\left(\boldsymbol{f}_{B}+\boldsymbol{\Omega}_{A B} \boldsymbol{l}\right)\right) \\
& =\frac{1}{\boldsymbol{l}^{2}}\left(\boldsymbol{l} \cdot \boldsymbol{f}_{B}\right)+\frac{1}{\boldsymbol{l}^{2}}\left(\boldsymbol{l} \cdot \boldsymbol{\Omega}_{A B} \boldsymbol{l}\right)=\frac{1}{\boldsymbol{l}^{2}}\left(\boldsymbol{l} \cdot \boldsymbol{f}_{B}\right) \\
& =
\end{aligned}
$$

und die sich durch den Wechsel des Bezugspunktes ergebenden senkrechten Anteile des freien Vektors heben sich heraus. Die Schraubsteigung ist ferner als Quotient zweier Skalarprodukte invariant unter Drehungen, denn es gilt etwa $(\boldsymbol{R} \boldsymbol{l}) \cdot \boldsymbol{R} \boldsymbol{f}=$ $\boldsymbol{l}^{T} \boldsymbol{R}^{T} \boldsymbol{R} \boldsymbol{f}=\boldsymbol{l}^{T} \boldsymbol{f}=\boldsymbol{l} \cdot \boldsymbol{f}$. Da sie unter Spiegelungen am Ursprung ihr Vorzeichen ändert, ist sie ein Pseudoskalar.

Ihre Invarianz unter der kompletten EukLIDschen Gruppe erklärt ihre Bedeutung, da sie in allen Koordinatensystemen denselben Wert annimmt.

2.1.2.3.2 Die Schraubachsaufpunkte. Die Schraubachsaufpunkte zu verschiedenen Bezugspunkten $A$ und $B$ transformieren sich wie folgt.

$$
\begin{aligned}
\boldsymbol{a}_{A} & =\frac{1}{\boldsymbol{l}_{A}^{2}}\left(\boldsymbol{l}_{A} \times \boldsymbol{f}_{A}\right)=\frac{1}{\boldsymbol{l}^{2}}\left(\boldsymbol{l} \times \boldsymbol{f}_{A}\right)=\frac{1}{\boldsymbol{l}^{2}}\left(\boldsymbol{l} \times\left(\boldsymbol{f}_{B}+\overrightarrow{A B} \times \boldsymbol{l}\right)\right) \\
& =\frac{1}{\boldsymbol{l}^{2}}\left(\boldsymbol{l} \times\left(\boldsymbol{f}_{B}-\boldsymbol{l} \times \overrightarrow{A B}\right)\right)=\frac{1}{\boldsymbol{l}^{2}}\left(\boldsymbol{l} \times \boldsymbol{f}_{B}\right)-\frac{1}{\boldsymbol{l}^{2}}(\boldsymbol{l} \times(\boldsymbol{l} \times \overrightarrow{A B})) \\
& =\boldsymbol{a}_{B}-\frac{1}{\boldsymbol{l}^{2}}\left(\boldsymbol{\Omega}_{l} \boldsymbol{\Omega}_{l} \overrightarrow{A B}\right) \stackrel{(A .2)}{=} \boldsymbol{a}_{B}-\frac{1}{\boldsymbol{l}^{2}}\left(-\boldsymbol{l}^{2} \boldsymbol{P}_{l}^{\perp} \overrightarrow{A B}\right) \\
& =\boldsymbol{a}_{B}+\boldsymbol{P}_{l}^{\perp} \overrightarrow{A B} .
\end{aligned}
$$

Diese Formel besitzt eine einfache geometrische Deutung. Es seien $P_{A}$ und $P_{B}$ die zu den Punkten $A$ und $B$ gehörenden Lotfußpunkte auf die Schraubachse. Dann gilt

$$
\vec{P}_{A}=\boldsymbol{P}_{l}^{\|} \overrightarrow{A B}
$$


und über einen geschlossenen Linienzug erhält man

$$
\begin{aligned}
\overrightarrow{A P}_{A} & =\overrightarrow{A B}+\overrightarrow{B P}_{B}+{\overrightarrow{P_{B} P_{A}}}_{=} \overrightarrow{A B}+\overrightarrow{B P}_{B}-{\overrightarrow{P_{A} P_{B}}}_{=} \overrightarrow{A B}+\overrightarrow{B P}_{B}-\boldsymbol{P}_{l}^{\|} \overrightarrow{A B} \\
& =\overrightarrow{B P}_{B}+\boldsymbol{P}_{l}^{\perp} \overrightarrow{A B} .
\end{aligned}
$$

Das ist mit $\overrightarrow{A P}_{A}=\boldsymbol{a}_{A}$ und $\overrightarrow{B P}_{B}=\boldsymbol{a}_{B}$ genau die obige Formel. Es werden über die Definition mit dem Kreuzprodukt die Aufpunkte $\boldsymbol{a}$ immer senkrecht zu $\boldsymbol{l}$ und damit senkrecht zur Schraubachse berechnet, was die Lotfußpunkte charakterisiert.

Da nach (A.16) das Kreuzprodukt zweier gedrehter Vektoren auch durch Drehung des Kreuzproduktes der ungedrehten Vektoren berechnet werden kann, ergibt sich in allen Koordinatensystemen dieselbe Gerade im Ortsraum.

\subsection{Die elastischen Matrizen}

In diesem Abschnitt sollen die einfachsten Objekte untersucht werden, die mit der bisher entwickelten Theorie verträglich sind. Es handelt sich also um Vorstellungen über ideale Meßergebnisse.

Vom Standpunkt der Elastizitätstheorie betrachtet handelt es sich beim elastisch aufgehängten starren Körper um ein Randwertproblem, bei dem die Werte der gesuchten Funktion auf den starren Berandungen vorgegeben und im Zwischenraum frei sind. Prinzipiell ließe sich das numerisch behandeln, etwa mit finiten Elementen. Da hier aber das Verhalten des elastischen Mediums selbst nicht interessiert, ist eine einfachere Beschreibungsweise möglich.

Gegenstand des Interesses ist das Verhalten der starren Berandung, genauer um den Zusammenhang zwischen der auf sie wirkenden Last und ihrer Lage. Unter Vernachlässigung der inneren Struktur der Aufhängung hat man demnach schematisch folgende Situation:

$$
f=\left(\begin{array}{c}
\boldsymbol{F} \\
\boldsymbol{T}
\end{array}\right) \stackrel{\text { Elastische Aufhängung }}{\longrightarrow} q
$$
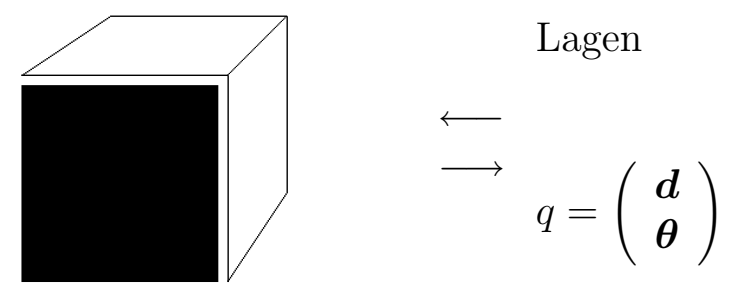

Dann wird eine Linearisierung durchgeführt und stellenweise die Existenz eines elastischen Potentials vorausgesetzt, aus dem spezielle Symmetrieeigenschaften abgeleitet werden können. Die den linearen Anteil beschreibenden Matrizen, Flexibilitätsmatrix und Steifheitsmatrix, die ich als elastische Matrizen bezeichnen möchte, sind nun die grundlegenden Objekte. 
Unter Benutzung der Transformationseigenschaften der Lasten und Lagen lassen sich aus der inneren Struktur der elastischen Matrizen Koordinatensysteme auszeichnen. Falls ein elastisches Potential existiert, ist das Koordinatensystem eindeutig; der Ursprung dieses Koordinatensystems ist das elastische Zentrum, seine Orientierung sind die funktionellen Achsen. Falls kein Potential existiert, verdoppelt sich die Anzahl der Koordinatensysteme: Eins für die Lasten und eins für die Lagen. Nach einer Transformation der elastischen Matrizen auf diese Koordinatensysteme ergibt sich dann die Normalform für Meßwerte, die auch auf reale Messungen anwendbar ist, die Realform.

\subsubsection{Die Flexibilitätsmatrix}

Folgende Situation sei gegeben: Eine im Punkt $A$ der starren Berandung angreifende Last verursacht im Punkt $B$ über die elastische Aufhängung eine Änderung der Lage. Die Lasten $f_{A}$ und Lagen $q_{B}$ werden dabei im raumfesten System gemessen. Für kleine Laständerungen kann man die sich nach Abklingen aller Ausgleichsprozesse einstellende statische Endlage in eine TAYLORreihe mit zeitunabhängigen Koeffizienten entwickeln:

$$
q_{B}\left(f_{A}\right)=q_{B}\left(f_{A}^{0}\right)+\mathcal{F}_{B A}\left(f_{A}-f_{A}^{0}\right)+\mathcal{O}\left(f_{A}-f_{A}^{0}\right)^{2},
$$

und für die Differenz der Lasten $\Delta f_{A}$ als Eingangsgrößen (= Input)

$$
\Delta f_{A}:=f_{A}-f_{A}^{0}
$$

erhält man für die Änderung der Lage $\Delta q_{B}$ als Ausgangsgrößen (= Output)

$$
\Delta q_{B}:=q_{B}\left(f_{A}\right)-q_{B}\left(f_{A}^{0}\right)
$$

ein lineares Input-Output-Modell:

$$
\Delta q_{B}=\mathcal{F}_{B A} \Delta f_{A}
$$

Die $6 \times 6$-Matrix $\mathcal{F}$ nennt man Flexibilitätsmatrix, ihre Einträge werden auch Einflußzahlen genannt [68]. Die Indizes $B A$ kennzeichnen die Bezugspunkte $A$ der Lasten und $B$ der Lagen. Für $f_{A}^{0}=0$ ist $q_{B}\left(f_{A}^{0}\right)$ die üblicherweise als Referenz dienende Ausgangslage der starren Berandung; daher können die Differenzen und mit ihnen die $\Delta$ 's in den Formeln auch fortgelassen werden. Spaltet man die Lasten und Lagen wieder in die grundlegenden Vektoren auf, so muß man $\mathcal{F}$ als Blockmatrix in vier $3 \times 3$-Matrizen, etwa $\boldsymbol{D}, \boldsymbol{S}_{2}, \boldsymbol{S}_{1}$ und $\boldsymbol{M}$, aufteilen. Der linearisierte Zusammenhang zwischen Lasten und Lagen lautet nun

$$
\left(\begin{array}{l}
\delta \boldsymbol{d} \\
\delta \boldsymbol{\theta}
\end{array}\right)_{B}=\underbrace{\left(\begin{array}{cc}
\boldsymbol{D} & \boldsymbol{S}_{2} \\
\boldsymbol{S}_{1} & \boldsymbol{M}
\end{array}\right)}_{\mathcal{F}_{B A}} \cdot\left(\begin{array}{c}
\boldsymbol{F} \\
\boldsymbol{T}
\end{array}\right)_{A}
$$


und mit ihm läßt sich der Effekt jeder angelegten Last auf die Lagen berechnen. Damit sind insbesondere Aussagen über Drehschrauben möglich, etwa indem man die Formel für die Schraubsteigung $\tau_{D}$ (2.7) mittels (2.10) durch $\boldsymbol{F}$ und $\boldsymbol{T}$ ausdrückt.

Damit die Dimensionen der Gleichungen zueinander passen, muß gelten

$$
\begin{aligned}
{[\boldsymbol{D}] } & =[\boldsymbol{d}] /[\boldsymbol{F}]=\mathrm{T}^{2} / \mathrm{M} \\
{\left[\boldsymbol{S}_{2}\right] } & =[\boldsymbol{d}] /[\boldsymbol{T}]=\mathrm{T}^{2} / \mathrm{ML} \\
{\left[\boldsymbol{S}_{1}\right] } & =[\boldsymbol{\theta}] /[\boldsymbol{F}]=\mathrm{T}^{2} / \mathrm{ML} \\
{[\boldsymbol{M}] } & =[\boldsymbol{\theta}] /[\boldsymbol{T}]=\mathrm{T}^{2} / \mathrm{ML}^{2}
\end{aligned}
$$

also lauten die Dimensionen der Blockmatrizen der Flexibilitätsmatrix

$$
[\mathcal{F}]=\frac{\mathrm{T}^{2}}{\mathrm{ML}^{2}}\left(\begin{array}{cc}
\mathrm{L}^{2} & \mathrm{~L} \\
\mathrm{~L} & 1
\end{array}\right)
$$

wobei hier die Einheit des Winkels, etwa rad, gleich 1 gesetzt wurde.

Zur Beschreibung zeitabhängiger Vorgänge sind kompliziertere konstitutive Gleichungen ${ }^{8}$ nötig. Weiterhin braucht man, um Beschleunigungen zu berücksichtigen, die Massenmatrix $\mathcal{M}$, welche die Masse, den Trägheitstensor und den Satz von SteINER zusammenfaßt. Mehr darüber im Kapitel 3.1] über Zustandsgleichungen und Dynamik.

\subsubsection{Transformationsverhalten und Fehlerfortpflanzung}

Das Transformationsverhalten der Flexibilitätsmatrix bei Verschiebung der Referenzpunkte von $A$ nach $A^{\prime}$ und von $B$ nach $B^{\prime}$ leitet sich aus dem der Lasten und Lagen ab. Im Sinne des linearen Input-Output-Modells $q(f)$ kann man die Gesamttransformation in drei Schritten durchführen:

1. Die Lasten werden vom Punkt $A^{\prime}$ wieder auf den Punkt $A$ bezogen, also $f_{A}=\mathcal{T}_{A A^{\prime}} f_{A^{\prime}}$.

2. Es wird der bekannte Zusammenhang zwischen $q_{A}$ und $f_{B}$ benutzt, d. h. $q_{B}=\mathcal{F}_{B A} f_{A}$.

3. Die Lagen werden nun auf den Punkt $B^{\prime}$ bezogen, also $q_{B^{\prime}}=\mathcal{T}_{B B^{\prime}}^{T} q_{B}$.

Insgesamt folgt für $q_{B^{\prime}}\left(f_{A^{\prime}}\right)$ die Gleichung

$$
q_{B^{\prime}}=\mathcal{T}_{B B^{\prime}}^{T} q_{B}=\mathcal{T}_{B B^{\prime}}^{T} \mathcal{F}_{B A} f_{A}=\mathcal{T}_{B B^{\prime}}^{T} \mathcal{F}_{B A} \mathcal{T}_{A A^{\prime}} f_{A^{\prime}}
$$

\footnotetext{
${ }^{8}$ Konstitutive Gleichungen sind meist phänomenologisch gewonnene Beziehungen wie das Hookesche Gesetz oder der NewTonsche Reibungsansatz, die das makroskopische Verhalten von Materialien charakterisieren.
} 
Das Transformationsverhalten der Flexibilitätsmatrix läßt sich diesen Überlegungen entsprechend der Abbildung 2.1] entnehmen.

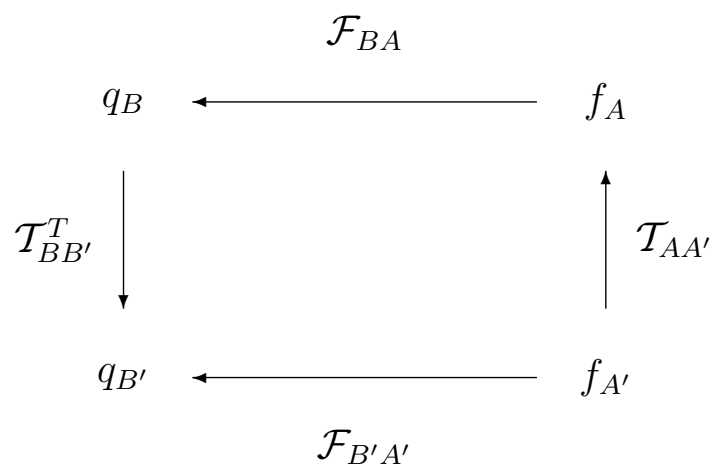

Abbildung 2.1: Das Transformationsverhalten der Flexibilitätsmatrix.

Im Formalismus der $6 \times 6$-Matrizen transformiert sich die Flexibilitätsmatrix also gemäß

$$
\mathcal{F}_{B^{\prime} A^{\prime}}=\mathcal{T}_{B B^{\prime}}^{T} \mathcal{F}_{B A} \mathcal{T}_{A A^{\prime}}
$$

Die Blockkomponenten der einzelnen Matrizen sind nun nach (2.1), (2.3) und (2.10) bekannt. Ihr Produkt lautet damit in Komponenten ${ }^{9}$

$$
\begin{aligned}
\mathcal{F}_{B^{\prime} A^{\prime}}=\left(\begin{array}{cc}
\boldsymbol{I} & \boldsymbol{\Omega}_{B^{\prime} B} \\
\mathbf{0} & \boldsymbol{I}
\end{array}\right) \cdot\left(\begin{array}{cc}
\boldsymbol{D} & \boldsymbol{S}_{2} \\
\boldsymbol{S}_{1} & \boldsymbol{M}
\end{array}\right) \cdot\left(\begin{array}{cc}
\boldsymbol{I} & \mathbf{0} \\
\boldsymbol{\Omega}_{A A^{\prime}} & \boldsymbol{I}
\end{array}\right) \\
=\left(\begin{array}{cc}
\boldsymbol{I} & \boldsymbol{\Omega}_{B^{\prime} B} \\
\mathbf{0} & \boldsymbol{I}
\end{array}\right) \cdot\left(\begin{array}{cc}
\boldsymbol{D}+\boldsymbol{S}_{2} \boldsymbol{\Omega}_{A A^{\prime}} & \boldsymbol{S}_{2} \\
\boldsymbol{S}_{1}+\boldsymbol{M} \boldsymbol{\Omega}_{A A^{\prime}} & \boldsymbol{M}
\end{array}\right) \\
=\left(\begin{array}{cc}
\boldsymbol{D}+\boldsymbol{S}_{2} \boldsymbol{\Omega}_{A A^{\prime}}+\boldsymbol{\Omega}_{B^{\prime} B} \boldsymbol{S}_{1}+\boldsymbol{\Omega}_{B^{\prime} B} \boldsymbol{M} \boldsymbol{\Omega}_{A A^{\prime}} & \boldsymbol{S}_{2}+\boldsymbol{\Omega}_{B^{\prime} B} \boldsymbol{M} \\
\boldsymbol{S}_{1}+\boldsymbol{M} \boldsymbol{\Omega}_{A A^{\prime}} & \boldsymbol{M}
\end{array}\right) \\
=\left(\begin{array}{cc}
\boldsymbol{D}+\boldsymbol{S}_{2} \boldsymbol{\Omega}_{A A^{\prime}}-\boldsymbol{\Omega}_{B B^{\prime}} \boldsymbol{S}_{1}-\boldsymbol{\Omega}_{B B^{\prime}} \boldsymbol{M} \boldsymbol{\Omega}_{A A^{\prime}} & \boldsymbol{S}_{2}-\boldsymbol{\Omega}_{B B^{\prime}} \boldsymbol{M} \\
\boldsymbol{S}_{1}+\boldsymbol{M} \boldsymbol{\Omega}_{A A^{\prime}} & \boldsymbol{M}
\end{array}\right) .
\end{aligned}
$$

Daher ist die Flexibilitätsmatrix kein Tensor!10

Die Komponenten der Flexibilitätsmatrix (=Einflußzahlen, Flexibilitätskonstanten) hängen also wegen des Transformationsverhaltens der freien Vektoren von den in der Meßapparatur gewählten Aufpunkten ab. Da verschiedene Apparaturen bezüglich des Meßobjektes unterschiedliche Referenzsysteme benutzen, ergeben

\footnotetext{
${ }^{9}$ Mit Blockmatrizen, auch Übermatrizen genannt, rechnet man, bis auf die einzuhaltende Reihenfolge der Multiplikation der Blockmatrixeinträge, genau wie mit Matrizen, vgl. etwa [73, 20.

${ }^{10}$ Bei einem Tensor wären die Transformationsmatrizen auf der linken und der rechten Seite entweder gleich oder invers zueinander, nicht aber transponiert (Drehmatrizen ausgenommen). Eine Matrix, wie z. B. die Volgtsche Anordnung der Elastizitätsmoduln, ist noch lange kein Tensor. Bei dieser wird das allgemeine HookEsche Gesetz $\sigma_{i j}=\Lambda_{i j k l} \epsilon_{k l}$ wegen der Symmetrie
} 
sich systematisch andere Zahlen. Zur Veranschaulichung der Situation vgl. Abb. 2.2 .

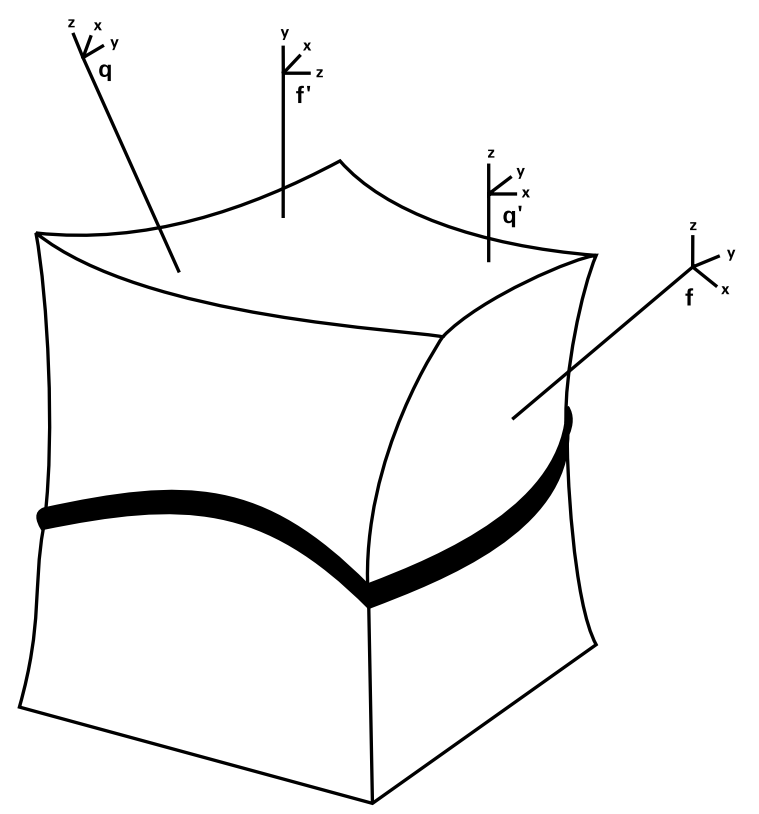

Abbildung 2.2: Die Abhängigkeit der Flexibilitätsmatrix von den Referenzsystemen. Nur im günstigsten Fall stimmen die Koordinatensysteme der Lasten und der Lagen überein. Ihre Ursprünge müssen aber nicht zusammenfallen, ihre Orientierung und Händigkeit kann verschieden sein, im schlimmsten Fall sind sie nicht einmal mehr orthogonal, oder die Achsen schneiden sich nicht mehr.

Daher:

Allein zum Vergleich von Messungen verschiedener Apparaturen am gleichen Meßobjekt benötigt man durch die Meßwerte eindeutig festgelegte Koordinatensysteme!

Prinzipiell wäre es möglich, Information über beliebige Referenzkoordinatensysteme, wie z. B. den anatomical landmarks der Biomechanik, auszutauschen. Eine Festlegung der Koordinatensysteme durch die Meßwerte hätte dem gegenüber den

von $\boldsymbol{\sigma}$ und $\boldsymbol{\epsilon}$ umsortiert zu

$$
\left(\begin{array}{l}
\sigma_{11} \\
\sigma_{22} \\
\sigma_{33} \\
\sigma_{23} \\
\sigma_{31} \\
\sigma_{12}
\end{array}\right)=\left(\begin{array}{cccccc}
c_{11} & c_{12} & c_{13} & c_{14} & c_{15} & c_{16} \\
& c_{22} & c_{23} & c_{24} & c_{25} & c_{26} \\
& & c_{33} & c_{34} & c_{35} & c_{36} \\
& & & c_{44} & c_{45} & c_{46} \\
& & & & c_{55} & c_{56} \\
& & & & & c_{66}
\end{array}\right) \cdot\left(\begin{array}{c}
\epsilon_{11} \\
\epsilon_{22} \\
\epsilon_{33} \\
\epsilon_{23} \\
\epsilon_{31} \\
\epsilon_{12}
\end{array}\right)
$$

Dabei wird der ursprünglich tensorielle Charakter der Gleichungen zerstört. 
Vorteil, ohne nachträglich nicht mehr zu bestimmende Gerätedaten oder Meßvorschriften auszukommen. Daher werde ich im Folgenden diesen Weg einschlagen.

Durch Fehlerfortpflanzung wachsen die Meßfehler der Matrixeinträge mit dem Abstand zum ursprünglich gewählten Koordinatensystem, wobei dieser Einfluß in den einzelnen Blockmatrizen unterschiedlich ist. Einzige Ausnahme ist die Messung des infinitesimalen Drehvektors $\delta \boldsymbol{\theta}$ in Abhängigkeit vom Drehmoment $\boldsymbol{T}$, also die von $\boldsymbol{M}$. Am ungünstigsten ist die Fehlerfortpflanzung bei $\boldsymbol{D}$ : Von der Aufhängung weit entfernt angreifende Kräfte erzeugen große Drehmomente, die wiederum große Drehungen hervorrufen, welche ihrerseits große zusätzliche Translationen in weit entfernten Meßsystemen erscheinen lassen. Dort besteht dann die Gefahr, daß die fortgepflanzten Meßfehler in die Größenordnung der Meßergebnisse geraten und die eigentlich interessierenden Effekte komplett überlagern.

\subsubsection{Die Steifheitsmatrix}

Die Steifheitsmatrix $\mathcal{S}$ ist definiert durch die inverse Beziehung $\Delta f_{A}=\mathcal{S}_{A B} \Delta q_{B}$. Sie transformiert sich entsprechend wie

$$
\mathcal{S}_{A^{\prime} B^{\prime}}=\mathcal{T}_{A^{\prime} A} \mathcal{S}_{A B} \mathcal{T}_{B^{\prime} B}^{T}
$$

In dieser Situation werden nun die Lageänderungen eingestellt und die erzeugten Lasten gemessen.11

Wegen $\mathcal{T}_{A B}^{-1}=\mathcal{T}_{B A}$ ist die Inversion $\mathcal{S}_{A B}^{-1}=\mathcal{F}_{B A}$ verträglich mit den obigen Transformationen der Referenzpunkte. $\frac{12}{}$

$$
\begin{aligned}
\mathcal{F}_{B^{\prime} A^{\prime}}^{-1} & =\left(\mathcal{T}_{B B^{\prime}}^{T} \mathcal{F}_{B A} \mathcal{T}_{A A^{\prime}}\right)^{-1}=\mathcal{T}_{A A^{\prime}}^{-1} \mathcal{F}_{B A}^{-1}\left(\mathcal{T}_{B B^{\prime}}^{T}\right)^{-1}=\mathcal{T}_{A^{\prime} A} \mathcal{S}_{A B} \mathcal{T}_{B^{\prime} B}^{T} \\
& =\mathcal{S}_{A^{\prime} B^{\prime}} .
\end{aligned}
$$

Daher ist auch eine Beschreibung der Meßergebnisse mit der Steifheitsmatrix in diesem Formalismus möglich. Vom theoretischen Standpunkt ist die Steifheitsmatrix sogar das geeignetere Objekt, da mit ihr Beschreibungen von Aufhängungen möglich sind, bei denen eine kleine Auslenkung keine Änderung der Last nach sich zieht (man denke an eine Einzelfeder im Raum, deren Ende senkrecht zu ihrer Längsrichtung bewegt wird). Die Flexibilitätsmatrix hätte in diesem Fall beliebig große Einträge. Da in dieser Arbeit vornehmlich dünne elastische Schichten betrachtet werden, für die solche Schwierigkeiten nicht auftreten, möchte ich sie auch nicht weiter betrachten.

\footnotetext{
${ }^{11}$ Von einer suggestiven Ursache-Wirkung-Beziehung sollte Abstand genommen werden. Solange keine Zeitabhängigkeit in den Gleichungen eingebaut ist, können Lasten und Lagen beide Ursache oder Wirkung sein.

${ }^{12}$ Offenbar ist es nicht zulässig, wie die experimentelle Bestimmung der Flexibilitätskonstanten aus einzelnen Geradensteigungen $m_{i j}$ von $q_{i}\left(f_{j}\right)$ nahelegen würde (vgl. 3.2.3), den Kehrwert der einzelnen Matrixeinträge zu verwenden: Dies wäre nicht mehr verträglich mit deren Transformationseigenschaften.
} 
Die Blöcke der Steifheitsmatrix möchte ich wie folgt bezeichnen

$$
\mathcal{S}=\left(\begin{array}{cc}
\boldsymbol{A} & \boldsymbol{B}_{2} \\
\boldsymbol{B}_{1} & \boldsymbol{C}
\end{array}\right)
$$

Sie haben die Dimensionen

$$
[\mathcal{S}]=\frac{\mathrm{M}}{\mathrm{T}^{2}}\left(\begin{array}{cc}
1 & \mathrm{~L} \\
\mathrm{~L} & \mathrm{~L}^{2}
\end{array}\right),
$$

und transformieren sich mit (2.12) gemäß

$$
\begin{aligned}
\mathcal{S}_{A^{\prime} B^{\prime}}=\mathcal{T}_{A^{\prime} A} \mathcal{S}_{A B} \mathcal{T}_{B^{\prime} B}^{T} & \\
= & \left(\begin{array}{cc}
\boldsymbol{I} & \mathbf{0} \\
\boldsymbol{\Omega}_{A^{\prime} A} & \boldsymbol{I}
\end{array}\right)\left(\begin{array}{cc}
\boldsymbol{A} & \boldsymbol{B}_{2} \\
\boldsymbol{B}_{1} & \boldsymbol{C}
\end{array}\right)\left(\begin{array}{cc}
\boldsymbol{I} & \boldsymbol{\Omega}_{B B^{\prime}} \\
\boldsymbol{0} & \boldsymbol{I}
\end{array}\right) \\
= & \left(\begin{array}{cc}
\boldsymbol{I} & \mathbf{0} \\
\boldsymbol{\Omega}_{A^{\prime} A} & \boldsymbol{I}
\end{array}\right)\left(\begin{array}{cc}
\boldsymbol{A} & \boldsymbol{B}_{2}+\boldsymbol{A} \boldsymbol{\Omega}_{B B^{\prime}} \\
\boldsymbol{B}_{1} & \boldsymbol{C}+\boldsymbol{B}_{1} \boldsymbol{\Omega}_{B B^{\prime}}
\end{array}\right) \\
= & \left(\begin{array}{cc}
\boldsymbol{A} & \boldsymbol{B}_{2}+\boldsymbol{A} \boldsymbol{\Omega}_{B B^{\prime}} \\
\boldsymbol{B}_{1}+\boldsymbol{\Omega}_{A^{\prime} A} \boldsymbol{A} & \boldsymbol{C}+\boldsymbol{B}_{1} \boldsymbol{\Omega}_{B B^{\prime}}+\boldsymbol{\Omega}_{A^{\prime} A} \boldsymbol{B}_{2}+\boldsymbol{\Omega}_{A^{\prime} A} \boldsymbol{A} \boldsymbol{\Omega}_{B B^{\prime}}
\end{array}\right) .
\end{aligned}
$$

Daraus ist die Transformationsinvarianz der Teilmatrix $\boldsymbol{A}$ sofort ersichtlich. Da kein einfacher Zusammenhang zwischen den Blöcken von $\mathcal{S}$ und $\mathcal{F}$ besteht, muß es auch für die Flexibilitätsmatrix neben der Transformationsinvarianz von $\boldsymbol{M}$ weitere transformationsinvariante Terme geben. Diese sollen jetzt untersucht werden, wodurch sich die innere Struktur der elastischen Matrizen weiter klärt.

Aus den Gleichungen $q=\mathcal{F} f$ und $f=\mathcal{S} q$ folgt

$$
\mathcal{F S}=\mathcal{I}=\mathcal{S F}
$$

wobei $\mathcal{I}$ die $6 \times 6$-Einheitsmatrix ist, also daß $\mathcal{S}$ und $\mathcal{F}$ zueinander rechts- und linksinvers sind. Das hat Konsequenzen für die einzelnen Blöcke der elastischen Matrizen. Mit den Zerlegungen

$$
\mathcal{S}=\left(\begin{array}{cc}
\boldsymbol{A} & \boldsymbol{B}_{2} \\
\boldsymbol{B}_{1} & \boldsymbol{C}
\end{array}\right) \quad \text { und } \quad \mathcal{F}=\left(\begin{array}{cc}
\boldsymbol{D} & \boldsymbol{S}_{2} \\
\boldsymbol{S}_{1} & \boldsymbol{M}
\end{array}\right)
$$

folgen daraus acht matrixwertige Gleichungen, und zwar vier für die verschwindenden Nebendiagonalen

$$
\begin{aligned}
& \mathbf{0}=\boldsymbol{A} \boldsymbol{S}_{2}+\boldsymbol{B}_{2} \boldsymbol{M} \\
& \mathbf{0}=\boldsymbol{S}_{1} \boldsymbol{A}+\boldsymbol{M} \boldsymbol{B}_{1} \\
& \mathbf{0}=\boldsymbol{D} \boldsymbol{B}_{2}+\boldsymbol{S}_{2} \boldsymbol{C} \\
& \mathbf{0}=\boldsymbol{B}_{1} \boldsymbol{D}+\boldsymbol{C} \boldsymbol{S}_{1}
\end{aligned}
$$


und vier für die Hauptdiagonalen

$$
\begin{aligned}
\boldsymbol{I} & =\boldsymbol{B}_{1} \boldsymbol{S}_{2}+\boldsymbol{C} \boldsymbol{M} \\
\boldsymbol{I} & =\boldsymbol{D} \boldsymbol{A}+\boldsymbol{S}_{2} \boldsymbol{B}_{1} \\
\boldsymbol{I} & =\boldsymbol{S}_{1} \boldsymbol{B}_{2}+\boldsymbol{M} \boldsymbol{C} \\
\boldsymbol{I} & =\boldsymbol{A} \boldsymbol{D}+\boldsymbol{B}_{2} \boldsymbol{S}_{1} .
\end{aligned}
$$

Unter der Annahme, daß $\boldsymbol{M}$ und $\boldsymbol{A}$ für sich invertierbar sind, folgen daraus zunächst die Beziehungen

$$
\begin{array}{lll}
\boldsymbol{S}_{2} \boldsymbol{M}^{-1} & \stackrel{(2.15)}{=} & -\boldsymbol{A}^{-1} \boldsymbol{B}_{2} \\
\boldsymbol{M}^{-1} \boldsymbol{S}_{1} \stackrel{(2.16)}{=} & -\boldsymbol{B}_{1} \boldsymbol{A}^{-1}
\end{array}
$$

und mit ihnen weiter

$$
\begin{array}{rll}
M^{-1} & \stackrel{(2.19)}{=} & C+B_{1} S_{2} M^{-1} \stackrel{(2.23)}{=} C-B_{1} A^{-1} B_{2} \\
A^{-1} & \stackrel{(2.20)}{=} & D+S_{2} B_{1} A^{-1} \stackrel{(2.24)}{=} D-S_{2} M^{-1} S_{1} .
\end{array}
$$

Damit ist je eine weitere transformationsinvariante Blockmatrix gefunden, die durch die Blöcke der Matrix selbst ausgedrückt werden kann. Im Fall der Matrix $\boldsymbol{D}-\boldsymbol{S}_{2} \boldsymbol{M}^{-1} \boldsymbol{S}_{1}$ ist gegenüber $\boldsymbol{M}$ wegen der experimentellen Schwierigkeiten beim Einstellen der Kraftwirkungslinien mit einem ungünstigeren Eingangsfehler zu rechnen.

Die Matrix $\boldsymbol{M}^{-1}$ ist genau das sog. Schur-Komplement von $\mathcal{S}$. Mit dem Invertierungslemm $2^{13}$ kann man nun auch weitere Blöcke der zueinander inversen Matrizen $\mathcal{S}$ und $\mathcal{F}$ durch die der jeweils anderen Matrix ausdrücken. Der Vollständigkeit halber möchte ich noch die restlichen Komponenten der Flexibilitätsmatrix durch die der für die Theorie natürlicheren Steifheitsmatrix (vgl. 2.2.3) ausdrücken. Neben (2.25) sind das noch:

$$
\begin{aligned}
& S_{1} \stackrel{(2.16)}{=} \quad M B_{1} A^{-1} \stackrel{(2.25)}{=}\left(C-B_{1} A^{-1} B_{2}\right)^{-1} B_{1} A^{-1} \\
& \boldsymbol{S}_{2} \stackrel{(2.15)}{=}-\boldsymbol{A}^{-1} \boldsymbol{B}_{2} \boldsymbol{M} \stackrel{(2.25)}{=}-\boldsymbol{A}^{-1} \boldsymbol{B}_{2}\left(\boldsymbol{C}-\boldsymbol{B}_{1} \boldsymbol{A}^{-1} \boldsymbol{B}_{2}\right)^{-1} \\
& D \stackrel{(2.20)}{=} A^{-1}-S_{2} B_{1} A^{-1} \\
& \boldsymbol{D} \stackrel{\sqrt{2.28})}{=} A^{-1}-\boldsymbol{A}^{-1} \boldsymbol{B}_{2}\left(\boldsymbol{C}-\boldsymbol{B}_{1} \boldsymbol{A}^{-1} \boldsymbol{B}_{2}\right)^{-1} \boldsymbol{B}_{1} \boldsymbol{A}^{-1} \text {. }
\end{aligned}
$$

Setzt man ferner die Invertierbarkeit von $C$ voraus, so erhält man mit einer zu (2.25) bzw. (2.29) analogen Rechnung einen weiteren Ausdruck für $\boldsymbol{D}$

$$
\boldsymbol{D}^{-1}=\boldsymbol{A}-\boldsymbol{B}_{2} \boldsymbol{C}^{-1} \boldsymbol{B}_{1}
$$

und erhält durch Gleichsetzen mit (2.29) die Identität von Schur, Frobenius und WoodBury, vgl. wieder [73] §22.5.

${ }^{13}$ Vgl. etwa [3], Teil 1: Grundlagen, $§ 22.4$ 


\subsubsection{Das elastische Potential}

Für HookEsche Verhältnisse, also falls das verallgemeinerte HookEsche Gesetz

$$
\sigma_{i j}=\Lambda_{i j k l} \epsilon_{k l}
$$

der Situation angemessen ist, existiert ein elastisches Potential $\Phi$ mit

$$
\Phi=\frac{1}{2} \int \sigma_{i j} \epsilon_{i j} d V=\frac{1}{2} \int \Lambda_{i j k l} \epsilon_{i j} \epsilon_{k l} d V
$$

Da der Dehnungstensor $\boldsymbol{\epsilon}$ stetig von den Randwerten, also von den Lagen $q$, abhängt, existiert ein funktionaler Zusammenhang $\Phi(q)$ zwischen Potential und Randwert, so daß die elastische Energie nur von der Lagedifferenz abhängt, also

$$
\Phi\left(\left(q_{2}\right)_{A}\right)-\Phi\left(\left(q_{1}\right)_{A}\right)=\int_{\left(\left(q_{1}\right)_{A}\right)}^{\left(\left(q_{2}\right)_{A}\right)} f_{A}^{T} d q_{A}
$$

Wegen

$$
f_{A}^{T} d q_{A}=\left(\mathcal{T}_{A B} f_{B}\right)^{T} \mathcal{T}_{B A}^{T} d q_{B}=f_{B}^{T} \underbrace{\mathcal{T}_{A B}^{T} \mathcal{T}_{B A}^{T}}_{=\mathcal{I}} d q_{B}=f_{B}^{T} d q_{B}
$$

hängt der Integrand nicht vom speziellen Koordinatensystem ab. Daher ist die Differenz der potentiellen Energien $\Phi\left(q_{2}\right)-\Phi\left(q_{1}\right)$ nur eine Funktion der äquivalenten Last- bzw. Lagesysteme; insbesondere folgt bei Existenz eines Potentials $\oint f^{T} d q=0$, d. h. es tauchen nur Hysteresen mit verschwindender Fläche auf. Bei dieser Herleitung wurde benutzt, daß das $d$ der Integration eine kleine Größe $\delta$ im Sinne der Transformation (2.3) ist.

Die Last $f$ läßt sich nun mit dem Potential über

$$
f_{A}^{T}=-\frac{\partial \Phi}{\partial q_{A}}
$$

berechnen ${ }^{14}$ Auch diese Formel ist konsistent mit dem Transformationsverhalten, denn es gilt

$$
\frac{\partial \Phi}{\partial q_{B}}=\left(\frac{\partial q_{B}}{\partial \Phi}\right)^{-1}=\left(\frac{\mathcal{T}_{A B}^{T} \partial q_{A}}{\partial \Phi}\right)^{-1}=\left(\frac{\partial q_{A}}{\partial \Phi}\right)^{-1}\left(\mathcal{T}_{A B}^{T}\right)^{-1}=\frac{\partial \Phi}{\partial q_{A}} \mathcal{T}_{B A}^{T}
$$

also $f_{B}=\mathcal{T}_{B A} f_{A}$. Wieder wurde benutzt, daß $\partial$ eine kleine Größe $\delta$ im Sinne der Transformation (2.3) beschreibt.

Aus diesem Zusammenhang läßt sich mit der Vertauschbarkeit der zweiten Ableitungen die Symmetrie der elastischen Matrizen $\mathcal{F}_{A A}$ und $\mathcal{S}_{A A}$ wie folgt ableiten:

$$
\left(\mathcal{S}_{A A}\right)_{i j}=\frac{\partial\left(f_{A}\right)_{i}}{\partial\left(q_{A}\right)_{j}}=-\frac{\partial \frac{\partial \Phi}{\partial\left(q_{A}\right)_{i}}}{\partial\left(q_{A}\right)_{j}}=-\frac{\partial^{2} \Phi}{\partial\left(q_{A}\right)_{i} \partial\left(q_{A}\right)_{j}}=-\frac{\partial^{2} \Phi}{\partial\left(q_{A}\right)_{j} \partial\left(q_{A}\right)_{i}}=\left(\mathcal{S}_{A A}\right)_{j i}
$$

\footnotetext{
${ }^{14}$ In der Technischen Mechanik ist dieser Zusammenhang auch als Satz von CAstigLiAnO bekannt, vgl. etwa 68, I. $§ 26$.
} 
Zusammen mit dem Transformationsverhalten der Lasten und Lagen (2.1) und (2.3) ergibt sich

$$
\mathcal{S}_{A B}^{T}=\left(\mathcal{S}_{A A} \mathcal{T}_{B A}^{T}\right)=\left(\mathcal{T}_{B A}^{T}\right)^{T} \mathcal{S}_{A A}^{T}=\mathcal{T}_{B A} \mathcal{S}_{A A}=\mathcal{S}_{B A}
$$

Diese Symmetrie ist Inhalt des Satzes von Maxwell und BetTi, auch Reziprozitätssatz von MAXwELL genannt!15 Sie besagt anschaulich, daß beim Anbringen der Lasten im Referenzsystem der Lagen und Messen der Lagen im Referenzsystem der Lasten die elastischen Eigenschaften dieses Systems in Form der elastischen Konstanten beibehalten werden. Bei einer Feder etwa entspricht das genau dem Vertauschen von Anfang und Ende. Da im linearen Input-Output-Modell Input und Output je ein Matrixindex zugeordnet ist, beschreibt die transponierte Matrix genau deren Vertauschen.

Da Invertierung und Transformation verträglich sind, gilt auch für die Flexibilitätsmatrix im Punkt $A\left(\mathcal{F}_{A A}\right)_{i j}=\left(\mathcal{F}_{A A}\right)_{j i}$. Auch diese Symmetrie ist wegen

$$
\mathcal{F}_{B B}=\mathcal{T}_{A B}^{T} \mathcal{F}_{A A} \mathcal{T}_{A B}=\mathcal{T}_{A B}^{T} \mathcal{F}_{A A}^{T} \mathcal{T}_{A B}=\mathcal{F}_{B B}^{T}
$$

verträglich mit dem Transformationsverhalten. Bei Messungen hat man bezüglich dieser Symmetrie auf die Einheiten besonders zu achten, denn es muß $\boldsymbol{T}^{T}=-\frac{\partial \Phi}{\partial \boldsymbol{\theta}}$ gelten. Die zum Drehmoment in $N m$ passende SI-Einheit ist der Radiant. Entsprechend müssen Vorsätze oder Vorfaktoren wie bei deg oder gon als Kehrwert bei den Drehmomenten berücksichtigt werden.

Wieder ist die Steifheitsmatrix gegenüber der Flexibilitätsmatrix vom theoretischen Standpunkt her bevorzugt, da sie die zweite Ableitung des elastischen Potentials nach den verallgemeinerten Koordinaten ist.

\subsubsection{Das Widerstandszentrum}

Für den Fall einer eben aufgehängten Scheibe ist bekannt, daß ein Mittelpunkt der Federkräfte existiert, mit der Eigenschaft,

„... daß bei einer Parallelverschiebung der Scheibe die Resultierende der Federkräfte durch ihn geht und daß bei einer Drehung der Scheibe um diesen Punkt die Federkräfte ein Kräftepaar bilden. ‘[16

Wegen der Analogie dieser Eigenschaften zu denen des Schwerpunktes eines starren Körpers heißt er auch elastischer Schwerpunkt, siehe wieder [34]. Im Rahmen meines dreidimensionalen Formalismus läßt sich dieses Ergebnis für ebene

\footnotetext{
${ }^{15}$ Vgl. wieder 68, I. $\S 26$.

${ }^{16}$ Zitat aus 34, Kap. 3.12 S.144. In der Einleitung 1.1] findet sich der Beweis dazu.
} 
Aufhängungen mit einer passenden Definition für diesen ebenen elastischen Schwerpunkt nachvollziehen, vgl. (B.2). Dieser in der englischsprachigen Literatur "center of resistance" genannte Punkt, das Widerstandszentrum, ist der Prototyp des für die Vergleichbarkeit von Messungen nötigen Referenzsystems.

Es wird sich zeigen, daß in drei Dimensionen kein Punkt mehr existiert, der die Eigenschaften des ebenen Widerstandszentrums hat. Das äußert sich in verschiedenen Unstimmigkeiten, in die man gerät, wenn man nach einem Punkt mit den gewünschten Eigenschaften sucht. FISH [17] drückt sich in seiner Definition tatsächlich vorsichtiger aus. An einem in der Mesialebene betrachteten einwurzeligen Zahn stellt er fest:

"Let us suppose that a horizontal force is to be applied toward the cheek. There is a point $C$ somewhere between the apex and the gingival margin, such that if the force be applied on a line passing through that point there will be no tipping and and no rotation of the tooth about its long axis. This point may be called the center of resistance. It is undoubtedly somewhere between the gingival margin and the point half way to the apex. Of course this point may vary with variation of the intensity of force or with change in direction of application of the force."

Die beiden ausgeschlossenen Rotationen sind senkrecht zur Richtung der Kraft gemeint, d. h. mit der Gleichung $\boldsymbol{F} \times \delta \boldsymbol{\theta}(\boldsymbol{F}, \boldsymbol{r})=\mathbf{0}$ wird implizit ein Ort $\boldsymbol{r}$ in Abhängigkeit von der Kraft $\boldsymbol{F}$ definiert. Diese drei Gleichungen für drei Unbekannte definieren eine Punktmenge, die mit meiner Abstandsfläche übereinstimmt, vgl. Abschnitt 2.3.2.

\subsubsection{Gewünschte Eigenschaften des Widerstandszentrums}

Es liegt nahe, für eine räumliche Aufhängung zunächst eine direkte Übertragung dieser Definition zu versuchen. Man sucht analog zum zweidimensionalen Fall nach einem Punkt $W$ mit den folgenden Eigenschaften

1. Eine reine Kraft (also eine Kraftschraube mit verschwindendem Drehmomentanteil) mit Wirkungslinie durch das Widerstandszentrum $W$ hat immer eine reine Translation zur Folge;

2. ein reines Drehmoment (also eine Drehschraube mit verschwindendem Translationsanteil) hat eine reine Rotation mit durch das Widerstandszentrum $W$ verlaufender Drehachse zur Folge.

Kurz gesagt soll gelten: 
1. Für alle Kraftwirkungslinien durch denselben Punkt $W$ gilt

$$
\boldsymbol{T}(W)=\mathbf{0} \Leftrightarrow \boldsymbol{\theta}=\mathbf{0} .
$$

2. Für alle Drehungen durch denselben Punkt $W$ gilt

$$
\boldsymbol{\delta}(W)=\mathbf{0} \quad \Leftrightarrow \quad \boldsymbol{F}=\mathbf{0} .
$$

Noch kürzer: Polare und axiale Vektoren entkoppeln im Sinne des Input-OutputModells.

Die Flexibilitätsmatrix hat wegen dieser Entkopplung in $W$ als lineare Abbildung zwischen $f$ und $q$ verschwindende Nebendiagonalelemente. Sie liegt daher in Blockdiagonalform

$$
\mathcal{F}_{W W}=\left(\begin{array}{cc}
\boldsymbol{D} & \mathbf{0} \\
\mathbf{0} & \boldsymbol{M}
\end{array}\right)
$$

vor. Nicht verschwindende Nebendiagonalmatrizen stehen im Widerspruch zu den obigen Wunscheigenschaften.

Die wichtigste aus der Existenz eines solchen Punktes $W$ ableitbare Eigenschaft ist das Reziprozitätstheorem: Für zwei Experimente ohne externe Drehmomente mit gleichen Kräften zu verschiedenen Angriffspunkten $K$ und $K^{\prime}$ mit

$$
\overrightarrow{W K^{\prime}}=\lambda \overrightarrow{W K}
$$

also bei Verlängerung des Hebels um den Faktor $\lambda$, gilt

$$
\boldsymbol{T}^{\prime}=\overrightarrow{W K^{\prime}} \times \boldsymbol{F}=\lambda \overrightarrow{W K} \boldsymbol{F}=\lambda \boldsymbol{T} .
$$

Die Ortsvektoren $\overrightarrow{W K^{\prime}}$ und $\overrightarrow{W K}$ sind gleichzeitig die Aufpunkte der Kraftschraube mit kürzestem Abstand zum Widerstandszentrum. Wegen (2.33) verlängern sich die axialen Vektoren $\boldsymbol{T}$ und damit $\boldsymbol{\theta}$ jeweils um den Faktor $\lambda$, denn aus

$$
\left(\begin{array}{c}
\boldsymbol{F}^{\prime} \\
\boldsymbol{T}^{\prime}
\end{array}\right)=\left(\begin{array}{c}
\boldsymbol{F} \\
\lambda \boldsymbol{T}
\end{array}\right)
$$

folgt

$$
\left(\begin{array}{c}
\delta \boldsymbol{d}^{\prime} \\
\delta \boldsymbol{\theta}^{\prime}
\end{array}\right)=\left(\begin{array}{cc}
\boldsymbol{D} & \mathbf{0} \\
\mathbf{0} & \boldsymbol{M}
\end{array}\right) \cdot\left(\begin{array}{c}
\boldsymbol{F}^{\prime} \\
\boldsymbol{T}^{\prime}
\end{array}\right)=\left(\begin{array}{cc}
\boldsymbol{D} & \mathbf{0} \\
\mathbf{0} & \boldsymbol{M}
\end{array}\right) \cdot\left(\begin{array}{c}
\boldsymbol{F} \\
\lambda \boldsymbol{T}
\end{array}\right)=\left(\begin{array}{c}
\delta \boldsymbol{d} \\
\lambda \delta \boldsymbol{\theta}
\end{array}\right) .
$$

Wegen (2.5) gilt für die Aufpunkte $D$ und $D^{\prime}$ auf die momentane Achsen der Drehschrauben mit kürzestem Abstand zum Widerstandszentrum

$$
\overrightarrow{W D^{\prime}}=\frac{1}{\left|\delta \boldsymbol{\theta}^{\prime}\right|^{2}}\left(\delta \boldsymbol{\theta}^{\prime} \times \delta \boldsymbol{d}\right)=\frac{1}{\lambda} \overrightarrow{W D}
$$

Damit folgt die Invarianz des Skalarproduktes der Ortsvektoren der Schraubachsaufpunkte:

$$
\overrightarrow{W K^{\prime}} \cdot \overrightarrow{W D^{\prime}}=\overrightarrow{W K} \cdot \overrightarrow{W D}
$$

wobei diese Eigenschaft für jede multilineare Abbildung von $\overrightarrow{W K^{\prime}}$ und $\overrightarrow{W D^{\prime}}$, also etwa auch für deren Kreuzprodukt gilt. 


\subsubsection{Unstimmigkeiten mit einem solchen Widerstandszentrum}

Die Realität ist leider komplexer, denn man hat Folgendes zu berücksichtigen:

1. Allein der etwaigen Meßfehler wegen müssen diese Gleichungen nicht mehr exakt erfüllbar sein, denn das Gleichungssystem für die Vektoren ist mit zwei vektorwertigen, also sechs Gleichungen für einen Ortsvektor, also drei Größen, überbestimmt. Falls keine Lösung existiert, läßt sich auch kein Punkt der elastischen Aufhängung mehr auszeichnen.

2. Um die beiden Nebendiagonalblockmatrizen mit je 9 Einträgen durch eine Transformation der Referenzkoordinatensysteme (2.1) und (2.3) zum Verschwinden zu bewegen, müßte man 18 Gleichungen durch 6 Größen erfüllen. Auch das ist ein überbestimmtes Gleichungssystem.

3. Im Unterschied zum Schwerpunkt ist hier die zur Masse analoge Größe einer elastischen Aufhängung ein Tensor zweiter Stufe, vgl. 4.1. Darin entspricht die formale mathematische Situation, bis auf die Signatur der Metrik und die Dimension, der in der Allgemeinen Relativitätstheorie. Von dort war mir bekannt, daß es problematisch sein würde, über den der Masse entsprechenden Energieimpulstensor einen Schwerpunkt zu definieren. Schon in der Speziellen Relativitätstheorie existieren keine starren Objekte mehr, und in der Allgemeinen Relativitätstheorie sind integrale Erhaltungsgrößen mit Mühe und manchmal nur unter Preisgabe der Kovarianz aufzufinden. Von Anfang an bestanden also Zweifel an der Existenz eines Widerstandszentrums.

4. Der Schluß vom Zwei- aufs Dreidimensionale ist auch vom Standpunkt der Analogie zur Bewegung gewagt: Da aus dem Momentanpol der ebenen Kinematik, einem Punkt also, in der räumlichen Kinematik eine momentane Schraubachse wird, ist kaum zu erwarten, daß der für eine ebene Aufhängung definierte elastische Schwerpunkt auch im räumlichen Fall als Punkt existiert. Ein analoges Objekt könnte schlicht die Eigenschaft verlieren, ein Punkt zu sein.

5. Es gibt Aufhängungen ohne ein solches Widerstandszentrum. Ein Beispiel ist eine Aufhängung aus zwei Federpaaren mit windschiefen Wirkungslinien der Kräfte der Einzelfedern, wobei der starre Körper jeweils zwischen den einzelnen Federn liegt, vgl. die Abb. 2.3 und 2.4: Genaueres in Kap. 5.1.4.

Die Existenz eines Punktes mit den Eigenschaften des Widerstandszentrums ist also nur eine Simplifizierung, die im dreidimensionalen Fall nichts mit der Realität zu tun haben muß.

Der Übergang von einer ebenen Aufhängung zu einer echt räumlichen geschieht mittels bestimmter Symmetrien. In Abschnitt 4.5 wird gezeigt, daß für bestimmte 


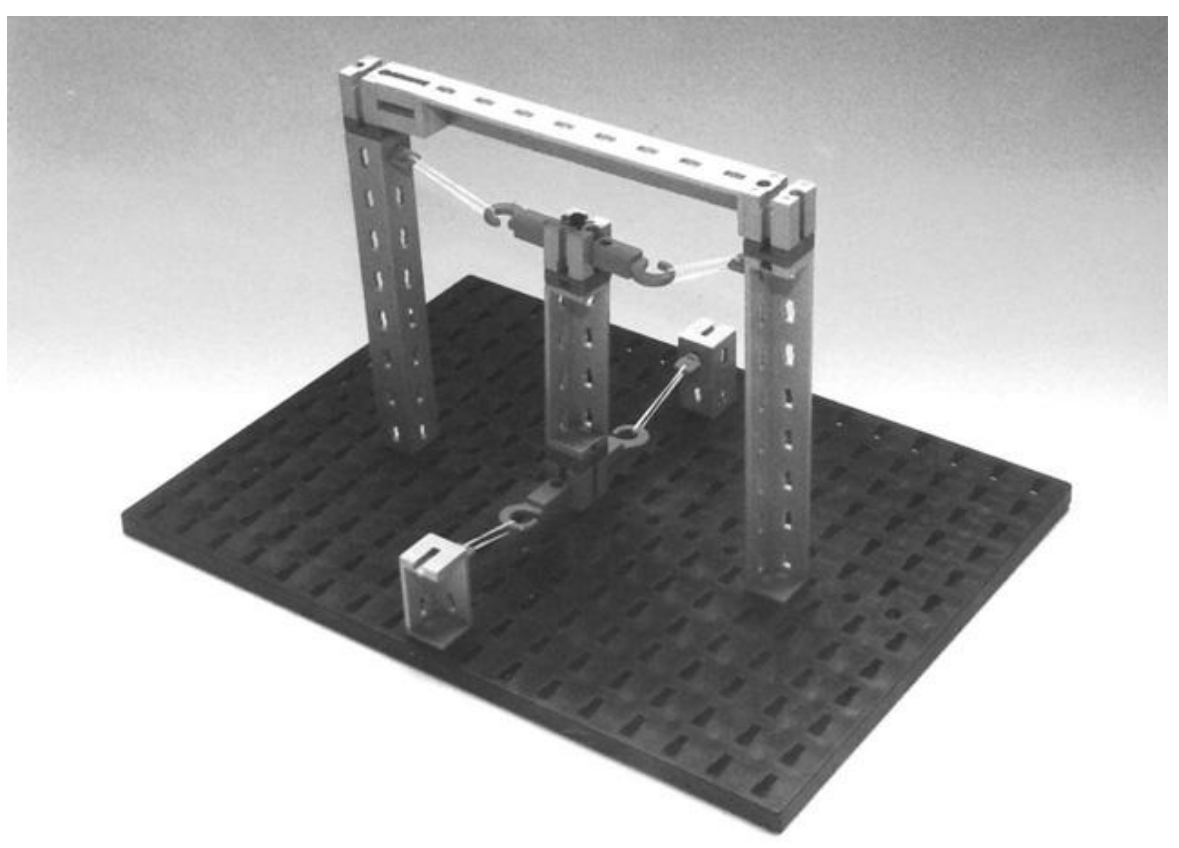

Abbildung 2.3: Das Schaumodell zur Nichtexistenz eines Widerstandszentrums. Die wesentliche Konstruktionsidee ist eine echt räumliche Aufhängung aus Federn mit windschiefen Wirkungslinien.

Symmetrien doch wieder ein Widerstandszentrum existiert. Da in vielen praktischen Fällen solche Symmetrien entweder exakt oder wenigstens näherungsweise vorliegen, bleibt das Widerstandszentrum eine oft zutreffende oder für praktische Belange ausreichende Vorstellung zur Beschreibung der hauptsächlichen Effekte einer elastischen Aufhängung. Um die Empfindlichkeit dieses Konzeptes gegenüber Störungen zu untersuchen, bedarf es einer übergreifenden quantitativen Theorie, also dieser Arbeit.

\subsubsection{Das elastische Zentrum}

Die Bedingungen (2.31) und (2.32) zur Definition eines ausgezeichneten Koordinatensystems müssen abgeschwächt werden. Da in einem Punkt nicht mehr beide polare bzw. axiale Vektoren gleichzeitig verschwinden können, folgt: einer ist exakt gleich Null und der andere ist betragsmäßig minimal. Das derart definierte Objekt bekommt zur begrifflichen Abgrenzung vom Widerstandszentrum einen neuen Namen: Das elastische Zentrum. Zur Unterscheidung vom Widerstandszentrum $W$ soll für das elastische Zentrum auch im Folgenden der Buchstabe $Z$, wie Zentrum, verwendet werden.

Die grundlegende Idee ist es, den linienflüchtigen Vektor gleich Null zu setzen und den Betrag seines freien Partners zu minimieren. Wenn man statt dessen den 

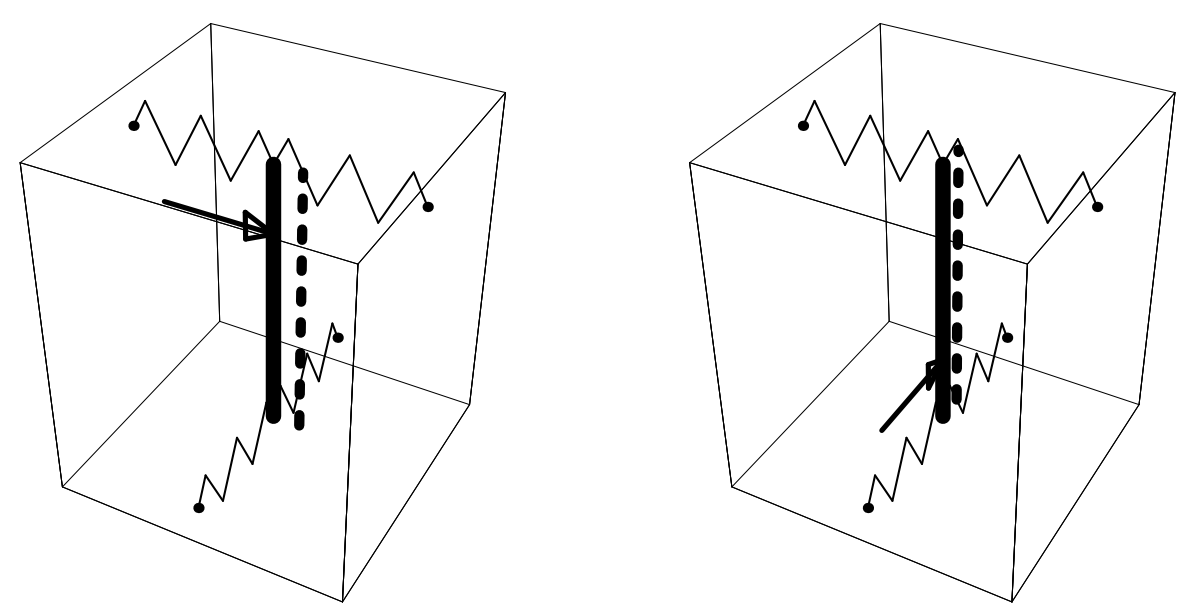

Abbildung 2.4: Der Schauversuch zur Nichtexistenz eines Widerstandszentrums. Eine Kraft in $x$-Richtung, die eine reine Translation hervorruft, und eine Kraft in $y$-Richtung, die ebenfalls eine reine Translation hervorruft, haben windschiefe Wirkungslinien. Die gestrichelte Linie ist die jeweilige Endlage des Stabes.

freien Vektor gleich Null setzen würde, wären zwei linienflüchtige Vektoren, also Geraden im Raum, an der Definition beteiligt, was später mathematische Probleme verursacht. Durch das Transformationsverhalten des freien Vektors wird dann je ein Punkt ausgezeichnet, einer vom Lastangriffspunkt $L$ und einer vom Meßpunkt $M$ aus.

Dadurch, daß ein linienflüchtiger Vektor gleich Null ist, folgt eine Beziehung zwischen den beiden Vektoren des anderen Systems. Der freie Vektor kann also in Abhängigkeit von seinem linienflüchtigen Partner geschrieben werden. Danach minimiert man seine Norm mit den Komponenten des Ortsvektors als Variablen. Da der Betrag des freien Vektors auch von dem Betrag seines linienflüchtigen Partners abhängt, ist dieser bei der Minimierung konstant zu halten. Für die Rechnung verwendet man demnach Einheitsvektoren.

\subsubsection{Ein Minimierungsproblem für freie Vektoren}

$\mathrm{Zu}$ lösen sind also

$$
|\delta \boldsymbol{d}(Z)|=\min _{Z} ! \quad \text { für } \quad \boldsymbol{F}=\mathbf{0}
$$

für das Meßsystem, also mit $\delta \boldsymbol{\theta}$ als Variable, und

$$
|\boldsymbol{T}(Z)|=\min _{Z} ! \quad \text { mit } \quad \delta \boldsymbol{\theta}=\mathbf{0}
$$

für das Lastsystem, also mit $\boldsymbol{F}$ als Variable. Nach den minimierten Größen sollen die zu den Lösungen gehörenden Punkte Translationszentrum $T$ und Drehmomentszentrum $D$ genannt werden. 
Die Komponenten von $q=\mathcal{F} f$ lauten mit (2.10) für die durch den experimentellen Aufbau festgelegten Koordinatensysteme Meßsystem $M$ und Lastsystem $L$

$$
\begin{aligned}
\delta \boldsymbol{d}_{M} & =\boldsymbol{D} \boldsymbol{F}_{L}+\boldsymbol{S}_{2} \boldsymbol{T}_{L} \\
\delta \boldsymbol{\theta}_{M} & =\boldsymbol{S}_{1} \boldsymbol{F}_{L}+\boldsymbol{M} \boldsymbol{T}_{L}
\end{aligned} .
$$

Es ergibt sich für $\boldsymbol{F}_{L}=\mathbf{0}$ zunächst $\delta \boldsymbol{\theta}_{M}=\boldsymbol{M} \boldsymbol{T}_{L}$ und damit $\delta \boldsymbol{d}_{M}=\boldsymbol{S}_{2} \boldsymbol{T}_{L}=$ $\boldsymbol{S}_{2} \boldsymbol{M}^{-1} \delta \boldsymbol{\theta}_{M}$ und weiter mit (2.3)

$$
\delta \boldsymbol{d}(D)=\delta \boldsymbol{d}_{L}+\boldsymbol{\Omega}_{D M} \delta \boldsymbol{\theta}_{M}=\left(\boldsymbol{S}_{2} \boldsymbol{M}^{-1}+\boldsymbol{\Omega}_{D M}\right) \delta \boldsymbol{\theta} .
$$

Für $\delta \boldsymbol{\theta}=\mathbf{0}$ ergibt sich ähnlich $\mathbf{0}=\boldsymbol{S}_{1} \boldsymbol{F}_{L}+\boldsymbol{M} \boldsymbol{T}_{L}$ und dann mit (2.1)

$$
\boldsymbol{T}(T)=\boldsymbol{T}_{L}+\boldsymbol{\Omega}_{T L} \boldsymbol{F}_{L}=\left(-\boldsymbol{M}^{-1} \boldsymbol{S}_{1}+\boldsymbol{\Omega}_{T L}\right) \boldsymbol{F} .
$$

Beide Minimierungsaufgaben sind damit vom Typ

$$
|\boldsymbol{x}(Z)|=\left|\left(\boldsymbol{A}+\boldsymbol{\Omega}_{Z}\right) \boldsymbol{y}\right|=\min _{Z} !,
$$

wobei $\boldsymbol{x}$ der freie Vektor, $\boldsymbol{y}$ der linienflüchtige Vektor und $\boldsymbol{A}$ die entsprechende Matrix sei. Wegen der Linearität der beteiligten Abbildungen ist die Norm des linienflüchtigen Vektors für das Minimierungsproblem unwichtig. Danach hat man sich noch von dem für das eigentliche Problem unwichtigen linienflüchtigen Einheitsvektors zu entledigen, genauer von dessen Richtung. Das geschieht mit der zusätzlichen Forderung nach Isotropie, bei der alle Raumrichtungen gleich stark beteiligt sind, durch die Mittelung

$$
\int_{|\boldsymbol{y}|=1}\left|\left(\boldsymbol{A}+\boldsymbol{\Omega}_{Z}\right) \boldsymbol{y}\right|^{2} d o=\min _{Z} !,
$$

über die Oberfläche der Einheitskugel mit dem Oberflächenelement do. Die Rechnung wird zweckmäßigerweise in Polarkoordinaten mit

$$
\boldsymbol{y}=\left(\begin{array}{c}
\sin \vartheta \cos \phi \\
\sin \vartheta \sin \phi \\
\cos \vartheta
\end{array}\right) \text { und } d o=\sin \vartheta d \vartheta d \phi
$$

in den Variablen $\vartheta$ und $\phi$ durchgeführt. Der Rechengang vereinfacht sich erheblich, wenn man erst nach den Komponenten von $\boldsymbol{x}$ differenziert und dann die Integration ausführt. Mit $\left(\boldsymbol{\Omega}_{x}\right)_{i j}=\varepsilon_{i k j} x_{k}$ erhält man durch Anwenden von $\frac{\partial}{\partial x_{k}}$ auf (2.36) mit der Kettenregel die Gleichung

$$
\begin{aligned}
0 & =\int_{|\boldsymbol{y}|=1}\left(\left(\boldsymbol{A}+\boldsymbol{\Omega}_{Z}\right) \boldsymbol{y}\right)_{i} y_{j} \varepsilon_{i k j} d o \\
& =\left(\boldsymbol{A}+\boldsymbol{\Omega}_{Z}\right)_{i l} \varepsilon_{i k j} \underbrace{\int_{|\boldsymbol{y}|=1} y_{l} y_{j} d o}_{=\frac{4 \pi}{3} \delta_{l j}},
\end{aligned}
$$


wobei das Ergebnis der Integration aus Isotropiegründen natürlich ein Vielfaches der Einheitsmatrix ist. Nach Ausführen der Kontraktion ergibt sich aus der Gleichung $\mathbf{0}=\left(\boldsymbol{A}+\boldsymbol{\Omega}_{Z}\right)_{i l} \varepsilon_{i k l}$ schließlich

$$
\Omega_{Z}=-\boldsymbol{A}^{A} \quad \text { bzw. } \quad \overrightarrow{O Z}=-\boldsymbol{A}^{D} .
$$

Die Minimierung der EUKLIDischen Matrixnorm von $\boldsymbol{A}+\boldsymbol{\Omega}_{Z}$ liefert dasselbe Ergebnis wie die integrale Betrachtungsweise, wie die folgende Rechnung zeigen soll. Von $\boldsymbol{y}$ entledigt man sich jetzt mit Hilfe einer zur EuKLIDischen Vektornorm, der in der Physik üblichen Länge eines Vektors, verträglichen Matrixnorm über

$$
\left|\left(\boldsymbol{A}+\Omega_{Z}\right) \boldsymbol{y}\right| \leq\left\|\boldsymbol{A}+\Omega_{Z}\right\| \cdot|\boldsymbol{y}|
$$

und minimiert fortan nur noch $\left\|\boldsymbol{A}+\boldsymbol{\Omega}_{Z}\right\|$. Da alle in Frage kommenden Normen äquivalent sind, d. h. durch Konstanten untereinander abgeschätzt werden können, fällt die Wahl der Norm aus Gründen der Einfachheit auf die EUKLIDische Matrixnorm

$$
\|\boldsymbol{A}\|=\sqrt{\sum_{i, j} a_{i j}^{2}}=\sqrt{S p\left(\boldsymbol{A}^{T} \boldsymbol{A}\right)} .
$$

Ableitungen dieser Norm besitzen lästige Terme mit Wurzeln; wird stattdessen ihr Quadrat minimiert, so kommen wegen $\partial_{x}\|*\|^{2}=2\|*\| \cdot \partial_{x}\|*\|$ höchstens neue Lösungen hinzu. Für Summen von solchen Termen gilt das natürlich nicht. Durch Differentiation nach den Koordinaten von $Z$ ergibt sich (die innere Ableitung von $\Omega_{Z}$ ist der $\boldsymbol{\varepsilon}$-Tensor) als Lösung des Problems der antisymmetrische Anteil der Matrix, gekennzeichnet durch den oberen Index A, also $*^{A}:=\frac{1}{2}\left(*-*^{T}\right)$. Das läßt sich auch wie folgt einsehen: Mit dem symmetrischen Anteil $*^{S}:=\frac{1}{2}\left(*+*^{T}\right)$ zerlegt man eine Matrix durch

$$
*=*^{S}+*^{A} \text {. }
$$

Für die EUKLIDische Norm folgt dann

$$
\begin{aligned}
\|\boldsymbol{A}\|^{2} & =\left\|\boldsymbol{A}^{S}+\boldsymbol{A}^{A}\right\|^{2}=\sum_{i, j}\left[\frac{1}{2}\left(a_{i j}+a_{j i}\right)+\frac{1}{2}\left(a_{i j}-a_{j i}\right)\right]^{2} \\
& =\left\|\boldsymbol{A}^{S}\right\|^{2}+\left\|\boldsymbol{A}^{A}\right\|^{2}+\frac{1}{4} \sum\left(a_{i j}+a_{j i}\right)\left(a_{i j}-a_{j i}\right) \\
& =\left\|\boldsymbol{A}^{S}\right\|^{2}+\left\|\boldsymbol{A}^{A}\right\|^{2}+\frac{1}{4} \sum\left(a_{i j}^{2}-a_{j i}^{2}\right) \\
& =\left\|\boldsymbol{A}^{S}\right\|^{2}+\left\|\boldsymbol{A}^{A}\right\|^{2}
\end{aligned}
$$

was als Satz des Pythagoras für Matrizen gesehen werden kann. Die antisymmetrische Matrix $\Omega_{Z}$ wirkt nur auf $\boldsymbol{A}^{A}$ und kann daher diesen (und nur diesen) Anteil der Norm zu exakt Null machen, es gilt also wieder (2.37).

Die Ergebnisse der obigen Minimierungen, hier noch einmal tabellarisch zusammengefaßt 


\begin{tabular}{c|c|c} 
System & $\begin{array}{c}\text { minimaler } \\
\text { freier Vektor }\end{array}$ & $\begin{array}{c}\text { reine } \\
\text { Größe }\end{array}$ \\
\hline Meßsystem $M$ & $\delta \boldsymbol{d}(Z)$ & $\boldsymbol{T}(\Leftrightarrow \boldsymbol{F}=\mathbf{0})$ \\
Lastsystem $L$ & $\boldsymbol{T}(Z)$ & $\delta \boldsymbol{d}(\Leftrightarrow \delta \boldsymbol{\theta}=\mathbf{0})$
\end{tabular}

können nun einfach abgelesen werden:

\begin{tabular}{c|c|c} 
Name des & $\begin{array}{c}\text { Ergebnis d. Minimierung } \\
\text { Flexibilitätsmatrix }\end{array}$ & $\begin{array}{c}\text { Ergebnis d. Minimierung } \\
\text { Steifheitsmatrix }\end{array}$ \\
\hline Drehmomentszentrum $D$ & $\boldsymbol{\Omega}_{M D}=\left(\boldsymbol{S}_{2} \boldsymbol{M}^{-1}\right)^{A}$ & $\boldsymbol{\Omega}_{M D}=-\left(\boldsymbol{A}^{-1} \boldsymbol{B}_{2}\right)^{A}$ \\
Translationszentrum $T$ & $\boldsymbol{\Omega}_{L T}=-\left(\boldsymbol{M}^{-1} \boldsymbol{S}_{1}\right)^{A}$ & $\boldsymbol{\Omega}_{L T}=\left(\boldsymbol{B}_{1} \boldsymbol{A}^{-1}\right)^{A}$
\end{tabular}

Dabei wurden (2.23) und (2.24) benutzt.

Im Folgenden sollen die Eigenschaften dieser neu berechneten Objekte untersucht werden. Dazu ist zunächst die Transformation auf das elastische Zentrum durchzuführen.

\subsubsection{Die direkte Transformation auf das elastische Zentrum}

Führt man nun die Transformation mit den obigen Transformationsmatrizen durch, so heben sich einige Terme aus Symmetriegründen weg. Definiert man nun für das Meßzentrum $M$ und das Lastzentrum $L$ die beiden symmetrischen Widerstandsmatrizen

$$
\begin{aligned}
\boldsymbol{W}_{M} & :=\left(\boldsymbol{S}_{2} \boldsymbol{M}^{-1}\right)^{S}=-\left(\boldsymbol{A}^{-1} \boldsymbol{B}_{2}\right)^{S} \\
\boldsymbol{W}_{L} & :=\left(\boldsymbol{M}^{-1} \boldsymbol{S}_{1}\right)^{S}=-\left(\boldsymbol{B}_{1} \boldsymbol{A}^{-1}\right)^{S}
\end{aligned}
$$

so lautet die auf das elastische Zentrum transformierte Matrix mit (2.11) und

$$
\boldsymbol{\Omega}_{M D}=\left(\boldsymbol{S}_{2} \boldsymbol{M}^{-1}\right)^{A}=\boldsymbol{S}_{2} \boldsymbol{M}^{-1}-\left(\boldsymbol{S}_{2} \boldsymbol{M}^{-1}\right)^{S}=\boldsymbol{S}_{2} \boldsymbol{M}^{-1}-\boldsymbol{W}_{M}
$$

sowie

$$
\boldsymbol{\Omega}_{L T}=\left(-\boldsymbol{M}^{-1} \boldsymbol{S}_{1}\right)^{A}=-\boldsymbol{M}^{-1} \boldsymbol{S}_{1}-\left(-\boldsymbol{M}^{-1} \boldsymbol{S}_{1}\right)^{S}=-\boldsymbol{M}^{-1} \boldsymbol{S}_{1}+\boldsymbol{W}_{L}
$$

insgesamt

$$
\begin{aligned}
\mathcal{F}_{D T} & =\mathcal{T}_{M D}^{T} \mathcal{F}_{M L} \mathcal{T}_{L T} \\
& =\left(\begin{array}{cc}
\boldsymbol{D}+\boldsymbol{S}_{2} \boldsymbol{\Omega}_{L T}-\boldsymbol{\Omega}_{M D} \boldsymbol{S}_{1}-\boldsymbol{\Omega}_{M D} \boldsymbol{M} \boldsymbol{\Omega}_{L T} & \boldsymbol{S}_{2}-\boldsymbol{\Omega}_{M D} \boldsymbol{M} \\
\boldsymbol{S}_{1}+\boldsymbol{M} \boldsymbol{\Omega}_{L T} & \boldsymbol{M}
\end{array}\right) \\
& =\left(\begin{array}{cc}
\boldsymbol{D}-\boldsymbol{S}_{2} \boldsymbol{M}^{-1} \boldsymbol{S}_{1}+\boldsymbol{W}_{M} \boldsymbol{M} \boldsymbol{W}_{L} & \boldsymbol{W}_{M} \boldsymbol{M} \\
\boldsymbol{M} \boldsymbol{W}_{L} & \boldsymbol{M}
\end{array}\right)
\end{aligned}
$$


Für HookEsche Verhältnisse ist die Ausgangsmatrix durch eine bloße Transformation der Bezugspunkte aufeinander symmetrisierbar. Für eine solche symmetrische Matrix gilt $\boldsymbol{S}_{2}=\boldsymbol{S}_{1}^{T}$ und $\boldsymbol{M}^{T}=\boldsymbol{M}$, woraus folgt

$$
\begin{aligned}
\boldsymbol{W}_{M} & =\left(\boldsymbol{S}_{2} \boldsymbol{M}^{-1}\right)^{S}=\left(\left(\boldsymbol{S}_{2} \boldsymbol{M}^{-1}\right)^{S}\right)^{T}=\left(\left(\boldsymbol{S}_{2} \boldsymbol{M}^{-1}\right)^{T}\right)^{S}=\left(\left(\boldsymbol{M}^{-1}\right)^{T}\left(\boldsymbol{S}_{2}\right)^{T}\right)^{S} \\
& =\left(\boldsymbol{M}^{-1} \boldsymbol{S}_{1}\right)^{S}=\boldsymbol{W}_{L}=: \boldsymbol{W} .
\end{aligned}
$$

Liegt sogar ein Widerstandszentrum vor, so gilt

$$
\boldsymbol{W}_{M}=\mathbf{0}=\boldsymbol{W}_{L}
$$

Im letzteren Fall sind die Matrizen $\boldsymbol{S}_{2} \boldsymbol{M}^{-1}$ und $\boldsymbol{M}^{-1} \boldsymbol{S}_{1}$ rein antisymmetrisch, und die aufs Widerstandszentrum transformierte Flexibilitätsmatrix lautet nur noch

$$
\mathcal{F}_{W W}=\left(\begin{array}{cc}
\boldsymbol{D}-\boldsymbol{S}_{2} \boldsymbol{M}^{-1} \boldsymbol{S}_{1} & \mathbf{0} \\
\mathbf{0} & \boldsymbol{M}
\end{array}\right) \stackrel{(2.26)}{=}\left(\begin{array}{cc}
\boldsymbol{A}^{-1} & \mathbf{0} \\
\mathbf{0} & \boldsymbol{M}
\end{array}\right) .
$$

Sie enthält nur noch die beiden transformationsinvarianten Blöcke.

Durch eine analoge Rechnung mit (2.14) sowie

$$
\boldsymbol{\Omega}_{M D}=-\boldsymbol{A}^{-1} \boldsymbol{B}_{2}-\boldsymbol{W}_{M} \quad \text { und } \quad \boldsymbol{\Omega}_{L T}=\boldsymbol{B}_{1} \boldsymbol{A}^{-1}+\boldsymbol{W}_{L}
$$

erhält man für die Steifheitsmatrix

$$
\begin{aligned}
\mathcal{S}_{T D} & =\mathcal{T}_{T L} \mathcal{S}_{L M} \mathcal{T}_{D M}^{T} \\
& =\left(\begin{array}{cc}
\boldsymbol{A} & -\boldsymbol{A} \boldsymbol{W}_{M} \\
-\boldsymbol{W}_{L} \boldsymbol{A} & \boldsymbol{C}-\boldsymbol{B}_{1} \boldsymbol{A}^{-1} \boldsymbol{B}_{2}+\boldsymbol{W}_{L} \boldsymbol{A} \boldsymbol{W}_{M}
\end{array}\right)
\end{aligned}
$$

mit den entsprechenden Spezialfällen wie oben.

Mit (2.25) und (2.26) kann den beiden elastischen Matrizen im elastischen Zentrum eine Form gegeben werden, die nur noch aus den transformationsinvarianten Matrizen $\boldsymbol{A}$ und $\boldsymbol{M}$ und den Widerstandsmatrizen $\boldsymbol{W}_{L}$ und $\boldsymbol{W}_{M}$ besteht

$$
\begin{aligned}
\mathcal{F}_{D T} & =\left(\begin{array}{cc}
\boldsymbol{A}^{-1}+\boldsymbol{W}_{M} \boldsymbol{M} \boldsymbol{W}_{L} & \boldsymbol{W}_{M} \boldsymbol{M} \\
\boldsymbol{M} \boldsymbol{W}_{L} & \boldsymbol{M}
\end{array}\right) \\
\mathcal{S}_{T D} & =\left(\begin{array}{cc}
\boldsymbol{A} & -\boldsymbol{A} \boldsymbol{W}_{M} \\
-\boldsymbol{W}_{L} \boldsymbol{A} & \boldsymbol{M}^{-1}+\boldsymbol{W}_{L} \boldsymbol{A} \boldsymbol{W}_{M}
\end{array}\right),
\end{aligned}
$$

und bei der man durch einfaches Nachrechnen überprüfen kann, daß sie invers zueinander sind.

\subsubsection{Eigenschaften des elastischen Zentrums}

Mit dieser Definition eines elastischen Zentrums sind die meisten Probleme mit dem Widerstandszentrum aus 2.2.4.2 überwunden. Die nunmehr eindeutig definierten elastischen Zentren besitzen folgende Eigenschaften: 
- Die Gleichungen sind kovariant und besitzen daher ein brauchbares Transformationsverhalten. Insbesondere werden sie nicht von den noch durchzuführenden Rotationen beeinflußt. Eine Drehung des Koordinatensystems der Lasten um $\boldsymbol{R}_{f}$ und das der Lagen um $\boldsymbol{R}_{q}$ bildet etwa $\boldsymbol{S}_{2} \boldsymbol{M}^{-1}$ ab auf

$$
\boldsymbol{R}_{q} \boldsymbol{S}_{2} \boldsymbol{R}_{f}^{T}\left(\boldsymbol{R}_{q} \boldsymbol{M} \boldsymbol{R}_{f}^{T}\right)^{-1}=\boldsymbol{R}_{q} \boldsymbol{S}_{2} \boldsymbol{R}_{f}^{T} \boldsymbol{R}_{f} \boldsymbol{M}^{-1} \boldsymbol{R}_{q}^{T}=\boldsymbol{R}_{q} \boldsymbol{S}_{2} \boldsymbol{M}^{-1} \boldsymbol{R}_{q}^{T}
$$

und wegen (A.15) wird $\overrightarrow{M D}$ tatsächlich nur auf $\boldsymbol{R}_{q} \overrightarrow{M D}$ gedreht. Der Beweis für $\overrightarrow{L T}$ verläuft analog.

- Wird das absolute Minimum angenommen, so verschwinden die freien Vektoren. Das ist genau die alte Definition des Widerstandszentrums, womit eine Grenzbeziehung zu den vorhergehenden Vorstellungen gefunden ist.

- Die Definition geschieht allein aus den Meßwerten heraus und ist immer möglich, also insbesondere auch retrospektiv.

- Der minimale Vektor und die zugehörige Variable gehören beide entweder zum Last- oder zum Meßsystem. Dadurch können deren Effekte, insbesondere bezüglich systematischer Fehler der Meßapparatur, unterschieden werden.

- Für eine symmetrische Ausgangsmatrix gilt $\boldsymbol{S}_{2}=\boldsymbol{S}_{1}^{T}$ und $\boldsymbol{M}=\boldsymbol{M}^{T}$ woraus

$$
\begin{aligned}
\boldsymbol{\Omega}_{M D} & =\left(\boldsymbol{S}_{2} \boldsymbol{M}^{-1}\right)^{A}=-\left(\boldsymbol{M}^{-1 T} \boldsymbol{S}_{2}^{T}\right)^{A}=-\left(\boldsymbol{M}^{T-1} \boldsymbol{S}_{2}^{T}\right)^{A}=-\left(\boldsymbol{M}^{-1} \boldsymbol{S}_{1}\right)^{A} \\
& =\boldsymbol{\Omega}_{L T}
\end{aligned}
$$

folgt, für übereinstimmendes Last- und Meßsystem fallen also Translationszentrum $T$ und Drehmomentszentrum $D$ zusammen. Bei Existenz eines elastischen Potentials und $M=L$ sind demnach beide Definitionen der elastischen Zentren gleichwertig. Für diesen Fall soll auch im Folgenden für das dann eindeutige elastische Zentrum die Abkürzung $Z$ verwendet werden.

- Die Extremaleigenschaften $|\boldsymbol{T}(Z)|=\min$ ! und $|\delta \boldsymbol{d}(Z)|=$ min! sind auch Eigenschaften der Achsen der Kraftschraube bzw. Drehschraube. Das erlaubt eine anschauliche Deutung des elastischen Zentrums als derjenige Ort, von dem über alle linienflüchtigen Einheitsvektoren gemittelte Schraubachsaufpunkte minimalen Abstand haben.

- Es läßt sich ein Bezug zur bisherigen Auswertepraxis („Rastermessung“, vgl. [3, 4]) herstellen: Das Lastsystem besteht aus einer Kraft in Koordinatenrichtung, also nacheinander $\boldsymbol{F} \|\left\{\boldsymbol{e}_{x}, \boldsymbol{e}_{y}, \boldsymbol{e}_{z}\right\}$, und zwei dazu senkrechten Drehmomenten, also die beiden jeweils dazu senkrechten Komponenten von $\boldsymbol{T}$. Mit diesen Drehmomenten ist dann die Last immer zu einer reinen Kraft äquivalent, deren Wirkungslinie aber kontrolliert werden kann. Die Stärke dieser Drehmomente wird so eingestellt, daß $\delta \boldsymbol{\theta} \approx \mathbf{0}$ ist. Pro Messung ergeben sich zwei Komponenten von $\overrightarrow{L T}$ als Lösung von $\mathbf{0}=\boldsymbol{T}_{T}=\boldsymbol{T}_{L}+\overrightarrow{T L} \times \boldsymbol{F}$. Existiert 
ein Widerstandszentrum, so sind die sechs sich ergebenden Größen miteinander konsistent und damit auf die drei Komponenten von $\overrightarrow{L T}$ reduzierbar. Um $\delta \boldsymbol{\theta}=\boldsymbol{0}$ exakt zu erreichen, benötigt man nach (2.35) aber noch zu $\boldsymbol{F}$ parallele Anteile von $\boldsymbol{T}$. Die Mittelung der sechs Werte entspricht unter Vernachlässigung dieser parallelen Anteile genau der Antisymmetrisierung von $-M^{-1} S_{1}$.

- Die obigen Lösungen der Minimierung haben die gleiche Gestalt, wenn das Minimierungsproblem für die Steifheitsmatrix formuliert wurde, sie sind demnach formäquivalent. Flexibilitäts- und Steifheitsmatrix sind wieder gleichberechtigt! 17

Einige der Eigenschaften, die aus der gewünschten Analogie zum Widerstandszentrum stammen, sind für ein elastisches Zentrum nicht mehr gegeben:

- Polare und axiale Vektoren entkoppeln nicht mehr. Die vom Schwerpunkt des starren Körpers herrührende Anschauung von Kräften, die Translationen, und Drehmomenten, die Rotationen nach sich ziehen, trifft nicht mehr zu.

- Das Reziprozitätstheorem gilt nicht mehr, da die Nebendiagonalblockmatrizen nicht mehr vollständig wegtransformierbar sind. Es kann daher als experimenteller Test auf die „Echtheit“ des Widerstandszentrums verwendet werden.

Zusammenfassend noch einmal die Definitionsgleichungen für das elastische Zentrum:

Das elastische Zentrum ist der Punkt, in dem die $\left\{\begin{array}{c}\text { Last } \\ \text { Auslenkung }\end{array}\right\}$ sich bei dem Vorliegen von reinen $\left\{\begin{array}{c}\text { Translationen } \\ \text { Drehmomenten }\end{array}\right\}$ durch minimale isotrop über alle Richtungen der $\left\{\begin{array}{c}\text { Kräfte } \\ \text { Drehungen }\end{array}\right\}$ gemittelte $\left\{\begin{array}{c}\text { Drehmomente } \\ \text { Translationen }\end{array}\right\}$ auszeichnet.

Oder mit Schraubachsen ausgedrückt: Das elastische Zentrum ist der Punkt, von dem die Achsen von $\left\{\begin{array}{c}\text { Kraftschrauben } \\ \text { Drehschrauben }\end{array}\right\}$ aller Richtungen beim $\left\{\begin{array}{c}\text { Vorliegen } \\ \text { Anlegen }\end{array}\right\}$

\footnotetext{
${ }^{17}$ Dies war das Kriterium, welches meine Entscheidung zu Gunsten der jetzigen Definition am stärksten beeinflußte. Da es zunächst unklar war, ob es sinnvoller sein würde, die Meßwerte über die Flexibilitäts- oder die Steifheitsmatrix auszuwerten, probierte ich beides an verschiedenen Beispielen und Definitionsversuchen aus. Bei nur einer Definition, der jetzigen, ergaben sich bei Anwendung derselben Rechenvorschrift auf Flexibilitäts- und Steifheitsmatrix dieselben Ergebnisse. Dieser Zufallsbefund führte mich dann zunächst über eine Untersuchung der Blockstruktur der beteiligten Matrizen auf den Formalismus mit den Transformationsmatrizen und auf die endgültige Definition des elastischen Zentrums.
} 
von reinen $\left\{\begin{array}{c}\text { Translationen } \\ \text { Drehmomenten }\end{array}\right\}$ minimalen Abstand (im Sinne des obigen Minimierungsproblemes) haben.

\subsection{Die Gesamtheit der Schraubachsen}

Die Analogie vom Widerstandszentrum zum Schwerpunkt konnte nicht aufrecht erhalten werden. Es gibt aber Aufhängungen, deren Eigenschaften beliebig nahe bei denen einer Aufhängung mit Widerstandszentrum liegen. Daher ist es naheliegend, ein Gebilde zu definieren, welches zwar kein Punkt mehr ist, das aber einige Eigenschaften des Widerstandszentrums beibehält und für den Grenzfall des Widerstandszentrums zu einem Punkt ausartet. Da die Minimalität der freien Vektoren aus der Definition des elastischen Zentrums eine Eigenschaft von Schraubachsen ist, lohnt es, die Gesamtheit der zugehörigen Schraubachsen zu untersuchen. Erster Definitionsversuch ist der Widerstandsbereich: Er ist der kleinste Bereich um das elastische Zentrum, innerhalb dessen alle Schraubachsen verlaufen, die sich ergeben, wenn das jeweils andere System aus reinen freien Vektoren besteht. In seinem Inneren sollte das elastische Zentrum als der Punkt größter Annäherung der Schraubachsen aneinander liegen.

Drei Fragen sind dabei von besonderem Interesse:

1. Wo liegen die Aufpunkte von Schraubachsen, die sich ergeben, wenn das andere System aus reinen freien Vektoren besteht?

2. Wie weit sind diese Schraubachsen vom elastischen Zentrum entfernt?

3. Wie groß sind die zugehörigen Schraubsteigungen?

Die Visualisierungen der Antworten auf diese Fragen sind die Aufpunktfläche, die Abstandsfläche und die Steigungsfläche. Mit ihnen kann das gedankliche Konzept des Widerstandszentrums innerhalb gewisser Toleranzgrenzen beibehalten werden. Im Folgenden wird dies näher erläutert.

\subsubsection{Die Aufpunktfläche}

Es gilt für $\boldsymbol{F}=\mathbf{0}$ im Drehmomentszentrum $D$ mit (2.34)

$$
\delta \boldsymbol{d}(D)=\left(\boldsymbol{S}_{2} \boldsymbol{M}^{-1}+\boldsymbol{\Omega}_{D M}\right) \delta \boldsymbol{\theta}=\left(\boldsymbol{S}_{2} \boldsymbol{M}^{-1}\right)^{S} \delta \boldsymbol{\theta}=\boldsymbol{W}_{M} \delta \boldsymbol{\theta}
$$

und für $\delta \boldsymbol{\theta}=\mathbf{0}$ im Translationszentrum $T$ mit (2.35)

$$
\boldsymbol{T}(T)=\left(-\boldsymbol{M}^{-1} \boldsymbol{S}_{1}+\boldsymbol{\Omega}_{T L}\right) \boldsymbol{F}=-\left(\boldsymbol{M}^{-1} \boldsymbol{S}_{1}\right)^{S} \boldsymbol{F}=-\boldsymbol{W}_{L} \boldsymbol{F} .
$$


Für die Aufpunkte $S$ und $K$ auf die Dreh- bzw. Kraftschraube mit kürzestem Abstand zu $D$ bzw. $T$ folgt dann

$$
\begin{aligned}
& \overrightarrow{D S}=\frac{1}{|\delta \boldsymbol{\theta}|^{2}}\left(\delta \boldsymbol{\theta} \times \boldsymbol{W}_{M} \delta \boldsymbol{\theta}\right) \quad \text { und } \\
& \overrightarrow{T K}=\frac{1}{|\boldsymbol{F}|^{2}}\left(\boldsymbol{F} \times\left(-\boldsymbol{W}_{L}\right) \boldsymbol{F}\right)
\end{aligned}
$$

geschrieben mit dem linienflüchtigen Einheitsvektor $\boldsymbol{x}:=\boldsymbol{l}^{0} \in\left\{\delta \boldsymbol{\theta}^{0}, \boldsymbol{F}^{0}\right\}$

$$
\overrightarrow{D S}=\left(\boldsymbol{x} \times \boldsymbol{W}_{M} \boldsymbol{x}\right) \quad \text { und } \quad \overrightarrow{T K}=-\left(\boldsymbol{x} \times \boldsymbol{W}_{L} \boldsymbol{x}\right)
$$

Diese Aufpunkte hängen nicht vom Betrag der linienflüchtigen Vektoren ab. Ferner werden den Vektoren $\boldsymbol{x}$ und $\boldsymbol{- x}$ dieselben Aufpunkte zugeordnet. Damit handelt es sich um eine Abbildung der Sphäre in den dreidimensionalen Raum mit der antipodalen Eigenschaft, also eine Realisierung der reellen projektiven Ebene, vgl. [22] 12.5 .

Um die Notation nicht unnötig zu verkomplizieren, soll im Folgenden von HooKEschen Verhältnissen ausgegangen werden, d. h. Translationszentrum $T$ und Drehmomentszentrum $D$ fallen zusammen zum elastischen Zentrum $Z$. Dann gilt aber $\boldsymbol{W}_{M}=\boldsymbol{W}_{L}=\boldsymbol{W}$, womit folgt:

Sind die beiden bei reinen freien Vektoren vorliegenden linienflüchtigen Vektoren $\delta \boldsymbol{\theta}$ und $\boldsymbol{F}$ parallel, so liegen die korrespondierenden Aufpunkte A der Schraubachsen von Kraft- bzw. Drehschraube einander bezüglich des elastischen Zentrums $Z$ genau gegenüber.

Die Formen der Aufpunktflächen gehen also für Last- und Meßsystem wegen

$$
\overrightarrow{Z A}= \pm(\boldsymbol{x} \times \boldsymbol{W} \boldsymbol{x})
$$

durch Punktspiegelung auseinander hervor. Für ein Widerstandszentrum fallen beide Achsen des Paares zusammen.

Da die Widerstandsmatrix symmetrisch ist, kann sie durch eine orthogonale Transformation auf Hauptdiagonalform gebracht werden. Mit $w_{i}$ sollen die Eigenwerte der Widerstandsmatrix bezeichnet werden, mit $w_{i j}:=w_{i}-w_{j}$ ihre Differenzen. Für die Hauptachsen $\boldsymbol{e}_{i}$ selber werden die Verhältnisse besonders einfach, denn für sie gilt

$$
\boldsymbol{e}_{i} \times\left(\boldsymbol{W} \boldsymbol{e}_{i}\right)=\boldsymbol{e}_{i} \times\left(w_{i} \boldsymbol{e}_{i}\right)=0,
$$

d. h. der Schraubachsaufpunkt liegt im elastischen Zentrum. 
Durch Parametrisierung des linienflüchtigen Einheitsvektors $\boldsymbol{x}$ in Koordinaten der Oberfläche der Einheitskugel

$$
\boldsymbol{x}=\left(\begin{array}{c}
\cos \phi \cos \theta \\
\sin \phi \cos \theta \\
\sin \theta
\end{array}\right)
$$

erhält man im System der Hauptachsen von $\boldsymbol{W}$ alle Schraubachsaufpunkte im Raum, also eine Parameterdarstellung der Aufpunktfläche

$$
\left(\begin{array}{l}
x \\
y \\
z
\end{array}\right)= \pm\left(\begin{array}{c}
w_{32} \sin \phi \sin \theta \cos \theta \\
w_{13} \cos \phi \sin \theta \cos \theta \\
w_{21} \sin \phi \cos \phi \cos ^{2} \theta
\end{array}\right)= \pm \frac{1}{2}\left(\begin{array}{c}
w_{32} \sin \phi \sin 2 \theta \\
w_{13} \cos \phi \sin 2 \theta \\
w_{21} \sin 2 \phi \cos ^{2} \theta
\end{array}\right)
$$

Diese genügt der impliziten Gleichung vierten Grades $\$ 18$

$$
x^{2} y^{2} w_{21}^{2}+y^{2} z^{2} w_{32}^{2}+z^{2} x^{2} w_{13}^{2} \mp x y z w_{32} w_{13} w_{21}=0
$$

Für etwa die Gegenbeispielfäche aus Abschnitt 2.2.4.2, berechnet in 5.1.4, ergibt sich eine Aufpunktfläche der in Abb. 2.5 gezeigten Form.
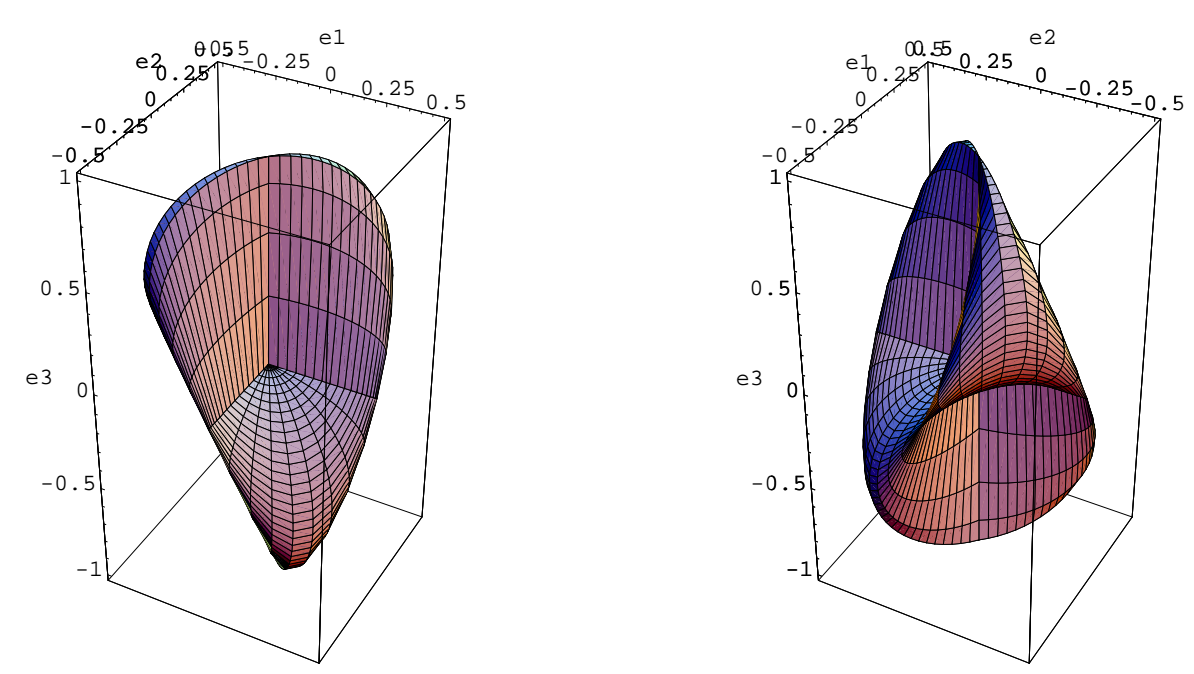

Abbildung 2.5: Zwei Ansichten der Aufpunktfläche mit $w_{1}=-1, w_{2}=1$ und $w_{3}=$ 0. Es wurde der Fall „,+" gewählt, also der der Drehschrauben. Weitere Einzelheiten in Abschnitt 5.1.4; der obere und untere Grat der Fläche korrespondiert jeweils mit der ursprünglichen Richtung der Einzelfedern.

${ }^{18}$ Diese erinnert an STEINERs Römische Fläche, vgl. [22] 12.5

$$
x^{2} y^{2}+y^{2} z^{2}+z^{2} x^{2}-x y z=0,
$$

wobei beide Flächen aber nur als Realisationen der reellen projektiven Ebene miteinander verwandt sind, da keine Lösung $\left(w_{1}, w_{2}, w_{3}\right)$ existiert mit $w_{21}^{2}=w_{32}^{2}=w_{13}^{2}=1$, was die eigentliche STEINERfläche kennzeichnet. 
Als Letztes soll gezeigt werden, daß die Aufpunktfläche dem Definitionsversuch von Fish [17] genügt, vgl. das Zitat auf S. 42. Für eine reine Kraft $\boldsymbol{F}$ soll die Kraftwirkungslinie derart gewählt werden, daß für einen Punkt $\boldsymbol{r}$ dieser Linie gilt $\boldsymbol{F} \times \delta \boldsymbol{\theta}(\boldsymbol{F}, \boldsymbol{r})=\mathbf{0}$, also

$$
\begin{aligned}
\mathbf{0} & =\boldsymbol{F} \times\left(\boldsymbol{S}_{1} \boldsymbol{F}+\boldsymbol{M}(\boldsymbol{r} \times \boldsymbol{F})\right)=\boldsymbol{F} \times\left(\boldsymbol{S}_{1} \boldsymbol{F}-\boldsymbol{M}(\boldsymbol{F} \times \boldsymbol{r})\right) \\
& =\boldsymbol{\Omega}_{F} \boldsymbol{S}_{1} \boldsymbol{F}-\boldsymbol{\Omega}_{F} \boldsymbol{M} \boldsymbol{\Omega}_{F} \boldsymbol{r} .
\end{aligned}
$$

Dieses lineare Gleichungssystem für $\boldsymbol{r}$ hängt nicht von der Stärke der Kraft ab, da diese in beiden Termen quadratisch auftritt. Derartige Effekte sind also in linearen Input-Output-Modellen nicht zu erwarten. Die Lösung $\boldsymbol{r}_{h}$ seines homogenen Teils ist die Kraftwirkunglinie selber, also $\boldsymbol{r}_{h}=\lambda \boldsymbol{F}^{0}$. Im Translationszentrum gilt $\boldsymbol{S}_{1}=$ $\boldsymbol{M} \boldsymbol{W}_{L}$ und es ist nun eine Lösung $\boldsymbol{r}_{i}$ des inhomogenen Gleichungssystems

$$
\boldsymbol{\Omega}_{F} \boldsymbol{M} \boldsymbol{\Omega}_{F} \boldsymbol{r}_{i}=\boldsymbol{\Omega}_{F} \boldsymbol{M} \boldsymbol{W}_{L} \boldsymbol{F}
$$

zu bestimmen. Hinreichend dafür ist $\boldsymbol{\Omega}_{F} \boldsymbol{r}_{i}=\boldsymbol{W}_{L} \boldsymbol{F}$, oder mit (A.2) gleichwertig $\boldsymbol{P}_{F}^{\perp} \boldsymbol{r}_{i}=-\boldsymbol{\Omega}_{F} \boldsymbol{W}_{L} \boldsymbol{F}$, demnach ist

$$
\boldsymbol{r}_{i}=-\boldsymbol{F}^{0} \times \boldsymbol{W}_{L} \boldsymbol{F}^{0}
$$

ein Punkt der Aufpunktfläche der Kraftschrauben und gleichzeitig Lösung des Gleichungssystems. Analog dazu erhält man für ein Gedankenexperiment, bei dem eine reine Drehung $\delta \boldsymbol{\theta}$ bezüglich einer Drehachse $\boldsymbol{r}$ vorgegeben ist so daß die hervorrufenden Kräfte parallel zu dieser Achse sind, ein Element der Aufpunktfläche der Drehschrauben. Hier wird die Rechnung mit $\delta \boldsymbol{\theta} \times \boldsymbol{F}(\delta \boldsymbol{\theta}, \boldsymbol{r})=\mathbf{0}$ vorteilhafterweise über die Steifheitsmatrix durchgeführt.

Die Aufpunktfläche beschreibt gewissermaßen die innere Struktur des Widerstandsbereiches, in dem Sinne, daß jeder Bereich, in dem die Aufpunktfläche liegt (z. B. ein Ellipsoid), als Widerstandsbereich gelten kann. Da die Aufpunktfläche nichtorientierbar ist, besitzt sie auch kein eigenes Inneres. Für ein Widerstandszentrum schrumpft sie auf einen Punkt zusammen.

\subsubsection{Die Abstandsfläche}

Die Abstandsfläche entsteht durch Zuordnung von linienflüchtigem Einheitsvektor zum minimalen Abstand der Schraubachse vom elastischen Zentrum. Sie verknüpft einen Aspekt der Lage und die Richtung der Schraubachsen im Raum. Daher ist die Abstandsfläche im Gegensatz zur Aufpunktfläche nicht im Ortsraum definiert und hängt deshalb auch nicht direkt mit der Anordnung der elastischen Aufhängung zusammen.

Für unsymmetrische elastische Matrizen sind die folgenden Überlegungen getrennt für Lastsystem und Meßsystem durchzuführen. Da nur der Betrag des Schraubachsabstandes diskutiert wird, gibt es für die hier angenommenen HookEschen 
Verhältnisse von der Abstandsfläche nur ein gemeinsames Exemplar für Last- und Meßsystem. Seine Gleichung ist

$$
\|\overrightarrow{Z A}\|^{2}=\|\boldsymbol{x} \times \boldsymbol{W} \boldsymbol{x}\|^{2}=\boldsymbol{x}^{T} \boldsymbol{W}^{2} \boldsymbol{x}-\left(\boldsymbol{x}^{T} \boldsymbol{W} \boldsymbol{x}\right)
$$

Man erhält wieder im Hauptachsensystem von $\boldsymbol{W}$ für den Abstand $r=|\overrightarrow{Z A}|$ die Gleichung

$$
\begin{aligned}
r^{2}(\phi, \theta) & =w_{32}^{2} \sin ^{2} \phi \sin ^{2} \theta \cos ^{2} \theta+w_{13}^{2} \cos ^{2} \phi \sin ^{2} \theta \cos ^{2} \theta+w_{21}^{2} \sin ^{2} \phi \cos ^{2} \phi \cos ^{4} \theta \\
& =\frac{1}{4} w_{32}^{2} \sin ^{2} \phi \sin ^{2} 2 \theta+\frac{1}{4} w_{13}^{2} \cos ^{2} \phi \sin ^{2} 2 \theta+\frac{1}{4} w_{21}^{2} \sin ^{2} 2 \phi \cos ^{4} \theta \quad \text { (2.48) }
\end{aligned}
$$

Interpretiert als Radialkomponente von Kugelkoordinaten liefert diese Gleichung mit $r^{2} \boldsymbol{x}(\phi \theta)$ eine Parameterdarstellung der gesamten Fläche. Auch hier existiert eine entsprechende implizite Gleichung, diesmal sechsten Grades

$$
x^{2} y^{2} w_{21}^{2}+y^{2} z^{2} w_{32}^{2}+z^{2} x^{2} w_{13}^{2}-\left(x^{2}+y^{2}+z^{2}\right)^{3}=0
$$

Am bisher mehrfach erwähnten Gegenbeispiel kann diese Fläche visualisiert werden, vgl. Abb. 2.6.

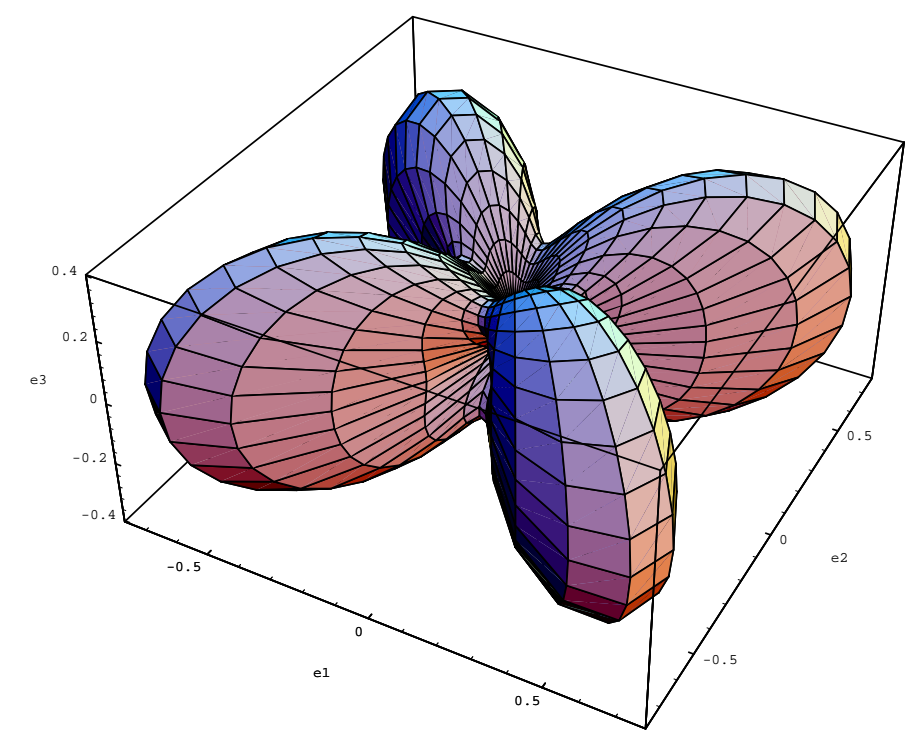

Abbildung 2.6: Die Abstandsfläche des Gegenbeispiels 5.1.4 mit $w_{1}=-1, w_{2}=1$ und $w_{3}=0$.

Durch Diskussion der Extrema dieses Schraubachsabstandes oder durch Abschätzen der einzelnen trigonometrischen Terme der Parameterdarstellung (2.45) durch 1 nach oben erhält man komponentenweise den begrenzenden Kasten

$$
\left(\begin{array}{l}
|x| \\
|y| \\
|z|
\end{array}\right) \leq \frac{1}{2}\left(\begin{array}{l}
\left|w_{32}\right| \\
\left|w_{13}\right| \\
\left|w_{21}\right|
\end{array}\right)
$$


und daraus eine vernünftige Definition der Größe des Widerstandsbereiches als eine Kugel mit Durchmesser

$$
\operatorname{Größe}(\mathrm{WB})=\max _{i, j} \frac{1}{2}\left|w_{i}-w_{j}\right| .
$$

Gestalt und Symmetrie von Aufpunkt- und Abstandsfläche rechtfertigen nachträglich noch einmal die getroffene Definition des elastischen Zentrums.

\subsubsection{Die Steigungsfläche}

Analog zu (2.44) ergibt sich für die Schraubsteigungen

$$
\tau_{M}=\left(\boldsymbol{x} \cdot \boldsymbol{W}_{M} \boldsymbol{x}\right) \quad \text { und } \quad \tau_{L}=-\left(\boldsymbol{x} \cdot \boldsymbol{W}_{L} \boldsymbol{x}\right) .
$$

Für $\boldsymbol{W}_{M}=\boldsymbol{W}_{L}=\boldsymbol{W}$ folgt daraus sofort:

Sind die beiden bei reinen freien Vektoren vorliegenden linienflüchtigen Vektoren $\delta \boldsymbol{\theta}$ und $\boldsymbol{F}$ parallel, so ergeben sich für die korrespondierenden Schraubachsen von Kraft- bzw. Drehschraube Schraubsteigungen mit entgegengesetzt gleichen Beträgen.

Eine Linksschraube im Lastsystem entspricht also einer Rechtsschraube im Meßsystem und umgekehrt. Die Formen der Steigungsflächen gehen also für Last- und Meßsystem wie die der Aufpunktflächen durch Punktspiegelung auseinander hervor. Für ein Widerstandszentrum entarten beide zu einem Punkt. Wie die Abstandsfläche, ist die Steigungsfläche nicht im Ortsraum definiert.

Man erhält im Hauptachsensystem von $\boldsymbol{W}$ für die Schraubsteigung $\tau$ die Gleichung

$$
\tau= \pm\left(w_{1} \cos ^{2} \phi \cos ^{2} \theta+w_{2} \sin ^{2} \phi \cos ^{2} \theta+w_{3} \sin ^{2} \theta\right) .
$$

Da $\tau$ auch negative Werte annehmen kann, ist eine Visualisierung im Raum der Art $\tau \boldsymbol{x}$, wie bei der Abstandsfläche, nicht sinnvoll. Statt dessen soll die einfache Auftragung $\tau(\phi, \theta)$ verwandt werden. Für die Gegenbeispielfläche ergeben sich die in den Abbildungen 2.7 und 2.8 gezeigten Plots.

Wegen der Extremaleigenschaft des RAYLEIGH-Quotienten ${ }^{19}$ werden die Maxima von $\tau$ angenommen für die Eigenvektoren von $\boldsymbol{W}$. Es gilt demnach

$$
\tau_{M A X}=\max _{i}\left(w_{i}\right) \quad \text { und } \quad \tau_{M I N}=\min _{i}\left(w_{i}\right) \quad .
$$

Fragt man nach den Richtungen zu gleichem $\tau$, so ist die Menge dieser Richtungen ein Schnitt der Einheitskugel mit der der symmetrischen Widerstandsmatrix

\footnotetext{
${ }^{19}$ Vgl. [73] $\$ 15.2$ und $\S 15.3$
} 


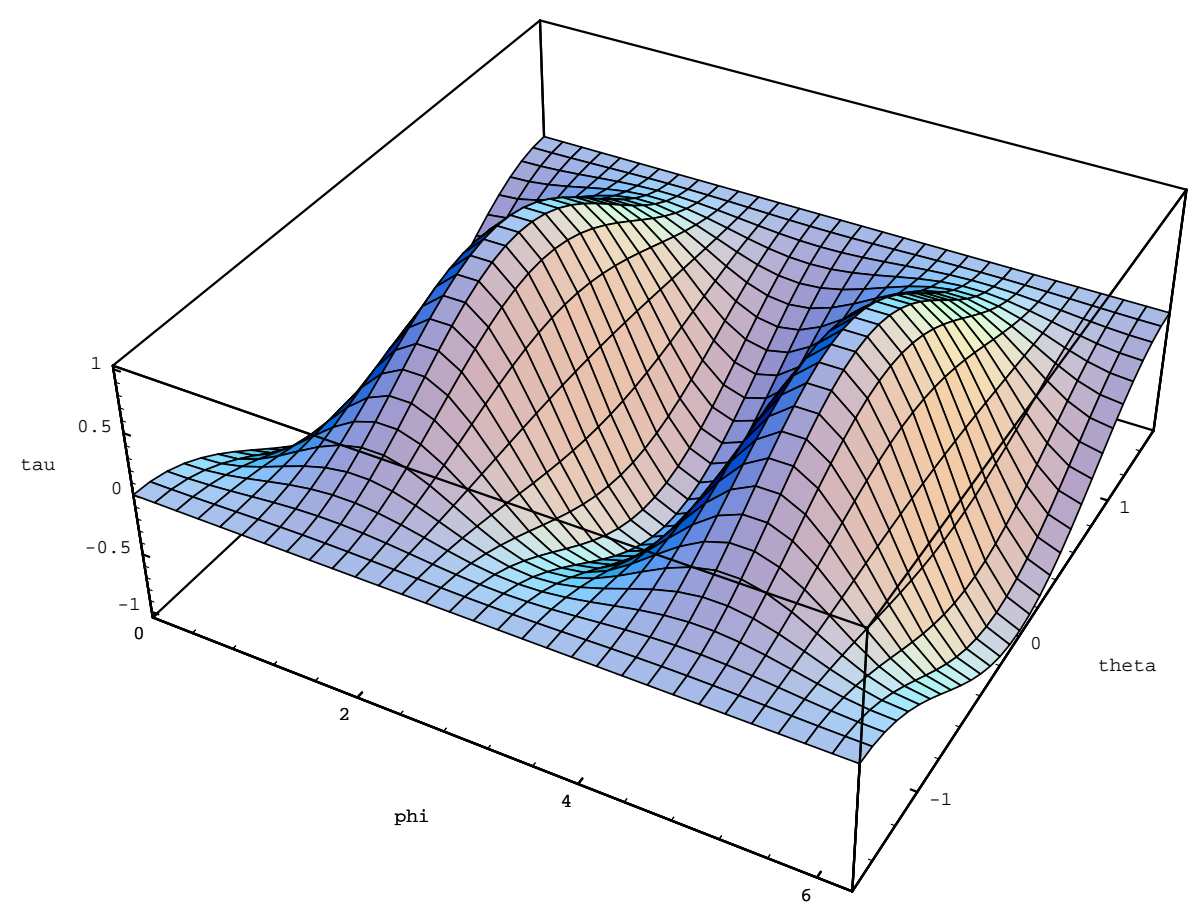

Abbildung 2.7: Die Schraubsteigung $\tau$ des Gegenbeispiels 5.1.4 mit den Werten $w_{1}=-1, w_{2}=1$ und $w_{3}=0$ in Abhängigkeit von $\phi$ und $\theta$.

zugeordneten Fläche zweiter Ordnung, also eine Kurve auf der Einheitskugel. Für $\operatorname{det} \boldsymbol{W} \neq 0$ handelt es sich bei dieser Fläche zweiter Ordnung um eine Mittelpunktsfläche. Für das Gegenbeispiel handelt es sich bei der Fläche zweiter Ordnung um einen hyperbolischen Zylinder, also keine Mittelpunktsfläche. Die Kurvenschar zu $\tau=$ const ergibt sich als Menge der Schnitte der Einheitskugel mit der entsprechenden Flächenschar. Sie überdeckt die Einheitskugel vollständig, da sich zu jeder Richtung zwangsläufig eine Schraubsteigung ergibt.

\subsubsection{Die Kongruenz der Schraubachsen}

Durch den zweiparametrigen linienflüchtigen Einheitsvektor, der ja Richtungsvektor der Schraubachse ist, ist zusammen mit der Aufpunktfläche eine zweiparametrige Schar von Geraden im Raum gegeben. Die Parameterdarstellung dieser Geradenschar ist also

$$
\boldsymbol{g}(\phi, \theta, \lambda)=\boldsymbol{x}(\phi, \theta) \times(\boldsymbol{W} \boldsymbol{x}(\phi, \theta))+\lambda \boldsymbol{x}(\phi, \theta) .
$$

Ein solches Gebilde der Form²0

$$
\boldsymbol{g}\left(u_{1}, u_{2}, u_{3}\right)=\boldsymbol{a}\left(u_{1}, u_{2}\right)+u_{3} \boldsymbol{r}\left(u_{1}, u_{2}\right)
$$

\footnotetext{
${ }^{20} \mathrm{Im}$ Gegensatz zu den sonst üblichen Konventionen möchte ich hier die Indizes der Flächenparameter unten anschreiben.
} 


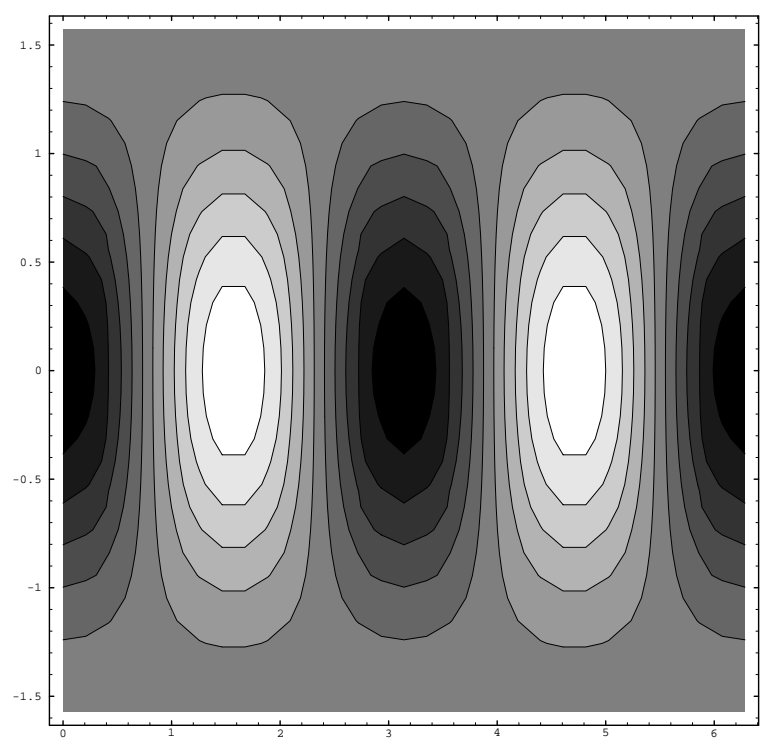

Abbildung 2.8: Die Schraubsteigung des Gegenbeispiels 5.1.4 mit $w_{1}=-1, w_{2}=1$ und $w_{3}=0$ in Abhängigkeit von $\phi$ und $\theta$ als Höhenlinien.

nennt man Geradenkongruenz oder Strahlsystem oder einfach kurz Kongruenz (vgl. auch im Folgenden [27], insbesondere Teil II).

Eine Kongruenz kann auch als einparametrige Schar von Regelflächen betrachtet werden. Die Extremallagen der Striktionspunkte dieser Regelflächen definieren für jede Gerade zwei Grenzpunkte, deren Gesamtheit die beiden Grenzflächen der Kongruenz definieren. Auch die Mitten zwischen den beiden Grenzpunkten bilden eine Fläche, die Mittenfläche. Um sie zu berechnen, bestimmt man zunächst die erste und zweite Fundamentalform der Kongruenz

$$
\begin{aligned}
g_{i k} & :=\boldsymbol{r}_{, i} \cdot \boldsymbol{r}_{, k} \\
G_{i k} & :=\boldsymbol{r}_{, i} \cdot \boldsymbol{a}_{, k},
\end{aligned}
$$

welche auch Funktionen der Parameter $u_{1}$ und $u_{2}$ sind. Eine Parameterdarstellung der Mittenfläche ergibt sich dann zu

$$
\boldsymbol{m}\left(u_{1}, u_{2}\right)=\boldsymbol{a}\left(u_{1}, u_{2}\right)-\frac{1}{2} G_{i k} g^{i k} \boldsymbol{r}\left(u_{1}, u_{2}\right),
$$

wobei $g^{i k}$ die Komponenten der zu $\left(g_{i k}\right)$ inversen Matrix sind. Die Grenzflächen erhält man, wenn man die Lösungen von

$$
\operatorname{det}\left(\boldsymbol{G}^{S}+u_{3} \boldsymbol{g}\right)=0
$$

wieder in die Gleichung der Kongruenz einsetzt. Die Brennflächen, das sind die Flächen aller Berührungspunkte der Kongruenzgeraden, erhält man ebenso aus den Lösungen der Gleichung

$$
u_{3}^{2}+u_{3} G_{i k} g^{i k}+\frac{\operatorname{det} \boldsymbol{G}}{\operatorname{det} \boldsymbol{g}}=0
$$


Mit Hilfe von Computeralgebra konnte ich allgemein zeigen, daß die Aufpunktfläche gleichzeitig die Mittenfläche der Schraubachskongruenz ist. Für die Grenzflächen ergeben sich leider keine einfachen Ausdrücke. Ferner ist die Kongruenz nicht isotrop, d. h. Mittenpunkte und Grenzpunkte fallen nicht zusammen. Auch die Brennflächen können ausgerechnet werden. Da sie aber keine einfache Parameterdarstellung mehr besitzen, möchte ich hier auf eine Darstellung verzichten.

Da sie mir gar keine physikalische Bedeutung mehr zu haben scheinen, habe ich weder die K-Hauptflächen noch die S-Hauptflächen dieser Kongruenz näher untersucht.

\subsection{Funktionelle Achsen und andere ausgezeich- nete Richtungen}

Die Freiheit der Wahl eines Koordinatensystems umfaßt die gesamte EuKLIDische Gruppe, d. h. Translationen und Drehungen. Da nach der Transformation der Meßwerte auf das elastische Zentrum die Orientierung der Koordinatensysteme noch frei ist, sind die Meßwerte noch nicht auf ihre Normalform gebracht. Das elastische Zentrum konnte durch ein eingehendes Studium des Gegensatzpaares „frei $\leftrightarrow$ linienflüchtig" bestimmt werden. Es wird sich zeigen, daß es nun sinnvoll ist, das Gegensatzpaar „polar $\leftrightarrow$ axial“ zur Definition von ausgezeichneten Richtungen heranzuziehen.

In der Mechanik folgt nach der Definition des Schwerpunktes meistens die der Hauptträgheitsachsen. Für eine räumliche elastische Aufhängung mit oder ohne Widerstandszentrum sollten sich auch ausgezeichnete Richtungen finden lassen. Im Gegensatz zu den Schraubachsen des letzten Abschnitts soll die Definition wie die des elastischen Zentrums wieder so gemacht werden, daß sie immer möglich und numerisch günstig ist.

Die funktionellen Achsen können nicht die Symmetrieachsen der Abstandsfläche sein, da diese für den Fall eines Widerstandszentrums zu einem Punkt ohne Symmetrieachsen degeneriert, was der obigen Anschauung widerspricht.

Genauso wenig darf man die Eigenvektoren von $\mathcal{F}_{D T}$ oder $\mathcal{S}_{T D}$ nehmen, da den Eigenwerten keine physikalische Einheit konsistent zugeordnet werden könnte: Wegen

$$
\{f\}=N\left(\begin{array}{c}
1 \\
m
\end{array}\right) \quad \text { und } \quad\{q\}=\left(\begin{array}{c}
m \\
1
\end{array}\right)
$$

kann es keinen Proportionalitätsfaktor zwischen $f$ und $q$ geben, wie die Eigenvektorgleichung es verlangte. Zudem sind aus physikalischen Gründen weder $\mathcal{F}$ noch $\mathcal{S}$ Endomorphismen. Weiter hätte man sich noch zu überlegen, wie man aus den formal gewonnenen sechs Eigenvektoren mit 36 Einträgen (normiert: 30, orthonor- 
miert: 15) drei bzw. sechs Orientierungen im Raum mit 3 bzw. 6 Freiheitsgraden gewinnt.

Das Problem mit den Einheiten läßt sich beheben - mit interessanten Konsequenzen. Statt wie eben „Polar parallel Polar“ und „Axial parallel Axial“ fordert man „Frei parallel Frei“ und „Linienflüchtig parallel Linienflüchtig“ mit demselben Proportionalitätsfaktor. Das hätte zur Folge, daß wegen (2.4) und (2.5) sowie (2.6) und (2.7) Kraft- und Drehschraube dieselben Aufpunkte, Richtungen und Schraubsteigungen haben. Formal geschieht dieses Vertauschen von axialem und polarem Vektor durch die Matrix

$$
\mathcal{J}:=\left(\begin{array}{cc}
0 & \boldsymbol{I} \\
\boldsymbol{I} & 0
\end{array}\right)
$$

und es ergibt sich das Eigenwertproblem $\mathcal{J S} q=\lambda q$ (oder entsprechend für die Flexibilitätsmatrix) mit einem einheitenbehafteten Eigenwert. Die in Eigenvektoren zu zerlegende Matrix hat die Komponenten

$$
\mathcal{J S}=\left(\begin{array}{cc}
\boldsymbol{B}_{1} & \boldsymbol{C} \\
\boldsymbol{A} & \boldsymbol{B}_{2}
\end{array}\right)
$$

und ist nicht mehr symmetrisch, weshalb nicht für alle Matrizen ein System aus reellen Eigenvektoren existieren kann. Selbst für ein Widerstandszentrum gilt $\mathcal{J S}=\left(\begin{array}{cc}\mathbf{0} & \boldsymbol{C} \\ \boldsymbol{A} & \mathbf{0}\end{array}\right)$, und das Eigenwertproblem lautet dann

$$
\boldsymbol{A} \delta \boldsymbol{d}=\lambda \delta \boldsymbol{\theta} \quad \text { und } \quad \boldsymbol{C} \delta \boldsymbol{\theta}=\lambda \delta \boldsymbol{d}=\lambda^{2} \boldsymbol{A}^{-1} \delta \boldsymbol{\theta} .
$$

Das ist eine verallgemeinerte Eigenwertaufgabe für die Matrizen $\boldsymbol{C}$ und $\boldsymbol{A}^{-1}$, wobei $\boldsymbol{A}^{-1}$ und $\boldsymbol{C}$ idealerweise symmetrisch und positiv definit sind.21 Nach 73 $\S 15$ Satz 5 gibt es dann ein gemeinsames Eigenvektorsystem mit positiven und daher radizierbaren Eigenwerten. Die Aufgabe ist also lösbar; die Eigenvektoren sind die der Matrix $\boldsymbol{A C}$. Beide beteiligten Matrizen eignen sich zur Definition eines Skalarproduktes; das gemeinsame Eigenvektorsystem ist orthogonal im Sinne dieses Skalarproduktes. Diese Achsen lassen sich auch aus einer energetischen Betrachtung herleiten: Hält man die Helizität $\tau_{H}=\delta \boldsymbol{d} \cdot \delta \boldsymbol{\theta}$ konstant, so ergibt das Minimierungsproblem mit Nebenbedingungen

$$
q^{T} \mathcal{S} q+\lambda\left(q^{T} \mathcal{J} q-2 \tau_{H}\right)=\min !
$$

genau die Gleichung von eben. Aus diesem Grunde möchte ich diese Achsen, für den Fall, daß sie existieren, die Achsen konstanter Helizität nennen. Aber selbst dann eignen sich diese Achsen nicht für die gesuchte Normalform, da sie i.A. nicht orthogonal sind. Ein schiefwinkliges Koordinatensystem erschwert zudem die Interpretier- und Vergleichbarkeit von Meßwerten.

\footnotetext{
${ }^{21}$ Das folgt aus der physikalischen Tatsache, daß die elastische Energie eine quadratische Form mit $q^{T} \mathcal{S} q>0$ für alle $q \neq 0$ ist - insbesondere für reine Rotationen oder Translationen.
} 
Statt ideale Fälle zu diskutieren, muß, wie bei der Definition des elastischen Zentrums, wieder auf der Ebene der Vektoren selber argumentiert werden; das löst außerdem das Problem, daß mit einem Eigenwertproblem sechs statt drei gleichberechtigte Achsen ausgezeichnet würden.

\subsubsection{Funktionelle Achspaare und Richtungen}

Wie beim elastischen Zentrum ist das zur Definition führende Gedankenexperiment so gemacht, daß jeweils einer der beiden linienflüchtigen Vektoren gleich Null ist. Zum einen gehört zu einem reinen Drehmoment eine Drehschraube und zum anderen korrespondiert eine reine Translation zu einer Kraftschraube. Dadurch folgt eine Gleichung zwischen den beiden die Schraube bildenden Vektoren des jeweils anderen Systems:

$$
\left\{\begin{array}{c}
\delta \boldsymbol{\theta}_{L}=\mathbf{0} \\
\boldsymbol{F}_{M}=\mathbf{0}
\end{array}\right\} \Rightarrow\left\{\begin{array}{c}
\boldsymbol{T}_{L}=\boldsymbol{B}_{1} \boldsymbol{A}^{-1} \boldsymbol{F}_{L} \\
\delta \boldsymbol{d}_{M}=-\boldsymbol{A}^{-1} \boldsymbol{B}_{2} \delta \boldsymbol{\theta}_{M}
\end{array}\right\} \Rightarrow\left\{\begin{array}{c}
\boldsymbol{T}_{D}=-\boldsymbol{W}_{L} \boldsymbol{F}_{D} \\
\delta \boldsymbol{d}_{T}=\boldsymbol{W}_{M} \delta \boldsymbol{\theta}_{T}
\end{array}\right\}
$$

und, je nachdem, ob der verschwindende linienflüchtige Vektor polar oder axial war, eine Bedingung zwischen den beiden nichtverschwindenden axialen bzw. polaren Vektoren

$$
\left\{\begin{array}{c}
\delta \boldsymbol{\theta}_{L}=\mathbf{0} \\
\boldsymbol{F}_{M}=\mathbf{0}
\end{array}\right\} \Rightarrow\left\{\begin{array}{c}
\boldsymbol{F}_{L}=\boldsymbol{A} \delta \boldsymbol{d}_{L} \\
\boldsymbol{T}_{M}=\left(\boldsymbol{C}-\boldsymbol{B}_{1} \boldsymbol{A}^{-1} \boldsymbol{B}_{2}\right) \delta \boldsymbol{\theta}_{M}
\end{array}\right\} .
$$

Diese Beziehungen sollen zur Definition der funktionellen Achsen und Richtungen verwandt werden: Die Wunschvorstellung vom Widerstandszentrum war, daß polare und axiale Vektoren entkoppeln. Also fordert man: Ist der $\left\{\begin{array}{c}\text { axiale } \\ \text { polare }\end{array}\right\}$ linienflüchtige Vektor gleich Null, so sind die beiden $\left\{\begin{array}{c}\text { polaren } \\ \text { axialen }\end{array}\right\}$ Vektoren der funktionellen $\left\{\begin{array}{c}\text { Richtungen } \\ \text { Achsen }\end{array}\right\}$ parallel. Die funktionellen $\left\{\begin{array}{c}\text { Richtungen } \\ \text { Achsen }\end{array}\right\}$ sind damit die Eigenvektoren der Matrizen $\left\{\begin{array}{c}\boldsymbol{A}=\left(\boldsymbol{D}-\boldsymbol{S}_{2} \boldsymbol{M}^{-1} \boldsymbol{S}_{1}\right)^{-1} \\ \boldsymbol{C}-\boldsymbol{B}_{1} \boldsymbol{A}^{-1} \boldsymbol{B}_{2}=\boldsymbol{M}^{-1}\end{array}\right\}$.

Diese Matrizen existieren auch für den Grenzfall des Widerstandszentrums. Beide sind transformationsinvariant. Zusammen mit den bei einer symmetrischen Ausgangsmatrix für Last- und Meßsystem gegenüberliegenden Schraubachsaufpunkten erhält man so die drei funktionellen Achspaare. Die Mittelgeraden dieser Achspaare verlaufen durch das elastische Zentrum. Das Achspaar trägt dem Umstand Rechnung, daß das dem Schwerpunkt analoge Objekt, der Widerstandsbereich, nun kein Punkt mehr ist.

Um insgesamt nur drei Richtungen im Raum auszuzeichnen, wäre es wünschenswert, für $\boldsymbol{A}$ und $\boldsymbol{M}^{-1}$ ein gemeinsames Eigenvektorsystem zu finden. Dieses käme 
den Achsen gleicher Helizität sehr nahe. Andererseits gehören beide Eigenvektoraufgaben zu verschiedenen Gedankenexperimenten, so daß sich keine so einfache anschauliche Bedeutung mehr ergibt. Fordert man etwa die Parallelität der linienflüchtigen Vektoren zu den beiden verschiedenen Gedankenexperimenten

$$
\boldsymbol{F}_{L} \| \boldsymbol{\theta}_{M}
$$

so ergibt sich zwischen den freien Vektoren wieder die verallgemeinerte Eigenwertaufgabe

$$
\boldsymbol{A} \delta \boldsymbol{d}_{L} \|\left(\boldsymbol{C}-\boldsymbol{B}_{1} \boldsymbol{A}^{-1} \boldsymbol{B}_{2}\right)^{-1} \boldsymbol{T}_{M}
$$

Wie im letzten Abschnitt kann die Theorie der Formenbüschel22 angewandt werden. Das verallgemeinerte Eigenwertproblem für zwei Matrizen wird dadurch gelöst, daß man durch die eine symmetrische Matrix ein Skalarprodukt auszeichnet, um dann im entsprechenden Basissystem weiterzuarbeiten. In diesen Koordinaten kann dann die verbleibende Matrix durch eine dort (und nur dort) orthogonale Transformation diagonalisiert werden. Die das Skalarprodukt auszeichnende Matrix ist hier $\boldsymbol{M}$. Es kann also eine gemeinsame Basis für die Matrizen $\boldsymbol{A}$ und $\left(\boldsymbol{C}-\boldsymbol{B}_{1} \boldsymbol{A}^{-1} \boldsymbol{B}_{2}\right)^{-1}=\boldsymbol{M}$ gefunden werden, bestehend aus den sog. Hauptvektoren des Büschels $\boldsymbol{A}+\lambda \boldsymbol{M}$, in der beide Matrizen diagonal sind. Da die beteiligten Matrizen hier invertierbar sind, folgt

$$
\left(\boldsymbol{C}-\boldsymbol{B}_{1} \boldsymbol{A}^{-1} \boldsymbol{B}_{2}\right) \boldsymbol{A} \delta \boldsymbol{d}_{L} \| \boldsymbol{T}_{M}
$$

und die Hauptvektoren sind einfach die Eigenvektoren der Matrix

$$
\left(\boldsymbol{C}-\boldsymbol{B}_{1} \boldsymbol{A}^{-1} \boldsymbol{B}_{2}\right) \boldsymbol{A} \text { bzw. } \quad \boldsymbol{M}^{-1}\left(\boldsymbol{D}-\boldsymbol{S}_{2} \boldsymbol{M}^{-1} \boldsymbol{S}_{1}\right)^{-1}
$$

oder für die Flexibilitätsmatrix passender $\left(\boldsymbol{D}-\boldsymbol{S}_{2} \boldsymbol{M}^{-1} \boldsymbol{S}_{1}\right) \boldsymbol{M}$. In diesem nichtorthogonalen Koordinatensystem fallen dann funktionelle Achsen und Richtungen zusammen. Von einer Definition der Normalform über ein nichtorthogonales Koordinatensystem muß aber, wieder wie oben, aus Gründen der Vergleichbarkeit von Meßwerten Abstand genommen werden. Noch schlimmer wird die Situation für gemessene Matrizen, da deren Eigenwerte nicht einmal mehr reell sein müssen. Dennoch werden die Ergebnisse dieses Abschnitts im Kapitel 5, welches Vorhersagen über Aufhängungen enthält, Verwendung finden.

\subsection{Standardformen der elastischen Matrizen}

Der Trägheitstensor im Schwerpunkt wird beim starren Körper dazu benutzt, Richtungen im Raum auszuzeichnen. Er verknüpft die beiden axialen Vektoren. Diese Definition ist translationsinvariant, da sie nur im Schwerpunkt gilt. Die Analogie zwischen Masse und Trägheitstensor auf der einen Seite und der Steifheitsmatrix

\footnotetext{
${ }^{22} \mathrm{Vgl}$. etwa 20] X. $\S 6$ oder wieder $[73$ \$13.8, $\S 14.4$ und $\S 15.5$.
} 
auf der anderen Seite wird in den Abschnitten 3.1.1 und dem Beginn von 4.5 noch klarer herausgearbeitet werden. Hier sind die Matrizen $\boldsymbol{A}$ und $\boldsymbol{M}$ translationsinvariant und kommen daher als Grundlage einer Definition von ausgezeichneten Richtungen in Frage, aber nur $\boldsymbol{M}$ verknüpft im Gegensatz zu $\boldsymbol{A}$ die beiden axialen Vektoren miteinander. Das tut zwar auch die Matrix $\boldsymbol{C}$, aber diese ist im Gegensatz zu $\boldsymbol{C}-\boldsymbol{B}_{1} \boldsymbol{A}^{-1} \boldsymbol{B}_{2}=\boldsymbol{M}^{-1}$ nicht translationsinvariant. Es muß demnach eine Definition gefunden werden, mit der man aus $\boldsymbol{M}$ und $\boldsymbol{M}^{-1}$ dieselben Richtungen herausbekommt. Genau das leisten die Eigenvektoren einer Matrix, also werden zur Definition dieser Normalform die funktionellen Achsen des letzten Abschnitts herangezogen $\stackrel{23}{23}$ Mit einer Tilde $\tilde{*}$ (oder genauer $\left.*\right|_{Z}$ bzw. $\left.*\right|_{T D}$ ) sollen im Folgenden die auf das elastische Zentrum transformierten Größen und mit einem Hut $\hat{*}$ die Standardformen der Größen gekennzeichnet werden.

Je nach Anwendungsbereich sollen diese Standardformen dabei verschiedene Namen tragen. Während die Realform auf beliebige Meßwerte angewandt werden kann, ist die Normalform für symmetrisierte oder unter Annahme eines Potentials berechnete Matrizen angemessen. Die Idealform wird, wie der Name schon sagt, nur in idealen Fällen benutzt, etwa wenn $\boldsymbol{M}$ und $\boldsymbol{A}$ von sich aus in kartesischen Koordinaten diagonal sind. Speziell bei Aufhängungen mit hoher Symmetrie wird das Sinn machen. Sie ist zur Auswertung von Meßwerten weniger geeignet. Ergeben sich weitere Symmetrien, etwa bei der Existenz eines Widerstandszentrums, so sollen weitere Namen verwandt werden, um diesen Umständen Rechnung zu tragen.

In der Theorie, also insbesondere für zueinander passende Koordinatensysteme, sind beide elastischen Matrizen symmetrisch. Es gilt also

$$
\begin{aligned}
\boldsymbol{D} & =\boldsymbol{D}^{S} \\
\boldsymbol{M} & =\boldsymbol{M}^{S} \\
\boldsymbol{S}_{2} & =\boldsymbol{S}_{1}^{T}=: \boldsymbol{S}
\end{aligned}
$$

für die Flexibilitätsmatrix und

$$
\begin{aligned}
\boldsymbol{A} & =\boldsymbol{A}^{S} \\
\boldsymbol{C} & =\boldsymbol{C}^{S} \\
\boldsymbol{B}_{2} & =\boldsymbol{B}_{1}^{T}=: \boldsymbol{B}
\end{aligned}
$$

für die Steifheitsmatrix.

Durch die symmetrische Matrix $\boldsymbol{M}=\left(\boldsymbol{C}-\boldsymbol{B} \boldsymbol{A}^{-1} \boldsymbol{B}^{T}\right)^{-1}$ werden nun Richtungen im Raum ausgezeichnet:

\footnotetext{
${ }^{23}$ Nicht nur die Analogie zu den Hauptträgheitsachsen hat bei dieser Wahl eine Rolle gespielt. Meinen Erfahrungen mit realen Meßwerten nach ist $\boldsymbol{M}$ von der Fehlerfortpflanzung her bei schlecht zur Theorie passenden Daten günstiger: Die Matrix $\boldsymbol{M}$ ist in allen mir zur Auswertung vorgelegten Fällen im Gegensatz zu $\boldsymbol{A}$ symmetrischer (im Sinne der Güte aus Anhang A.2.3) und darüber hinaus meistens sogar diagonaldominant.
} 
Die charakteristischen Richtungen der elastischen Aufhängung sind die funktionellen Achsen, also die normierten Eigenvektoren $\boldsymbol{e}_{i}$ der symmetrischen Matrix $\boldsymbol{M}$. Die Transformationsmatrix $\boldsymbol{R}$ setzt sich aus den in einem Rechtssystem angeordneten Spaltenvektoren über $\boldsymbol{R}=\left(\boldsymbol{e}_{1}, \boldsymbol{e}_{2}, \boldsymbol{e}_{3}\right)$ zusammen; sie ist eine Drehmatrix. Die reellen und positiven Eigenvektoren $m_{i}$ sind dabei der Größe nach angeordnet; zur Veranschaulichung am Meßobjekt kann optional ihre Reihenfolge dem ursprünglichen Koordinatensystem $\left(\boldsymbol{e}_{x}, \boldsymbol{e}_{y}, \boldsymbol{e}_{z}\right)$ angeglichen werden .

Damit ist die Normalform der Steifheitsmatrix bis auf eine prinzipiell noch mögliche zyklische Permutation der Eigenvektoren eindeutig bestimmt. Da die Matrix $\boldsymbol{M}$ symmetrisch ist, läßt sie sich durch die Drehung $\boldsymbol{R}$ auf Hauptachsen- bzw. Diagonalgestalt diag $\left(m_{i}\right)$ transformieren

$$
\hat{\boldsymbol{M}}=\operatorname{diag}\left(m_{i}\right)=\boldsymbol{R} \boldsymbol{M} \boldsymbol{R}^{T},
$$

womit die Normalform der elastischen Matrizen gefunden ist:

In der Normalform der Flexibilitäts- bzw. Steifheitsmatrix sind die transformationsinvariante Teilmatrix $\boldsymbol{M}$ und ihre Inverse $\boldsymbol{C}-\boldsymbol{B} \boldsymbol{A}^{-1} \boldsymbol{B}^{T}$ diagonal.

In $Z$ gilt $\left(\tilde{\boldsymbol{M}}^{-1} \tilde{\boldsymbol{S}}\right)^{D}=\mathbf{0}$, also ist $\boldsymbol{S}_{Z}=\boldsymbol{W} \boldsymbol{M}$, wobei $\boldsymbol{W}$ symmetrisch ist vgl. 2.2.5.2 und über das elastische Zentrum definiert ist, also $\boldsymbol{W} \equiv \tilde{\boldsymbol{W}}$. In Blockkomponenten sieht die Normalform von $\mathcal{F}$ damit so aus:24

$$
\hat{\mathcal{F}}=\underbrace{\left(\begin{array}{cc}
\boldsymbol{R} & \mathbf{0} \\
\mathbf{0} & \boldsymbol{R}
\end{array}\right)}_{=: \mathcal{R}} \mathcal{F}_{Z}\left(\begin{array}{cc}
\boldsymbol{R}^{T} & \mathbf{0} \\
\mathbf{0} & \boldsymbol{R}^{T}
\end{array}\right)=\left(\begin{array}{cc}
\hat{\boldsymbol{D}}^{S} & \operatorname{diag}\left(m_{i}\right) \hat{\boldsymbol{W}}^{S} \\
\hat{\boldsymbol{W}}^{S} \operatorname{diag}\left(m_{i}\right) & \operatorname{diag}\left(m_{i}\right)
\end{array}\right) .
$$

Damit sind die ursprünglich 21 Komponenten einer symmetrischen $6 \times 6$-Matrix auf 3 Diagonaleinträge in der transformationsinvarianten Teilmatrix $\boldsymbol{M}$ plus zweimal 6 Komponenten der beiden symmetrischen Teilmatrizen $\hat{D}$ und $\hat{\boldsymbol{W}}$ reduziert. Die Differenz von 6 Größen sind genau die 6 Freiheitsgrade der Translation und Rotation - hier festgelegt durch die Transformation auf das elastische Zentrum

${ }^{24}$ Für $\hat{\mathcal{S}}=\mathcal{R}^{T} \mathcal{S}_{Z} \mathcal{R}$ folgt keine so einfache Gestalt. Allenfalls für die Teilmatrix $\hat{\boldsymbol{C}}$ gilt

$$
\begin{aligned}
\hat{C_{Z}} & =\boldsymbol{R}^{T}\left(\boldsymbol{C}_{Z}\right) \boldsymbol{R} \stackrel{\sqrt{2.41}=}{=} \boldsymbol{R}^{T}\left(\boldsymbol{C}-\boldsymbol{B} \boldsymbol{A}^{-1} \boldsymbol{B}^{T}-\boldsymbol{W} \boldsymbol{A W}\right) \boldsymbol{R} \\
\stackrel{(2.25)}{=} & \boldsymbol{R}^{T}\left(\boldsymbol{M}^{-1}-\boldsymbol{W} \boldsymbol{A} \boldsymbol{W}\right) \boldsymbol{R} \\
= & \operatorname{diag}\left(m_{i}^{-1}\right)-\hat{\boldsymbol{W}} \hat{\boldsymbol{A}} \hat{\boldsymbol{W}} .
\end{aligned}
$$

d. h. falls ein Widerstandszentrum existiert, ist auch $\hat{\boldsymbol{C}}$ diagonal. 
und die Drehung auf die funktionellen Achsen. Als Nächstes soll diese Definition auf nicht symmetrische Ausgangsmatrizen verallgemeinert werden.

Bei fehlerbehafteten Matrizen versagt eine Definition über die Eigenvektoren, da diese dann nicht mehr reell sein müssen. Um die verbleibenden Freiheitsgrade der Rotation in den Referenzsystemen der Lasten und Lagen zu berücksichtigen, wird statt der Eigenvektoren von $\boldsymbol{M}$ die Zerlegung in ihre singulären Werte ${ }^{25}$ verwendet. Aus zwei Drehungen $\boldsymbol{R}_{f}$ und $\boldsymbol{R}_{q}$ mit der Eigenschaft, daß $\operatorname{diag}\left(m_{i}\right)=\boldsymbol{R}_{q} \boldsymbol{M} \boldsymbol{R}_{f}^{T}$, setzt man nun zwei Drehungen

$$
\mathcal{R}_{q}:=\left(\begin{array}{cc}
\boldsymbol{R}_{q} & \mathbf{0} \\
\mathbf{0} & \boldsymbol{R}_{q}
\end{array}\right) \quad \text { und } \quad \mathcal{R}_{f}:=\left(\begin{array}{cc}
\boldsymbol{R}_{f} & \mathbf{0} \\
\mathbf{0} & \boldsymbol{R}_{f}
\end{array}\right)
$$

für die verallgemeinerten Lagen $\hat{q}=\mathcal{R}_{q} q$ und Lasten $\hat{f}=\mathcal{R}_{f} f$ zusammen und verfährt weiter wie oben. Damit nutzt man die Freiheit aus, daß die Orientierung von Last- und Meßsystem prinzipiell unabhängig voneinander wählbar ist 26 Man erhält für Meßwerte die Normalform, für reale Meßwerte die Realform:

Wie in der Normalform ist in der Realform der Flexibilitätsmatrix die transformationsinvariante Teilmatrix $\hat{\boldsymbol{M}}$ bzw. ihre Inverse $\hat{\boldsymbol{C}}-$ $\hat{\boldsymbol{B}}_{1} \hat{\boldsymbol{A}}^{-1} \hat{\boldsymbol{B}}_{2}$ diagonal.

Das hat dieselben Konsequenzen für die Matrizen $\boldsymbol{S}_{1}$ und $\boldsymbol{S}_{2}$ wie in der Normalform für $\boldsymbol{S}$. Die ursprünglich 36 Komponenten einer $6 \times 6$-Matrix sind nun auf 3 Diagonaleinträge in der transformationsinvarianten Teilmatrix $\boldsymbol{M}$ plus zweimal 6 Komponenten der symmetrischen Matrizen $\boldsymbol{W}_{M}$ und $\boldsymbol{W}_{L}$ plus 9 Komponenten der Teilmatrix $\boldsymbol{D}$ reduziert. Die Differenz von 12 Größen sind genau zweimal die 6 Freiheitsgrade der Translation und Rotation - hier festgelegt durch die beiden Transformationen auf das elastische Zentrum und die beiden Drehungen der

\footnotetext{
${ }^{25}$ Vgl. [73] \$16.5. Der Zusammenhang zwischen der Hauptachsentransformation und der Zerlegung in die singulären Werte ergibt sich wie folgt. Sei $\boldsymbol{M}$ zerlegt in

$$
\boldsymbol{M}=\boldsymbol{R}_{q}^{T} \hat{\boldsymbol{M}} \boldsymbol{R}_{f}=\boldsymbol{R}_{q}^{T} \operatorname{diag}\left(m_{i}\right) \boldsymbol{R}_{f}, \quad \text { also } \quad \hat{\boldsymbol{M}}=\operatorname{diag}\left(m_{i}\right)=\boldsymbol{R}_{q} \boldsymbol{M} \boldsymbol{R}_{f}^{T}
$$

Auch die symmetrischen Matrizen $\boldsymbol{M} \boldsymbol{M}^{T}$ und $\boldsymbol{M}^{T} \boldsymbol{M}$ werden dadurch zerlegt

$$
\begin{aligned}
& \boldsymbol{M} \boldsymbol{M}^{T}=\boldsymbol{R}_{q}^{T} \operatorname{diag}\left(m_{i}\right) \boldsymbol{R}_{f} \boldsymbol{R}_{f}^{T} \operatorname{diag}\left(m_{i}\right) \boldsymbol{R}_{q}=\boldsymbol{R}_{q}^{T} \operatorname{diag}\left(m_{i}^{2}\right) \boldsymbol{R}_{q} \\
& \boldsymbol{M}^{T} \boldsymbol{M}=\boldsymbol{R}_{f}^{T} \operatorname{diag}\left(m_{i}\right) \boldsymbol{R}_{q} \boldsymbol{R}_{q}^{T} \operatorname{diag}\left(m_{i}\right) \boldsymbol{R}_{f}=\boldsymbol{R}_{f}^{T} \operatorname{diag}\left(m_{i}^{2}\right) \boldsymbol{R}_{f}
\end{aligned}
$$

die beiden Drehmatrizen der Zerlegung in die singulären Werte sind also die der Hauptachsen von $\boldsymbol{M} \boldsymbol{M}^{T}$ und $\boldsymbol{M}^{T} \boldsymbol{M}$.

${ }^{26}$ Die Situation ist vergleichbar mit der, die zur Definition des elastischen Zentrums führte. Dort konnte die Wunschvorstellung eines Widerstandszentrums nur für symmetrische Matrizen in das elastische Zentrum umgedeutet werden. Für unsymmetrische Matrizen ergaben sich zwei Ausfertigungen des elastischen Zentrums: Translations- und Drehmomentszentrum. Entsprechend erhält man für die beteiligten Koordinatensysteme jeweils eine mögliche Orientierung.
} 
Zerlegung in die singulären Werte. Die Komponenten der Realform sind

$$
\hat{\mathcal{F}}=\mathcal{R}_{q} \mathcal{F}_{D T} \mathcal{R}_{f}^{T} \stackrel{(2.40)}{=}\left(\begin{array}{cc}
\hat{\boldsymbol{D}} & \operatorname{diag}\left(m_{i}\right) \hat{\boldsymbol{W}}_{M}^{S} \\
\hat{\boldsymbol{W}}_{L}^{S} \operatorname{diag}\left(m_{i}\right) & \operatorname{diag}\left(m_{i}\right)
\end{array}\right)
$$

und wieder ist $\hat{\boldsymbol{C}}$ diagonal, falls ein Widerstandszentrum vorliegt.

Störend an diesen beiden Definitionen ist nur die fehlende Forminvarianz der Standardform von Flexibilitäts- und Steifheitsmatrix. Unter Preisgabe der Orthogonalität des Referenzsystems oder in speziellen Situationen erhält man im gemeinsamen Eigensystem von $\boldsymbol{A}$ und $\boldsymbol{M}$, also in dem der Achsen konstanter Helizität, die Idealform der Matrizen, die dieses Problem überwindet:

In der Idealform der Flexibilitäts- bzw. Steifheitsmatrix sind die beiden translationsinvarianten Teilmatrizen $\boldsymbol{M}=\left(\boldsymbol{C}-\boldsymbol{B}_{1} \boldsymbol{A}^{-1} \boldsymbol{B}_{2}\right)^{-1}$ und $\boldsymbol{A}=\left(\boldsymbol{D}-\boldsymbol{S}_{2} \boldsymbol{M}^{-1} \boldsymbol{S}_{1}\right)^{-1}$ diagonal .

Mit (2.43) folgt nun

$$
\boldsymbol{D}_{D T}=\boldsymbol{A}^{-1}+\boldsymbol{W}_{M} \boldsymbol{M} \boldsymbol{W}_{L}
$$

weshalb, falls ein Widerstandszentrum existiert, auch $\boldsymbol{D}=\boldsymbol{A}^{-1}$ diagonal ist.

Nun sind die ursprünglich 36 Komponenten einer symmetrischen $6 \times 6$-Matrix auf 3 Diagonaleinträge in den transformationsinvarianten Teilmatrizen $\boldsymbol{A}$ und $\boldsymbol{M}$ plus zweimal 6 Komponenten der beiden symmetrischen Matrizen $\boldsymbol{W}_{L}$ und $\boldsymbol{W}_{M}$, also insgesamt 21 Einträge, reduziert. Die Idealformen der elastischen Matrizen lauten

$$
\begin{gathered}
\hat{\mathcal{S}}=\left(\begin{array}{cc}
\operatorname{diag}\left(a_{i}\right) & -\operatorname{diag}\left(a_{i}\right) \hat{\boldsymbol{W}}_{M} \\
-\hat{\boldsymbol{W}}_{L} \operatorname{diag}\left(a_{i}\right) & \operatorname{diag}\left(m_{i}^{-1}\right)-\hat{\boldsymbol{W}}_{L} \operatorname{diag}\left(a_{i}\right) \hat{\boldsymbol{W}}_{M}
\end{array}\right) \\
\hat{\mathcal{F}}=\left(\begin{array}{cc}
\operatorname{diag}\left(a_{i}^{-1}\right)+\hat{\boldsymbol{W}}_{M} \operatorname{diag}\left(m_{i}\right) \hat{\boldsymbol{W}}_{L} & \hat{\boldsymbol{W}}_{M} \operatorname{diag}\left(m_{i}\right) \\
\operatorname{diag}\left(m_{i}\right) \hat{\boldsymbol{W}}_{L} & \operatorname{diag}\left(m_{i}\right)
\end{array}\right),
\end{gathered}
$$

und für ein Widerstandszentrum sind dann beide gleich auf Diagonalform gebracht. Die Differenz von 3 weiteren Größen gegenüber der Normalform liegt an der Festlegung von drei nichtorthogonalen Achsen: Diese haben dreimal 2 Freiheitsgrade statt der Orientierung allein.

Natürlich ist die Idealform auch auf symmetrische Matrizen anwendbar, wobei sich dann wegen $\boldsymbol{W}_{L}=\boldsymbol{W}_{M}$ die Anzahl der unabhängigen Komponenten von 21 auf 12 statt von 36 auf 21 reduziert: 3 für das elastische Zentrum und 6 für die Achsen fehlen.

Über diese drei Definitionen hinaus sind noch - immer spezieller werdend - die folgenden Situationen sinnvoll mit Namen zu belegen: die Blockdiagonalform, bei 
der ein Widerstandszentrum vorliegt, und die Diagonalform, bei der zusätzlich die Idealform vorliegt. Ein Sonderfall ist noch die Skalarform, die die Form der Massenmatrix (vgl. 3.1.1) ist, bei der die Stärke der elastische Aufhängung, wie die Trägheit eines Massenpunktes, durch ein Vielfaches der Einheitsmatrix beschrieben wird, vgl. 4.5. 


\section{Kapitel 3}

\section{Effekte realer Messungen}

An biologischen Objekten gewonnene Meßwerte stimmen leider nur selten mit der Vorhersage einer strengen Theorie überein. In diesem Kapitel sollen einige Besonderheiten realer Meßdaten besprochen werden, die nicht mit den idealisierten Vorstellungen des letzten Kapitels übereinstimmen. Diese Diskrepanzen können mehrere Ursachen haben:

1. im Meßobjekt selber, bzw. in einer geeigneten Zustandsgleichung, die beispielsweise auch eine Beschreibung von Relaxationseffekten einschließt (Abschnitt 3.1),

2. in der Auswertung, die die Rohdaten in eine Flexibilitäts- bzw. Steifheitsmatrix umwandelt (Abschnitt [3.2), und schließlich

3. im Versuchsaufbau bzw. in der Durchführung der Messung (Abschnitt 3.3).

Nicht alle Effekte lassen sich mit den von mir erdachten Auswertungsmethoden getrennt aus den Meßdaten ablesen. Als einzigen Vorschlag kann ich dann eine plausible Reihenfolge der Größenordnungen der fehlerverursachenden Effekte anbieten.

\subsection{Zustandsgleichungen und Dynamik}

Eine Gleichung der Form

$$
q=\mathcal{F} f
$$

besagt, daß eine Last $f(t)$ ohne zeitliche Verzögerung eine Auslenkung $q(t)$ hervorruft. Dies entspricht dem sich in der Realität erst allmählich einstellenden Gleichgewichtszustand. Das Hookesche Gesetz ist demnach der Idealzustand. Die Auftragung von $q(t)$ gegen $f(t)$ bei realen Messungen ergibt oft Hysteresekurven mit von 
der Art des Kraftstoßes abhängenden Verläufen, [3, 4] und [72]. Je nach Verhalten unter Wechsellast unterscheidet man

1. Echte Hysteresen mit $\oint \boldsymbol{F} d \boldsymbol{s} \geq 0$, es wird Energie dissipiert;

2. Achtförmige Kurven mit Doppelpunkt im Ursprung und $\oint \boldsymbol{F} d \boldsymbol{s} \approx 0$. In diesem Fall existieren zusätzlich zum elastischen Potential noch zusätzlich weitere innere Freiheitsgrade, die während eines Umlaufes elastische Energie aufnehmen und wieder abgeben können.

Zeitabhängige Probleme werden durch Bewegungsgleichungen beschrieben. Daher werden als Erstes zusätzliche Kräfte untersucht: Die der Trägheit und die der inneren Reibung.

\subsubsection{Kinetische Energie}

Ein ausgezeichneter Punkt des starren Körpers, der als Ursprung des körperfesten Koordinatensystems dient, bewegt sich auf der Bahn $\boldsymbol{d}(t)$. Die Koordinatenachsen drehen sich mit der Drehmatrix $\boldsymbol{R}(t)$. Ein Punkt des starren Körpers, beschrieben durch den Ortsvektor $\boldsymbol{\xi}$, bewegt sich dann auf der Bahn

$$
\boldsymbol{x}(t)=\boldsymbol{d}(t)+\boldsymbol{R}(t) \boldsymbol{\xi} .
$$

Für kleine Bewegungen reicht der Drehvektor $\boldsymbol{\theta}$ wegen $\boldsymbol{R}(t)=\boldsymbol{I}+\boldsymbol{\Omega}_{\xi}+\mathcal{O}\left(\theta^{2}\right)$ zur Beschreibung der Bewegung aus. Die Bahngeschwindigkeit lautet also $\mathbb{1}$

$$
\dot{\boldsymbol{x}}(t)=\dot{\boldsymbol{d}}(t)+\dot{\boldsymbol{\theta}}(t) \times \boldsymbol{\xi} .
$$

Nun kann die Bahngeschwindigkeit in den verallgemeinerten Koordinaten ausgedrückt werden mittels

$$
\begin{aligned}
\dot{\boldsymbol{x}}(\boldsymbol{\xi}, t) & =\dot{\boldsymbol{d}}(t)-\boldsymbol{\Omega}_{\xi} \dot{\boldsymbol{\theta}}(t)=\underbrace{\left(\boldsymbol{I} \vdots-\boldsymbol{\Omega}_{\xi}\right)}_{=: \boldsymbol{X}} \dot{q}(t) \\
& =\boldsymbol{X}(\boldsymbol{\xi}) \dot{q}(t) .
\end{aligned}
$$

\footnotetext{
${ }^{1}$ Für endliche Bewegungen erhält man die Ableitung $\dot{\boldsymbol{R}}$ einer Drehmatrix aus $\boldsymbol{I}=\boldsymbol{R}^{T} \boldsymbol{R}$ über die Produktregel$$
\mathbf{0}=\dot{\boldsymbol{R}}^{T} \boldsymbol{R}+\boldsymbol{R}^{T} \dot{\boldsymbol{R}}=\dot{\boldsymbol{R}}^{T} \boldsymbol{R}+\boldsymbol{R}^{T} \dot{\boldsymbol{R}}=\left(\boldsymbol{R}^{T} \dot{\boldsymbol{R}}\right)^{T}+\boldsymbol{R}^{T} \dot{\boldsymbol{R}}
$$

Da offenbar $\boldsymbol{R}^{T} \dot{\boldsymbol{R}}$ eine antisymmetrische Matrix $\boldsymbol{\Omega}$ ist, folgt

$$
\dot{R}=R \Omega,
$$

nach einem Vektorprodukt im Gangsystem folgt also die Drehung ins Rastsystem. Einen analogen Ausdruck mit einem Vektorprodukt im Rastsystem erhält man analog aus $\boldsymbol{I}=\boldsymbol{R} \boldsymbol{R}^{T}$.
} 
Die kinetische Energie ist eine Funktion der Zeit $T(t)$, gegeben durch eine Integration über alle Massenelemente $d m$ des Körpers. Mit der Massendichte $\rho(\boldsymbol{\xi})$ bekommt man die kinetische Energie

$$
\begin{aligned}
T(t) & =\frac{1}{2} \iiint \dot{\boldsymbol{x}}^{2} d m=\frac{1}{2} \iiint \dot{\boldsymbol{x}}^{T} \dot{\boldsymbol{x}} \rho(\boldsymbol{\xi}) d V=\frac{1}{2} \iiint \dot{q}^{T} \boldsymbol{X}^{T} \boldsymbol{X} \dot{q} \rho(\boldsymbol{\xi}) d V \\
& =\frac{1}{2} \dot{q}^{T} \underbrace{\left(\iiint \boldsymbol{X}^{T} \boldsymbol{X} \rho(\boldsymbol{\xi}) d V\right)}_{=: \mathcal{M}} \dot{q}
\end{aligned}
$$

als quadratische Form $T=\frac{1}{2} \dot{q}^{t} \mathcal{M} \dot{q}$ der verallgemeinerten Geschwindigkeiten mit der Massenmatrix $\mathcal{M}$. Die innere Struktur der Massenmatrix läßt sich nun berechnen über

$$
\begin{aligned}
\mathcal{M} & =\iiint\left(\begin{array}{c}
\boldsymbol{I} \\
\boldsymbol{\Omega}_{\xi}
\end{array}\right)\left(\begin{array}{lll}
\boldsymbol{I} & \vdots & -\boldsymbol{\Omega}_{\xi}
\end{array}\right) \rho(\boldsymbol{\xi}) d V \\
& =\left(\begin{array}{cc}
\iint \boldsymbol{I} \rho(\boldsymbol{\xi}) d V & -\iint \boldsymbol{\Omega}_{\xi} \rho(\boldsymbol{\xi}) d V \\
\iint \boldsymbol{\Omega}_{\xi} \rho(\boldsymbol{\xi}) d V & -\iint \boldsymbol{\Omega}_{\xi}^{2} \rho(\boldsymbol{\xi}) d V
\end{array}\right) \\
& \stackrel{\text { A.2. }}{=}\left(\begin{array}{cc}
\iint \boldsymbol{I} \rho(\boldsymbol{\xi}) d V & -\iint \boldsymbol{\Omega}_{\xi} \rho(\boldsymbol{\xi}) d V \\
\iint \boldsymbol{\Omega}_{\xi} \rho(\boldsymbol{\xi}) d V & \iint \boldsymbol{\xi}^{2} \boldsymbol{P}_{\xi}^{\perp} \rho(\boldsymbol{\xi}) d V
\end{array}\right) \\
& =\left(\begin{array}{cc}
m \boldsymbol{I} & -m \boldsymbol{\Omega}_{s} \\
m \boldsymbol{\Omega}_{s} & \boldsymbol{J}
\end{array}\right),
\end{aligned}
$$

worin $m$ die Gesamtmasse, $\boldsymbol{s}$ der Ortsvektor des Schwerpunktes und $\boldsymbol{J}$ der Trägheitstensor sind. Liegt der Koordinatenursprung im Schwerpunkt, so verschwinden die Nebendiagonalmatrizen. Gedreht auf die Hauptträgheitsachsen, ist $\boldsymbol{J}$ und damit ganz $\mathcal{M}$ diagonal. Das ist das von der Massenmatrix bevorzugte Koordinatensystem. Dieselbe Rechenvorschrift wie für das elastische Zentrum ergibt formal angewandt auf $\mathcal{M}$

$$
-\left[(m \boldsymbol{I})^{-1}\left(-m \boldsymbol{\Omega}_{s}\right)\right]^{D}=+\boldsymbol{\Omega}_{s}^{D}=\boldsymbol{s},
$$

was die Analogie des elastischen Zentrums zum Schwerpunkt noch einmal unterstreicht?

Das Transformationsverhalten der Massenmatrix $\mathcal{M}$ läßt sich aus einer Translation der Integrationsvariablen $\boldsymbol{\xi}$ ableiten; da die Transformationsmatrizen aus (2.1) und (2.3) keine Funktionen der Zeit sind, transformieren sich die Ableitungen der

\footnotetext{
${ }^{2}$ Noch deutlicher wird dies, wenn man statt der Matrizen $\boldsymbol{X}$ wieder die Transformationsmatrizen $\mathcal{T}$ verwendet. Die Massenmatrix ergibt sich dann aus

$$
\begin{aligned}
\mathcal{M} & =\iiint d V\left(\begin{array}{cc}
\boldsymbol{I} & \mathbf{0} \\
\boldsymbol{\Omega}_{\xi} & \boldsymbol{I}
\end{array}\right)\left(\begin{array}{cc}
\rho(\boldsymbol{\xi}) \boldsymbol{I} & \mathbf{0} \\
\mathbf{0} & \mathbf{0}
\end{array}\right)\left(\begin{array}{cc}
\boldsymbol{I} & -\boldsymbol{\Omega}_{\xi} \\
\mathbf{0} & \boldsymbol{I}
\end{array}\right) \\
& =\iiint d V \rho(\boldsymbol{\xi})\left(\begin{array}{cc}
\boldsymbol{I} & -\boldsymbol{\Omega}_{\xi} \\
\boldsymbol{\Omega}_{\xi} & -\boldsymbol{\Omega}_{\xi}^{2}
\end{array}\right)=\left(\begin{array}{cc}
m \boldsymbol{I} & -m \boldsymbol{\Omega}_{s} \\
m \boldsymbol{\Omega}_{s} & \boldsymbol{J}
\end{array}\right) .
\end{aligned}
$$
}


Lasten oder Lagen wie die Lasten oder Lagen selber. Unter Wechsel des Koordinatenursprunges transformiert sich die Massenmatrix $\mathcal{M}$ daher wie die Steifheitsmatrix $\mathcal{S}$. In Komponenten kann man nun etwa $\mathcal{M}_{O}$ bezüglich $O$ durch die Form im Schwerpunkt $S$ ausdrücken. Es ergibt sich

$$
\begin{aligned}
\mathcal{M}_{O} & =\mathcal{T}_{O S} \mathcal{M}_{S} \mathcal{T}_{S O}^{T} \\
& =\left(\begin{array}{cc}
\boldsymbol{I} & \mathbf{0} \\
\boldsymbol{\Omega}_{s} & \boldsymbol{I}
\end{array}\right)\left(\begin{array}{cc}
m \boldsymbol{I} & \mathbf{0} \\
\mathbf{0} & \boldsymbol{J}_{S}
\end{array}\right)\left(\begin{array}{cc}
\boldsymbol{I} & -\boldsymbol{\Omega}_{s} \\
\mathbf{0} & \boldsymbol{I}
\end{array}\right) \\
& =\left(\begin{array}{cc}
m \boldsymbol{I} & -m \boldsymbol{\Omega}_{s} \\
m \boldsymbol{\Omega}_{s} & \boldsymbol{J}_{S}-m \boldsymbol{\Omega}_{s}^{2}
\end{array}\right),
\end{aligned}
$$

also speziell für den Trägheitstensor mit (A.2)

$$
\boldsymbol{J}_{O}=\boldsymbol{J}_{S}+m s^{2} \boldsymbol{P}_{s}^{\perp}
$$

besser bekannt in der Koordinatenform $\left(\boldsymbol{J}_{O}\right)_{i j}=\left(\boldsymbol{J}_{S}\right)_{i j}+m\left(s^{2} \delta_{i j}-s_{i} s_{j}\right)$ als Satz von STEINER - ein weiterer Beleg für die Leistungsfähigkeit des hier verwendeten Formalismus.

Die Bewegungsgleichung eines reibungsfrei elastisch aufgehängten starren Körpers ergibt sich nun aus dem Kräftegleichgewicht

$$
0=\mathcal{M} \ddot{q}+\mathcal{S} q
$$

Die Matrizen $\mathcal{M}$ und $\mathcal{S}$ müssen nicht im selben Bezugssystem diagonal sein, d. h. die zu erwartenden Bewegungsformen müssen nicht einfach sein. Eine harmonische Bewegung $q=q_{0} e^{i \omega t}$ führt über $0=\left(-\mathcal{M} \omega^{2}+\mathcal{S}\right) q_{0} e^{i \omega t}$ auf die charakteristische Gleichung

$$
\operatorname{det}\left(-\mathcal{M} \omega^{2}+\mathcal{S}\right)=0
$$

eine Gleichung sechsten Grades für die Quadrate der Eigenfrequenzen, d. h. für die Eigenfrequenzen selber liegen immer Doppelwurzeln vor. Die zu den $\omega_{i}, i=$ $1 \ldots 6$ gehörenden Lösungen $\left(q_{0}\right)_{i}$ nennt man Normal- oder Hauptkoordinaten des Systems.

Die Vorstellung, daß $q=q_{0} e^{i \omega t}$ eine harmonische Schraubschwingung darstellt, muß noch präzisiert werden. In den Formeln (2.4) und (2.5) für die Aufpunkte, sowie (2.6) und (2.7) für die Steigungen der Schrauben stehen in den Zählern Produkte aus zwei Größen $\sim e^{i \omega t}$ und in den Nennern ein Betrag. Es ergeben sich also für eine Normalmode Schraubsteigungen oder Schraubachsaufpunkte, die mit der doppelten Frequenz harmonisch schwingen, etwa

$$
\frac{1}{\left|\boldsymbol{\theta} e^{i \omega t}\right|^{2}}\left(\boldsymbol{\theta} e^{i \omega t} \circ \boldsymbol{d} e^{i \omega t}\right)=\frac{1}{|\boldsymbol{\theta}|^{2}}(\boldsymbol{\theta} \circ \boldsymbol{d}) e^{i 2 \omega t},
$$

und entsprechende Ausdrücke für die Schraubparameter der Kraftschraube. Bei einer Superposition von Normalmoden können die zeitabhängigen Terme im Nenner 
nicht mehr gekürzt werden, es ergeben sich dann keine harmonischen Bewegungen mehr.

Um Hysteresen beschreiben zu können, muß noch ein Modell für die Dämpfung gefunden werden, was im nächsten Abschnitt geschehen soll.

\subsubsection{Dissipation}

Statt die Überlegungen auf der Existenz eines elastischen Potentials aufzubauen, muß Thermodynamik betrieben werden. Die Effekte müssen über die Gleichung

$$
d U=T d S+\sigma_{i k} d \epsilon_{i k} \quad \text { bzw. } \quad d U=T d S+f d q
$$

oder für den Fall weiterer Freiheitsgrade durch entsprechende Beziehungen berechnet werden. Ein thermodynamischer Prozeß ist aber nur dann reversibel, wenn er mit beliebig kleiner Geschwindigkeit verläuft; nur dann stellt sich zu jedem Zeitpunkt ein Gleichgewichtszustand ein.

Unter Außerachtlassung der auf Wärmeleitung beruhenden Anteile der dissipierten mechanischen Energie wird diese allein durch Prozesse der inneren Reibung erklärt, vgl. ([40], §33). Statt dem HookEschen Gesetz $\sigma_{i j}=\lambda_{i j k l} \epsilon_{k l}$ besteht nun noch zusätzlich ein Anteil des Spannungstensors, der linear von der zeitlichen Ableitung der Dehnung abhängt, also insgesamt

$$
\sigma_{i j}=\lambda_{i j k l} \epsilon_{k l}+\eta_{i j k l} \dot{\epsilon}_{k l}
$$

Da in unserem Fall der elastisch aufgehängte starre Körper durch weniger Freiheitsgrade beschrieben werden kann, gilt einfach

$$
f_{i}=\mathcal{S}_{i j} q_{j}+\mathcal{D}_{i j} \dot{q}_{j}
$$

wobei $\mathcal{D}$ die zur Dissipationsfunktion gehörende symmetrische $6 \times 6$-Matrix ist, vgl. [38] §25. Die Bewegungsgleichung eines solchen Mediums unter Einfluß der äußeren Last $f(t)$ lautet nun

$$
f(t)=\mathcal{S} q(t)+\mathcal{D} \dot{q}(t)+\mathcal{M} \ddot{q}(t)
$$

Dieses ist die einfachste allgemeine Gleichung, mit der ein Meßvorgang am elastisch aufgehängten starren Körper beschrieben werden kann.

Der Ansatz $q=q_{0} e^{k t}$ für das homogene System führt nun auf die Säkulargleichung

$$
\operatorname{det}\left(\mathcal{S}+k \mathcal{D}+k^{2} \mathcal{M}\right)=0,
$$

eine Gleichung 12-ten Grades in der komplexen Variable $k$. Mit steigender Dämpfung findet eine zunehmende Aufspaltung der Doppelwurzeln des ungedämpften Systems statt. Durch Variation der Konstanten kann schließlich eine allgemeine Lösung für die Inhomogenität $f(t)$ gefunden werden. 


\subsubsection{Zeitabhängigkeit und Superpositionsprinzip}

Die Frage ist also, wie man auch in solchen Situationen den HookEschen Anteil, also die elastischen Matrizen $\mathcal{F}$ bzw. $\mathcal{S}$, der Aufhängung mißt. Üblich sind folgende Methoden ( $\Theta$ ist dabei die HEAvisIDEsche Stufenfunktion):

1. Kriechversuch: $f=f_{0} \cdot \Theta\left(t-t_{0}\right)$ und $q(t \rightarrow \infty)$ wird gemessen,

2. Relaxationsversuch: $q=q_{0} \cdot \Theta\left(t-t_{0}\right)$ und $f(t \rightarrow \infty)$ wird gemessen.

Es kann zu unsinnigen Meßzeiten führen, $\frac{3}{3}$ im Rahmen der Genauigkeit bis zum vollständigen Einstellen des thermischen Gleichgewichtes zu warten. Zu kleine Meßzeiten gegenüber den Relaxationszeiten führen aber beim Kriechversuch auf systematisch zu niedrige Auslenkungen und damit auf zu niedrige Matrixeinträge. Eine numerische Extrapolation auf den Funktionswert der Asymptote ist ebenfalls schwierig durchzuführen.

Eine denkbare Methode zur Verkürzung der Meßzeit nutzt die Abhängigkeit von der Form des Kraftstoßes: Durch eine anfängliche Überhöhung $\Delta f$ der Last auf $f+\Delta f$ wird $q(f+\Delta f)$ auf den erwarteten asymptotischen Wert von $q(f)$ gebracht. Danach wird die Last wieder auf $f$ verringert und nur noch gewartet, bis sich die inneren Spannungen des Materials abgebaut haben. Das Problem hierbei ist dabei die Bestimmung des zu erwartenden asymptotischen Wertes. Ebenso könnte man versuchen, durch eine aktive Regelung des Kraftsystems einen konstanten Wert für die Lage einzustellen oder durch Veränderung der Lage einen konstanten Wert für die Last zu erhalten.

Eine weitere Methode soll im Folgenden beschrieben werden. Für eine große Klasse von realen Materialien gilt das Superpositionsprinzip $(\alpha \in \boldsymbol{R})$

$$
\begin{aligned}
q\left(f_{1}(t)+f_{2}(t)\right) & =q\left(f_{1}(t)\right)+q\left(f_{2}(t)\right) \\
q(\alpha f(t)) & =\alpha q(f(t))
\end{aligned}
$$

oder der umgekehrte Zusammenhang für $f(q)$, in der Mathematik besser bekannt als Linearität. Entsprechend nennt man ein solches Materialverhalten linear. Die zeitliche Abhängigkeit der Last $f(t)$ von der Auslenkung $q(t)$ läßt sich dann mit dem linearen Differentialoperator $\mathcal{L}$ formulieren als

$$
f(t)=\mathcal{L} q(t)
$$

in Übereinstimmung mit der obigen Bewegungsgleichung. Der HookEsche Anteil, also die Steifheitsmatrix $\mathcal{S}$, ist einfach der asymptotische Wert von $\mathcal{L}$ für $f=$ const.

\footnotetext{
${ }^{3}$ Beispielsweise ergaben die Messungen zur initialen Zahnbeweglichkeit [3, 4] Zeitkonstanten in der Größenordnung von 30s. Dabei ist noch unklar, ob überhaupt eine Relaxation im eigentlichen Sinne stattfindet. Eine Bewegung des Zahnes wie bei einer kieferorthopädischen Behandlung kann nur mit einem Flüssigkeitsmodell beschrieben werden. Für eine linear viskoelastische Flüssigkeit ist aber der Kriechversuch unsinnig.
} 
Um lineare Fluide mathematisch beschreiben zu können, benötigt man einen zweiten Differentialoperator der auf die Lasten wirkt. Die konstitutive Gleichung hat dann die Form

$$
\mathcal{L}_{f} f(t)=\mathcal{L}_{q} q(t)
$$

welche für alle linear viskoelastischen Medien gilt. Die hier interessierenden Gleichungen sind analog zum KeLvin-VoigT-Modell, vgl. Abschnitt 3.1.2, oder zum Drei-Parameter-Modell 1 .

Sei $f_{B}(t)$ eine bis zum Maximum $f_{M A X}$ wachsende Belastungsfunktion und $f_{E}(t)=$ $f_{M A X}-f_{B}(t)$ die zugehörige Entlastungsfunktion, sowie $q_{\infty}$ die asymptotisch zu $f_{\text {MAX }}$ gehörende Auslenkung. Die Be- und Entlastungsexperimente werden zu verschiedenen Zeitpunkten $t_{1}$ und $t_{2}$ durchgeführt, also

$$
\begin{aligned}
& \mathcal{L}_{f} f_{B}\left(t-t_{1}\right)=\mathcal{L}_{q} q_{B}\left(t-t_{1}\right) \quad \text { und } \\
& \mathcal{L}_{f} f_{E}\left(t-t_{2}\right)=\mathcal{L}_{q} q_{E}\left(t-t_{2}\right)
\end{aligned}
$$

Ausnutzen der Linearitätseigenschaften liefert weiter

$$
\begin{aligned}
\mathcal{L}_{f}\left(f_{M A X}-f_{B}\left(t-t_{2}\right)\right) & =\mathcal{L}_{q} q_{E}\left(t-t_{2}\right) \\
\mathcal{L}_{f} f_{M A X}-\mathcal{L}_{f} f_{B}\left(t-t_{2}\right) & =\mathcal{L}_{q} q_{E}\left(t-t_{2}\right) \\
\mathcal{L}_{f} f_{M A X}-\mathcal{L}_{q} q_{B}\left(t-t_{2}\right) & =\mathcal{L}_{q} q_{E}\left(t-t_{2}\right) .
\end{aligned}
$$

Wegen des Verschwindens der Ableitungen von $f_{\text {MAX }}$ gilt $\mathcal{L}_{f} f_{\text {MAX }}=f_{\text {MAX }}$ und schließlich

$$
f_{\text {MAX }}=\mathcal{L}_{f} f_{\text {MAX }}=\mathcal{L}_{q} q_{E}\left(t-t_{2}\right)+\mathcal{L}_{q} q_{B}\left(t-t_{2}\right)=\mathcal{L}_{q}\left(q_{E}\left(t-t_{2}\right)+q_{B}\left(t-t_{2}\right)\right) \quad .
$$

Bringt man nachträglich für die aufgezeichneten Verläufe die Zeitnullpunkte $t_{1}$ und $t_{2}$ in Übereinstimmung, etwa durch einen klar erkennbaren Knick im Kurvenverlauf, so erhält man den asymptotischen Wert über

$$
f_{M A X}=\mathcal{L}_{q}\left(q_{B}(t)+q_{E}(t)\right)=\mathcal{L}_{q} q_{\infty}=\mathcal{S} q_{\infty} .
$$

Mit einer Durchführung von Be- und Entlastungsexperiment kann man so gleichzeitig zum einen die Linearität der zu Grunde liegenden Gleichungen überprüfen und zum anderen direkt den HookEschen Anteil ermitteln. Die Brauchbarkeit dieses Modells kann an der zu verschiedenen Zeitnullpunkten berechenbaren Konstanz von $q_{B}\left(t-t_{1}+t_{1}\right)+q_{E}\left(t-t_{2}+t_{2}\right)$ überprüft werden. Über eine Mittelung der Kurvenverläufe kann dann auf den asymptotischen Wert mit erhöhter Genauigkeit geschlossen werden.

Dadurch kann der Einfluß folgender Fehlerquellen beseitigt werden:

- Es wurde nicht auf Sättigung extrapoliert, und die Relaxationszeiten der zu den Matrixelementen gehörenden Prozesse sind unterschiedlich, d. h. sie setzen sich aus verschiedenen Normalmoden zusammen.

\footnotetext{
${ }^{4}$ Näheres zur linearen Viskoelastizität findet sich in 19 .
} 
- Das elastische Medium ist in Wirklichkeit eine sehr zähe Flüssigkeit, daher liefert der Versuch einer Extrapolation auf den asymptotischen Wert kein sinnvolles Ergebnis.

Die Schwierigkeit besteht nun in der Bestimmung des geeigneten Zeitnullpunktes, so daß $q_{B}(t)+q_{E}(t)=$ const möglichst gut erfüllt ist.

Unter Annahme einer bestimmten Zeitabhängigkeit lassen sich genauere Aussagen treffen als mit der Linearität allein. Das soll im nächsten Abschnitt erfolgen.

\subsubsection{Spezielle Lastsysteme}

In diesem Abschnitt sollen Effekte spezieller Lastsysteme besprochen werden. Dazu gehören die zeitlich konstante Last mit Be- und Entlastungsexperiment und die lineare Last mit gleichmäßig anwachsender Last. Der Einfluß der Trägheit sei als gering vorausgesetzt. Die Gleichung (3.2) vereinfacht sich nun zu

$$
f(t)=\mathcal{S} q(t)+\mathcal{D} \dot{q}(t) .
$$

Bei gegebener Lastfunktion $f(t)$ und vorgegebenen Anfangswerten $q_{0}:=q\left(t=t_{0}\right)$ ist das ein lineares System von Differentialgleichungen von erster Ordnung

$$
\dot{q}(t)=-\mathcal{D}^{-1} \mathcal{S} q(t)+\mathcal{D}^{-1} f(t)
$$

mit konstanten Koeffizienten. Die allgemeine Lösung läßt sich entweder durch Variation der Konstanten gewinnen oder mit der Propagatormethode. Mit dem Propagator

für welchen

$$
\mathcal{P}(t, \tau):=\exp \left(-(t-\tau) \mathcal{D}^{-1} \mathcal{S}\right),
$$

$$
\dot{\mathcal{P}}=-\mathcal{D}^{-1} \mathcal{S P}
$$

gilt, lautet die allgemeine Lösung zu einer gegebenen Lastfunktion $f(t)$

$$
q(t)=\mathcal{P}\left(t, t_{0}\right) q_{0}+\int_{t_{0}}^{t} P(t, \tau) \mathcal{D}^{-1} f(\tau) d \tau .
$$

Die Exponentialfunktion einer Matrix ist über ihre Potenzreihe definiert, ihre praktische Berechnung erfolgt über ihre Zerlegung in Eigenvektoren oder über ihr Minimalpolynom, vgl. [73] $§ 20.3{ }^{5}$ Die Methode über die Eigenvektoren des homogenen Systems, also über die Säkulargleichung, ist mit der Propagatormethode identisch.

Es sollen ferner die durch die angelegten Lastfunktionen erzeugten Drehschrauben untersucht werden, und zwar sowohl die absoluten (auch endlich oder finit genannt) als auch die momentanen, welche differentiell zu berechnen sind.

\footnotetext{
${ }^{5}$ Zum Nachrechnen der Lösung benötigt man, daß eine Matrix und ihre Exponentialabbildung miteinander vertauschen, was aber nach der Definition über ihre Potenzreihe klar ist.
} 


\subsubsection{Die konstante Last}

Eine konstante Last ist einfach gegeben durch

$$
f\left(t>t_{0}\right)=f_{0}
$$

und beinhaltet unter Vernachlässigung der Trägheitseffekte Be- und Entlastungsexperiment. Mit $\mathcal{F}=\mathcal{S}^{-1}$ erhält man nun die Lösung der Differentialgleichung (3.3) $\mathrm{zu}$

$$
q(t)=\mathcal{P}\left(t, t_{0}\right) q_{0}+\left(\mathcal{I}-\mathcal{P}\left(t, t_{0}\right)\right) \mathcal{F} f_{0}
$$

Für große Zeiten ergibt sich das asymptotische Verhalten

$$
q(t \rightarrow \infty)=\mathcal{F} f_{0}
$$

und nahe $t_{0}$ ergibt sich das lineare Verhalten

$$
\begin{aligned}
q\left(t \approx t_{0}\right) & =q_{0}+\left(t-t_{0}\right) \mathcal{D}^{-1} \mathcal{S}\left(\mathcal{F} f_{0}-q_{0}\right) \\
& =q_{0}+\left(t-t_{0}\right) \mathcal{D}^{-1}\left(f_{0}-\mathcal{S} q_{0}\right)=q_{0}+\left(t-t_{0}\right) q_{1}
\end{aligned}
$$

Die Flexibilitätsmatrix ergibt sich also nach Abklingen der zeitabhängigen Terme aus mehreren Belastungsexperimenten. Dazu ist das Gleichungssystem $q_{\infty}=\mathcal{F} f_{0}$ mit einem passenden statistischen Verfahren zu lösen, vgl. Abschnitt [3.2, Die Dämpfungsmatrix erhält man dann ebenso aus dem Verhalten nahe $t_{0}$ entweder mit gemessenem bekanntem $q_{0}$ und $C S$ oder, falls $q_{0} \mathrm{oBdA}$. zu $q_{0}=0$ gewählt werden kann, aus $q_{1}=\mathcal{D}^{-1} f_{0}$.

Die Schraubparameter ergeben sich nun mit der Formel (2.8), wobei sich die Zeitentwicklung von $\boldsymbol{d}$ und $\boldsymbol{\theta}$ aus der von $q$ durch Dekomposition ergibt. Im asymptotischen Fall sind die Lagen konstant und es existieren keine momentanen Schraubachsen mehr. Die absoluten Schraubachsen hingegen sind konstant. Werden die momentanen Schraubachsen zu $q_{0}=0$ durch eine endliche Differenz berechnet, so ergibt sich in der Nähe des Nullpunktes

$$
\Delta q=-\mathcal{D}^{-1} f_{0} \Delta t
$$

d. h. am Anfang der Bewegung bleiben die momentanen Schraubachsen konstant; der gemeinsame Faktor $\Delta t$ kürzt sich heraus. Die allgemeine Zeitentwicklung lautet

$$
\begin{aligned}
\dot{q}(t) & =\dot{\mathcal{P}}\left(q_{0}-\mathcal{F} f_{0}\right)=-\mathcal{D}^{-1} \mathcal{S P}(t)\left(q_{0}-\mathcal{F} f_{0}\right)=-\mathcal{P}(t) \mathcal{D}^{-1} \mathcal{S}\left(q_{0}-\mathcal{F} f_{0}\right) \\
& =-\mathcal{P}(t) \mathcal{D}^{-1}\left(\mathcal{S} q_{0}-f_{0}\right)
\end{aligned}
$$

Ist nun $\mathcal{D}^{-1}\left(\mathcal{S} q_{0}-f_{0}\right)$ eine Fundamentalmode des Systems, so wird die Zeitentwicklung durch eine einzelne Exponentialfunktion beschrieben, welche sich bei der Berechnung der momentanen Schraubparameter wiederum heraushebt. Es gibt also 6 Lastfälle, zu denen ein Belastungsexperiment konstante Momentanschrauben 
mit jeweils nicht notwendig gleichen Konstanten liefert. Da schon für etwa zwei Fundamentalmoden gilt

$$
\frac{\left(\boldsymbol{d}_{1} e^{i \omega_{1} t}+\boldsymbol{d}_{2} e^{i \omega_{2} t}\right) \circ\left(\boldsymbol{\theta}_{1} e^{i \omega_{1} t}+\boldsymbol{\theta}_{2} e^{i \omega_{2} t}\right)}{\left|\boldsymbol{\theta}_{1} e^{i \omega_{1} t}+\boldsymbol{\theta}_{2} e^{i \omega_{2} t}\right|^{2}}=\frac{\left(\boldsymbol{d}_{1}+\boldsymbol{d}_{2} e^{i\left(\omega_{2}-\omega_{1}\right) t}\right) \circ\left(\boldsymbol{\theta}_{1}+\boldsymbol{\theta}_{2} e^{i\left(\omega_{2}-\omega_{1}\right) t}\right)}{\left|\boldsymbol{\theta}_{1}+\boldsymbol{\theta}_{2} e^{i\left(\omega_{2}-\omega_{1}\right) t}\right|^{2}},
$$

ergibt sich für eine allgemeine Mode eine beliebig komplizierte Zeitabhängigkeit der Schraubparameter.

\subsubsection{Die lineare Last}

Die nächst einfachere Last nach der konstanten ist eine linear mit der Zeit ansteigende (oder fallende) Last. Zusammen mit einer konstanten Last ergibt sich die lineare Last

$$
f\left(t>t_{0}\right)=f_{0}+\left(t-t_{0}\right) f_{1}
$$

wobei $f_{0}$ und $f_{1}$ räumlich nicht proportional zueinander sein müssen $[$ [

Die Lösung der Differentialgleichung (3.3) lautet nun

$$
q(t)=\mathcal{P}\left(t, t_{0}\right) q_{0}+\left(t-t_{0}\right) \mathcal{F} f_{1}+\left(\mathcal{I}-\mathcal{P}\left(t, t_{0}\right)\right)\left(\mathcal{F} f_{0}-\mathcal{F} \mathcal{D} \mathcal{F} f_{1}\right)
$$

mit der asymptotischen Lösung

$$
q(t \rightarrow \infty)=\mathcal{F} f_{1} t+\mathcal{F} f_{0}-\mathcal{F} \mathcal{D} \mathcal{F} f_{1}=q_{\infty}+q_{1} t
$$

Die Flexibilitätsmatrix ergibt sich aus den Steigungen zu verschiedenen Lasten $f_{1}$. Mit ihr läßt sich aus den Achsabschnitten die Dämpfungsmatrix berechnen, vgl. auch 3.2 .3 ,

Nach Abklingen aller Relaxationsprozesse ergeben sich absolute Schraubachsen mit der Zeitabhängigkeit

$$
\frac{\left(\boldsymbol{d}_{\infty}+\boldsymbol{d}_{1} t\right) \circ\left(\boldsymbol{\theta}_{\infty}+\boldsymbol{\theta}_{1} t\right)}{\left|\left(\boldsymbol{\theta}_{\infty}+\boldsymbol{\theta}_{1} t\right)\right|^{2}}
$$

aber differentiell, sowie mit endlichen Differenzen nach Kürzen der Zeit, konstante momentane Schraubachsen. Fundamentalmoden ergeben nun keine konstanten Schraubachsen mehr, da aus $q(t)=q_{\infty}+q_{1} t+q_{2} e^{i \omega t}$ auch nach Differentiation kein gemeinsamer Faktor mehr herausgezogen werden kann. Zu Beginn der Lastaufbringung ergeben sich, wie gehabt, konstante Momentanschrauben.

Durch eine abgebrochene TAYLORentwicklung erhält man im allgemeinen Fall in erster Näherung linear und in zweiter Näherung quadratisch von der Zeit abhängende Schraubparameter. Die zu verwendende Ordnung der Entwicklung wird durch die Größe der einzelnen Terme und die erforderliche Genauigkeit bestimmt.

\footnotetext{
${ }^{6}$ Im Gegenteil: Wählt man etwa als Last eine reine Kraftschraube mit konstantem Anteil $F \boldsymbol{e}_{z}$ und linearem Anteil $T \boldsymbol{e}_{z}$, so entstehen je nach Wahl der Kraftwirkungslinie zusätzliche Drehmomente in der $y x$-Ebene. $\boldsymbol{F}_{0}$ und $\boldsymbol{F}_{1}$ sowie $\boldsymbol{T}_{0}$ und $\boldsymbol{T}_{1}$ stehen also senkrecht aufeinander.
} 
Interessant ist ferner die Näherung nahe $t_{0}$. Wieder ergibt sich

$$
q\left(t \approx t_{0}\right)=q_{0}+\left(t-t_{0}\right) \mathcal{D}^{-1}\left(f_{0}-\mathcal{S} q_{0}\right)+\mathcal{O}\left(\left(t-t_{0}\right)^{2}\right)
$$

Ist nun $q_{0}=0$ als Anfangswert vorgegeben und eine Last ohne konstanten Anteil $f_{0}=0$ angelegt, so verläuft die Lageänderung nicht mehr linear sondern quadratisch mit der Zeit. Genauer ergibt sich dann

$$
q\left(t \approx t_{0}\right)=\frac{1}{2}\left(t-t_{0}\right)^{2} \mathcal{D}^{-1} f_{1}
$$

Diese Gleichung taugt aber nur begrenzt zur Auswertung verrauschter Daten, da der Zeitnullpunkt nicht mehr ohne weiteres aus der Lageänderung abzulesen ist.

\subsection{Der Einfluß der Auswertung}

Einer Messung liegt immer eine Vorstellung über das Meßobjekt zu Grunde, etwa eine Zustandsgleichung, welche gleichzeitig Ziel dieser Messung ist. Da über viele Einzelmessungen gemittelt wird, sind meistens Annahmen statistischer Natur, etwa über die Verteilung der Meßwerte, beteiligt.

Im Regelfall wird man Matrizen messen, die nicht durch eine einfache Transformation der Bezugspunkte vollständig zu symmetrisieren sind. Auch die Normalform der elastischen Matrizen wird dann nicht symmetrisch sein. Nur für HookEsche Verhältnisse, also falls Drehmomentszentrum und Translationszentrum dieselben Orte sind, hängt eine Symmetrisierung der Steifheitsmatrix

$$
\mathcal{S}^{S}=\frac{1}{2}\left(\mathcal{S}+\mathcal{S}^{T}\right)
$$

nicht von der Wahl des Bezugspunktes ab. Anderenfalls wird durch die Symmetrisierung Information vernichtet. Möglicherweise ist aber die zu diskutierende Matrix im Rahmen der fortgepflanzten Eingangsfehler von ihrer symmetrisierten Variante statistisch nicht verschieden. Dies kann etwa dann entschieden werden, wenn man statt eines Punktschätzers für die Meßwerte einen Intervallschätzer benutzt, etwa in Form eines Konfidenzintervalls, und den weiteren Rechenweg dann über das Gausssche Fehlerfortpflanzungsgesetz kontrolliert.

Systematische Fehler können nur durch experimentelle Sorgfalt vermieden werden, herausrechnen lassen sie sich nachträglich kaum ${ }^{7}$ Hier sollen verschiedene statistische Verfahren vorgestellt werden, mit denen versucht wird, aus mehreren Messungen die zufälligen, nicht vermeidbaren Einzelfehler zu minimieren.

Die einfachste und schon in der Einleitung beschriebene Methode zum Bestimmen einer Flexibilitätsmatrix besteht darin, einzeln die kartesischen Komponenten der

\footnotetext{
${ }^{7}$ Vgl. etwa die gegen Ende von 6.4 angewandte Methode.
} 
Last anzulegen und die Änderungen der Endlagen durch die jeweilige Last zu dividieren. Man erhält dann die Flexibilitätsmatrix aus den sechs Lastfällen $f 1 \ldots f 6$ über

$$
\begin{aligned}
\mathcal{F} & =\left(\begin{array}{cccccc}
d 1_{x} & d 2_{x} & d 3_{x} & d 4_{x} & d 5_{x} & d 6_{x} \\
d 1_{y} & d 2_{y} & d 3_{y} & d 4_{y} & d 5_{y} & d 6_{y} \\
d 1_{z} & d 2_{z} & d 3_{z} & d 4_{z} & d 5_{z} & d 6_{z} \\
\theta 1_{x} & \theta 2_{x} & \theta 3_{x} & \theta 4_{x} & \theta 5_{x} & \theta 6_{x} \\
\theta 1_{y} & \theta 2_{y} & \theta 3_{y} & \theta 4_{y} & \theta 5_{y} & \theta 6_{y} \\
\theta 1_{z} & \theta 2_{z} & \theta 3_{z} & \theta 4_{z} & \theta 5_{z} & \theta 6_{z}
\end{array}\right)\left(\begin{array}{cccccc}
F_{x} & 0 & 0 & 0 & 0 & 0 \\
0 & F_{y} & 0 & 0 & 0 & 0 \\
0 & 0 & F_{z} & 0 & 0 & 0 \\
0 & 0 & 0 & T_{x} & 0 & 0 \\
0 & 0 & 0 & 0 & T_{y} & 0 \\
0 & 0 & 0 & 0 & 0 & T_{z}
\end{array}\right)^{-1} \\
& =(q 1, q 2, \ldots q 6)(f 1, f 2, \ldots f 6)^{-1}
\end{aligned}
$$

Diese Methode ist schnell und liefert sicherlich eine gute Abschätzung der Größenordnung der Meßwerte. Sie eignet sich insbesondere - immer unter Annahme der Gültigkeit des Superpositionsprinzipes - auch zum Gewinnen einer Steifheitsmatrix mit numerischen Methoden. Die sechs Lastfälle müssen dazu einzeln simuliert werden. Die Methode hat den Nachteil, alle dynamischen Effekte, die durch das Einschalten der Kräfte ins Spiel kommen, zu vernachlässigen, d. h. das zu messende Objekt darf keine zu großen Zeitkonstanten besitzen.

\subsubsection{Lineare Regression und Fehlerfortpflanzung}

Regressionsmodelle hängen über das Transformationsverhalten der verallgemeinerten Lasten und Lagen vom implementierten Ursprung des Koordinatensystems ab. Die dadurch verursachten Fehler können vermieden werden, indem man die Transformation auf die elastischen Zentren auf die Daten selbst anwendet und die Schätzung so oft wiederholt, bis sich ein konsistentes Bild ergibt.

Ein weiteres Problem liegt in der Linearität der Regression selbst verborgen. Wie etwa bei der Bandscheibe 6.2 kann aufgrund der Symmetrie der Aufhängung der lineare Anteil von einzelnen Komponenten verschwinden. Wendet man auf solche Daten über einen nicht zu Null symmetrischen Bereich eine lineare Regression an, so entstehen Werte, die kaum mehr mit dem Meßobjekt zu tun haben - es würde ein Parabelast durch eine Gerade ersetzt. Diese Werte können dann über die Fehlerfortpflanzung ein grob verzerrtes Bild der Situation vorspiegeln.

Das folgende Iterationsverfahren könnte diesem Problem abhelfen.

1. Aus den Daten bezüglich der Koordinatensysteme $M$ und $L$ werden die Koeffizienten einer quadratischen Regression bestimmt, etwa

$$
q_{i}(f)=F_{i j} f_{j}+F_{i j k} f_{j} f_{k} \quad .
$$

Prinzipiell sind auch andere Schätzverfahren möglich, etwa Einzelregressionen, die nicht einmal ein korrektes Transformationsverhalten besitzen müssen. 
2. Nur der lineare Anteil $F_{i j}$ wird zur Berechnung der elastischen Zentren $\overrightarrow{M D}_{1}$ und $\overrightarrow{L T}_{1}$ verwandt.

3. Die Daten selbst werden nun auf $D_{1}$ und $T_{1}$ transformiert.

4. Mit den transformierten Daten wird die Schätzung wiederholt und die elastischen Zentren ${\overrightarrow{D_{1} D_{2}}}_{2}$ und $\vec{T}_{1}$ berechnet.

5. Die Daten werden nun auf $\overrightarrow{M D}_{2}=\overrightarrow{M D}_{1}+{\overrightarrow{D_{1} D_{2}}}_{\text {und }} \overrightarrow{L T}_{2}=\overrightarrow{L T}_{1}+{\overrightarrow{T_{1} T_{2}}}$ transformiert und der Vorgang so lange wiederholt, bis $\overrightarrow{M D}_{i}$ und $\overrightarrow{L T}_{i}$ gegen einen festen Wert konvergieren.

Die so gewonnene elastische Matrix ist dann der mit den Meßwerten am besten übereinstimmende Kandidat im elastischen Zentrum.

In Abschnitt 6.4 wird sich am Beispiel zeigen, daß mit den verallgemeinerten Linearen Modellen der multivariaten Statistik die Rekursion nach einem Schritt beendet ist, eine Folge der Linearität der verwandten Gleichungen. Bei anderen Schätzern, etwa nichtparametrischen, wird das nicht mehr der Fall sein.

\subsubsection{Herausrechnen mittels Zeitentwicklung}

Nimmt man an, daß eine ganz bestimmte Differentialgleichung dem Verhalten des Meßobjektes gerecht wird, so kann ihre bekannte Zeitentwicklung zur Auswertung der Meßdaten herangezogen werden. Beispielsweise kann die anteilige Mischung der zu $f_{0}$ und $f_{1}$ der linearen Last aus Abschnitt 3.1.4.2 gehörenden Moden umgangen werden.

Mit einem zweiten Experiment zur doppelten Last $2 f_{1}$ erhält man aus

$$
f_{a}=f_{0}+f_{1} t \quad \text { und } \quad f_{b}=f_{0}+2 f_{1} t
$$

mit der Zeitentwicklung (3.6) durch Subtraktion

$$
q_{b}(t)-q_{a}(t)=\mathcal{F} f_{1}\left(t-t_{0}\right)-\left(\mathcal{I}-\mathcal{P}\left(t, t_{0}\right)\right) \mathcal{F} \mathcal{D} \mathcal{F} f_{1},
$$

und hat damit den Einfluß der konstanten Last $f_{0}$ eliminiert. In jeder Komponente liegt damit eine Summe aus einer Geraden und bis zu sechs Exponentialfunktionen vom Typ $1-e^{-\lambda t}$ vor. Die Vorfaktoren können etwa durch einen Kurvenfit bestimmt werden. Der zur Geraden gehörende Anteil wird dann nach der eingangs beschriebenen Methode zur Flexibilitätsmatrix zusammengesetzt.

Gilt es lediglich, den Einfluß der langsamsten Mode zu eliminieren, so kann die Steigung der Kurve $q_{b}(t)-q_{a}(t)$ am Ende, also für große $t$, bestimmt werden. Diese stellt dann einen verläßlicheren Wert für die Flexibilitätskonstante dar als eine lineare Regression über die Gesamtdauer oder gar das anfängliche Verhalten liefert. 


\subsubsection{Ausgleichsrechnung mit sechs Freiheitsgraden}

Nach Problemen mit Relaxationszeiten ist das genaue Einstellen der Koordinatenachse die experimentell schwierigste Aufgabe. Daher können auch die Lasten als fehlerbehaftet angesehen werden. Es wäre demnach meßtechnisch von Vorteil, $\mathrm{zu}$ einer beliebigen Situation gleichzeitig $q$ und $f$ messen zu können. Da dann Justierarbeiten für die Einzelmessung entfielen, würde der Meßvorgang vereinfacht und so die Gesamtmeßzeit verkürzt. Das führt auf eine im Folgenden vorgeführte überbestimmte Version des eben genannten Verfahrens. Aus $n$ Messungen $\left(q_{1}, f_{1}\right),\left(q_{2}, f_{2}\right), \ldots\left(q_{n}, f_{n}\right)$ werden die Matrizen

$$
\boldsymbol{L}:=\left(f_{1}, f_{2}, \ldots f_{n}\right) \quad \text { und } \quad \boldsymbol{Q}:=\left(q_{1}, q_{2}, \ldots q_{n}\right)
$$

gebildet. Das überbestimmte Gleichungssystem lautet nun $\boldsymbol{Q}=\mathcal{F} \boldsymbol{L}$. Die Flexibilitätsmatrix wird nun so bestimmt, daß $\|\boldsymbol{Q}-\mathcal{F} \boldsymbol{L}\|^{2}=$ min!, was auf das lineare Gleichungssystem $\boldsymbol{Q} \boldsymbol{L}^{T}=\mathcal{F} \boldsymbol{L} \boldsymbol{L}^{T}$ führt. Man erhält schließlich

$$
\mathcal{F}=\left(\boldsymbol{Q} \boldsymbol{L}^{T}\right)\left(\boldsymbol{L} \boldsymbol{L}^{T}\right)^{-1}=\left(\sum q f^{T}\right)\left(\sum f f^{T}\right)^{-1}
$$

und ist bei der Aufbringung der Lasten nicht mehr an die Koordinatenachsen gebunden.

Bei dieser Methode wurde, wie auch bei anderen, stillschweigend ein statistisches Modell eingeführt. Die Verwendung eines gewöhnlichen Kleinste-Quadrate-Schätzers für $\mathcal{F}$ setzt implizit voraus, daß $q-\mathcal{F} f$ normalverteilt mit Streuung 1 ist 8 Das Hauptproblem der obigen Minimierungsaufgabe aber liegt hier in der Natur der verallgemeinerten Koordinaten: Es werden Größen unterschiedlicher physikalischer Einheiten zusammengefaßt. Das läßt sich durch einheitenbehaftete Wichtungsfaktoren vermeiden. Eine solche Skalierung der einzelnen Lastkomponenten, etwa mit der empirischen Varianz-Kovarianzmatrix $\boldsymbol{\Sigma}$, entspricht einer für alle Komponenten individuellen Wahl der Einheiten. Diese Skalierung wird dann hinterher bei den Schätzern rückgängig gemacht. In der angewandten Statistik erhält man so den verallgemeinerten Kleinste-Quadrate-Schätzer

$$
\mathcal{F}=\left(\boldsymbol{Q} \boldsymbol{\Sigma}^{-1} \boldsymbol{L}^{T}\right)\left(\boldsymbol{L} \boldsymbol{\Sigma}^{-1} \boldsymbol{L}^{T}\right)^{-1}
$$

Dieses Verfahren kann analog angewendet werden für ein Experiment, bei dem die Auslenkungen vorgegeben und die Lasten gemessen werden. Statt der Flexibilitätsmatrix bestimmt man damit dann die Steifheitsmatrix.

Die bisherige Meßpraxis in der Arbeitsgruppe ([3, 4] und [72]) bestand darin, die Last in Richtung einer Koordinatenachse aufzubringen, langsam stufenweise zu erhöhen und kurz vor einem erneuten Anstieg die Lage zu messen. Die Steigung der Regressionsgeraden der Lagen in Abhängigkeit von der Last ergab dann die

\footnotetext{
${ }^{8}$ Vgl. auch im Folgenden [9] 1.4.
} 
jeweiligen Flexibilitätskonstanten. Unter Vernachlässigung der Effekte, die durch die treppenförmig aufgebrachte Last entstehen, hat man die linear mit der Zeit anwachsende Last

$$
f=f_{0}+t g_{0}
$$

und, falls der Lastzuwachs langsamer als die Relaxationseffekte erfolgt, eine ebenso linear in der Zeit anwachsende Lage

$$
q=q_{0}+t p_{0}+\mathcal{O}\left(t^{2}\right)
$$

Dieser Ansatz nimmt die Beschleunigungen und daher die Trägheitseffekte als vernachlässigbar an, das Experiment verläuft also quasistatisch. Durch Einsetzen in (3.2) fällt die Massenmatrix erwartungsgemäß heraus, man erhält zunächst

$$
f_{0}+t g_{0}=\mathcal{S} q_{0}+t \mathcal{S} p_{0}+\mathcal{D} p_{0}
$$

und durch einen Koeffizientenvergleich in $t$ weiter

$$
g_{0}=\mathcal{S} p_{0} \quad \text { und } \quad f_{0}=\mathcal{S} q_{0}+\mathcal{D} p_{0}
$$

Faßt man wieder sechs solcher Messungen zu einer Matrix zusammen, so erhält man

$$
\boldsymbol{G}=\mathcal{S P} \quad \text { und } \quad \boldsymbol{L}=\mathcal{S} \boldsymbol{Q}+\mathcal{D} \boldsymbol{P}
$$

Daraus ergeben sich Steifheitsmatrix und Dissipationsmatrix zu

$$
\mathcal{S}=\boldsymbol{G} \boldsymbol{P}^{-1} \quad \text { und } \quad \mathcal{D}=\left(\boldsymbol{L}-\boldsymbol{G} \boldsymbol{P}^{-1} \boldsymbol{Q}\right) \boldsymbol{P}^{-1}
$$

Es wurde meines Wissens noch nirgends versucht, die Achsabschnitte quantitativ zur Bestimmung von $\mathcal{D}$ einzusetzen. Dazu müßte aber die Messung sehr genau sein, um nach einer Inversion und drei Multiplikationen der Matrizen noch verläßliche Resultate liefern zu können. Auch hier ist eine Übertragung auf den überbestimmten Fall möglich, das entspricht mehreren Messungen mit verschiedenen linearen Anteilen. Man erhält die Steifheitsmatrix als Lösung von

$$
\boldsymbol{P} \boldsymbol{G}^{T}-\boldsymbol{P} \boldsymbol{P}^{T} \mathcal{S}^{T}=0
$$

und die Dissipationsmatrix als diejenige von

$$
\boldsymbol{P} \boldsymbol{L}^{T}-\boldsymbol{P} \boldsymbol{Q}^{T} \mathcal{S}-\boldsymbol{P} \boldsymbol{P}^{T} \mathcal{D}^{T}=0
$$

wobei $\mathcal{S}$ aus der ersten Gleichung eingesetzt wird.

Eine weitere Möglichkeit zur Gewinnung einer elastischen Matrix besteht in der Erweiterung des Regressionsmodells auf den multivariaten Fall. Statt der Bestimmung der einzelnen Regressionsgeraden gilt es nun, die Parameter $b$ und $\mathcal{F}$ der Gleichung

$$
q=b+\mathcal{F} f
$$


an möglichst viele Meßdatenpaare $(q, f)_{n}$ anzupassen. Bei einer guten Justierung der Koordinatenmeßapparatur sollte der Vektor $b$, wie auch die Achsabschnitte der einzelnen Regressionen, nahezu Null sein.

Die Normalgleichungen des passenden Minimierungsproblems nach der Methode der Fehlerquadrate

$$
\sum_{n}\left(q_{n}-\left(b+\mathcal{F} f_{n}\right)\right)^{2}=\min !
$$

führen auf ein lineares Gleichungssystem mit mehreren rechten Seiten. Mit der Schreibweise $[*]:=\sum_{n} *$ erhält man $b$ und $\mathcal{F}$ als Lösung von

$$
\left(\begin{array}{cc}
{[1]} & {\left[f^{T}\right]} \\
{[f]} & {\left[f f^{T}\right]}
\end{array}\right) \cdot\left(\begin{array}{c}
b^{T} \\
\mathcal{F}^{T}
\end{array}\right)=\left(\begin{array}{c}
{\left[q^{T}\right]} \\
{\left[f q^{T}\right]}
\end{array}\right)
$$

Auch für diese Methode läßt sich ein verallgemeinerter Kleinste-Quadrate-Schätzer angeben.

Der Vorteil dieses Verfahrens besteht im korrekten Transformationsverhalten der Schätzer. Der Nachteil der Methoden, bei denen man $f$ und $q$ in allen Freiheitsgraden gleichzeitig mißt, liegt darin, daß Lasten meist über das elastische Verhalten von Medien, also mittels des HookEschen Gesetzes über Auslenkungen, bestimmt werden. Schaltet man etwa eine Kraftmeßdose und das eigentliche Meßobjekt in Reihe, so beeinflussen sich, wie bei der Durchbiegung einer Meßapparatur, beide Messungen gegenseitig. Dieser Effekt läßt sich zwar herausrechnen, vgl. Abschnitt 3.3.1, bleibt aber dennoch eine potentielle Fehlerquelle. Geschickter ist es, den apparativen Aufbau so zu konzipieren, daß derartige unerwünschte Kopplungen der Lasten mit den Lagen vermieden werden.

\subsection{Der Einfluß der Meßapparatur}

Unter einer Meßapparatur soll im Folgenden das eigentliche Meßgerät und die unmittelbar nachgeschaltete Auswertung bis hin zum Datensatz von einander zugeordneten Zahlen-6-Tupeln $f$ und $q$ verstanden werden. Mit einem geeigneten statistischen Verfahren, vgl. den letzten Abschnitt 3.2, werden aus diesen Paaren von Lasten und Lageänderungen die eigentlichen Meßwerte berechnet, also die Koeffizienten der elastischen Matrizen $\mathcal{S}$ oder $\mathcal{F}$.

Außer fortgepflanzten Meßfehlern kommen noch andere Ursachen für antisymmetrische Anteile in Frage. In diesem Abschnitt sollen drei Eigenschaften der Apparatur besprochen werden, deren Effekte aus den Messungen eliminierbar sind: Die Eigenelastizität der Meßapparatur, die Verwendung inkompatibler Einheitensysteme für Lasten und Lagen und eine Verdrehung der Vektoren innerhalb der Lasten oder Lagen. Einen vierten Effekt verursacht eine große aber konstante raumfeste Vorlast. Es wird sich zeigen, daß dieser Effekt nicht nachträglich aus den Daten herauszurechnen ist. 


\subsubsection{Der Einfluß der Elastizität der Meßapparatur}

Die Eigenelastizität einer Meßapparatur kann die Ergebnisse verfälschen. Dieser Effekt läßt sich aber zusammen mit einer Nullmessung herausrechnen. Jede zusammengesetzte Aufhängung, also auch Meßobjekt plus Meßapparatur, kann in hintereinander oder parallel geschaltete Aufhängungen zerlegt werden. Bei solchen $\left\{\begin{array}{c}\text { hintereinander } \\ \text { parallel }\end{array}\right\}$ geschalteten elastischen Aufhängungen addieren sich deren
$\left\{\begin{array}{c}\text { Auslenkungen } \\ \text { Lasten }\end{array}\right\}$ für gleiche $\left\{\begin{array}{c}\text { Lasten } \\ \text { Auslenkungen }\end{array}\right\}$, d. h. $\left\{\begin{array}{l}\mathcal{F} \\ \mathcal{S}\end{array}\right\}$ ist additiv. Ist also bei einer derartigen $\left\{\begin{array}{c}\text { Hintereinanderschaltung } \\ \text { Parallelschaltung }\end{array}\right\}$ die Matrix $\left\{\begin{array}{l}\mathcal{F}_{0} \\ \mathcal{S}_{0}\end{array}\right\}$ bei einem völlig $\left\{\begin{array}{c}\text { steifen } \\ \text { losen }\end{array}\right\}$ Meßobjekt aufgenommen worden, so erhält man die Matrix des tatsächlichen Effektes über

$$
\left\{\begin{array}{c}
\mathcal{F}=\mathcal{F}_{M E S S}-\mathcal{F}_{0} \\
\mathcal{F}=\mathcal{S}^{-1}=\left(\mathcal{S}_{M E S S}-\mathcal{S}_{0}\right)^{-1}=\left(\mathcal{F}_{M E S S}^{-1}-\mathcal{F}_{0}^{-1}\right)^{-1}
\end{array}\right\} .
$$

Diese Formeln sind äquivalent zu denen der Kombination von Federn oder den KIRCHHOFFschen Regeln der Elektrotechnik. Hier sind statt der skalaren Federkonstanten die elastischen Matrizen zu nehmen und entsprechend zu invertieren. Damit ist eine Beschreibung zusammengesetzter Aufhängungen durch Einzelmessungen möglich, vgl. dazu auch 4.5.

Damit ist das multivariate Verfahren aus 3.2 .3 mit einer passend geeichten kombinierten Last- und Lagemeßapparatur realisierbar.

\subsubsection{Inkompatible Einheitensysteme}

Prinzipiell können für jede Messung die Einheiten und damit die Zahlenwerte von Kraft, Drehmoment, Länge und Winkel frei gewählt werden. Die Einheiten der Blöcke der Steifheitsmatrix ergeben sich mit ihrer Definition daher zu

$$
\begin{aligned}
\{\boldsymbol{D}\} & =\{\boldsymbol{d}\} /\{\boldsymbol{F}\} \\
\left\{\boldsymbol{S}_{2}\right\} & =\{\boldsymbol{d}\} /\{\boldsymbol{T}\} \\
\left\{\boldsymbol{S}_{1}\right\} & =\{\boldsymbol{\theta}\} /\{\boldsymbol{F}\} \\
\{\boldsymbol{M}\} & =\{\boldsymbol{\theta}\} /\{\boldsymbol{T}\},
\end{aligned}
$$

womit sich

$$
\begin{aligned}
\left\{\boldsymbol{W}_{M}\right\} & =\{\overrightarrow{M D}\}=\left\{\boldsymbol{S}_{2}\right\} /\{\boldsymbol{M}\}=\{\boldsymbol{d}\} /\{\boldsymbol{\theta}\} \\
\left\{\boldsymbol{W}_{L}\right\} & =\{\overrightarrow{L T}\}=\left\{\boldsymbol{S}_{1}\right\} /\{\boldsymbol{M}\}=\{\boldsymbol{T}\} /\{\boldsymbol{F}\}
\end{aligned}
$$


ableiten läßt. Eine ungeschickte Wahl der Einheiten hat als skalarer Faktor keinen Einfluß auf die Symmetrie der Teilmatrizen auf der Hauptdiagonalen. Auch die Transformation auf das Drehmoment- und Translationszentrum wird nicht beeinflußt, da sie mit den Zahlenwerten allein funktioniert. Hingegen kann die Symmetrie der Gesamtmatrix ungünstig beeinflußt werden.

Falls ein Potential vorliegt, sollte eine elastische Matrix durch eine einzige Transformation von Last- und Meßsystem aufeinander symmetrisiert werden können, etwa durch

$$
\boldsymbol{S}_{2}-\boldsymbol{\Omega}_{M L} \boldsymbol{M}=\boldsymbol{S}_{1}^{T} \quad \text { oder } \quad \boldsymbol{S}_{1}+\boldsymbol{M} \boldsymbol{\Omega}_{L M}=\boldsymbol{S}_{2}^{T} .
$$

Diese Gleichungen stimmen aber nur dann in ihren Zahlenwerten überein, falls

$$
\{\overrightarrow{M L}\}=\{\boldsymbol{T}\} /\{\boldsymbol{F}\} \quad \text { und } \quad\{\boldsymbol{d}\} /\{\boldsymbol{\theta}\}=\{\boldsymbol{T}\} /\{\boldsymbol{F}\}
$$

Sind, wie es für die Theorie günstig ist, kanonisch konjugierte Variablen gewählt worden, so folgt die letzte Gleichung aus $\{\boldsymbol{T}\}\{\boldsymbol{\theta}\}=\{\boldsymbol{d}\}\{\boldsymbol{F}\}$.

Die Abweichung von einem konsistenten Einheitensystem sei nun durch den dimensionslosen Parameter

$$
\lambda:=\frac{\{\boldsymbol{T}\}\{\boldsymbol{\theta}\}}{\{\boldsymbol{F}\}\{\boldsymbol{d}\}}
$$

beschrieben.

Die Matrix des Abstands der beiden Bezugssysteme läßt sich nun mit

$$
\left(\lambda \boldsymbol{S}_{2}-\boldsymbol{S}_{1}^{T}\right) \boldsymbol{M}^{-1}=\boldsymbol{\Omega}_{M L} \quad \text { und } \quad \boldsymbol{M}^{-1}\left(\lambda \boldsymbol{S}_{2}^{T}-\boldsymbol{S}_{1}\right)=\boldsymbol{\Omega}_{L M}
$$

berechnen. Ist die Transformation auf das elastische Zentrum bereits durchgeführt, so erhält man stattdessen mit (2.42)

$$
\begin{aligned}
& \boldsymbol{\Omega}_{D T}=\left(\lambda \boldsymbol{W}_{M} \boldsymbol{M}-\left(\boldsymbol{M} \boldsymbol{W}_{L}\right)^{T}\right) \boldsymbol{M}^{-1}=\lambda \boldsymbol{W}_{M}-\boldsymbol{W}_{L} \quad \text { und } \\
& \boldsymbol{\Omega}_{T D}=\boldsymbol{M}^{-1}\left(\left(\lambda \boldsymbol{W}_{M} \boldsymbol{M}\right)^{T}-\boldsymbol{M} \boldsymbol{W}_{L}\right)=\lambda \boldsymbol{W}_{M}-\boldsymbol{W}_{L} \quad ;
\end{aligned}
$$

Faktoren übertragen sich von den Matrizen $\boldsymbol{S}_{i}$ auf die $\boldsymbol{W}_{i}$.

Da beide rechten Seiten symmetrisch sind, können sie nicht gleich den linken sein; das Gleichungssystem macht also nur Sinn, falls alle beteiligten Matrizen verschwinden. Das ist nur der Fall, wenn ein Widerstandszentrum vorliegt. Ein skalarer Faktor $\lambda \neq 1$ könnte hingegen die Ursache für die Widersprüchlichkeit des Gleichungssystems sein, und zwar dann, wenn $\boldsymbol{W}_{L} \approx \lambda \boldsymbol{W}_{M}$. Nun sind das neun Gleichungen für nur eine Größe. Die einfachste Möglichkeit, diese Gleichung zu erfüllen und dabei die Norm, um die es ja letztlich geht, zu verwenden, ist

$$
\lambda=\frac{\left\|\boldsymbol{W}_{L}\right\|}{\left\|\boldsymbol{W}_{M}\right\|} .
$$

Sie hat darüber hinaus noch folgende Eigenschaften: 
- Sie ist eine Invariante der Widerstandsmatrizen,

- sie benutzt alle Einträge der Matrizen und

- sie ist forminvariant unter der Vertauschung von Meß- und Lastsystem, also der Symmetrie $M \leftrightarrow L$; der gesuchte Faktor hätte sicher auch vor der Matrix $\boldsymbol{W}_{L}$ eingeführt werden können $\stackrel{9}{9}$

Obwohl $\left[\boldsymbol{W}_{M}\right]=\left[\boldsymbol{W}_{L}\right]=\mathrm{L}$, steckt in $\boldsymbol{W}_{M}$ gegenüber $\boldsymbol{W}_{L}$ noch zusätzlich der Umrechnungsfaktor der verwendeten Winkeleinheit in das Bogenmaß rad. Ein Wert für $\lambda$ also, der einer Zehnerpotenz nahekommt oder einer des Faktors $\pi / 180 \approx 0.0175$, deutet dann auf das Vorliegen inkompatibler Einheitensysteme hin. Hingegen kann das Ergebnis $\lambda \approx 1$ als Bestätigung der Vertrauenswürdigkeit der Meßwerte gewertet werden.

Da diese Formel für $\boldsymbol{W}_{M} \neq 0$ immer ein Ergebnis liefert, kann nicht mehr automatisch eine Aussage darüber getroffen werden, ob tatsächlich eine ungeschickte Wahl der Größe zu Grunde lag. Es muß daher in jedem Einzelfall anhand vorliegender Fehlerabschätzungen der Messung geprüft werden, daß es sich um keine Fehlinterpretation von Meßfehlern handelt. Um aus den Widerstandsmatrizen noch Information ziehen zu können, sollte in jedem Fall die relative Genauigkeit der Messung die Quotienten

$$
\frac{\left\|\boldsymbol{W}_{M} \boldsymbol{M}\right\|}{\left\|\boldsymbol{S}_{2}\right\|} \text { und } \frac{\left\|\boldsymbol{M} \boldsymbol{W}_{L}\right\|}{\left\|\boldsymbol{S}_{1}\right\|},
$$

gebildet aus den Normen der Nebendiagonalmatrizen nach und vor der Transformation auf das elastische Zentrum, deutlich übersteigen.

\subsubsection{Verdrehung der Referenzsysteme}

Eine Verdrehung vom Meßsystem gegenüber dem Lastsystem würde durch die Realform wieder ausgeglichen. Eine Verdrehung innerhalb des Last- oder Meßsystems kann hingegen so nicht mehr kompensiert werden. Da die Lasten in unserer Arbeitsgruppe „von Hand“ aufgebracht wurden und für jeden Lastfall neu justiert

\footnotetext{
${ }^{9}$ Als Minimierungsproblem $\left\|\boldsymbol{W}_{L}-\lambda \boldsymbol{W}_{M}\right\|^{2}=\min$ ! formuliert, liefert die Differentation nach $\lambda$ die Normalgleichung $0=\sum_{i j}\left(\boldsymbol{W}_{M}\right)_{i j}\left(\boldsymbol{W}_{L}\right)_{i j}-\lambda\left(\boldsymbol{W}_{M}\right)_{i j}\left(\boldsymbol{W}_{M}\right)_{i j}$, mit dem Ergebnis

$$
\lambda=\frac{\operatorname{Sp}\left(\boldsymbol{W}_{M} \boldsymbol{W}_{L}\right)}{\left\|\boldsymbol{W}_{M}\right\|^{2}} .
$$

Ebenso erhält man für $\left\|\bar{\lambda} \boldsymbol{W}_{L}-\boldsymbol{W}_{M}\right\|^{2}=\min$ ! das Resultat

$$
\bar{\lambda}=\frac{\operatorname{Sp}\left(\boldsymbol{W}_{L} \boldsymbol{W}_{M}\right)}{\left\|\boldsymbol{W}_{L}\right\|^{2}},
$$

welches wegen $\lambda \bar{\lambda} \neq 1$ nicht mit dem ersten verträglich ist. Diese Lösungen sind daher nicht forminvariant. Das geometrische Mittel $\sqrt{\lambda / \bar{\lambda}}$ beider entspricht aber genau der obigen Wahl.
} 
wurden, ist es denkbar, daß für Kräfte und Drehmomente leicht verschiedene Koordinatenachsen benutzt wurden. Dagegen ist es unwahrscheinlich, daß im Meßsystem eine solche Verdrehung auftritt, da sich der so entstehende Effekt systematisch auf sämtliche Messungen auswirken würde, und daher schon beim endgültigen Aufbau der Apparatur eliminiert sein sollte. Als Arbeitshypothese und als Verfahren zum Aufspüren solcher Fehler ist eine solche Annahme aber dennoch sinnvoll.

Unter der Annahme also, daß eine Drehung mit eventuell einer Vertauschung der Koordinatenachsen vorliegt, daß also nur orthogonale Transformationen vorliegen, läßt sich der dadurch entstehende Effekt nachträglich herausrechnen.

Die Verdrehungen von Kräften gegenüber den Drehmomenten im Lastsystem und Translationen gegenüber Drehungen im Meßsystem sind unabhängig voneinander.

Die tatsächlich angreifende Last ist dann

$$
f^{\prime}=\underbrace{\left(\begin{array}{cc}
\boldsymbol{R}_{F} & \mathbf{0} \\
\mathbf{0} & \boldsymbol{I}
\end{array}\right)}_{=: \mathcal{R}_{L}}\left(\begin{array}{c}
\boldsymbol{F} \\
\boldsymbol{T}
\end{array}\right)=\mathcal{R}_{L} f
$$

und die tatsächlich gemessene Lage

$$
q^{\prime}=\underbrace{\left(\begin{array}{cc}
\boldsymbol{R}_{d} & \mathbf{0} \\
\mathbf{0} & \boldsymbol{I}
\end{array}\right)}_{=: \mathcal{R}_{M}}\left(\begin{array}{c}
\boldsymbol{d} \\
\boldsymbol{\theta}
\end{array}\right)=\mathcal{R}_{M} q
$$

Der Zusammenhang der tatsächlichen Flexibilitätsmatrix $q^{\prime}=\mathcal{F}^{\prime} f^{\prime}$ mit der gemessenen $q=\mathcal{F} f$ lautet wegen

$$
q^{\prime}=\mathcal{R}_{M} q=\mathcal{R}_{M} \mathcal{F} f=\underbrace{\mathcal{R}_{M} \mathcal{F} \mathcal{R}_{L}^{T}}_{=: \mathcal{F}^{\prime}} f^{\prime}
$$

einfach

$$
\mathcal{F}^{\prime}=\mathcal{R}_{M} \mathcal{F} \mathcal{R}_{L}^{T}
$$

oder analog für die Steifheitsmatrix

$$
\mathcal{S}^{\prime}=\mathcal{R}_{L} \mathcal{S} \mathcal{R}_{M}^{T}
$$

Nun sollten selbst inkonsistente Koordinatensysteme nicht vom gewählten Bezugspunkt abhängen. Nur dann können die auftretenden Effekte von denen der Koordinatensysteme getrennt werden. Die einzige noch dafür in Frage kommende Matrix ist $\boldsymbol{A}$, da $\boldsymbol{M}$ schon zur Definition der Normal- bzw. Realform herangezogen wurde. In Komponenten hat man

$$
\mathcal{S}^{\prime}=\left(\begin{array}{ll}
\boldsymbol{A}^{\prime} & \boldsymbol{B}_{2}^{\prime} \\
\boldsymbol{B}_{1}^{\prime} & \boldsymbol{C}^{\prime}
\end{array}\right)
$$




$$
\begin{aligned}
& =\left(\begin{array}{cc}
\boldsymbol{R}_{F} & \mathbf{0} \\
\mathbf{0} & \boldsymbol{I}
\end{array}\right)\left(\begin{array}{cc}
\boldsymbol{A} & \boldsymbol{B}_{2} \\
\boldsymbol{B}_{1} & \boldsymbol{C}
\end{array}\right)\left(\begin{array}{cc}
\boldsymbol{R}_{d}^{T} & \mathbf{0} \\
\mathbf{0} & \boldsymbol{I}
\end{array}\right)=\left(\begin{array}{cc}
\boldsymbol{R}_{F} & \mathbf{0} \\
\mathbf{0} & \boldsymbol{I}
\end{array}\right)\left(\begin{array}{cc}
\boldsymbol{A} \boldsymbol{R}_{d}^{T} & \boldsymbol{B}_{2} \\
\boldsymbol{B}_{1} \boldsymbol{R}_{d}^{T} & \boldsymbol{C}
\end{array}\right) \\
& =\left(\begin{array}{cc}
\boldsymbol{R}_{F} \boldsymbol{A} \boldsymbol{R}_{d}^{T} & \boldsymbol{R}_{F} \boldsymbol{B}_{2} \\
\boldsymbol{B}_{1} \boldsymbol{R}_{d}^{T} & \boldsymbol{C}
\end{array}\right)
\end{aligned}
$$

also muß die Matrix $\boldsymbol{A}^{\prime}=\boldsymbol{R}_{F} \boldsymbol{A} \boldsymbol{R}_{d}^{T}$ die Information über eine mögliche Verdrehung der Referenzsysteme enthalten.

Solche Verdrehungen wirken sich auf die Berechnung der elastischen Zentren aus. Das über die Lagen definierte Drehmomentszentrum ändert sich wegen

$$
-\left(\boldsymbol{A}^{-1} \boldsymbol{B}_{2}\right)^{\prime}=-\left(\boldsymbol{R}_{F} \boldsymbol{A} \boldsymbol{R}_{d}^{T}\right)^{-1} \boldsymbol{R}_{F} \boldsymbol{B}_{2}=-\boldsymbol{R}_{d} \boldsymbol{A}^{-1} \boldsymbol{B}_{2}
$$

unter Verdrehungen der Lagen, und das über die Lasten definierte Translationszentrum ändert sich analog wegen

$$
\left(\boldsymbol{B}_{1} \boldsymbol{A}^{-1}\right)^{\prime}=\boldsymbol{B}_{1} \boldsymbol{R}_{d}^{T}\left(\boldsymbol{R}_{F} \boldsymbol{A} \boldsymbol{R}_{d}^{T}\right)^{-1}=\boldsymbol{B}_{1} \boldsymbol{A}^{-1} \boldsymbol{R}_{F}^{T}
$$

unter Verdrehungen der Lasten. Eine schlechte Güte dieser Matrizen im Sinne von A.2.3 kann also ein Hinweis auf eine derartige Störung im Aufbau sein.

Nimmt man eine Verdrehung nur im Meß- oder Lastsystem an, so ist entweder $\boldsymbol{R}_{F}=\boldsymbol{I}$ oder $\boldsymbol{R}_{q}=\boldsymbol{I}$, und $\boldsymbol{A}^{\prime}$ ist damit als Produkt einer symmetrischen und einer orthogonalen Matrix dargestellt. Diese Symmetrisierung einer Matrix durch Multiplikation mit einer Drehmatrix nennt man Polarzerlegung ${ }^{10}$ Sei

$$
\boldsymbol{A}^{\prime}=\boldsymbol{R}_{L}^{T} \operatorname{diag}\left(a_{i}\right) \boldsymbol{R}_{R}
$$

die Zerlegung von $\boldsymbol{A}$ in ihre singulären Werte, vgl. auch Fußnote 25 des vorangegangenen Kapitels, dann wird $\boldsymbol{A}^{\prime}$ sowohl durch Multiplikation von links als auch von rechts mit $\boldsymbol{R}:=\boldsymbol{R}_{R}^{T} \boldsymbol{R}_{L}$ symmetrisiert. Die erhaltenen symmetrischen Matrizen lauten dann

$$
\boldsymbol{A}_{L}=\boldsymbol{R}_{R}^{T} \operatorname{diag}\left(a_{i}\right) \boldsymbol{R}_{R} \quad \text { und } \quad \boldsymbol{A}_{R}=\boldsymbol{R}_{L}^{T} \operatorname{diag}\left(a_{i}\right) \boldsymbol{R}_{L} .
$$

\footnotetext{
${ }^{10} \mathrm{Vgl}$. etwa [20, Bd. 1 IX $\S 14$. Die Polarzerlegung läßt sich auch folgendermaßen durchführen: Seien $\lambda_{i}$ die Eigenwerte der Matrix $\boldsymbol{A}$, die Vektoren $\boldsymbol{v}_{i}$ die zugehörigen normierten Eigenvektoren und $\boldsymbol{P}_{v_{i}}^{\|}:=\boldsymbol{v}_{i} \boldsymbol{v}_{i}^{T}$ die Parallelprojektoren auf die entsprechenden Eigenräume. Die Wurzel einer positiv semidefiniten symmetrischen Matrix $\boldsymbol{A}$ kann über deren Spektralzerlegung definiert bzw. berechnet werden:

$$
\boldsymbol{A}=\sum_{i} \lambda_{i} \cdot \boldsymbol{P}_{v_{i}}^{\|} \Rightarrow \sqrt{\boldsymbol{A}}=\sum_{i} \sqrt{\lambda_{i}} \cdot \boldsymbol{P}_{v_{i}}^{\|} .
$$
}

Mit dieser Wurzel erhält man den korrekt gedrehten und symmetrischen Anteil von $\boldsymbol{A}^{\prime}$

$$
\sqrt{A^{\prime} A^{\prime T}}=\sqrt{A R R^{T} A^{T}}=\sqrt{A^{2}}=A
$$

und damit weiter die Drehmatrix über

$$
\boldsymbol{R}=\boldsymbol{A}^{-1} \boldsymbol{A}^{\prime}
$$


Je nach Arbeitshypothese kann die Drehung entweder mit $\boldsymbol{R}_{F}=\boldsymbol{I}$ und $\boldsymbol{R}_{d}=$ $\boldsymbol{R}_{R}^{T} \boldsymbol{R}_{L}$ den Lagen oder mit $\boldsymbol{R}_{q}=\boldsymbol{I}$ und $\boldsymbol{R}_{F}=\boldsymbol{R}_{R}^{T} \boldsymbol{R}_{L}$ den Lasten zugeordnet werden.

Den zugehörigen Drehwinkel erhält man aus $\operatorname{Sp} \boldsymbol{R}=1+2 \cos \theta$. Er dient zur Überprüfung der Zulässigkeit der Arbeitshypothese: Da das obige Verfahren immer funktioniert (es liefert sogar mit uneigentlich orthogonalen Matrizen Information über eventuell vertauschte Koordinatenachsen), bekommt man für unsinnige Eingangsdaten Scheinergebnisse. Dadurch werden alle möglichen anderen Effekte, welche Ursache antisymmetrischer Anteile sind, falsch interpretiert und sind einer weiteren Auswertung nicht mehr zugänglich. Falls also der berechnete Drehwinkel zu groß ist, sollten andere Erklärungen für gemessene antisymmetrische Anteile der unter Translationen transformationsinvarianten Matrix $\boldsymbol{A}=\left(\boldsymbol{D}-\boldsymbol{S}_{2} \boldsymbol{M}^{-1} \boldsymbol{S}_{1}\right)^{-1}$ herangezogen werden.

\subsubsection{Der Einfluß der Vorlast}

Rein mathematisch äußert sich eine große Vorlast in einem anderen Entwicklungspunkt für das lineare Input-Output-Modell (2.9). Um die zu diskutierenden physikalischen Effekte besser verständlich zu machen, möchte ich einige Beispiele (frei nach [43]) voranstellen.

Gegeben sei ein einfaches mathematisches Pendel der Länge $l$ mit der Masse $m$ und damit der Gewichtskraft $G=m g$. Die Auslenkung von der Ruhelage ist $x=l \phi$, die Beschleunigung ist also $\ddot{x}=l \ddot{\phi}$. Das Pendel werde nacheinander der Gewichtskraft, einer lateral steifen Feder und einer längs der Schwere wirkenden vorgespannten Feder ausgesetzt.

Die Bewegungsgleichung für das Pendel im Schwerefeld allein lautet

$$
m l \ddot{\phi}+G \sin \phi=0
$$

und für kleine Auslenkungswinkel ergibt sich

$$
m \ddot{\phi}+\frac{G}{l} \phi=0
$$

Bei einer lateral steifen Feder ohne Vorlast gilt für die rücktreibende Kraft das HookEsche Gesetz

$$
m \ddot{\phi}+k^{\perp} \phi=0
$$

wobei $k^{\perp}$ die Lateralsteifigkeit ist. Ist eine lange Feder in dieselbe Richtung wie die Gewichtskraft vorgespannt, so übernimmt die Vorspannung $F_{0}^{\|}$die Rolle der 
Gewichtskraft, und man erhältt11

$$
m \ddot{\phi}+\frac{F_{0}^{\|}}{l} \phi=0
$$

Alle Effekte superponiert ergeben die Bewegungsgleichung

$$
m \ddot{\phi}+\left(\frac{m g}{l}+\frac{F_{0}^{\|}}{l}+k^{\perp}\right) \phi=0,
$$

die eine harmonische Schwingung mit der Schwingfrequenz

$$
\omega=\sqrt{\frac{g}{l}+\frac{F_{0}^{\|}}{m l}+\frac{k^{\perp}}{m}}
$$

beschreibt. Drei Effekte sind hier auffällig:

1. Die Vorlast steht im Steifigkeitskoeffizienten

$$
k=k^{\perp}+F_{0}^{\|} / l+G / l,
$$

wobei große ${ }^{12}$ Vorlasten versteifend wirken (Stress Stiffening).

2. Die Richtungen von Koeffizient und Vorlast stehen senkrecht aufeinander, was im räumlichen Formalismus durch Kreuzprodukte oder antisymmetrische Matrizen beschrieben werden wird.

3. Die Vorspannung einer langen Feder ist als raumfest anzusehen. Eine Änderung der Lage ist gleichzeitig eine Änderung der Orientierung des Referenzkoordinatensystems und verschiebt damit die Kraftwirkungslinie bezüglich des geänderten Koordinatensystems. Es gibt keine Notwendigkeit anzunehmen, daß das nur Effekte in zweiter Ordnung zur Folge hat, speziell für große Vorlasten.

Im Folgenden sollen diese Erscheinungen für das räumliche Problem abgeleitet werden. Der entscheidende Punkt ist eine als zeitlich konstant und mit im Raum festen Richtungen angenommene große Vorlast, die sich über durch Lageänderungen hervorgerufene Koordinatentransformationen auf die Steifigkeitskoeffizienten auswirkt.

\footnotetext{
${ }^{11}$ Dies entspricht den Vorstellungen zu Beginn des Entstehens der Theorie der schwingenden Saite, s. 67] IV A.

${ }^{12}$ In der Biomechanik liegen typische Werte für den E-Modul im GPa-Bereich. Zusammen mit Abmessungen im $\mathrm{cm}$-Bereich ergeben sich mit den Federkonstanten vergleichbare Vorlasten in einer Größenordnung oberhalb von $k N$ oder $k N m$. Kleinere Vorlasten rufen demnach kaum spürbare Effekte hervor. Bei einer Saite mit vernachlässigbarer Lateralsteifigkeit ist das natürlich nicht der Fall.
} 


\subsubsection{Lagewechsel als Koordinatentransformation}

Für ein räumliches Problem tragen drei Effekte zur tatsächlich wirkenden Laständerung bei:

- Die angelegte Laständerung $\delta f_{L}$ selber,

- die Änderung des Hebels $\left(\delta \mathcal{T}_{L^{\prime} L}\right) f_{L}$ und

- die Wirkung der als raumfest angenommenen Vorlast $f^{0}$ im geänderten Koordinatensystem, $\delta f^{0}$.

Es sind vier Koordinatensysteme beteiligt: Das Lastsystem $L$ und das Meßsystem $M$ vor der Last/Lageänderung und beide nach der Änderung, bezeichnet mit $L^{\prime}$ und $M^{\prime}$. Es gilt nun, die beiden Beziehungen

$$
\delta f_{L}=\mathcal{S}_{L M} \delta q_{M} \quad \text { und } \quad \delta f_{L^{\prime}}=\mathcal{S}_{L^{\prime} M^{\prime}} \delta q_{M^{\prime}}
$$

auszunutzen, um $\mathcal{S}_{L^{\prime} M^{\prime}}$ und $\mathcal{S}_{L M}$ miteinander in Beziehung zu setzen. Das soll in den folgenden Abschnitten geschehen.

\subsubsection{Die Transformation der Laständerungen}

Mit der Produktregel erhält man aus (2.1) für kleine Laständerungen

$$
\delta f_{L}=\delta\left(\mathcal{T}_{L L^{\prime}} f_{L^{\prime}}\right)=\left(\delta \mathcal{T}_{L L^{\prime}}\right) f_{L^{\prime}}+\mathcal{T}_{L L^{\prime}} \delta f_{L^{\prime}}
$$

Der zweite Term ist die Laständerung selber; ihr Beitrag zur Gesamtlaständerung $\left.\delta f_{L}\right|_{L}$ ist

$$
\begin{aligned}
\left.\delta f_{L}\right|_{L} & =\mathcal{T}_{L L^{\prime}} \delta f_{L^{\prime}}=\mathcal{T}_{L L^{\prime}} \mathcal{S}_{L^{\prime} M^{\prime}} \delta q_{M^{\prime}} \\
& =\mathcal{T}_{L L^{\prime}} \mathcal{S}_{L^{\prime} M^{\prime}} \mathcal{T}_{M M^{\prime}}^{T} \delta q_{M}
\end{aligned}
$$

Der erste Term ist die Änderung des Hebels. Mit der Abkürzung

$$
\mathcal{T}_{L L^{\prime}}=\left(\begin{array}{cc}
\boldsymbol{I} & \mathbf{0} \\
\boldsymbol{\Omega}_{L L^{\prime}} & \boldsymbol{I}
\end{array}\right)=\underbrace{\left(\begin{array}{cc}
\boldsymbol{I} & \mathbf{0} \\
\mathbf{0} & \boldsymbol{I}
\end{array}\right)}_{\mathcal{I}}+\underbrace{\left(\begin{array}{cc}
\mathbf{0} & \mathbf{0} \\
\boldsymbol{\Omega}_{L L^{\prime}} & \mathbf{0}
\end{array}\right)}_{=: \mathcal{N}_{L L^{\prime}}}
$$

erhält man seinen Beitrag $\left.\delta f_{L}\right|_{H} \mathrm{zu}$

$$
\begin{aligned}
\left.\delta f_{L}\right|_{H} & =\left(\delta \mathcal{T}_{L L^{\prime}}\right) f_{L^{\prime}}=\left(\delta \mathcal{N}_{L L^{\prime}}\right) f_{L^{\prime}}=\mathcal{N}_{\delta L L^{\prime}} f_{L^{\prime}} \\
& =\left(\begin{array}{cc}
\mathbf{0} & \mathbf{0} \\
\boldsymbol{\Omega}_{\delta L L^{\prime}} & \mathbf{0}
\end{array}\right)\left(\begin{array}{c}
\boldsymbol{F} \\
\boldsymbol{T}_{L^{\prime}}
\end{array}\right)=\left(\begin{array}{c}
\mathbf{0} \\
\boldsymbol{\Omega}_{\delta L L^{\prime}} \boldsymbol{F}
\end{array}\right)=\left(\begin{array}{c}
\mathbf{0} \\
-\boldsymbol{\Omega}_{F} \delta L L^{\prime}
\end{array}\right) \\
& =\left(\begin{array}{c}
\mathbf{0} \\
\boldsymbol{\Omega}_{F} \delta L^{\prime} L
\end{array}\right)=\left(\begin{array}{cc}
\mathbf{0} & \mathbf{0} \\
\boldsymbol{\Omega}_{F} & \mathbf{0}
\end{array}\right)\left(\begin{array}{c}
\delta \overrightarrow{L^{\prime} L} \\
0
\end{array}\right) .
\end{aligned}
$$


Mit $\delta \overrightarrow{L^{\prime} L}=\delta\left(\overrightarrow{O L}-\overrightarrow{O L^{\prime}}\right)=\delta \boldsymbol{d}_{L}-\delta \boldsymbol{d}_{L^{\prime}}$ und $0=\delta\left(\theta_{L}-\theta_{L^{\prime}}\right)=\delta \theta_{L}-\delta \theta_{L^{\prime}}$ folgt weiter

$$
\begin{aligned}
\left.\delta f_{L}\right|_{H} & =\mathcal{N}_{F}\left(\delta q_{L}-\delta q_{L^{\prime}}\right)=\mathcal{N}_{F}\left(\mathcal{T}_{M L}^{T}-\mathcal{T}_{M L^{\prime}}^{T}\right) \delta q_{M} \\
& =\mathcal{N}_{F}\left(\mathcal{N}_{M L}^{T}-\mathcal{N}_{M L^{\prime}}^{T}\right) \delta q_{M}=\mathcal{N}_{F}\left(\mathcal{N}_{\overrightarrow{M L}-\overrightarrow{M L^{\prime}}}^{T}\right) \delta q_{M}=\mathcal{N}_{F}\left(\mathcal{N}_{L^{\prime} L}^{T}\right) \delta q_{M} \\
& =\left(\begin{array}{cc}
\mathbf{0} & \mathbf{0} \\
\boldsymbol{\Omega}_{F} & \mathbf{0}
\end{array}\right)\left(\begin{array}{cc}
\mathbf{0} & \boldsymbol{\Omega}_{L L^{\prime}} \\
\mathbf{0} & \mathbf{0}
\end{array}\right) \delta q_{M}=\left(\begin{array}{cc}
\mathbf{0} & \mathbf{0} \\
\mathbf{0} & \boldsymbol{\Omega}_{F} \boldsymbol{\Omega}_{L L^{\prime}}
\end{array}\right) \delta q_{M}
\end{aligned}
$$

woraus ersichtlich ist, daß die Hebeländerung nur einen Effekt zweiter Ordnung bewirkt.

\subsubsection{Die Vorlastmatrix}

Die Lageänderungen

$$
\overrightarrow{L L^{\prime}}=\overrightarrow{O L^{\prime}}-\overrightarrow{O L}=\boldsymbol{d}_{L^{\prime}}-\boldsymbol{d}_{L}=\delta \boldsymbol{d}_{L}
$$

und

$$
\boldsymbol{\theta}_{L L^{\prime}}=\delta \boldsymbol{\theta}_{L}
$$

spielen eine Doppelrolle als Transformationen des Koordinatensystems. Für eine raumfeste Vorlast hat das zwei Effekte zur Folge:

1. Die Translation verändert die wirkenden Drehmomente nach dem Hebelgesetz $f_{L^{\prime}}=\mathcal{T}_{L^{\prime} L} f_{L}$ und

2. die Rotation ändert die Richtung der Kraftwirkungslinie in der Art, daß in diese der um $\boldsymbol{R}_{\delta \theta}$ gedrehten Situation nach Aufbringen der Laständerung um $\boldsymbol{R}_{-\delta \theta}=\boldsymbol{R}_{\delta \theta}^{T}$ gedreht erscheint. Diese Rückdrehung besagt gerade, daß die raumfeste Kraftwirkungslinie nicht mitgedreht wird.

Die durch die Lageänderung verursachte Änderung der Vorlast ist damit

$$
\begin{aligned}
\delta f_{L}^{0} & =\mathcal{R}_{\theta_{L L^{\prime}}} \mathcal{T}_{-L L^{\prime}} f_{L}^{0}-f_{L}^{0} \\
& =\left(\begin{array}{cc}
\boldsymbol{R}_{\delta \theta}^{T} & \mathbf{0} \\
\mathbf{0} & \boldsymbol{R}_{\delta \theta}^{T}
\end{array}\right)\left(\begin{array}{cc}
\boldsymbol{I} & \mathbf{0} \\
-\boldsymbol{\Omega}_{\delta d} & \mathbf{0}
\end{array}\right) f_{L}^{0}-\left(\begin{array}{cc}
\boldsymbol{I} & \mathbf{0} \\
\mathbf{0} & \mathbf{I}
\end{array}\right) f_{L}^{0} \\
& =\left(\begin{array}{cc}
\boldsymbol{R}_{\delta \theta}^{T}-\boldsymbol{I} & \mathbf{0} \\
-\boldsymbol{R}_{\delta \theta}^{T} \boldsymbol{\Omega}_{\delta d} & \boldsymbol{R}_{\delta \theta}^{T}-\boldsymbol{I}
\end{array}\right) f_{L}^{0},
\end{aligned}
$$

und bis auf Terme zweiter Ordnung ergibt sich

$$
\begin{aligned}
& \delta f_{L}^{0}=\left(\begin{array}{cc}
-\boldsymbol{\Omega}_{\delta \theta} & \mathbf{0} \\
-\boldsymbol{\Omega}_{\delta d} & -\boldsymbol{\Omega}_{\delta \theta}
\end{array}\right)\left(\begin{array}{c}
\boldsymbol{F}_{L}^{0} \\
\boldsymbol{T}_{L}^{0}
\end{array}\right)=\left(\begin{array}{c}
-\boldsymbol{\Omega}_{\delta \theta} \boldsymbol{F}_{L}^{0} \\
-\boldsymbol{\Omega}_{\delta d} \boldsymbol{F}_{L}^{0}-\boldsymbol{\Omega}_{\delta \theta} \boldsymbol{T}_{L}^{0}
\end{array}\right) \\
& =\left(\begin{array}{c}
\boldsymbol{\Omega}_{F_{L}^{0}} \delta \boldsymbol{\theta} \\
\boldsymbol{\Omega}_{F_{L}^{0}} \delta \boldsymbol{d}+\boldsymbol{\Omega}_{T_{L}^{0}} \boldsymbol{\theta}
\end{array}\right)=\underbrace{\left(\begin{array}{cc}
\mathbf{0} & \boldsymbol{\Omega}_{F^{0}} \\
\boldsymbol{\Omega}_{F^{0}} & \boldsymbol{\Omega}_{T^{0}}
\end{array}\right)_{L}}_{=: \mathcal{V}_{L}}\left(\begin{array}{c}
\delta \boldsymbol{d} \\
\delta \boldsymbol{\theta}
\end{array}\right)_{L},
\end{aligned}
$$


also insgesamt

$$
\delta f_{L}^{0}=\mathcal{V}_{L} \delta q_{L}
$$

mit der antisymmetrischen Vorlastmatrix

$$
\mathcal{V}_{L}=\left(\begin{array}{cc}
0 & \boldsymbol{\Omega}_{F^{0}} \\
\boldsymbol{\Omega}_{F^{0}} & \boldsymbol{\Omega}_{T^{0}}
\end{array}\right)_{L},
$$

die den gesuchten Zusatzterm zur Steifheitsmatrix darstellt.

\subsubsection{Die Transformation der Vorlastmatrix}

Bei einer kompletten Transformation beider Bezugssysteme transformiert sich die Vorlastmatrix wie die Steifheitsmatrix

$$
\mathcal{V}_{B}=\mathcal{T}_{B A} \mathcal{V}_{A} \mathcal{T}_{B A}^{T}
$$

Dabei bleibt ihre Antisymmetrie wegen

$$
\mathcal{V}_{B}^{T}=\left(\mathcal{T}_{B A} \mathcal{V}_{A} \mathcal{T}_{B A}^{T}\right)^{T}=\mathcal{T}_{B A}^{T} \mathcal{V}_{A}^{T} \mathcal{T}_{B A}=-\mathcal{T}_{B A}^{T} \mathcal{V}_{A} \mathcal{T}_{B A}=-\mathcal{V}_{B}
$$

erhalten. Aber auch die Beträge transformieren sich korrekt wie äquivalente Lastsysteme:

$$
\begin{aligned}
\mathcal{V}_{B} & =\mathcal{T}_{B A} \mathcal{V}_{A} \mathcal{T}_{B A}^{T} \\
& =\left(\begin{array}{cc}
\boldsymbol{I} & \mathbf{0} \\
\boldsymbol{\Omega}_{B A} & \boldsymbol{I}
\end{array}\right)\left(\begin{array}{cc}
\mathbf{0} & \boldsymbol{\Omega}_{F^{0}} \\
\boldsymbol{\Omega}_{F^{0}} & \boldsymbol{\Omega}_{T_{A}^{0}}
\end{array}\right)\left(\begin{array}{cc}
\boldsymbol{I} & \boldsymbol{\Omega}_{A B} \\
\mathbf{0} & \boldsymbol{I}
\end{array}\right) \\
& =\left(\begin{array}{cc}
\boldsymbol{I} & \mathbf{0} \\
\boldsymbol{\Omega}_{B A} & \boldsymbol{I}
\end{array}\right) \underbrace{\left(\begin{array}{cc}
\mathbf{0} & \Omega_{F^{0}} \\
\boldsymbol{\Omega}_{F^{0}} & \boldsymbol{\Omega}_{F^{0}} \boldsymbol{\Omega}_{A B}+\boldsymbol{\Omega}_{T_{A}^{0}}
\end{array}\right)}_{=: \mathcal{V}_{A B}} \\
& =\left(\begin{array}{cc}
\mathbf{0} & \boldsymbol{\Omega}_{F^{0}} \\
\boldsymbol{\Omega}_{F^{0}} & \boldsymbol{\Omega}_{B A} \boldsymbol{\Omega}_{F^{0}}+\boldsymbol{\Omega}_{F^{0}} \boldsymbol{\Omega}_{A B}+\boldsymbol{\Omega}_{T_{A}^{0}}
\end{array}\right) \\
& =\left(\begin{array}{cc}
\mathbf{0} & \boldsymbol{\Omega}_{F^{0}} \\
\boldsymbol{\Omega}_{F^{0}} & \boldsymbol{\Omega}_{T_{B}^{0}}
\end{array}\right),
\end{aligned}
$$

denn wegen $(\mathrm{A} .2 .2$ gilt

$$
\begin{aligned}
\Omega_{T_{A}^{0}}+\Omega_{B A} \Omega_{F^{0}}+\Omega_{F^{0}} \Omega_{A B} & =\Omega_{T_{A}^{0}}+\left[\Omega_{B A}, \Omega_{F^{0}}\right] \\
& =\Omega_{T_{A}^{0}}+\Omega_{\overrightarrow{B A} \times F^{0}}=\Omega_{T_{A}^{0}+\overrightarrow{B A} \times F^{0}} \\
& =\Omega_{T_{B}^{0}} .
\end{aligned}
$$

Wie am Gang der Rechnung zu erkennen ist, entstehen für ungleiche Referenzsysteme Zusatzterme, welche die Antisymmetrie von $\mathcal{V}$ zerstören.

Der Struktur der Vorlastmatrix sind noch zwei Dinge anzumerken: 
1. Wegen der Antisymmetrie $\mathcal{V}^{T}=-\mathcal{V}$ tragen die Vorlasten nicht zusätzlich zu ihrem Beitrag über die Verschiebung des Gleichgewichtes zur potentiellen Energie bei. Da diese ein Skalar ist, gilt nämlich

$$
\begin{aligned}
\Phi^{\prime} & =\frac{1}{2} \delta q^{T}(\mathcal{S}+\mathcal{V}) \delta q=\Phi+\frac{1}{2} \delta q^{T} \mathcal{V} \delta q \\
& =\Phi+\frac{1}{2}\left(\delta q^{T} \mathcal{V} \delta q\right)^{T}=\Phi-\frac{1}{2} \delta q^{T} \mathcal{V} \delta q \\
& =\Phi .
\end{aligned}
$$

Die elastische Situation wird demnach für kleine Lageänderungen nicht geändert, obwohl sie von der ohne Vorlast sicherlich deutlich verschieden ist.

2. Da die Blöcke der Vorlastmatrix selber antisymmetrisch sind, beeinflußt eine Vorlast nur senkrecht zu ihr liegende Komponenten. Das sieht man aus den ausgeschriebenen Gleichungen (3.11) und (3.12):

$$
\begin{aligned}
& \boldsymbol{F}_{L}=\boldsymbol{F}^{0} \times \delta \boldsymbol{\theta} \\
& \boldsymbol{T}_{L}=\boldsymbol{F}^{0} \times \delta \boldsymbol{d}+\boldsymbol{T}^{0} \times \delta \boldsymbol{\theta} .
\end{aligned}
$$

Daher trägt die Vorlast, wie die in der Mechanik durch Zwangskräfte hervorgerufenen Verrückungen, nicht direkt zur elastischen Energie bei. Es würde auch für eine lineare Theorie keinen Sinn ergeben, wenn eine Normalkraft sich unter Einwirkung einer äußeren Kraft selbst beeinflußte.

\subsubsection{Auswertung von Meßdaten unter Vorlast}

Unter der Annahme, daß alle störenden Terme weitgehend durch die Vorlast erklärbar sind, gilt $\mathcal{S}_{T D}=\mathcal{S}_{Z Z}+\mathcal{V}_{Z}$ bzw. in Komponenten

$$
\left(\begin{array}{cc}
\boldsymbol{A} & \boldsymbol{B}_{2} \\
\boldsymbol{B}_{1} & \boldsymbol{C}
\end{array}\right)_{T D} \approx\left(\begin{array}{cc}
\boldsymbol{A}_{Z} & \boldsymbol{B}_{Z} \\
\boldsymbol{B}_{Z}^{T} & \boldsymbol{C}_{Z}
\end{array}\right)+\left(\begin{array}{cc}
\mathbf{0} & \boldsymbol{\Omega}_{F} \\
\boldsymbol{\Omega}_{F} & \boldsymbol{\Omega}_{T}
\end{array}\right)
$$

und die Abschätzung für die maximale Vorlast lautet einfach

$$
\boldsymbol{F}_{\max } \approx \frac{1}{2}\left(\boldsymbol{B}_{2}-\boldsymbol{B}_{1}^{T}\right)^{D}
$$

und

$$
\boldsymbol{T}_{\max } \approx C^{D}
$$

Da $\boldsymbol{A}$ im Gegensatz zu $\boldsymbol{A}_{Z}$ nicht symmetrisch sein muß, können diese Formeln nur näherungsweise gelten. Ferner hängt diese „Definition“ von der Wahl des verwendeten Koordinatensystems und - im Gegensatz zur direkten Auswertung von dem verwendeten Einheitensystem (speziell dem der Winkel) ab. Ein weiteres Problem ergibt sich nun daraus, daß die Normal- und Realform der Matrix über 
die Matrix $\boldsymbol{M}=\left(\boldsymbol{C}-\boldsymbol{B}_{1} \boldsymbol{A}^{-1} \boldsymbol{B}_{2}\right)^{-1}$ definiert sind. Daher beeinflußt der hier untersuchte Effekt über die Matrizen $\boldsymbol{B}$ und $\boldsymbol{C}$ die Normalform, was im nächsten Abschnitt näher diskutiert werden soll.

Die antisymmetrischen Anteile der Matrizen $\boldsymbol{B}_{2}$ und $\boldsymbol{B}_{1}$ transformieren sich wie folgt:

$$
\begin{aligned}
& \boldsymbol{B}_{1}^{\prime D}=\left(\boldsymbol{B}_{1}+\boldsymbol{\Omega}_{1} \boldsymbol{A}\right)^{D}=\boldsymbol{B}_{1}^{D}+\boldsymbol{A}^{F} \boldsymbol{x}_{1} \\
& \boldsymbol{B}_{2}^{\prime D}=\left(\boldsymbol{B}_{2}+\boldsymbol{A} \boldsymbol{\Omega}_{2}\right)^{D}=\boldsymbol{B}_{2}^{D}+\boldsymbol{A}^{T F} \boldsymbol{x}_{2}
\end{aligned}
$$

sie können daher insbesondere durch eine einfache Transformation nach

$$
\begin{aligned}
& \boldsymbol{x}_{1}^{0}=-\boldsymbol{A}^{F-1} \boldsymbol{B}_{1}^{D} \\
& \boldsymbol{x}_{2}^{0}=-\boldsymbol{A}^{T F-1} \boldsymbol{B}_{2}^{D}
\end{aligned}
$$

zu Null gemacht werden!13 Jede Abweichung von diesen Koordinatensystemen liefert bei der obigen Interpretation der antisymmetrischen Terme scheinbare Vorlasten der Größe

$$
\begin{aligned}
& \boldsymbol{F}_{1}:=\boldsymbol{F}\left(\boldsymbol{x}_{1}+\boldsymbol{x}_{1}^{0}\right)=\boldsymbol{A}^{F} \boldsymbol{x}_{1} \\
& \boldsymbol{F}_{2}:=\boldsymbol{F}\left(\boldsymbol{x}_{2}+\boldsymbol{x}_{2}^{0}\right)=\boldsymbol{A}^{T F} \boldsymbol{x}_{2},
\end{aligned}
$$

welche bei steifen Aufhängungen erhebliche Werte annehmen kann. Insbesondere steht $\mathrm{zu}$ befürchten, daß die Koeffizientenmatrix, die wegen (2.26) auch die Einträge der Matrix $\boldsymbol{D}$ enthält, deren ungünstigen Eigenschaften hinsichtlich der Fehlerfortpflanzung erbt.

Da offenbar die Bestimmung der Vorlast und die Transformation auf das elastische Zentrum koppeln, möchte ich als Nächstes auf die bewährten Definitionsgleichungen in der Form aus Abschnitt 2.2.5.1 zurückkommen. Die um die Vorlast als zusätzliche Variable erweiterten Minimierungsprobleme lauten

$$
\begin{aligned}
& \min !=\left\|-\boldsymbol{A}^{-1}\left(\boldsymbol{B}_{2}-\boldsymbol{\Omega}_{F}\right)-\boldsymbol{\Omega}_{D Z}\right\|^{2} \\
& \min !=\left\|\left(\boldsymbol{B}_{1}-\boldsymbol{\Omega}_{F}\right) \boldsymbol{A}^{-1}-\boldsymbol{\Omega}_{T Z}\right\|^{2} .
\end{aligned}
$$

Die dazugehörigen Normalgleichungen sind in der Schreibweise von (A.2.2)

$$
\left(\begin{array}{cc}
\boldsymbol{I} & -\boldsymbol{A}^{-1 F T} \\
-\boldsymbol{A}^{-1 F} & \boldsymbol{A}^{-1 G F}
\end{array}\right)\left(\begin{array}{c}
\overrightarrow{D Z} \\
\boldsymbol{F}
\end{array}\right)=\left(\begin{array}{c}
-\left(\boldsymbol{A}^{-1} \boldsymbol{B}_{2}\right)^{D} \\
\left(\boldsymbol{A}^{-1 G} \boldsymbol{B}_{2}\right)^{D}
\end{array}\right)
$$

\footnotetext{
${ }^{13}$ Dieser so festgelegte Ort ist einer meiner früheren Definitionsversuche für das elastische Zentrum. Da er die Nebendiagonalmatrizen symmetrisiert, hatte ich ihn das Symmetriezentrum genannt. Die analoge Rechenvorschrift für die Flexibilitätsmatrix lieferte aber andere Werte; die Definitionsgleichung war also, im Gegensatz zur jetzigen, nicht forminvariant - ein weiteres Indiz dafür, daß die hier schließlich getroffene Definition vor allen anderen Möglichkeiten ausgezeichnet ist.
} 
und

$$
\left(\begin{array}{cc}
\boldsymbol{I} & \boldsymbol{A}^{-1 F} \\
\boldsymbol{A}^{-1 F T} & \boldsymbol{A}^{-1 T G F}
\end{array}\right)\left(\begin{array}{c}
\overrightarrow{T Z} \\
\boldsymbol{F}
\end{array}\right)=\left(\begin{array}{c}
\left(\boldsymbol{B}_{1} \boldsymbol{A}^{-1}\right)^{D} \\
\left(\boldsymbol{B}_{1} \boldsymbol{A}^{-1 T G}\right)^{D}
\end{array}\right)
$$

Da auch hier die rechten Seiten vom gewählten Koordinatensystem abhängen, besitzt diese Berechnungsart dieselben konzeptionellen Schwierigkeiten wie die obige naive Methode. Insbesondere können wieder Koordinatensysteme gefunden werden, in denen die scheinbare Vorlast verschwindet. Die Gleichungen (3.15) und (3.16) haben eine interessante Eigenschaft: Wenn man die rechten Seiten auf die der Lösung entsprechenden Koordinatensysteme transformiert, verschwindet der Kandidat für das elastische Zentrum erwartungsgemäß und der Kandidat für die Vorlast bleibt unverändert. Die Lösungen sind daher für alle Ausgangskoordinatensysteme selbstkonsistent und demzufolge nicht iterativ verbesserbar.

In den elastischen Zentren der direkten Auswertung 2.2.5.2 waren die mit (3.15) und (3.16) bestimmten Kräfte im Vergleich zu anderen Koordinatensystemen relativ gering. Es macht also Sinn, diese Formeln im durch die direkte Auswertung bestimmten elastischen Zentrum anzuwenden. Die beiden resultierenden Exemplare für die Vorlast könnten dann zur weiteren Auswertung gemittelt werden.

Falls das immer noch zu große Vorlasten liefert, bliebe nur noch die Hoffnung, die Vorlast durch ein passendes statistisches Modell aus den Rohdaten zu bestimmen. Dann wäre eine nachträgliche Bestimmung der Vorlast unnötig, und die obigen Schwierigkeiten entfielen. Das erforderte aber die sinnvolle Entkopplung der Steifheitsmatrix in einen symmetrischen und einen antisymmetrischen Anteil, wie sie nur im elastischen Zentrum möglich ist. Da dessen Definition aber aus den Daten selber geschieht, entfällt auch diese Möglichkeit.

\subsubsection{Vorlasten, Fehlerfortpflanzung und die Standardformen}

Alle Änderungen an den Matrizen $\boldsymbol{B}$ und $\boldsymbol{C}$, wie sie ja durch Subtraktion von Vorlasten entstehen, beeinflussen wegen (2.25)

$$
\boldsymbol{M}^{-1}=\boldsymbol{C}-\boldsymbol{B}_{1} \boldsymbol{A}^{-1} \boldsymbol{B}_{2}
$$

auch die bezüglich der Fehlerfortpflanzung günstigste und zur Definition der Standardformen aus Abschnitt 2.5 herangezogene Matrix $\boldsymbol{M}$. Dasselbe gilt für eine Modifikation von $\boldsymbol{A}$ im Rahmen der fortgepflanzten Eingangsfehler. In diesem Abschnitt soll eine Klasse von solchen Vorlasten besprochen werden, die an $\boldsymbol{M}$ keine Veränderung hervorruft. Diese könnten nützlich sein, die nur vage bestimmbare Vorlast weiter einzugrenzen. Durch Abzug der Vorlasten erhält man

$$
\begin{aligned}
\boldsymbol{M}^{-1} & =\boldsymbol{C}-\boldsymbol{B}_{1} \boldsymbol{A}^{-1} \boldsymbol{B}_{2} \\
& \mapsto \boldsymbol{C}-\boldsymbol{\Omega}_{T}-\left(\boldsymbol{B}_{1}-\boldsymbol{\Omega}_{F}\right) \boldsymbol{A}^{-1}\left(\boldsymbol{B}_{2}-\boldsymbol{\Omega}_{F}\right) \\
& =\boldsymbol{M}^{-1}-\boldsymbol{\Omega}_{T}+\underbrace{\boldsymbol{\Omega}_{F} \boldsymbol{A}^{-1} \boldsymbol{B}_{2}+\boldsymbol{B}_{1} \boldsymbol{A}^{-1} \boldsymbol{\Omega}_{F}-\boldsymbol{\Omega}_{F} \boldsymbol{A}^{-1} \boldsymbol{\Omega}_{F}}_{=: \boldsymbol{M}_{F}} .
\end{aligned}
$$


Ein Drehmoment vom Betrag

$$
\boldsymbol{T}=\boldsymbol{M}_{F}^{D}
$$

läßt offenbar den antisymmetrischen Anteil von $\boldsymbol{M}^{-1}$ und damit den von $\boldsymbol{M}$ unverändert. Als Nächstes sollen daher Lösungen von $\boldsymbol{M}_{F}^{S}=\mathbf{0}$ bestimmt werden. Es ergibt sich

$$
\begin{aligned}
\mathbf{0}= & 2 \boldsymbol{M}_{F}^{S} \\
= & \boldsymbol{\Omega}_{F} \boldsymbol{A}^{-1} \boldsymbol{B}_{2}+\boldsymbol{B}_{1} \boldsymbol{A}^{-1} \boldsymbol{\Omega}_{F}-\boldsymbol{\Omega}_{F} \boldsymbol{A}^{-1} \boldsymbol{\Omega}_{F} \\
& +\boldsymbol{B}_{2}^{T} \boldsymbol{A}^{-1 T} \boldsymbol{\Omega}_{F}^{T}+\boldsymbol{\Omega}_{F}^{T} \boldsymbol{A}^{-1 T} \boldsymbol{B}_{1}^{T}-\boldsymbol{\Omega}_{F}^{T} \boldsymbol{A}^{-1 T} \boldsymbol{\Omega}_{F}^{T} \\
= & \boldsymbol{\Omega}_{F} \underbrace{\left(\boldsymbol{A}^{-1} \boldsymbol{B}_{2}-\boldsymbol{A}^{-1 T} \boldsymbol{B}_{1}^{T}\right)}_{=: \boldsymbol{U}}+\underbrace{\left(\boldsymbol{B}_{1} \boldsymbol{A}^{-1}-\boldsymbol{B}_{2}^{T} \boldsymbol{A}^{-1 T}\right)}_{=-\boldsymbol{U}^{T}} \boldsymbol{\Omega}_{F} \\
& -\boldsymbol{\Omega}_{F}\left(\boldsymbol{A}^{-1}+\boldsymbol{A}^{-1 T}\right) \boldsymbol{\Omega}_{F} \\
= & \boldsymbol{\Omega}_{F}\left(\boldsymbol{U}-\boldsymbol{A}^{-1} \boldsymbol{\Omega}_{F}\right)-\left(\boldsymbol{U}-\boldsymbol{A}^{-1} \boldsymbol{\Omega}_{F}\right)^{T} \boldsymbol{\Omega}_{F} .
\end{aligned}
$$

Hinreichend für das Verschwinden des gesamten Terms ist 14

$$
\mathbf{0}=\boldsymbol{\Omega}_{F}\left(\boldsymbol{U}-\boldsymbol{A}^{-1} \boldsymbol{\Omega}_{F}\right)
$$

mit den Lösungen $\boldsymbol{\Omega}_{F}=0$ oder $\boldsymbol{U}=\boldsymbol{A}^{-1} \boldsymbol{\Omega}_{F}$ oder $\left(\boldsymbol{U}-\boldsymbol{A}^{-1} \boldsymbol{\Omega}_{F}\right)_{i j}=F_{i} x_{j}$ mit $\boldsymbol{x}$ beliebig. Dualisieren der letzten Gleichung liefert zunächst

$$
\boldsymbol{U}^{D}-\boldsymbol{A}^{-1 T F} \boldsymbol{F}=\frac{1}{2} \boldsymbol{x} \times \boldsymbol{F}
$$

Mit dem Entwicklungssatz folgt weiter für den zu $\boldsymbol{F}$ senkrechten Anteil von $\boldsymbol{x}$

$$
\boldsymbol{x}^{\perp}=\frac{2}{\boldsymbol{F}^{2}} \boldsymbol{F} \times\left(\boldsymbol{U}^{D}-\boldsymbol{A}^{-1 T F} \boldsymbol{F}\right)
$$

Damit ist eine spezielle Lösung $\boldsymbol{x}^{\perp}$ gefunden. Die Kraft $\boldsymbol{F}$ muß aber auch Lösung von $\boldsymbol{U}^{D}-\boldsymbol{A}^{-1 T F} \boldsymbol{\Omega}_{F}=\frac{1}{2} \boldsymbol{\Omega}_{x} \boldsymbol{F}$ sein. Wenn man also statt der ermittelten Kraft den entsprechend modifizierten Abkömmling, also die Lösung von

$$
\boldsymbol{U}^{D}=\left(\frac{1}{2} \boldsymbol{\Omega}_{x}-\boldsymbol{A}^{-1 T F}\right) \boldsymbol{F}
$$

benutzt, ändert sich die Matrix $\boldsymbol{M}$ nicht.

Da die Matrix $\boldsymbol{A}$ diejenige mit der schlechtesten Fehlerfortpflanzung ist, liegt es nahe, sie im Rahmen der Eingangsfehler „passend“ zu machen. Dies hat aber immer Auswirkungen auf die Matrix $\boldsymbol{M}$, da der Anteil $\boldsymbol{B}_{1} \boldsymbol{A}^{-1} \boldsymbol{B}_{2}$ von (2.25) für alle

${ }^{14}$ Wegen $\left(\lambda \boldsymbol{A}^{-1}+(1-\lambda) \boldsymbol{A}^{-1 T}\right)^{S}=\boldsymbol{A}^{S}$ ist auch eine solche Linearkombination an dieser Stelle zulässig; das vergrößert die Menge aller möglichen Lösungen um einen weiteren freien Parameter $\lambda$. 
Bezugssysteme verschwinden müßte. Im elastischen Zentrum etwa lautet er aber $\left(-\boldsymbol{W}_{L} \boldsymbol{A}\right) \boldsymbol{A}^{-1}\left(-\boldsymbol{A} \boldsymbol{W}_{M}\right)=\boldsymbol{W}_{L} \boldsymbol{A} \boldsymbol{W}_{M}$ und ist nur im Fall eines Widerstandszentrums zum Verschwinden zu bewegen. Hingegen ergeben sich beim Abziehen der Vorlast gewisse Freiheiten: Zusatzterme der Art $\boldsymbol{A}^{-1} \mapsto \boldsymbol{A}^{-1}+\boldsymbol{x}_{1} \boldsymbol{F}^{T}+\boldsymbol{F} \boldsymbol{x}_{2}^{T}$ mit $\boldsymbol{x}_{1}, \boldsymbol{x}_{2}$ beliebig, lassen $\left(\boldsymbol{B}_{1}+\boldsymbol{\Omega}_{F}\right) \boldsymbol{A}^{-1}\left(\boldsymbol{B}_{2}+\boldsymbol{\Omega}_{F}\right)$ ungeändert.

Meiner Erfahrung mit realen Meßwerten nach führen solche „Verbesserungen“ nur zu unbefriedigenden Ergebnissen, weshalb diese im Folgenden auch nicht zum Einsatz kommen werden.

\subsection{Nicht symmetrisierbare elastische Matrizen}

Im vorigen Kapitel wurde eine Transformation der ermittelten Steifheitsmatrix $\mathcal{S}_{L M}$ oder ihrer Inversen, der Flexibilitätsmatrix $\mathcal{F}_{M L}$, vom Lastsystem $L$ bzw. Meßsystem $M$ auf die beiden Kandidaten für ein elastisches Zentrum, Translationszentrum $T$ und Drehmomentszentrum $D$, hergeleitet. In der Praxis hatten die so erhaltenen elastischen Matrizen $\mathcal{S}_{T D}=\mathcal{T}_{T L} \mathcal{S}_{L M} \mathcal{T}_{D M}^{T}$ und $\mathcal{F}_{D T}=\mathcal{T}_{M D}^{T} \mathcal{F}_{M L} \mathcal{T}_{L T}$ zwar deutlich kleinere Nebendiagonaleinträge, waren aber immer noch nicht symmetrisch. Auch mit der Transformation auf die Normalform konnten die verbleibenden antisymmetrischen Terme nicht beseitigt werden.

Die zuerst untersuchte Quelle dieses Fehlers liegt im statistischen Verfahren, und ist für retrospektive Auswertungen nicht relevant. Inkompatible Einheitensysteme kommen allenfalls während des Aufbaus einer Meßapparatur vor. Selbst unter Annahme von verdrehten Referenzsystemen, also nach Durchführung der Polarzerlegung für die transformationsinvarianten Teilmatrizen, sind die Nebendiagonalmatrizen noch nicht gleich und daher die Steifheitsmatrix insgesamt nicht symmetrisch. Als letzter und mathematisch anspruchsvollster Effekt kam eine dem Meßobjekt innewohnende Vorlast in Frage. Ihre Kopplung mit allen Blockmatrizen bis auf die am schwersten zu bestimmende, $\boldsymbol{A}$, machte es mir unmöglich, aus den mir vorliegenden Daten sinnvolle Schlüsse zu ziehen.

Ein Überblick der zu Grunde liegenden gegenseitigen Beeinflussungen der Transformationen wird in den folgenden Tabellen gegeben; das statistische Modell ist hierbei ausgenommen, da es selbstverständlich mit allen Matrixeinträgen koppelt.

\begin{tabular}{l||c|c|c|c|c|} 
Kopplung & $\begin{array}{c}\text { Translation } \\
\text { Meßsystem }\end{array}$ & $\begin{array}{c}\text { Translation } \\
\text { Lastsystem }\end{array}$ & $\begin{array}{c}\text { Rotation } \\
\text { Meßsyst. }\end{array}$ & $\begin{array}{c}\text { Rotation } \\
\text { Lastsyst. }\end{array}$ & Einheiten \\
\hline \hline Transl. Meßs. & $\mathrm{x}$ & - & - & - & - \\
\hline Rot. Meßs. & - & $\mathrm{x}$ & - & - & - \\
\hline Transl. Lasts. & - & - & $\mathrm{x}$ & - & - \\
\hline Rot. Lasts. & - & - & - & $\mathrm{x}$ & - \\
\hline Einheiten & - & - & - & - & $\mathrm{x}$ \\
\hline
\end{tabular}


Diese ersten nicht miteinander koppelnden Rechenschritte möchte ich im Folgenden die direkte Auswertung nennen. Sie führten bei allen mir zur Auswertung vorgelegten Matrizen zu sinnvollen Ergebnissen. Bei den übrigen ist die Sachlage deutlich verwickelter, wie unschwer aus der folgenden Tabelle zu ersehen ist! 15

\begin{tabular}{l||c|c|} 
Kopplung & Verdrehung Lastsystem & Vorlast \\
\hline \hline Translation Meßsystem & - & $\mathrm{x}$ \\
\hline Rotation Meßsystem & - & $\mathrm{x}$ \\
\hline Translation Lastsystem & $\mathrm{x}$ & $\mathrm{x}$ \\
\hline Rotation Lastsystem & $\mathrm{x}$ & $\mathrm{x}$ \\
\hline Einheiten & - & $\mathrm{x}$ \\
\hline Verdrehung Lastsystem & $\mathrm{x}$ & $\mathrm{x}$ \\
\hline Vorlast & $\mathrm{x}$ & $\mathrm{x}$ \\
\hline
\end{tabular}

Da die mir vorliegenden Matrizen keine zweifelsfreie Zuordnung zu einem fehlerverursachenden Effekt zulassen, wurden diese Effekte standardmäßig nicht zur Erklärung irgendwelcher Abweichungen von der Symmetrie herangezogen.

Prinzipielle Probleme treten nur noch in folgenden Situationen auf:

- Das verwendete statistische Modell war ungeeignet, und die Originaldaten liegen nicht mehr vor.

- Das verwendete Koordinatensystem war nicht orthogonal oder teilweise ein Linkssystem, und die Anordnung ist nicht mehr zu rekonstruieren.

- Die Auslenkungen sind endlich, und daher stimmt das Transformationsverhalten der Matrix nicht mehr.

- Das Meßobjekt besitzt bislang unberücksichtigte Zwangsbedingungen, vgl. B.3.

- Dem Material hätte ein Flüssigkeitsmodell zu Grunde gelegt werden müssen, dennoch wurde ein Extrapolationsverfahren auf einen nicht existierenden asymptotischen Zustand eingesetzt.

- Das Superpositionsprinzip ist grob verletzt; die zu Grunde liegende Theorie muß nichtlinear sein.

In diesen Fällen wird der Gültigkeitsbereich der bisherigen linearen Theorie übertreten, sie sind daher nicht mehr Gegenstand dieser Arbeit.

\footnotetext{
${ }^{15}$ Beispielsweise geht im Gegensatz zu den Überlegungen aus Abschnitt 2.2.5.3 in die Definition von $\overrightarrow{L T}$ nur eine Drehmatrix in die Rechnung ein, weshalb der Beweis der Entkopplung von Translation und Rotation im Lastsystem unter Verwendung von (A.15) nicht mehr gelingt.
} 


\section{Kapitel 4}

\section{Das elastische Potential einer dünnen Schicht}

Im ersten Kapitel wurde eine Methode vorgestellt, mit der Meßdaten von Flexibilitäts- bzw. Steifigkeitskoeffizienten des elastisch aufgehängten starren Körpers in eine miteinander vergleichbare Form gebracht werden konnten. Um über diese bloße Vergleichbarkeit hinaus mit den so gewonnenen Aussagen etwas anfangen zu können, braucht man auf der anderen Seite theoretische Vorhersagen über einfache Aufhängungen, die dann entweder quantitativ als Probekörper für Messungen zur Validierung der Theorie dienen, oder einfach qualitativ eine Vorstellung über zu erwartende Meßergebnisse sind. Um derartige Vorhersagen zu bekommen, werden üblicherweise bestimmte vereinfachende modellhafte Annahmen eingesetzt. Inwieweit die so ersonnenen Objekte zur Beschreibung der Beobachtungen brauchbar sind, entscheidet dann letztlich wieder der Vergleich mit der Messung. Bei einer solchen Modellbildung muß es immer möglich sein, einerseits unbrauchbare Messungen zu identifizieren und andererseits zu stark vereinfachte Modelle zu falsifizieren.

Das Problem ist es, aus der Geometrie der starren Berandung und der inneren Struktur des elastischen Mediums eine konkrete Flexibilitäts- bzw. Steifheitsmatrix zu berechnen. Die Grundgleichungen der Elastostatik wären sicher zu einer Beschreibung geeignet. Dann handelte es sich um ein gemischtes Randwertproblem, da auf der starren Berandung die Verschiebungen und im Zwischenraum die hier verschwindenden Spannungen vorgegeben sind. Exakte Lösungen für solche Probleme liegen nur in Ausnahmefällen vor, zu denen die für diese Arbeit interessanten Objekte leider nicht gehören. Um aber ohne Finite Elemente oder vergleichbare numerische Methoden auskommen zu können, werde ich einige vereinfachte Modelle vorführen: Über das Studium einer HookEschen Feder in einer räumlichen Anordnung wird die dünne elastische Schicht als lokal ebenes Problem mit den Mitteln der Darstellungstheorie von Invarianten behandelt. Das so entwickelte Modell wird zunächst mit Gedankenexperimenten verfeinert, um dann mit komplexeren Betrachtungsweisen aus der Kontinuumsmechanik verglichen und abschließend für 
gut befunden zu werden. Die sich so ergebende prinzipielle Vorgehensweise ist in Abb. 4.1 illustriert.

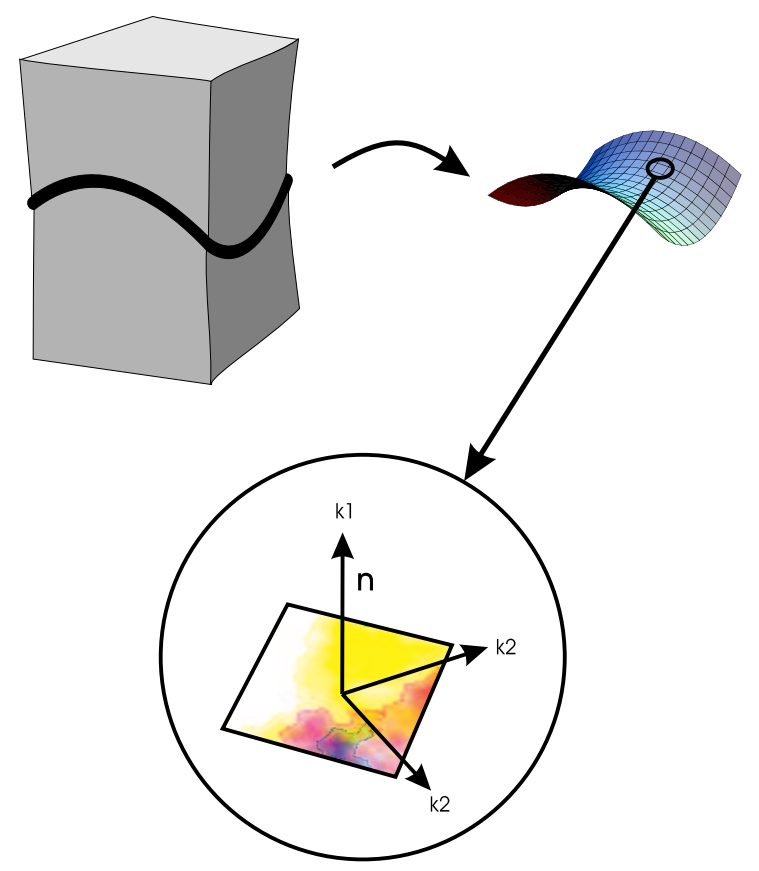

Abbildung 4.1: Die Idee des Federmodells. Eine starr berandete dünne elastische Schicht wird zunächst zu einer Fläche abstrahiert. Diese wird parametrisiert; ein differentielles Flächenelement ist dann eben. Kann das Material als isotrop genähert werden, so wird das elastische Verhalten durch zwei von der lokalen Schichtdicke abhängenden Schichtgrößen beschrieben: Eine parallel zur Schichtnormale $\boldsymbol{n}$ und zwei gleiche senkrecht zu dieser.

\subsection{Federn im Raum}

Es lohnt sich, wie gesagt, zunächst die mathematische Beschreibung einer Einzelfeder mit Hookeschen Verhalten zu betrachten. Systeme solcher Federn sind eine nicht nur für Demonstrationszwecke brauchbare Approximation an das Verhalten elastischer Kontinua.

\subsubsection{Die Einzelfeder}

Das Potential einer HookEschen Feder wird üblicherweise angegeben als

$$
\Phi=\frac{1}{2} k x^{2}
$$


wobei die Richtgröße $k$ die Dimension Energie/Länge ${ }^{2}$, also formal Masse/Zeit ${ }^{2}$ und mehr physikalisch Energiedichte $\times$ Länge hat. Diese Formel gilt natürlich nur eindimensional und mit dem Koordinatenursprung im Federende und Koordinatenachse in Federrichtung. Im Raum gilt für eine Feder der Ruhelänge $l$, deren elastische Energie nur von dem Abstand zwischen den Federenden abhängt, wobei ein Federende im Koordinatenursprung und das andere bei $\boldsymbol{x}$ liegt

$$
\Phi=\frac{1}{2} k(|\boldsymbol{x}|-l)^{2}
$$

Das Potential hat die Ableitungen

$$
\Phi_{, i}=k(|\boldsymbol{x}|-l) \frac{x_{i}}{|\boldsymbol{x}|}=k\left(1-\frac{l}{|\boldsymbol{x}|}\right) x_{i}
$$

und

$$
\begin{aligned}
\Phi_{, i, j} & =k\left(\left(1-\frac{l}{|\boldsymbol{x}|}\right) \delta_{i j}+\frac{l}{|\boldsymbol{x}|^{3}} x_{i} x_{j}\right) \\
& =k\left(\left(1-\frac{l}{|\boldsymbol{x}|}\right)\left(\boldsymbol{P}^{\perp}+\boldsymbol{P}^{\|}\right)_{i j}+\frac{l}{|\boldsymbol{x}|} \boldsymbol{P}_{i j}^{\|}\right) \\
& =k\left(\left(1-\frac{l}{|\boldsymbol{x}|}\right) \boldsymbol{P}^{\perp}+\boldsymbol{P}^{\|}\right)_{i j},
\end{aligned}
$$

wobei $\boldsymbol{P}^{\|}$und $\boldsymbol{P}^{\perp}$ Parallel- und Orthogonalprojektor zur Richtung $\frac{\boldsymbol{x}}{|\boldsymbol{x}|}$ der Feder sind. Im entspannten Gleichgewicht gilt $l=|\boldsymbol{x}|$, und so lautet die harmonische Näherung um die Gleichgewichtsposition $\boldsymbol{l}$

$$
\Phi=\frac{1}{2} k(\boldsymbol{x}-\boldsymbol{l})^{T} \boldsymbol{P}_{l}^{\|}(\boldsymbol{x}-\boldsymbol{l}) .
$$

Dieser Parallelprojektor besagt, daß in erster Näherung nur der zur Feder parallele Anteil der Verschiebung wirksam ist und eine Feder im Raum durch eine symmetrische Matrix beschrieben werden kann!

Der einfachste vorgespannte Fall sei etwa realisiert durch eine Aufhängung aus zwei gegenüberliegenden Federn gleicher Federstärke $k$ und Ruhelänge $l$. Der Ursprung der ersten Feder liege im Nullpunkt und der der zweiten im Punkt $\boldsymbol{L}$. Das Gesamtpotential lautet demnach

$$
\Phi=\frac{1}{2} k\left((|\boldsymbol{x}|-l)^{2}+(|\boldsymbol{x}-\boldsymbol{L}|-l)^{2}\right),
$$

und aus Symmetriegründen verschwindet im Punkt $\boldsymbol{x}_{0}=\boldsymbol{L} / 2$ der Gradient $\Phi_{, i}$. Benutzt man diese Gleichgewichtslage als Entwicklungspunkt, so ergibt sich mit $L:=|\boldsymbol{L}| / 2$ näherungsweise

$$
\Phi=\frac{1}{2} 2 k(\boldsymbol{x}-\boldsymbol{L} / 2)^{T}\left(\left(1-\frac{l}{L / 2}\right) \boldsymbol{P}^{\perp}+\boldsymbol{P}^{\|}\right)(\boldsymbol{x}-\boldsymbol{L} / 2)
$$

\footnotetext{
${ }^{1}$ Vgl. auch im Folgenden [7] $§ 12.5$.
} 
Gilt nun $L / 2=\left|\boldsymbol{x}_{0}\right|>l$, so enthält die harmonische Näherung um die Gleichgewichtsposition Anteile senkrecht zur Richtung der Feder. Schon für eine Anordnung aus zwei Federn mit verschiedenen Anfangspunkten ist also die Situation komplizierter. Der nächste Abschnitt gilt daher solchen Anordnungen.

\subsubsection{Systeme von Federn mit gleichen Endpunkten}

Betrachtet werde eine Anordnung von mehreren Federn mit verschiedenen Anfangspunkten $\boldsymbol{a}_{n}$ und Ruhelängen $l_{n}$. Das Gesamtpotential ist jetzt die Summe der einzelnen Potentiale

$$
\Phi=\frac{1}{2} \sum_{n} k_{n}\left(\left|\boldsymbol{x}-\boldsymbol{a}_{n}\right|-l_{n}\right)^{2},
$$

und die Ableitungen lauten nun

$$
\Phi_{, i}=\sum_{n} k_{n}\left(1-\frac{l_{n}}{\left|\boldsymbol{x}-\boldsymbol{a}_{n}\right|}\right)\left(\boldsymbol{x}-\boldsymbol{a}_{n}\right)_{i}
$$

und

$$
\Phi_{, i, j}=\sum_{n} k_{n}\left(\left(1-\frac{l_{n}}{\left|\boldsymbol{x}-\boldsymbol{a}_{n}\right|}\right) \boldsymbol{P}_{x-a_{n}}^{\perp}+\boldsymbol{P}_{x-a_{n}}^{\|}\right)_{i j} .
$$

Um den Anteil der Orthogonalprojektoren zum Verschwinden zu bewegen, hätte man die i.a. nichtlineare Gleichung $\sum_{n} k_{n}\left(1-\frac{l_{n}}{\left|\boldsymbol{x}-\boldsymbol{a}_{n}\right|}\right) \boldsymbol{P}_{x-a_{n}}^{\perp}=0$ zu lösen, welche nicht mehr identisch mit der Gleichgewichtsbedingung $\Phi_{, i}=0$ ist. Falls aber für die Einzelfedern gilt $\left|\boldsymbol{x}-\boldsymbol{a}_{n}\right|=l_{n}$, dann verschwindet neben dem Gradienten auch diese Summe; das entspricht einer Anordnung von nicht vorgespannten Federn mit gleichem Endpunkt. In diesem Fall bekommt man demnach bei der harmonischen Näherung des Potentials lauter reine Parallelprojektoren als Summanden, welche als Summe aber wieder eine symmetrische Matrix mit bis zu vollem Rang haben. Bei zudem übereinstimmenden Federanfängen entsteht eine Summe von gleichen Projektoren, und die Gesamtmatrix wird, wie beim Gesetz der Parallelschaltung von Federn, durch Summation der einzelnen Federkonstanten gebildet.

Im allgemeinen Fall von vorgespannten Federn wird das Potential also auch von den Richtungen senkrecht zu den einzelnen Federn abhängen. Anschaulich heißt das nur, daß eine Bewegung um die Gleichgewichtslage in eine beliebige Raumrichtung eine Feder oder gar eine ganze Gruppe spannt und daher eine Zunahme der potentiellen Energie hervorruft. Da eine Summe von Parallelprojektoren bzw. Orthogonalprojektoren nicht wieder ein solcher Projektor sein muß, wird die harmonische Näherung für ein System von Federn allgemein beschrieben mit der symmetrischen Matrix $\boldsymbol{K}$ durch

$$
\Phi=\frac{1}{2}(\boldsymbol{x}-\boldsymbol{l})^{T} \boldsymbol{K}_{l}(\boldsymbol{x}-\boldsymbol{l}),
$$

wobei der Index $\boldsymbol{l}$ der Gleichgewichtslage an die Abhängigkeit von $\boldsymbol{K}$ von dem Entwicklungspunkt erinnern soll. Diese Matrix $\boldsymbol{K}$ möchte ich ab jetzt die Federmatrix 
nennen. Der Rang von $\boldsymbol{K}$ ist also aus den Symmetrien und der inneren Struktur der Aufhängung bestimmt.

Im Fall des vorgespannten Federpaares aus dem vorigen Abschnitt etwa gilt

$$
\boldsymbol{K}=2 k \boldsymbol{P}_{L}^{\|}+2 k\left(1-\frac{l}{L / 2}\right) \boldsymbol{P}_{L}^{\perp}=k_{1} \boldsymbol{P}_{L}^{\|}+k_{2} \boldsymbol{P}_{L}^{\perp}
$$

mit $0<k_{2}<k_{1}$.

\subsection{Die dünne elastische Schicht zwischen zwei starren Berandungen als lokal ebenes Pro- blem}

Im letzten Abschnitt wurde gezeigt, daß ein System aus HookEschen Federn durch eine symmetrische Matrix beschrieben werden kann. Als Nächstes soll eine dünne elastische Schicht untersucht werden. Wie sich zeigen wird, ist auch für diese eine Beschreibung durch eine symmetrische $3 \times 3$-Matrix möglich.

\subsubsection{Die Federmatrix eines isotropen Materials}

Ein isotropes Medium besitzt zwei elastische Konstanten, beispielsweise den Elastizitätsmodul $E$ und den Schubmodul $G$ oder die beiden LAmÉ-Koeffizienten. Das läßt sich begründen mit der allgemeinen Form der linearen Abbildung, die die Spannungen $\sigma_{i j}$ mit den Dehnungen $\varepsilon_{i j}$ verknüpft: Das verallgemeinerte HooKEsche Gesetz $\varepsilon_{i j}=\Lambda_{(i j)(k l)} \sigma_{k l}$ läßt sich im isotropen Fall durch die Forderung nach Forminvarianz unter orthogonalen Abbildungen ausdrücken durch

$$
\Lambda_{(i j)(k l)}=\lambda_{1} \delta_{i j} \delta_{k l}+\lambda_{2} \delta_{i(k} \delta_{l) j}
$$

Diese etwas abstrakte Vorgehensweise, bei der zunächst keine Rede von physikalischen Phänomenen wie longitudinaler und transversaler Schallausbreitung ist, benutzt wesentlich das Transformationsverhalten der an den Grundgleichungen beteiligten Größen.

Allgemein besteht diese Methode, die sogenannte Invariantentheorie, darin, für einen gegebenen Variablensatz und eine gegebene Symmetrie- bzw. Transformationsgruppe allgemeine Ausdrücke für unter der Gruppenoperation forminvariante Ansätze zu finden. Diese sogenannten Darstellungen sind dann Funktionen in den aus den Variablen bildbaren Invarianten. Im günstigsten Fall oder unter zusätzlichen Annahmen sind sie endliche Multinome. Insbesondere interessiert man sich für kleinste Mengen von Objekten, mit denen ein allgemeiner Ansatz möglich ist, 
sog. Basen. Von diesem Verfahren wird in der Kontinuumsphysik ausgiebig Gebrauch gemacht, wobei die verwendeten Symmetriegruppen meist die Drehgruppe $S O(3)$ oder die ganze orthogonale Gruppe $O(3)$ sind. Ausführlicheres findet sich in [14] und [71].

Hier ist die Aufgabe, die allgemeine Form der Federmatrix einer dünnen isotropen Schicht mit nicht zu stark variierender Schichtdicke zu bestimmen. Die Federmatrix ist ein symmetrischer Tensor zweiter Stufe, und die Geometrie der dünnen isotropen Schicht ist als lokal ebenes Problem allein durch ihren Normalenvektor $\boldsymbol{n}$, als einzig ausgezeichneter Richtung, bestimmt. Daher besteht der Ansatz für $K_{i j}$ aus den Zutaten $\delta_{i j}$ sowie $n_{i}$ und lautet allgemein $K_{i j}=k_{0} n_{i} n_{j}+k_{2} \delta_{i j}$, oder gleichwertig

$$
\boldsymbol{K}=k_{1} \boldsymbol{P}_{n}^{\|}+k_{2} \boldsymbol{P}_{n}^{\perp}
$$

analog zum vorgespannten Federpaar. Damit besteht eine anzahlmäßige 1-zu-1Korrespondenz zwischen den Schichtgrößen $k_{1}$ und $k_{2}$ und den zwei elastischen Konstanten. Anschaulich erwartet man eine Proportionalität zwischen $k_{2}$ und dem Torsionsmodul bzw. Schubmodul $G$, da Drehungen mit $\boldsymbol{n}$ als Achse nur Auslenkungen senkrecht zur Schichtnormale hervorrufen. Zwischen $k_{1}$ und $E$ besteht keine direkte Proportionalität, da die elastische Schicht unter einer senkrecht zu ihr wirkenden Kraft in ihren Querdimensionen unverändert bleibt. $k_{1}$ ist demnach besser proportional zum Koeffizienten der einseitigen Kompression, vgl. [40] §5, anzunehmen. Die restliche in den Schichtgrößen enthaltene Information muß dann eine reine Funktion der Schichtgeometrie mit dimensionslosen Argumenten sein.

\subsubsection{Die Federmatrix eines faserigen Materials}

Eine ursprüngliche Motivation dieser Arbeit war die Modellierung des Periodonts, also einer faserigen Struktur. Um darauf zurückzukommen, möchte ich eine Abhängigkeit der Federmatrix von einer zusätzlichen Richtung $\boldsymbol{e}$ im Inneren des elastischen Mediums diskutieren. Da im Periodont die Fasern nur unter starker funktioneller Belastung ausgebildet werden und dann noch zu großen Teilen in einem festen Bezug zu Schichtnormale und Zahnachse stehen,2 muß dieses Modell keine zusätzliche Genauigkeit liefern. Da die Darstellungstheorie keine Frage nach der Herkunft der zusätzlichen Richtung stellt, könnten mit derartigen Ansätzen etwa über den Gradienten der Schichtdicke auch wieder Phänomene wie eine variable Schichtdicke näherungsweise beschrieben werden.

Die analoge Fragestellung aus der Kontinuumsmechanik ist die Theorie der mesomorphen Phase, also die der flüssigen Kristalle bzw. der kristallinen Flüssigkeiten (vgl. 40] Kap. VI). Die elastische Energie möge nicht von dem Vorzeichen von $\boldsymbol{e}$ abhängen, d. h. es sind invariante Darstellungen unter der vollen $O(3)$ gefordert. Physikalisch entspricht das einer nematischen Flüssigkeit. Ein Tensor vierter

\footnotetext{
${ }^{2}$ Vgl. [5].
} 
Stufe einer nematischen Flüssigkeiten hat gegenüber dem einer isotropen Flüssigkeit entsprechend den drei FRANKschen Moduln drei weitere linear unabhängige Kombinationen der Komponenten der Zutaten $\boldsymbol{I}$ und $\boldsymbol{e}$

$$
\begin{aligned}
\Lambda_{(i j)(k l)=} & \lambda_{1} \delta_{i j} \delta_{k l}+\lambda_{2} \delta_{i(k} \delta_{l) j} \\
& +\lambda_{3}\left(e_{i} e_{j} \delta_{k l}+e_{k} e_{l} \delta_{i j}\right)+\lambda_{4} e_{(i} \delta_{j)(l} e_{k)}+\lambda_{5} e_{i} e_{j} e_{k} e_{l},
\end{aligned}
$$

wobei die zusätzliche Symmetrie $\Lambda_{(i j)(k l)}=\Lambda_{(k l)(i j)}$ eine Folge der Onsagerschen Reziprozitätsbeziehungen ist.

Für das Problem der Bestimmung der Federmatrix heißt das, einen Tensor zweiter Stufe aus den Komponenten von $\boldsymbol{I}, \boldsymbol{n}$ und $\boldsymbol{e}$ zusammenzusetzen, der zudem forminvariant unter Punktspiegelungen ist. Diese Darstellung lautet:

$$
\boldsymbol{K}=k_{1} \boldsymbol{P}_{n}^{\|}+k_{2} \boldsymbol{P}_{n}^{\perp}+k_{3} \boldsymbol{P}_{e}^{\|}+k_{4}\left(\boldsymbol{n} \boldsymbol{e}^{T}+\boldsymbol{e} \boldsymbol{n}^{T}\right) .
$$

Terme mit $\boldsymbol{P}_{e}^{\perp}$ tauchen als Linearkombination der ersten drei Summanden nicht auf; Terme mit $\boldsymbol{n}(\boldsymbol{e} \times \boldsymbol{n})^{T}$ oder $\boldsymbol{e}(\boldsymbol{e} \times \boldsymbol{n})^{T}$ sind nicht invariant unter Punktspiegelungen. Der Parallelprojektor $\boldsymbol{P}_{e \times n}^{\|}$ist das zwar, aber mit (A.18) kann man zeigen, daß sich auch dieser als Linearkombination der anderen Terme schreiben läßt. Damit ist keine anzahlmäßige Korrespondenz der beiden Ansätze vorhanden, eine Folge der durch die Annahme einer dünnen Schicht gegenüber einem Volumen erhöhten Symmetrie des Problems.

Nimmt man ein Medium mit drei unabhängigen inneren Richtungen an, also etwa eine Schichtnormale und zwei Faserrichtungen $\boldsymbol{e}_{1}$ und $\boldsymbol{e}_{2}$, so bilden diese eine Basis des Raumes. Daher stellt

$$
\boldsymbol{K}=k_{1} \boldsymbol{P}_{n}^{\|}+k_{2} \boldsymbol{P}_{1}^{\|}+k_{3} \boldsymbol{P}_{2}^{\|}+k_{4}\left(\boldsymbol{n} \boldsymbol{e}_{1}^{T}+\boldsymbol{e}_{1} \boldsymbol{n}^{T}\right)+k_{5}\left(\boldsymbol{n} \boldsymbol{e}_{2}^{T}+\boldsymbol{e}_{2} \boldsymbol{n}^{T}\right)+k_{6}\left(\boldsymbol{e}_{1} \boldsymbol{e}_{2}^{T}+\boldsymbol{e}_{2} \boldsymbol{e}_{1}^{T}\right)
$$

in der entsprechenden Basis den vollständigen Zugriff auf die sechs einzelnen Komponenten der symmetrischen Federmatrix $\boldsymbol{K}$ dar.

\subsubsection{Das Federmodell}

Dem Federmodell dieser Arbeit liegt folgende Vorstellung zu Grunde:

Jedem Punkt des körperfesten Systems der gemeinsamen Oberfläche des starren Körpers mit dem elastischen Medium wird eine symmetrische Federmatrix zugeordnet. Die diesem Punkt zugeordnete potentielle Energie hängt außer von dessen Ort über seine Verschiebung noch von den verallgemeinerten Koordinaten der starren Berandung im ortsfesten System ab. Das elastische Potential der gesamten Aufhängung wird, da es sich um eine Parallelschaltung handelt, als Integral bzw. 
für diskrete Anordnungen als Summe über die Einzelfedern berechnet. Durch zweimalige Differentiation nach den verallgemeinerten Koordinaten erhält man daraus die Steifheitsmatrix.

Der Einfachheit halber soll vorausgesetzt werden, daß die Anordnung von Federn kein Getriebe bildet, oder - in der Terminologie der Getriebelehre - daß die Federn, ersetzt durch eine entsprechende Anordnung von starren Stäben, ein übergeschlossenes Getriebe bilden. Für eine einzelne Feder mit kugelgelenkartiger Aufhängung der Federenden trifft das beispielsweise nicht zu. Ein weniger simples übergeschlossenes Getriebe besteht aus einer kreisförmigen Ansammlung von gleich langen Federn, welche die Erzeugenden eines einschaligen Hyperboloiden bilden. Solche Fälle sind als entartet zu betrachten und, falls nötig, gesondert zu diskutieren. Verglichen mit der obigen Vorstellung von Federn im Raum heißt das, daß die elastische Schicht unter äußerer Last einer Anordnung von Federn mit Vorspannung entsprechen sollte und daß schon von daher die Federmatrizen im allgemeinen vollen Rang haben.

In Ruhe mögen raumfestes und körperfestes System übereinstimmen. Unter Last verschiebe sich die Aufhängung bzw. die starre Berandung auf die im raumfesten System $A$ gemessene Position $\left(\boldsymbol{d}_{A}, \boldsymbol{\theta}_{A}\right)$. Die Verschiebung eines Punktes $B$ des starren Körpers entspricht der Längung der Feder, also der Auslenkung $\boldsymbol{x}$ des vorigen Abschnitts. Diese berechnet sich im raumfesten System $A$ mit den Ergebnissen aus dem ersten Kapitel als Translationsanteil von $q_{B} \mathrm{zu}$

$$
\boldsymbol{x}(B) \equiv \boldsymbol{d}_{B}=\boldsymbol{d}_{A}+\boldsymbol{\Omega}_{B A} \boldsymbol{\theta}_{A}
$$

Integriert wird über alle Punkte $B$ der Berandung, also bezogen auf den ruhenden Körper über die Ortsvektoren $\overrightarrow{A B}=: \boldsymbol{\xi}$. Damit ergibt sich der Zusammenhang mit den verallgemeinerten Koordinaten

$$
\begin{aligned}
\boldsymbol{x}(\boldsymbol{\xi}) & =\boldsymbol{d}_{A}-\boldsymbol{\Omega}_{\xi} \boldsymbol{\theta}_{A}=\underbrace{\left(\boldsymbol{I} \vdots-\boldsymbol{\Omega}_{\xi}\right)}_{=: \boldsymbol{X}} q_{A} \\
& =\boldsymbol{X}(\boldsymbol{\xi}) q_{A} .
\end{aligned}
$$

Entsprechend gibt die Gleichgewichtslage $q_{0}$ die Ausgangslagen $\boldsymbol{l}(\boldsymbol{\xi})$ der Federn vor durch $\boldsymbol{l}(\boldsymbol{\xi})=\boldsymbol{X}(\boldsymbol{\xi}) q_{0}$. Damit lautet das Potential insgesamt

$$
\begin{aligned}
\Phi & =\frac{1}{2} \oiint(\boldsymbol{x}(\boldsymbol{\xi})-\boldsymbol{l}(\boldsymbol{\xi}))^{T} \boldsymbol{K}(\boldsymbol{\xi})(\boldsymbol{x}(\boldsymbol{\xi})-\boldsymbol{l}(\boldsymbol{\xi})) d \boldsymbol{\xi} \\
& =\frac{1}{2} \mathscr{\Psi f}\left(q-q_{0}\right)^{T} \boldsymbol{X}(\boldsymbol{\xi})^{T} \boldsymbol{K}(\boldsymbol{\xi}) \boldsymbol{X}(\boldsymbol{\xi})\left(q-q_{0}\right) d \boldsymbol{\xi} \\
& =\frac{1}{2}\left(q-q_{0}\right)^{T} \underbrace{\left(\boldsymbol{y} \boldsymbol{X}(\boldsymbol{\xi})^{T} \boldsymbol{K}(\boldsymbol{\xi}) \boldsymbol{X}(\boldsymbol{\xi}) d \boldsymbol{\xi}\right)}_{\mathcal{S}}\left(q-q_{0}\right),
\end{aligned}
$$


und die Steifheitsmatrix $\mathcal{S}$ läßt sich somit aus der Federmatrix durch Integration über die Aufhängung gewinnen. Schließlich kann die innere Struktur der Steifheitsmatrix des Federmodells bestimmt werden:

$$
\begin{aligned}
& \mathcal{S}=\oiiint \boldsymbol{X}^{T} \boldsymbol{K} \boldsymbol{X} \\
& =\left\{f\left(\begin{array}{c}
\boldsymbol{I} \\
\boldsymbol{\Omega}_{\xi}
\end{array}\right) \boldsymbol{K}(\boldsymbol{\xi})\left(\begin{array}{lll}
\boldsymbol{I} & \vdots & -\boldsymbol{\Omega}_{\xi}
\end{array}\right) d \boldsymbol{\xi}\right. \\
& =\left(\begin{array}{cc}
\mathbb{Z} \boldsymbol{K}(\boldsymbol{\xi}) d \boldsymbol{\xi} & -\mathbb{Z C} \boldsymbol{K}(\boldsymbol{\xi}) \boldsymbol{\Omega}_{\xi} d \boldsymbol{\xi} \\
\mathbb{J} \boldsymbol{\Omega}_{\xi} \boldsymbol{K}(\boldsymbol{\xi}) d \boldsymbol{\xi} & -\mathbb{\int} \boldsymbol{\Omega}_{\xi} \boldsymbol{K}(\boldsymbol{\xi}) \boldsymbol{\Omega}_{\xi} d \boldsymbol{\xi}
\end{array}\right) .
\end{aligned}
$$

Eine besondere Eigenschaft der Steifheitsmatrix des Federmodells ist die Spurfreiheit der Nebendiagonalmatrizen, denn es gilt

$$
\operatorname{Sp}\left(\oiiint \int \boldsymbol{\Omega}_{\xi} \boldsymbol{K}(\boldsymbol{\xi}) d \boldsymbol{\xi}\right)=\oiiint \operatorname{Sp}\left(\boldsymbol{\Omega}_{\xi} \boldsymbol{K}(\boldsymbol{\xi})\right) d \boldsymbol{\xi}=\oiiint\left(\Omega_{i j} K_{i j}\right) d \boldsymbol{\xi}=0
$$

da die Spur eines Produktes aus einer antisymmetrischen und einer symmetrischen Matrix immer gleich Null ist.

\subsection{Schichtgrößen, Materialkonstanten und Ab- messungen}

Im letzten Abschnitt konnte die Federmatrix $\boldsymbol{K}$, je nach Material verschieden, zerlegt werden in eine Summe aus Schichtgrößen $k_{i}$ und symmetrischen Matrizen (genauer Projektoren und Dyaden) $\boldsymbol{\Psi}_{i}$

$$
\boldsymbol{K}=\sum k_{i} \Psi_{i}
$$

In diesem Abschnitt soll die Abhängigkeit der Schichtgrößen von den elastischen Konstanten des Materials und der Geometrie der Schicht untersucht werden. Dabei geht es eher darum, passende Modelle für einfache aber wichtige Spezialfälle zu finden als eine allgemeine und damit zur Elastizitätstheorie konkurrierende Methode zu entwickeln. Letzteres ist wegen der Komplexität der beteiligten Gleichungen ohnehin ein aussichtsloses Unterfangen - sonst hätten Finite-Elemente- oder vergleichbare ausgereifte numerische Methoden der Elastizitätstheorie, wie etwa in [33] beschrieben, wohl kaum ihre derzeitige Bedeutung.

In diesem Abschnitt wird ausgiebig von Gedankenexeperimenten Gebrauch gemacht. Solche Überlegungen sind typisch für die sog. Dimensionsanalyse, vgl. auch im Folgenden [51]. 


\subsubsection{Dimensionsbetrachtungen}

Gegeben sind die beiden elastischen Konstanten $E$ und $G$ des Materials, die Schichtdicke $d$ und die Fläche $F$ der Berandung. Gesucht ist die Schichtgröße $k$ mit

- der richtigen Dimension Energiedichte $\times$ Länge $=\mathrm{M} \mathrm{T}^{-2}$

- einer Abhängigkeit nur noch von dimensionslosen Größen wie etwa der Querdehnzahl $\sigma=\frac{E}{2 G}-1$ oder $\frac{F}{d^{2}}$.

Wegen $[E]=[G]=\mathrm{M} \mathrm{T}^{-2} \mathrm{~L}^{-1}=$ Energiedichte muß gelten $k \sim E$, was, da die Proportionalitätskonstante von $\sigma$ abhängen kann, gleichbedeutend mit $k \sim$ $G$ ist. Physikalisch bedeutet das, daß bei Verwendung eines anderen Materials, sagen wir mit höherer Energiedichte, bei Beibehaltung der übrigen Parameter eine proportional vergrößerte Federkonstante bzw. Federmatrix vorliegt. Es bleibt also noch eine charakteristische Länge zu bestimmen.

Eine Vergrößerung der Dicke von $d$ auf $2 d$ entspricht der Hintereinanderschaltung zweier Federn gleicher Federkonstanten. Die neue Federkonstante $k^{\prime}$ ergibt sich als harmonisches Mittel der beiden alten, also

$$
k^{\prime}=k(2 d)=\left(\frac{1}{k}+\frac{1}{k}\right)^{-1}=\frac{k(d)}{2} .
$$

Ebenso folgert man für ganzzahlige $m$, daß $k(m d)=k / m d$ und weiter mit der Substitution $d \mapsto d / n$ die umgekehrte Proportionalität für rationale Proportionalitätsfaktoren. Mit der Stetigkeit der Funktion $k(d)$ ist dann endgültig für die Federkonstante die Proportionalität

$$
k \sim \frac{1}{d}
$$

für reelle Zahlen bewiesen. Dieselbe Aussage überträgt sich auf die Federmatrix.

Eine Verdoppelung der Fläche $F$ entspricht einer Parallelschaltung von Federn, also

$$
k^{\prime}=k(2 F)=(k+k)=2 k(F),
$$

und mit demselben Gedankengang von eben ergibt sich

$$
k \sim F \quad .
$$

Die Größe $E \cdot F / d$ hat nun genau die richtige Dimension und ist bei allen Gedankenexperimenten proportional zu $k$. Für die Schichtgröße $k$ gilt also

$$
k=\frac{F}{d} \cdot \underbrace{E \cdot \varphi(\sigma, \boldsymbol{e} \cdot \boldsymbol{n})}_{=: K},
$$


und eine mögliche allgemeine Form der Federmatrix lautet insgesamt

$$
\boldsymbol{K}=\sum \frac{F}{d} \cdot K \cdot \Psi
$$

Der Ansatz für die symmetrische dimensionslose Matrix $\boldsymbol{\Psi}$ hat nach den Ergebnissen des letzten Abschnitts entsprechend der Symmetrie innerhalb der Schicht zu erfolgen. $\varphi$ und damit $K$ sind beliebige stetige Funktionen ihrer Variablen und den aus den geometrischen Größen bildbaren skalaren Invarianten. Nach den obigen Überlegungen dürfte keine Abhängigkeit von $F / d^{2}$ vorliegen, also bei etwas vorsichtigerer Deutung der Gedankenexperimente kaum ausgeprägt sein. Wegen $G=\frac{E}{2(1+\sigma)}$ ist auch derselbe Ansatz mit $G$ statt $E$ formal korrekt, nur die Funktion $\varphi$ ändert sich. Dasselbe gilt natürlich auch für den Koeffizienten der einseitigen Kompression.

Die Proportionalität von $k$ zu $F$ und damit die von $\boldsymbol{K}$ zu $F$ hat noch einen weiteren Vorzug: Beim Übergang zu einer Integration über eine Oberfläche bekommt man die gesamte Federmatrix und damit die potentielle Energie rechnerisch leicht über $d \boldsymbol{K} \sim d F$.

\subsection{2 Ähnlichkeitsbetrachtungen}

In den folgenden zwei Abschnitten sollen genauere Aussagen getroffen werden für die Fälle, in denen die Konstanz der Schichtdicke oder die Gleichheit von Grundund Deckfläche nicht mehr gegeben ist. Nach dem Zwischenwertsatz sollte jeder Mittelwert den maximalen Fehler reduzieren, den man durch Auswahl des ungünstigeren der Werte bekäme. Grundidee ist hierbei, durch ein Gedankenexperiment einen geeigneten Mittelwert für die beteiligten Größen zu finden. Da für jede vorgegebene Genauigkeit eine beliebige Fläche immer durch Dreiecke approximierbar ist, genügt es zur Beschreibung der dünnen elastischen Schicht, Aussagen über Prismen oder Pyramidenstümpfe mit dreieckiger Grundseite zu treffen.

\subsubsection{Veränderliche Schichtdicke}

Ein dreieckiges Prisma mit Grundfläche $F$ und der Höhe $h$ werde durchgeschnitten. Es entstehen zwei hintereinander geschaltete dreiseitige Prismen mit den Kantenlängen $\left(d_{1}, d_{2}, d_{3}\right)$ und $\left(h-d_{1}, h-d_{2}, h-d_{3}\right)$. Da sich die Kehrwerte der einzelnen Federkonstanten zum Kehrwert der gesamten Federkonstante addieren, muß für den Mittelwert $d\left(d_{1}, d_{2}, d_{3}\right)$ gelten

$$
\frac{h}{F}=\frac{d\left(d_{1}, d_{2}, d_{3}\right)}{F}+\frac{d\left(h-d_{1}, h-d_{2}, h-d_{3}\right)}{F} .
$$

Dies tut der arithmetische Mittelwert $d_{A}$ der Dicken, also

$$
d\left(d_{1}, d_{2}, d_{3}\right)=d_{A}\left(d_{1}, d_{2}, d_{3}\right):=\frac{1}{3}\left(d_{1}+d_{2}+d_{3}\right),
$$


der auch gleichzeitig einfachste Lösung der obigen Funktionalgleichung ist.

\subsubsection{Grund- und Deckfläche}

Die Situation für verschieden große Grund- und Deckflächen ist leider nicht so einfach. Für ähnliche Flächen, also insbesondere für einen Pyramidenstumpf, sollte nach dem Zwischenwertsatz ein $\lambda \in[0,1]$ existieren, so daß sich die Federkonstante des Gesamtkörpers bezüglich der gemittelten Fläche ergibt aus der Hintereinanderschaltung zweier Körper mit Dicke $\lambda d$ und Fläche $F_{1}$ sowie Dicke $(1-\lambda) d$ und Fläche $F_{2}$. Nach Kürzen der Dicke ergibt sich

$$
F\left(F_{1}, F_{2}\right)=\left(\frac{\lambda}{F_{1}}+\frac{(1-\lambda)}{F_{2}}\right)^{-1},
$$

also ein gewichtetes harmonisches Mittel. Damit beide Aufhängungen denselben Energiegehalt besitzen, sollten sie gleiches Volumen haben. Für einen Pyramidenstumpf (und daher allgemein für Flächen mit ähnlicher Grund- und Deckfläche wie Kegelstumpf und Kugelschale) ist das Volumen $V$ außer von der Schichtdicke nur eine Funktion von Grund- und Deckfläche:

$$
V=\frac{1}{3}\left(F_{1}+F_{2}+\sqrt{F_{1} F_{2}}\right) d,
$$

und das muß gleich dem Volumen der Vergleichsanordnung $\lambda F_{1} d+(1-\lambda) F_{2} d$ sein. Daraus erhält man $\lambda$ und durch Einsetzen in (4.8) schließlich

$$
F\left(F_{1}, F_{2}\right)=\frac{3 F_{1} F_{2}}{2\left(F_{1}+F_{2}\right)-\sqrt{F_{1} F_{2}}}
$$

oder ausgedrückt mit dem dimensionslosen Flächenverhältnis $x:=\frac{F_{2}}{F_{1}}$

$$
F\left(F_{1}, F_{2}\right)=F_{1} \frac{3 x}{2(1+x)-\sqrt{x}}
$$

Diese Funktion ist positiv, monoton und nach oben hin durch 1.5 beschränkt; der Mittelwert begünstigt also die kleinere Fläche. Das ist auch anschaulich zu erwarten, da die kleinere Fläche einer schwächeren Feder entspricht, welche beim gewichteten harmonischen Mittel stärker ins Gewicht fällt. Für gleiche Flächen, also für $x=1$, ergibt sich erwartungsgemäß diese Fläche zurück. Da dann $\lambda=1 / 2$ gilt, entspricht das für annähernd gleiche Flächen dem gewöhnlichen harmonischen Mittel $\frac{2 x}{1+x}$, welches bis $x=2$ die Funktion (4.9) mit einem absoluten Fehler $\leq 0.025$ approximiert, vgl. Abb. 4.2 .

Für kleinere Werte von $x$ wird diese Approximation sogar noch deutlich besser, so daß

$$
F\left(F_{1}, F_{2}\right)=F_{H}\left(F_{1}, F_{2}\right):=\left(\frac{F_{1}+F_{2}}{2 F_{1} F_{2}}\right)^{-1}=F_{1} \frac{2 x}{1+x}
$$




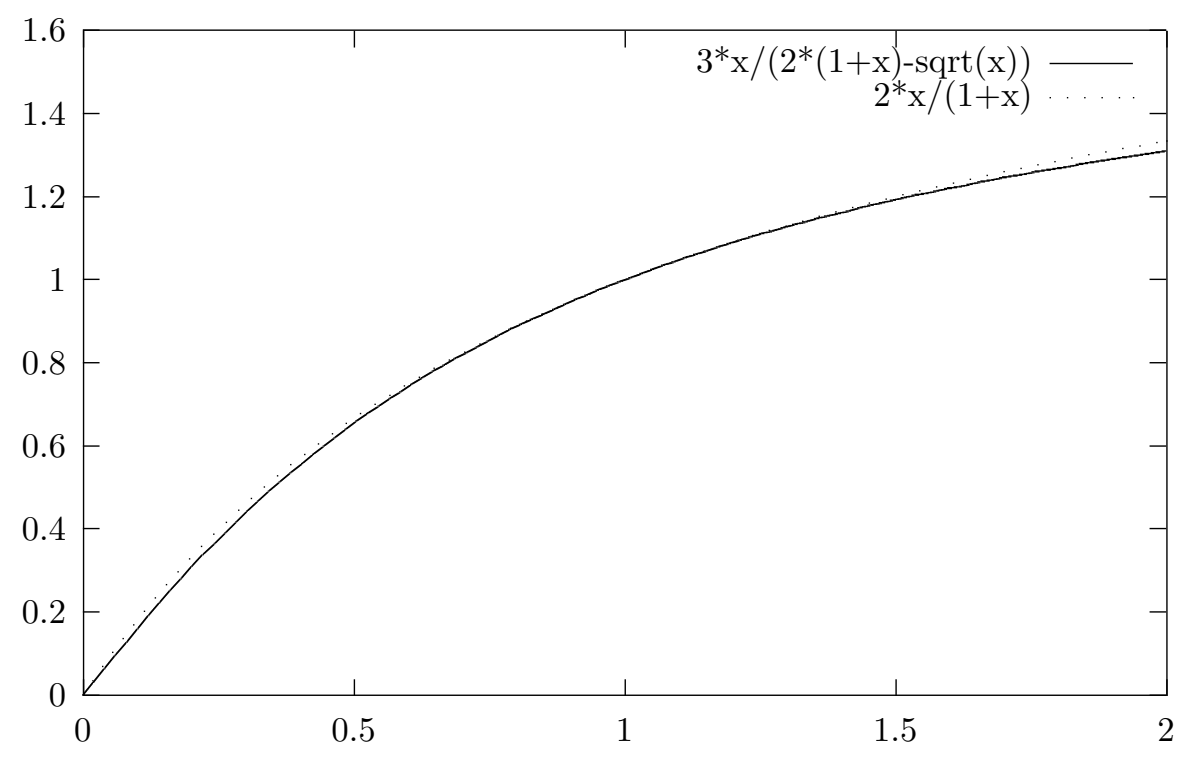

Abbildung 4.2: Die Mittelung der Flächen

ein für alle relevanten Fälle hinreichend genaues, forminvariantes und genügend einfaches Mittel der Flächen darstellt.

Die Grenzen dieser Näherungen sind schnell erreicht. Schon bei einfachen Körpern mit nicht ähnlicher Grund- und Deckfläche hängt das Gesamtvolumen nicht mehr allein von diesen Flächen und der Schichtdicke ab. Ein Obelisk beispielsweise, das ist ein Polyeder mit trapezförmigen Seitenflächen, bei dem Grund- und Deckfläche nicht ähnliche Rechtecke mit parallelen Normalen sind, ist das einfachste Beispiel für einen solchen Körper. Bezeichnen $a$ und $b$ die jeweiligen Kantenlängen von Grund- und Deckfläche, dann gilt für das Volumen

$$
V=\frac{1}{3}\left(F_{1}+F_{2}+\frac{1}{2}\left(a_{1} b_{2}+a_{2} b_{1}\right)\right) d
$$

und schon zur Beschreibung der elastischen Gesamtenergie sind weitere dimensionslose Größen notwendig. Nichtsdestoweniger liegt das harmonische Mittel der Flächen wahrscheinlich nahe genug an dem tatsächlichen Wert, aber eine Überprüfung der Genauigkeit dieser Näherung kann dann nur noch durch allgemeinere Theorien erfolgen.

Noch komplizierter wird die Situation für nicht parallele und ungleich große Grundund Deckflächen. Noch im vorigen Beispiel konnte man von einer gegebenen Triangulierung auf einer der Flächen über den gemeinsamen Normalenvektor eine passende Triangulierung der Gegenfläche finden. Das ist nun nicht mehr möglich, so daß man endgültig auf geschicktes Raten oder Numerik angewiesen ist.

Für alle Fälle, in denen die obigen Näherungen die Situation hinreichend genau 
beschreiben, möchte ich also

$$
\boldsymbol{K}=\sum \frac{F_{H}}{d_{A}} \cdot K \cdot \Psi
$$

als Ansatz für die Federmatrix verwenden, welcher genauer als der für Grund- oder Deckfläche allein ist.

\subsection{Stab- und Federmodell in der Kontinuums- mechanik}

Im Allgemeinen ist die Energie, hier die potentielle Energie, eine quadratische Form in ihren Variablen. Im Federmodell wurde aber nur der translatorische Anteil genutzt, der rotatorische wirkte nur mittelbar über den Abstand. In den folgenden Abschnitten soll diese Näherung genauer untersucht und dazu probehalber fallen gelassen werden. In Abschnitt 4.4.3 wird sie sich aber dennoch als zutreffend erweisen.

\subsubsection{Die Flexibilitätsmatrix eines Stabes}

Für die Anwendung wichtig und daher bereits untersucht sind der elastische Stab bzw. die beliebig gekrümmte Biegelinie. Beispiele für solche Anwendungen sind technische Schraubenfedern und Federn an kieferorthopädischen Geräten. Für einen dünnen einseitig eingespannten Stab kann trotz lokal kleiner Deformationen die Änderung der Position seines belasteten Endes erheblich sein. In diesem Fall treten zur elastischen Energie noch Anteile der Biegung und der Torsion hinzu; in bestimmten Fällen dominieren sie sogar das Geschehen ([40], §16 ff). Da nun jeder verallgemeinerten Kraft vom Stab ein Widerstand entgegengesetzt wird, tragen alle mit zur elastischen Energie bei. Statt der Federmatrix $\boldsymbol{K}$ ist daher nun eine $6 \times 6$-Matrix $\mathcal{K}$ zur Beschreibung des lokalen Beitrages zur elastischen Energie notwendig. Ebenso hat man statt der Matrix $\boldsymbol{X}$, welche die lokalen Verschiebungen mit den verallgemeinerten Koordinaten der starren Berandung $q_{A}$ verknüpft, die gesamte Transformationsmatrix $\mathcal{T}^{T}$ zu benutzen, da diese die vollständigen Positionen zu verschiedenen Aufpunkten ineinander transformiert.

Da man einen Stab als Hintereinanderschaltung von Elementarstäben verstehen kann, bekommt man seine Flexibilitätsmatrix zu einem festen Koordinatensystem durch folgendes Verfahren:

1. Translation auf die neutrale Faser des Stabes, das ist der Mittelpunkt der Querkräfte, 
2. Drehung auf den Normalschnitt des Stabes, so daß seine Hauptträgheitsachsen mit den neuen Koordinatenachsen zusammenfallen,

3. Ansatz einer Flexibilitätsmatrix $\mathcal{E}=\mathcal{K}^{-1}$ für die Elementarzellen bzw. Linienelemente, welche die infinitesimalen Lasten mit den (hier additiven) infinitesimalen Auslenkungen verknüpft,

4. Verfeinerungen mittels differentialgeometrischer Invarianten wie etwa Krümmungs- und Windungskorrekturen,

5. Berücksichtigung der Effekte der endlichen Stabdicke,

6. Rückdrehung und Rücktranslation auf das Ausgangskoordinatensystem, und schließlich

7. Integration aller Beiträge über den Stab bzw. die Bogenlänge der neutralen Faser.

Die Transformationen zwischen Ausgangs- und lokalem Koordinatensystem für die verallgemeinerten Kräfte bzw. Koordinaten können beschrieben werden durch die Matrizen

$$
\underbrace{\mathcal{U}_{A B}^{f}}_{=: \mathcal{U}_{A B}}=\mathcal{R} \mathcal{T}_{A B}=\left(\begin{array}{cc}
\boldsymbol{R} & \mathbf{0} \\
\mathbf{0} & \boldsymbol{R}
\end{array}\right)\left(\begin{array}{cc}
\boldsymbol{I} & \mathbf{0} \\
\boldsymbol{\Omega}_{A B} & \boldsymbol{I}
\end{array}\right)=\left(\begin{array}{cc}
\boldsymbol{R} & \mathbf{0} \\
\boldsymbol{R} \boldsymbol{\Omega}_{A B} & \boldsymbol{R}
\end{array}\right)
$$

für die Lasten und

$$
\mathcal{U}_{B A}^{q}=\mathcal{T}_{A B}^{T} \mathcal{R}^{T}=\left(\mathcal{U}_{A B}\right)^{T}
$$

für die Positionen. Die Matrix $\boldsymbol{R}$ kann für Biegelinien und Stäbe mit zwei gleichen Flächenträgheitsmomenten ausgedrückt werden durch das begleitende Dreibein der neutralen Faser mittels

$$
\boldsymbol{R}=(\boldsymbol{t}, \boldsymbol{n}, \boldsymbol{b})
$$

wobei $\boldsymbol{t}, \boldsymbol{n}$ und $\boldsymbol{b}$ wie üblich Tangente, Normale und Binormale sind.

Bei Stäben mit asymmetrischen Querschnitten fallen die Bezugspunkte der Achsen der Hauptträgheitsmomente und die neutrale Faser nicht mehr zusammen, und man bekommt ([6], Kap. II, §2 Ziff. 8) für eine schwach gekrümmte Linie (die $\kappa$ hier sind Materialkonstanten wie die $k$, und nicht mit der Krümmung zu verwechseln)

$$
\mathcal{E}=\left(\begin{array}{cccccc}
\frac{1}{E F} & 0 & 0 & 0 & 0 & 0 \\
0 & \frac{\kappa}{G F} & \frac{\kappa^{*}}{G F} & 0 & 0 & 0 \\
0 & \frac{\kappa^{*}}{G F} & \frac{\kappa^{\prime}}{G F} & 0 & 0 & 0 \\
0 & 0 & 0 & \frac{\kappa^{\prime \prime}}{G J_{0}} & 0 & 0 \\
0 & 0 & 0 & 0 & \frac{1}{E J_{y}} & 0 \\
0 & 0 & 0 & 0 & 0 & \frac{1}{E J_{z}}
\end{array}\right)
$$


wobei $J_{y, z}$ die Trägheitsmomente bezüglich der y- bzw. z-Achse, $J_{0}$ das polare Trägheitsmoment des Querschnittes bezüglich dessen Schwerpunkt, und die $\kappa$ für schwach gekrümmte Linien nur von der Querschnittsform und sonst auch von den differentialgeometrischen Korrekturen abhängige Beiwerte sind. Hat der Querschnitt eine Symmetrieachse, so fallen Querkraftmittelpunkt und Querschnittsschwerpunkt zusammen, und es gilt $\kappa^{*}=0$.

Ein Beispiel einer solchen elementaren Flexibilitätsmatrix, hier einer Schraubenfeder mit kreisförmigem Querschnitt, also $J_{y}=J_{z}=: I$ ist ([41] S. 43 ff.)

$$
\mathcal{E}=\left(\begin{array}{cc}
\operatorname{diag}\left(k_{i}\right) & \mathbf{0} \\
\mathbf{0} & \operatorname{diag}\left(g_{i}\right)
\end{array}\right)=\left(\begin{array}{cccccc}
\frac{1}{E F} & 0 & 0 & 0 & 0 & 0 \\
0 & \frac{1}{k G F} & 0 & 0 & 0 & 0 \\
0 & 0 & \frac{1}{k G F} & 0 & 0 & 0 \\
0 & 0 & 0 & \frac{1}{G K} & 0 & 0 \\
0 & 0 & 0 & 0 & \frac{1}{E I} & 0 \\
0 & 0 & 0 & 0 & 0 & \frac{1}{E J}+\frac{1}{E \varrho^{2} F}
\end{array}\right)
$$

mit den dimensionsbehafteten Größen $E$ und $G$ sowie der Querschnittsfläche $F$,

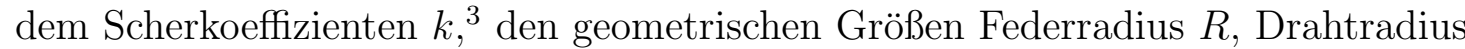
$r$ und Pitchwinkel $p$ mit $\tan (p)=h / R$ und den abgeleiteten geometrischen Größen

$$
\begin{aligned}
\varrho & =R\left(1+\tan ^{2} p\right)=R\left(1+\left(\frac{h}{R}\right)^{2}\right) \\
F & =\pi R^{2} \\
I & =2 \pi R^{4}=J_{y}=J_{z} \\
J & =2 \pi R^{4}\left(1-\frac{1}{2}\left(\frac{r}{R}\right)^{2}-\sqrt{1-\left(\frac{r}{R}\right)^{2}}\right)=I\left(\frac{1}{8}\left(\frac{r}{R}\right)^{2}+\mathcal{O}\left(\frac{r}{R}\right)^{4}\right) \\
K & =\frac{\pi r^{4}}{2}\left(1+\frac{3\left(\frac{r}{\varrho}\right)^{2}}{16\left(1-\left(\frac{r}{\varrho}\right)^{2}\right)}\right) .
\end{aligned}
$$

Hierbei sind die Terme zu $k_{z}$ und $g_{y}$ laut [41] S. 44

"... as no established theory for their contribution to the elastic energy seems to exist..."

ausdrücklich als Ansätze zu verstehen.

\footnotetext{
${ }^{3}$ Für kreisförmige Querschnitte gilt, lt. [41] S. 78, $k \approx 9 / 10$, vgl. auch [6] Kap. V, $\S 1$, Ziff. 3 ...11. Solche Bei- oder Korrekturwerte der Technischen Mechanik werden uneinheitlich gehandhabt; sie sind eine Folge des meist eindimensional formulierten Problems des Biegebalkens, vgl. [54.
} 
Um beide Matrizen vergleichen zu können, müßten noch die Krümmungs- und Windungskorrekturen (und damit auch die der Vorlast) sowie die Effekte von Abweichungen von Biegelinienmodell in die allgemeine Form (4.12) eingearbeitet werden. Diese dürfen nur von der lokalen Geometrie und dem lokalen Drahtquerschnitt abhängen, können also nach dem Fundamentalsatz der Differentialgeometrie der Raumkurven nur Funktionen der Invarianten Krümmung $\kappa$ und Torsion $\tau$ sein. Für eine Spiralfeder, also den Fall einer Schraubenlinie

$$
\boldsymbol{x}=\left(\begin{array}{c}
R \cos t \\
R \sin t \\
h t
\end{array}\right)
$$

sind das

$$
\kappa=\frac{R}{R^{2}+h^{2}} \quad \text { und } \quad \tau=\frac{h}{R^{2}+h^{2}}
$$

und das zusätzliche dimensionslose Argument $r / R \stackrel{4}{\text { प Wegen }}$

$$
R=\frac{\kappa}{\kappa^{2}+\tau^{2}} \quad \text { und } \quad h=\frac{\tau}{\kappa^{2}+\tau^{2}}
$$

können sämtliche von $R$ und $h$ abhängenden Größen durch $\kappa$ und $\tau$ ausgedrückt werden. Insbesondere ist $\varrho=1 / \kappa$, was den Namen Krümmungskorrektur rechtfertigt.

Insgesamt ergibt sich schließlich die gesamte Flexibilitätsmatrix zum Koordinatensystem $B$

$$
\begin{aligned}
\mathcal{F}_{\mathcal{B B}} & =\int \mathcal{U}_{B A}^{q}(s) \mathcal{E}_{A A}(s) \mathcal{U}_{A B}^{f}(s) d s \\
& =\int \mathcal{U}_{\mathcal{A B}}{ }^{T}(s) \mathcal{E}_{A A}(s) \mathcal{U}_{A B}(s) d s,
\end{aligned}
$$

wobei $s$ die Bogenlänge der neutralen Faser im Punkt $A(s)$ ist.

Beide Matrizen haben Blockdiagonal- oder sogar Diagonalform. Anschaulich ist das klar, da man ein Widerstandszentrum immer im Inneren der Aufhängung erwartet; für eine beliebig kleine Aufhängung existiert demnach immer ein Widerstandszentrum. Die Achsen konstanter Helizität aus Abschnitt 2.4 waren als Eigenwertproblem der Matrix $\boldsymbol{A C}=\boldsymbol{D}^{-1} \boldsymbol{M}^{-1}=(\boldsymbol{M D})^{-1}$ definiert. Für eine elementare Flexibilitätsmatrix sollten sie existieren und nur von der Geometrie der Anordnung abhängen. Daher darf auch $\boldsymbol{K} \boldsymbol{G}$ von den dimensionsbehafteten elastischen Konstanten nur als Gesamtfaktor abhängen. In der Tat erhält man für die allgemeine Form nach [6]

$$
\boldsymbol{K} \boldsymbol{G}=\frac{1}{E G F}\left(\begin{array}{ccc}
\kappa^{\prime \prime} / J_{0} & 0 & 0 \\
0 & \kappa / J_{y} & \kappa^{*} / J_{z} \\
0 & \kappa^{*} / J_{y} & \kappa^{\prime} / J_{z}
\end{array}\right)
$$

\footnotetext{
${ }^{4}$ Der Quotient $h / R$ ist wegen $h / R=\tau / \kappa$ kein zusätzliches Argument.
} 
und für die Schraubenfeder nach 41

$$
\boldsymbol{K} \boldsymbol{G}=\frac{1}{E G F}\left(\begin{array}{ccc}
1 / K & 0 & 0 \\
0 & 1 / k I & 0 \\
0 & 0 & 1 / k J+1 / k \varrho^{2} F
\end{array}\right)
$$

Eine konsequent differentialgeometrisch aufgebaute Theorie einer Schraubenfeder bzw. eines Biegedrahtes mit entsprechender Verwendung der Tensorrechnung konnte ich in der Literatur nicht finden, obwohl mir ein solches Unterfangen lohnend erscheint. Seinen Grund mag das darin haben, daß es neben dem begleitenden Dreibein immer noch ein weiteres Koordinatensystem gibt, nämlich das der Hauptachsen der Flächenträgheitsmomente. Eine Folge von immer genauer werdenden Näherungslösungen findet sich wieder in der sog. strengen Federtheorie [6] Kap. V, $\S 1$, Ziff. 3 bis 11, welche zu großen Teilen wohl auf HARINGx [25] zurückgeht.

\subsubsection{Die allgemeine Form der elastischen Energie}

Die potentielle Energie eines physikalischen Systems läßt sich immer als quadratische Form ihrer Koordinaten schreiben, also mit dem lokalen Beitrag

$$
\Phi=\frac{1}{2} q^{T} \mathcal{K} q
$$

mit der diesmal vollbesetzten Matrix

$$
\mathcal{K}=\left(\begin{array}{cc}
\boldsymbol{K} & \boldsymbol{N}^{T} \\
\boldsymbol{N} & \boldsymbol{G}
\end{array}\right)
$$

bei der alle Komponenten der verallgemeinerten Koordinaten miteinander koppeln. So ist es beispielsweise anschaulich klar, daß bei einem Biegebalken in $x$-Richtung die zu $F_{y}$ und $\theta_{z}, F_{z}$ und $\theta_{y}, T_{y}$ und $d_{z}$ sowie $T_{z}$ und $\theta_{y}$ gehörenden Komponenten miteinander gekoppelt sind. Das ist aber teilweise eine Folge des gewählten Koordinatensystems, welches hier am freien Ende des Balkens und damit sicher nicht in seinem elastischen Zentrum liegt.

Ein hinreichend klein gewähltes Teilelement eines homogenen Materials besitzt keine innere Struktur mehr und damit ein Widerstandszentrum. Damit verschwinden die Nebendiagonalmatrizen, und es gilt nur noch

$$
\mathcal{K}=\left(\begin{array}{cc}
K & 0 \\
0 & G
\end{array}\right)
$$

Das entkoppelt axiale und polare Vektoren wieder. Durch Transformation auf ein passendes Koordinatensystem, beim Stab etwa die Hauptträgheitsachsen, kann eine der beiden Matrizen diagonalisiert werden, beim Stab ist das $\boldsymbol{G}=\operatorname{diag}\left(g_{i}\right)$. 
Die Matrix $\boldsymbol{G}$ ist dann zur Beschreibung eines Systems nötig, wenn, wie beim Torsionsstab, auch bei kleinen lokalen Deformationen insgesamt große Drehungen zu erwarten sind.

Eine aus solchen Elementen parallel zusammengesetzte Aufhängung hat dann die Steifheitsmatrix

$$
\begin{aligned}
& \mathcal{S}=\oiiint \mathcal{T} \mathcal{K} \mathcal{T}^{T} \\
& =\left\{f\left(\begin{array}{cc}
\boldsymbol{I} & \mathbf{0} \\
\Omega_{\xi} & \boldsymbol{I}
\end{array}\right)\left(\begin{array}{cc}
\boldsymbol{K}(\boldsymbol{\xi}) & \mathbf{0} \\
\mathbf{0} & \boldsymbol{G}(\boldsymbol{\xi})
\end{array}\right)\left(\begin{array}{cc}
\boldsymbol{I} & -\boldsymbol{\Omega}_{\xi} \\
\mathbf{0} & \boldsymbol{I}
\end{array}\right) d \boldsymbol{\xi}\right. \\
& =\left(\begin{array}{cc}
\mathbb{\int C} \boldsymbol{K}(\boldsymbol{\xi}) d \boldsymbol{\xi} & -\mathbb{I C} \boldsymbol{K}(\boldsymbol{\xi}) \boldsymbol{\Omega}_{\xi} d \boldsymbol{\xi} \\
\mathbb{\int} \boldsymbol{\Omega}_{\xi} \boldsymbol{K}(\boldsymbol{\xi}) d \boldsymbol{\xi} & \mathbb{Z} \boldsymbol{G}(\boldsymbol{\xi}) d \boldsymbol{\xi}-\mathbb{Z C} \boldsymbol{\Omega}_{\xi} \boldsymbol{K}(\boldsymbol{\xi}) \boldsymbol{\Omega}_{\xi} d \boldsymbol{\xi}
\end{array}\right)
\end{aligned}
$$

und erfüllt wegen $\operatorname{Sp}\left(\mathbb{X} \boldsymbol{K}(\boldsymbol{\xi}) \boldsymbol{\Omega}_{\xi} d \boldsymbol{\xi}=\right) \operatorname{\int C} \operatorname{Sp}\left(\boldsymbol{K}(\boldsymbol{\xi}) \boldsymbol{\Omega}_{\xi}\right) d \boldsymbol{\xi}=0$ wieder die Spurbedingung der Nebendiagonalmatrizen. Ein Vergleich dieses Ansatzes - ich möchte ihn das Stabmodell nennen - mit dem Federmodell ist also nur anhand eines konkreten Beispieles möglich.

Falls zwischen den einzelnen Koordinatensystemen zusätzlich auch Drehungen vorkommen erhält man

$$
\begin{aligned}
& \mathcal{S}=\oiiint \mathcal{U K U}^{T} \\
& =\mathscr{f}\left(\begin{array}{cc}
\boldsymbol{R} & \mathbf{0} \\
\boldsymbol{R} \Omega & \boldsymbol{R}
\end{array}\right)\left(\begin{array}{cc}
\boldsymbol{K} & \mathbf{0} \\
\mathbf{0} & \boldsymbol{G}
\end{array}\right)\left(\begin{array}{cc}
\boldsymbol{R}^{T} & -\boldsymbol{\Omega} \boldsymbol{R}^{T} \\
\mathbf{0} & \boldsymbol{R}^{T}
\end{array}\right) d \boldsymbol{\xi} \\
& =\left(\begin{array}{cc}
\mathbb{I} \boldsymbol{R} \boldsymbol{K} \boldsymbol{R}^{T} d \boldsymbol{\xi} & -\mathbb{X} \boldsymbol{R} \boldsymbol{K} \boldsymbol{\Omega} \boldsymbol{R}^{T} d \boldsymbol{\xi} \\
\mathbb{I} \boldsymbol{R} \boldsymbol{\Omega} \boldsymbol{K} \boldsymbol{R}^{T} d \boldsymbol{\xi} & \mathbb{I} \boldsymbol{R} \boldsymbol{G} \boldsymbol{R}^{T} d \boldsymbol{\xi}-\mathbb{I} \boldsymbol{R} \boldsymbol{\Omega} \boldsymbol{K} \boldsymbol{\Omega} \boldsymbol{R}^{T} d \boldsymbol{\xi}
\end{array}\right),
\end{aligned}
$$

was wegen der Zyklizität der Spur nichts an der Spurbedingung ändert.

Im allgemeinen Fall mit nichtverschwindenden Nebendiagonalen - hier ohne Drehungen - gälte

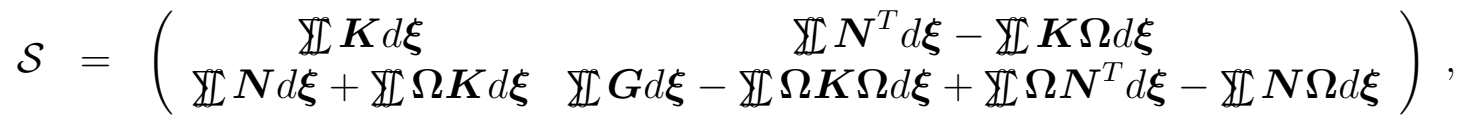

und die Spurbedingung wäre dahin. Damit ist es prinzipiell möglich, anhand der Meßwerte das Stabmodell und dadurch auch das Federmodell zu falsifizieren.

Da aber bei den interessierenden Aufhängungen weder große Drehungen noch ein starker Einfluß einer inneren Struktur des Mediums zu erwarten ist, wird das Federmodell in genau diesen Fällen die Messungen in guter Näherung beschreiben.

\subsubsection{Identifikation der Schichtgrößen}

In diesem Abschnitt soll eine Begründung des Federmodells mit den Methoden der Elastizitätstheorie gesucht werden, also eine mit den Mittelwertsätzen der Konti- 
nuumsmechanik ableitbare Beziehung der Form $f_{i}=\mathcal{S}_{i j} q_{j}$. Die dazu notwendigen Beziehungen und die üblichen Bezeichnungen der Elastizitätstheorie sind etwa in [40] nachzulesen.

Gegeben sei die pro Oberflächeneinheit des elastischen Mediums wirkende äußere Kraft $\boldsymbol{P}$. Die Gesamtkraft $\boldsymbol{F}$ der starren Berandung ist dann gegeben durch

$$
F_{i}=\oiint P_{i} d o=\oiint \sigma_{i j} n_{j} d o
$$

wobei $\boldsymbol{n}$ der Einheitsvektor der äußeren Normalen der Berandung ist und do die Metrik der Fläche enthält. Ebenso erhält man für das gesamte Drehmoment $\boldsymbol{T}$ mit dem Aufpunktvektor $\boldsymbol{\xi}$

$$
T_{i}=\oiint(\boldsymbol{\xi} \times \boldsymbol{P})_{i} d o=\oiint\left(\boldsymbol{\Omega}_{\xi}\right)_{i j} P_{j} d o=\oiint\left(\boldsymbol{\Omega}_{\xi}\right)_{i j} \sigma_{j k} n_{k} d o
$$

also insgesamt für die verallgemeinerten Kräfte

$$
f_{i}=\oiint \underbrace{\left(\begin{array}{c}
\boldsymbol{I} \\
\boldsymbol{\Omega}_{\xi}
\end{array}\right)_{i j}}_{=\left(\boldsymbol{X}^{T}\right)_{i j}} \sigma_{j k} n_{k} d o
$$

also kurz $f=\oiint \boldsymbol{X}^{T} \boldsymbol{\sigma} \boldsymbol{n} d o$.

Auf der Deckfläche lassen sich die Auslenkungen $u_{i}$ durch die verallgemeinerten Koordinaten ausdrücken: Bezüglich des gewählten Koordinatensystems gilt $u_{i}=$ $d_{i}+(\boldsymbol{\theta} \times \boldsymbol{\xi})_{i}=d_{i}-\left(\boldsymbol{\Omega}_{\xi}\right)_{i j} \theta_{j}$, also insgesamt

$$
u_{i}=\underbrace{\left(\delta_{i j}:-\left(\Omega_{\xi}\right)_{i j}\right)}_{=(\boldsymbol{X})_{i j}}\left(\begin{array}{c}
d_{j} \\
\theta_{j}
\end{array}\right),
$$

oder kurz $\left.\boldsymbol{u}\right|_{\partial V}=\boldsymbol{X} q$.

Das verallgemeinerte HooKEsche Gesetz lautet $\sigma_{i j}=\Lambda_{i j k l} \epsilon_{k l}$, und mit $\epsilon_{i j}=u_{i, j}$ folgt aus (4.14) und (4.15)

$$
\begin{aligned}
f_{i} & =\oiint X_{i j}^{T} \sigma_{j k} n_{k} d o=\oiint X_{i j}^{T} \Lambda_{j k l m} \epsilon_{l m} n_{k} d o \\
& =\oiint X_{i j}^{T} \Lambda_{j k l m} u_{l, m} n_{k} d o=\oiint X_{i j}^{T} \Lambda_{j k l m}\left(X_{l n} q_{n}\right)_{, m} n_{k} d o \\
& =\oiint X_{i j}^{T} \Lambda_{j k l m}\left(X_{l n, m} q_{n}+X_{l n} q_{n, m}\right) n_{k} d o
\end{aligned}
$$

Mit $X_{l n, m}=\left(\delta_{l n, m} \vdots-\left(\varepsilon_{l k n} \xi_{k}\right)_{, m}\right)=\left(0_{l m n} \vdots-\varepsilon_{l m n}\right)$ und der Symmetrie $\Lambda_{j k l m}=$ $\Lambda_{(j k)(l m)}$ verschwindet der erste Summand. Da die verallgemeinerten Koordinaten 
zu einem festen Koordinatensystem gehören, hängen sie nicht vom Aufpunkt ab und es gilt einfach $q_{n, m}=0$. Mit dem zweiten Summand verschwindet somit das gesamte Integral, sofern es über die ganze Berandung ausgeführt wird. Das bedeutet, daß sich die Beiträge von Grund- und Deckfläche sowie der freien Berandung gegenseitig aufheben, also daß der Körper sich im Gleichgewicht befindet. Um die Kraft auf nur einer Seite zu erhalten, muß das Oberflächenintegral in drei Teile zerlegt werden, und zwar in die Bereiche

1. Grundfläche $G$ oder Deckfläche $D$,

2. eine Fläche $F$ in der Mitte und

3. der freie Rand der dünnen elastischen Schicht.

Für genügend dünne Schichten kann der Beitrag des freien Randes vernachlässigt werden, und die Beiträge von Grundfläche $G$ und Mittenfläche $F$ sind entgegengesetzt gleich groß. Jeder für sich ist gleich der angelegten Last, d. h.

$$
\begin{aligned}
f_{i} & =\iint_{G}\left(\boldsymbol{X}^{T} \boldsymbol{P}\right)_{i} d o \\
& =-\iint_{F}\left(\boldsymbol{X}^{T} \boldsymbol{P}\right)_{i} d o \\
& =-\iint_{F} X_{i j}^{T} \Lambda_{j k l m} u_{l, m} n_{k} d o .
\end{aligned}
$$

Nun muß eine Aussage über die Verschiebungen $u_{l}$ auf der Fläche $F$ getroffen werden. Nach dem in Abschnitt 4.3.2.2 Gesagten ist klar, daß es im Inneren der elastischen Aufhängung eine Höhe gibt, in der die Verschiedenheit von Grund- und Deckfläche mit (4.11) lokal korrekt berücksichtigt wird. Diese so zusammengesetzte Fläche möchte ich, analog zur neutralen Faser in der Theorie des Biegebalkens, die neutrale Fläche nennen.

Seien $u$ und $v$ zwei Parameter, welche die zum bewegten System gehörende Oberfläche, also die Deckfläche, orthogonal parametrisieren. Die zugehörigen Tangentenvektoren $\boldsymbol{t}_{u}$ und $\boldsymbol{t}_{v}$ bilden dann zusammen mit der Normale $\boldsymbol{n}$ ein orthonormiertes System, d. h. $\boldsymbol{t}_{i} \cdot \boldsymbol{t}_{j}=\delta_{i j}$ und $\boldsymbol{t}_{i} \cdot \boldsymbol{n}=0$. Dieses ist das begleitende Dreibein der Fläche. Die Punkte $\boldsymbol{\xi}_{F}$ der neutralen Fläche können also immer beschrieben werden durch einen nahegelegenen Punkt $\boldsymbol{\xi}$ der Deckfläche

$$
\boldsymbol{\xi}_{F}(u, v, h)=\boldsymbol{\xi}\left(u_{0}, v_{0}, h_{0}=0\right)+\left(u-u_{0}\right) \boldsymbol{t}_{u}+\left(v-v_{0}\right) \boldsymbol{t}_{v}+h \boldsymbol{n} .
$$

In diesem Koordinatensystem ist der Gradient gegeben durch

$$
\boldsymbol{\nabla}=\boldsymbol{t}_{u} \frac{\partial}{\partial u}+\boldsymbol{t}_{v} \frac{\partial}{\partial v}+\boldsymbol{n} \frac{\partial}{\partial h}
$$

wobei der Gradient auf der Fläche allein gegeben ist durch

$$
\boldsymbol{\nabla}_{F}=\boldsymbol{t}_{u} \frac{\partial}{\partial u}+\boldsymbol{t}_{v} \frac{\partial}{\partial v}
$$


also insgesamt

$$
\boldsymbol{\nabla}=\boldsymbol{\nabla}_{F}+\boldsymbol{n} \frac{\partial}{\partial h}
$$

In nullter Näherung ist die Auslenkung auf der neutralen Fläche gegeben durch eine lineare Interpolation der Werte zwischen Grund- und Deckfläche, also mit der Schichtdicke $d(\boldsymbol{\xi})$ von der Deckfläche aus gesehen durch

$$
u_{l}^{0}=\left(\boldsymbol{X}_{q}\right)_{l}\left(1-\frac{h}{d}\right) .
$$

Dabei ist aber noch nicht die „Verkippung“ der Deckfläche durch die Dickenänderung der Schicht berücksichtigt. Mit dem in der Fläche liegenden Vektor

$$
\Delta \boldsymbol{\xi}:=\left(u-u_{0}\right) \boldsymbol{t}_{u}+\left(v-v_{0}\right) \boldsymbol{t}_{v}
$$

erhält man die relative Dickenänderung in dieser Richtung als mit dem Flächengradienten gebildete Richtungsableitung zu $\Delta \boldsymbol{\xi} \cdot \nabla_{F} d / d$, vgl. Abbildung 4.3 ,

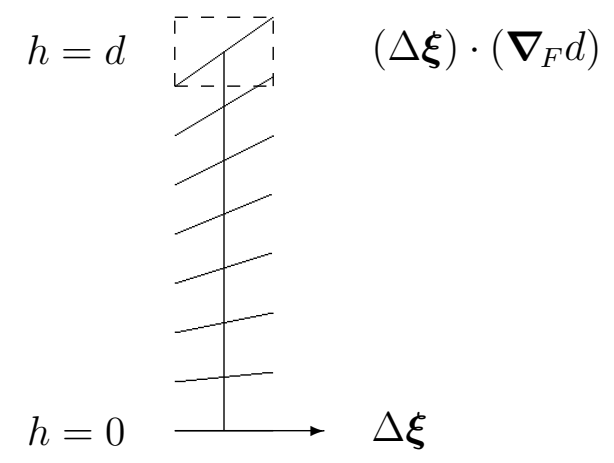

Abbildung 4.3: Der Ansatz für die Deformation.

Damit lautet die erste Näherung der Auslenkung auf der neutralen Fläche unter Berücksichtigung dieses Korrekturfaktors

$$
\begin{aligned}
u_{l}(\boldsymbol{\xi}, h) & =(\boldsymbol{X}(\boldsymbol{\xi}) q)_{l}\left(1-\frac{h}{d(\boldsymbol{\xi})}\left(1+\frac{\Delta \boldsymbol{\xi} \cdot \nabla_{F} d(\boldsymbol{\xi})}{d(\boldsymbol{\xi})}\right)\right) \\
& =(\boldsymbol{X}(\boldsymbol{\xi}) q)_{l}\left(1-\frac{h}{d(\boldsymbol{\xi})}-\frac{h \Delta \boldsymbol{\xi} \cdot \nabla_{F} d(\boldsymbol{\xi})}{d^{2}(\boldsymbol{\xi})}\right)
\end{aligned}
$$

Die Komponenten des Spannungstensors können nun daraus gewonnen werden über

$$
\epsilon_{l m}=u_{l, m}=\left[\left(\nabla_{F}\right)_{m}+(\boldsymbol{n})_{m} \frac{\partial}{\partial h}\right] u_{l}
$$

\footnotetext{
5 Mit dieser Schreibweise braucht das Koordinatensystem der Fläche nicht einmal mehr orthogonal zu sein.
} 
Der Flächengradient $\boldsymbol{\nabla}_{F}$ wirkt nun auf $\Delta \boldsymbol{\xi}$ wie die Einheitsmatrix auf der Fläche, denn es gilt

$$
\left(\boldsymbol{t}_{u} \frac{\partial}{\partial u}+\boldsymbol{t}_{v} \frac{\partial}{\partial v}\right)_{m}\left(\left(u-u_{0}\right) \boldsymbol{t}_{u}+\left(v-v_{0}\right) \boldsymbol{t}_{v}\right)_{n}=\left(\boldsymbol{t}_{u}\right)_{m}\left(\boldsymbol{t}_{u}\right)_{n}+\left(\boldsymbol{t}_{v}\right)_{m}\left(\boldsymbol{t}_{v}\right)_{n}
$$

da die Summe von Parallelprojektoren aus orthonormalen Eigenvektoren die identische Abbildung ergibt. Es ergibt sich für den Spannungstensor

$$
\begin{aligned}
\epsilon_{l m}= & u_{l, m} \\
= & X_{l n, m} q_{n}\left(1-\frac{h}{d}-\frac{h(\Delta \boldsymbol{\xi}) \cdot\left(\boldsymbol{\nabla}_{F} d\right)}{d^{2}}\right) \\
& +(X q)_{l}\left(-\frac{1}{d} n_{m}+\frac{h}{d^{2}}\left(\boldsymbol{\nabla}_{F} d\right)_{m}\right) \\
& +(X q)_{l}\left(-\frac{(\Delta \boldsymbol{\xi}) \cdot\left(\boldsymbol{\nabla}_{F} d\right)}{d^{2}} n_{m}-\frac{h}{d^{2}}\left(\boldsymbol{\nabla}_{F} d\right)_{m}\right. \\
& \left.-\frac{h}{d^{2}}(\Delta \boldsymbol{\xi})_{n}\left(\boldsymbol{\nabla}_{F} d\right)_{n, m}+2 \frac{h(\Delta \boldsymbol{\xi}) \cdot\left(\boldsymbol{\nabla}_{F} d\right)}{d^{3}}\left(\boldsymbol{\nabla}_{F} d\right)_{m}\right) .
\end{aligned}
$$

In der Grenze beliebig kleiner Oberflächenelemente, also für $\Delta \boldsymbol{\xi} \rightarrow \mathbf{0}$, verschwinden die meisten Terme und diejenigen mit dem Schichtdickegradienten $\left(\nabla_{F} d\right)_{m}$ heben sich genau heraus. Man erhält damit den Integranden

$$
\epsilon_{l m}=u_{l, m}=X_{l n, m} q_{n}\left(1-\frac{h}{d}\right)-X_{l n} q_{n} \frac{1}{d} n_{m}
$$

Alle Ableitungen von $\boldsymbol{X}$ oder $\boldsymbol{X}^{T}$ tragen wegen der Symmetrie von $\Lambda_{(i j)(k l)}$ mit demselben Argument wie eben nichts bei, weshalb der erste Term vernachlässigt werden kann. Die Integration über die neutrale Fläche (4.16) wurde durch die obige Parametrisierung in eine über die Deckfläche überführt:

$$
\begin{aligned}
f_{i} & =\iint_{D} X_{i j}^{T} \Lambda_{j k l m} \frac{1}{d} X_{l n} q_{n} n_{k} d o \\
& =\underbrace{\iint_{D} X_{i j}^{T} \Lambda_{j k l m} \frac{1}{d} X_{l n} n_{m} n_{k} d o}_{=\mathcal{S}_{i n}} q_{n},
\end{aligned}
$$

es ergeben sich schließlich die Komponenten der Steifheitsmatrix zu

$$
\mathcal{S}_{i n}=\iint_{D} X_{i j}^{T} n_{k} \Lambda_{j k l m} \frac{1}{d} X_{l n} n_{m} d o
$$

Der Vergleich mit dem Federmodell (4.4) ergibt nun sofort die Federmatrix

$$
K_{j l}(\boldsymbol{\xi})=\frac{1}{d(\boldsymbol{\xi})} \Lambda_{j k l m}(\boldsymbol{\xi}) n_{k}(\boldsymbol{\xi}) n_{m}(\boldsymbol{\xi})
$$


die nun die einfache $3 \times 3$-Gestalt hat. Es brauchen also im Rahmen der getroffenen Näherungen keine Rotationsanteile, wie die allgemeine Form der elastischen Energie aus 4.4 .2 vermuten ließ, berücksichtigt zu werden. Insbesondere konnte die aus Dimensionsbetrachtungen abgeleitete Abhängigkeit $\boldsymbol{K} \sim 1 / d$ genauer begründet werden.

Obwohl streng genommen bei Anwesenheit äußerer Drehmomente eine Richtung ausgezeichnet und damit die Isotropie zerstört wird, möchte ich im Folgenden die nur für isotrope Medien gültige Zerlegung

$$
\Lambda_{i j k l}=\lambda \delta_{i j} \delta_{k l}+2 \mu \delta_{i(l)} \delta_{k) j}=\lambda \delta_{i j} \delta_{k l}+\mu\left(\delta_{i l} \delta_{k j}+\delta_{i k} \delta_{l j}\right)
$$

mit den beiden LAMÉ-Moduln $\lambda$ und $\mu$ verwenden, um damit die resultierende Steifheitsmatrix zu berechnen. In Komponenten ergibt sich mit den entsprechenden Anteilen der Blockmatrix $\boldsymbol{X}$

$$
\begin{aligned}
A_{i j} & =\iint d o \frac{1}{d}\left(\lambda \delta_{i j} \delta_{k l}+\mu\left(\delta_{i l} \delta_{k j}+\delta_{i k} \delta_{l j}\right)\right) \delta_{i k} n_{l} \delta_{m j} n_{n} \\
& =\iint d o \frac{1}{d}\left(\lambda n_{i} n_{j}+\mu\left(\delta_{i j}+n_{i} n_{j}\right)\right) \\
B_{i j} & =\iint d o \frac{1}{d}\left(\lambda \delta_{i j} \delta_{k l}+\mu\left(\delta_{i l} \delta_{k j}+\delta_{i k} \delta_{l j}\right)\right) \delta_{i k} n_{l} \varepsilon_{m o j} \xi_{o} n_{n} \\
& =\iint d o \frac{1}{d}\left(\lambda n_{i} \varepsilon_{m o j} \xi_{o} n_{m}+\mu\left(\varepsilon_{i o j} \xi_{o}+n_{i} \varepsilon_{l o j} \xi_{o} n_{l}\right)\right) \\
C_{i j} & =-\iint d o \frac{1}{d}\left(\lambda \delta_{i j} \delta_{k l}+\mu\left(\delta_{i l} \delta_{k j}+\delta_{i k} \delta_{l j}\right)\right) \varepsilon_{i o k} \xi_{o} n_{l} \varepsilon_{m p j} \xi_{p} n_{n} \\
& =-\iint d o \frac{1}{d}\left(\lambda \varepsilon_{i o l} \varepsilon_{n p j} \xi_{o} \xi_{p} n_{l} n_{m}+\mu\left(\varepsilon_{i o m} \xi_{o} \varepsilon_{m p j} \xi_{p}+\varepsilon_{i o n} \xi_{o} n_{n} \varepsilon_{l p j} \xi_{p} n_{l}\right)\right)
\end{aligned}
$$

bzw. in Matrizenschreibweise

$$
\begin{aligned}
\boldsymbol{A} & =\iint d o \frac{1}{d}\left((\lambda+2 \mu) \boldsymbol{P}_{n}^{\|}+\mu \boldsymbol{P}_{n}^{\perp}\right) \\
\boldsymbol{B} & =\iint d o \frac{1}{d}\left((\lambda+2 \mu) \boldsymbol{P}_{n}^{\|} \boldsymbol{\Omega}_{\xi}+\mu \boldsymbol{P}_{n}^{\perp} \boldsymbol{\Omega}_{\xi}\right) \\
\boldsymbol{C} & =\iint d o \frac{1}{d}\left((\lambda+2 \mu) \boldsymbol{\Omega}_{\xi} \boldsymbol{P}_{n}^{\|} \boldsymbol{\Omega}_{\xi}+\mu \boldsymbol{\Omega}_{\xi} \boldsymbol{P}_{n}^{\perp} \boldsymbol{\Omega}_{\xi}\right) .
\end{aligned}
$$

Das ist genau das Federmodell mit

$$
\begin{aligned}
k_{1} & =(\lambda+2 \mu) / d \\
k_{2} & =\mu / d \\
\boldsymbol{K} & =\frac{1}{d}\left[(\lambda+2 \mu) \boldsymbol{P}_{n}^{\|}+\mu \boldsymbol{P}_{n}^{\perp}\right] .
\end{aligned}
$$

Diese Entsprechung folgt leichter mit (4.21) aus (4.20). Da der LAMÉ-Modul $\mu$ gleich dem Schubmodul $G$ ist, ist das für $k_{2}$ genau das gewünschte Ergebnis

$$
k_{2}=\frac{G}{d}=\frac{E / d}{2(1+\sigma)} .
$$


Die Schichtgröße $k_{1}$ muß noch im Elastizitätsmodul $E$ ausgedrückt werden. Es ergibt sich

$$
\begin{aligned}
k_{1} \cdot d & =\lambda+2 \mu=K-\frac{2}{3} \mu+2 \mu=K+\frac{4}{3} \mu=\frac{E}{3(1-2 \sigma)}+\frac{4}{3} \frac{E}{2(1+\sigma)} \\
& =E \frac{(1-\sigma)}{(1-2 \sigma)(1+\sigma)}
\end{aligned}
$$

mit dem Kompressionsmodul $K$ (Youngsche Zahl) und der Querdehnzahl $\sigma \in$ $[-1,1 / 2]{ }^{6}$ Dies ist genau der Koeffizient der einseitigen Kompression, vgl. [40] $\S 5$. Auch dieser Koeffizient ist das Wunschergebnis, da eine dünne Schicht unter Vernachlässigung der Randeffekte keine Änderung der Querdimension zuläßt. Für $\sigma=0$, also bei verschwindender Querdehnung, ergibt sich $k_{1} d=E=3 K$, dann ist kein Unterschied mehr zwischen der einfachen Streckung und der einseitigen Kompression.

Von Interesse ist ferner das dimensionslose Verhältnis

$$
v:=\frac{k_{2}}{k_{1}}=\frac{1-2 \sigma}{2-2 \sigma}
$$

welches eine reine Funktion der Querdehnzahl ist. Im Intervall $\sigma \in[0,1 / 2]$ ist die Abweichung von der Näherung

$$
v \approx \frac{1}{2}-\sigma
$$

immer kleiner als 0.086; am Rand ist sie exakt. Obwohl die größte Abweichung bei dem realistischen Wert von $\sigma=0.29$ angenommen wird, ist diese Näherung für biologische Materialien gerade zum Verständnis von $v$ ausreichend. Eine bessere Näherung für Werte um $\sigma=1 / 3$ erhält man über eine abgebrochene TAYLORreihe zu $v \approx\left(7-27 \sigma^{2}\right) / 16$. Die durch das Verhältnis $v$ ausgedrückte Querdehnzahl lautet

$$
\sigma=\frac{1-2 v}{2-2 v}
$$

und hat dieselbe Form wie $v(\sigma)$.

Aus den Graphen von $k_{1} / E$ und $k_{2} / E$ in Abhängigkeit von der Querdehnzahl, Abb. 4.4, läßt sich nun für $\sigma>0$

$$
\begin{aligned}
k_{1} d / E & >1 \\
k_{2} d / E & \in(1 / 4,1 / 2) \quad \text { und } \\
k_{1} & >2 k_{2}
\end{aligned}
$$

ersehen. Da in Tabellenwerken oft nur der Elastizitätsmodul $E$ angegeben ist, kann man so mit einer passablen Annahme über die Querdehnzahl $\sigma$ Werte für die Schichtgrößen bekommen.

\footnotetext{
${ }^{6}$ Körper mit $\sigma<0$ sind nach [40] nicht bekannt. Der Kehrwert der Querdehnzahl ist die Poisson-Zahl, in der Literatur wird aber manchmal auch die Querdehnzahl selbst so genannt.
} 


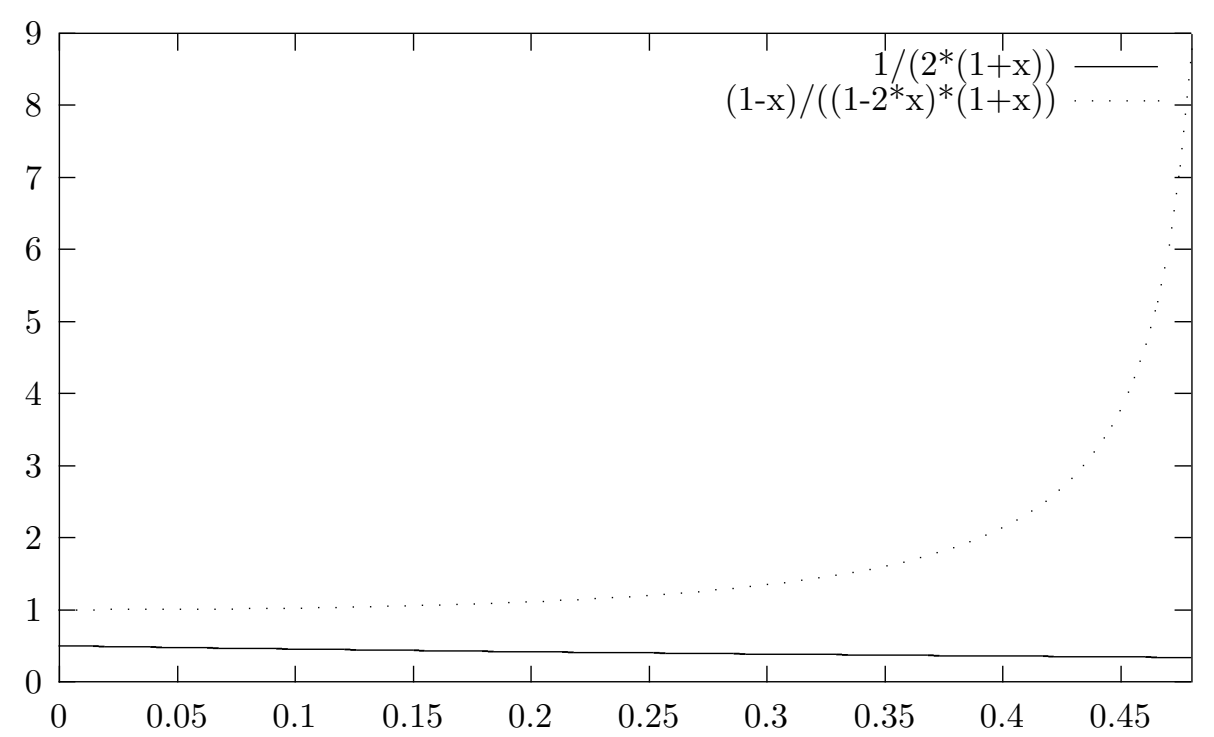

Abbildung 4.4: Die auf $E / d$ normierten Schichtgrößen des Federmodells als Funktion der Querdehnzahl.

Wie beim vorgespannten Federpaar aus Abschnitt 4.1 gilt allgemein für eine Querdehnzahl $\sigma \in[-1,1 / 2]$

$$
0<k_{2}<k_{1}
$$

da die folgenden Ungleichungen äquivalent sind:

$$
\begin{aligned}
\frac{E / d}{2(1+\sigma)} & <\frac{(1-\sigma) E / d}{(1-2 \sigma)(1+\sigma)} \\
\frac{1}{2} & <\frac{1-\sigma}{1-2 \sigma} \\
1-2 \sigma & <2(1-\sigma) \\
0 & <1 .
\end{aligned}
$$

Der formale Nachweis für die ersten Ungleichungen verläuft analog.

Für ein nicht isotropes Medium, wie in 4.2 .2 beschrieben, ist der Ansatz für die Deformation im Inneren der Schicht streng genommen nicht mehr gültig. Dennoch möchte ich die aus (4.20) folgende Identifikation der Parameter angeben. Der Vergleich von (4.2) und (4.3) ergibt

$$
\begin{aligned}
k_{1} d & =\lambda_{1}+\lambda_{2}+\frac{1}{4}(\text { en })^{2} \lambda_{4} \\
k_{2} d & =\frac{1}{2} \lambda_{2}+\frac{1}{4}(\text { en })^{2} \lambda_{4} \\
k_{3} d & =\frac{1}{4} \lambda_{4}+(\text { en })^{2} \lambda_{5} \\
k_{4} d & =(\text { en })\left(\lambda_{3}+\frac{1}{4} \lambda_{4}\right) .
\end{aligned}
$$


Die fünf elastischen Konstanten werden also, wie vorausgesagt, auf vier Schichtgrößen reduziert. Der Quotient $k_{2} / k_{1}$ hängt nun nicht mehr allein von der Querdehnzahl ab.

Das Stabmodell liefert eine weitere Deutung des Koeffizienten $v$. Dieser entspricht, abgesehen von den Beiwerten oder Scherkoeffizienten, den mit Formelsammlungen verträglichen Wert $v=k_{2} / k_{1}=G / E=1 / 2(1+\sigma)$. Je nach innerer Struktur des Mediums ergeben sich folgende Umrechnungen und Wertebereiche für die Querdehnzahl

\begin{tabular}{l||l|l}
$v \leftrightarrow \sigma \quad \backslash \quad$ Modell & Einseitige Kompression & Elastizitätsmodul \\
\hline \hline$v=k_{2} / k_{1}$ & $(1-2 \sigma) /(2-2 \sigma)$ & $1 /(2+2 \sigma)$ \\
\hline$\sigma$ & $(1-2 v) /(2-2 v)$ & $1 / 2 v-1$ \\
\hline$\sigma \in(0,1 / 2)$ & $v \in(0,1 / 2)$ & $v \in(1 / 3,1 / 2)$ \\
\hline$\sigma \in(-1,1 / 2)$ & $v \in(0,3 / 2)$ & $v \in(1 / 3, \infty)$
\end{tabular}

Die Graphen sind in Abb. 4.5 dargestellt. Die stärksten Unterschiede sind für $\sigma \approx$ $0.5 \mathrm{zu}$ erwarten.

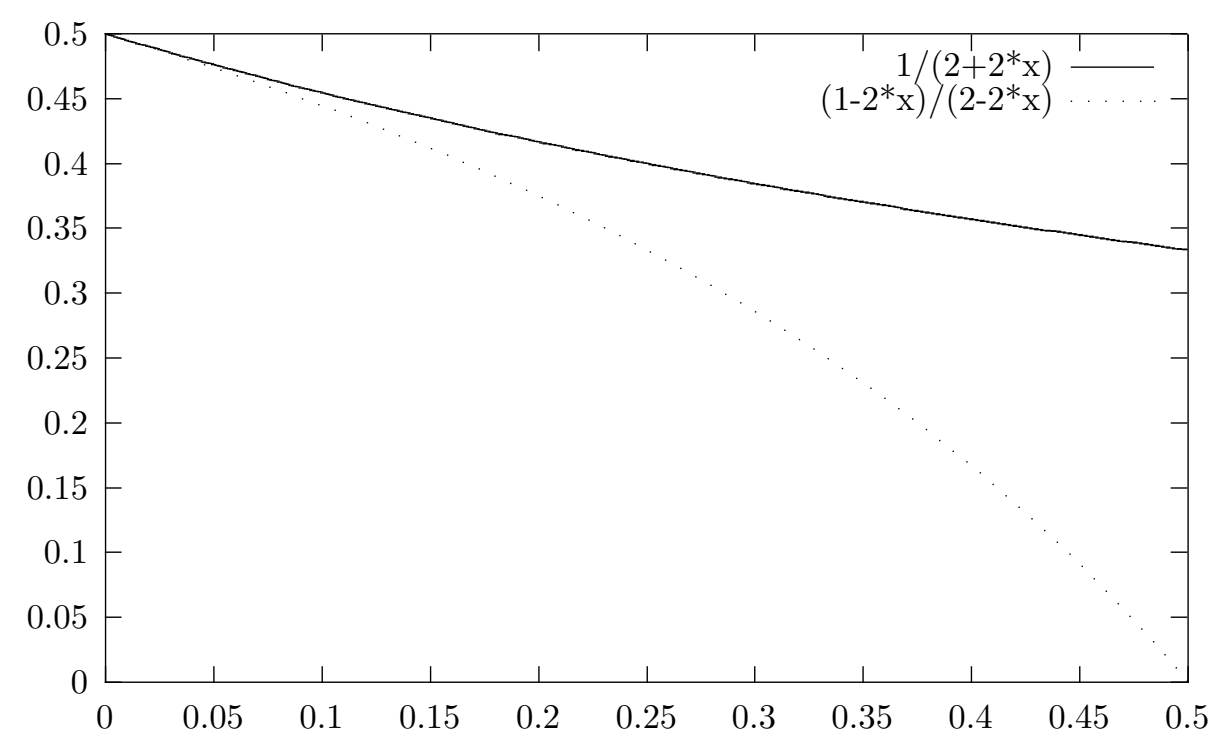

Abbildung 4.5: Der Quotient $v=k_{2} / k_{1}$ als Funktion der Querdehnzahl $\sigma$ für Federund Stabmodell.

Ein Wert von $v \leq 1 / 3$ stellt damit eine klare Aussage gegen das Stabmodell dar, d. h. es lassen sich auch aus an makroskopischen Aufhängungen gewonnenen Meßdaten Schlüsse auf die innere Struktur des Mediums ziehen. Leider ist so keine Entscheidung für ein Modell zu treffen, zum einen aus erkenntnistheoretischen Gründen, und zum anderen wegen der verdoppelten Parameterzahl des nächst einfacheren Modells der nicht isotropen Schicht.

Obwohl die elastische Energie allein eine Vielzahl von Modellen erlaubt, ist das Federmodell mit zwei Parametern und dem Modul der einseitigen Kompression als 
Materialkonstante in Richtung der Schichtnormale für eine dünne isotrope Schicht angemessen und hinreichend begründet. Die anderen Modelle, mit oder ohne Rotationsanteil $\boldsymbol{G}$, mit zwei oder mehr Parametern oder mit einer anderen Deutung der Schichtgrößen, bleiben demnach anderen Situationen vorbehalten. Das Problem der passenden Wahl eines Modells stellt sich, abhängig von der jeweiligen Anwendung, auch bei anderen Methoden wie etwa der der Finiten Elemente. Damit ist diese Modellvielfalt kein prinzipielles Manko der vorliegenden Theorie, sondern ein Weg zu möglichen Erweiterungen.

\subsection{Zusammengesetzte Aufhängungen}

Für den Schwerpunktsvektor $\boldsymbol{s}$ eines aus zwei Punktmassen $m_{1}$ und $m_{2}$ mit Schwerpunktsvektoren $\boldsymbol{s}_{1}$ und $\boldsymbol{s}_{2}$ zusammengesetzten Körpers gilt bekanntlich der Schwerpunktsatz

$$
\boldsymbol{s}=\frac{m_{1} \boldsymbol{s}_{1}+m_{2} \boldsymbol{s}_{2}}{m_{1}+m_{2}}
$$

Durch den Übergang zum Kontinuum mit Massendichte $\rho(\boldsymbol{x})$ erhält man daraus die allgemeine Definition des Schwerpunktes

$$
\boldsymbol{s}:=\frac{\int \rho(\boldsymbol{x}) \boldsymbol{x} d^{3} x}{\int \rho(\boldsymbol{x}) d^{3} x},
$$

aus der man durch Einführung $\delta^{3}$-förmiger Dichten wieder zur Ausgangsformel zurückkommt. Der Trägheitstensor ist ähnlich über

$$
\boldsymbol{J}:=\int \rho(\boldsymbol{x})\left(\boldsymbol{x}^{2} \boldsymbol{I}-\boldsymbol{x} \boldsymbol{x}^{T}\right) d^{3} x=-\int \boldsymbol{x}^{2} \rho(\boldsymbol{x}) \boldsymbol{P}_{x}^{\perp} d^{3} x
$$

definiert.

Die den Schwerpunkt und den Trägheitstensor definierenden Teilintegrale erinnern an die Komponenten der Steifheitsmatrix

$$
\begin{aligned}
\boldsymbol{A} & =\mathscr{H} \boldsymbol{K} d \boldsymbol{\xi} \\
\boldsymbol{B} & =-\mathscr{\int} \boldsymbol{K} \boldsymbol{\Omega}_{\xi} d \boldsymbol{\xi} \\
\boldsymbol{C} & =-\mathscr{\int} \boldsymbol{\Omega}_{\xi} \boldsymbol{K} \boldsymbol{\Omega}_{\xi} d \boldsymbol{\xi},
\end{aligned}
$$

und zwar über die Analogie

$$
\begin{aligned}
\text { Masse } & \longleftrightarrow \text { Federmatrix } \\
\text { Ortsvektor } & \longleftrightarrow \text { zum Ortsvektor duale Matrix. }
\end{aligned}
$$

In der Tat lautet die Definition des elastischen Zentrums

$$
\boldsymbol{z}=-\left(\boldsymbol{A}^{-1} \boldsymbol{B}\right)^{D}=\left((\oiiint \boldsymbol{K} d \boldsymbol{\xi})^{-1} \oiiint \boldsymbol{K} \boldsymbol{\Omega}_{\xi} d \boldsymbol{\xi}\right)^{D}
$$


und diese Formel ist für eine zum Skalar entartete Federmatrix genau die alte Definition des Schwerpunktes. Wegen (A.2) stimmen dann sogar die Formeln für $\boldsymbol{C}$ und für $\boldsymbol{J}$ überein. Damit kann die vorliegende Theorie auch als Mechanik mit einer nichtskalaren Masse gedeutet werden: Wie in der Allgemeinen Relativitätstheorie wird die Masse durch einen Tensor zweiter Stufe beschrieben.

Mit diesen Analogien im Hinterkopf sollen im Folgenden allgemeine Aussagen über elastische Aufhängungen mit obigen Komponenten abgeleitet werden. Da Integrationen auf Grenzwerte von Summationen zurückgeführt werden können, ist es zunächst sinnvoll, die Effekte einfacher Summen zu studieren.

\subsubsection{Die Analogie zum Schwerpunktsatz und ihre Grenzen}

Für zusammengesetzte Aufhängungen folgt aus ihrer Definition über die Integrale die Additivität der Teilmatrizen $\boldsymbol{A}, \boldsymbol{B}$ und $\boldsymbol{C}$. Das läßt sich auch aus der Additivität des Potentials $\Phi=\Phi_{1}+\Phi_{2}$ für die Steifheitsmatrix herleiten:

$$
\mathcal{S}_{i j}=\frac{\partial^{2} \Phi}{\partial q_{i} \partial q_{j}}=\frac{\partial^{2}\left(\Phi_{1}+\Phi_{2}\right)}{\partial q_{i} \partial q_{j}}=\frac{\partial^{2} \Phi_{1}}{\partial q_{i} \partial q_{j}}+\frac{\partial^{2} \Phi_{2}}{\partial q_{i} \partial q_{j}}=\left(\mathcal{S}_{1}\right)_{i j}+\left(\mathcal{S}_{2}\right)_{i j}
$$

Daraus kann man unmittelbar die Nicht-Additivität der Flexibilitätsmatrix für zusammengesetzte Aufhängungen ablesen, denn es gilt

$$
\mathcal{F}=\mathcal{S}^{-1}=\left(\mathcal{S}_{1}+\mathcal{S}_{2}\right)^{-1} \neq \mathcal{S}_{1}^{-1}+\mathcal{S}_{2}^{-1}=\mathcal{F}_{1}+\mathcal{F}_{2}
$$

Für die elastischen Zentren einer aus zwei Teilen zusammengesetzten Aufhängung gilt dann

$$
\begin{aligned}
\boldsymbol{z}_{1} & =-\left(\boldsymbol{A}_{1}^{-1} \boldsymbol{B}_{1}\right)^{D} \\
\boldsymbol{z}_{2} & =-\left(\boldsymbol{A}_{2}^{-1} \boldsymbol{B}_{2}\right)^{D} \\
\boldsymbol{z} & =-\left(\left(\boldsymbol{A}_{1}+\boldsymbol{A}_{2}\right)^{-1}\left(\boldsymbol{B}_{1}+\boldsymbol{B}_{2}\right)\right)^{D},
\end{aligned}
$$

und nur in Ausnahmefällen läßt sich wegen der matrixwertigen „Wichtungsfaktoren ${ }^{\alpha} \boldsymbol{\nabla}$ eine einfache Linearkombination von $\boldsymbol{z}_{1}$ und $\boldsymbol{z}_{2}$ finden, so daß $\boldsymbol{z} \in\left\langle\boldsymbol{z}_{1}, \boldsymbol{z}_{2}\right\rangle$.

\footnotetext{
${ }^{7}$ Mit der Formel (A.20) und der Rechenregel A.1 für den $\varepsilon$-Tensor läßt sich die Definition des Widerstandszentrums als (multi)lineare Abbildung zwischen $\boldsymbol{B}$ und $\boldsymbol{z}$ besser verstehen:

$$
\begin{aligned}
\boldsymbol{z}_{p} & =-\frac{1}{2 \operatorname{det} \boldsymbol{A}} \varepsilon_{j l m} \varepsilon_{i k n} A_{l k} A_{m n} B_{j q} \varepsilon_{q i p} \\
& =\frac{1}{\operatorname{det} \boldsymbol{A}} A_{m[p} A_{q] l}^{T} \varepsilon_{l m j} B_{j q} .
\end{aligned}
$$

Bei einer Summe wie $\boldsymbol{A}_{1}+\boldsymbol{A}_{2}$ entstehen gemischte Terme, die ein Aufschreiben von $\boldsymbol{z}$ als Funktion von $\boldsymbol{z}_{1}$ und $\boldsymbol{z}_{2}$ allein unmöglich machen. Dasselbe Problem hat man mit Tensoren vierter Stufe, wenn man die Gleichungen für $\boldsymbol{\Omega}_{z}$ aufschreibt.
} 
Diese Ausnahmefälle zeichnen sich durch eine besondere Struktur der Matrizen $\boldsymbol{A}$ und $\boldsymbol{B}$ oder, wie im Fall der skalaren Masse, eine besonders einfache Vertauschungsregel für die Matrix $\boldsymbol{A}$ aus. Der Grund für das Scheitern einer Analogie zum Schwerpunktsatz liegt zum einen an den nicht mehr skalaren Größen, und zum anderen an der Dualisierung bzw. Antisymmetrisierung: Wegen

$$
\left(\boldsymbol{A}^{-1} \boldsymbol{B}\right)^{A}=\frac{1}{2}\left(\boldsymbol{A}^{-1} \boldsymbol{B}-\left(\boldsymbol{A}^{-1} \boldsymbol{B}\right)^{T}\right)=\frac{1}{2}\left(\boldsymbol{A}^{-1} \boldsymbol{B}-\boldsymbol{B}^{T} \boldsymbol{A}^{-1}\right)
$$

tauchen die „Massen“ einmal links und einmal rechts in diesem Produkt auf, woraus folgt, daß es keine matrixwertigen Wichtungsfaktoren geben kann, die diese Inversion wieder rückgängig machen.

Da der Fall des Widerstandszentrums von besonderem Interesse ist, soll er im nächsten Abschnitt näher betrachtet werden.

\subsubsection{Superposition von Widerstandszentren}

Das elastische Zentrum einer Superposition zweier Aufhängungen mit Widerstandszentren hat die besonders einfache Form

$$
\begin{aligned}
\boldsymbol{z} & =-\left(\left(\boldsymbol{A}_{1}+\boldsymbol{A}_{2}\right)^{-1}\left(-\boldsymbol{A}_{1} \boldsymbol{\Omega}_{1}-\boldsymbol{A}_{2} \boldsymbol{\Omega}_{2}\right)\right)^{D} \\
& =\left(\left(\boldsymbol{A}_{1}+\boldsymbol{A}_{2}\right)^{-1}\left(\boldsymbol{A}_{1} \boldsymbol{\Omega}_{1}\right)\right)^{D}+\left(\left(\boldsymbol{A}_{1}+\boldsymbol{A}_{2}\right)^{-1}\left(\boldsymbol{A}_{2} \boldsymbol{\Omega}_{2}\right)\right)^{D} \\
& =\left(\left(\boldsymbol{A}_{1}+\boldsymbol{A}_{2}\right)^{-1} \boldsymbol{A}_{1}\right)^{T F} \boldsymbol{z}_{1}+\left(\left(\boldsymbol{A}_{1}+\boldsymbol{A}_{2}\right)^{-1} \boldsymbol{A}_{2}\right)^{T F} \boldsymbol{z}_{2}
\end{aligned}
$$

welche einer Mittelung mit im Gegensatz zum Schwerpunktsatz matrixwertigen Gewichten entspricht. Im Fall von vielen infinitesimal kleinen Teilelementen existiert wegen dem Mittelwertsatz lokal immer ein Widerstandszentrum, nämlich der Ortsvektor der Oberfläche der Berandung. Die Teilmatrizen $\boldsymbol{A}_{i}$ werden dann zu den Federmatrizen $\boldsymbol{K}$, und insgesamt ergibt sich für das elastische Zentrum

$$
\begin{aligned}
\boldsymbol{z} & =-\left((\oiint \boldsymbol{K})^{-1}\left(-\oiint f \boldsymbol{K} \boldsymbol{\Omega}_{\xi}\right)\right)^{D} \\
& =\left(\boldsymbol{A}^{-1}\left(-\oiint f \boldsymbol{K} \boldsymbol{\Omega}_{\xi}\right)\right)^{D} \\
& =\oiint\left(\boldsymbol{A}^{-1} \boldsymbol{K}\right)^{T F} \boldsymbol{\xi} .
\end{aligned}
$$

Ein elastisches Zentrum existiert also immer und kann geschlossen angegeben werden. Wann aber existiert ein Widerstandszentrum? Die direkte Berechnung des Widerstandszentrums der Gesamtaufhängung erfordert die Berechnung der Inversen einer Matrixsumme, welche für theoretische Untersuchungen nicht immer günstig ist. Daher sollen zwei weitere Größen vorgestellt werden, an denen zu testen ist, ob die Aufhängung ein Widerstandszentrum hat. 
Im folgenden Abschnitt soll untersucht werden, unter welchen Umständen eine Superposition zweier Aufhängungen mit Widerstandszentrum wieder eine Aufhängung mit Widerstandszentrum ergibt. Wegen der Definition der Steifheitsmatrix als Integral über die Berandung lassen sich dann allgemeine Klassen von Aufhängungen mit einem Widerstandszentrum finden.

Nach Voraussetzung haben die beiden Teilaufhängungen und die Gesamtaufhängung ein Widerstandszentrum, weshalb alle Matrizen vom Typ $\boldsymbol{A}^{-1} \boldsymbol{B}$ antisymmetrisch sind, also $\boldsymbol{A}^{-1} \boldsymbol{B}=-\boldsymbol{B}^{T} \boldsymbol{A}^{-1}$. Rechts- und Linksmultiplikation mit $\boldsymbol{A}$ ergibt $\boldsymbol{B} \boldsymbol{A}=-\boldsymbol{A} \boldsymbol{B}^{T}$, bzw. ausmultipliziert

$$
\begin{aligned}
\boldsymbol{B}_{1} \boldsymbol{A}_{1} & =-\boldsymbol{A}_{1} \boldsymbol{B}_{1}^{T} \\
\boldsymbol{B}_{2} \boldsymbol{A}_{2} & =-\boldsymbol{A}_{2} \boldsymbol{B}_{2}^{T} \\
\boldsymbol{B}_{1} \boldsymbol{A}_{1}+\boldsymbol{B}_{1} \boldsymbol{A}_{2}+\boldsymbol{B}_{2} \boldsymbol{A}_{1}+\boldsymbol{B}_{2} \boldsymbol{A}_{2} & =-\boldsymbol{A}_{1} \boldsymbol{B}_{1}^{T}-\boldsymbol{A}_{1} \boldsymbol{B}_{2}^{T}-\boldsymbol{A}_{2} \boldsymbol{B}_{1}^{T}-\boldsymbol{A}_{2} \boldsymbol{B}_{2}^{T} .
\end{aligned}
$$

Die Terme der dritten Gleichung mit gleichen Subskripten in $\boldsymbol{A}$ und $\boldsymbol{B}$ heben sich wegen den ersten beiden Gleichungen weg, und es bleibt übrig

$$
\boldsymbol{B}_{1} \boldsymbol{A}_{2}+\boldsymbol{B}_{2} \boldsymbol{A}_{1}=-\boldsymbol{A}_{1} \boldsymbol{B}_{2}^{T}-\boldsymbol{A}_{2} \boldsymbol{B}_{1}^{T}
$$

bzw. kürzer

$$
\left(\boldsymbol{B}_{1} \boldsymbol{A}_{2}+\boldsymbol{B}_{2} \boldsymbol{A}_{1}\right)^{S}=\mathbf{0} .
$$

Mit $\boldsymbol{\Omega}=-\boldsymbol{A}^{-1} \boldsymbol{B}$, also $\boldsymbol{B}=-\boldsymbol{A} \boldsymbol{\Omega}$, erhält man weiter

$$
\begin{aligned}
\mathbf{0} & =\left(\boldsymbol{A}_{1} \boldsymbol{\Omega}_{1} \boldsymbol{A}_{2}+\boldsymbol{A}_{2} \boldsymbol{\Omega}_{2} \boldsymbol{A}_{1}\right)^{S} \\
& =\frac{1}{2}\left(\boldsymbol{A}_{1} \boldsymbol{\Omega}_{1} \boldsymbol{A}_{2}-\boldsymbol{A}_{2} \boldsymbol{\Omega}_{1} \boldsymbol{A}_{2}+\boldsymbol{A}_{2} \boldsymbol{\Omega}_{2} \boldsymbol{A}_{1}-\boldsymbol{A}_{1} \boldsymbol{\Omega}_{2} \boldsymbol{A}_{2}\right) \\
& =\frac{1}{2}\left(\boldsymbol{A}_{1}\left(\boldsymbol{\Omega}_{1}-\boldsymbol{\Omega}_{2}\right) \boldsymbol{A}_{2}+\boldsymbol{A}_{2}\left(\boldsymbol{\Omega}_{2}-\boldsymbol{\Omega}_{1}\right) \boldsymbol{A}_{1}\right) \\
& =\left(\boldsymbol{A}_{1}\left(\boldsymbol{\Omega}_{1}-\boldsymbol{\Omega}_{2}\right) \boldsymbol{A}_{2}\right)^{S} .
\end{aligned}
$$

Aus dieser letzten Gleichung können nun wie folgt zwei wichtige Klassen von Aufhängungen mit Widerstandszentrum abgelesen werden.

\subsubsection{Aufhängungen mit gleichem Widerstandszentrum}

Für eine Superposition von Aufhängungen mit gleichem Widerstandszentrum gilt $\Omega_{1}=\Omega_{2}=\Omega$ und folglich ist (4.32) für beliebige $\boldsymbol{A}$ erfüllt. Das Gesamtwiderstandszentrum ist nun gegeben durch

$$
\begin{aligned}
-\left(\left(\boldsymbol{A}_{1}+\boldsymbol{A}_{2}\right)^{-1}\left(\boldsymbol{B}_{1}+\boldsymbol{B}_{2}\right)\right)^{D} & =-\left(\left(\boldsymbol{A}_{1}+\boldsymbol{A}_{2}\right)^{-1}\left(-\boldsymbol{A}_{1} \boldsymbol{\Omega}-\boldsymbol{A}_{2} \boldsymbol{\Omega}\right)\right)^{D} \\
& =\left(\left(\boldsymbol{A}_{1}+\boldsymbol{A}_{2}\right)^{-1}\left(\boldsymbol{A}_{1}+\boldsymbol{A}_{2}\right) \boldsymbol{\Omega}\right)^{D} \\
& =\boldsymbol{\Omega}^{D} \\
& =\boldsymbol{z}
\end{aligned}
$$

also folgt insgesamt: 
Eine Superposition von Aufhängungen mit gleichem Widerstandszentrum ergibt wieder eine Aufhängung mit eben diesem Widerstandszentrum,

was auch anschaulich zu erwarten war.

\subsubsection{Gleichartige Aufhängungen}

Für einander ähnliche, aber ungleich starke Aufhängungen ist die eine Federmatrix ein Vielfaches der anderen. Für die gesamte Aufhängung gilt dann $\boldsymbol{A}_{2}=\lambda \boldsymbol{A}_{1}$ mit dem reellen Parameter $\lambda$, und die Gleichung (4.32) ist wieder erfüllt. Dieses liegt bei

$$
\begin{aligned}
\boldsymbol{z} & =-\left(\left(\boldsymbol{A}_{1}+\lambda \boldsymbol{A}_{1}\right)^{-1}\left(\boldsymbol{B}_{1}+\boldsymbol{B}_{2}\right)\right)^{D}=-\frac{1}{1+\lambda}\left(\boldsymbol{A}_{1}^{-1}\left(\boldsymbol{B}_{1}+\boldsymbol{B}_{2}\right)\right)^{D} \\
& =\frac{1}{1+\lambda}\left(-\boldsymbol{A}_{1}^{-1} \boldsymbol{B}_{1}-\boldsymbol{A}_{1}^{-1} \boldsymbol{B}_{2}\right)^{D}=\frac{1}{1+\lambda}\left(\boldsymbol{\Omega}_{1}-\lambda\left(\lambda \boldsymbol{A}_{1}^{-1}\right) \boldsymbol{B}_{2}\right)^{D} \\
& =\frac{1}{1+\lambda}\left(\boldsymbol{\Omega}_{1}-\lambda \boldsymbol{A}_{2}^{-1} \boldsymbol{B}_{2}\right)^{D}=\frac{1}{1+\lambda}\left(\boldsymbol{\Omega}_{1}+\lambda \boldsymbol{\Omega}_{2}\right)^{D} \\
& =\frac{1}{1+\lambda}\left(\boldsymbol{z}_{1}+\lambda \boldsymbol{z}_{2}\right),
\end{aligned}
$$

es gilt also:

Eine Superposition von gleichartigen Aufhängungen mit nicht notwendigerweise gleichem Widerstandszentrum ergibt insgesamt eine Aufhängung mit Widerstandszentrum. Dieses ergibt sich analog dem Schwerpunktsatz, wobei die Rolle der Masse von der Stärke der Aufhängung übernommen wird.

Beispielsweise hat daher eine Superposition von ähnlichen ebenen Aufhängungen mit Widerstandszentrum unter Beibehaltung der Ebenennormale wieder ein Widerstandszentrum.

Eine Aufhängung mit konstanten Schichtgrößen kann durch eine elastische Schicht aus überall gleichem Material mit konstanter Schichtdicke realisiert werden. Grundund Deckfläche sind dann Parallelfächen [28]. Wird nun die Schichtdicke überall gleichmäßig erhöht, so erhält man aus $\boldsymbol{K}_{2}=\lambda \boldsymbol{K}_{1}$ unter der üblichen Vernachlässigung von Randeffekten und geometrischen Korrekturen $\boldsymbol{A}_{2}=\lambda \boldsymbol{A}_{1}$ und $B_{2}=\lambda \boldsymbol{B}_{1}$, woraus

$$
\boldsymbol{z}_{2}=-\left(\boldsymbol{A}_{2}^{-1} \boldsymbol{B}_{2}\right)^{D}=-\left(\left(\lambda \boldsymbol{A}_{1}\right)^{-1}\left(\lambda \boldsymbol{B}_{1}\right)\right)^{D}=-\left(\boldsymbol{A}_{1}^{-1} \boldsymbol{B}_{1}\right)^{D}=\boldsymbol{z}_{1}
$$

folgt. Wie im ebenen Fall der Einleitung 1.1 hängt auch hier die Lage des elastischen Zentrums nicht von der „Stärke “ der Aufhängung ab — vorausgesetzt, 
daß diese durch einen einzigen skalaren Parameter beschrieben werden kann. Eine gleichmäßige Veränderung der Schichtdicke hat somit keinen Einfluß auf die Lage des elastischen Zentrums, eine Veränderung des Materials hingegen durchaus. Insbesondere sollten unterschiedliche Querdehnzahlen auf verschiedene elastische Zentren führen.

\subsubsection{Aufhängungen mit identischer Gegenfeder}

Ein weiteres wichtiges Kriterium zum Entscheiden, ob ein Widerstandszentrum vorliegt, kann direkt aus der Definition des elastischen Zentrums abgelesen werden: Wenn ein Koordinatensystem gefunden werden kann, so daß $\boldsymbol{B}_{1}+\boldsymbol{B}_{2}=\mathbf{0}$, dann befindet sich nicht nur der Koordinatenursprung im elastischen Zentrum, sondern es liegt wegen der Blockdiagonalform von $\mathcal{S}$ sogar ein Widerstandszentrum vor.

Die Gleichung $\boldsymbol{B}_{1}=-\boldsymbol{B}_{2}$ kann aber dadurch erreicht werden, daß die beiden Teilaufhängungen gleich stark und gegenüber liegend sind. Da wegen $\boldsymbol{B}=-\mathbb{\int} \boldsymbol{K} \boldsymbol{\Omega}_{\xi} d \boldsymbol{\xi}$ die Matrix $\boldsymbol{B}$ eine ungerade Funktion in $\boldsymbol{\xi}$ ist, folgt aus $\boldsymbol{K}(-\boldsymbol{\xi})=\boldsymbol{K}(\boldsymbol{\xi})$ für alle $\boldsymbol{\xi}$ des Integrationsbereiches, also wenn die Federmatrix eine gerade Funktion in $\boldsymbol{\xi}$ ist, mit einer Variablensubstitution $\boldsymbol{\xi} \mapsto-\boldsymbol{\xi}$ sofort $\boldsymbol{B}=-\boldsymbol{B}$, also $\boldsymbol{B}=\mathbf{0}$. Ausformuliert bedeutet das:

Eine punktsymmetrische Aufhängung, also eine, bei der zu jeder Feder eine gegenüber liegende gleich starke Gegenfeder existiert, besitzt im Symmetriezentrum ein Widerstandszentrum.

Dieser Fall hat weit reichende Konsequenzen: Eine Kombination dieser Erkenntnis mit dem Ergebnis über gleichartige Aufhängungen ergibt eine große Klasse von Aufhängungen die ein Widerstandszentrum besitzen. So hat beispielsweise ein elliptisches Paraboloid mit zur Mittelachse senkrechter Aufhängung, etwa als genähertes Modell einer Frontzahnwurzel, in jedem Höhenschnitt und damit insgesamt ein Widerstandszentrum. Eine nicht zur Mittelachse, sondern zur Oberflächennormale senkrechte Aufhängung hat diese Eigenschaft zunächst nicht und muß daher gesondert untersucht werden. 


\section{Kapitel 5}

\section{Verschiedene Aufhängungen}

In diesem Kapitel soll die Struktur von Steifheitsmatrizen verschiedener Aufhängungen vorhergesagt werden. Dazu wird ihre Darstellung als Integral über die Oberfläche der starren Berandung des letzten Kapitels verwendet. Dabei wird wie folgt vorgegangen:

1. Der Ursprung des Koordinatensystems wird, falls eines existiert, in das Symmetriezentrum der Aufhängung gelegt, da dieses ein guter Kandidat für das Widerstandszentrum oder das elastische Zentrum ist.

2. Die Koordinaten werden so gewählt, daß die Integration über die Oberfläche einfach ist, d. h. die Oberfläche sollte durch eine konstante Koordinate beschreibbar sein.

3. Die kartesischen Komponenten des Normalenvektors werden in den neuen Koordinaten ausgedrückt und in die Darstellung der Federmatrix $\boldsymbol{K}$ des Federmodells eingesetzt. Dadurch umgeht man die Verwendung von Drehmatrizen.

4. Die Blockkomponenten der Steifheitsmatrix ergeben sich durch Integration über die Matrixkomponenten (ggf. nach vorheriger Multiplikation mit den zu den Aufpunkten der Oberfläche dualen antisymmetrischen Matrizen).

5. Diese Integrale sind Oberflächenintegrale erster Art und erfordern die Berechnung der Determinante der Metrik der Fläche; in den üblichen Bezeichnungen der Flächentheorie lautet diese

$$
g=\sqrt{\operatorname{det}\left(\boldsymbol{x}_{, i} \cdot \boldsymbol{x}_{, j}\right)}=\sqrt{E F-G^{2}}=\left\|\boldsymbol{x}_{, 1} \times \boldsymbol{x}_{, 2}\right\| .
$$

Die Integrationen werden, falls sie zu umfangreich und damit fehlerträchtig sind, mit Hilfe eines Computeralgebra-Systems durchgeführt. 


\subsection{Einfache Aufhängungen und elementare Re- chenmethoden}

In diesem Kapitel soll die Struktur von Steifheitsmatrizen von einfachen aber für Anwendungen wichtigen Aufhängungen berechnet werden. Dazu werden zunächst elementare Rechenmethoden vorgestellt.

\subsubsection{Die rechteckige Scheibe}

Am einfachsten Beispiel, nämlich dem einer ebenen rechteckigen Scheibe mit konstanten Schichtgrößen, sollen die Integrationen im Matrixformalismus vorgeführt werden.

Die Scheibe habe die Abmessungen $a \times b$ und das Koordinatensystem liege in der Scheibenmitte. Mit

$$
\boldsymbol{n}=\left(\begin{array}{l}
0 \\
0 \\
1
\end{array}\right)
$$

erhält man

$$
\boldsymbol{K}=k_{1} \boldsymbol{P}_{n}^{\|}+k_{2} \boldsymbol{P}_{n}^{\perp}=k_{1}\left(\begin{array}{ccc}
0 & 0 & 0 \\
0 & 0 & 0 \\
0 & 0 & 1
\end{array}\right)+k_{2}\left(\begin{array}{ccc}
1 & 0 & 0 \\
0 & 1 & 0 \\
0 & 0 & 0
\end{array}\right)=\left(\begin{array}{ccc}
k_{2} & 0 & 0 \\
0 & k_{2} & 0 \\
0 & 0 & k_{1}
\end{array}\right)
$$

und

$$
\boldsymbol{\Omega}_{\xi}=\left(\begin{array}{ccc}
0 & 0 & y \\
0 & 0 & -x \\
-y & x & 0
\end{array}\right)
$$

Da das Oberflächenelement einfach durch $d o=d x d y$ gegeben ist, erhält man die Komponenten der Steifheitsmatrix für konstante $k_{i} \mathrm{zu}$

$$
\boldsymbol{A}=\int_{x=-a / 2}^{a / 2} \int_{y=-b / 2}^{b / 2} \boldsymbol{K} d x d y=\boldsymbol{K} \int_{x=-a / 2}^{a / 2} \int_{y=-b / 2}^{b / 2} d x d y=\left.\left.\boldsymbol{K} x y\right|_{x=-a / 2} ^{a / 2}\right|_{y=-b / 2} ^{b / 2}=a b \boldsymbol{K}
$$

und da der Integrand ungerade und der Integrationsbereich symmetrisch zum Ursprung liegt, folgt

$$
\boldsymbol{B}=-\int_{x=-a / 2}^{a / 2} \int_{y=-b / 2}^{b / 2} \boldsymbol{K} \boldsymbol{\Omega}_{\xi} d x d y=0
$$


und es existiert daher ein Widerstandszentrum im Koordinatenursprung, also in der Scheibenmitte. Schließlich folgt

$$
\begin{aligned}
& \boldsymbol{C}=-\int_{x=-a / 2}^{a / 2} \int_{y=-b / 2}^{b / 2} \Omega_{\xi} \boldsymbol{K} \boldsymbol{\Omega}_{\xi} d x d y \\
& =-\int_{x=-a / 2}^{a / 2} \int_{y=-b / 2}^{b / 2}\left(\begin{array}{ccc}
0 & 0 & y \\
0 & 0 & -x \\
-y & x & 0
\end{array}\right)\left(\begin{array}{ccc}
k_{2} & 0 & 0 \\
0 & k_{2} & 0 \\
0 & 0 & k_{1}
\end{array}\right)\left(\begin{array}{ccc}
0 & 0 & y \\
0 & 0 & -x \\
-y & x & 0
\end{array}\right) d x d y \\
& =-\int_{x=-a / 2}^{a / 2} \int_{y=-b / 2}^{b / 2}\left(\begin{array}{ccc}
0 & 0 & k_{1} y \\
0 & 0 & -k_{1} x \\
-k_{2} y & k_{2} x & 0
\end{array}\right)\left(\begin{array}{ccc}
0 & 0 & y \\
0 & 0 & -x \\
-y & x & 0
\end{array}\right) d x d y \\
& =-\int_{x=-a / 2}^{a / 2} \int_{y=-b / 2}^{b / 2}\left(\begin{array}{ccc}
-k_{1} y^{2} & k_{1} x y & 0 \\
k_{1} x y & -k_{1} x^{2} & 0 \\
0 & 0 & -k_{2} y^{2}-k_{2} x^{2}
\end{array}\right) d x d y \\
& =\left(\begin{array}{ccc}
k_{1} a b^{3} / 12 & 0 & 0 \\
0 & k_{1} b a^{3} / 12 & 0 \\
0 & 0 & k_{2}\left(a b^{3}+b a^{3}\right) / 12
\end{array}\right) \\
& =\frac{a b}{12}\left(\begin{array}{ccc}
k_{1} b^{2} & 0 & 0 \\
0 & k_{1} a^{2} & 0 \\
0 & 0 & k_{2}\left(b^{2}+a^{2}\right)
\end{array}\right) \text {. }
\end{aligned}
$$

Die Achsen konstanter Helizität sind die Eigenvektoren der Matrix $\boldsymbol{A C}$. Da die Matrizen $\boldsymbol{A}$ und $\boldsymbol{C}$ beide diagonal sind, ist es auch deren Produkt

$$
\boldsymbol{A C}=k_{1} k_{2} \frac{(a b)^{2}}{12}\left(\begin{array}{ccc}
b^{2} & 0 & 0 \\
0 & a^{2} & 0 \\
0 & 0 & a^{2}+b^{2}
\end{array}\right)
$$

und diese Eigenvektoren sind (bis auf die Entartung für $a=b$ ) genau die Koordinatenachsen. Da die Materialkonstanten nur als gemeinsame Faktoren der Matrixelemente auftauchen, hängt das Eigenvektorproblem wie erwartet nicht von diesen ab. Da die Achsen konstanter Helizität spezielle zusätzliche Eigenschaften gegenüber den funktionellen Achsen und Richtungen haben, sind mit ihnen die beiden letzteren bestimmt und stimmen überein.

\subsubsection{Die Kreisscheibe}

Mit diesem Beispiel möchte ich an dieser Stelle einen weiteren Formalismus vorstellen, an dem die komponentenweise Integration in manchen Fällen (speziell bei spärlich besetzten Tensoren höherer Stufe) überschaubarer wird: Die Rechnung in Komponenten unter Zuhilfenahme der Summationskonvention. Wieder lauten der 
Normalenvektor

$$
n_{i}=\delta_{i}^{3}
$$

und die Federmatrix

$$
\begin{aligned}
K_{i j} & =k_{1} n_{i} n_{j}+k_{2}\left(\delta_{i j}-n_{i} n_{j}\right)=k_{1} \delta_{i}^{3} \delta_{j}^{3}+k_{2}\left(\delta_{i j}-\delta_{i}^{3} \delta_{j}^{3}\right) \\
& =k_{1} \delta_{i}^{3} \delta_{j}^{3}+k_{2}\left(\delta_{i}^{1} \delta_{j}^{1}+\delta_{i}^{2} \delta_{j}^{2}\right) .
\end{aligned}
$$

Der antisymmetrische Tensor der Aufpunkte des Randes der Fläche lautet in Polarkoordinaten

$$
\begin{aligned}
\Omega_{i j} & =\varepsilon_{i k j} x_{k}=\varepsilon_{i k j}\left(\delta_{k}^{1} r \cos \phi+\delta_{k}^{2} r \sin \phi\right) \\
& =r \cos \phi\left(\delta_{i}^{3} \delta_{j}^{2}-\delta_{i}^{2} \delta_{j}^{3}\right)+r \sin \phi\left(\delta_{i}^{1} \delta_{j}^{3}-\delta_{i}^{3} \delta_{j}^{1}\right)
\end{aligned}
$$

und die Integranden erhält man als Produkte

$$
\begin{aligned}
K_{i j} \Omega_{j l}= & \left(k_{1} \delta_{i}^{3} \delta_{j}^{3}+k_{2}\left(\delta_{i}^{1} \delta_{j}^{1}+\delta_{i}^{2} \delta_{j}^{2}\right)\right) \cdot \\
& \cdot\left(r \cos \phi\left(\delta_{j}^{3} \delta_{l}^{2}-\delta_{j}^{2} \delta_{l}^{3}\right)+r \sin \phi\left(\delta_{j}^{1} \delta_{l}^{3}-\delta_{j}^{3} \delta_{l}^{1}\right)\right) \\
= & r \cos \phi\left(k_{1} \delta_{i}^{3} \delta_{l}^{2}-k_{2} \delta_{i}^{2} \delta_{l}^{3}\right)+r \sin \phi\left(-k_{1} \delta_{i}^{3} \delta_{l}^{1}+k_{2} \delta_{i}^{1} \delta_{l}^{3}\right)
\end{aligned}
$$

und

$$
\begin{aligned}
\Omega_{i j} K_{j m} \Omega_{m l}= & \left(r \cos \phi\left(\delta_{i}^{3} \delta_{j}^{2}-\delta_{i}^{2} \delta_{j}^{3}\right)+r \sin \phi\left(\delta_{i}^{1} \delta_{j}^{3}-\delta_{i}^{3} \delta_{j}^{1}\right)\right) \\
& \cdot\left(r \cos \phi\left(k_{1} \delta_{j}^{3} \delta_{l}^{2}-k_{2} \delta_{j}^{2} \delta_{l}^{3}\right)+r \sin \phi\left(-k_{1} \delta_{j}^{3} \delta_{l}^{1}+k_{2} \delta_{j}^{1} \delta_{l}^{3}\right)\right) \\
= & -r^{2} \cos ^{2} \phi\left(k_{1} \delta_{i}^{2} \delta_{l}^{2}+k_{2} \delta_{i}^{3} \delta_{l}^{3}\right)-r^{2} \sin ^{2} \phi\left(k_{1} \delta_{i}^{1} \delta_{l}^{1}+k_{2} \delta_{i}^{3} \delta_{l}^{3}\right) \\
& +r^{2} \sin \phi \cos \phi\left(k_{1} \delta_{i}^{2} \delta_{l}^{1}+k_{2} \delta_{i}^{1} \delta_{l}^{2}\right) .
\end{aligned}
$$

Wegen des Oberflächenelementes $d o=r d r d \phi$ hat man nur noch die Integrationen über die Integranden $r, r^{2} \sin \phi, r^{2} \cos \phi, r^{3} \sin ^{2} \phi, r^{3} \cos ^{2} \phi$ und $r^{3} \sin \phi \cos \phi$ in den Grenzen $r=0 \cdots R$ und $\phi=0 \cdots 2 \pi$ auszuführen. Wegen der Periodizität der Integranden gilt $\int_{0}^{2 \pi} \sin \phi d \phi=\int_{0}^{2 \pi} \cos \phi d \phi=\int_{0}^{2 \pi} \sin \phi \cos \phi d \phi=0$, und die Integrale der Komponenten von $\boldsymbol{B}$ verschwinden ganz. Mit $\boldsymbol{B}=\mathbf{0}$ existiert also wieder ein Widerstandszentrum im Koordinatenursprung d. h. in der Scheibenmitte. Mit $\int_{0}^{2 \pi} \sin ^{2} \phi d \phi=\int_{0}^{2 \pi} \cos ^{2} \phi d \phi=1-\int_{0}^{2 \pi} \sin ^{2} \phi d \phi=\pi$ und $\int_{0}^{R} r^{3} d r=R^{4} / 4$ erhält man sofort

$$
A_{i j}=\pi R^{2}\left(k_{1} \delta_{i}^{3} \delta_{j}^{3}+k_{2}\left(\delta_{i}^{1} \delta_{j}^{1}+\delta_{i}^{2} \delta_{j}^{2}\right)\right)
$$

und

$$
\begin{aligned}
C_{i j} & =\pi \frac{R^{4}}{4}\left(\left(k_{1} \delta_{i}^{2} \delta_{j}^{2}+k_{2} \delta_{i}^{3} \delta_{j}^{3}\right)+\left(k_{1} \delta_{i}^{1} \delta_{j}^{1}+k_{2} \delta_{i}^{3} \delta_{j}^{3}\right)\right) \\
& =\pi \frac{R^{4}}{4}\left(k_{1}\left(\delta_{i}^{1} \delta_{j}^{1}+\delta_{i}^{2} \delta_{j}^{2}\right)+2 k_{2} \delta_{i}^{3} \delta_{j}^{3}\right) .
\end{aligned}
$$

Die Matrix zur Bestimmung der Achsen konstanter Helizität

$$
(\boldsymbol{A C})_{i j}=k_{1} k_{2} \frac{\pi^{2} R^{6}}{4}\left(\delta_{i}^{1} \delta_{j}^{1}+\delta_{i}^{2} \delta_{j}^{2}+2 \delta_{i}^{3} \delta_{j}^{3}\right)
$$


ist nun wieder diagonal, d. h. abgesehen von der zu erwartenden Entartung in der $x y$-Ebene können wieder die Koordinatenachsen als funktionelle Achsen betrachtet werden.

\subsubsection{Vergleich von Quadrat und Kreis}

Eine der im Federmodell implizit enthaltenen Aussagen ist, daß die elastischen Eigenschaften im wesentlichen von der Fläche, nicht aber von deren Form abhängen. Ein Vergleich von Quadrat und Kreisscheibe, also

$$
\boldsymbol{A}_{\square}=a^{2}\left(\begin{array}{ccc}
k_{2} & 0 & 0 \\
0 & k_{2} & 0 \\
0 & 0 & k_{1}
\end{array}\right) \longleftrightarrow \boldsymbol{A}_{\circ}=\pi R^{2}\left(\begin{array}{ccc}
k_{2} & 0 & 0 \\
0 & k_{2} & 0 \\
0 & 0 & k_{1}
\end{array}\right)
$$

und

$$
\boldsymbol{C}_{\square}=\frac{a^{4}}{12}\left(\begin{array}{ccc}
k_{1} & 0 & 0 \\
0 & k_{1} & 0 \\
0 & 0 & 2 k_{2}
\end{array}\right) \quad \longleftrightarrow \boldsymbol{C}_{\circ}=\pi \frac{R^{4}}{4}\left(\begin{array}{ccc}
k_{1} & 0 & 0 \\
0 & k_{1} & 0 \\
0 & 0 & 2 k_{2}
\end{array}\right)
$$

ergibt bei gleichen Flächen $a^{2}=\pi R^{2}$ eine vollständige Übereinstimmung in den Matrizen $\boldsymbol{A}_{\square}$ und $\boldsymbol{A}_{\circ}$. Daraus ergeben sich für die Matrizen $\boldsymbol{C}_{\square}$ und $\boldsymbol{C}_{\circ}$ die Vorfaktoren $\frac{a^{4}}{12}=\pi^{2} \frac{R^{4}}{12} \approx \pi \frac{R^{4}}{4}$. Der Unterschied von $\frac{\pi^{2}}{12} \approx 0.82 \mathrm{zu} \frac{\pi}{4} \approx 0.79$ liegt bei weniger als $5 \%$. In diesem Fall also gilt die Übereinstimmung der Steifheitsmatrizen näherungsweise sogar für endliche Flächen, und nicht nur, wie für das Federmodell erforderlich, für infinitesimale Flächenelemente.

\subsubsection{Eine Aufhängung ohne Widerstandszentrum}

Eine weitere einfache Möglichkeit, zu Steifheitsmatrizen zu gelangen, bietet die Superposition von bekannten Steifheitsmatrizen der Teilaufhängungen.

In diesem Abschnitt soll eine aus Aufhängungen mit Widerstandszentrum zusammengesetzte Anordnung besprochen werden, welche selbst kein Widerstandszentrum mehr besitzt. Nach dem im Kapitel 4.5 über zusammengesetzte Aufhängungen gesagten muß folgendes berücksichtigt sein:

- Die Komponenten dürfen sich nicht ähnlich sein, also beispielsweise weder isotrop (d. h. $\boldsymbol{A}=a \boldsymbol{I}$ ) noch eben und gleich vorgespannt,

- die einzelnen Widerstandszentren müssen verschieden sein und

- die Anordnung darf keine einfache Symmetrie aufweisen. 
Das läßt sich wie folgt realisieren: Die Anordnung besteht aus zwei Federpaaren die gegeneinander $90^{\circ}$ verdreht und verschoben sind. Ihre Widerstandszentren liegen bei $\left(0,0, z_{1}\right)$ und $\left(0,0, z_{2}\right)$ und ihre Federn weisen in $x$-bzw. $y$-Richtung. Durch die unterschiedliche Ausrichtung der Komponenten ist die Anordnung insgesamt räumlich anisotrop. Rein geometrisch liegt ihr ein Paar von Kräften mit windschiefen Wirkungslinien zu Grunde. Diese Anordnung wurde, mit ihren qualitativen Eigenschaften, schon in Abschnitt 2.2.4.2 mit den Abbildungen 2.3 und 2.4 vorgestellt. Hier soll nun die Rechnung folgen.

Es soll die Gleichung (4.32) zur Überprüfung der Existenz eines Widerstandszentrums verwendet werden, d. h. es sind lediglich $\boldsymbol{A}_{1,2}$ und $\boldsymbol{\Omega}_{1,2}$ zu berechnen.

Mit den Teilmatrizen

$$
\begin{array}{rlr}
\boldsymbol{A}_{1}=\left(\begin{array}{ccc}
k_{1} & 0 & 0 \\
0 & k_{2} & 0 \\
0 & 0 & k_{2}
\end{array}\right) & \text { und } & \boldsymbol{\Omega}_{1}=\left(\begin{array}{ccc}
0 & -z_{1} & 0 \\
z_{1} & 0 & 0 \\
0 & 0 & 0
\end{array}\right) \\
\boldsymbol{A}_{2}=\left(\begin{array}{ccc}
k_{2} & 0 & 0 \\
0 & k_{1} & 0 \\
0 & 0 & k_{2}
\end{array}\right) & \text { und } & \Omega_{1}=\left(\begin{array}{ccc}
0 & -z_{2} & 0 \\
z_{2} & 0 & 0 \\
0 & 0 & 0
\end{array}\right)
\end{array}
$$

in $x$ - und $y$-Richtung folgt, eingesetzt in die Gleichung (4.32), daß die Matrix

$$
\begin{aligned}
\boldsymbol{A}_{1}\left(\boldsymbol{\Omega}_{1}-\boldsymbol{\Omega}_{2}\right) \boldsymbol{A}_{2} & =\left(\begin{array}{ccc}
k_{1} & 0 & 0 \\
0 & k_{2} & 0 \\
0 & 0 & k_{2}
\end{array}\right)\left(\begin{array}{ccc}
0 & -z_{1}+z_{2} & 0 \\
z_{1}-z_{2} & 0 & 0 \\
0 & 0 & 0
\end{array}\right)\left(\begin{array}{ccc}
k_{2} & 0 & 0 \\
0 & k_{1} & 0 \\
0 & 0 & k_{2}
\end{array}\right) \\
& =\left(\begin{array}{ccc}
k_{1} & 0 & 0 \\
0 & k_{2} & 0 \\
0 & 0 & k_{2}
\end{array}\right)\left(\begin{array}{ccc}
0 & -k_{1} & 0 \\
k_{2} & 0 & 0 \\
0 & 0 & 0
\end{array}\right)\left(z_{1}-z_{2}\right) \\
& =\left(\begin{array}{ccc}
0 & -k_{1}^{2} & 0 \\
k_{2}^{2} & 0 & 0 \\
0 & 0 & 0
\end{array}\right)\left(z_{1}-z_{2}\right)
\end{aligned}
$$

nur für $k_{1}=k_{2}$ antisymmetrisch ist, oder für $z_{1}=z_{2}$ sogar ganz verschwindet. Realisiert man nun die Aufhängungen so, daß keine dieser beiden Bedingungen erfüllt ist, so erhält man die gewünschte Anordnung ohne Widerstandszentrum.

Die Blöcke der Steifheitsmatrix ergeben sich durch Addition zu

$$
\begin{aligned}
\boldsymbol{A} & =\boldsymbol{A}_{1}+\boldsymbol{A}_{2}=\left(\begin{array}{ccc}
k_{1}+k_{2} & 0 & 0 \\
0 & k_{1}+k_{2} & 0 \\
0 & 0 & 2 k_{2}
\end{array}\right) \\
\boldsymbol{B} & =-\boldsymbol{A}_{1} \boldsymbol{\Omega}_{1}-\boldsymbol{A}_{2} \boldsymbol{\Omega}_{2} \\
& =-\left(\begin{array}{ccc}
0 & -k_{1} z_{1} & 0 \\
k_{2} z_{1} & 0 & 0 \\
0 & 0 & 0
\end{array}\right)-\left(\begin{array}{ccc}
0 & -k_{2} z_{2} & 0 \\
k_{1} z_{2} & 0 & 0 \\
0 & 0 & 0
\end{array}\right)
\end{aligned}
$$




$$
\begin{aligned}
= & \left(\begin{array}{ccc}
0 & k_{1} z_{1}+k_{2} z_{2} & 0 \\
-\left(k_{2} z_{1}+k_{1} z_{2}\right) & 0 & 0 \\
0 & 0 & 0
\end{array}\right) \\
\boldsymbol{C}= & -\boldsymbol{\Omega}_{1} \boldsymbol{A}_{1} \boldsymbol{\Omega}_{1}-\boldsymbol{\Omega}_{2} \boldsymbol{A}_{2} \Omega_{2} \\
= & -\left(\begin{array}{ccc}
0 & -z_{1} & 0 \\
z_{1} & 0 & 0 \\
0 & 0 & 0
\end{array}\right)\left(\begin{array}{ccc}
0 & -k_{1} z_{1} & 0 \\
k_{2} z_{1} & 0 & 0 \\
0 & 0 & 0
\end{array}\right) \\
& -\left(\begin{array}{ccc}
0 & -z_{1} & 0 \\
z_{1} & 0 & 0 \\
0 & 0 & 0
\end{array}\right)\left(\begin{array}{ccc}
0 & -k_{2} z_{2} & 0 \\
k_{1} z_{2} & 0 & 0 \\
0 & 0 & 0
\end{array}\right) \\
= & \left(\begin{array}{ccc}
k_{2} z_{1}^{2}+k_{1} z_{2}^{2} \\
0 & k_{1} z_{1}^{2}+k_{2} z_{2}^{2} & 0 \\
0 & 0
\end{array}\right),
\end{aligned}
$$

wobei $C_{33}=0$ eine Folge der auf der $z$-Achse angenommenen Enden der Federn ist. In dieser Feinheit stimmen realisiertes Modell und Rechnung demnach nicht überein. Das elastische Zentrum liegt aus Symmetriegründen in der Mitte bei $z=$ $\frac{z_{1}+z_{2}}{2}$. Mit $z_{1}^{\prime}:=z_{1}-z$ und $z_{2}^{\prime}:=z_{2}-z$ gilt dort $z_{1}^{\prime}=-z_{2}^{\prime}=\frac{z_{1}-z_{2}}{2}$. Man erhält so

$$
\boldsymbol{B}_{Z}=\frac{z_{1}-z_{2}}{2}\left(\begin{array}{ccc}
0 & k_{1}-k_{2} & 0 \\
-k_{2}+k_{1} & 0 & 0 \\
0 & 0 & 0
\end{array}\right)=\frac{1}{2}\left(z_{1}-z_{2}\right)\left(k_{1}-k_{2}\right)\left(\begin{array}{ccc}
0 & 1 & 0 \\
1 & 0 & 0 \\
0 & 0 & 0
\end{array}\right)
$$

und mit

$$
\begin{aligned}
\boldsymbol{W}_{Z} & =-\left(\boldsymbol{A}^{-1} \boldsymbol{B}\right)^{S}=-\boldsymbol{A}^{-1} \boldsymbol{B} \\
& =\frac{1}{2}\left(z_{1}-z_{2}\right)\left(k_{1}-k_{2}\right)\left(\begin{array}{ccc}
\left(k_{1}+k_{2}\right)^{-1} & 0 & 0 \\
0 & \left(k_{1}+k_{2}\right)^{-1} & 0 \\
0 & 0 & \left(2 k_{2}\right)^{-1}
\end{array}\right)\left(\begin{array}{ccc}
0 & 1 & 0 \\
1 & 0 & 0 \\
0 & 0 & 0
\end{array}\right) \\
& =\frac{z_{1}-z_{2}}{2} \frac{k_{1}-k_{2}}{k_{1}+k_{2}}\left(\begin{array}{ccc}
0 & 1 & 0 \\
1 & 0 & 0 \\
0 & 0 & 0
\end{array}\right)
\end{aligned}
$$

lassen sich wie folgt Aufpunktfläche und Abstandsfläche berechnen. Das Rechtssystem der Matrix $\boldsymbol{W}_{Z}$ aus Eigenvektoren ist

$$
\boldsymbol{e}_{1}=\frac{1}{\sqrt{2}}\left(\begin{array}{c}
1 \\
-1 \\
0
\end{array}\right), \quad \boldsymbol{e}_{2}=\frac{1}{\sqrt{2}}\left(\begin{array}{l}
1 \\
1 \\
0
\end{array}\right) \quad \text { und } \quad \boldsymbol{e}_{3}=\left(\begin{array}{l}
0 \\
0 \\
1
\end{array}\right),
$$

und die zugehörigen Eigenwerte sind mit $\lambda:=\frac{z_{1}-z_{2}}{2} \frac{k_{1}-k_{2}}{k_{1}+k_{2}}$

$$
w_{1}=-\lambda, \quad w_{2}=\lambda \quad \text { und } \quad w_{3}=0
$$

Das System der Hauptachsen ergibt sich aus den kartesischen Koordinatenachsen durch eine Drehung um $\boldsymbol{e}_{z}$ um $45^{\circ}$. Insbesondere ist es möglich, den Längenmaßstab 
so zu wählen, daß ein bestimmter Zahlenwert für $\lambda$ eingenommen wird, etwa $\lambda=1$. Aus $w_{1}=-1, w_{2}=1$ und $w_{3}=0$ folgt $w_{32}=-1, w_{13}=-1$ und $w_{21}=2$. Mit $\overrightarrow{D S}=x \boldsymbol{e}_{1}+y \boldsymbol{e}_{2}+z \boldsymbol{e}_{3}$ ergeben sich im neuen Koordinatensystem die folgenden Gleichungen

$$
\overrightarrow{D S} \stackrel{(2.45)}{=} \pm \frac{1}{2}\left(\begin{array}{c}
-\sin \phi \sin 2 \theta \\
-\cos \phi \sin 2 \theta \\
2 \sin 2 \phi \cos ^{2} \theta
\end{array}\right)=-\overrightarrow{T K}
$$

für die Aufpunktfläche, s. Abb. 2.5.

$$
\begin{aligned}
\|\overrightarrow{Z A}\|^{2} \stackrel{(2.48)}{=} & \frac{1}{4} \sin ^{2} \phi \sin ^{2} 2 \theta+\frac{1}{4} \cos ^{2} \phi \sin ^{2} 2 \theta+\sin ^{2} 2 \phi \cos ^{4} \theta \\
= & \frac{1}{4} \sin ^{2} 2 \theta+\sin ^{2} 2 \phi \cos ^{4} \theta
\end{aligned}
$$

für die Abstandsfläche, s. Abb. 2.6, und für die Steigungsfläche die Gleichung

$$
\tau_{M} \stackrel{(2.52)}{=}\left(-\cos ^{2} \phi \cos ^{2} \theta+\sin ^{2} \phi \cos ^{2} \theta\right)=-\cos ^{2} \theta \cos 2 \phi=-\tau_{L},
$$

mit den zugehörigen Abbildungen 2.7 und 2.8.

Die Untersuchung einer Aufhängung mit $C_{33} \neq 0$ ergibt keine prinzipiell neuen Eigenschaften, sondern erschwert höchstens die Rechnung, da diese aus vier Teilaufhängungen zusammengesetzt ist.

\subsubsection{Die rechteckige Scheibe mit variabler Schichtdicke}

Die einfachste Methode die erforderlichen Integrationen durchzuführen besteht in der Verwendung eines Computeralgebrasystems, etwa ReduCE oder MATHEMATICA. Die von mir verwendeten Prozeduren für Mathematica sind in Anhang A.3 beschrieben. Sie werden eingelesen mit

$$
\begin{aligned}
& <<\mathrm{d}: \backslash \text { math } \backslash \text { mproc } \\
& <<\mathrm{d}: \backslash \text { math } \backslash \text { mintproc }
\end{aligned}
$$

und erlauben verschiedene Lösungswege, von denen ich einen am Beispiel der rechteckigen Scheibe mit veränderlicher Schichtdicke vorführen möchte. Die Bezeichnungen werden dabei aus Abschnitt 5.1.1 übernommen.

Zunächst sind der Normalenvektor $\boldsymbol{n}$ der Schicht und der Aufpunktvektor $\boldsymbol{\xi}$

$$
\begin{aligned}
& \mathrm{n}=\operatorname{DVec}[0,0,1] \\
& \mathrm{Xi}=\operatorname{Vektor}[\mathrm{X}]
\end{aligned}
$$

mit 
$\{\{0\},\{0\},\{1\}\}$

$\{\{X x\},\{X y\},\{X z\}\}$

als Output zu definieren. Die zu $\boldsymbol{\xi}$ gehörende antisymmetrische Matrix $\Omega_{\xi}$ wird über

OmXi=Meps $[\mathrm{Xi}]$

definiert. Die Schichtdicke $h(\boldsymbol{\xi})$ variiere nur in $x$-Richtung von $h(-a / 2)=d$ bis $h(a / 2)=D$. Schichtdicke und Federmatrix sind also Funktionen

$$
\begin{aligned}
& \mathrm{h}\left[\mathrm{x}_{-}\right]:=(\mathrm{d}+\mathrm{D}) / 2+\mathrm{x}(\mathrm{D}-\mathrm{d}) /(2 \mathrm{a}) \\
& \mathrm{K}\left[\mathrm{x}_{-}\right]:=\text {FedIsoMat }[\mathrm{n}, \mathrm{k} 1 / \mathrm{h}[\mathrm{x}], \mathrm{k} 2 / \mathrm{h}[\mathrm{x}]]
\end{aligned}
$$

der Variable $x$. Die Matrix $\boldsymbol{A}$ bekommt man nun einfach über

$$
\begin{aligned}
& \text { aint=Integrate }[\mathrm{K}[\mathrm{Xx}], \quad\{\mathrm{Xx},-\mathrm{a} / 2, \mathrm{a} / 2\}] \\
& \mathrm{A}=\text { Simplify [Integrate[aint }, \quad\{\mathrm{Xy},-\mathrm{b} / 2, \mathrm{~b} / 2\}]]
\end{aligned}
$$

mit dem Ergebnis

$$
\boldsymbol{A}=\frac{a b}{D}\left(\begin{array}{ccc}
k_{2} & 0 & 0 \\
0 & k_{2} & 0 \\
0 & 0 & k_{1}
\end{array}\right) f(d / D)
$$

wobei die Funktion

$$
f(x):=2 \frac{\ln \frac{3 x+1}{x+3}}{x-1}
$$

eine mittels

$$
\operatorname{Limit}[2 \log [(3 x+1) /(x+3)] /(x-1), x->1]
$$

berechenbare hebbare Unstetigkeit bei $x=1$ mit $f(x \rightarrow 1)=1$ besitzt. Dieser Grenzfall ist genau der einer Scheibe mit konstanter Dicke. Ebenso erhält man die Matrix $\boldsymbol{B}$ über

$$
\begin{aligned}
& \text { bint=Integrate }[-0 m X i . K[X x],\{X x,-a / 2, a / 2\}] \\
& \text { B=Simplify [Integrate[bint, }\{X y,-b / 2, b / 2\}] / / . \mathrm{Xz}->0]
\end{aligned}
$$

nach einigen Vereinfachungen per Hand zu

$$
\boldsymbol{B}=\frac{a^{2} b}{D}\left(\begin{array}{ccc}
0 & 0 & 0 \\
0 & 0 & k_{1} \\
0 & -k_{2} & 0
\end{array}\right) g(d / D)
$$


mit

$$
g(x):=2 \frac{1-x+(1+x) \ln \frac{3 x+1}{x+3}}{(1-x)^{2}} .
$$

Für den Spezialfall konstanter Schichtdicke verschwindet wegen $g(x \rightarrow 1)=0$ die Gesamtmatrix, und es existiert ein Widerstandszentrum. Ist $d \neq D$, so liegt das elastische Zentrum nicht mehr im Ursprung, sondern mit

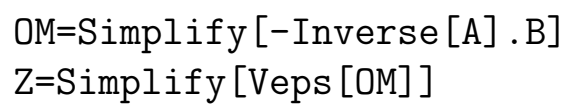

in $x$-Richtung verschoben bei

$$
Z_{x}=\frac{1}{2} \frac{g(d / D)}{f(d / D)} a \frac{k_{1}^{2}+k_{2}^{2}}{k_{1} k_{2}} .
$$

Entweder mit

$$
\begin{aligned}
& W=\text { Simplify }[\operatorname{Sym}[\mathrm{OM}]] \\
& \text { Series }[W,\{d, D, 1\}]
\end{aligned}
$$

oder per Hand an

$$
-\boldsymbol{A}^{-1} \boldsymbol{B}=-\frac{g(x)}{f(x)} a\left(\begin{array}{ccc}
0 & 0 & 0 \\
0 & 0 & k_{1} / k_{2} \\
0 & -k_{2} / k_{1} & 0
\end{array}\right)
$$

erkennt man, daß diese doch noch recht einfache, fast ebene Aufhängung kein Widerstandszentrum mehr besitzt.

Der Vollständigkeit halber erhält man für die Matrix $\boldsymbol{C}$ Diagonalform mit $x=d / D$

$$
\begin{aligned}
C_{11} & =\frac{a b^{3}}{12 D} k_{1} f(x) \\
C_{22} & =\frac{a^{3} b}{k_{1}} \frac{x+1}{x-1} g(x) \\
C_{33} & =\frac{a b^{3}}{12 D} k_{2} f(x)+\frac{a^{3} b}{k} \frac{x+1}{2 x-1} g(x),
\end{aligned}
$$

welche wegen

$$
\lim _{x \rightarrow 1} \frac{x+1}{x-1} g(x)=\frac{1}{12}
$$

wieder eine hebbare Unstetigkeit mit dem korrekten Grenzwert hat.

Insgesamt zeigt sich, daß schon einfache Annahmen über die Aufhängung zu recht komplizierten Ausdrücken für die Steifheitsmatrix führen. 


\subsubsection{Flächen- und Dickenkorrektur}

Wegen der komplizierten Form der Ausdrücke sind ihre linearen Näherungen von Interesse. Es ergibt sich

$$
\begin{aligned}
\boldsymbol{A} & \approx \frac{a b}{D}\left(\begin{array}{ccc}
k_{2} & 0 & 0 \\
0 & k_{2} & 0 \\
0 & 0 & k_{1}
\end{array}\right)\left(1-\frac{d-D}{2 D}\right) \\
\boldsymbol{B} & \approx \frac{a^{2} b}{24 D}\left(\begin{array}{ccc}
0 & 0 & 0 \\
0 & 0 & k_{1} \\
0 & -k_{2} & 0
\end{array}\right) \frac{d-D}{D} \\
\boldsymbol{C} & \approx \frac{a b}{12}\left(\begin{array}{ccc}
k_{1} b^{2} & 0 & 0 \\
0 & k_{1} a^{2} & 0 \\
0 & 0 & k_{2}\left(a^{2}+b^{2}\right)
\end{array}\right)\left(1-\frac{d-D}{2 D}\right) \\
\boldsymbol{\Omega}_{\xi} & \approx \frac{a}{24}\left(\begin{array}{ccc}
0 & 0 & 0 \\
0 & 0 & -k_{1} / k_{2} \\
0 & k_{2} / k_{1} & 0
\end{array}\right) \frac{d-D}{D} .
\end{aligned}
$$

Diese Ergebnisse sollen nun mit den Korrekturen nach den Ähnlichkeitsbetrachtungen aus Abschnitt 4.3.2 verglichen werden. Nach (4.11) geschieht das durch die Ersetzungen $F_{H}$ und $d_{A}$ im Ergebnis für die rechteckige Scheibe mit konstanter Schichtdicke.

Für das harmonische Mittel von Grund- und Deckfläche ergibt sich mit dem Satz des Pythagoras

$$
F_{G}=a b \quad \text { und } \quad F_{D}=b \sqrt{a^{2}+\left(D-d^{2}\right)}=a b \sqrt{1+\left(\frac{D-d}{a}\right)^{2}}
$$

also

$$
F_{H}=\frac{2 F_{G} F_{D}}{F_{G}+F_{D}}=a b \frac{2 \sqrt{1+\left(\frac{D-d}{a}\right)^{2}}}{1+\sqrt{1+\left(\frac{D-d}{a}\right)^{2}}}
$$

und bis auf Terme vierter Ordnung

$$
F_{H}=a b\left(1+\frac{1}{4}\left(\frac{D-d}{a}\right)^{2}\right) .
$$

Die etwas vergrößerte Deckfläche ruft demnach nur Effekte zweiter Ordnung hervor.

Das arithmetische Mittel der Dicken ist

$$
d_{A}=\frac{D+d}{2}=\frac{2 D+(d-D)}{2}=D\left(1+\frac{d / D-1}{2}\right)
$$


und bis zu quadratischen Termen hin entwickelt ergibt sich

$$
\frac{1}{d_{A}}=\frac{1}{D}\left(1-\frac{1}{2}(d / D-1)+\frac{1}{4}(d / D-1)^{2}\right)
$$

Die Vorfaktoren bei $\boldsymbol{A}$ und $\boldsymbol{C}$ in der linearen Näherung können daher wegen

$$
1-\frac{d-D}{2 D}=1-\frac{1}{2}(d / D-1)
$$

ohne Integrationen allein aus der Betrachtung der veränderlichen Schichtdicke abgeleitet werden. Sie erklären damit die Terme bis hin zur linearen Näherung, womit die Überlegungen des Abschnitts 4.3.2 am Beispiel bestätigt sind.

Durch dieses Beispiel ist klar, daß mit komplizierten Dickefunktionen $d(\boldsymbol{\xi})$ nur selten geschlossene Lösungen zu erwarten sind. Dem könnte man aber für empirische Dickefunktionen möglicherweise dadurch begegnen, die Funktion 1/d geeignet zu approximieren.

\subsubsection{Ebene Aufhängungen mit konstanten Schichtgrößen}

Im Gegensatz zu den in der Einleitung behandelten zweidimensionalen Synarthrosen, welche durch eine Linie verbunden werden, verbindet eine ebene Aufhängung zwei Körper durch eine ebene Fläche. Die Oberflächenintegrale sind dann Integrale über ebene Gebiete.

Sind die Berandungen der Flächen in rechtwinkligen Koordinaten gegeben, also $x \in[a, b]$ und $y \in[c(x), d(x)]$, so ergeben sich mit dem Satz von Fubini und dem Prinzip des CAVALIERI die Integrale als gewöhnliche Doppelintegrale über ein ebenes Gebiet zu

$$
\oiiint * d o=\int_{x=a}^{b}\left(\int_{y=c(x)}^{d(x)} * d y\right) d x
$$

wobei $*$ der jeweilige matrixwertige Integrand ist. Die rechteckige Scheibe ist genau in dieser Weise berechnet worden, wobei dann $c(x)$ und $d(x)$ einfach Konstanten sind.

Für konstante Schichtgrößen, also falls überall dasselbe Material mit derselben Dicke vorliegt, kann die Federmatrix wegen $\boldsymbol{K}=$ const aus den Integralen herausgezogen werden. Da die Integration dann nur noch über

$$
\begin{aligned}
\boldsymbol{A} & =\boldsymbol{K} \iint d x d y=\boldsymbol{K} O \\
\boldsymbol{B} & =-\boldsymbol{K}\left(\iint \boldsymbol{\Omega}_{\xi} d x d y\right)=-\boldsymbol{K}\left(\iint \boldsymbol{\xi} d x d y\right)^{D} \\
(\boldsymbol{C})_{i l} & =-\left(\iint \boldsymbol{\Omega}_{\xi} \boldsymbol{K} \boldsymbol{\Omega}_{\xi} d x d y\right)_{i l}=-\varepsilon_{i m j} \varepsilon_{k n l}(\boldsymbol{K})_{j k} \iint \xi_{m} \xi_{n} d x d y
\end{aligned}
$$


erfolgt, sind rechentechnisch nur die Momente der „Dichte“ $\boldsymbol{\xi}$ bis hin zum zweiten zu berechnen. Das elastische Zentrum ist für diesen Spezialfall, vgl. auch B.2.3, tatsächlich gleich dem Flächenschwerpunkt

$$
\boldsymbol{z}=-\left(\boldsymbol{A}^{-1} \boldsymbol{B}\right)^{D}=\frac{\iint \boldsymbol{\xi} d x d y}{\iint d x d y}
$$

Insbesondere kann an der Formel für $\boldsymbol{B}$ abgelesen werden, daß für eine punktsymmetrische Fläche ein Widerstandszentrum im Symmetriezentrum existiert.

Die Berechnung der Momente über das Gebiet $G$ kann noch weiter vereinfacht werden, wenn eine Parameterdarstellung des Randes $\partial G$ vorliegt, etwa

$$
\left.\left(\begin{array}{l}
x \\
y
\end{array}\right)\right|_{\partial G}=\left(\begin{array}{c}
f(\varphi) \\
g(\varphi)
\end{array}\right),
$$

und das Gebiet sternförmig ist. Mit dem allgemeinen Satz von STOKES $\int_{G} \partial \omega=$ $\int_{\partial G} \omega$ erhält man dann etwa die folgenden Formeln:

$$
\begin{aligned}
\iint_{G} d x d y & =\oint_{\partial G} x d y=\int_{\varphi} f g_{, \varphi} d \varphi \\
\iint_{G} x d x d y & =\oint_{\partial G} \frac{1}{2} x^{2} d y=\frac{1}{2} \int_{\varphi} f^{2} g_{, \varphi} d \varphi \\
\iint_{G} y d x d y & =\oint_{\partial G} x y d y=\int_{\varphi} f g g_{, \varphi} d \varphi \\
\iint_{G} x^{2} d x d y & =\oint_{\partial G} \frac{1}{3} x^{3} d y=\frac{1}{3} \int_{\varphi} f^{3} g_{, \varphi} d \varphi \\
\iint_{G} y^{2} d x d y & =\oint_{\partial G} x y^{2} d y=\int_{\varphi} f g^{2} g_{, \varphi} d \varphi \\
\iint_{G} x y d x d y & =\oint_{\partial G} \frac{1}{2} x^{2} y d y=\frac{1}{2} \int_{\varphi} f^{2} g g_{, \varphi} d \varphi .
\end{aligned}
$$

Die Bestimmung der Einträge der Steifheitsmatrix ist damit auf eine Integration von Funktionen einer reellen Variablen über den Rand des Gebietes zurückgeführt! Auch für nicht-konstante Federmatrizen kann die Anwendung des Stokesschen Satzes vorteilhaft sein.

Mit diesen Gleichungen ist die allgemeine Gestalt der Steifheitsmatrix einer ebenen Aufhängung wie folgt gegeben: Mit den Abkürzungen für Fläche, elastisches Zentrum und zweites Moment

$$
\begin{aligned}
\iint_{G} d x d y & =: \quad O \\
\iint_{G} \boldsymbol{\xi} d x d y & =: O \quad\left(\begin{array}{c}
z_{1} \\
z_{2}
\end{array}\right) \\
\iint_{G} \xi_{m} \xi_{n} d x d y & =: \quad c_{1} \delta_{m}^{1} \delta_{n}^{1}+c_{2} \delta_{m}^{2} \delta_{n}^{2}+c_{3}\left(\delta_{m}^{1} \delta_{n}^{2}+\delta_{m}^{2} \delta_{n}^{1}\right)
\end{aligned}
$$

\footnotetext{
${ }^{1}$ Das scheint zunächst sonderbar, da in Abschnitt 4.4.3 genau die Randanteile vernachlässigt wurden. Hier erhält man über die zur Rechnung nötigen Ableitungen auf dem Rand Kenntnis über das Innere des Gebietes.
} 
erhält man mit dem Tensorformalismus 5.1.2 nach ein wenig Rechnung die Matrizen

$$
\begin{aligned}
& \boldsymbol{A}=O\left(\begin{array}{ccc}
k_{2} & 0 & 0 \\
0 & k_{2} & 0 \\
0 & 0 & k_{1}
\end{array}\right) \\
& \boldsymbol{B}=O\left(\begin{array}{ccc}
0 & 0 & -z_{2} k_{2} \\
0 & 0 & z_{1} k_{2} \\
z_{2} k_{1} & -z_{1} k_{1} & 0
\end{array}\right) \\
& \boldsymbol{C}=\left(\begin{array}{ccc}
c_{2} k_{1} & -c_{3} k_{1} & 0 \\
-c_{3} k_{1} & c_{1} k_{1} & 0 \\
0 & 0 & \left(c_{1}+c_{2}\right) k_{2}
\end{array}\right)
\end{aligned}
$$

welche die Frage nach der Struktur der elastischen Matrix einer ebenen sternförmigen Aufhängung mit konstanten Schichtgrößen beantwortet. Die Transformation auf das Widerstandszentrum läßt $\boldsymbol{B}$ verschwinden und ändert die Komponenten von $C \mathrm{zu}$

$$
\begin{aligned}
c_{2} k_{1} & \mapsto k_{1}\left(c_{2}-O z_{2}^{2}\right) \\
c_{1} k_{1} & \mapsto k_{1}\left(c_{1}-O z_{1}^{2}\right) \\
\left(c_{1}+c_{2}\right) k_{2} & \mapsto k_{2}\left(c_{1}+c_{2}-O z_{1}^{2}-O z_{2}^{2}\right) \\
-c_{3} k_{1} & \mapsto k_{1}\left(-c_{3}+O z_{1} z_{2}\right) .
\end{aligned}
$$

Insbesondere bleibt das Verhältnis

$$
\frac{C_{33}}{C_{11}+C_{22}}=\frac{k_{2}}{k_{1}}=v
$$

unter Translationen ungeändert.

Ist eine Gleichung des Randes $\partial G$ in Polarkoordinaten als $R(\varphi)$ gegeben, so ist

$$
\left.\left(\begin{array}{l}
x \\
y
\end{array}\right)\right|_{\partial G}=\left(\begin{array}{c}
R(\varphi) \cos \varphi \\
R(\varphi) \sin \varphi
\end{array}\right)
$$

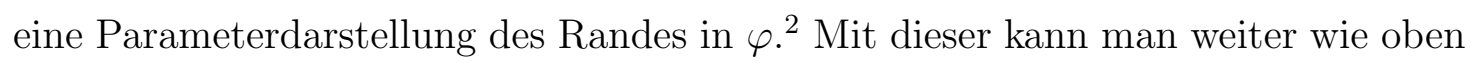
verfahren. Ist darüber hinaus das Gebiet bezüglich des Koordinatenursprunges sternförmig, dann stellt

$$
\left(\begin{array}{l}
x \\
y
\end{array}\right)=\left(\begin{array}{l}
r R(\varphi) \cos \varphi \\
r R(\varphi) \sin \varphi
\end{array}\right)
$$

mit $r \in[0,1]$ eine Parametrisierung des gesamten Gebietes dar. Das erlaubt eine weitere Berechnungmethode der Integrale. Da die Funktionaldeterminate gegeben ist durch

$$
\frac{\partial(x, y)}{\partial(r, \varphi)}=R^{2} r
$$

\footnotetext{
${ }^{2}$ Es muß insbesondere gelten $\varphi=\arctan \frac{y}{x}$, was etwa für die Parameterdarstellung der Ellipse nicht zutrifft.
} 
können nun die $r$-Integrationen ausgeführt werden. Mit

$$
\xi^{0}:=\left(\begin{array}{c}
\cos \varphi \\
\sin \varphi \\
0
\end{array}\right)
$$

ergibt sich

$$
\begin{aligned}
\boldsymbol{A} & =\boldsymbol{K} \iint R^{2} r d r d \varphi=\frac{1}{2} \boldsymbol{K} \int R^{2} d \varphi \\
\boldsymbol{B} & =-\boldsymbol{K}\left(\iint \boldsymbol{\xi}^{0} R^{3} r^{2} d r d \varphi\right)^{D}=-\frac{1}{3} \boldsymbol{K}\left(\int \boldsymbol{\xi}^{0} R^{3} d \varphi\right)^{D} \\
(\boldsymbol{C})_{i l} & =-\varepsilon_{i m j} \varepsilon_{k n l}(\boldsymbol{K})_{j k} \iint \xi_{m}^{0} \xi_{n}^{0} R^{4} r^{3} d r d \varphi=-\frac{1}{4} \varepsilon_{i m j} \varepsilon_{k n l}(\boldsymbol{K})_{j k} \int \xi_{m}^{0} \xi_{n}^{0} R^{4} d \varphi .
\end{aligned}
$$

Wie bei der Benutzung des Satzes von Stokes vereinfachen sich Winkelintegrationen (wie im Beispiel der Kreisscheibe) meist aus Symmetriegründen erheblich; etwa ergibt eine Integration einer ungeraden Funktion über einen symmetrischen Bereich um den Ursprung einfach ohne Rechnung den Wert Null.

\subsubsection{Die Ellipse}

Normalenvektor und Federmatrix stimmen mit denen der Kreisscheibe überein. Die Berandung ist nun parametrisiert durch

$$
(x, y)=(a \cos \varphi, b \sin \varphi) \equiv\left(\begin{array}{l}
f \\
g
\end{array}\right)
$$

wobei $\varphi \in[0,2 \pi)$. Mit

$$
\begin{aligned}
\iint_{G} d x d y & =\int_{0}^{2 \pi} f g_{, \varphi} d \varphi=a b \int_{0}^{2 \pi} \cos ^{2} \varphi d \varphi=\pi a b \\
\iint_{G} x d x d y & =\frac{1}{2} \int_{0}^{2 \pi} f^{2} g_{, \varphi} d \varphi=\frac{1}{3} a^{2} b \int_{0}^{2 \pi} \cos ^{3} \varphi d \varphi=0 \\
\iint_{G} y d x d y & =\int_{0}^{2 \pi} f g g_{, \varphi} d \varphi=a b^{2} \int_{0}^{2 \pi} \cos ^{2} \varphi \sin \varphi d \varphi=0 \\
\iint_{G} x^{2} d x d y & =\frac{1}{3} \int_{0}^{2 \pi} f^{3} g_{, \varphi} d \varphi=a^{3} b \int_{0}^{2 \pi} \cos ^{4} \varphi d \varphi=\frac{\pi}{4} a^{3} b \\
\iint_{G} y^{2} d x d y & =\int_{0}^{2 \pi} f g^{2} g_{, \varphi} d \varphi=a b^{3} \int_{0}^{2 \pi} \cos ^{2} \varphi \sin ^{2} \varphi d \varphi=\frac{\pi}{4} a b^{3} \\
\iint_{G} x y d x d y & =\frac{1}{2} \int_{0}^{2 \pi} f^{2} g g_{, \varphi} d \varphi=\frac{1}{2} a^{2} b^{2} \int_{0}^{2 \pi} \cos ^{2} \varphi \sin \varphi d \varphi=0
\end{aligned}
$$

ergibt sich für $\boldsymbol{A}$ mit der eben berechneten Fläche der Ellipse

$$
A_{i j}=\pi a b\left(k_{2}\left(\delta_{i}^{1} \delta_{j}^{1}+\delta_{i}^{2} \delta_{j}^{2}\right)+k_{1} \delta_{i}^{3} \delta_{j}^{3}\right) \quad,
$$


und da die ersten Momente verschwinden folgt $\boldsymbol{B}=\mathbf{0}$, was auch aus Symmetriegründen klar ist. Für $\boldsymbol{C}$ erhält man, da die Matrizen $\boldsymbol{K}$ und die der zweiten Momente diagonal sind, nach einiger Rechnung

$$
C_{i j}=\pi \frac{a b}{4}\left(b^{2} k_{1} \delta_{i}^{1} \delta_{j}^{1}+a^{2} k_{1} \delta_{i}^{2} \delta_{j}^{2}+\left(a^{2}+b^{2}\right) k_{2} \delta_{i}^{3} \delta_{j}^{3}\right) \quad .
$$

Die Matrix $\boldsymbol{A C}$ zur Bestimmung der Achsen konstanter Helizität enthält den gemeinsamen Vorfaktor $k_{1} k_{2}$ und hängt somit nicht vom Material ab. Da auch sie diagonal ist, können wieder die Koordinatenachsen als funktionelle Achsen betrachtet werden - wie es zu erwarten war.

Der Vergleich mit einer rechteckigen Scheibe mit den Kantenlängen $a \sqrt{\pi}$ und $b \sqrt{\pi}$ ergibt wie in 5.1 .3 bei gleichen Flächen wieder die Faktoren $\frac{\pi^{2}}{12} \leftrightarrow \frac{\pi}{4}$, für die Matrix $\boldsymbol{C}$. Die Art der "Länglichkeit" $\frac{a}{b}$ des Bereiches hat demnach für endliche Bereiche keinen Einfluß auf die Federmatrix. Für infinitesimale Flächenelemente bedeutet das, daß die Wahl der Flächenparameter beliebig ist - wie es auch sein soll.

\subsubsection{Die Pascalsche Schnecke}

Die PAScalsche Schnecke ist gegeben durch die Gleichung

$$
R(\varphi)=a+b \cos \varphi
$$

Für $b<a<2 b$ liefert sie eine realistische erste Näherung zur Beschreibung der Form einer Bandscheibe, vgl. Abb. 5.1, die mit geeigneten Werten zu

$$
R(\varphi)=a+b \cos \varphi+c \cos 2 \varphi+d \cos 3 \varphi+\ldots
$$

verfeinert werden kann, vgl. etwa [8].

Mit den Ergebnissen der letzten Abschnitte erhält man

$$
\begin{aligned}
\boldsymbol{A} & =\frac{\pi}{2}\left(2 a^{2}+b^{2}\right)\left(\begin{array}{ccc}
k_{2} & 0 & 0 \\
0 & k_{2} & 0 \\
0 & 0 & k_{1}
\end{array}\right) \\
\boldsymbol{B} & =\frac{\pi}{4} b\left(4 a^{2}+b^{2}\right)\left(\begin{array}{ccc}
0 & 0 & 0 \\
0 & 0 & k_{2} \\
0 & -k_{1} & 0
\end{array}\right) \\
\boldsymbol{C} & =\frac{\pi}{32}\left(\begin{array}{ccc}
c_{1} k_{1} & 0 & 0 \\
0 & c_{2} k_{1} & 0 \\
0 & 0 & \left(c_{1}+c_{2}\right) k_{2}
\end{array}\right)
\end{aligned}
$$

wobei

$$
c_{1}=8 a^{4}+12 a^{2} b^{2}+b^{4} \quad \text { und } \quad c_{2}=8 a^{4}+36 a^{2} b^{2}+5 b^{4} \text {. }
$$




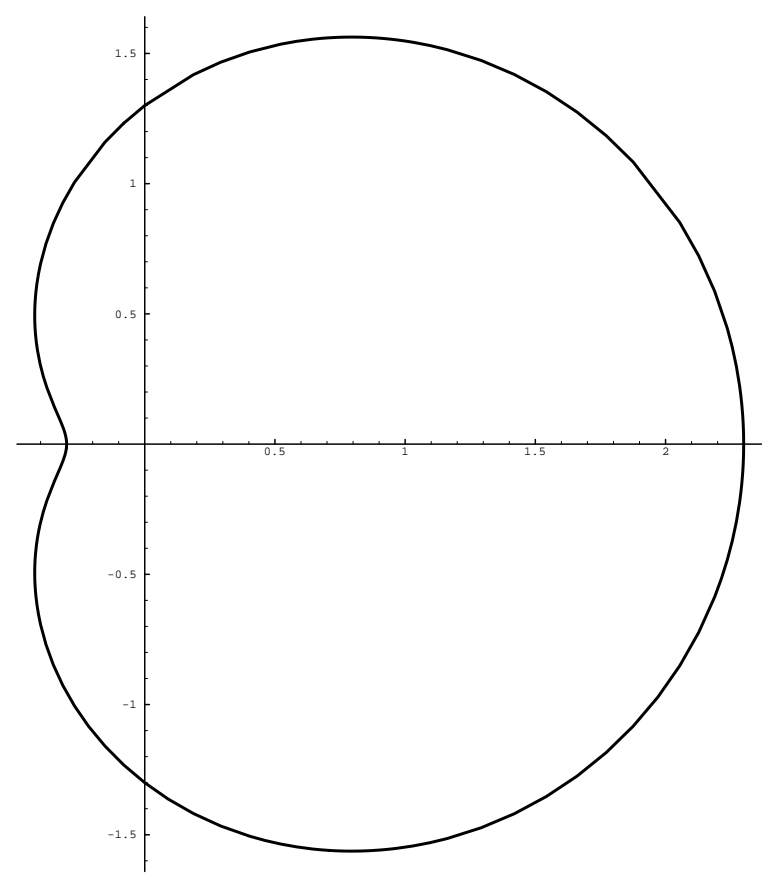

Abbildung 5.1: Die PASCALsche Schnecke $R(\varphi)=a+b \cos \varphi$ zu den Werten $a=1.3$ und $b=1$.

Das elastische Zentrum der Anordnung liegt auf der $x$-Achse bei

$$
Z=\frac{b\left(4 a^{2}+b^{2}\right)\left(k_{1}^{2}+k_{2}^{2}\right)}{2\left(2 a^{2}+b^{2}\right) k_{1} k_{2}},
$$

was mit $v:=k_{2} / k_{1}$ geschrieben werden kann als

$$
Z=\frac{b\left(4 a^{2}+b^{2}\right)\left(1+v^{2}\right)}{2\left(2 a^{2}+b^{2}\right) v}
$$

Seine Lage hängt nicht von der „Stärke“ der Aufhängung ab. Da nur für $k_{1}=k_{2}$ oder ein $k_{i}=0$ ein Widerstandszentrum existiert, ist diese Aufhängung, obwohl sie relativ einfach und symmetrisch um die $x$-Achse ist, ein Beispiel dafür, daß eine ebene Aufhängung nicht automatisch ein Widerstandszentrum besitzen muß.

Die analytische Transformation auf das elastische Zentrum ist zwar möglich, liefert aber zu lange und zu wenig aussagekräftige Terme, als daß sie an dieser Stelle sinnvoll sei.

\subsection{Aufhängungen mit hoher Symmetrie}

Aufhängungen mit hoher Symmetrie sind solche, deren Aufbau so einfach ist, daß eine geschlossene Lösung wahrscheinlich ist. Es wird insbesondere der wichtige Spe- 
zialfall konstanter Schichtgrößen betrachtet. Darunter fallen neben den im letzten Abschnitt diskutierten ebenen Aufhängungen solche mit Kugel- oder Zylindersymmetrie.

\subsubsection{Voll- und Halbkugel}

Die Vollkugel besitzt perfekte sphärische Symmetrie und wird daher betrachtet, obwohl sie kaum ein Modell einer Synarthrose sein kann. Die Halbkugel liefert die notwendige theoretische Vorarbeit für einen Vergleich mit noch durchzuführenden Messungen an der Pfanne einer Hüftendoprothese. Es wird wieder von einer konstanten Schichtdicke und überall gleichem Material, also von konstanten Schichtgrößen ausgegangen. Die Dicke der Schicht wird, wie in 4.3 .2 beschrieben, durch das harmonische Mittel aus Grund- und Deckfläche berücksichtigt. Dadurch ist der effektive Radius gleich dem harmonischen Mittel aus minimalem und maximalem Radius.

Der die gesamte Oberfläche einer Kugel mit Radius $R$ parametrisierende Vektor lautet

$$
\boldsymbol{\xi}=R\left(\begin{array}{c}
\cos \varphi \cos \vartheta \\
\sin \varphi \cos \vartheta \\
\sin \vartheta
\end{array}\right)
$$

mit $\varphi \in[0,2 \pi]$ und $t \in[-\pi / 2, \pi / 2]$. Entsprechend wird eine obere Halbkugel durch $\varphi \in[0,2 \pi]$ und $t \in[0, \pi / 2]$ parametrisiert.

Die auf den Mittelpunkt bezogene Steifheitsmatrix der Vollkugel lautet

$$
\mathcal{S}_{V}=\frac{R^{2} \pi}{3}\left(\begin{array}{c|c}
\left(k_{1}+2 k_{2}\right) \boldsymbol{I} & \mathbf{0} \\
\hline \mathbf{0} & 2 R^{2} k_{2} \boldsymbol{I}
\end{array}\right)
$$

Sie besitzt damit erwartungsgemäß perfekte Skalarform, vgl. Abschnitt 2.5, Für $\rho=k_{1}=k_{2}$ ist dies genau die Massenmatrix einer Kugelschale mit der Flächendichte $\rho$. Schon für $k_{1} \neq k_{2}$ weicht das Verhalten einer elastisch aufgehängten Kugel, der Aufhängung mit der höchst möglichen Symmetrie, von der einer flächig verteilten Masse ab.

Die Steifheitsmatrix der Halbkugel lautet

$$
\mathcal{S}_{H}=\frac{R^{2} \pi}{3}\left(\begin{array}{ccc|ccc}
2 k_{1}+4 k_{2} & 0 & 0 & 0 & 3 R k_{2} & 0 \\
0 & 2 k_{1}+4 k_{2} & 0 & -3 R k_{2} & 0 & 0 \\
0 & 0 & 2 k_{1}+4 k_{2} & 0 & 0 & 0 \\
\hline 0 & -3 R k_{2} & 0 & 4 R^{2} k_{2} & 0 & 0 \\
3 R k_{2} & 0 & 0 & 0 & 4 R^{2} k_{2} & 0 \\
0 & 0 & 0 & 0 & 0 & 4 R^{2} k_{2}
\end{array}\right) .
$$


Für sie existiert ein Widerstandszentrum $W$, welches auf der Mittelachse bei

$$
W=\frac{3 R}{2} \frac{1}{\frac{k_{1}}{k_{2}}+2}
$$

liegt. Dieser Ort hängt nur von der Querdehnzahl, also nicht mehr von einer wie auch immer definierten „Stärke“ der Aufhängung ab.

Die auf das Widerstandszentrum transformierte Matrix lautet

$$
\mathcal{S}_{W}=\frac{2 \pi R^{2}}{3}\left(\begin{array}{ccc|ccc}
k_{1}+2 k_{2} & 0 & 0 & 0 & 0 & 0 \\
0 & k_{1}+2 k_{2} & 0 & 0 & 0 & 0 \\
0 & 0 & k_{1}+2 k_{2} & 0 & 0 & 0 \\
\hline 0 & 0 & 0 & \frac{R^{2} k_{2}\left(8 k_{1}+7 k_{2}\right)}{4 k_{1}+8 k_{2}} & 0 & 0 \\
0 & 0 & 0 & 0 & \frac{R^{2} k_{2}\left(8 k_{1}+7 k_{2}\right)}{4 k_{1}+8 k_{2}} & 0 \\
0 & 0 & 0 & 0 & 0 & 2 R^{2} k_{2}
\end{array}\right)
$$

und hat entsprechend ihrer hohen Symmetrie Diagonal- oder sogar Skalarform. Nur in der Wirkung auf Drehmomente ändert sich das skalare Verhalten gegenüber dem der Vollkugel.

\subsubsection{Der Kreiszylinder}

Eine zylindrische Anordnung ist nach der sphärischen die zweitwichtigste symmetrische Aufhängung im Raum. Der Kreiszylinder ist das räumliche Pendant zu einfachen Zahnmodellen wie dem Stabmodell und dem Scheibenmodell [3]. An dieser Stelle möchte ich außerdem vorführen, wie das Federmodell zu Untersuchungen der Form nütze ist.

Die Parameterdarstellung des Kreiszylinders mit Radius $r$ und Höhe $h$ mit Ursprung in der Zylindermitte ist

$$
\boldsymbol{\xi}=\left(\begin{array}{c}
r \cos \varphi \\
r \sin \varphi \\
t
\end{array}\right)
$$

mit $\varphi \in[0,2 \pi]$ und $t \in[-h / 2, h / 2]$. Für den gesamten Zylinder ergibt sich

$$
\begin{aligned}
\boldsymbol{A} & =\pi h r\left(\begin{array}{ccc}
k_{1}+k_{2} & 0 & 0 \\
0 & k_{1}+k_{2} & 0 \\
0 & 0 & 2 k_{2}
\end{array}\right) \\
\boldsymbol{B} & =\mathbf{0} \\
\boldsymbol{C} & =\frac{\pi h r}{12}\left(\begin{array}{ccc}
h^{2}\left(k_{1}+k_{2}\right)+12 r^{2} k_{2} & 0 & 0 \\
0 & h^{2}\left(k_{1}+k_{2}\right)+12 r^{2} k_{2} & 0 \\
0 & 0 & 24 r^{2} k_{2}
\end{array}\right)
\end{aligned}
$$

Da $\boldsymbol{A} \boldsymbol{C}$ diagonal ist, sind die funktionellen Achsen wieder die Koordinatenachsen, obwohl die Matrixeinträge diesmal von der Querdehnzahl abhängen. 


\subsubsection{Der Kreiskegel}

Gegeben sei ein auf der Spitze stehender Kreiskegel, dessen Rand zum Radius $r$ die Höhe $h$ habe. Als Modell des Periodonts eines Frontzahnes ist er in nullter Näherung brauchbar [64, 65] und bekannt unter dem Namen „Zipfelmützenmodell“. Seine Parametrisierung lautet

$$
\boldsymbol{\xi}=\left(\begin{array}{c}
r t \cos \varphi \\
r t \sin \varphi \\
h t
\end{array}\right)
$$

mit $\varphi \in[0,2 \pi]$ und $t \in[0,1]$. Es ergibt sich

$$
\begin{aligned}
\boldsymbol{A} & =\frac{\pi r}{2 \sqrt{h^{2}+r^{2}}}\left(\begin{array}{ccc}
h^{2}\left(k_{1}+k_{2}\right)+2 r^{2} k_{2} & 0 & 0 \\
0 & h^{2}\left(k_{1}+k_{2}\right)+2 r^{2} k_{2} & 0 \\
0 & 0 & 2\left(r^{2} k_{1}+h^{2} k_{2}\right)
\end{array}\right) \\
\boldsymbol{B} & =\frac{\pi h r}{3} \sqrt{h^{2}+r^{2}}\left(k_{1}+k_{2}\right)\left(\begin{array}{ccc}
0 & 1 & 0 \\
-1 & 0 & 0 \\
0 & 0 & 0
\end{array}\right) \\
\boldsymbol{C} & =\frac{\pi r}{4} \sqrt{h^{2}+r^{2}}\left(\begin{array}{ccc}
h^{2}\left(k_{1}+k_{2}\right)+r^{2} k_{1} & 0 & 0 \\
0 & h^{2}\left(k_{1}+k_{2}\right)+r^{2} k_{1} & 0 \\
0 & 0 & 2 r^{2} k_{2}
\end{array}\right) .
\end{aligned}
$$

Der Kreiskegel besitzt ein Widerstandszentrum auf der Mittelachse bei

$$
W=\frac{2 h\left(h^{2}+r^{2}\right)\left(k_{1}+k_{2}\right)}{3\left(h^{2}\left(k_{1}+k_{2}\right)+2 r^{2} k_{2}\right)} .
$$

Für einen beliebig engen Kegel gilt $r \rightarrow 0$ und nach Kürzen von $k_{1}+k_{2}$ folgt

$$
W(r \rightarrow 0)=\frac{2}{3} h
$$

Ein beliebig weiter Kegel mit festem $r$ nähert sich einer Kreisscheibe an; man erhält erwartungsgemäß $W(h \rightarrow 0)=0$. Vernachlässigt man die Scherelastizität, so erhält man mit $k_{2} \rightarrow 0$ nach Kürzen von $k_{1}$ den Grenzwert

$$
W\left(k_{2} \rightarrow 0\right)=\frac{2}{3} \frac{h^{2}+r^{2}}{h}
$$

welcher für kleine $h$ beliebig große Werte annehmen kann. Die genaue Abhängigkeit des Widerstandszentrums von der Form des Kegels soll im Folgenden diskutiert werden.

Mit den dimensionslosen Größen $v:=k_{2} / k_{1}$ und dem halben Öffnungswinkel $\alpha$ läßt sich nun die Lage des Widerstandszentrums als Funktion dieser beiden Variablen und einer charakteristischen Länge diskutieren. Um die beiden Spezialfälle $r \rightarrow 0$ 
und $h \rightarrow 0$ mit einschließen zu können, bietet sich die Seitenlänge $\sqrt{r^{2}+h^{2}}$ als geeigneter Bezug an.

Mit $\cos (\alpha)=h / \sqrt{r^{2}+h^{2}}, \sin (\alpha)=r / \sqrt{r^{2}+h^{2}}$ und $\tan (\alpha)=r / h$ ergibt sich nach Division durch $k_{1}+k_{2}$ zunächst

$$
W=\frac{2}{3} \sqrt{r^{2}+h^{2}} \frac{\cos \alpha}{\cos ^{2} \alpha+2 \sin ^{2} \alpha k_{2} /\left(k_{1}+k_{2}\right)},
$$

und in dimensionsloser Form mit $w:=W / \sqrt{r^{2}+h^{2}}$

$$
w=\frac{2}{3} \frac{\cos \alpha}{\cos ^{2} \alpha+2 \sin ^{2} \alpha v /(1+v)} .
$$

Von Interesse ist, ob und unter welchen Bedingungen dieser Wert größer als Eins werden kann; dann nämlich läge das Widerstandszentrum außerhalb der Aufhängung. Mit (4.30) lautet $w$ in der Querdehnzahl ausgedrückt

$$
w(\sigma, \alpha)=\frac{4 \cos \alpha(-3+4 \sigma)}{3(-5+8 \sigma-\cos 2 \alpha)}
$$

und $w=1$ ergibt nach $\sigma$ aufgelöst den kritischen Fall

$$
\sigma_{w=1}=\frac{3(5-4 \cos \alpha+3 \cos 2 \alpha)}{24-16 \cos \alpha}
$$

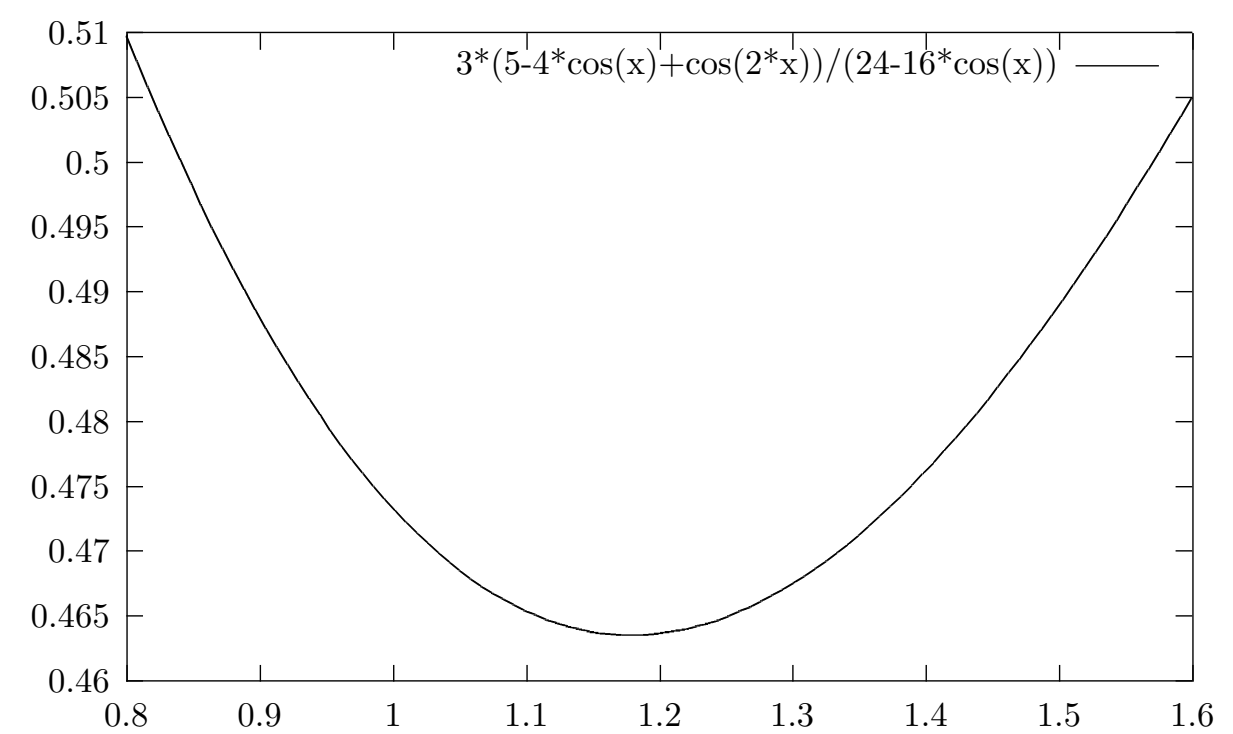

Abbildung 5.2: Der kritische Bereich von $\sigma$ als Funktion des halben Öffnungswinkels des Kreiskegels. Das Widerstandszentrum liegt außerhalb der Aufhängung.

In der Tat erhält man in einem Bereich oberhalb der Kurve (s. Abb. 5.2) mit $\sigma<0.5$ Werte mit $w>1$ ! Der kleinste dazu nötige Wert von $\sigma$ liegt bei $\sigma_{w=1}=0.464$ bei 
einem Winkel von $\alpha_{w=1}=1.179 \mathrm{rad}=67.5^{\circ}$. Da die Klammer im Nenner von (5.5) für $\sigma \rightarrow 0.5$ den Wert $2 \cos ^{2} \alpha$ annimmt, können sich für $\alpha \rightarrow \pi$ sogar beliebig große Werte für $w$ ergeben. Der Grenzfall $\sigma \rightarrow 0.5$ entspricht physikalisch einem Schermodul, der klein im Vergleich zum Kompressionsmodul ist, wie etwa bei Gummi. Demnach sollte eine solche Aufhängung tatsächlich konstruierbar sein; ihre mechanischen Eigenschaften könnten dann am Experiment verifiziert werden. Probekörper mit Wunscheigenschaften, daß etwa das Widerstandszentrum innerhalb der Aufhängung zu liegen habe, sind den obigen Erkenntnissen zufolge tunlichst nicht aus Gummi herzustellen.

Insbesondere wird deutlich, daß die Anschauung vom Widerstandszentrum als Schwerpunkt der Aufhängung zu einfach ist. Die Abweichung von dieser idealisierten Vorstellung liegt in der durch das tensorielle Verhalten der Federmatrizen transformierten Scherelastizität begründet.

\subsection{Komplexere Aufhängungen}

Komplexere Aufhängungen erhält man beispielsweise durch Zusammensetzung von zwei einfachen Aufhängungen. Ist eine Fläche als Ganzes in einer Parameterdarstellung gegeben, so ist immer noch eine numerische Integration möglich - allerdings unter Preisgabe der Vorteile der exakten Lösung mittels Computeralgebra.

\subsubsection{Der zweiseitige Keil}

Der zweiseitige Keil ist das Bindeglied zwischen zwei- und dreidimensionalen Zahnmodellen, seine Form ist genau das Winkelzahnmodell ("wedge-shape model") aus [64]. Diese Fläche ist aus zwei Rechtecken zusammengesetzt.

Gegeben sei ein zweiseitiger Keil mit den Längen $a$ und Breite $b$ der beiden begrenzenden Seiten. Eine Berandung entsteht dadurch, daß die rechteckige Scheibe mit den Seiten $a \times b$ der $x-y$-Ebene in $x$-Richtung um die Strecke $\pm a / 2$ auf ihren Rand translatiert und dann um die $y$-Achse um den Winkel $\pm(\pi / 2-\alpha)$ rotiert wird. Der Winkel $\alpha$ ist dann der halbe Öffnungswinkel des Keils.

Bezüglich der Keilspitze ergeben sich die Komponenten

$$
\begin{aligned}
\boldsymbol{A}= & a b\left(\left(\begin{array}{ccc}
k_{1}+k_{2} & 0 & 0 \\
0 & 2 k_{2} & 0 \\
0 & 0 & k_{1}+k_{2}
\end{array}\right)+\right. \\
& \left.+\cos 2 \alpha\left(k_{1}-k_{2}\right)\left(\begin{array}{ccc}
1 & 0 & 0 \\
0 & 0 & 0 \\
0 & 0 & -1
\end{array}\right)\right)
\end{aligned}
$$




$$
\begin{aligned}
\boldsymbol{B}= & a^{2} b \cos \alpha\left(\begin{array}{ccc}
0 & -k_{1} & 0 \\
k_{2} & 0 & 0 \\
0 & 0 & 0
\end{array}\right) \\
\boldsymbol{C}= & \frac{a b}{6}\left(\left(\begin{array}{ccc}
b^{2} k_{1} & 0 & 0 \\
0 & 4 a^{2} k_{1} & 0 \\
0 & 0 & \left(4 a^{2}+b^{2}\right) k_{2}
\end{array}\right)+\right. \\
& \left.+\cos ^{2} \alpha\left(\left(4 a^{2}+b^{2}\right) k_{2}-b^{2} k_{1}\right)\left(\begin{array}{ccc}
1 & 0 & 0 \\
0 & 0 & 0 \\
0 & 0 & -1
\end{array}\right)\right) .
\end{aligned}
$$

Für $\alpha=\pi / 4$ ergibt sich der Grenzfall einer ebenen Scheibe mit den Abmessungen $2 a \times b$ zurück. Das elastische Zentrum der Anordnung liegt auf der $z$-Achse bei

$$
Z=\frac{a \cos \alpha}{2} \frac{3 k_{1}+k_{2}+\left(k_{1}-k_{2}\right) \cos 2 \alpha}{k_{1}+k_{2}+\left(k_{1}-k_{2}\right) \cos 2 \alpha}
$$

Wie bei dem Kreiskegel ergeben sich wieder für kleine $v=k_{2} / k_{1}=(1-2 \sigma) /(2-2 \sigma)$ oder für $\sigma$ nahe bei $1 / 2$ Werte von $Z / a>1$. Die Auflösung von $Z / a=1 \mathrm{mit}$

$$
\frac{Z}{a}=\frac{\cos \alpha}{2} \frac{7-8 \sigma+\cos 2 \alpha}{3-4 \sigma+\cos 2 \alpha}
$$

ergibt

$$
\sigma_{Z=a}=\frac{1}{8}(5-2 \cos \alpha+\cos 2 \alpha)
$$

vgl. Abb. 5.3, mit einem Extremum bei $\alpha=60^{\circ}=\pi / 3 \mathrm{rad}$ mit $\sigma=7 / 16=0.4375$.

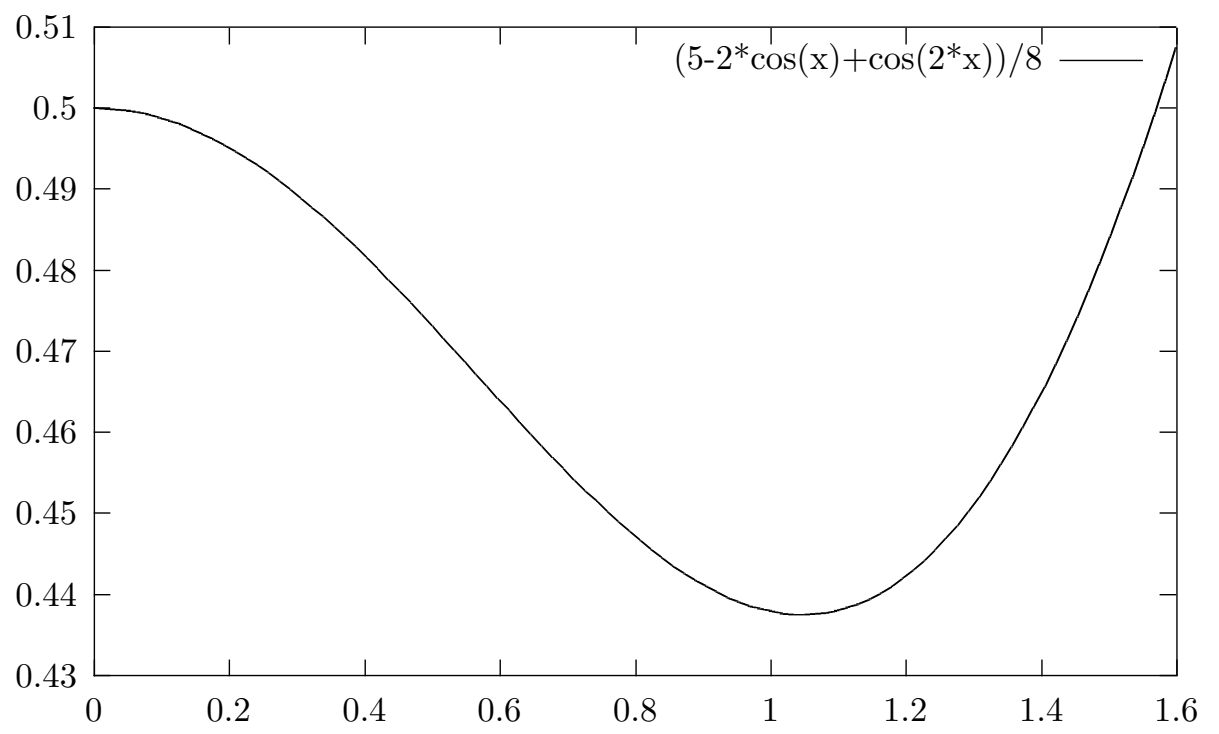

Abbildung 5.3: Der kritische Bereich von $\sigma$ als Funktion des halben Öffnungswinkels des Keils. Das elastische Zentrum liegt dann oberhalb der Aufhängung. 
Der Winkelbereich, in dem das elastische Zentrum außerhalb der Aufhängung liegt, ist erheblich größer als der des Kreiskegels. Ein Keil mit einem Öffnungswinkel von $120^{\circ}$ und mit einem Gummi mit $\sigma \approx 0.5$ bietet sich demnach als Probekörper zur Überprüfung dieses Phänomens an. Einsetzen dieser Werte ergibt aber nur $Z / a=5 / 4$, so daß zur Erzielung des größtmöglichen Effektes eher mit einem größeren Winkel gearbeitet werden sollte. In der Tat ergibt sich für $\sigma=0.5$ wieder ein Nenner von $1-\cos 2 \alpha=2 \cos ^{2} \alpha$, der $Z / a$ für $\alpha \rightarrow \pi / 4$ divergieren läßt.

\subsubsection{Der Kreiszylinder mit Boden}

Der Zylinder mit Boden wurde in unserer Arbeitsgruppe als Probekörper verwandt. Als becherförmiges Modell eines Zahnes kann er die Frage beantworten, wie weit sich das elastische Zentrum durch den Einfluß des Bodens von der Mitte entfernt. Als Grenzfall $a \rightarrow \infty$ der Hyperellipsen $x^{a}+y^{a}=1$ gibt er ferner einen Anhaltspunkt, wie stark die Form die berechnete Steifheitsmatrix beeinflußt.

Durch Superposition des Kreiszylinders aus 5.2.2 und der auf den Punkt $z=-h / 2$ translatierten Kreisscheibe aus 5.1.2 erhält man mit der Abkürzung

$$
c:=\frac{\pi r}{12}\left(h^{3}\left(k_{1}+k_{2}\right)+\left(3 h^{2} r+12 h r^{2}\right) k_{2}+3 r^{3} k_{1}\right)
$$

bezüglich der Zylindermitte

$$
\begin{aligned}
\boldsymbol{A} & =\pi r\left(\begin{array}{ccc}
h\left(k_{1}+k_{2}\right)+r k_{2} & 0 & 0 \\
0 & h\left(k_{1}+k_{2}\right)+r k_{2} & 0 \\
0 & 0 & 2 h k_{2}+r k_{1}
\end{array}\right) \\
\boldsymbol{B} & =\frac{\pi}{2} h k_{2} r^{2}\left(\begin{array}{ccc}
0 & -1 & 0 \\
1 & 0 & 0 \\
0 & 0 & 0
\end{array}\right) \\
\boldsymbol{C} & =\left(\begin{array}{ccc}
c & 0 & 0 \\
0 & c & 0 \\
0 & 0 & \frac{\pi}{2} r^{3}(4 h+r) k_{2}
\end{array}\right) .
\end{aligned}
$$

Diese Aufhängung besitzt ein Widerstandszentrum auf der Mittelachse unterhalb der Mitte bei

$$
W=-\frac{h r k_{2}}{2\left(h\left(k_{1}+k_{2}\right)+r k_{2}\right)}
$$

oder, wenn man $W$ auf die Höhe $h$ normiert und auf den Boden bezieht, mit den dimensionslosen Größen $v=k_{2} / k_{1}$ und $u:=h / r$

$$
w:=\frac{W}{h}+\frac{1}{2}=\frac{u+u v}{2(u+v+u v)} .
$$

Ersetzt man $v=(1-2 \sigma) /(2-2 \sigma)$ wieder durch $\sigma$, so erhält man

$$
w=u \frac{-3+4 \sigma}{-2+4 \sigma-6 u+8 \sigma u} .
$$


Die Grenzfälle dieser Funktion entsprechen den Erwartungen: Für eine Scheibe mit $u \rightarrow 0$ ergibt sich $w=0$ und für einen beliebig kleinen Deckel folgt mit $u \rightarrow \infty$ der Wert $w=1 / 2$. Für eine vernachlässigte Scherelastizität erhält man mit $\sigma \rightarrow 1 / 2$ ebenfalls $w=1 / 2$. Werte von $w$, die größer als $1 / 2$ sind, werden nicht angenommen; das Widerstandszentrum liegt in jedem Fall durch den Einfluß des hinzugekommenen Bodens unterhalb der Zylindermitte.

Werte von $w \geq 1$ tauchen nicht auf, da

$$
\sigma_{w=1}=\frac{2+3 u}{4(1+u)}
$$

für $v>0$ nur Werte größer als $1 / 2$ annimmt.

\subsubsection{Das Rotationsparaboloid}

Diese Aufhängung soll als das einfachste aber dennoch echt räumliche Zahnmodell dienen. Es wird auch von anderen Autoren im Rahmen numerischer Modelle [7] verwendet. Dieses Modell soll außerdem klären, ob das Widerstandszentrum außerhalb der Aufhängung beim Kreiskegel 5.2.3 und beim zweiseitigem Keil 5.3.1 ein Artefakt des Knickes in der Spitze ist.

Die Oberfläche des Paraboloids werde mit

$$
\boldsymbol{x}=\left(\begin{array}{c}
R t \cos (\varphi) \\
R t \sin (\varphi) \\
H t^{2}
\end{array}\right),
$$

wobei $\varphi \in[0,2 \pi)$ und $t \in[0,1]$ parametrisiert. Der obere Rand liege bei $x=y=R$ und $z=H$. Das Problem vereinfacht sich durch die Einführung der dimensionslosen Parameter $h:=H / R$ und $v:=k_{2} / k_{1}$.

Die Rechnung wurde mit Mathematica unter Verwendung der die Integrationsroutinen zusammenfassenden Prozeduren AUnFed, BUnFed und CUnFed, vgl. A.3.2 durchgeführt. Da die Grenzen der einen Variablen nicht die andere Variable enthalten, kann eine gemeinsame Stammfunktion angegeben werden, in welche die gewünschten Grenzen dann jeweils einzusetzen sind. Mit den Abkürzungen

$$
\begin{aligned}
a & =\frac{\pi H^{2} k_{1}}{6 h^{4}}\left(1-2 v+\sqrt{1+4 h^{2}}\left(-1+2 h^{2}+2 v+2 h^{2} v\right)\right) \\
a_{z} & =\frac{\pi H^{2} k_{1}}{6 h^{4}}\left(-3+2 v+\sqrt{1+4 h^{2}}\left(3-2 v+4 h^{2} v\right)\right) \\
b & =\frac{\pi H^{3} k_{1}}{60 h^{6}}\left(3-2 v+\sqrt{1+4 h^{2}}\left(-3+6 h^{2}+12 h^{4}+2 v-4 h^{2} v+12 h^{4} v\right)\right) \\
c & =\frac{\pi H^{4} k_{1}}{840 h^{8}}\left(13-8 v+\sqrt{1+4 h^{2}}\left(-13+26 h^{2}+132 h^{4}+120 h^{6}+\right.\right.
\end{aligned}
$$




$$
\begin{gathered}
\left.\left.+8 v-16 h^{2} v+48 h^{4} v+120 h^{6} v\right)\right) \\
c_{z}=\frac{\pi H^{4} k_{1}}{60 h^{8}}\left(v+\sqrt{1+4 h^{2}}\left(-v+2 h^{2} v+24 h^{4} v\right)\right)
\end{gathered}
$$

lautet die Steifheitsmatrix mit Ursprung des Koordinatensystems im Scheitel der Parabel

$$
\mathcal{S}=\left(\begin{array}{ccc|ccc}
a & 0 & 0 & 0 & -b & 0 \\
0 & a & 0 & b & 0 & 0 \\
0 & 0 & a_{z} & 0 & 0 & 0 \\
\hline 0 & b & 0 & c & 0 & 0 \\
-b & 0 & 0 & 0 & c & 0 \\
0 & 0 & 0 & 0 & 0 & c_{z}
\end{array}\right)
$$

Für diese Aufhängung existiert ein Widerstandszentrum und liegt bei $W=\frac{b(h, v)}{a(h, v)}$, oder explizit

$$
\begin{aligned}
& W(h, v) / H= \\
& \quad\left(-24 h^{2}+12 h^{4}+48 h^{6}-9 v+36 h^{2} v+56 h^{4} v+96 h^{6} v+6 v^{2}+44 h^{4} v^{2}+48 h^{6} v^{2}\right. \\
& \left.\quad+\sqrt{1+4 h^{2}}\left(-6 h^{2}+9 v+6 h^{2} v-6 v^{2}+12 h^{2} v^{2}\right)\right) \\
& \quad /\left(20 h^{2}\left(-3 h^{2}+4 h^{4}-3 v+6 h^{2} v+8 h^{4} v+6 v^{2}+9 h^{2} v^{2}+4 h^{4} v^{2}\right)\right) .
\end{aligned}
$$

Im Gegensatz zu numerischen Modellen kann diese Funktion analytisch diskutiert werden - wenn auch bequem nur unter Zuhilfenahme von Computeralgebra. Eine beliebig enge Parabel ergibt den Grenzfall

$$
W(h \mapsto \infty, v) / H=\frac{3}{5}=0.6 \quad,
$$

und eine beliebig weite Parabel als Annäherung an eine Scheibe ergibt mit

$$
W(h \mapsto 0, v) / H=\frac{1}{2 v}
$$

erneut in Abhängigkeit von der Querdehnzahl beliebig große Werte für $v \mapsto 0$. Für eine Kreisscheibe ist aber eine Normierung auf $R$ sinnvoller. Mit $W / R=h W / H$ ergibt sich für $v \neq 0$ erwartungsgemäß

$$
W(h \mapsto 0, v) / R=0 \quad .
$$

Die Plots der Funktion $W / R$ in Abb. 5.4 zeigen, daß sich in einem Bereich nahe $(h, v) \approx(0,0)$ wieder beliebig große Werte ergeben können. Es lag demnach nicht am Knick in der Spitze. Für große $h$ ist das zwar auch der Fall, aber dann ist $W / R$ auch die ungeeignete Normierung. Der Bereich mit $W / R>1$ für $v$ und $h$ läßt sich zwar analytisch festlegen, da das aber einiges Hantieren mit quadratischen Ungleichungen erfordert, soll es hier nicht vorgeführt werden. 

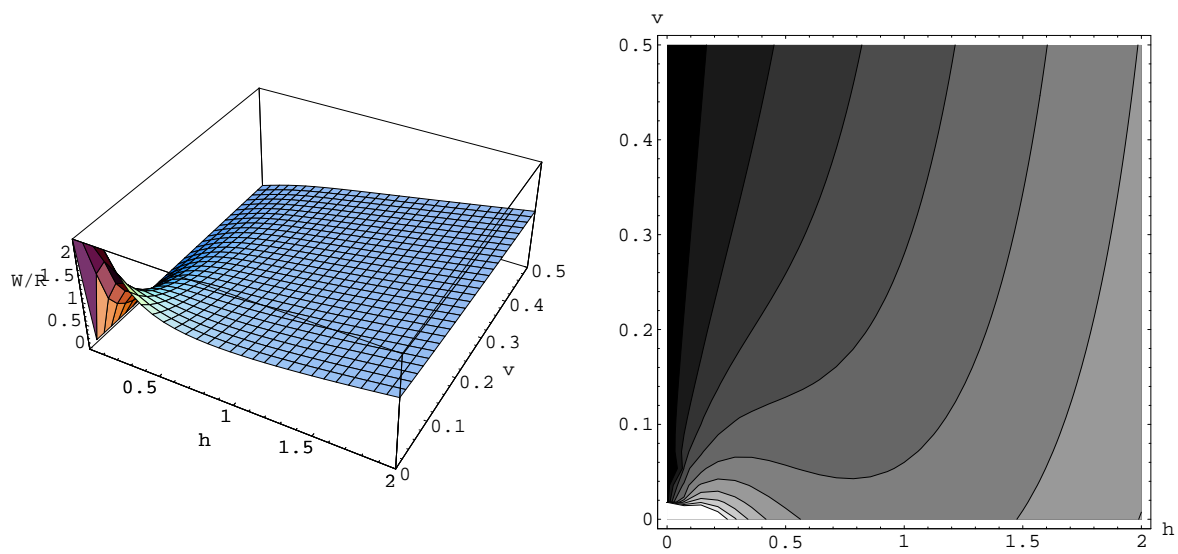

Abbildung 5.4: 3D-Plot und Höhenliniendarstellung der normierten Lage des Widerstandszentrums eines Rotationsparaboloides.

Die einzigen Änderungen der Steifheitsmatrix nach Transformation auf das Widerstandszentrum $W$ sind: $b \mapsto 0$ und $c \mapsto c-\frac{b^{2}}{a}$. Dadurch ergibt sich der Ausdruck

$$
\sigma^{2}=\frac{c-b^{2} / a}{a}
$$

für die Konstante des Reziprozitätstheorems, vgl. Abschnitt 5.4, welche als $\sigma^{2} / H^{2}$ auch wieder untersucht werden kann. Für $h \rightarrow \infty$ etwa ergibt sich der asymptotische Wert

$$
\sigma^{2}(h \rightarrow \infty) / H^{2}=\frac{12}{175} \approx 0.0686
$$

und für eine Kreisscheibe folgt

$$
\sigma^{2}(h \rightarrow 0) / R^{2}=h^{2} \frac{\sigma^{2}}{H^{2}}(h \rightarrow 0)=\frac{1}{4 v} .
$$

Für flache Aufhängungen und kleine $v$ sind demnach auch für die Konstante des Reziprozitätstheorems große Werte zu erwarten.

\subsubsection{Die allgemeine rotationssymmetrische Aufhängung}

In diesem Abschnitt soll auf drei Weisen gezeigt werden, daß die allgemeine rotationssymmetrische Aufhängung mit konstanten Schichtgrößen ein Widerstandszentrum besitzt.

Die Existenz eines Widerstandszentrums folgt nach den Erkenntnissen der Abschnitte 4.5.2.3, nach dem eine Aufhängung mit kreisförmigem Querschnitt pro Schnittfläche ein Widerstandszentrum auf der Symmetrielinie hat, und 4.5.2.2, nach dem eine Superposition von gleichartigen Aufhängungen wieder ein Widerstandszentrum hat. Es bleibt noch zu zeigen, daß die Neigung des Randes nicht 
verhindert, daß eine einzelne Schicht ein Widerstandszentrum besitzt. Das ist aber ebenfalls klar, da diese, ein kleiner Kegelstumpf, als Superposition zweier Kegel mit leicht unterschiedlichen Höhen betrachtet werden kann. Diese werden zwar mit negativem Gewicht superponiert, aber da in dem Abschnitt über gleichartige Aufhängungen lediglich die Linearität vorausgesetzt wurde, ist das zulässig. Demnach besitzt ein Kegelstumpf und mit ihm die gesamte rotationssymmetrische Aufhängung mit konstanten Schichtgrößen ein Widerstandszentrum auf der Mittelachse.

Auch aus der Superposition von zwei Steifheitsmatrizen mit Widerstandszentrum kann die Existenz eines Widerstandszentrums der gemeinsamen Aufhängung geschlossen werden. Sei die $z$-Achse die Symmetrieachse. Es ergeben sich die Matrizen

$$
\boldsymbol{A}_{1}=\left(\begin{array}{ccc}
a_{1} & 0 & 0 \\
0 & a_{1} & 0 \\
0 & 0 & a_{1 z}
\end{array}\right) \quad \text { und } \quad \boldsymbol{A}_{2}=\left(\begin{array}{ccc}
a_{2} & 0 & 0 \\
0 & a_{2} & 0 \\
0 & 0 & a_{2 z}
\end{array}\right)
$$

sowie, da jeweils ein Widerstandszentrum auf der z-Achse existiert,

$$
\boldsymbol{B}_{1}=\boldsymbol{A}_{1} \boldsymbol{\Omega}_{z_{1}}=\left(\begin{array}{ccc}
a_{1} & 0 & 0 \\
0 & a_{1} & 0 \\
0 & 0 & a_{1 z}
\end{array}\right)\left(\begin{array}{ccc}
0 & -z_{1} & 0 \\
z_{1} & 0 & 0 \\
0 & 0 & 0
\end{array}\right)=a_{1} z_{1} \boldsymbol{\Omega}_{e_{z}}
$$

und $\boldsymbol{B}_{2}=a_{2} z_{2} \boldsymbol{\Omega}_{e_{z}}$ entsprechend. Das Widerstandszentrum ergibt sich damit zu

$$
\begin{aligned}
\boldsymbol{z} & =-\left(\left(\boldsymbol{A}_{1}+\boldsymbol{A}_{2}\right)^{-1}\left(\boldsymbol{B}_{1}+\boldsymbol{B}_{2}\right)\right)^{D}=\left(\left(\boldsymbol{A}_{1}+\boldsymbol{A}_{2}\right)^{-1}\left(a_{1} z_{1}+a_{2} z_{2}\right) \boldsymbol{\Omega}_{e_{z}}\right)^{D} \\
& =\frac{a_{1} z_{1}+a_{2} z_{2}}{a_{1}+a_{2}} \boldsymbol{e}_{z} .
\end{aligned}
$$

Die Superposition erfolgt damit analog zum Schwerpunktsatz 4.5, wobei die Masse den Anteilen der Federmatrix innerhalb der Schicht entspricht. Mittels Induktion folgt nun die Existenz des Widerstandszentrums einer Anordnung von beliebig vielen solcher Schichten, und wegen der Stetigkeit der verwendeten Operationen auch die von einer rotationssymmetrischen Berandung.

Als drittes soll dieser Nachweis mit Computeralgebra erbracht werden. Mit den Prozeduren aus A.3 und dem Normalenvektor

$$
x\left[p_{-}, t_{-}\right]:=\operatorname{DVec}[r[t] \operatorname{Cos}[p], r[t] \operatorname{Sin}[p], h t]
$$

mit $p \in[0,2 \pi)$ und $t \in[0,1]$ läßt man die Abhängigkeit des Radius $r$ von der relativen Höhe $t$ noch offen. Die nötigen geometrischen Hilfsgrößen bekommt man über

$$
\mathrm{xx}=\mathrm{x}[\mathrm{p}, \mathrm{t}]
$$




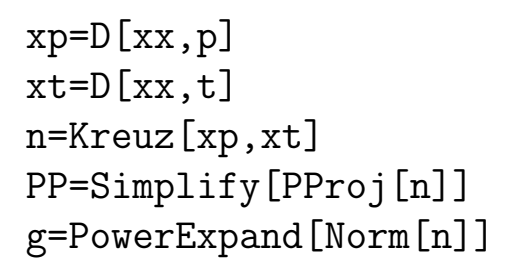

und die zur Integration vorbereitete elementare Federmatrix wird dann mit

Integrand=Simplify [PowerExpand[Apart [g (k1 PP + k2 (Edrei-PP))]]]

bestimmt. Die Matrizen $\boldsymbol{A}$ und $\boldsymbol{B}$ ergeben sich damit einfach zu

$$
\begin{aligned}
& A=\text { Integrate }[\text { Integrand, }\{\mathrm{p}, 0,2 \mathrm{P} i\},\{t, 0,1\}] \\
& \mathrm{B}=\text { Integrate }[-\operatorname{Meps}[\mathrm{xx}] . \text { Integrand, }\{\mathrm{p}, 0,2 \mathrm{P} i\},\{t, 0,1\}]
\end{aligned}
$$

als bestimmte Integrale in Abhängigkeit der Funktionen $r(t)$ und $r^{\prime}(t)$. Mit den Abkürzungen

$$
\begin{aligned}
a & :=\pi \int_{0}^{1} d t r(t) \frac{h^{2}\left(k_{1}+k_{2}\right)+2 k_{2} r^{\prime 2}(t)}{\sqrt{h^{2}+r^{\prime 2}(t)}} \\
a_{z} & :=2 \pi \int_{0}^{1} d t r(t) \frac{h^{2} k_{2}+k_{1}{r^{\prime}}^{2}(t)}{\sqrt{h^{2}+r^{\prime 2}(t)}} \\
b & :=h \pi \int_{0}^{1} d t r(t) \frac{h^{2}\left(k_{1}+k_{2}\right) t+\left(k_{1}-k_{2}\right) r(t) r^{\prime}(t)+2 k_{2} t r^{\prime 2}(t)}{\sqrt{h^{2}+r^{\prime 2}(t)}}
\end{aligned}
$$

ergibt sich einfach

$$
\boldsymbol{A}=\left(\begin{array}{ccc}
a & 0 & 0 \\
0 & a & 0 \\
0 & 0 & a_{z}
\end{array}\right) \quad \text { und } \quad \boldsymbol{B}=b\left(\begin{array}{ccc}
0 & 1 & 0 \\
-1 & 0 & 0 \\
0 & 0 & 0
\end{array}\right)
$$

die Winkelintegration über die Variable $\mathrm{p}$ konnte demnach ausgeführt werden. Es existiert folglich ein Widerstandszentrum auf der $z$-Achse bei $Z=b / a$. Mit

$$
\boldsymbol{C}=\left(\begin{array}{ccc}
c & 0 & 0 \\
0 & c & 0 \\
0 & 0 & c_{z}
\end{array}\right)
$$

wobei

$$
\begin{aligned}
c & :=\pi \int_{0}^{1} d t r \frac{h^{4}\left(k_{1}+k_{2}\right) t^{2}+h^{2} k_{2} r^{2}+2 h^{2}\left(k_{1}-k_{2}\right) t r r^{\prime}+2 h^{2} k_{2} t^{2} r^{\prime 2}+k_{1} r^{2} r^{\prime 2}}{\sqrt{h^{2}+r^{\prime 2}}} \\
c_{z} & :=2 k_{2} \pi \int_{0}^{1} d t r^{3} \sqrt{h^{2}+r^{\prime 2}}
\end{aligned}
$$


ist die Struktur der rotationssymmetrischen Aufhängungen mit konstanten Schichtgrößen aus 5.2.2, 5.2.3, 5.3.2 und 5.3.3 durch Rückführung auf eindimensionale Integrale abschließend allgemein geklärt:

Eine rotationssymmetrische Aufhängung besitzt ein Widerstandszentrum und wird durch eine elastische Matrix in Diagonalform beschrieben.

\subsubsection{Der Schaft einer Hüftendoprothese}

Dieses Beispiel soll veranschaulichen, daß die vorliegende Theorie auch auf komplexere Aufhängungen anwendbar ist. Der Preis ist hoch: Die Integrationen können nur noch numerisch durchgeführt werden, was, angesichts der Kompliziertheit der auftretenden Terme, das Computeralgebra-System mehrfach zu Abstürzen veranlaßte. Dafür existiert aber eine echt räumliche Messung [72], mit der die Ergebnisse dieses Abschnitts in 6.5 verglichen werden können.

Der Prothesenschaft sei modelliert durch

$$
x(\varphi, t):=\left(t \cos (\varphi), 2 t \sin (\varphi)+t^{4} / 160,2 t^{2}\right) / 2000
$$

wobei $\varphi \in[0,2 \pi)$ und $t \in[0,10]$, vgl. Abb. 5.5. Alle Angaben über Zahlenwerte sind in SI. Der Koordinatenursprung befindet sich an der Spitze des Schaftes.
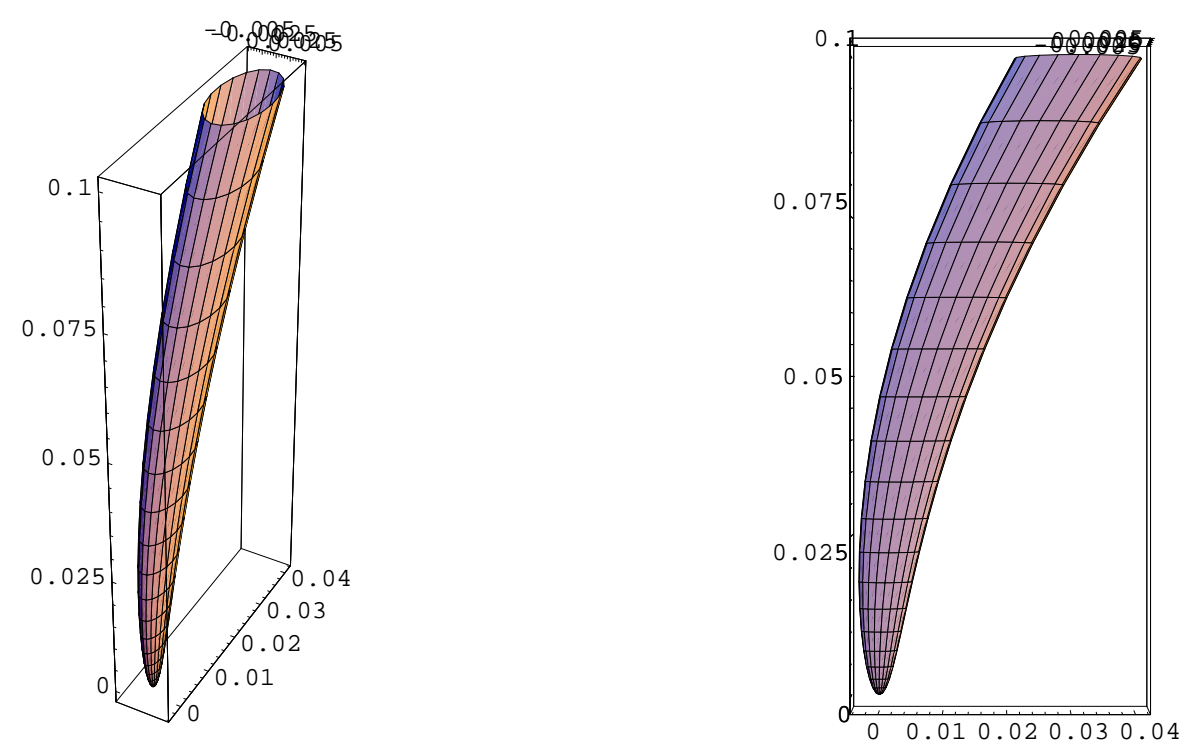

Abbildung 5.5: Das geometrische Modell eines Hüftendoprothesenschaftes. Alle Längenangaben in $m$. 
Die Elastizitätsmodule des Implantatmaterials mit 200 GPa und der Kortikalis mit $18 G P a$ liegen immer noch um einen Faktor 6 über der des Knochenzementes mit $3 G P a$ oder dem der Spongiosa mit 0.08 ...1GPa. Die Näherung einer starr berandeten elastischen Schicht sollte demnach zutreffend sein. Die elastische Schicht ist eine Hintereinanderschaltung von Spongiosa und Knochenzement. Die Spongiosa habe die Dicke

$$
d_{\text {Spong }}=\left(1+2 \sin ^{2}(\varphi)\right) 10^{-3}+10^{-4} t
$$

welche zwischen 1 und $3 \mathrm{~mm}$ variiert und besitze die Materialkonstanten Querdehnzahl und E-Modul

$$
\sigma_{\text {Bone }}=0.3 \quad \text { und } \quad E_{\text {Spong }}=0.08 \cdot 10^{9} .
$$

Der aus Polymetamethylacrylat bestehende Knochenzement habe die Dicke

$$
d_{\mathrm{pmma}}=4 \cdot 10^{-3}
$$

und die Materialkonstanten $\sigma_{\mathrm{pmma}}=0.41$ und $E_{\mathrm{pmma}}=3 \cdot 10^{9}$. Es sei dahingestellt, ob die Beschreibung von Spongiosa mit einem Kontinuumsmodell wirklich zulässig ist. Die einzelnen Schichtgrößen werden dann unter Benutzung von (4.28) und (4.29) berechnet, und mit dem harmonischen Mittel

$$
k=\left(k_{\text {Spong }}^{-1}+k_{\text {pmma }}^{-1}\right)^{-1}
$$

hintereinander geschaltet. Es ergeben sich die Schichtgrößen als folgende Funktionen der Flächenparameter

$$
\begin{aligned}
& k_{1}=\frac{1}{9.86 \cdot 10^{-12}+9.29 \cdot 10^{-12} t+1.86 \cdot 10^{-11} \sin ^{2} \varphi} \\
& k_{2}=\frac{1}{3.63 \cdot 10^{-11}+3.25 \cdot 10^{-12} t+6.5 \cdot 10^{-11} \sin ^{2} \varphi},
\end{aligned}
$$

und mit einer möglichst Ressourcen schonenden Anordnung von algebraischen und numerischen Operationen erhält man im Koordinatensystem der Parametrisierung die Steifheitsmatrix

$$
\mathcal{S}=10^{6}\left(\begin{array}{ccc|ccc}
121 . & 0 . & 0 . & 0 . & 7.00 & -1.53 \\
0 . & 58.9 & -6.76 & -3.53 & 0 . & 0 . \\
0 . & -6.76 & 43.3 & 1.05 & 0 . & 0 . \\
\hline 0 . & -3.53 & 1.05 & 0.271 & 0 . & 0 . \\
7.00 & 0 . & 0 . & 0 . & 0.491 & -0.118 \\
-1.53 & 0 . & 0 . & 0 . & -0.118 & 0.0317
\end{array}\right)
$$

Das elastische Zentrum der Anordnung liegt bei

$$
Z=\left(\begin{array}{c}
0 \\
0.01389 \\
0.05796
\end{array}\right)
$$


und die Translation auf das elastische Zentrum liefert die elastischen Matrizen

$$
\mathcal{S}_{Z}=10^{6}\left(\begin{array}{ccc|ccc}
121 . & 0 . & 0 . & 0 . & -0.0336 & 0.155 \\
0 . & 58.9 & -6.76 & -0.0249 & 0 . & 0 . \\
0 . & -6.76 & 43.3 & 0.0572 & 0 . & 0 . \\
\hline 0 . & -0.0249 & 0.0572 & 0.0499 & 0 . & 0 . \\
-0.0336 & 0 . & 0 . & 0 . & 0.0875 & -0.0297 \\
0.155 & 0 . & 0 . & 0 . & -0.0297 & 0.0126
\end{array}\right)
$$

und

$$
\mathcal{F}_{Z}=10^{-6}\left(\begin{array}{ccc|ccc}
0.00884 & 0 . & 0 . & 0 . & -0.169 & -0.508 \\
0 . & 0.0173 & 0.00269 & 0.00556 & 0 . & 0 . \\
0 . & 0.00269 & 0.0235 & -0.0257 & 0 . & 0 . \\
\hline 0 . & 0.00556 & -0.0257 & 20.1 & 0 . & 0 . \\
-0.169 & 0 . & 0 . & 0 . & 60.7 & 145 \\
-0.508 & 0 . & 0 . & 0 . & 145 & 429
\end{array}\right)
$$

Die funktionellen Achsen sind die Vektoren

$$
\left(\begin{array}{l}
1 \\
0 \\
0
\end{array}\right), \quad\left(\begin{array}{c}
0 \\
0.9447 \\
-0.3280
\end{array}\right) \text { und }\left(\begin{array}{c}
0 \\
0.3280 \\
0.9447
\end{array}\right) \text {. }
$$

Ihre Verdrehung gegenüber den Koordinatenachsen beträgt damit $19^{\circ}$ und findet entsprechend der Symmetrie in der yz-Ebene statt. Mit der Drehmatrix aus den funktionellen Achsen kann die Transformation auf die Realform durchgeführt werden, die Flexibilitätsmatrix lautet dann

$$
\hat{\mathcal{F}}=10^{-6}\left(\begin{array}{ccc|ccc}
0.0245 & 0 . & 0.000182 & 0 . & -0.0224 & 0 . \\
0 . & 0.00884 & 0 . & -0.535 & 0 . & 0.00696 \\
0.000182 & 0 . & 0.0163 & 0 . & 0.0137 & 0 . \\
\hline 0 . & -0.535 & 0 . & 480 . & 0 . & 0 . \\
-0.0224 & 0 . & 0.0137 & 0 . & 20.1 & 0 . \\
0 . & 0.00696 & 0 . & 0 . & 0 . & 10.2
\end{array}\right) .
$$

Die Realform vertauscht, da sie die Eigenwerte von $\boldsymbol{M}$ ihrer Größe nach anordnet, im Wesentlichen die $x$ - mit der $z$-Achse.

Bis auf die absolute Stärke der Aufhängung, welche sich in skalaren Vorfaktoren ausdrückt, führen andere Modelle zu vergleichbaren Ergebnissen. Daher sollen in 6.5 genau diese Zahlen mit der Messung verglichen werden.

An diesem Beispiel zeigt sich, wie schnell der sinnvolle Einsatz eines Computeralgebrasystems, also die symbolische Manipulation von Ausdrücken, an seine Grenzen gelangt. Eine komplizierte Dickenfunktion der Spongiosa führt, da sie im Nenner des Integranden auftaucht, auf nicht geschlossen integrierbare Ausdrücke. Prinzipiell kann dann der Versuch weiterhelfen, die Dicke durch einen Ausdruck im Nenner 
zu approximieren, dessen Kehrwert wieder einfach wäre und der allein auf integrable Ausdrücke führte. Hier allerdings resultieren aus der Hintereinanderschaltung mit dem Knochenzement über das harmonische Mittel Ausdrücke, die wieder einen komplizierten Nenner haben und demzufolge nicht geschlossen integrabel sind. Das zweite Hemmnis gegen geschlossene Integrabilität ist die Metrik der Fläche, welche als Wurzel der Determinante des metrischen Tensors eine Folge der verwandten Oberfläche ist. Beides schränkt die Anwendbarkeit des analytischen Federmodells bezüglich Formgebung und Dickenfunktion der behandelbaren Probleme stark ein. Der Weg über die Numerik steht aber weiterhin offen.

\subsection{Diagonalform, Transversalkraft und Rezipro- zitätstheorem}

In diesem Kapitel wurde unter der Voraussetzung konstanter Schichtgrößen für viele Aufhängungen eine elastische Matrix in Diagonalform berechnet. Dazu gehören unter den ebenen Aufhängungen die rechteckige Scheibe, Kreisscheibe und Ellipse, sowie die Aufhängungen mit Rotationssymmetrie, also etwa Voll- und Halbkugel, Kreiszylinder mit und ohne Boden, Kreiskegel und Rotationsparaboloid.

Diese hohe Symmetrie der elastischen Matrix erlaubt es, unter der Annahme einer speziellen Last, nämlich einer zur Symmetrieachse transversalen Kraft, die Konstante des Reziprozitätstheorems direkt aus den Matrixkomponenten abzulesen.

Die transversale Kraft $F$ greife im Abstand $h$ auf der Symmetrieachse, hier der $z$-Achse, an und weise in $x$-Richtung. Das entspricht der Last

$$
f=F(1,0,0,0, h, 0)^{T}
$$

mit dem Aufpunkt der Kraftschraube

$$
\overrightarrow{T K}=h \boldsymbol{e}_{z}
$$

Zusammen mit der Flexibilitätsmatrix in Diagonalform

$$
\mathcal{F}=\operatorname{diag}\left(D_{x x}, D_{y y}, D_{z z}, M_{x x}, M_{y y}, M_{z z}\right)
$$

ergibt sich die Lage

$$
q=\mathcal{F} f=F\left(D_{x x}, 0,0,0, h M_{y y}, 0\right)^{T}
$$

und daraus die Drehschraube

$$
\overrightarrow{D S}=\frac{1}{|\boldsymbol{\theta}|^{2}}(\boldsymbol{\theta} \times \boldsymbol{d})=\frac{1}{\left|F h M_{y y} \boldsymbol{e}_{y}\right|^{2}}\left(\left(F h M_{y y} \boldsymbol{e}_{y}\right) \times\left(F D_{x x} \boldsymbol{e}_{x}\right)\right)=-\boldsymbol{e}_{z} \frac{D_{x x}}{h M_{y y}}
$$


Das negative Vorzeichen besagt, daß die beiden Schraubachsaufpunkte einander gegenüber liegen. Für die Konstante des Reziprozitätstheorems ergibt sich dann einfach

$$
\sigma^{2}=-\overrightarrow{D S} \cdot \overrightarrow{T K}=\frac{D_{x x}}{M_{y y}}=\frac{A_{x x}^{-1}}{C_{y y}^{-1}}=\frac{C_{y y}}{A_{x x}} .
$$

Für andere Richtungen der Kraft ergeben sich durch analoge Rechnung andere Konstanten. Da dieser Lastfall aber in der Geschichte der Untersuchung der initialen Zahnbeweglichkeit eine herausragende Rolle spielt, vgl. Abschnitt 6.4, wurde er hier explizit vorgestellt. 


\section{Kapitel 6}

\section{Anwendung auf biomechanische Messungen}

In diesem Kapitel sollen die Erkenntnisse aus den vorangegangenen Abschnitten dieser Arbeit auf Meßwerte angewandt werden. Messungen aus der eigenen Arbeitsgruppe stehen dabei im Mittelpunkt. An diesen Reanalysen werden Leistungsfähigkeit und Grenzen des von mir erdachten Formalismus gezeigt.

\subsection{Die Auswertung im Überblick}

Um zu den einzelnen Beispielen nicht immer dieselben Erläuterungen abgeben zu müssen, sollen zunächst die Rechenschritte der Auswertung in ihrer Abfolge stichwortartig dargestellt werden. Darüber hinaus werden nur die für den jeweiligen Versuch relevanten Teile der Auswertung durchgeführt.

\subsubsection{In jedem Fall durchzuführende Schritte}

1. Messen oder Berechnen einer elastischen Matrix mittels eines statistischen Modells oder einer Theorie für die Linearisierung

- Steifheitsmatrix $q_{M}=\mathcal{S}_{M L} f_{L}$

- Flexibilitätsmatrix $f_{L}=\mathcal{F}_{L M} q_{L}$

2. Dekomposition von Steifheitsmatrix oder Flexibilitätsmatrix

$$
\mathcal{S}_{M L}=\left(\begin{array}{cc}
\boldsymbol{A} & \boldsymbol{B}_{2} \\
\boldsymbol{B}_{1} & \boldsymbol{C}
\end{array}\right) \quad \text { und } \quad \mathcal{F}_{L M}=\mathcal{S}_{M L}^{-1}=\left(\begin{array}{cc}
\boldsymbol{D} & \boldsymbol{S}_{2} \\
\boldsymbol{S}_{1} & \boldsymbol{M}
\end{array}\right)
$$

$\boldsymbol{M}$ und $\boldsymbol{A}$ sind translationsinvariant. 


\subsubsection{Der Translationsanteil}

1. Berechnung der Matrizen $-\boldsymbol{A}^{-1} \boldsymbol{B}_{2}=\boldsymbol{S}_{2} \boldsymbol{M}^{-1}$ und $\boldsymbol{B}_{1} \boldsymbol{A}^{-1}=-\boldsymbol{M}^{-1} \boldsymbol{S}_{1}$

2. Aufspalten in einen symmetrischen und einen antisymmetrischen Anteil

- Antisymmetrischer Anteil: Transformationsmatrizen

$$
\begin{aligned}
\boldsymbol{\Omega}_{M D} & =-\left(\boldsymbol{A}^{-1} \boldsymbol{B}_{2}\right)^{A}=\left(\boldsymbol{S}_{2} \boldsymbol{M}^{-1}\right)^{A} \\
\boldsymbol{\Omega}_{L T} & =\left(\boldsymbol{B}_{1} \boldsymbol{A}^{-1}\right)^{A}=-\left(\boldsymbol{M}^{-1} \boldsymbol{S}_{1}\right)^{A}
\end{aligned}
$$

- Symmetrischer Anteil: Widerstandsmatrizen

$$
\begin{aligned}
& \boldsymbol{W}_{M}=-\left(\boldsymbol{A}^{-1} \boldsymbol{B}_{2}\right)^{S}=\left(\boldsymbol{S}_{2} \boldsymbol{M}^{-1}\right)^{S} \\
& \boldsymbol{W}_{L}=-\left(\boldsymbol{B}_{1} \boldsymbol{A}^{-1}\right)^{S}=\left(\boldsymbol{M}^{-1} \boldsymbol{S}_{1}\right)^{S}
\end{aligned}
$$

3. Berechnung der Güten

$$
G_{M}=\frac{2\left\|\boldsymbol{W}_{M}\right\|^{2}-3\left\|\boldsymbol{\Omega}_{M D}\right\|^{2}}{2\left\|\boldsymbol{W}_{M}\right\|^{2}+3\left\|\boldsymbol{\Omega}_{M D}\right\|^{2}} \quad \text { und } \quad G_{L}=\frac{2\left\|\boldsymbol{W}_{L}\right\|^{2}-3\left\|\boldsymbol{\Omega}_{L T}\right\|^{2}}{2\left\|\boldsymbol{W}_{L}\right\|^{2}+3\left\|\boldsymbol{\Omega}_{L T}\right\|^{2}}
$$

und Beurteilung der elastischen Matrix.

\subsubsection{Information im antisymmetrischen Anteil}

Untersuchung der Transformationsmatrizen aus Punkt 2 in 6.1.2.

1. Ablesen der Ortsvektoren der elastischen Zentren

- Drehmomentszentrum $\overrightarrow{M D}=\mathbf{\Omega}_{M D}^{D}$

- Translationszentrum $\overrightarrow{L T}=\Omega_{L T}^{D}$

2. Zusammensetzen der $6 \times 6$ Transformationsmatrizen

- der Lasten $f_{T}=\mathcal{T}_{T L} f_{L}$

$$
\mathcal{T}_{T L}=\left(\begin{array}{cc}
\boldsymbol{I} & \mathbf{0} \\
\boldsymbol{\Omega}_{T L} & \boldsymbol{I}
\end{array}\right)
$$

- der Lagen $q_{D}=\mathcal{T}_{M D}^{T} q_{M}$

$$
\mathcal{T}_{M D}^{T}=\left(\begin{array}{cc}
\boldsymbol{I} & \boldsymbol{\Omega}_{D M} \\
\mathbf{0} & \boldsymbol{I}
\end{array}\right)
$$


3. Translation der Koordinatenursprünge auf die elastischen Zentren

- Steifheitsmatrix: $\mathcal{S}_{T D}=\mathcal{T}_{T L} \mathcal{S}_{L M} \mathcal{T}_{D M}^{T}$

- Flexibilitätsmatrix: $\mathcal{F}_{D T}=\mathcal{T}_{M D}^{T} \mathcal{F}_{M L} \mathcal{T}_{L T}$

4. Stärke des Effektes der Transformation: Blocknorm

$$
\|*\|_{6 \times 6}=\left(\begin{array}{cccc}
\| *_{1.3,1 . .3} & \|_{3 \times 3} & \| *_{1 . .3,4.6} & \|_{3 \times 3} \\
\| *_{4 . .6,1 . .3} & \|_{3 \times 3} & \| *_{4 . .6,4.6} & \|_{3 \times 3}
\end{array}\right)
$$

vorher und nachher.

\subsubsection{Information im symmetrischen Anteil}

Untersuchung der Widerstandsmatrizen aus Punkt 2 in 6.1.2,

1. - Größe des Widerstandsbereiches $\frac{1}{2} \max _{i, j}\left|w_{i}-w_{j}\right|$

- Aufpunktflächen: Parameterdarstellung $\overrightarrow{Z A}= \pm \boldsymbol{x} \times \boldsymbol{W} \boldsymbol{x}$ $\operatorname{mit} \boldsymbol{x}(\phi, \theta) \in S^{2}$

- Abstandsflächen $|\overrightarrow{Z A}|^{2}=\boldsymbol{x}^{T} \boldsymbol{W}^{2} \boldsymbol{x}-\left(\boldsymbol{x}^{T} \boldsymbol{W} \boldsymbol{x}\right)^{2}$

- Steigungsflächen $\tau= \pm \boldsymbol{x}^{T} \boldsymbol{W} \boldsymbol{x}$

2. Schraubachskongruenzen $\boldsymbol{x}(\phi, \theta) \times( \pm \boldsymbol{W} \boldsymbol{x}(\phi, \theta))+\lambda \boldsymbol{x}(\phi, \theta)$

\subsubsection{Der Rotationsanteil}

1. Zerlegung von $\boldsymbol{M}$ in die singulären Werte $\boldsymbol{M}=\boldsymbol{R}_{f}^{T} \operatorname{diag}\left(m_{i}\right) \boldsymbol{R}_{q}$

2. Spalten sind Bilder der Einheitsvektoren

3. Untersuchung der Drehwinkel $\cos \alpha=\frac{1}{2}(\operatorname{Sp} \boldsymbol{R}-1)$

4. Untersuchung der Drehrichtungen $\boldsymbol{n}=\left(\boldsymbol{R}^{D}\right)^{0}$

5. Zusammensetzung der $6 \times 6$ Rotationsmatrizen

$$
\mathcal{R}_{q}=\left(\begin{array}{cc}
\boldsymbol{R}_{q} & \mathbf{0} \\
\mathbf{0} & \boldsymbol{R}_{q}
\end{array}\right) \quad \text { und } \quad \mathcal{R}_{f}=\left(\begin{array}{cc}
\boldsymbol{R}_{f} & \mathbf{0} \\
\mathbf{0} & \boldsymbol{R}_{f}
\end{array}\right)
$$

6. Berechnung der Normalform

- der Flexibilitätsmatrix $\tilde{\mathcal{F}}=\mathcal{R}_{q} \mathcal{F}_{D T} \mathcal{R}_{f}^{T}$

- der Steifheitsmatrix $\tilde{\mathcal{S}}=\mathcal{R}_{f} \mathcal{S}_{T D} \mathcal{R}_{q}^{T}$ 


\subsubsection{Optionale Schritte}

- Manuelle Angleichung der Orientierung an das Laborkoordinatensystem durch Permutation der Koordinaten

- Test des Federmodells mit $\operatorname{Sp}(\boldsymbol{B})=0$

- Inkompatible Einheitensysteme? Untersuche $\left\|\boldsymbol{W}_{L}\right\| /\left\|\boldsymbol{W}_{M}\right\|$

- Verdrehung der Referenzsysteme? Untersuche Singulärwertzerlegung

$$
\boldsymbol{A}=\boldsymbol{R}_{L}^{T} \operatorname{diag}\left(a_{i}\right) \boldsymbol{R}_{R}
$$

oder Polarzerlegung

- Vorlast? Untersuche antisymmetrische Anteile von $S_{T D}$

\subsection{Das Wirbelsäulensegment}

Die Wirbelsäule ist das vom Kopf bis zum Becken reichende Achsenskelett des menschlichen Körpers. Sie besteht aus den knöchernen Wirbelkörpern, den knorpeligen Bandscheiben dazwischen und dem Bandapparat. Nach Morphologie und Funktion unterscheidet man die Halswirbel $C_{1}-C_{7}$, die Brustwirbel $T_{1}-T_{12}$, die Lendenwirbel $L_{1}-L_{5}$ sowie Kreuzbein und Steißbein. Untereinander sind je zwei Wirbelkörper neben dem Bandapparat über eine Bandscheibe als Synarthrose flächig und durch je zwei Diarthrosen, die Wirbelbogengelenke, gelenkig miteinander verbunden. Eine solche Einheit zu zwei benachbarten Wirbelkörpern wird Bewegungssegment genannt. Es bestimmt maßgeblich das mechanische Verhalten der gesamten Wirbelsäule. Eine Bandscheibe setzt sich aus dem aus Bindegewebe und Knorpel bestehenden äußeren Faserring, dem Annulus fibrosus, und dem zentral gelegenen inneren Gallertkern, dem Nucleus pulposus zusammen. Der Faserring besteht in der Aufsicht aus konzentrischen Schichten kollagener Fasern und Faserknorpel, wobei die Fasern in der Seitsicht einer Schicht eine gekreuzte Struktur aufweisen, ähnlich den Erzeugenden eines einschaligen Hyperboloides. Zwischen der Bandscheibe und den Wirbelkörpern befindet sich hyaliner Knorpel, dennoch wird das Bewegungssegment insgesamt zu den Symphysen gezählt [31].

In diesem Abschnitt soll eine von PANJABI et. al. 53] gemessene gemittelte Steifheitsmatrix der in die beiden angrenzenden Wirbelkörper eingebetten Bandscheibe der Lendenwirbelsäule besprochen werden. Der Vergleich mit der Theorie findet unter Benutzung der Form einer allgemeinen ebenen Aufhängung mit konstanten Schichtgrößen 5.1.6 statt. Einige Werte sollen danach mit einer Messung von Stefan SpIERING aus der eigenen Arbeitsgruppe [59] verglichen werden. Um die Überlegungen abzurunden, werden den Messungen und der eigenen Theorie die numerischen Resultate BROBERGs [8] gegenübergestellt. 
Das Koordinatensystem liegt in der Mitte des oberen Wirbelkörpers, die $y$-Achse weist nach cranial, die $z$-Achse nach frontal und die $x$-Achse in Richtung von $\boldsymbol{e}_{y} \times \boldsymbol{e}_{z}$. Unter der Annahme der Symmetrie in der Sagittalebene (hier also $y z$ ) wird nur eine Auswahl der 36 möglichen Matrixeinträge betrachtet. Diese Reduktion ist zulässig unter der Voraussetzung, daß das Koordinatensystem tatsächlich die Symmetrieebene als Koordinatenebene hat: Kräfte in der Ebene und Drehmomente senkrecht zu ihr sollten die Ebene in ihrer Lage unverändert lassen; es finden also weder Translationen senkrecht zur Ebene noch Rotationen mit in der Ebene verlaufenden Drehachsen statt. Eine Bestätigung dieser Symmetrieannahme durch die Meßwerte hätte letztere noch validiert, das fand jedoch leider nicht statt.

Um viskoelastische Effekte auszuschalten, wurden die Lagen 4 min nach Aufbringen der Last gemessen, was etwa 97\% des asymptotischen Wertes und damit einer Zeitkonstanten von gut 1 min entspricht. Die Steifheitsmatrizen wurden aus den Flexibilitätskonstanten mittels Matrixinversion berechnet und über die Elemente $T_{1-2}$ bis $T_{11-12}$ gemittelt. Die Steifheitsmatrix für positive Lasten lautet dann

$$
\mathcal{S}^{+}=10^{4}\left(\begin{array}{ccc|ccc}
0.011 & 0 & 0 & 0 & 0.058 & 0.015 \\
0 & 0.078 & 0.005 & -0.076 & 0 & 0 \\
0 & 0.005 & 0.01 & -0.164 & 0 & 0 \\
\hline 0 & -0.076 & -0.164 & 15.2 & 0 & 0 \\
0.058 & 0 & 0 & 0 & 15.3 & -0.804 \\
0.015 & 0 & 0 & 0 & -0.804 & 14.8
\end{array}\right),
$$

und die für negative Lasten

$$
\mathcal{S}^{-}=10^{4}\left(\begin{array}{ccc|ccc}
0.011 & 0 & 0 & 0 & 0.062 & 0.164 \\
0 & 0.124 & -0.002 & -0.073 & 0 & 0 \\
0 & -0.002 & 0.01 & -0.156 & 0 & 0 \\
\hline 0 & -0.073 & -0.156 & 18.6 & 0 & 0 \\
0.062 & 0 & 0 & 0 & 14.5 & 0.2 \\
0.164 & 0 & 0 & 0 & 0.2 & 19.7
\end{array}\right)
$$

beide in den Einheiten

$$
\left(\begin{array}{c|c}
N / m m & N / r a d \\
\hline N m m / m m & N m m / r a d
\end{array}\right),
$$

d. h. die Translationsrichtgrößen sind in der Größenordnung $0.1-1 \mathrm{MN} / \mathrm{mm}$ und die der Rotation $150-200 \mathrm{Nm} / \mathrm{rad}$.

Die Güten der Transformationsmatrizen liegen mit

$$
G^{+}=-0.34 \text { und } \quad G^{-}=-0.97
$$

im ersten Fall recht weit entfernt vom Idealwert $G=-1$, vermutlich eine Folge der 
getroffenen Vereinfachungen! ${ }^{1}$ Das elastische Zentrum liegt bei

$$
\boldsymbol{z}^{+}=\left(\begin{array}{c}
0 \\
-8.90 \\
2.60
\end{array}\right) m m \quad \text { und } \quad \boldsymbol{z}^{-}=\left(\begin{array}{c}
0 \\
-15.34 \\
3.24
\end{array}\right) m m
$$

also im ersten Fall eher auf der Unterseite des Wirbelkörpers und im zweiten eher in der Mitte der Bandscheibe. Der Widerstandsbereich hat eine Größe von

$$
B^{+}=8.00 \mathrm{~mm} \quad \text { und } \quad B^{-}=2.43 \mathrm{~mm} \text {. }
$$

Die Matrix für negative Lasten kommt demnach einer Synarthrose mit einem Widerstandszentrum recht nahe. In diesem Fall, der sich ja aus verschiedenen Einzellasten zusammensetzt, sind die Wirbelbogengelenke offenbar gleich gekoppelt oder sogar ganz ohne Kontakt, vgl. B.3. Diese Matrix allein soll nun weiter untersucht werden.

Die Transformation auf das elastische Zentrum ergibt

$$
\tilde{\mathcal{S}}_{Z}^{-}=10^{4}\left(\begin{array}{ccc|ccc}
0.011 & 0 . & 0 . & 0 . & 0.0264 & -0.00473 \\
0 . & 0.124 & -0.002 & 0.298 & 0 . & 0 . \\
0 . & -0.002 & 0.01 & -0.00909 & 0 . & 0 . \\
\hline 0 . & 0.298 & -0.00909 & 16.8 & 0 . & 0 . \\
0.0264 & 0 . & 0 . & 0 . & 14.2 & -0.736 \\
-0.00473 & 0 . & 0 . & 0 . & -0.736 & 17.3
\end{array}\right) .
$$

Eine Rotation um etwa $12^{\circ}$ um die $x$-Achse beendet den Weg zur Realform. Es ergeben sich schließlich die Flexibilitätsmatrix

$$
\hat{\mathcal{F}}^{-}=10^{-4}\left(\begin{array}{ccc|ccc}
91.3 & 0 & 0 & 0 & -0.161 & 0.0539 \\
0 & 13.4 & 20.8 & -0.14 & 0 & 0 \\
0 & 20.8 & 95.4 & 0.0584 & 0 & 0 \\
\hline 0 & -0.14 & 0.0584 & 0.0622 & 0 & 0 \\
-0.161 & 0 & 0 & 0 & 0.0715 & 0 \\
0.0539 & 0 & 0 & 0 & 0 & 0.0574
\end{array}\right)
$$

in den Einheiten

$$
\left(\begin{array}{c|c}
m m / N & m m / N m m \\
\hline \mathrm{rad} / N & \mathrm{rad} / \mathrm{Nmm}
\end{array}\right)
$$

und in den obigen Einheiten die Steifheitsmatrix

$$
\hat{\mathcal{S}}^{-}=10^{4}\left(\begin{array}{ccc|ccc}
0.011 & 0 . & 0 . & 0 . & 0.0247 & -0.0103 \\
0 . & 0.118 & -0.0259 & 0.289 & 0 . & 0 . \\
0 . & -0.0259 & 0.0162 & -0.0734 & 0 . & 0 . \\
\hline 0 . & 0.289 & -0.0734 & 16.8 & 0 . & 0 . \\
0.0247 & 0 . & 0 . & 0 . & 14 . & -0.0232 \\
-0.0103 & 0 . & 0 . & 0 . & -0.0232 & 17.4
\end{array}\right)
$$

\footnotetext{
${ }^{1}$ In der zitierten Publikation befindet sich zudem ein Druckfehler: Bei der auf S. 190 in $[53$ ] angegebenen Matrix $\mathcal{F}^{+}$muß es heißen -5.62 statt -56.2 , vgl. die Einheiten im Plot $F_{23}$ in Fig. 8. Mit dieser Matrix bekäme $\boldsymbol{A}$ auf der Hauptdiagonale negative Einträge, eine Kraft in $y$-Richtung hätte dann eine Translation in die Gegenrichtung zur Folge, was offenbar nicht vernünftig ist.
} 
Die Steifheitsmatrix der allgemeinen ebenen Aufhängung aus 5.1.6 hat in diesen Koordinaten die schachbrettartige Gestalt

$$
\mathcal{S}=\left(\begin{array}{ccc|ccc}
* & 0 & * & 0 & * & 0 \\
0 & * & 0 & * & 0 & * \\
* & 0 & * & 0 & * & 0 \\
\hline 0 & * & 0 & * & 0 & * \\
* & 0 & * & 0 & * & 0 \\
0 & * & 0 & * & 0 & *
\end{array}\right)
$$

Die Spurbedingung (4.6) ist zwar erfüllt, aber durch die Vernachlässigung einiger Terme wenig aussagekräftig. Wegen der unterschiedlich vorausgesetzten Symmetrien können nur die Diagonalelemente mit denen der allgemeinen ebenen Aufhängung (5.1) bis (5.4) verglichen werden. Diese waren unter der Voraussetzung der konstanten Schichtgrößen abgeleitet worden. Ich möchte hier nur Verhältnisse der elastischen Konstanten betrachten.

Man erhält für die translationsinvariante Teilmatrix $\boldsymbol{A}$ mit (5.1)

$$
\frac{\left(A_{11}+A_{22}\right) / 2}{A_{33}}=\frac{k_{2}}{k_{1}}=v_{T},
$$

hier also

$$
v_{T}=\frac{0.014}{0.118}=0.119
$$

wodurch das Stabmodell mit Werten von $v \geq 1 / 3$ als hier nicht brauchbar ausgeschlossen werden kann, vgl. Abb. 4.5. Mit (4.30) entspricht das im Federmodell einer Querdehnzahl von $\sigma_{T} \approx 0.43$. Gegenüber Translationen ändert sich also ihr Volumen kaum, die Bandscheibe kommt eher einem Körper aus Gummi nahe.

Anders ist die Situation bei den Rotationen. Aus (5.4) folgt

$$
\frac{C_{33}}{C_{11}+C_{22}}=\frac{\left(c_{1}+c_{2}-O z_{1}^{2}-O z_{2}^{2}\right) k_{2}}{\left(c_{1}+c_{2}-O z_{1}^{2}-O z_{2}^{2}\right) k_{1}}=\frac{k_{2}}{k_{1}}=v_{R}
$$

hier mit den Zahlenwerten

$$
v_{R}=\frac{14}{16.8+17.4}=0.409,
$$

die, wieder mit (4.30), einer Querdehnzahl von $\sigma_{R} \approx 0.15$ entsprechen. Gegenüber Rotationen ändert sich also das Volumen der Bandscheibe im Wesentlichen durch die Längenänderung.

Diese starke Diskrepanz in den Querdehnzahlen läßt sich durch die innere Struktur der Bandscheibe erklären, da dann die Voraussetzungen zur Ableitung von (5.1) bis (5.3), also insbesondere die konstanten Schichtgrößen, nicht mehr gegeben sind. Der Teil der inneren Struktur, welcher dieses Phänomen erklärt, ist der gallertartige Kern der Bandscheibe, der Nucleus pulposus. Seine Funktion ist, legt man die 
obigen Meßwerte zu Grunde, die gesamte Bandscheibe gegenüber Translationen inkompressibler als gegenüber Rotationen zu gestalten. Das ermöglicht die Beweglichkeit der gesamten Wirbelsäule als Hintereinanderschaltung von Drehungen mit räumlich versetzten Achsen und verhindert gleichzeitig eine zu starke Verschiebung der einzelnen Wirbelsegmente gegeneinander.

Sicherlich kann man auch versuchen, kompliziertere Modelle, etwa die mit einer Faserstruktur oder die dem Biegebalken verwandten mit einem expliziten lokalen Beitrag der Rotationsanteile zur elastischen Energie, im Hinblick auf eine Erklärung der Werte heranzuziehen. Da die Phänomene aber schon mit dem einfacheren Modell gedeutet werden können, ist das nicht nötig.

Die Messung von [59] an einem konservierten Bewegungssegment ergab nach Entfernung der Wirbelbogengelenke bezüglich axialer Rotationen (also in $y$-Richtung) eine Flexibilität von $0.021 \mathrm{rad} / \mathrm{Nm}$ gegenüber hier $0.007 \mathrm{rad} / \mathrm{Nm}$. Demnach hätte die Konservierung keine versteifende, sondern eine um den Faktor drei flexibilisierende Wirkung. Das kann durch die starke individuelle Streuung der elastischen Konstanten erklärt werden - es handelt sich ja um eine Messung zu einem Einzelpräparat. Plausibler ist dieser Effekt mit einer erhöhten Durchlässigkeit der Zellmembranen gegenüber der Konservierungsflüssigkeit im Vergleich zur originalen Gewebsflüssigkeit erklärbar. Dennoch sollte diese „Stärke“ der Aufhängung als genereller Vorfaktor nur geringen Einfluß auf die Struktur der Bewegung haben.

Ferner sollte ein variables axiales Drehmoment zusammen mit einer konstanten Last parallel dazu eine reine Drehung mit durch das Widerstandszentrum verlaufender Achse zur Folge haben. Alle meßbaren Translationen stehen dann senkrecht auf dieser Achse. In der Tat waren die Aufpunkte der Drehschrauben erwartungsgemäß konstant. Hingegen ergab die Messung statt einer verschwindenden eine linear mit dem Drehmoment anwachsende Schraubsteigung! Das könnte zwar ein Effekt der in 3.1.4.2 besprochenen Relaxationseffekte sein, ist hier aber m. E. anders zu deuten: Wenn eine flächige Aufhängung auf ein zu ihr senkrechtes Drehmoment tatsächlich mit einer Translation parallel zu diesem antwortet, dann muß dieses aus Symmetriegründen einen von dem Vorzeichen der Drehung unabhängigen Wert haben. Da das mit einer linearen Abhängigkeit unvereinbar ist, ergeben sich im einfachsten Fall Ursprungsparabeln für die Translation. Auch das läßt sich auf den Bau der Bandscheibe zurückführen: Eine axiale Kompression/Extension der Bandscheibe unter einem axialen Drehmoment hat unter Berücksichtigung der gekreuzt verlaufenden Fasern des Annulus fibrosus für ein entgegengesetzt gerichtetes Drehmoment wieder eine Kompression/Extension zur Folge. Die Messung ergab ein Anwachsen der Bandscheibe von $0.1 \mathrm{~mm}$ auf $2^{\circ}$. Dieses Anwachsen kann mit dem Hyperboloidmodell erklärt werden. Der theoretischen Verkleinerung des Volumens im Inneren bei Drehungen wirken sowohl der inkompressible Kern als auch die Volumenerhöhung der Faserschichten entgegen. Der Faserring ist demnach derart gestaltet, daß der Druck im Kern den Faserring nicht nach außen ausbeult, sondern die einzelnen Fasern längt - ein komplexes Zusammenspiel aus Geometrie 
und Elastizität.

Alle Messungen mit Wirbelbogengelenken hatten nur noch kompliziertere Effekte zur Folge. Diese ließen sich so interpretieren, daß nicht der lineare Zwangskontakt aus B.3, sondern für einen einzelnen Kontakt die dimere Kette, und für beide Gelenke im Kontakt das Viergelenk, vgl. [49], angemessene Modelle zum Beschreiben der Bewegung sind. Da diese Zwangsbedingungen quadratisch sind, wird der Gültigkeitsbereich dieser linearen Theorie verlassen.

Als Letztes soll noch der Vergleich mit dem komplexen geometrischen Modell der Bandscheibe Brobergs [8] vorgenommen werden. Dort werden die Lageänderungen bei verschiedenen axialen Vorlasten vorgegeben und die resultierenden Lasten über die einzelnen Bestandteile der Bandscheibe numerisch aufsummiert. Die Bandscheibe wird aus zwei Phasen zusammengesetzt. Die eine Phase sind die Fasern; sie sind ohne Verbindung untereinander, tragen aber über ihre Krümmung zu den Lasten bei. Dieser Beitrag koppelt über die Ausbeulung der konzentrisch angeordneten Faserschichten mit dem Gallertkern. In den aufeinander folgende Schichten sind die Fasern entgegengesetzt geneigt, wodurch die gekreuzte Struktur modelliert wird. Für die Fasern selbst wird eine nichtlineare Kraft-Auslenkungs-Beziehung zu Grunde gelegt. Die zweite Phase sind der Kern und der Raum zwischen den Faserschichten, welche als inkompressibel angenommen werden. Aus den Graphen wurden die Diagonalelemente der differentiellen Steifheitsmatrix zu den axialen Vorlasten 700 und $3000 N$ bestimmt. Es ergaben sich die Steifigkeiten

$$
\mathcal{S}_{700}=((.093, .080,2.2) \mathrm{MN} / \mathrm{m},(9.2,12,360) \mathrm{Nm} / \mathrm{rad})
$$

und

$$
\mathcal{S}_{3000}=((0.27,0.20,6.3) \mathrm{MN} / \mathrm{m},(21,27,690) \mathrm{Nm} / \mathrm{rad}) \quad .
$$

Zunächst ist klar erkennbar, daß ein Stress-Stiffening um etwa den Faktor 3 für Translationen und den Faktor 2 für Rotationen stattgefunden hat, vgl. auch [43]. Interessanterweise ergibt sich für axiale Kompression eine Kopplung zwischen axialen Kräften und Drehmomenten, was einerseits auf eine nicht verschwindene Steigung der Kraftschraube und andererseits auf Nebendiagonalelemente in der Steifheitsmatrix führt. Diese lassen sich nicht durch eine Transformation auf das elastische Zentrum wegtransformieren, so daß kein Widerstandszentrum sondern ein Widerstandsbereich vorliegt. Als Nächstes sollen wieder die Verhältnisse der Größen betrachtet werden. Für Translationen ergibt sich beide Male

$$
v_{T}=0.04,
$$

was das reine Stabmodell wieder ausschließt und einer Querdehnzahl von etwa 0.47 entspricht. Die extreme Anisotropie bei den Rotationssteifigkeiten führt auf

$$
v_{R}=18 \text { und } 15 \text {, }
$$

was, da der Wert erheblich größer ist als 1, nur durch ein Stabmodell mit einer expliziten Abhängigkeit der Federmatrix $\mathcal{K}$ von den Rotationen erklärt werden 
kann, vgl. (4.13). Das entspricht m. E. auch den im Modell verwandten Annahmen. Wahrscheinlich würde eine elastische Kopplung der Faserringe untereinander einen geringeren Einfluß der inkompressiblen Anteile zur Folge haben und die Isotropie des Rotationsanteils erhöhen.

Insgesamt ist das Modell einer linearen Abhängigkeit der Lagen von den Lasten selbst für eine Bandscheibe ohne Wirbelbogengelenke zu einfach, obwohl mit ihm Aussagen getroffen werden können. Demnach müßte auch das multivariate Regressionsmodell aus Abschnitt 3.2.3 mindestens um quadratische Terme erweitert werden, etwa

$$
q_{i}=\mathcal{F}_{i j} f_{j}+F_{i j k} f_{j} f_{k} .
$$

Dabei ist zu beachten, daß quadratische Terme auch bei ungenügender Relaxation entstehen. Nur Messungen an frischem Material werden entscheiden können, ob die absoluten Steifigkeiten eine Folge des generellen Einflusses der Konservierung auf die Bestandteile der Bandscheibe sind, oder aber ob es sich um eine unterschiedliche Wirkung auf die einzelnen Komponenten handelt, die eine geänderte Kopplung zwischen elastischen und flüssigen Anteilen nach sich zieht.

\subsection{Ein zylindrischer Probekörper}

Dieser Probekörper diente BenEDIKT BeCKER [4] der Überprüfung der Meßapparatur aus dem nächsten Abschnitt. Da die Steifheitsmatrix einer solchen Aufhängung in Abschnitt 5.2 .2 berechnet wurde, soll zunächst diese Messung betrachtet und mit der Vorhersage verglichen werden. Ferner soll exemplarisch vorgeführt werden, wie bei bekannten Abmessungen gleichzeitig Elastizitätsmodul und Querdehnzahl aus der Steifheitsmatrix bestimmt werden können.

Es handelte sich um einen ein Kreiszylinder mit $4 \mathrm{~mm}$ innerem Radius und $18.5 \mathrm{~mm}$ Höhe, bei dem die Berandungen und die elastische Schicht durch Polymerisation miteinander verbunden waren. Die Schichtdicke betrug $1 \mathrm{~mm}$. Der geometrische Mittelpunkt des Zylinders lag bei $(3.8,0.76,0.75) \mathrm{cm}$ und die Zylinderachse wies in $x$-Richtung.

Es wurden drei vollständige Messungen mit verschiedenen Lastbezugspunkten und ein vom Experimentator restringierter Datensatz als Rohdaten verwendet. Die Bestimmung der Flexibilitätskonstanten erfolgte durch univariate lineare Regression. Die Flexibilitätsmatrizen wurden einzeln auf Realform gebracht. Dabei änderte sich die Blocknorm von

$$
\left(\begin{array}{c|c}
8 \ldots 120 & 31 \ldots 49 \\
\hline 1 \ldots 36 & 24 \ldots 37
\end{array}\right) \quad \text { auf } \quad\left(\begin{array}{c|c}
7 \ldots 11 & 3 \ldots 5 \\
\hline 2 \ldots 4 & 24 \ldots 37
\end{array}\right)
$$

in den Einheiten

$$
\left(\begin{array}{c|c}
\mu \mathrm{m} / \mathrm{N} & \mu \mathrm{m} / \mathrm{Ncm} \\
\hline 10^{-4} \mathrm{rad} / \mathrm{N} & 10^{-4} \mathrm{rad} / \mathrm{Ncm}
\end{array}\right)=\left(\begin{array}{c|c}
\mu \mathrm{m} / \mathrm{N} & 10^{-4} / \mathrm{N} \\
\hline 10^{-4} \mathrm{rad} / \mathrm{N} & 10^{-4} \mathrm{rad} / \mathrm{Ncm}
\end{array}\right),
$$


welche konsistent sind und auch im Folgenden beibehalten werden sollen. Die Güte für das Meßsystem lag einheitlich bei -0.997, die für das Lastsystem bei zwei Messungen bei -0.994 und -0.976 . Zwei Experimente waren so gestaltet, daß der Lastangriff in der Zylindermitte erfolgte, bei diesen lag die Güte erwartungsgemäß nahe +1 bei +0.918 und +0.926 . Das Drehmomentszentrum liegt für alle Matrizen bei

$$
\overrightarrow{M D}=\left(\begin{array}{l}
3.81 \\
0.82 \\
0.62
\end{array}\right) \mathrm{cm}
$$

mit Streuungen im Bereich 0.6mm. Nach Auskunft der Flexibilitätsmatrizen wurden die Bezugspunkte der Last dabei im Bereich

$$
\overrightarrow{L T}=\left(\begin{array}{c}
0 \ldots 3.75 \\
-0.99 \ldots 0.67 \\
-0.94 \ldots 0.65
\end{array}\right) \mathrm{cm}
$$

variiert. Die Größe des Widerstandsbereiches ergab $0.15 \mathrm{~cm}$ für das Meßsystem und $0.18 \pm 0.08 \mathrm{~cm}$ für das Lastsystem. Zylindermitte und Drehmomentszentrum stimmen in $x$ - und $y$-Richtung recht gut überein, nur in $z$-Richtung ergibt sich eine im Rahmen der Genauigkeit tolerable Abweichung von 1.3 $\mathrm{mm}$. Die Rotation auf die Realform erfolgte einheitlich um etwa die $x$-Achse um $62^{\circ}$ und $61^{\circ}$. Die Güte der Matrix $\boldsymbol{A}$ reichte von +0.39 bis hin zu +0.98 und lag im Mittel bei 0.65 .

Da Realformen dieser Matrizen alle miteinander vergleichbar sind, dürfen sie komponentenweise diskutiert werden. Die Mittelwerte der einzelnen Komponenten der Flexibilitätsmatrizen in Realform lauten

$$
\left(\begin{array}{ccc|ccc}
6.39 & 0.831 & -0.901 & 0.0158 & 0.396 & 0.399 \\
-0.542 & 4.48 & 0.306 & 2.44 & -0.692 & -0.00921 \\
-0.393 & -0.981 & 2.77 & 2.9 & -0.0112 & -0.332 \\
\hline-0.0339 & 0.183 & 1.04 & 28.2 & 0 & 0 \\
0.0332 & -1.17 & -0.191 & 0 & 4.57 & 0 \\
0.149 & -0.162 & 0.201 & 0 & 0 & 3.88
\end{array}\right)
$$

mit den Streuungen

$$
\left(\begin{array}{ccc|ccc}
0.412 & 1.08 & 0.69 & 0.00353 & 0.0784 & 0.0772 \\
0.48 & 2.22 & 1.3 & 0.581 & 0.122 & 0.0725 \\
1.08 & 1.86 & 0.418 & 0.659 & 0.085 & 0.0639 \\
\hline 0.292 & 2.18 & 1.62 & 6.38 & 0 & 0 \\
0.362 & 0.382 & 0.0645 & 0 & 0.861 & 0 \\
0.224 & 0.0556 & 0.618 & 0 & 0 & 0.75
\end{array}\right) .
$$

Die zeitliche Abfolge der Messungen zeigte, daß diese Streuung teils durch eine langsam fortschreitende Auspolymerisation der elastischen Schicht und teils durch die Restriktion des Datensatzes hervorgerufen wurde. Bis auf die Komponente $D_{22}$ 
verursacht das im Wesentlichen nur einen skalaren Effekt, also entspricht die obige gemittelte Matrix etwa einem zeitlichen Zwischenzustand.

Bei drei Werten lehnt ein beidseitiger t-Test auf gleiche Mittelwerte zum 10\%Niveau bei einer Veränderung des empirischen Mittels um $s / \mu=\sqrt{n} / t_{4,10 \%}=0.9$ Standardabweichungen nicht ab. Als Faustformel dürfen demnach die Werte um etwa eine Standardabweichung verändert werden, ohne signifikante Veränderungen vorgenommen zu haben. Dennoch sind mit 12 von 21 Werten außerhalb der Diagonalen nur rund die Hälfte überhaupt statistisch von Null verschieden. Der Rest liegt, wie die Veränderung der Blocknorm zeigt, im Bereich des absoluten Fehlers der Apparatur. Da die ursprünglichen Flexibilitätskoeffizienten durch die Rotation auf Realform untereinander vermischt werden, können deren Streuungen auch andere Komponenten stören. Prinzipiell erlaubt das eine Interpretation der Nebendiagonalmatrizen als Vorliegen eines Widerstandszentrums. Hier soll etwas behutsamer zunächst eine Symmetrisierung der Matrix unter Berücksichtigung der Streuungen erfolgen?2 Es ergibt sich die symmetrische Matrix

$$
\left(\begin{array}{ccc|ccc}
6.39 & -0.119 & -0.702 & 0.0152 & 0.331 & 0.335 \\
& 4.48 & -0.225 & 1.97 & -0.809 & -0.0957 \\
& & 2.77 & 2.36 & -0.113 & -0.282 \\
\hline & & & 28.2 & 0 & 0 \\
& & & & 4.57 & 0 \\
& & & & & 3.88
\end{array}\right)
$$

mit der Streuung

$$
\left(\begin{array}{ccc|ccc}
0.412 & 0.665 & 0.84 & 0.00697 & 0.129 & 0.115 \\
& 2.22 & 1.53 & 0.917 & 0.186 & 0.0629 \\
& & 0.418 & 0.936 & 0.0733 & 0.116 \\
\hline & & & 6.38 & 0 & 0 \\
& & & & 0.861 & 0 \\
& & & & & 0.75
\end{array}\right) .
$$

Eine formale abermalige Transformation auf das elastische Zentrum mit einer Veränderung des Bezugspunktes im sub- $m m$ Bereich liefert schließlich die Flexi-

\footnotetext{
${ }^{2}$ Das gewichtete Mittel zweier Meßreihen mit Mittelwerten und Standardabweichungen $\mu_{1} \pm s_{1}$ und $\mu_{2} \pm s_{2}$ ist

$$
\frac{\mu_{1} s_{2}+\mu_{2} s_{1}}{s_{1}+s_{2}}
$$

Dieses Mittel entfernt sich von den anfänglichen Mittelwerten gleich weit, gemessen in der jeweiligen Streuung. Die Streuung für den neuen Wert ergibt sich nach der Fehlerfortpflanzung $\mathrm{zu}$

$$
\frac{\sqrt{2} s_{1} s_{2}}{s_{1}+s_{2}}
$$

also aus dem durch $\sqrt{2}$ dividierten harmonischen Mittel der einzelnen Streuungen. Insgesamt werden so Werte mit kleinen Streuungen begünstigt - wie es auch sein soll.
} 
bilitätsmatrix

$$
\left(\begin{array}{ccc|ccc}
6.39 & -0.118 & -0.702 & 0.0152 & 0.325 & 0.33 \\
& 4.49 & -0.22 & 2 . & -0.809 & -0.096 \\
& 2.78 & 2.39 & -0.113 & -0.282 \\
\hline & & 28.2 & 0 . & 0 . \\
& & & & 4.57 & 0 . \\
& & & & & 3.88
\end{array}\right)
$$

Obwohl immer noch die Terme der Nebendiagonalmatrizen statistisch von Null verschieden sind, zeigt die Betrachtung der Normen der absoluten Fehler der Originaldaten zusammen mit der Fehlerfortpflanzung über die Abstände, daß ihnen kaum eine sinnvolle Information zu entnehmen ist. Eine Deutung der antisymmetrischen Anteile der Nebendiagonalmatrizen $\boldsymbol{B}_{i}$ der Steifheitsmatrix ist wegen der Werte bis zu $20 k N$ auch nicht sinnvoll. Die über alle Messungen konstante Drehung auf Realform erlaubt zusammen mit der aus den Hauptdiagonaltermen ablesbaren Anisotropie die Deutung, daß der Probekörper leicht asymmetrisch bzw. azentrisch gestaltet ist und daher kein Widerstandszentrum mehr besitzt, aber daß der Widerstandsbereich mit $1.4 \mathrm{~mm}$ klein und in derselben Größenordnung wie die Meßgenauigkeit ist. Der Ausreißer bei $D_{22}$ zeigt ferner, daß an den Translationsanteil einer Einzelmessung keine allzu großen Erwartungen zu stellen sind.

Im Folgenden sollen die Werte mit der Vorhersage des Kreiszylinders verglichen werden, um die Schichtgrößen zu bestimmen. Dazu wendet man die mit der Streuung gewichtete Mittelung auf die zur $y$ - und $z$-Achse gehörenden Einträge an. Das wird von einem entsprechenden t-Test nicht verworfen. Es ergibt sich die mit dem Ergebnis aus Abschnitt 5.2.2 zu vergleichende Diagonalmatrix

$\mathcal{F}=\operatorname{diag}(6.39 \pm 0.41,3.05 \pm 0.50,3.05 \pm 0.50,28.2 \pm 6.4,4.20 \pm 0.75,4.20 \pm 0.75)$

und mit dieser das überbestimmte Gleichungssystem

$$
\begin{aligned}
\pi h r 2 k_{2} & =1 /(6.39 \mu \mathrm{m} / \mathrm{N}) \\
\pi h r\left(k_{1}+k_{2}\right) & =1 /(3.05 \mu \mathrm{m} / \mathrm{N}) \\
2 \pi h r^{3} k_{2} & =1 /\left(28.2 \cdot 10^{-4} \mathrm{rad} / \mathrm{Ncm}\right) \\
\frac{\pi h r}{12}\left(h^{2}\left(k_{1}+k_{2}\right)+12 r^{2} k_{2}\right) & =1 /\left(4.20 \cdot 10^{-4} \mathrm{rad} / \mathrm{Ncm}\right),
\end{aligned}
$$

welches in zwei Teilsysteme für Rotation und Translation zerfällt. Wegen der Flächenkorrektur (4.10) ist mit der Dicke $1 \mathrm{~mm}$ in diesen Formeln der Radius

$$
r=(0.5(1 / 4+1 / 5))^{-1} m m=4.44 m m
$$

anzusetzen. Unter Benutzung des Rotationsanteils ergeben sich die Schichtgrößen

$$
k_{1}=2.61 \cdot 10^{9} \mathrm{~N} / \mathrm{m}^{3} \mathrm{rad} \quad \text { und } \quad k_{2}=3.34 \cdot 10^{8} \mathrm{~N} / \mathrm{m}^{3} \mathrm{rad},
$$


und mit dem Translationsanteil folgt

$$
k_{1}=9.54 \cdot 10^{8} \mathrm{~N} / \mathrm{m}^{3} \quad \text { und } \quad k_{2}=2.99 \cdot 10^{8} \mathrm{~N} / \mathrm{m}^{3} \text {. }
$$

Das Verhältnis beider Werte sollte in beiden Fällen dasselbe sein, was nicht zutrifft. Diese Diskrepanz kann behoben werden, indem man annimmt, daß die nahe an der Grenze der absoluten Genauigkeit liegenden Werte für $\mathcal{F}_{22}$ und $\mathcal{F}_{33}$ wegen der Eigenelastizität der Apparatur überschätzt wurden. Ist der tatsächliche Wert gleich $1.25 \mu \mathrm{m} / \mathrm{N}$, welcher außerhalb der absoluten Meßgenauigkeit liegt, und ist der effektive Radius mit $4.5 \mathrm{~mm}$ etwas größer, so erhält man zusammen mit dem nach oben korrigierten Wert $\mathcal{F}_{44}=0.003 \mathrm{rad} / N \mathrm{~cm}$ annähernd übereinstimmend

$$
k_{1}=2.8 \cdot 10^{9} \mathrm{~N} / \mathrm{m}^{3} \quad \text { und } \quad k_{2}=3.1 \cdot 10^{8} \mathrm{~N} / \mathrm{m}^{3} \quad \text {, }
$$

was $v=0.11$, also einer Querdehnzahl von $\sigma=0.44$, und mit einer Dicke von $1 \mathrm{~mm}$ einem Elastizitätsmodul von $E=0.88 \mathrm{MPa}$ entspricht.

Da diese Werte für Elastomere nicht untypisch sind, stimmen Messung und Theorie im Rahmen der Genauigkeiten von Messung und Herstellung des Probekörpers überein. Zur Bestimmung der elastischen Konstanten ist, wegen der günstigen Fehlerfortpflanzung, einer Messung mit reinen Drehmomenten an einem gut bekannten Körper der Vorzug zu geben.

Zusammenfassend lieferte die Meßapparatur in etwa 0.5 bis max. $1.5 \mathrm{~mm}$ genaue Orte. Die große Streuung der Flexibilitätskonstanten ergibt sich aus vier Hauptquellen:

- Aus dem Lastsystem, welches nur mit großer manueller Geschicklichkeit an die Koordinatenachsen des ruhenden Meßobjektes angepaßt werden kann,

- aus dem Meßsystem, da die verwendete 6-Taster-Anordnung wegen der unbekannten Nullagen der Taster Fehler erster Ordnung bei der Bestimmung der Translationsanteile der Lagen hervorruft,

- aus einer Restriktion des Datensatzes auf einseitige oder erst ab einem Mindestwert wirkende Lasten und

- aus der Dauer der gesamten Messung, da sich das Meßobjekt gegen Ende der Messung durch chemische Veränderungen von seinem Anfangszustand entfernt hat.

Dieser letzte Effekt ist gerade bei an biologischen Objekten gewonnenen Daten als mögliche Fehlerquelle im Auge zu behalten. Außerdem zeigt sich, daß die Lösungen der Gleichungen für die Materialkonstanten sehr stark von der Probengeometrie abhängen. Daher sind extrem genaue Probekörper vonnöten, denn eine kleine absolute Veränderung der Schichtdicke entspricht bei einer dünnen Schicht einer großen relativen. 


\subsection{Initiale Zahnbeweglichkeit}

In diesem Abschnitt sollen von BENEDIKT BECKER in unserer Arbeitsgruppe durchgeführte Messungen zur initialen Zahnbeweglichkeit [3, 4] zur Sprache kommen. An ihnen soll die Notwendigkeit der Dreidimensionalität der Betrachtung einer Synarthrose und die entscheidende Rolle der Scherelastizität gezeigt werden - gleich ob Messung oder Theorie. Die Genauigkeit und Zuverlässigkeit der Meßapparatur wurde bereits im letzten Abschnitt betrachtet.

Zunächst sollen die bekanntesten Theorien und Vorstellungen zum elastischen Verhalten des Periodonts, des bewegungsbestimmenden Elementes des Zahnhalteapparates, vgl. [44, 7], vorgestellt werden.

\subsubsection{Elastische Faser vs. inkompressible Flüssigkeit?}

Der Zahnhalteapparat verbindet die Zahnwurzel federnd mit der Alveole des Kiefers. Seine wesentlichen Bestandteile sind das Periodont, die bindegewebige Wurzelhaut des Zahnes, das, wie auch die Knochenhaut des Alveolarkamms, kontinuierlich in das Zahnfleisch übergeht. Der innere Aufbau des Periodonts ist ausführlich in [5] beschrieben. Auch der Bandapparat des Zahnfleisches besitzt eine komplexe räumliche Faserstruktur, vgl. [15]. Die Verbindung der Zähne untereinander erfolgt außer dem Bandapparat, einer Synarthrose, auch durch ihre Approximalkontakte, einer Diarthrose. Das Periodont enthält neben den Fasern die interstitielle Flüssigkeit und ein umfangreiches Blut- und Lymphgefäßsystem. Seine reichliche Nervenversorgung mit Mechanorezeptoren erlaubt es, den Zahn als Tastorgan einzusetzen.

Die auf das in dieser Arbeit verwendete Federmodell passenden Fasern sind die flächig angeordneten kollagenen. Die Faserstruktur selbst wird aus Gründen der Einfachheit zunächst außer acht gelassen. Wichtiger für das Federmodell ist die Verteilung der Dicke des Periodonts über die Wurzeloberfläche. Der dünnste Teil des Periodonts befindet sich von der Mitte der Zahnwurzel aus gesehen etwas in Richtung zur Wurzelspitze hin, man spricht auch von einer „Sanduhrform“. In der Nähe des dünnsten Teiles werden auch die Rotationsachsen zu physiologischen Lasten vermutet. Die Dicke des Periodontalspaltes variiert interindividuell von $50 \mu \mathrm{m}$ bis $300 \mu m$, als mittlere Dicken sind etwa 200-140-200 $\mu \mathrm{m}$ anzunehmen [12]. Eine Streuung der elastischen Eigenschaften um einen Faktor drei ist daher durchaus zu erwarten.

Hier interessiert die Bewegung eines Frontzahnes unter Last. Nach anfänglich naiven Modellen des Zahnes als ein- oder zweiseitigem Hebel mit entweder in der Wurzelspitze oder im Alveolareingang gelegener Drehachse bildeten sich um 1930 zwei konkurrierende Theorien aus. Die erste zieht die Faserstuktur des Periodonts zur Erklärung heran und die zweite dessen Flüssigkeitsanteil, vgl. [66]. Die Vor- 
stellungen der elastic cord theory, im Folgenden als ECT abgekürzt, geht auf A. M. Schwarz [55] zurück und wurde von H. Thirring und V. Weisskopf erstmalig durchgerechnet. Die Ergebnisse sind, leider ohne Angabe des Rechenganges, in [56] dargestellt. Die Incompressible Membrane Theory, abgekürzt IMT, beruht auf Ideen von A. Gysi [23] und wurde von J. L. Synge [64, 65] ausgearbeitet. Der wesentliche Unterschied beider Theorien ist die Gültigkeit für verschiedene Zeitskalen: Während zu Beginn der Lasteinwirkung die Flüssigkeitsanteile sicher eine Rolle spielen, sollten diese Effekte durch Diffusion proportional zum anfänglich aufgebauten Druckgradienten nach einer gewissen Zeit abgeklungen sein. Die Flüssigkeit muß daher in einer Theorie, welche die Dämpfungseffekte modelliert, berücksichtigt werden. Hier noch abschließend ein grober tabellarischer Überblick über die am elastischen oder kinematischen Geschehen beteiligten Gewebe:

\begin{tabular}{l||l|l} 
Dauer $\backslash$ Last & niedrig & hoch \\
\hline \hline kurz & Flüssigkeit & Flüssigkeit, Alveolarkamm \\
\hline mittel & bindegewebige Fasern & Fasern, Alveolarkamm \\
\hline lang & Knochenzellen & Nekrose
\end{tabular}

Das von mir entwickelte Federmodell ist demnach für niedrige Lasten und mittlere Dauern angemessen.

Es existieren in der späteren Literatur die unterschiedlichsten Vorstellungen über Art und Auswirkung der faserigen und kontinuierlichen Anteile der Gewebe, vgl. wieder etwa [5]. So ist beispielsweise eine Abhängigkeit der Zahnstellung vom Blutdruck zu beobachten, weshalb eine Veränderung des Gesamtvolumens des Periodonts nicht dauerhaft mit einer Veränderung des Druckes einhergehen muß; das System ist thermodynamisch offen. Zur Deutung des Zahndurchbruchs und der Möglichkeit orthodontischer Zahnbewegungen werden dem Periodont als Kontinuum statt elastischer oder viskoelastischer gar thixotrope Eigenschaften zugeschrieben [32], die Dauern solcher Bewegungen übersteigen freilich die der hier vorgestellten Untersuchung. Andere wiederum stellen den mikroskopischen Aufbau des Faserapparates mit seiner gewellten Struktur und seinem chemomechanischen Verhalten in den Vordergrund [10. Da alle diese Vorstellungen gedanklich auf der ECT oder der IMT aufbauen, möchte ich diese nur schwer konkret mathematisch handhabbaren Erweiterungsmöglichkeiten hier vollständig außer Acht lassen.

Um die beiden elementaren Theorien mit der meinigen vergleichen zu können, werde ich zunächst die bekanntere, die ECT, in einer nach [13] von mir modifizierten Fassung vorstellen. Sie enthält einige Annahmen und Ansätze, die m. E. vom Autor nicht klar genug herausgearbeitet sind, und kann, ganz ohne Benutzung eines Computers, analytisch gelöst werden. Danach soll in chronologischer Reihenfolge ein Überblick der wichtigsten Arbeiten gegeben werden.

Die Grundidee der ECT ist es, die Gesamtkraft als Summe der Einzelkräfte von Teilsegmenten der Zahnwurzel und das Gesamtdrehmoment als Summe der Ein- 
zeldrehmomente zu berechnen. Diese Teilsegmente werden an einem einwurzeligen Zahn über eine Achse längs des Zahnes gewonnen. Es wird meist nur der transversale Lastfall betrachtet, vgl. Abb. 6.1.

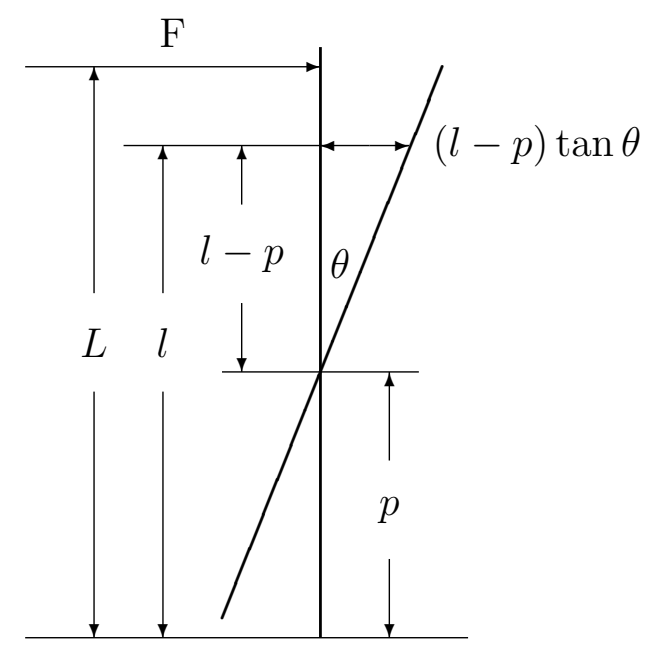

Abbildung 6.1: Die Grundfigur der Elastic Cord Theory.

1. Annahme: Die Transversalkraft ruft nur eine reine, kleine Drehung hervor. Das ist insofern eine zulässige Näherung, als die Translationsanteile gegenüber den Rotationsanteilen klein sind. Der Drehpol $p$ liegt dann auf der Zahnachse. In zwei Dimensionen ist das korrekt, die allgemeine räumliche Bewegung ist aber eine Schraubung.

2. Ansatz: Die i-te Einzelkraft $f_{i}$ ist proportional zur Einzelfläche $a_{i}$ mal der Auslenkung im i-ten Punkt. Mit dem Drehwinkel $\theta$ (kleine Drehungen: $\tan \theta \approx$ $\theta$ ) erhält man

$$
f_{i}=K \theta\left(l_{i}-p\right) a_{i}
$$

Der Proportionalitätsfaktor $K$, ein Skalar, ist eine Materialkonstante des Periodonts. Hier wird eine Zustandsgleichung für das Periodont vorausgesetzt. Diese enthält Informationen über die Dimension des Problems, die Scherelastizität und das Transformationsverhalten der beteiligten Größen.

3. Das Einzeldrehmoment $t_{i}$ bezüglich des Pols ist gegeben als Produkt der Einzelkraft $f_{i}$ mit dem Abstand vom Pol.

$$
t_{i}=\left(l_{i}-p\right) f_{i}=K \theta\left(l_{i}-p\right)^{2} a_{i}
$$

Hier werden die Scherkräfte vernachlässigt, da eine sehr spezielle Auslenkung vorausgesetzt wird und benachbarte Flächenstücke nicht miteinander wechselwirken. 
4. Annahme: Die Einzelfläche $a_{i}$ ist bekannt als Funktion der Längsachse. Ist der Wurzelquerschnitt kreisförmig, so kann die Einzelfläche etwa aus einer Röntgenaufnahme bestimmt werden. Der Wurzelumfang $u_{i}$ ergibt sich dann aus der Wurzeldicke $d_{i}$ als $u_{i}=\pi d_{i}$. Die als zylindrisch genäherte Einzelfläche erhält man mit der Einzelhöhe $h$ zu

$$
a_{i}=\pi d_{i} h
$$

Die Einzelfläche kann auch aus Modellen der Zahnwurzelgeometrie wie $d_{i} \sim l_{i}$ oder $d_{i}^{2} \sim l_{i}$ gewonnen werden; die Summation wird dann zur Integration.

Die Gesamtkraft ist nun

$$
\begin{aligned}
F & =K \theta \sum_{i=1}^{n}\left(l_{i}-p\right) a_{i}=K \theta(\underbrace{\sum_{i=1}^{n} l_{i} a_{i}}_{=: B}-p \underbrace{\sum_{i=1}^{n} a_{i}}_{=: A}) \\
& =K \theta(B-p A),
\end{aligned}
$$

und das Gesamtdrehmoment ergibt sich zu

$$
\begin{aligned}
T & =K \theta \sum_{i=1}^{n}\left(l_{i}-p\right)^{2} a_{i}=K \theta \sum_{i=1}^{n}\left(l_{i}^{2}-2 p l_{i}+p^{2}\right) a_{i}=K \theta(\underbrace{\sum_{i=1}^{n} l_{i}^{2} a_{i}}_{=: C}-2 p B+p^{2} A) \\
& =K \theta\left(C-2 p B+p^{2} A\right) .
\end{aligned}
$$

Da die Kraft $F$ rein transversal ist, wird das gesamte Drehmoment durch diese erzeugt

$$
T=F(L-p)
$$

Durch Einsetzen der Werte für $F$ und $T$ der ECT folgt daraus

$$
\begin{aligned}
K \theta\left(C-2 p B+p^{2} A\right) & =K \theta(B-p A)(L-p) \\
C-2 p B+p^{2} A & =B L-p(A L+B)+p^{2} A \\
C-2 p B & =B L-p(A L+B) \\
p(A L-B) & =B L-C,
\end{aligned}
$$

und die quadratischen Terme heben sich weg - unabhängig von der Verteilung der Federstärke oder der Einzelflächen über die Zahnachse. Das Resultat ist die Lage $p$ des Pols als gebrochen-rationale Funktion des Hebelarmes $L$ mit linearem Zähler und Nenner

$$
p(L)=\frac{B L-C}{A L-B} .
$$

Diese Hyperbel hat die folgenden Eigenschaften:

- Die Lage des Drehpols hängt weder von der Stärke $F$ der Kraft, noch von der Materialkonstante $K$ der Aufhängung, noch vom Ausmaß $\theta$ der Drehung ab. 
- Die Abhängigkeit der Lage $p$ des Pols vom Angriffspunkt $L$ der Kraft ist durch die Gleichung einer Hyperbel gegeben.

- Für $\mathrm{L}=\mathrm{B} / \mathrm{A}$ hat die Hyperbel ihre Asymptote, es liegt also eine reine (hier vernachlässigte) Translation vor. Die Anordnung besitzt damit ein Widerstandszentrum bei

$$
W:=\frac{B}{A}=\frac{\sum_{i=1}^{n} l_{i} a_{i}}{\sum_{i=1}^{n} a_{i}}
$$

Das ist genau die Formel für den Schwerpunkt der Massen $a_{i}$.

- Durch elementare Umformungen läßt sich die Hyperbel auf das Widerstandszentrum beziehen

$$
\begin{aligned}
p\left(L-\frac{B}{A}\right) & =\frac{B}{A} L-\frac{C}{A} \\
p(L-W) & =W L-\frac{C}{A} \\
(p-W)(L-W) & =W L-\frac{C}{A}-W(L-W)=W^{2}-\frac{C}{A} \\
& =:-\sigma^{2} .
\end{aligned}
$$

Das ist das Reziprozitätstheorem mit der Konstanten

$$
\sigma^{2}=\frac{C}{A}-W^{2}=\frac{A C-B^{2}}{A^{2}} .
$$

Das negative Vorzeichen vor $\sigma^{2}$ besagt, daß sich $p$ und $L$ bezüglich $W$ gegenüber liegen. Wegen

$$
\begin{aligned}
\sum_{i=1}^{n}\left(l_{i}-W\right)^{2} a_{i} & =\sum_{i=1}^{n}\left(l_{i}^{2}-2 l_{i} W+W^{2}\right) a_{i} \\
& =\underbrace{\sum_{i=1}^{n} l_{i}^{2} a_{i}}_{=C}-2 W \underbrace{\sum_{i=1}^{n} l_{i} a_{i}}_{=B=W A}+W^{2} \underbrace{\sum_{i=1}^{n} a_{i}}_{A} \\
& =C-W^{2} A
\end{aligned}
$$

entspricht die Konstante genau dem zweiten zentrierten und normierten Moment der Verteilung der Massen $a_{i}$ und ist mit $a_{i}>0$ selbst positiv.

Eine solche hyperbelartige Abhängigkeit, zuerst in [56] dargestellt, ergibt sich nun auch aus der IMT und späteren Theorien. Dadurch können diese Theorien miteinander verglichen werden, $\stackrel{3}{,}$ indem diese Größen auf eine charakteristische Länge,

\footnotetext{
${ }^{3}$ Zur Vereinfachung dieses Vergleichs sei noch die Formel für die Konstante einer allgemeinen Hyperbel gegeben. Aus

$$
p(L)=\frac{D L-C}{A L-B} \quad \text { folgt } \quad \sigma^{2}=\frac{A C-B D}{A^{2}} .
$$
}


hier die Zahnwurzellänge $w$, bezogen werden. Damit lassen sich diese Theorien durch nur zwei dimensionslose Zahlen beschreiben: $W / w$ und $\sigma^{2} / w^{2}$. Es sei noch angemerkt, daß sich auch für die auf den Alveolarrand als Ursprung bezogenen Längen eine Beziehung der obigen Form ergibt.

Aus Schwarz (1928), [56] S. 400, folgt $W / w=1 / 2$ und $\sigma^{2} / w^{2}=1 / 12$. Das ist demnach das in [50] besprochene Modell eines eindimensionalen Stockes. Es geht zurück auf die Vorstellungen des CAsEschen Pflockversuches, vgl. [55] S. 65.

SyNGE erhält 1933 für sein einfachstes Modell, den Keilzahn mit dem halben Öffnungswinkel $\alpha$, vgl. [64] $\S 11$, ein Widerstandszentrum bei $W / w=1 /\left(2 \cos ^{2} \alpha\right)$. Er berechnet die Lage des Drehpols für unterschiedliche Kraftwirkungslinien und findet für nicht-transversale Lasten an der Inzisalkante einen Pol abseits der Wurzelachse. Für eine rein transversale Last ist die Lage des Drehpoles auf der Zahnlängsachse eine Funktion von $L$ allein, die Konstante des Reziprozitätstheorems ist $\sigma^{2}=1 /\left(60 \cos ^{4} \alpha\right)$. Beide divergieren für den Fall einer flachen Scheibe. Der kritische Winkel, für den $\mathrm{W} / \mathrm{w}=1$ gilt, ist $\alpha_{k}=\arccos \sqrt{1 / 2}=45^{\circ}$. Dieser Wert ist zwar kleiner als die $60^{\circ}$ bei meinem durchgerechneten Keil aus 5.3.1, hängt aber auch nicht von der Querdehnzahl ab. Als Zahlenwert für den Winkel gibt SYNGE $15^{\circ} 25^{\prime}=0.2662 \mathrm{rad}$ an, dem entsprechen $W / w=0.53$ und $\sigma^{2} / w^{2}=0.0187$.

Als zweites Beispiel seiner allgemeinen Theorie für rotationssymmetrische Aufhängungen, 64] Part III, berechnet SYNGE in $\$ 14$ einen Rotationskegel. Es ergibt sich mit $14^{\circ} 35^{\prime}=0.2505 \mathrm{rad}$

$$
\frac{W}{w}=\frac{4(1+2 \sin \alpha)}{5 \cos ^{2} \alpha(1+3 \cos \alpha)} \approx 0.7312
$$

und

$$
\frac{\sigma^{2}}{w^{2}}=\frac{2(1+2 \sin \alpha)^{2}}{75 \cos ^{4} \alpha(1+3 \sin \alpha)^{2}} \approx 0.0223 .
$$

Der kritische Winkel des Modells liegt bei $41.1^{\circ}=0.717 \mathrm{rad}$. Als Besonderheit sei noch erwähnt, daß die Funktion $\frac{W}{w}(\alpha)$ ein Minimum bei ziemlich genau dem realistischen Wert $12^{\circ} \mathrm{zu}$ dem Wert 0.73 annimmt. Der kritische Winkel meines Kreiskegels 5.2 .3 liegt, wieder abhängig von der Querdehnzahl, bei 67.5 .

Die Theorie von Synge ist aber umfangreicher, da sie für einen ebenen Lastfall die vollständigen elastischen Matrizen angibt. Als bedeutendster Unterschied zu meiner Theorie erweist sich die Abhängigkeit der Steifheitsmatrix von der Schichtdicke $d$ : Bei Synge gilt $\mathcal{S} \sim d^{-3}$ und hier $\mathcal{S} \sim d^{-1}$. Die fehlenden physikalischen Einheiten werden von der Wurzellänge $w$ geliefert, der Unterschied ist also der dimensionslose Faktor $(w / d)^{2}$. Unter der Annahme der Elastizität von Gummi für das Periodont und gemittelter Abmessungen eines oberen Schneidezahnes erhält er zur Veranschaulichung die Flexibilitäten für einen Lastangriff an der Schneidezahnkante. Unter axialer Last ergibt $\operatorname{sich}^{\llbracket} 2.8 \cdot 10^{-7} \mathrm{in} / 0.38 \mathrm{lbs}=0.0042 \mu \mathrm{m} / \mathrm{N}$ und

\footnotetext{
${ }^{4} 1 \mathrm{lbs}=453.59237 \mathrm{~g}$ entsprechend etwa $4.45 \mathrm{~N}$.
} 
bei transversaler Last $8.9 \cdot 10^{-6} \mathrm{in} / 0.19 \mathrm{lbs}=0.267 \mu \mathrm{m} / N$. Rechnet man den Wert für die Transversallast auf Drehmomente und Drehungen bezüglich des Widerstandszentrums um, so ergibt sich $0.267 \mu \mathrm{m} /(1.33 \mathrm{~cm})^{2} N=0.151 \cdot 10^{-4} \mathrm{rad} / \mathrm{N} \mathrm{cm}$. Eine Berechnung der Flexibilität unter axialen Drehmomenten findet nicht statt, vermutlich divergierte diese aufgrund der angenommenen Rotationssymmetrie.

Motiviert durch dieses Problem bei der Berechnung der Rotationsflexibilität und die Geometrie eines oberen Schneidezahnes, dessen Querschnitt oft als Kreisbogendreieck beschrieben wird, ist das dritte Beispiel SyNGES, nach der allgemeinen Behandlung des verallgemeinerten Kegels in [65], ein Kegel mit gleichseitigen Dreiecken als Querschnitt. Er erhält ebenfalls 1933 die vollständige Steifheitsmatrix als FOURIERreihe

$\mathcal{S}=\frac{12 G}{d^{3}} \psi c^{4}\left(\begin{array}{ccc|ccc}\sum_{n} R_{11}^{(n)} & & & & & \\ & \sum_{n} R_{22}^{(n)} & & \\ & & & \\ & & \sum_{n} S_{21}^{(n)} & \\ & & \sum_{n} R_{33}^{(n)} & & \\ \hline c \sum_{n} S_{21}^{(n)} & c \sum_{n} S_{12}^{(n)} & & c \sum_{n} T_{11}^{(n)} & \\ & & & c \sum_{n} T_{22}^{(n)} & \\ & & & c \sum_{n} T_{33}^{(n)}\end{array}\right)$,

wobei $G$ der Schermodul, $d$ die als konstant angenommene Schichtdicke, $\psi=$ $6 \arctan (\sqrt{3} \sin \alpha)$ mit dem Winkel zwischen Wurzelachse und Mittelsenkrechte der Seitenfläche $\alpha$, sowie $c$ die als konstant angenommene Seitenlänge des verallgemeinerten Zylinders ist. Die Summanden sind Funktionen von $\alpha$ und $n$ allein. Bei transversaler Belastung liegt der Drehpol $p$ der Anordnung bei

$$
\frac{p}{c}=\frac{(L / c) \sum_{n} S_{12}^{(n)}-\sum_{n} T_{22}^{(n)}}{(L / c) \sum_{n} R_{11}^{(n)}-\sum_{n} S_{12}^{(n)}}
$$

und ist daher von der gewünschten Bauart. Die gemittelten Geometriedaten ergeben

$$
\sum_{n} R_{11}^{(n)}=0.00223, \quad \sum_{n} S_{12}^{(n)}=0.00157, \quad \sum_{n} T_{22}^{(n)}=0.00115
$$

also gilt

$$
\frac{W}{w} \approx \frac{W}{c}=0.70 \quad \text { und } \quad \frac{\sigma^{2}}{w^{2}} \approx \frac{\sigma^{2}}{c^{2}}=0.020
$$

Für ein Periodont als weiches Gummi mit $G=1.6 \cdot 10^{7} \mathrm{dyn} / \mathrm{cm}^{2}=1.6 \mathrm{MPa}$ und $d=240 \mu \mathrm{m}$ ergibt sich unter axialen Rotationen als Abschätzung eine Flexibilität von $3.9 \cdot 10^{-8} \mathrm{rad} / \mathrm{g} \mathrm{mm}=4.0 \mu \mathrm{rad} / \mathrm{N} \mathrm{mm}=0.4 \cdot 10^{-4} \mathrm{rad} / \mathrm{N} \mathrm{cm}$.

BuRstone erweitert gegen 1962 die ECT um eine wurzelförmige Verteilung der Federstärke $a \sim \sqrt{l}$. Es ergeben sich die Werte [11]

$$
\frac{W}{w}=\frac{5}{3}=0.60 \quad \text { und } \quad \frac{\sigma^{2}}{w^{2}}=\frac{12}{175}=0.0686
$$


DAVIDIAN benutzt 1971 die ECT zusammen mit einer empirischen Verteilung der Federstärke, gewonnen aus Röntgenaufnahmen der Zähne. Eine Reanalyse seiner Daten, [13] Table III, ergibt

$$
\frac{W}{w}=0.60 \text { und } \quad \frac{\sigma^{2}}{w^{2}}=0.071 \quad .
$$

Der Mittelwert über vier Patienten ergibt $W / w=0.58 \pm 0.01$. Aufschlußreich ist eine Auftragung der Quadrate der in inch gemessenen Einzelflächen $a_{i}^{2} / i n^{2}$ gegen ihre Position $l_{i} / 0.04$ in auf der Zahnachse s. Abb. 6.2.

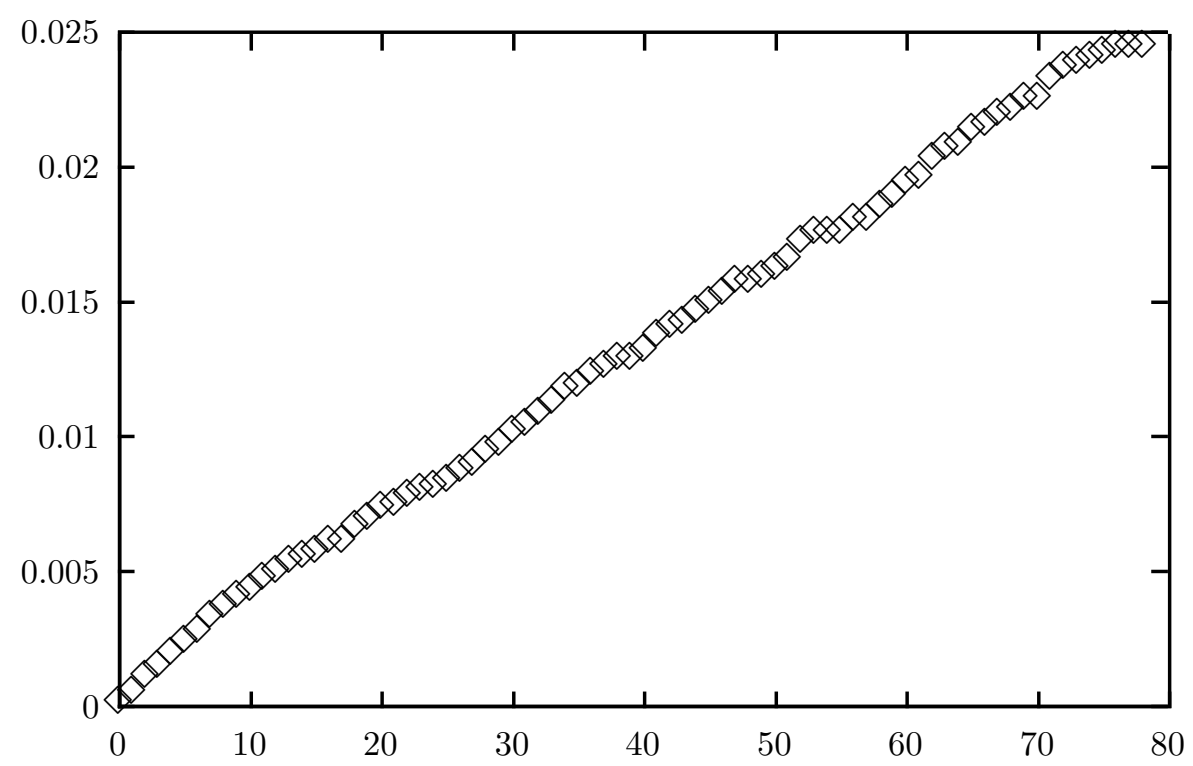

Abbildung 6.2: Die Quadrate der Einzelflächen als Funktion der Wurzellänge.

Die gute Proportionalität legt eine Abhängigkeit der Form $a^{2} \sim l$ bzw. $a(l) \sim \sqrt{l}$ nahe. Das erklärt die mit BuRsTone vergleichbaren Zahlenwerte.

NiKOLAI berechnet in [47] 1974 einen Zahn mit trapezförmigem Querschnitt und zur Wurzelspitze hin linear dünner werdenden Periodont mit Hilfe der Elastostatik. In der ECT würden sich beide Effekte exakt kompensieren und sich eine Gleichverteilung ergeben. In der Tat erhält man mit Hilfe eines in [50] angegebenen Fits an den hyperbelförmigen Graphen die Werte

$$
\frac{W}{w}=0.48 \quad \text { und } \quad \frac{\sigma^{2}}{w^{2}}=0.08
$$

welche etwa dem Pflockmodell entsprechen. Es wird in NikOLAIs Arbeit zum ersten Mal deutlich, wie wichtig die Verteilung der Dicke des Periodonts ist.

STEYN et al. verwenden in 61] einen konischen Zahn mit gemittelten Abmessungen. Unter Vernachlässigung von Scherspannungen ergibt sich bezüglich des Alveolarrandes eine Hyperbel, in den Bezeichnungen hier

$$
\frac{p}{w}=\frac{L / w-0.71}{1.73 L / w-1}
$$


Die relevanten Konstanten sind also

$$
\frac{W}{w}=0.58 \quad \text { und } \quad \frac{\sigma^{2}}{w^{2}}=0.076
$$

Die dort verwendete Federstärke in Normalrichtung ist genau der Koeffizient der einseitigen Kompression $2 \mu+\lambda$ geteilt durch die Schichtdicke und entspricht daher meinem $k_{1}$.

SutCliffe und ATHERTON berechnen 1980 auch einen solchen konischen Zahn und vernachlässigen die Scherspannungen [63]. Das entspricht der ECT. Sie bekommen einen komplizierten Ausdruck, der sich aber bei näherem Hinsehen als Hyperbel entpuppt. In ihrer Formel (6) des Anhangs läßt sich zunächst einmal der gemeinsame Faktor $(b+c)$ kürzen. Ersetzt man dann ihre Größen $b$ und $c$ durch $w-p$ und $p$, so ergibt sich

$$
\frac{p}{w}=\frac{1}{2} \cdot \frac{(6 d+4 w) L-(4 d+3 w) w}{(6 d+3 w) L-(3 d+2 w) w},
$$

wobei $d$ die Distanz von der Wurzelspitze bis zur Kegelspitze ist. Insbesondere gilt

$$
\frac{W}{w}=\frac{2+3 d / w}{3+6 d / w}
$$

und es liegt, wie bei Synge, eine explizite Abhängigkeit von der Form vor. Bei geeigneter Wahl von $d$ lassen sich so die Formeln von BuRstone und STEYN zusammenfassen. Für die Konstante des Reziprozitätstheorems ergibt sich

$$
\frac{\sigma^{2}}{w^{2}}=\frac{1}{18} \cdot \frac{1+6(d / w)+6(d / w)^{2}}{(1+2(d / w))^{2}} .
$$

Alle diese von elastischen Fasern ausgehenden Theorien - ausgenommen die NiKOLAIs - können zusammengefaßt diskutiert werden als Klasse von Theorien mit zwei freien Parametern: Dem Exponenten $\kappa$ der Abhängigkeit $a \sim l^{\kappa}$ und den Integrationsgrenzen $h$ und $H$. Eine Proportionalitätskonstante hebt sich ohnehin heraus; der zweite relevante Parameter ist das dimensionslose Verhältnis $\lambda:=H / h$. Mit

$$
A=\int_{h}^{H} l^{\kappa} d l \quad \text { und } \quad B=\int_{h}^{H} l^{\kappa+1} d l \quad \text { und } \quad C=\int_{h}^{H} l^{\kappa+2} d l
$$

ergibt sich nach Ausführen der Integrationen das Widerstandszentrum bei

$$
\frac{W}{w}=\frac{B / A-h}{H-h}=\frac{\kappa+1}{\kappa+2} \cdot \frac{(\kappa+1)(1-\lambda)-\lambda\left(1-\lambda^{\kappa+1}\right)}{(\kappa+1)(1-\lambda)\left(1-\lambda^{\kappa+1}\right)},
$$

also insbesondere für $\lambda \rightarrow 0$ bei $W / w=(\kappa+1) /(\kappa+2)$. Für die Konstante des Reziprozitätstheorems ergibt sich der Ausdruck

$$
\frac{\sigma^{2}}{w^{2}}=\frac{A C-B^{2}}{A^{2}(H-h)^{2}}=\frac{\kappa+1}{(\kappa+2)^{2}(\kappa+3)} \cdot \frac{\left(1-\lambda^{\kappa+2}\right)^{2}-\lambda^{\kappa+1}(1-\lambda)^{2}(\kappa+2)^{2}}{(1-\lambda)^{2}\left(1-\lambda^{\kappa+1}\right)^{2}} .
$$

Für den wichtigen Spezialfall $h \rightarrow 0$ seien hier noch tabellarisch einige Ergebnisse angegeben. 


\begin{tabular}{r||c|c|c|c|c}
$\kappa$ & 0 & $1 / 2$ & 1 & $3 / 2$ & 2 \\
\hline \hline$W / w$ & $1 / 2$ & $3 / 5$ & $2 / 3$ & $5 / 7$ & $3 / 4$ \\
\hline$\sigma^{2} / w^{2}$ & $1 / 12$ & $12 / 175$ & $1 / 18$ & $20 / 441$ & $3 / 80$
\end{tabular}

Die Lage des Widerstandszentrums wächst mit steigendem Exponenten $\kappa$ und fällt mit zunehmendem $\lambda$, da die Aufhängung sich für $\lambda \rightarrow 1$ der Gleichverteilung annähert. Die Konstante des Reziprozitätstheorems fällt mit wachsendem $\kappa$ und steigt mit zunehmendem $\lambda$ wieder.

NÄGERL et. al. untersuchen 1991 in [50] den Einfluß der Scherelastizität auf die ECT. Die Scherelastizität wird, wie die Federstärke selbst, durch eine Verteilung über die Wurzelänge modelliert. Zunächst wird der eindimensionale Fall betrachtet, der später auf einen zweidimensionalen Fall erweitert wird. Seit SyngE werden erstmalig wieder verschiedene Richtungen der Kraftwirkungslinie diskutiert. Dabei zeigt sich, daß der Gesamtverschiebungsvektor nicht mehr dieselbe Richtung wie der Kraftvektor hat, und daß das Reziprozitätstheorem daher eine Folge des speziellen Ansatzes der Transversallast ist. Im danach allgemein, also ohne Annahme einer speziellen Verteilung, durchgerechneten Fall eines elastisch aufgehängten Rechteckes ergibt sich ein quadratisch mit dem Verhältnis von Breite zu Wurzellänge wachsender Zusatzterm zur Konstante $\sigma^{2} / w^{2}$. Diese Theorie wurde von mir auf beliebige zweidimensionale Berandungen verallgemeinert, die Rechnung findet sich im Teil1.1 der Einleitung. Dieses Anwachsen der Schichtkonstante wurde in [3] experimentell bestätigt, die Konstante liegt für eine haftende Einbettung bei $\sigma^{2} / w^{2}=0.24$, also mehr als dreimal größer als bei der eindimensionalen ECT!

Damit ist die Struktur einer Theorie klar, welche die ECT verallgemeinert und der IMT mathematisch ebenbürtig ist. Sie muß

- dreidimensional sein,

- die Scherelastizität berücksichtigen,

- kompliziertere als skalare Zustandsgleichungen erlauben,

- ein korrektes Transformationsverhalten besitzen,

- eine Verallgemeinerung auf ein anisotropes Medium zulassen

- und dabei möglichst einfach bleiben.

Das genau leistet das in dieser Arbeit vorgestellte Federmodell - auch wenn der Name zunächst etwas irreführend ist, da er an die naive ECT erinnert. Eine Verallgemeinerung der hier besprochenen zweidimensionalen Theorien auf eine mit Scherelastizität findet sich wieder bereits in der Einleitung 1.1 dieser Arbeit. Darüber hinaus müssen die zu diskutierenden Objekte nach meinen Erkenntnissen modifiziert werden. Das elastische Zentrum $Z$ und der Widerstandsbereich $B$ ersetzen nun 
das didaktische Konzept des Widerstandszentrums. Wie die durchgerechneten Beispiele zeigen, ist die Anwendbarkeit der Theorie nicht auf Modelle des Periodonts beschränkt.

Als Abschluß sollen noch halbwegs realistische Zahlen eingesetzt werden, um eine Abschätzung der Größenordnung der nach dieser dreidimensionalen Theorie mit Scherelastizität zu erwartenden Terme für einen oberen Frontzahn zu treffen.

Für den Rotationsparaboloiden aus 5.3 .3 mit konstanten Schichtgrößen ergaben sich für den Grenzfall einer beliebig engen Parabel die BuRstonEschen Werte $W / H=3 / 5$ und $\sigma^{2} / H^{2}=12 / 175$. Mit den Zahlenwerten

$$
\begin{aligned}
d & =200 \mu \mathrm{m} \\
R & =3.5 \mathrm{~mm} \\
H & =13 \mathrm{~mm} \\
E & =1.2 \mathrm{~N} / \mathrm{mm}^{2} \\
\sigma & =0.45,
\end{aligned}
$$

vgl. Abb. 6.3, und der Identifikation von $d k_{1}$ mit dem Koeffizienten der einseitigen Kompression erhält man eine Flexibilitätsmatrix von

$$
\mathcal{F}=\left(\begin{array}{lll|lll}
0.43 & & & & & \\
& 0.43 & & & & \\
& & 1.7 & & & \\
\hline & & & 3.5 & & \\
& & & & 3.5 & \\
& & & & & 34
\end{array}\right)
$$

in den Einheiten

$$
\left(\begin{array}{c|c}
\frac{\mu m}{N} & \\
\hline & 10^{-4} \frac{\mathrm{rad}}{\mathrm{Ncm}}
\end{array}\right)
$$

bezüglich des Widerstandszentrums bei $61.7 \%$ der Wurzellänge. Da die Flexibilitätsmatrix in Diagonalform vorliegt, gilt das Reziprozitätstheorem, allerdings mit von der Richtung der Kraft abhängenden Konstanten. Für eine Transversalkraft etwa, also eine, die zentral und senkrecht zur Mittellinie angreift, ergibt sich als Konstante

$$
\sigma^{2}=\frac{D_{x x}}{M_{y y}}=\frac{0.43 \mu m / N}{3.5 \cdot 10^{-4} / N c m}=0.13 \mathrm{~cm}^{2},
$$

also bezogen auf die Höhe bzw. Wurzellänge

$$
\frac{\sigma^{2}}{H^{2}}=0.072
$$


Für den anderen Extremfall $k_{1}=E / d$ ergibt sich analog in denselben Einheiten

$$
\mathcal{F}=\left(\begin{array}{ccc|ccc}
1.3 & & & & & \\
& 1.3 & & & & \\
& & 2.3 & & & \\
\hline & & & 9.5 & & \\
& & & & 9.5 & \\
& & & & & 34
\end{array}\right)
$$

mit einer Konstanten von

$$
\frac{\sigma^{2}}{H^{2}}=0.081
$$

Bis auf das Verhältnis der Matrixeinträge unterscheiden sich beide Theorien wenig, da die absoluten Werte leicht durch eine Modifikation der Schichtdicke oder des E-Moduls verändert werden können. Die Werte für die Konstante des Reziprozitätstheorems liegen zwischen denen der ECT in der ScHWARZschen oder BURSTONEschen Variante.

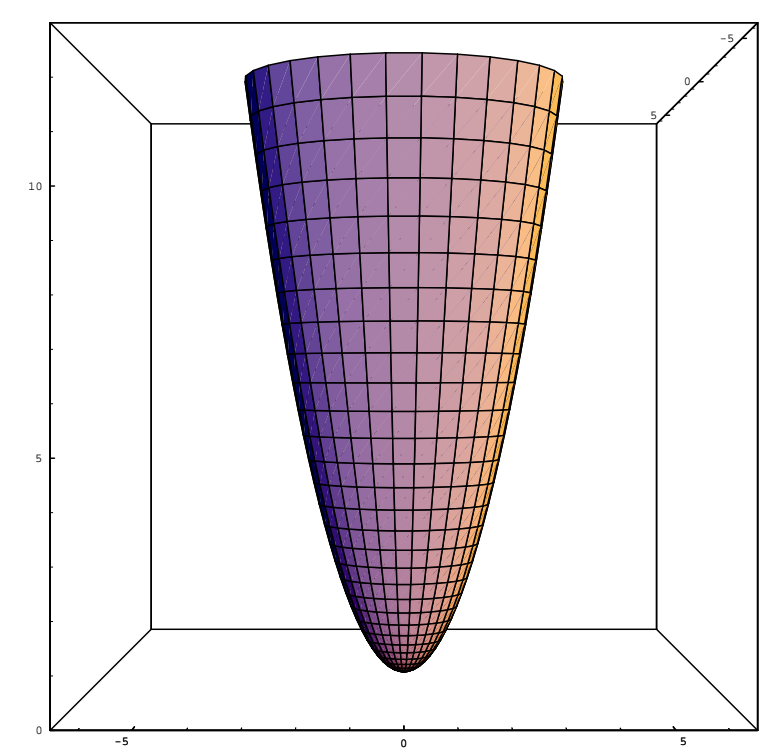

Abbildung 6.3: Das Paraboloidmodell der Wurzel eines oberen Frontzahnes.

Als Letztes soll noch ein halbnumerisches Resultat für eine veränderliche Schichtdicke vorgestellt werden. Da die Dicke mit $1 / d$ in die Rechnungen eingeht, ist es für symbolische Rechnungen sinnvoll, eine Funktion zu finden, deren Kehrwert möglichst einfach ist und die die Sanduhrform des Periodonts mit 200-140-200 $\mu \mathrm{m}$ approximiert. Ein brauchbarer Ansatz mit ganzen Zahlen ist

$$
d_{P} / m m=\frac{2}{10+17 t-17 t^{2}}
$$


wobei $t \in[0,1]$ der Integrationsparameter der Höhe ist. Bezüglich des Widerstandszentrums ergibt sich unter alleiniger Berücksichtigung der gemittelten Abmessungen durch Verwendung der Längeneinheit $\mathrm{mm}$

$$
\boldsymbol{A}_{W} / m m=K_{1} \operatorname{diag}(583.9+642.8 v, \%, 58.88+1.167 .8 v)
$$

und, etwas komplizierter,

$$
\boldsymbol{C}_{W} / \mathrm{mm}^{3}=K_{1} \operatorname{diag}\left(\frac{11645(0.4839+v)(1.0532+v)}{0.9084+v}, \%, 8553 v\right) .
$$

Hierin ersetzt die Materialkonstante $K_{1}$ die lokale Größe $k_{1}=K_{1} / d_{P}(h)$. Die Quotienten aus axialen und radialen Komponenten können nun als Funktion des Parameters $v$ diskutiert werden. Für $v \in(0.01,0.5)$ variieren die Verhältnisse etwa zwischen 3 und 10. Die Form der Matrixeinträge zeigt, daß die durch Wahl von $k_{1}$ als $E / d$ oder als Modul der einseitigen Kompression hervorgerufenen Unterschiede auf Wahl des Parameters $v$ zurückgeführt werden können. Ferner ist es prinzipiell möglich, wie beim Kreiszylinder, diese Ausdrücke zum Bestimmen der Materialparameter zu nutzen, also insbesondere auch auf die von $v$.

\subsubsection{In vivo}

An einem männlichen 26 Jahre alten Probanden wurden die Auslenkungen des Inzisivus 11 unter nacheinander allen sechs Lasten gemessen [4]. Die Messung erforderte einen hohen apparativen und zeitlichen Aufwand. Die lange Meßdauer ergab sich aus der großen Zeitkonstante bei $\tau=40 \mathrm{~s}$ für die langsamsten Relaxationseffekte. Der maximale Fehler der Flexibilitätskonstanten wurde bezüglich der im Aufbau realisierten Koordinatenursprünge mit 10\% angegeben.

Als Nächstes sollen die Größenordnungen der zu erwartenden Effekte untersucht werden. Die Dicke des Periodontalspaltes wurde mit $200 \mu m$ an der Wurzelspitze angenommen. Lineare Effekte liegen höchstens bis zu einer Deformation auf $75 \%$ des Ausgangswertes vor, das ergibt eine für die lineare Theorie maximal zulässige Translation von $50 \mu \mathrm{m}$. Zusammen mit einer Wurzellänge von $13 \mathrm{~mm}$ und einem bei 60\% dieser Länge angenommenen Widerstandszentrum ergibt sich eine Drehung um den „Hebel“ $7.8 \mathrm{~mm}$, also eine maximal zulässige Rotation um den Winkel

$$
\theta \approx \tan \theta=\frac{50 \mu m}{7.8 m m}=6.4 \mathrm{mrad}=0.37^{\circ} .
$$

Apparativ war es möglich, Drehmomente bis $5 \mathrm{Nm}$ und Kräfte bis $2.4 \mathrm{~N}$ zu erzeugen. Am Probanden wurden Drehmomente bis $2.7 \mathrm{Nm}$ und Kräfte bis $1.6 \mathrm{~N}$ appliziert, wobei das Koordinatensystem der Kräfte von dem durch ein OberkieferGipsmodell abgeschätzten Widerstandszentrum nicht mehr als $2.5 \mathrm{~cm}$ in Koordinatenrichtung entfernt war. Das maximale durch Kräfte erzeugte Drehmoment bezüglich dieses Widerstandszentrums lag dann bei $3.8 \mathrm{Ncm}$. Berücksichtigt man 
witerhin eine Zahnachse von ca. $30^{\circ}$ gegenüber der Frontalebene ergeben sich maximale transversale Drehmomente von $2.4 \mathrm{Ncm}$ für die aufgebrachten Drehmomente und $3.8 \mathrm{Ncm}$ für die aufgebrachten Kräfte, beide Male tangential zum Alveolarkamm. Orthodontische Lasten liegen etwa bei $2 / 3$ dieser Werte, werden aber länger appliziert. Insgesamt ergibt sich daraus eine a-priori-Abschätzung für die Flexibilitätskoeffizienten von

$$
\frac{6.4 \mathrm{mrad}}{2.4 \mathrm{Ncm}}=2.7 \mathrm{mrad} / \mathrm{Ncm} \quad \text { bzw. } \quad \frac{6.4 \mathrm{mrad}}{3.8 \mathrm{Ncm}}=1.7 \mathrm{mrad} / \mathrm{Ncm}
$$

für die Rotationsanteile und

$$
\frac{50 \mu m}{1.6 N}=31 \mu m / N
$$

für die Translationen. Ergäben sich aus der Messung größere Flexibilitäten, so kann der lineare Bereich der Bewegung verlassen worden sein, was die Meßdaten in Frage stellte.

Vom Autor, Benedikt BeckeR, wurde mir der auf Lagen umgerechnete und um die Elastizität der Meßapparatur korrigierte Originaldatensatz dankenswerterweise zur Verfügung gestellt. Dadurch ist es möglich, den Einfluß des statistischen Modells auf die Schätzwerte der elastische Matrizen exemplarisch zu untersuchen. Dabei wurde das Statistik-Programmpaket SAS eingesetzt.

Da bei verschwindenden Lasten auch die Lagen zu Null geeicht werden, ist eine Ursprungsgerade das einfachste angebrachte statistische Modell. Alle 36 Steigungen der Abhängigkeit $q(f)$ ergeben die Flexibilitätsmatrix. Durch eine Nulldrift des Meßgerätes oder eine Abhängigkeit der Eichkurve des Gleichstrommotors zur Aufbringung der Drehmomente oder eine tatsächliche Veränderung der Nullage des Zahnes kann es sinnvoll sein, auch Achsabschnitte mit zu schätzen, aber nur die Steigungen zu berücksichtigen. Bei geeigneten Voraussetzungen kann, wie in 3.2.3 beschrieben, die Dissipationsmatrix über die Achsabschnitte bestimmt werden. Da die Aufhängung eines Frontzahnes, wie die Bandscheibe, ein nahezu symmetrisches Gebilde ist, sind quadratische Effekte zu erwarten. Auch hier bestünde die Flexibilitätsmatrix aus den Koeffizienten zu den linearen Anteilen. Die Approximation eines Parabelastes durch eine Gerade lieferte dann falsche Werte. Eine weitere Ursache quadratischer Terme könnte in der Stärke der aufgebrachten Last liegen. Wird etwa die Auslenkung an der Wurzelspitze zu groß gegenüber der Dicke des Periodonts, so macht sich das in einer Sättigung der Lageänderungen bei wachsenden Lasten bemerkbar. Das wäre dann daran zu erkennen, daß der Vorfaktor vor dem quadratischen Term genau das umgekehrte Vorzeichen zu dem linearen besitzt.

Neben dem Grad des statistischen Modells sind wegen der Fehlerfortpflanzung die Bezugspunkte der Daten von großer Wichtigkeit. Um die sich ergebenden Mehrdeutigkeiten einzugrenzen, bietet es sich an, die Bezugspunkte des Modells derart zu bestimmen, daß die geschätzten elastischen Matrizen bereits auf Drehmoments- 
und Translationszentrum transformiert herauskommen. Werden nur die Lagen allein transformiert, genügt weiterhin ein univariates Modell. Transformiert man hingegen auch die Lasten, so muß eine multivariate Regression durchgeführt werden. Diese unterscheidet sich von der univariaten durch die Anzahl der Freiheitsgrade, da nun für sechs Lasten nur ein gemeinsamer Achsabschnitt (Intercept) zur Verfügung steht. Auch hier können quadratische Terme mit berücksichtigt werden.

Der experimentelle Aufbau gab die Lasten vor und bestimmte die Lageänderungen, was über eine Ursache-Wirkung-Beziehung ein Modell $q(f)$ suggeriert. Dennoch können die Fehler bei der Realisation der Lasten die der Messung der Lagen überschreiten. Dann aber bietet sich ein Umdrehen des multivariaten Modells an, und die Steifheitsmatrix wird geschätzt über ein Regressionsmodell $f(q)$.

Als letzte Fehlerquellen kommen der Approximalkontakt oder eine sonstwie geartete Vorspannung im Zahnhalteapparat in Frage. Dann ergäben sich zusammengesetzte Geradenstücke, die, als Parabeln interpretiert, falsche Schätzer lieferten. Auch eine Lockerung der Befestigung der Apparatur am Zahn kann solche Effekte verursachen, so daß bei quadratischen Modellen immer Vorsicht angebracht sein sollte. Einzige Abhilfe wäre dann eine Restriktion des Datensatzes von Hand auf einen nach vernünftigen und reproduzierbaren Kriterien ausgewählten sinnvollen Bereich.

\subsubsection{Statistik vs. Theoriekonformität}

Für alle eben vorgestellten Auswertemethoden gibt es Für- und Gegenargumente. Es können nun zweierlei Kriterien zur Beurteilung der Flexibilitätsmatrix herangezogen werden: Die Statistik selbst oder die „Stimmigkeit" der Realform der geschätzten Matrix im Hinblick auf das Wunschergebnis eines Widerstandszentrums. Eine statistische Beurteilung multivariater Modelle, die die Anzahlen der Freiheitsgrade berücksichtigt, erlaubt der F-Test, der die durch das Modell erklärten Streuungen mit denen des Restfehlers ins Verhältnis setzt. Eine Beurteilung der Stimmigkeit der Matrix im Sinne dieser Theorie erfolgt im Wesentlichen über

- die nur abseits des elastischen Zentrums sinnvolle Güte der Transformationsmatrizen, die idealerweise bei -1 liegt,

- die mit den anatomischen Gegebenheiten verträgliche Lage der elastischen Zentren,

- die Blocknorm ${ }^{5}$ vor- und nach der Transformation, wobei kleine Nebendiagonalwerte zu bevorzugen sind,

\footnotetext{
${ }^{5}$ Diese war definiert über $\sqrt{\sum_{i j} a_{i j}^{2}}$, und ist das dreifache des quadratischen Mittels der Matrixeinträge.
} 
- die Größe der Widerstandsbereiche, die höchstens im Millimeterbereich liegen sollte,

- den mit der Zahnachse übereinstimmenden Drehwinkel der Realform und

- die Güte der translationsinvarianten Matrix $\boldsymbol{A}$.

Um einen Überblick der Auswirkungen des verwendeten statistischen Modells auf die geschätzten Flexibilitätskonstanten zu geben, sind diese in Tabelle 6.1 zusammengefaßt. Die $x z$-Ebene entsprach etwa der Sagittalebene, wobei $\boldsymbol{e}_{z}$ waagerecht nach vorn, und $\boldsymbol{e}_{x}$ senkrecht nach unten wiesen. Die $y$-Achse verlief entsprechend horizontal und tangential zum Alveolarkamm.

Als erstes soll die Norm der geschätzten Matrizen betrachtet werden. Das univariat quadratische Modell lieferte für $\boldsymbol{D}$ und $\boldsymbol{S}_{2}$, also für die aufgebrachten Kräfte, die betragsmäßig größten Schätzer. Bei ihm arbeiten zu viele Freiheitsgrade gegeneinander. Es wurde daher verworfen. Des weiteren besitzen die Schätzer der univariaten Modelle nicht das korrekte Transformationsverhalten, d. h. sie sind nicht verträglich mit einer Gleichung des Typs $q_{i}=\left(q_{0}\right)_{i}+\mathcal{F}_{i j} f_{j}+\mathcal{O}_{2}$. Da das univariate lineare Modell genau so viele freie Parameter hat wie das quadratische ohne Intercept, aber dennoch den beiden anderen linearen Ansätzen vergleichbare Schätzer liefert, wurde es für die folgende Überlegung noch beibehalten. Gemittelt über alle nicht verworfenen Modelle ergibt sich als Übersicht der Größenordnungen die Flexibilitätsmatrix

$$
\hat{\mathcal{F}}=\left(\begin{array}{ccc|ccc}
69.3 & 13.9 & -79.3 & -0.363 & -40.3 & 4.56 \\
85.8 & 254 . & -249 . & 62 . & -19.4 & 25.2 \\
-41.8 & -9.02 & 59.2 & -6.11 & 17.5 & -3.95 \\
\hline 17.1 & 42.4 & -40.6 & 10.6 & -2.27 & 3.94 \\
-10.2 & -2 . & 10.2 & -0.277 & 6.17 & -0.63 \\
6.56 & 22.6 & -13.3 & 3.15 & -0.317 & 5.14
\end{array}\right)
$$

mit den Streuungen

$$
\left(\begin{array}{ccc|ccc}
3.52 & 93.9 & 48.7 & 3.01 & 13.9 & 4.6 \\
4.89 & 52.6 & 120 . & 29.3 & 7.2 & 5.61 \\
1.75 & 26.4 & 26.6 & 3.53 & 8.11 & 2.72 \\
\hline 1.44 & 8.87 & 18.7 & 4.89 & 1.36 & 1.74 \\
0.416 & 14 . & 7.43 & 0.255 & 1.98 & 0.65 \\
0.623 & 2.26 & 4.88 & 2.06 & 0.57 & 1.13
\end{array}\right)
$$

in den Einheiten

$$
\left(\begin{array}{c|c}
\mu \mathrm{m} / N & \mu \mathrm{m} / \mathrm{N} \mathrm{cm} \\
\hline 10^{-4} \mathrm{rad} / N & 10^{-4} \mathrm{rad} / \mathrm{Ncm}
\end{array}\right) .
$$

Die verbleibenden quadratischen Modelle liefern durchweg kleinere Schätzer als die dazugehörigen linearen, d. h. die Streuung wird im Wesentlichen durch den Grad des Modells bestimmt! 


\begin{tabular}{|c|c|c|c|c|c|c|c|}
\hline \multirow{2}{*}{$\begin{array}{l}\text { Kompo- } \\
\text { nente }\end{array}$} & \multicolumn{3}{|c|}{ Ursprungsgraph } & \multicolumn{2}{|c|}{ multivariat } & \multicolumn{2}{|c|}{ univariat } \\
\hline & linear & $\mathcal{S}_{l}^{-1}$ & quadr. & linear & quadr. & linear & quadr. \\
\hline$\overline{\bar{D} / \frac{\mu m}{N}}$ & 418 & 2434 & 282 & 419 & 290 & ב 592 & 99 \\
\hline$d_{x} F_{x}$ & 67.0 & 72.5 & 69.2 & 71.6 & 72.2 & 63.6 & 31.3 \\
\hline$d_{x} F_{y}$ & -15.4 & -4.2 & 117.5 & -8.5 & 121.6 & -127.7 & -167.1 \\
\hline$d_{x} F_{z}$ & -94.6 & -94.7 & -25.7 & -88.1 & -21.8 & -151.0 & -154.3 \\
\hline$d_{y} F_{x}$ & 80.9 & 86.9 & 83.9 & 91.5 & 91.1 & 80.3 & 109.8 \\
\hline$d_{y} F_{y}$ & 262.5 & 265.1 & 189.3 & 278.5 & 199.5 & 330.7 & 612.7 \\
\hline$d_{y} F_{z}$ & -281.5 & -299.4 & -116.1 & -266.5 & -106.5 & -422.9 & -633.4 \\
\hline$d_{z} F_{x}$ & -40.0 & -44.0 & -39.7 & -43.5 & -41.7 & -41.7 & -68.4 \\
\hline$d_{z} F_{y}$ & -0.7 & 0.6 & -37.5 & -6.0 & -40.4 & 30.0 & 26.7 \\
\hline$d_{z} F_{z}$ & 67.3 & 69.3 & 30.4 & 62.4 & 27.6 & 98.3 & 127.5 \\
\hline $\boldsymbol{S}_{2} / \frac{\mu m}{N c m}$ & 95.5 & 96.5 & 37.2 & 108 & 46.3 & 118 & 53 \\
\hline$d_{x} T_{x}$ & 0.5 & -1.6 & -4.6 & 3.8 & -2.2 & 1.9 & -5.6 \\
\hline$d_{x} T_{y}$ & -45.5 & -47.1 & -21.7 & -48.8 & -24.1 & -54.7 & -31.1 \\
\hline$d_{x} T_{z}$ & 5.9 & 6.1 & -2.2 & 8.9 & 0.0 & 8.7 & -1.3 \\
\hline$d_{y} T_{x}$ & 73.8 & 74.4 & 22.3 & 81.5 & 28.2 & 92.0 & 31.9 \\
\hline$d_{y} T_{y}$ & -20.3 & -18.8 & -8.6 & -28.0 & -14.5 & -25.9 & -19.2 \\
\hline$d_{y} T_{z}$ & 25.8 & 25.8 & 16.4 & 32.7 & 21.7 & 28.6 & 15.3 \\
\hline$d_{z} T_{x}$ & -7.1 & -7.2 & -0.9 & -9.7 & -2.6 & -9.1 & -0.8 \\
\hline$d_{z} T_{y}$ & 20.5 & 20.6 & 6.6 & 23.0 & 8.3 & 26.2 & 13.8 \\
\hline$d_{z} T_{z}$ & -4.7 & -4.8 & 0.1 & -7.0 & -1.4 & -6.0 & 1.7 \\
\hline $\boldsymbol{S}_{1} / \frac{10^{-4} \mathrm{rad}}{N}$ & 72.3 & 76.5 & 51 & 73.2 & 52.4 & 99.2 & 182 \\
\hline$\theta_{x} F_{x}$ & 15.8 & 18.5 & 17.5 & 17.7 & 18.3 & 14.9 & 21.9 \\
\hline$\theta_{x} F_{y}$ & 43.6 & 44.6 & 31.7 & 46.6 & 32.8 & 55.1 & 114.5 \\
\hline$\theta_{x} F_{z}$ & -45.7 & -48.4 & -19.8 & -43.0 & -18.8 & -67.8 & -101.9 \\
\hline$\theta_{y} F_{x}$ & -9.9 & -10.5 & -10.2 & -10.4 & -10.5 & -9.4 & -5.4 \\
\hline$\theta_{y} F_{y}$ & 2.2 & 1.1 & -17.6 & 1.4 & -18.0 & 19.0 & 25.0 \\
\hline$\theta_{y} F_{z}$ & 12.6 & 11.6 & 2.0 & 11.8 & 1.6 & 21.4 & 23.8 \\
\hline$\theta_{z} F_{x}$ & 6.1 & 6.8 & 6.0 & 7.2 & 7.3 & 5.9 & 3.9 \\
\hline$\theta_{z} F_{y}$ & 21.9 & 24.4 & 19.3 & 23.7 & 21.1 & 25.4 & 79.6 \\
\hline$\theta_{z} F_{z}$ & -14.5 & -16.9 & -8.6 & -12.9 & -7.0 & -19.8 & -37.8 \\
\hline$M / \frac{10^{-4} \mathrm{rad}}{\mathrm{Ncm}}$ & 16.7 & 16.6 & 6.6 & 19 & 7.93 & 20.5 & 9.6 \\
\hline$\theta_{x} T_{x}$ & 12.6 & 12.6 & 4.2 & 14.0 & 4.8 & 15.6 & 5.8 \\
\hline$\theta_{x} T_{y}$ & -2.5 & -1.9 & -0.5 & -3.9 & -1.1 & -3.6 & -2.7 \\
\hline$\theta_{x} T_{z}$ & 4.6 & 4.4 & 1.5 & 5.8 & 2.1 & 5.3 & 0.0 \\
\hline$\theta_{y} T_{x}$ & -0.3 & -0.2 & 0.1 & -0.7 & -0.1 & -0.3 & 0.4 \\
\hline$\theta_{y} T_{y}$ & 6.9 & 7.3 & 3.6 & 7.3 & 3.8 & 8.2 & 5.1 \\
\hline$\theta_{y} T_{z}$ & -0.9 & -0.9 & 0.3 & -1.2 & 0.1 & -1.2 & 0.5 \\
\hline$\theta_{z}^{y} T_{x}$ & 4.0 & 3.3 & 0.2 & 4.9 & 1.2 & 5.4 & 0.9 \\
\hline$\theta_{z} T_{y}$ & -0.3 & 0.0 & 0.6 & -1.1 & -0.5 & -0.6 & -0.1 \\
\hline$\theta_{z} T_{z}$ & 5.3 & 5.6 & 3.3 & 6.1 & 4.2 & 6.2 & 4.8 \\
\hline
\end{tabular}

Tabelle 6.1: Geschätzte Flexibilitätskoeffizienten zu verschiedenen Modellen. 
Die auf die elastischen Zentren transformierte Form der über die verschiedenen Modelle gemittelten Matrix lautet

$$
\left(\begin{array}{ccc|ccc}
5.97 & 6.16 & -4.59 & -5.19 & 1.61 & -0.919 \\
-41.9 & 6.37 & 5.88 & 3.44 & -3.65 & 5.64 \\
19.6 & 16.1 & 33.6 & -3.39 & 9.76 & -1.27 \\
\hline-14.6 & 16.6 & -21.9 & 12.7 & 0 & 0 \\
7.79 & 4.69 & 5.96 & 0 & 5.95 & 0 \\
-5.93 & 3.44 & 3.72 & 0 & 0 & 3.44
\end{array}\right)
$$

ihre Komponenten geben Auskunft über die erforderliche absolute Genauigkeit der Schätzer. Sind sie insbesondere kleiner als die entsprechenden Unsicherheiten der Schätzungen, so ist aus ihnen keine Aussage mehr zu treffen. Dabei wurde die Fehlerfortpflanzung noch nicht berücksichtigt. Die Messung ist demnach im Vergleich zu den zu treffenden Aussagen an der Grenze der Meßgenauigkeit.

Eine weitere Fehlerquelle stellt die Eigenelastizität der Apparatur dar. Die Daten der Messung wurden um diese punktweise korrigiert. In denselben Einheiten wie die der Meßdaten lautet die Flexibilitätsmatrix der Apparatur [4] allein

$$
\mathcal{F}_{0}=\left(\begin{array}{ccc|ccc}
9.12 & -34.8 & -38.2 & 0.52 & -11.3 & 1.91 \\
-0.56 & 5.66 & -4.37 & 4.3 & 0.21 & -8.7 \\
-3.49 & 5.62 & 12 . & -0.7 & 5.38 & -0.21 \\
\hline 0.16 & -0.86 & -1.08 & 0.91 & -0.08 & -0.06 \\
-1.34 & 3.91 & 4 . & -0.07 & 2.1 & -0.07 \\
0.38 & -1.67 & -1.91 & -0.04 & -0.13 & 3.33
\end{array}\right) ;
$$

auch sie befindet sich in der Größenordnung der interessanten Effekte.

Als Nächstes soll, wie eingangs erwähnt, die Beurteilung der Schätzer nach Gesichtspunkten dieser Theorie erfolgen. Die Ergebnisse sind in Tabelle 6.2 zusammengefaßt.

Die Güte des Meßsystems ist durchweg besser als die des Lastsystems, was auf eine Störung bei der Aufbringung der Lasten hindeutet. Daher wurde versucht, unabhängige und abhängige Variablen zu vertauschen, also die Steifheitsmatrix zu schätzen. Die Ergebnisse unterschieden sich aber auch nach Inversion der Matrix nicht wesentlich von den bisherigen. Bei den linearen Modellen sind die Güten besser als bei den quadratischen. Das Widerstandszentrum sollte nach einer Abschätzung (70\% der Wurzellänge) am Oberkiefer-Gipsmodell des Probanden [4] bei etwa

$$
\overrightarrow{M D}=\left(\begin{array}{l}
3.7 \\
0.9 \\
7.8
\end{array}\right) \mathrm{cm}
$$

liegen. Der Lastangriff erfolgte bezüglich des Meßsystems [4] bei

$$
\overrightarrow{M L}=\left(\begin{array}{l}
2.34 \\
0.20 \\
5.30
\end{array}\right) \mathrm{cm}
$$




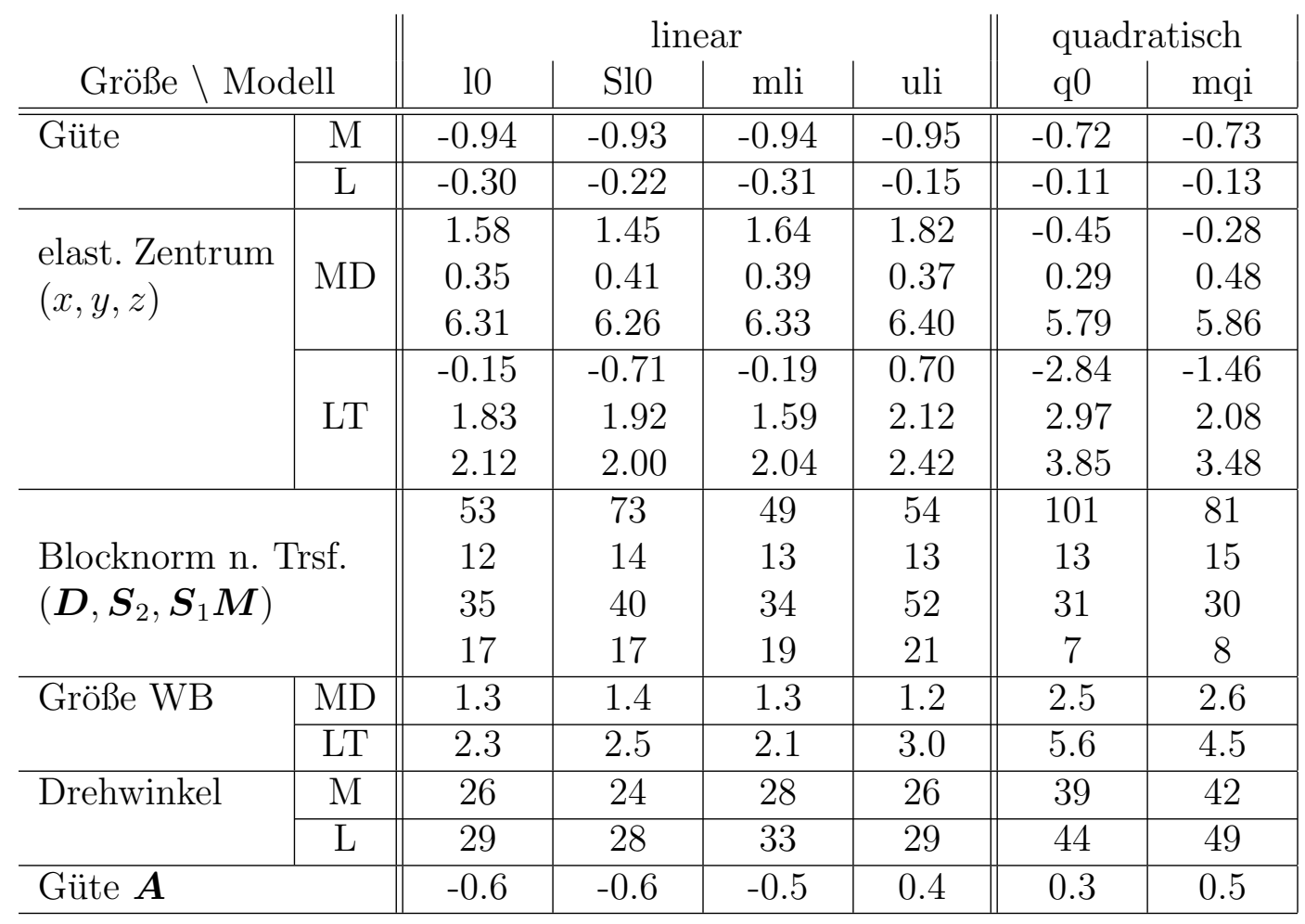

Tabelle 6.2: Wichtige Parameter zu verschiedenen Modellen. Die Abkürzungen bedeuten: multivariat, univariat, linear, quadratisch, $\mathbf{0}$ ohne absolutes Glied, invertierte Steifheitsmatrix, mit intercept. 
so daß das Translationszentrum bei

$$
\overrightarrow{L T}=\left(\begin{array}{l}
3.7 \\
0.9 \\
7.8
\end{array}\right) \mathrm{cm}-\left(\begin{array}{l}
2.3 \\
0.2 \\
5.3
\end{array}\right) \mathrm{cm}=\left(\begin{array}{l}
0.4 \\
0.7 \\
2.5
\end{array}\right) \mathrm{cm}
$$

liegen sollte. Beides ist für die linearen Modelle besser erfüllt als für die quadratischen, wobei es bemerkenswert ist, daß, obwohl die Einträge der Matrizen von Modell zu Modell recht verschieden sind, die Werte für die elastischen Zentren in derselben Größenordnung liegen. Die Blocknorm nach Transformation ist am kleinsten für die linearen Modelle mit wenig Freiheitsgraden. Interessant ist, daß die quadratischen Modelle wesentlich kleinere Werte für die Flexibilitäten der Matrix $\boldsymbol{M}$ vorhersagen. Da diese Matrix aber schon ganz zu Beginn der Berechnungen wesentlich ist, kann ein Eingangsfehler bei den Flexibilitäten großen Einfluß auf den weiteren Verlauf der Rechnung haben. Korrigiert man die Einträge der linearen Modelle nach unten, so ergeben sich größere Werte für die Position der elastischen Zentren, welche mit den Abmessungen der Apparatur besser übereinstimmen. Die Größe der Widerstandsbereiche liegt überraschenderweise im $\mathrm{cm}$-Bereich, es ergeben sich etwas kleinere Werte für die linearen Modelle. Die Drehwinkel der Realform sollten bei $30^{\circ}$ liegen, der Neigung des Alveolarkamms gegen die Vertikale. Auch hier liefern die linearen Modelle die besseren Werte. Die Güte der Matrix $\boldsymbol{A}$ ist am günstigsten für die Modelle mit zwei Freiheitsgraden pro Variable, aber nach dem Gesagten über die Fehlerfortpflanzung, vermutlich kein aussagekräftiger Parameter zur Beurteilung eines Modells mehr.

Die linearen Modelle sind aus der Sicht der Theorie die günstigeren. Anders ist das aus statistischer Sicht. Ein Maß für die Güte der Anpassung unter Berücksichtigung der Anzahl der Parameter des Modells liefert der F-Test der Varianzanalyse. Man zerlegt die Gesamtstreuung der Meßwerte $\sigma_{G}$ in die durch das Modell erklärte $\sigma_{M}$ und die restliche Streuung $\sigma_{E}$ durch

$$
\sigma_{G}^{2}=\sigma_{M}^{2}+\sigma_{E}^{2}
$$

und bildet den Quotienten

$$
F:=\frac{\sigma_{M}}{\sigma_{E}}
$$

Große Werte erklären höhere Anteile der Streuung durch das Modell. Die sich ergebenden Werte sind in Tabelle 6.3 dargestellt. Zum Vergleich wurden den verschiedenen Werten je Lagekomponente ein Rang zugeordnet und dieser für jedes Modell summiert. Die quadratischen Modelle liefern die besseren Anpassungen der Kurven an die Meßwerte. Bis auf eine Komponente ist damit das quadratische Modell ohne Intercept das statistisch sinnvollste - was auch durch den visuellen Eindruck der Auftragung der Meßwerte bestätigt werden kann.

Nun sind gerade die im statistisch sinnvollsten Modell (mq0) geschätzten Größen nur schlecht mit der Theorie verträglich, es scheidet daher aus. Ebenso spricht die Statistik gegen die von der Theorie bevorzugte univariate lineare Regression (uli). 


\begin{tabular}{l||r|r|r|r|}
\multicolumn{1}{c||}{ Komponente } & \multicolumn{4}{c|}{ Modell } \\
\hline \hline$d_{x}$ & 776 & 705 & 1084 & 898 \\
$d_{y}$ & 1201 & 1127 & 2048 & 1611 \\
$d_{z}$ & 872 & 856 & 1228 & 972 \\
\hline$\theta_{x}$ & 1051 & 988 & 1410 & 1090 \\
$\theta_{y}$ & 754 & 637 & 913 & 728 \\
$\theta_{z}$ & 737 & 660 & 595 & 430 \\
\hline$\Sigma$ Ränge & 15 & 22 & 8 & 15 \\
\hline
\end{tabular}

Tabelle 6.3: Werte des F-Testes zu den einzelnen Lageänderungen und verschiedenen Modellen. Die Abkürzungen entsprechen denen der Tabelle 6.2.

Da sich die Schätzer der übrigen Modelle bis auf ihren Grad nur wenig voneinander unterscheiden, stehen demnach aus Gründen der Einfachheit nur noch die Modelle ohne Achsabschnitt als Kompromiß zwischen Statistik und Theoriekonformität zur Debatte.

Eine Vortransformation des Datensatzes auf die elastischen Zentren des jeweiligen Modells ergab keinen Unterschied zu den transformierten Schätzern. Das ist insofern verständlich, als daß es sich bei den Modellen um sog. Verallgemeinerte Lineare Modelle handelt und die Transformationen auch auf linearen Operationen beruhen.

Die großen Widerstandsbereiche deuten darauf hin, daß außer dem Periodont noch eine andere elastische Struktur an der Bewegung beteiligt sein könnte. Diese könnte die Meßapparatur selbst oder etwa der Alveolarkamm sein. Auf die Apparatur deuten die zu kleinen Werte für das Widerstandszentrum hin. Eine Beteiligung des Alveolarkamms könnte durch zu hohe Lasten hervorgerufen sein. In der Tat reichten die angelegten Lasten bis hin $\mathrm{zu} 1.7 \mathrm{~N}$ und $2.7 \mathrm{~N} \mathrm{~cm}$ gegenüber orthodontischen Lasten von $0.3 \mathrm{~N}$ und $0.8 \mathrm{~N} \mathrm{~cm}$, wobei von Geraden abweichende Verläufe im Wesentlichen bei den Drehmomenten zu beobachten waren. Es bestand also die Hoffnung, daß, wenn die Kräfte auf etwa $0.9 \mathrm{~N}$ und die Drehmomente auf $1.5 \mathrm{~N} \mathrm{~cm}$ beschränkt würden, sich die Diskrepanz zwischen den Schätzern der verschiedenen Modelle verringert. Dieses Eintreten der Sättigung konnte hauptsächlich (in 11 von 18 Fällen) bei den Kräften beobachtet werden, bei den Drehmomenten waren die Parabeln in 10 von 18 Fällen vom Ursprung weg gekrümmt. Ob es sich bei diesem Phänomen um biologische Prozesse während der Messung oder aber um ein Problem des Meßaufbaus handelte, ist nachträglich nicht mehr zu entscheiden. Um außerdem einen Einfluß der Eichfunktion der Gleichstrommotoren auszuschließen, wurden Drehmomente mit Beträgen unter $0.3 \mathrm{~N} \mathrm{~cm}$ nicht berücksichtigt, denn bei etwa $0.3 \mathrm{~N} \mathrm{~cm}$ trafen sich alle Fitparabeln auf der Nullinie. Als Arbeitshypothese wäre es demnach sinnvoll, dort den Nullpunkt der Drehmomente nachträglich anzusetzen. Das würde das gute Abschneiden der univariaten Modelle erklären. Das 
Ergebnis dieses Versuches war, daß die Schätzwerte der quadratischen Modelle für die Matrix $\boldsymbol{M}$ praktisch verschwanden, so daß mit ihnen keine sinnvolle Aussage mehr möglich war. Die Ergebnisse der linearen Modelle hingegen veränderte sich kaum, so daß im Folgenden von einer Restriktion des Datensatzes abgesehen wurde.

Als letztes kommt noch eine Black-Box-Methode zur Elimination eines unbekannten systematischen Fehlers ungeachtet seiner Ursache in Frage ${ }^{6}$ Dazu verwendet man zwei ungleich gute Schätzer, sagen wir $\mathcal{F}_{1}$ und $\mathcal{F}_{2}$, mit einem jeweils unterschiedlichen Anteil der vermuteten Störterme. Durch Bildung des gewichteten Mittels

$$
\mathcal{F}=(1+\lambda) \mathcal{F}_{1}-\lambda \mathcal{F}_{2}
$$

bei dem das Gewicht zum schlechteren Wert negativ ist, heben sich bei geeigneter Wahl von $\lambda$ die Störterme heraus. Das äußert sich in günstigeren Werten für die Parameter dieser Theorie. Für zu große $\lambda$ überwiegen dann die zufälligen Fehler, und die Parameter verschlechtern sich wieder. Da alle Matrixeinträge gleichzeitig manipuliert werden, bleibt das Transformationsverhalten einer solchen Linearkombination korrekt. In der Tat erhält man für

$$
\hat{\mathcal{F}}=2.5 \mathcal{F}_{l 0}-1.5 \mathcal{F}_{S l 0}
$$

eine Matrix mit den Güten -0.95 und -0.45, den elastischen Zentren

$$
\overrightarrow{M D}=\left(\begin{array}{l}
1.83 \\
0.20 \\
6.39
\end{array}\right) \quad \text { und } \quad \overrightarrow{L T}=\left(\begin{array}{l}
1.00 \\
1.79 \\
2.41
\end{array}\right)
$$

und den Blocknormen

$$
\left(\begin{array}{c|c}
395 & 94 \\
\hline 66 & 17
\end{array}\right) \quad \text { und } \quad\left(\begin{array}{c|c}
34 & 11 \\
\hline 28 & 17
\end{array}\right)
$$

vor und nach der Transformation. Die Größe der Widerstandsbereiche ergibt sich zu $1.27 \mathrm{~cm}$ und $2.03 \mathrm{~cm}$, mit dieser Größenordnung hat man sich offenbar abzufinden. Die Drehwinkel der Realform sind $29^{\circ}$ und $30^{\circ}$, die Drehung findet um die $y$-Achse statt. Selbst die Güte der Matrix $\boldsymbol{A}$ ist nun mit 0.41 im deutlich positiven Bereich. In allen Kriterien übertrifft diese Matrix die einzelnen Schätzer, vgl. Tabelle 6.2. Das bekräftigt die Vermutung, daß ein noch unverstandener systematischer Fehler vorlag.

Die Realform dieser interpolierten Flexibilitätsmatrix eines oberen Frontzahnes, die ich daher für den vertrauenswürdigsten Schätzer halte und im Folgenden verwenden

\footnotetext{
${ }^{6}$ Dieses Verfahren kommt etwa zum Einsatz in Verfahren der numerischen Integration, bei denen die Fehlerordnung bekannt ist. Die beiden Schätzer unterscheiden sich durch die Anzahl der Stützstellen. Der systematische Fehler ist dann durch die Form der zu integrierenden Funktion bedingt.
} 
möchte, lautet

$$
\hat{\mathcal{F}}=\left(\begin{array}{ccc|ccc}
6.18 & -11 . & 7.05 & -2.64 & 0.682 & -1.08 \\
-10.8 & 21.5 & -0.183 & 1.77 & 0.343 & 3.08 \\
12.9 & 10.8 & 8.2 & -6.48 & 7.12 & -0.227 \\
\hline-18.7 & 14.3 & -9.72 & 15.6 & 0 & 0 \\
5.48 & 9.03 & 2.77 & 0 & 6.00 & 0 \\
-1.61 & 1.2 & 5.74 & 0 & 0 & 2.59
\end{array}\right)
$$

Im Rahmen der Meßgenauigkeit kann sie symmetrisiert werden, was aber für die weitere Diskussion nicht von Belang ist.

Die größte Flexibilität gegenüber Rotationen ergibt sich mit $16 \cdot 10^{-4} \mathrm{rad} / \mathrm{Ncm}$ erwartungsgemäß in Richtung der Zahnlängsachse $\boldsymbol{e}_{x}$, gefolgt von $6 \cdot 10^{-4} \mathrm{rad} / \mathrm{Ncm}$ tangential zum Alveolarkamm. Am wenigsten flexibel ist der Frontzahn mit 3 . $10^{-4} \mathrm{rad} / \mathrm{Ncm}$ gegenüber Rotationen, deren Achsen sagittal und senkrecht zur Längsachse liegen, die also Verschiebungen der Zahnwurzel entlang des Alveolarkamms hervorrufen. Die Werte liegen unterhalb des kritischen Wertes von 17 . $10^{-4} \mathrm{rad} / \mathrm{Ncm}$ für die Transversallasten, die Messung fand demnach im zulässigen Bereich statt. Verglichen mit den Werten SyngeS von $0.15 \cdot 10^{-4} \mathrm{rad} / \mathrm{Ncm}$ in transversaler Richtung und $0.4 \cdot 10^{-4} \mathrm{rad} / \mathrm{Ncm}$ axial ergibt sich etwa ein Faktor $30 \ldots 40$. Dieser Faktor belegt, daß tatsächlich die elastischen Anteile gemessen wurden, daß also die Messung langsam genug erfolgte und die IMT nicht mehr gültig ist. Ein anschaulicher Grund dafür, daß die IMT eine zu steife Zahnaufhängung vorhersagt, liegt in der reichlichen Versorgung des Periodonts mit Blutgefäßen: Zumindest in diesem Flüssigkeitsanteil kann sich der Druck nur bis zum systolischen Wert aufbauen; ebenso würde er erhöht, falls er für Teile des Periodonts unterhalb des diastolischen Wertes fiele.

Über die Translationsrichtgrößen kann angesichts der erforderlichen Genauigkeit nur ausgesagt werden, daß sie unterhalb etwa $15 \mu \mathrm{m} / N$ liegen. Damit sind sie wieder kleiner als der für die Linearität kritische Wert von $31 \mu m / N$. Der Wert SyngEs von $0.3 \mu \mathrm{m} / N$ für eine Transversallast an der Zahnkante befindet sich damit weit außerhalb der Nachweismöglichkeit, erst recht der von $0.004 \mu m / N$ für axiale Lasten.

Auch die Konstante für das Reziprozitätstheorem kann für eine an der Krone im Abstand $h$ angreifende Transversallast, hier also in die neue $\boldsymbol{e}_{z}$-Richtung, nach oben abgeschätzt werden. Das zusätzliche Drehmoment ist dann $\boldsymbol{T}=h F \boldsymbol{e}_{y}$. Unter Vernachlässigung der Nebendiagonalterme erhält man

$$
\sigma^{2}=\frac{(\boldsymbol{D})_{z z}}{(\boldsymbol{M})_{y y}}=1.4 \mathrm{~cm}^{2},
$$

also ergibt sich bezogen auf die Wurzellänge etwa ein Wert von $\sigma^{2} / w^{2} \leq 1$. Dieser Wert ist um etwa den Faktor 10 größer als erwartet; es ist demnach anzunehmen, 
daß der wahre Wert für $(\boldsymbol{D})_{z z}$ etwa eine Größenordnung kleiner ist. Das Reziprozitätstheorem soll im Folgenden näher untersucht werden. Aus der transversalen Einheitslast

$$
f(h)=(0,0,1,0, h, 0)^{T}
$$

ergibt sich durch $q(h)=\mathcal{F} f(h)$ eine einparametrige Schar von Drehschrauben. Ihre Aufpunkte definieren demnach eine Raumkurve. Diese besteht aus drei ganzrationalen Funktionen vom Grad 2 in Zähler und Nenner. Da der Aufpunkt der Kraftschraube bei $-h$ auf der $x$-Achse liegt, ergibt sich selbst für das Skalarprodukt der Aufpunkte der Schrauben wieder eine ganzrationale Funktion, hier

$$
\boldsymbol{a}_{f} \cdot \boldsymbol{a}_{q}=-\frac{h\left(23.8+67.0 h+42.7 h^{2}\right)}{135.2+33.3 h+36.0 h^{2}} .
$$

Diese ist klar von einer Konstanten verschieden; nicht einmal die lineare Näherung für kleine oder große $h$ ist konstant. Ohne die Nebendiagonalmatrizen künstlich zu vernachlässigen, gilt das Reziprozitätstheorem hier also nicht.

Der Vergleich mit der eigenen Theorie ist auch mit großen Unsicherheiten behaftet. Zum einen sind die elastischen Parameter des Periodonts nur ungenau bekannt, zum anderen gibt es keine klaren Aussagen über die genaue Verteilung der Dicke des Periodonts über die Zahnwurzel, geschweige denn über den Verlauf der Fasern. Zusammmen mit einer ohnehin nicht integrablen elliptischen Grundform, die durch die Meßwerte nahegelegt wird, ergäbe sich erst ein realistisches Zahnmodell. Eine analytische Beschreibung ist daher nicht realisierbar. Dennoch möchte ich den Vergleich mit dem Rotationsparaboloiden aus 5.3.3 und den daraus in diesem Kapitel gewonnenen Zahlenwerten wagen. Die Werte der Matrix $\boldsymbol{M}$ liegen mit $\boldsymbol{M}=\operatorname{diag}(34,3.5,3.5) \mathrm{rad} / \mathrm{Ncm}$ bzw. $\boldsymbol{M}=\operatorname{diag}(34,9.5,9.5) \mathrm{rad} / \mathrm{Ncm}$, im Gegensatz zu der Vorhersage von Synge, in derselben Größenordnung wie die Meßergebnisse. Damit ist die ECT, oder besser gesagt die Theorie meiner Arbeit, für die Vorhersage geeignet und prinzipiell durch das Experiment bestätigt. Die Unterschiede der Werte deuten klar darauf hin, daß der Querschnitt des Frontzahnes kein Kreis ist. Ferner sind die elastischen Parameter nur ungenau bekannt, und es wurde eine gemittelte Geometrie zu Grunde gelegt, was die Abweichungen erklärt. Das Verhältnis der axialen zu den radialen Steifigkeiten lautet gemittelt $15.6 /((6+2.6) / 2)=15.6 / 4.3=3.6$ und spricht eher für das Stabmodell, nach dem Federmodell sollte es bei 10 liegen. Der Vergleich mit dem Verhältnis $C_{22} / C_{33}$ der Rechnung mit veränderlicher Dicke des Periodonts (6.1) ergibt $v=0.35$, was entwe$\operatorname{der} \sigma=0.23$ oder nach dem Stabmodell $\sigma_{S}=0.42$ ergibt. Als Materialkonstanten erhält man aus $C_{33}=1 / 15.6 N \mathrm{~cm}$

$$
\begin{aligned}
K_{2} \equiv G & =0.75 \mathrm{~N} / \mathrm{mm}^{2} \\
K_{1} & =2.1 \mathrm{~N} / \mathrm{mm}^{2} \quad \text { und } \\
E & =0.31 \mathrm{~N} / \mathrm{mm}^{2} \quad \mathrm{bzw} \\
E \equiv K_{1} & =2.1 \mathrm{~N} / \mathrm{mm}^{2}
\end{aligned}
$$


Da die Werte für das Stabmodell näher den Ausgangswerten der Vorhersage sind, erklärt das die scheinbare Bestätigung des Stabmodells. Ob nun das Periodont weicher und die Querdehnzahl kleiner ist als angenommen, oder ob tatsächlich das Stabmodell auf die Zahnaufhängung zutrifft, ist aus den Daten für die Rotationsflexibilitäten allein nicht zu entscheiden.

Die Flexibilitäten für die Matrix $\boldsymbol{D}$ zwischen 0.43 und $2.2 \mu m / N$ liegen zwar zwei bis drei Größenordnungen über denen von Synge, aber leider auch eine Größenordnung unterhalb der Grenze der Meßgenauigkeit. Das erklärt die diesbezüglich schlecht zu interpretierenden Ergebnisse.

Alles in allem geben diese Werte klare Auskunft über die Anforderungen an eine Meßapparatur zur Bestimmung der elastischen Konstanten der initialen Zahnbeweglichkeit. Diese halte ich unvereinbar mit den während einer in-vivo-Messung auftretenden Störgrößen. Ferner wäre noch eine Meßvorschrift zu ersinnen, die dem durch den Blutdruck getriebenen System die rein elastischen Anteile entnähme. Eine passende Theorie müßte elastische und flüssige Bestandteile des Periodonts miteinander koppeln, wobei die letzteren dem eben Gesagten zufolge noch in passive und aktive Anteile zu unterscheiden sind. Eine Entscheidung zu Gunsten des Feder- oder des Stabmodells zur Beschreibung der Zahnaufhängung kann demnach aus den vorliegenden Daten nicht getroffen werden. Es wäre immerhin denkbar, daß die oben bestimmten Materialkonstanten den Einfluß der Flüssigkeitsanteile in der Kontinuumsnäherung mit berücksichtigen.

\subsubsection{In vitro}

Die Messungen an Leichenzähnen fanden vor denen am lebenden Probanden statt, um die Größenordnung der zu erwartenden Effekte festzustellen. Da nur die über univariate lineare Regression gewonnenen Werte für alle gemessenen Zähne zur Verfügung standen, sollen ihre Realformen hier kurz vorgestellt werden, um einen Einblick in die Unterschiede von Zahn zu Zahn zu geben. Die Indizes geben die Position der Zähne in der zahnmedizinisch üblichen Nomenklatur des internationalen Zahnschemas $^{7}$ an: Die erste Zahl kennzeichnet den Quadranten und die zweite die Nummer des Zahnes von der Mitte des Zahnbogens aus gerechnet. Eine erste Ziffer 4 bedeutet also eine Messung an der rechten Hälfte des Unterkiefers. Es ergeben sich in den Einheiten

$$
\left(\begin{array}{c|c}
\mu \mathrm{m} / N & \mu \mathrm{m} / \mathrm{Ncm} \\
\hline 10^{-4} \mathrm{rad} / N & 10^{-4} \mathrm{rad} / \mathrm{Ncm}
\end{array}\right)
$$

\footnotetext{
${ }^{7}$ Vgl. etwa [26].
} 
die Realformen

$$
\mathcal{F}_{43}=\left(\begin{array}{ccc|ccc}
24 . & -21.4 & 3.67 & -8.02 & -0.442 & -5.5 \\
-28 . & 47 . & -1.46 & -0.822 & 8.69 & 9.05 \\
16.2 & -1.35 & 21.3 & -10.7 & 9.51 & -3.66 \\
\hline 2.7 & 4.51 & -0.345 & 59.1 & 0 & 0 \\
2.43 & -10.1 & 1.5 & 0 & 31.8 & 0 \\
-0.177 & 1.42 & 11.2 & 0 & 0 & 30.3
\end{array}\right)
$$

sowie

$$
\mathcal{F}_{44}=\left(\begin{array}{ccc|ccc}
0.233 & -0.675 & -4.01 & -1.56 & -0.537 & 0.626 \\
1.63 & -1.62 & -4.06 & -1.26 & 1.53 & -0.985 \\
-1.99 & 5.87 & 8.92 & 1.86 & -1.25 & -1.1 \\
\hline-1.77 & 9.46 & 3.14 & 31.7 & 0 & 0 \\
4.04 & 13.2 & 5.65 & 0 & 13.5 & 0 \\
1.05 & 4.44 & 3.29 & 0 & 0 & 10.6
\end{array}\right)
$$

und

$$
\mathcal{F}_{45}=\left(\begin{array}{ccc|ccc}
1.39 & 0.397 & -0.354 & -0.519 & 0.0216 & 0.399 \\
4.52 & 4.57 & -6.02 & 0.0837 & 0.998 & 0.69 \\
-1.55 & 4.31 & 9.72 & 1.93 & 0.86 & 0.25 \\
\hline 3.73 & 7.15 & 2.24 & 25.4 & 0 & 0 \\
1.85 & -3.12 & -1.04 & 0 & 6.55 & 0 \\
0.464 & -0.833 & -2.59 & 0 & 0 & 5.25
\end{array}\right)
$$

Wie bei der in-vivo-Messung sind die Werte der $\boldsymbol{M}$-Matrizen die verläßlichsten. Die gemessenen Flexibilitäten nehmen erwartungsgemäß vom Eckzahn bis zum zweiten kleinen Backenzahn zu. Wie auch beim Wirbelsegment ist die Flexibilität beim konservierten Gewebe größer.

Die Güten im Meßsystem reichen von -0.988 bis -0.998 und im Lastsystem von -0.942 bis -0.984 , sind also alle sehr gut. Die anderen Flexibilitätskonstanten bewegen sich nach Transformation wieder im 10\%-Bereich der Ausgangszahlen oder sogar darunter. Entsprechend variiert die Größe des Widerstandsbereiches von 0.16 bis $0.42 \mathrm{~cm}$ für das Meßsystem und von 0.35 bis $0.69 \mathrm{~cm}$ für das Lastsystem. Die kleineren Werte sind eher realistisch.

Aufgrund ihrer hohen Flexibilität liefert die Matrix $\mathcal{F}_{43}$ am ehesten brauchbare Werte für die Translationen. An ihr möchte ich abschließend exemplarisch vorführen, mit welchen Standardinformationen die Aussagen über Flexibilitätsmatrizen bezüglich der Theorie getroffen wurden. Zusammen mit den 6 elastischen Matrizen (Flexibilitätsmatrix, Steifheitsmatrix $) \times($ Original, bzgl. Elastisches Zentrum, Realform) fassen sie die hier in Text gekleideten Ergebnisse für eine Messung zusammen. Die Einheiten sind, falls sie sich wie hier nicht durch die Rechnung mit dem Computeralgebra-System ergeben, aus den entsprechenden Definitionsgleichungen abzulesen. 
File: d: \math \43.mat vom (Y, M, D, h, m, s) $\{1999,10,18,17,45,46\}$

1. Information im Translationsanteil.

Guete. (M, D, S2,-B2): -0.987867 (L, T, $-\mathrm{S} 1, \mathrm{~B} 1):-0.979216$

a) Antisymmetrischer Anteil

Ortsvektor MD: $\{\{4.25399\},\{0.713976\},\{0.831578\}\}$

LT: $\{\{2.71718\},\{0.604588\},\{0.594781\}\}$

Abstand. MD: 4.39291 LT: 2.84646

Blocknorm vorher $\{\{710 ., 238\},.\{153 ., 73.6\}\}$

und nachher $\{\{69 ., 21.7\},\{16.3,73.6\}\}$

b) Symmetrischer Anteil

Eigenwerte der Widerstandsmatrizen

$(\mathrm{MD}):\{0.452038,-0.382388,-0.0529665\}$

(LT) : $\{-0.372311,0.336022,-0.0610061\}$

Groesse des Widerstandsbereiches

(MD) : 0.417213 (LT) : 0.354166

2. Information im Rotationsanteil.

Funktionelle Achsen: Matrix M

Guete: 1

Drehwinkel in Grad. Mess: 37.3668 Last:36.589

Drehmatrizen

Mess: $\{\{0.84,-0.41,0.36\},\{0.28,0.89,0.36\},\{-0.47,-0.2,0.86\}\}$

Last: $\{\{0.84,0.3,-0.46\},\{-0.41,0.9,-0.16\},\{0.37,0.33,0.87\}\}$

Drehrichtungen Mess: $\{\{-0.455112\},\{0.681249\},\{0.573388\}\}$

Last: $\{\{0.411285\},\{-0.695195\},\{-0.589532\}\}$

3. Miscellana (Realform liegt vor).

Spurbedingung Spur/Norm. (B2): 0.255808 (B1): -0.43875

Vorfaktor Einheitensysteme (L/M): 0.849903

Stimmigkeit der Kraftkomponenten: Matrix A

Guete: 0.729878

Orthogonale Matrizen

Polar: $\{\{0.95,0.036,-0.31\},\{-0.099,0.98,-0.19\},\{0.3,0.21,0.93\}\}$ 


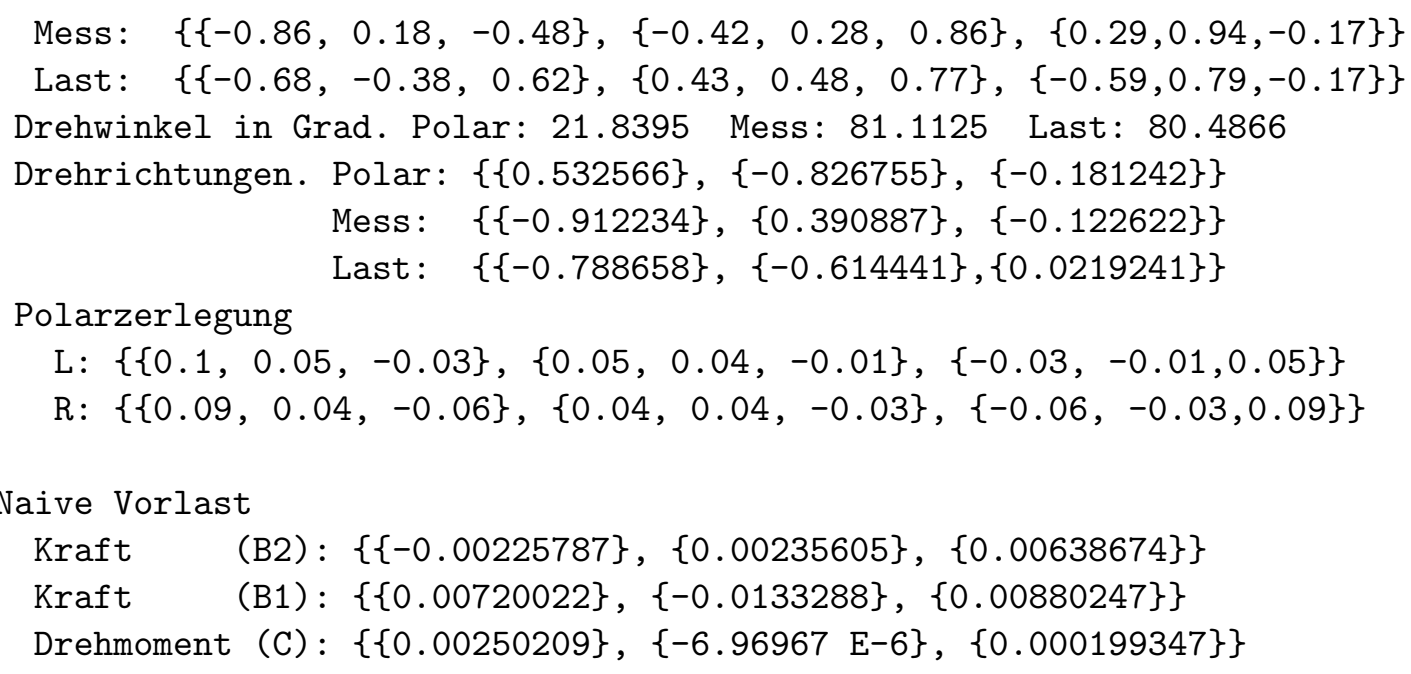

Wenn man die Flexibilitätskoeffizienten mit einem skalaren Faktor multipliziert, welcher die elastischen Eigenschaften der unterschiedlichen Gewebe näherungsweise ineinander umrechnet, bekommt man eine brauchbare Vorstellung von den bei noch durchzuführenden in-vivo-Messungen zu erwartenden Werten. Nach den in Abschnitt 6.2 gewonnenen Einsichten und den Werten für den Frontzahn sollte dieser Faktor unterhalb von 1/3 liegen. Vermutlich liegt dieser Effekt im fehlenden Blutdruck und in der vergrößerten Durchlässigkeit der Zellwände gegenüber der die anatomischen Präparate konservierenden Flüssigkeiten begründet.

\subsection{Statik eines Hüftendoprothesenschaftes}

Die Werte entstammen der Diplomarbeit Wolfgang Schäfer [72, verheiratet GöRTZ. Es handelt sich um einen festsitzenden Hüftendoprothesenschaft. Alle Zahlenwerte in den Matrizen sind im SI mit den Einheiten

$$
\left(\begin{array}{c|c}
\mu m / N & \mu m / N m \\
\hline \mu r a d / N & \mu r a d / N m
\end{array}\right)
$$

angegeben. Die ursprünglichen Meßwerte lauten demnach

$$
\mathcal{F}=10^{-6}\left(\begin{array}{ccc|ccc}
0.517 & 0.058 & -0.006 & 0.71 & 8.39 & 1.12 \\
-0.033 & 0.207 & -0.005 & -3.52 & -0.46 & -1.6 \\
0.089 & 0.005 & 0.008 & -0.1 & 1.11 & 0.2 \\
\hline 0.53 & -4 . & 0.152 & 71.4 & 10.2 & -2.3 \\
9.07 & -0.52 & 0.038 & 10.2 & 155.2 & 6.5 \\
0.49 & 0.02 & -0.003 & 0.3 & 14 . & 197.8
\end{array}\right)
$$


wobei der Vorfaktor $10^{-6}=\mu$ so gewählt ist, daß die in der Matrix stehenden Zahlen den obigen Einheiten entsprechen. Die Norm der Blöcke ist

$$
10^{-6}\left(\begin{array}{c|c}
0.568 & 9.41 \\
\hline 9.95 & 262
\end{array}\right)
$$

Nach Translation auf Drehmoments- und Translationszentrum ergeben sich die folgenden Zahlenwerte

$$
\tilde{\mathcal{F}}=10^{-6}\left(\begin{array}{ccc|ccc}
0.028 & 0.095 & -0.008 & 0.184 & 0.355 & 0.156 \\
-0.012 & 0.010 & 0.003 & 0.158 & 0.177 & -0.142 \\
0.024 & -0.001 & 0.008 & 0.046 & -0.095 & 0.141 \\
\hline-0.059 & 0.093 & 0.017 & 71.4 & 10.2 & -2.3 \\
0.186 & 0.064 & 0.023 & 10.2 & 155.2 & 6.5 \\
0.061 & 0.032 & -0.003 & 0.3 & 14 . & 197.8
\end{array}\right)
$$

mit den Normen

$$
10^{-6}\left(\begin{array}{c|c}
0.104 & 0.540 \\
\hline 0.238 & 262
\end{array}\right) .
$$

Von den ursprünglichen Einträgen der Nebendiagonalmatrizen bleiben nur etwa $6 \%$ für $\boldsymbol{S}_{2}$ und $2 \%$ für $\boldsymbol{S}_{1}$ übrig, d. h., die gemessenen Translationen sind im Wesentlichen eine Folge des Hebelgesetzes der Auslenkungen. Bei einer Meßgenauigkeit von um die 5\% darf daher von einem Widerstandszentrum gesprochen werden. Die Güten der Transformationsmatrizen sind -0.996 für das Meßsystem und -0.999 für das Lastsystem. Dieses liegt bei

$$
\overrightarrow{M D}=\left(\begin{array}{c}
0.0080 \\
0.0032 \\
-0.0515
\end{array}\right) \quad \text { und } \quad \overrightarrow{L T}=\left(\begin{array}{c}
-0.0000 \\
-0.0019 \\
-0.0573
\end{array}\right)
$$

also mit Abweichungen im $m m$-Bereich bei $5 \mathrm{~cm}$ in $-z$-Richtung. Die Norm der Matrix $\boldsymbol{D}$ fällt auf $18 \%$ ihres Ausgangswertes, so daß sie im Rahmen der Meßgenauigkeit brauchbar sein sollte. Ihre Güte ändert sich hingegen von ursprünglich 0.92 auf -0.35, weshalb ich ihren absoluten Komponenten nur noch wenig Aussagekraft zumesse. Die Anordnung ist daher bezüglich reiner Kräfte durch das Drehmomentszentrum erheblich steifer, als es nach den ursprünglichen Zahlenwerten aussieht.

Nach Drehungen um $14^{\circ}$ bzw. $15^{\circ}$ für Meß- und Lastsystem ergibt sich schließlich die Realform

$$
\hat{\mathcal{F}}=10^{-6}\left(\begin{array}{ccc|ccc}
0.009 & -0.002 & -0.021 & 0.094 & 0.066 & -0.087 \\
-0.005 & 0.020 & -0.016 & 0.086 & 0.282 & 0.135 \\
-0.014 & 0.096 & 0.019 & -0.249 & 0.296 & 0.126 \\
\hline 0.014 & -0.055 & -0.093 & 200.196 & 0 & 0 \\
-0.043 & 0.078 & 0.151 & 0 & 154.154 & 0 \\
-0.033 & 0.069 & -0.089 & 0 & 0 & 70.140
\end{array}\right)
$$


Die verwendeten Drehvektoren lauten

$$
\left(\begin{array}{l}
0.216 \\
0.007 \\
0.124
\end{array}\right) \text { und }\left(\begin{array}{c}
-0.238 \\
0.000 \\
-0.122
\end{array}\right) \text {, }
$$

also beseitigt eine einfache Drehung den Hauptanteil der Abweichung der funktionellen Achsen von denen des Koordinatensystems. Diese Matrix kann nun mit der numerischen Rechnung verglichen werden. Da die Matrix $\boldsymbol{M}$ stark diagonaldominant ist, befinden sich auf ihrer Hauptdiagonale näherungsweise ihre Eigenwerte. Da die Realform diese Eigenwerte von $\boldsymbol{M}$ der Größe nach anordnet, sind im Wesentlichen die $x$ - und die $z$-Achse vertauscht worden. Die Normen der Matrizen ändern sich wegen der Invarianz der Norm unter Drehungen gegenüber den vorigen Werten nicht.

Da die Nebendiagonalmatrizen im Rahmen der Meßgenauigkeit verschwinden, erübrigt sich auch eine Untersuchung der Vorlasten. Die Anwendung der Formeln aus 3.3 .4 ergäbe auch unsinnige Werte in der Größenordnung $k N$ bzw. $k N m$.

Die Rechnung mit einem vereinfachten geometrischen Modell mit passenden Abmessungen aus 5.3.5 ergab die Realform der Flexibilitätsmatrix

$$
\hat{\mathcal{F}}=10^{-6}\left(\begin{array}{ccc|ccc}
0.0245 & 0 . & 0.000182 & 0 . & -0.0224 & 0 . \\
0 . & 0.00884 & 0 . & -0.535 & 0 . & 0.00696 \\
0.000182 & 0 . & 0.0163 & 0 . & 0.0137 & 0 . \\
\hline 0 . & -0.535 & 0 . & 480 . & 0 . & 0 . \\
-0.0224 & 0 . & 0.0137 & 0 . & 20.1 & 0 . \\
0 . & 0.00696 & 0 . & 0 . & 0 . & 10.2
\end{array}\right)
$$

mit der Blocknorm

$$
10^{-6}\left(\begin{array}{c|c}
0.0308 & 0.536 \\
\hline 0.536 & 480 .
\end{array}\right)
$$

Die Einträge der Nebendiagonalmatrizen $\boldsymbol{S}_{2}$ und $\boldsymbol{S}_{1}$ entsprechen ihrer Größenordnung denen der Messung. Im Rahmen der Meßgenauigkeit können sie vernachlässigt werden. Die Einträge der Matrix $\boldsymbol{D}$ sind kleiner als die gemessenen. Dies legt zusammen mit der durch die Transformationen gestörten Symmetrie des gemessenen Exemplars die Deutung nahe, daß die interessierenden Effekte durch das Zusammenspiel aus Meßfehlern und Fehlerfortpflanzung überdeckt wurden. Immerhin sind, bis auf die 0.096 beim gemessenen Exemplar, typische Matrixeinträge innerhalb eines Faktors 2 miteinander vergleichbar. Damit ist der Prothesenschaft gegenüber reinen Translationen durch das Widerstandszentrum etwas nachgiebiger als erwartet.

Unerwartet sind hingegen die Einträge der Matrix $\boldsymbol{M}$. Die gemessene Nachgiebigkeit gegenüber Drehmomenten in $x$ - und $y$-Richtung übertrifft die Erwartungen um den Faktor 7. Das kann dadurch erklärt werden, daß der Schaft durch die angelegten Drehmomente in sich verbogen wird und demnach nicht als starrer Körper 
hätte genähert werden dürfen. Das Experiment konnte diesen Effekt bestätigen. Bezüglich der Drehmomente um die Schaftachse sollte der Rechnung nach der Schaft mehr als doppelt so nachgiebig sein. Das kann eine Folge des Modells sein, der Schaft wäre an seinen Enden mit der Kortikalis in engerem Kontakt, als modelliert wurde. Wäre der Schaft in der Kortikalis gleichmäßig eingeklemmt, so sollte sich das in einer stärkeren Asymmetrie der Rotationen senkrecht zur Schaftachse äußern. Andere Modelle und Dickenfunktionen lieferten aber immer Werte mit einem Verhältnis von etwa $50: 2: 1$ gegenüber dem der Messung von $3: 2: 1$. Diese Diskrepanz der Messung gegenüber dem Synarthrosenmodell blieb ungeklärt: $\mathrm{Ob}$ die verwandte Dickenfunktion oder die Materialkonstanten zu wenig der Realität entsprachen oder ob gar das Stabmodell zuständig gewesen wäre, ist selbst eine umfangreiche Untersuchung, zu der hier nur ein Ansatz vorgestellt werden sollte.

Insgesamt ergibt sich, daß das Synarthrosenmodell für den Schaft einer Hüftendoprothese unangemessen ist. Im Experiment [72] zeigte sich, wie gesagt, eine Durchbiegung des teilweise in die Kortikalis eingeklemmten Schaftes. Ferner ist die Bälkchenstruktur der Spongiosa, welche der WoLfFschen Hypothese nach den Hauptspannungslinien folgt, nur unzureichend durch ein Kontinuumsmodell beschrieben. Beides allein ist Grund genug für die Nichtanwendbarkeit der Theorie auf den vorliegenden Fall.

\subsection{Schraubenfedern}

In diesem Abschnitt sollen zwei von L. LINDKVIST mit einer verläßlichen Methode [41] berechneten Steifheitsmatrizen untersucht werden. Die Genauigkeit der berechneten Matrizen entspricht dem aktuellen Stand der Technik, ferner liegt eine Diskussion der modellbedingten Fehler vor. Das Rechenverfahren ähnelt dem Federmodell, nur daß dort, wie für eine linienförmige Anordnung angemessen, elementare Flexibilitätsmatrizen mittels Integration hintereinander geschaltet werden, vgl. Abschnitt 4.4. Ferner ist, da die Abmessungen exakt bekannt sind, eine Visualisierung im Ortsraum möglich. Obwohl es sich dabei um kein Beispiel aus der Biomechanik handelt, möchte ich diese Beispiele hier dennoch vorführen, um die Nützlichkeit der Konzepte dieser Arbeit auch in der Technischen Mechanik zu zeigen.

Es handelt sich um zwei Schraubenfedern mit einer Windung, vgl. Abb. 6.4, Sie haben die Abmessungen

\begin{tabular}{|l|c|c|}
\hline Größe & Feder 1 & Feder 2 \\
\hline \hline Drahtradius $r / m m$ & 1.75 & 10 \\
\hline Radius der Schraubenlinie $R / m m$ & 15 & 15 \\
\hline Höhe der Schraubenlinie $l / m m$ & 7 & 50 \\
\hline
\end{tabular}


und die elastischen Konstanten $E=206 G P a$ und $\sigma=0.3$.
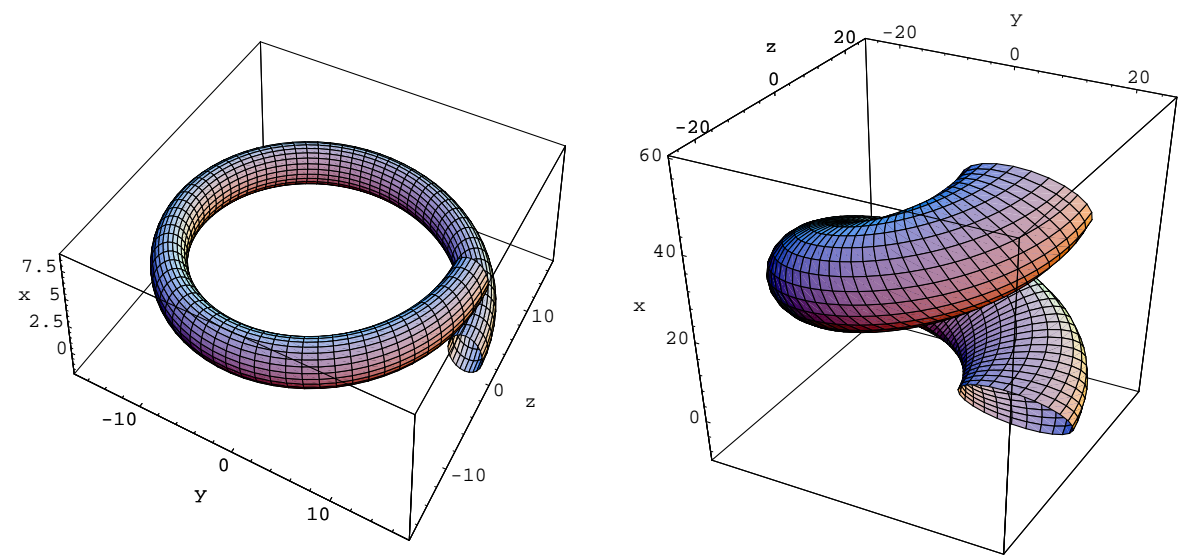

Abbildung 6.4: Die untersuchten Schraubenfedern. Die Längenangaben sind in $m m$. Die $x$-Achse ist die Schraubenlängsachse und auf der $y$-Achse beginnt und endet die Feder. Der Ursprung des Koordinatensystems liegt auf dieser Achse in Höhe des Endes der Schraubenlinie.

Die Einheiten sind derart gewählt, daß alle Matrixeinträge die gleiche Dimension $m / N$ haben; das geschieht, indem Drehmomente durch eine charakteristische Länge geteilt und Drehungen mit ihr multipliziert werden. Diese Länge ist der Federradius $R=15 \mathrm{~mm}$. Nach den Überlegungen von Abschnitt 3.3 .2 ist das Einheitensystem konsistent; die Auswertung darf demnach mit den Zahlen allein durchgeführt werden. Längen sind dann auf $R$ skaliert. Die Zahlen wurden auf vier absolute Nachkommastellen aus der Originalarbeit übernommen, hier aber immer nur auf drei relative Stellen gerundet angegeben.

Die Flexibilitätsmatrizen lauten

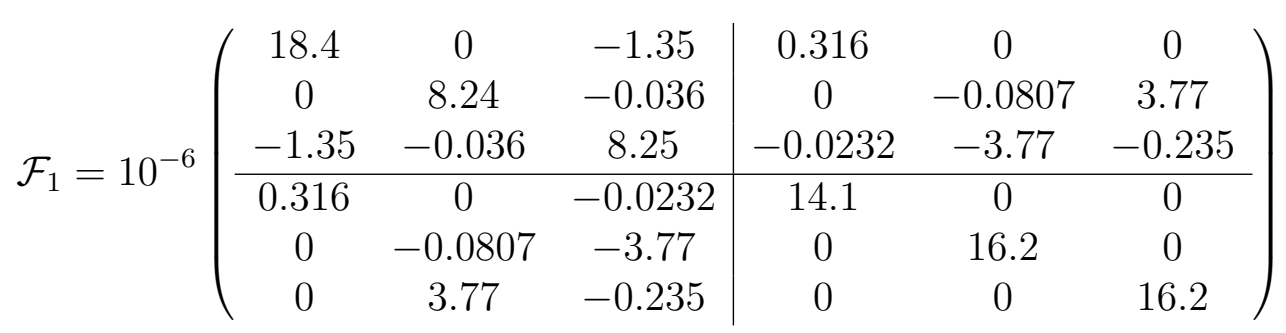

und

$$
\mathcal{F}_{2}=10^{-6}\left(\begin{array}{ccc|ccc}
20.7 & 0 & -8.96 & 2.32 & 0 & 0 \\
0 & 68.9 & -1 . & 0 & -0.857 & 26.7 \\
-8.96 & -1 . & 69.8 & -0.945 & -26.7 & -1.46 \\
\hline 2.32 & 0 & -0.945 & 14.8 & 0 & 0 \\
0 & -0.857 & -26.7 & 0 & 16.1 & 0 \\
0 & 26.7 & -1.46 & 0 & 0 & 16 .
\end{array}\right)
$$


Die Güten der Matrizen $\boldsymbol{S} \boldsymbol{M}^{-1}$ betragen jeweils -0.991. Die elastischen Zentren befinden sich bei

$$
\boldsymbol{z}_{1}=\left(\begin{array}{c}
-3.50 \\
0.0124 \\
0
\end{array}\right) m m \quad \text { und } \quad \boldsymbol{z}_{2}=\left(\begin{array}{c}
-24.923 \\
0.480 \\
0
\end{array}\right) m m
$$

und die Widerstandsbereiche haben die Größe

$$
B_{1}=0.278 m m \text { und } \quad B_{1}=1.924 m m \text {. }
$$

Nach Translation auf die elastischen Zentren ergeben sich die Flexibilitätsmatrizen

$$
\mathcal{F}_{1 z}=10^{-6}\left(\begin{array}{ccc|ccc}
18.4 & 0 & -1.35 & 0.316 & 0 & -0.0133 \\
0 & 7.36 & 0 & 0 & -0.0807 & 0 \\
-1.35 & 0 & 7.37 & -0.0116 & 0 & -0.235 \\
\hline 0.316 & 0 & -0.0116 & 14.1 & 0 & 0 \\
0 & -0.0807 & 0 & 0 & 16.2 & 0 \\
-0.0133 & 0 & -0.235 & 0 & 0 & 16.2
\end{array}\right)
$$

und

$$
\mathcal{F}_{2 z}=10^{-6}\left(\begin{array}{ccc|ccc}
20.7 & -0.00265 & -8.84 & 2.32 & 0 & -0.513 \\
-0.00265 & 24.3 & -0.003 & 0 & -0.857 & 0.0828 \\
-8.84 & -0.003 & 25.5 & -0.472 & 0.0833 & -1.46 \\
\hline 2.32 & 0 & -0.472 & 14.8 & 0 & 0 \\
0 & -0.857 & 0.0833 & 0 & 16.1 & 0 \\
-0.513 & 0.0828 & -1.46 & 0 & 0 & 16 .
\end{array}\right),
$$

wobei sich die Blocknormen von

$$
10^{-6}\left(\begin{array}{c|c}
22 & 5 \\
\hline 5 & 27
\end{array}\right) \quad \text { und } \quad 10^{-6}\left(\begin{array}{c|c}
101 & 38 \\
\hline 38 & 27
\end{array}\right)
$$

auf

$$
10^{-6}\left(\begin{array}{c|c}
21 & 0.4 \\
\hline 0.4 & 27
\end{array}\right) \quad \text { und } \quad 10^{-6}\left(\begin{array}{c|c}
43 & 3 \\
\hline 3 & 27
\end{array}\right)
$$

verkleinern. Die Spurbedingung, die ja ein Test des Federmodells darstellt, ist mit $\mathrm{Sp} \boldsymbol{B}_{1}=0.0015 \cdot 10^{6}$ und $\operatorname{Sp} \boldsymbol{B}_{2}=-0.0014 \cdot 10^{6}$ nicht erfüllt, eine Folge des anderen Ansatzes. Da hier elementare Flexibilitätsmatrizen addiert bzw. integriert werden, sollte die Spurbedingung, welche ja eine Folge des Transformationsverhaltens ist, für die Matrizen $\boldsymbol{S}$ gelten. In der Tat erhält man $\operatorname{Sp} \boldsymbol{S}_{1}=-0.0001 \cdot 10^{-6}$ und $\operatorname{Sp} \boldsymbol{S}_{2}=0$, womit die Spurbedingung bis auf Rundungsfehler bestätigt ist. Eine Drehung um $120^{\circ}$ mit der Drehmatrix

$$
\boldsymbol{R}=\left(\begin{array}{ccc}
0 & -1 & 0 \\
0 & 0 & 1 \\
-1 & 0 & 0
\end{array}\right)
$$


welche $x^{-}, y$ - und $z$ - Achse auf die $-y$-, $z$ - und $-x$-Achse abbildet, bringt die Flexibilitätsmatrizen schließlich auf die Realform, was hier aber nicht weiter erhellend ist.

Erwartungsgemäß liegen die elastischen Zentren der Federn mit nur geringen Abweichungen auf Höhe der Federmitten. Sie sind in Richtung auf Federbeginn und -ende hin leicht abseits der Mittellinie gelegen. Diese Abweichung von der Symmetrie soll nun etwas näher beleuchtet werden. Um beide Abweichungen vergleichbar zu machen, müssen sie durch eine charakteristische Länge dividiert werden. Ist die Höhe $l=0$, so ist die Feder eine Kreistorus und hat ein Widerstandszentrum, die Abweichungen verschwinden dann. Daher ist $l$ eine besonders geeignete Länge zum Untersuchen des Effektes. Ferner ist zu erwarten, daß dieser Verschiebungseffekt mit der Drahtdicke $d=2 r$, genauer dem dimensionslosen Quotienten $r / R$ wächst. In der Tat erhält man für die $y$-Komponenten der elastischen Zentren

$$
\frac{y_{1}}{l_{1} \frac{r_{1}}{R}}=0.0152 \quad \text { und } \quad \frac{y_{2}}{l_{2} \frac{r_{2}}{R}}=0.0144
$$

also zwei etwa gleiche Zahlen, womit der Effekt in erster Näherung verstanden ist. Die Werte auf der rechten Seite können nur noch eine Funktion dimensionsloser Argumente sein.

Auch die Größe des Widerstandsbereiches ist eine Länge und sollte trotz ihrer komplizierten Definition einfach skalieren. Mit

$$
\frac{B_{1}}{l_{1}}=0.0397 \text { und } \quad \frac{B_{2}}{l_{2}}=0.0385
$$

ist auch der hauptsächliche Effekt erklärt. Die Größe des Widerstandsbereiches wächst demnach im Wesentlichen mit der Federlänge, die hier gleich der Schraubsteigung ist.

Für Federn mit mehreren Windungen sind für die Verschiebung des elastischen Zentrums keine höheren Werte zu erwarten, da der Einfluß von Federanfang und -ende gleich bleibt. Der Widerstandsbereich sollte sich vergrößern, da Anfang und Ende nun weiter von der Mitte entfernt sind; der Quotient $B / l$ sollte dennoch konstant bleiben.

Da die Herstellerangaben über Fertigungstoleranzen für die axiale Steifheit der Federn bei $13 \%$ liegen,, 8 sind die hier untersuchten Effekte eher von theoretischem Interesse.

${ }^{8}$ 41] S. 65. 


\section{Kapitel 7}

\section{Zusammenfassung}

Hier sollen zunächst die wesentlichen Aussagen der vorliegenden Arbeit thesenartig zusammengefaßt werden. Dabei werden in erster Linie die Ergebnisse von zwei der drei Hauptteile dieser Arbeit zur Sprache kommen: Das elastische Zentrum mit der Normalform der Meßwerte und das Federmodell samt Beispielen. Sie ergeben die Hauptlinie dieser Arbeit. Der dritte Hauptteil, die Anwendung der ersten beiden auf Meßdaten, illustriert zwar die Nützlichkeit des Formalismus und gibt einen Einblick in treffbare praktische Aussagen - er kann aber keine neuen theoretischen Resultate liefern.

Danach sollen, als Ausblick auf kommende Untersuchungen, die mir außerdem wichtig erscheinenden Aspekte aufgeführt werden, die hier nicht mehr mit der ihnen meines Erachtens gebührenden Aufmerksamkeit behandelt werden konnten.

\subsection{Thesen}

1. Eine elastische Matrix ist die einfachste mathematische Abbildungsvorschrift zwischen kleinen Lasten und Lageänderungen einer starr berandeten elastischen Aufhängung.

2. Die elastische Matrix selbst ${ }^{1]}$ enthält die Information über zwei ausgezeichnete Koordinatensysteme im Raum.

\footnotetext{
${ }^{1}$ Diese Eigenschaft ist kein Ergebnis, sondern war eine a-priori-Forderung an das zu definierende Koordinatensystem. Aus der Überzeugung heraus, daß die Meßwerte alle zum Beschreiben des Systems nötige Information beinhalten, nicht zuletzt motiviert durch meine positivistisch beeinflußte Grundhaltung, vgl. etwa 24, dort insbesondere $§ 2$, wollte ich von Anfang an ein aus den Meßwerten selbst heraus definiertes Koordinatensystem. Dieses hat den ästhetischen Vorteil der Abgeschlossenheit, d. h. es werden keine anderen Objekte, als aus den Meßdaten selbst gewonnen werden können, zu ihrer Erklärung herangezogen. Die Steifheitsmatrix ist gewissermaßen die mit den Augen des Meßgerätes gesehene elastische Aufhängung.
} 
3. Ist die elastische Matrix symmetrisch, so genügt ein Koordinatensystem.

4. Die Symmetrie der Steifheitsmatrix kann aus der Existenz eines Potentials gefolgert werden.

5. Mit dieser Information läßt sich eine ausgezeichnete und unter Wechseln des Koordinatensystems invariante Form der elastischen Matrix finden.

6. Diese Form erlaubt die Vergleichbarkeit von Meßwerten und/oder Vorhersagen; insbesondere taugt damit die elastische Matrix als Meßobjekt.

7. Bezüglich dieser invarianten Form lassen sich wesentliche Eigenschaften der Aufhängung durch aussagekräftige Parameter ausdrücken oder als geometrische Gebilde visualisieren.

8. Auch Meßobjekten, die diesen idealisierten Vorstellungen nicht genügen, läßt sich durch eine geeignete Meß- und Auswertevorschrift eine elastische Matrix zuordnen.

9. Eine hinreichend dünne elastische Schicht kann als Fläche betrachtet werden.

10. Ein isotropes Medium kann dann durch zwei Materialkonstanten beschrieben werden; diese modellieren das Verhalten senkrecht zur und innerhalb der Schicht.

11. Für genügend dünne Schichten können die Schichtgrößen aus der Elastizitätstheorie hergeleitet werden.

12. Aus den zwei Materialkonstanten, der Flächennormale und der Schichtdicke, kann eine Matrix zusammengesetzt werden, über die der Beitrag des Flächenelementes zur elastischen Energie berechnet werden kann.

13. Je nach Anwendung sind verschiedene solcher Federmatrizen als Ausgangspunkt der weiteren Rechnung zulässig.

14. Die Integration der Federmatrix über die gesamte Fläche liefert die Steifheitsmatrix der ganzen Aufhängung.

15. Ist die Integration numerisch durchführbar, so erlaubt das die Beschreibung aller Lastfälle; ist sie analytisch durch bestimmte Integrale möglich, so können darüber hinaus die Schichtgrößen untersucht werden; als unbestimmte Integrale sind zudem Variationen der Form beschreibbar.

16. Für einfache oder hinreichend symmetrische Aufhängungen sind die Integrale geschlossen lösbar und es ergibt sich eine analytische Form der Steifheitsmatrix.

17. Das ausgezeichnete Koordinatensystem ist nur in sehr speziellen Fällen mit dem Flächenschwerpunkt der Aufhängung identisch. Es kann sogar außerhalb der konvexen Hülle der Aufhängung liegen. 
18. Es können allgemeine Aussagen über Aufhängungen bestimmter Symmetrien getroffen werden.

19. Der Vergleich von Meßdaten mit solchen Aussagen läßt Schlüsse auf die innere Struktur des Meßobjektes zu.

20. Stimmen Federmodell und Meßdaten nicht überein, so ist das eher ein Hinweis auf eine von den Voraussetzungen des Modells abweichende Struktur, als ein Versagen des Modells an sich.

21. Der Vergleich von Meßdaten mit einem analytischen Modell erlaubt prinzipiell die Bestimmung der elastischen Konstanten des Materials.

22. Die Auswertung von Aufhängungen unterschiedlichster Herkunft ist möglich und sinnvoll.

23. Die Genauigkeit der bisher überhaupt durchgeführten Messungen reicht nicht aus, um zweifelsfrei eine Aussage für oder gegen das Federmodell zu treffen.

\subsection{Was zu tun bleibt}

Die im ersten Teil definierten Flächen können sicher noch ausführlicher eingesetzt werden. Insbesondere ihre Rolle als Visualisierung kann bei Serienmessungen an ähnlichen Meßobjekten ausgebaut werden. Dies ist aber nur sinnvoll für außerordentlich genaue Messungen.

Dasselbe gilt für die Kongruenz der Schraubachsen und die aus ihr abgeleiteten Flächen. Im Gegensatz zu den eben genannten Flächen ist die Kongruenz selbst weniger als Visualisierung geeignet, dafür aber mehr zu mathematischen Untersuchungen.

Eine Präzisierung des Reziprozitätstheorems oder des ihm zu Grunde liegenden Gedankenexperimentes in Form einer Abhängigkeit der Aufpunkte von Kraftschrauben und Drehschrauben steht ebenfalls noch aus. Gibt man etwa die Kraftwirkungslinie einer reinen Kraft vor, so erhält man über das äquivalente Lastsystem im elastischen Zentrum, die Flexibilitätsmatrix und (2.5) sofort die Drehschraube. Der sich so ergebende Ausdruck hängt aber neben der Richtung der Kraft $\boldsymbol{F}^{0}$ auch wegen $\boldsymbol{T}_{Z}=\boldsymbol{r} \times \boldsymbol{F}$ noch vom Abstand $\boldsymbol{r}$ der Kraftwirkungslinie ab. Damit ist eine konkrete Situation zwar immer beschreibbar, aber ein allgemeiner der Theorie zugänglicher Zusammenhang ist so nicht mehr erkennbar.

Bislang fehlt eine grundlegende statistische Untersuchung, die für ein Meßobjekt mit bekannten oder vermuteten Eigenschaften ein passendes optimales Verfahren anbietet. Je nach Meßobjekt ist damit untrennbar die Notwendigkeit einer nichtlinearen Beschreibung verknüpft. Dennoch hoffe ich, mit meinen Überlegungen einen ersten Schritt in die richtige Richtung getan zu haben. 
Es wäre ferner wünschenswert, einen Rechenweg zu ersinnen, der die Vorlast von den übrigen Effekten entkoppelt. Dieses Problem ist aufgrund der Kleinheit der störenden Terme vermutlich nur gemeinsam mit den Erwägungen zur statistischen Vorgehensweise zu lösen.

Im Zusammenhang mit den eben erwähnten Genauigkeitsfragen steht auch ein prinzipielles Problem: Ist die ermittelte Realform einer Matrix im Rahmen der Genauigkeit nicht symmetrisch, so bedürfte es eines tieferen Argumentes, daß auch dann Drehmomentszentrum und Translationszentrum denselben Ort beschreiben. Diese Frage ist aber nur für der direkten Auswertung nachgeschaltete Transformationen von Bedeutung, d. h. es handelt sich um Effekte höherer Ordnung.

Auch die Federmatrix eines faserigen Materials fand in dieser Arbeit keine Anwendung mehr. Mit ihren vier Materialkonstanten bei nicht ausreichend bekanntem Faserverlauf erschien sie mir im Rahmen der derzeitigen Meßgenauigkeit doch zu spekulativ. Mit Anzahl der freien Parameter steigt zwar die Anpassungsfähigkeit an Meßdaten, aber ebenso wächst die Gefahr einer Fehlinterpretation.

Ein Vergleich des Federmodells mit erprobten numerischen Verfahren (wie etwa der FEM) mußte ausbleiben, da aus den zur Verfügung gestellten Basiselementen (truss, beam, beam3d,...) keine isotrope Schicht zusammengesetzt werden kann. Das liegt daran, daß die Federmatrix im Grunde ein neues solches Basiselement ist. Ferner sollte ein derartiger Vergleich auch eine Optimierung des Verfahrens im Hinblick auf Laufzeit und Ressourcen beinhalten. Da der Einsatz von Computeralgebra in der Mechanik zusammen mit der Entwicklung geeigneter Algorithmen und der Vergleich zu den klassischen Methoden ein aktuelles Forschungsgebiet darstellt (vgl. etwa [37, 35]), können dessen Ergebnisse hier nur gestreift werden.

Die Auswertung der Meßdaten an biologischen Objekten zeigte, daß die Flüssigkeitsanteile der Gewebe eine nicht zu vernachlässigende Rolle im elastischen Geschehen spielen. Um für diese Anteile eine angemessene Theorie zu finden, muß die ausgearbeitete Theorie noch mit einem hydrostatischen Pendant gekoppelt werden. Eine von Mow und LAI für Gelenkknorpel vorgeschlagene Theorie [45] mag dazu als Ausgangspunkt dienen. Mir erscheint der Weg über die Erweiterte Thermodynamik [46] plausibel.

Alle diese ausstehenden Untersuchungen möchte ich ausdrücklich als nicht abgeschlossen wissen, hoffe aber dennoch, in dieser Arbeit für sie einen Anstoß oder sogar brauchbare Anregungen gegeben zu haben.

\subsection{Danksagung}

Allen voran danke ich meinen Eltern, Eva und Wolfgang Dathe, für die Ermöglichung des Studiums, das in dieser Arbeit seinen vorläufigen Abschluß findet. 
Ich danke dem Vorsteher der kieferorthopädischen Abteilung des Zentrums ZahnMund-Kiefer des Universitätsklinikums Göttingen, Herrn Prof. Dr. Kubein-Meesenburg, für die Bereitstellung einer Stelle, die diese Arbeit erst ermöglicht hat. Weiter danke ich meinem Betreuer Herrn Prof. Dr. Nägerl für den Anstoß zu dieser Arbeit und die vielfältigen Diskussionen, Anregungen und didaktischen Modelle, die großen Einfluß auf den Werdegang der Arbeit hatten, insbesondere auf die biomechanischen Aspekte. Meinem Korreferenten, Herrn Prof. Dr. Hegerfeldt aus dem Institut für Theoretische Physik Göttingen, gebührt der Dank, sich mit diesem doch etwas abseits des Vorlesungskanons liegenden Gebiet intensiv und konstruktiv auseinandergesetzt zu haben.

Den Mitgliedern meiner Arbeitsgruppe danke ich für ständige Diskussionsbereitschaft und viele brauchbare Vorschläge und Anregungen. Insbesondere Herrn Dipl.Phys. Stefan Spiering danke ich für die tatkräftige Unterstützung bei Programmierproblemen. Herrn Dipl.-Phys. Benedikt Becker möchte ich ausdrücklich dafür danken, mir seinen Datensatz der Messung der initialen Zahnbeweglichkeit zur Verfügung gestellt zu haben. Herrn Dipl.-Math. Gerhard Sattler danke ich für die konstante Unterstützung bei allgemeinen und spezielleren Computerproblemen in den alltäglichen Krisensituationen derer, die mit Rechnern zu tun haben. Leider hat er den Abschluß dieser Arbeit nicht mehr erlebt.

Das Weiteren danke ich Herrn Dr. rer. nat. Gunther Helms für die intensive kritische Durchsicht einer Vorabversion des ersten Teils, aus der viele konkrete Anregungen stammen, diesen prägnanter zu formulieren. Frau Dr. rer. nat. Kirsten M. Thieme danke ich ebenfalls für die kritische Durchsicht einer frühen Teilversion, die mich zum Anlegen des Symbolverzeichnisses veranlaßte. Meiner Freundin, Frau Dipl.-Designerin Stephanie Willeke, danke ich, neben der seelischen Unterstützung, besonders für Hinweise, die zu Verbesserungen des Layouts führten. Ihr und hauptsächlich meiner Mutter verdanke ich ferner die Minimierung der Anzahl der in dieser Arbeit enthaltenen Rechtschreibfehler. 


\section{Anhang A}

\section{Verwendete Symbole und einfache Zusammenhänge}

Es wurde versucht, die auftauchenden Symbole im Text selbst an der Stelle ihres ersten Auftauchens zu definieren und erläutern. Da eine Arbeit wie diese nur selten linear von Anfang bis Ende durchgelesen wird, ist eine Zusammenstellung der verwendeten Symbole mit ihrer Systematik für den Quereinsteiger unverzichtbar.

\section{A.1 Notation}

\section{A.1.1 Allgemeine Symbole}

\begin{tabular}{l|l|l} 
Symbol & Name & Typ \\
\hline \hline[] & Dimension einer phys. Größe, Mittelung & Dimension, Operator \\
{$[],,\{\}$,} & Kommutator, Antikommutator & Operator \\
\{\} & Einheit & Phys. Größe \\
$\left\{\left.\right|_{\mid} \mid \|\right.$ & Aufgespannter Unterraum & Vektoroperator \\
$\|$ & Betrag, Länge & Abb. nach $\boldsymbol{R}^{+}$ \\
$*$ & Norm & Abb. nach $\boldsymbol{R}^{+}$ \\
$\%$ & beliebige Größe & Platzhalter \\
$\delta$ & Prozent, Faulenzer & $1 / 100$, letzter Ausdruck \\
$\Delta$ & kleine Differenz & Operator \\
$\circ$ & endliche Differenz & Operator \\
$\left.*_{1}\right|_{*_{2}}$ & Skalar- oder Vektorprodukt & Vektoroperator \\
$*_{1} \| *_{2}$ & $*_{1}$ bezüglich $*_{2}$ & Spezifikation \\
$\tilde{*}$ & $*_{1}$ parallel $*_{2}$ & Vektorrelation \\
$\hat{*}$ & $*$ bezüglich elastischer Zentren & Spezifikation \\
\hline
\end{tabular}




\section{A.1.2 Sub- und Superskripte}

\section{A.1.2.1 Subskripte}

\begin{tabular}{l|l} 
Symbol & Bedeutung \\
\hline \hline$i, j, k \ldots$ & Laufindizes von Vektoren/Tensoren \\
$x, y, z$ & Kartesische Koordinaten \\
$1,2, \ldots$ & Durchnumerierung \\
$A, B, \ldots, Z$ & Zugehörigkeit zum Punkt $A, B, \ldots, Z$ \\
$x, y, F, T, \ldots$ & Zugehörigkeit zum Vektor $\boldsymbol{x}, \boldsymbol{y}, \boldsymbol{F}, \boldsymbol{T}, \ldots$ \\
$A, H$ & Arithmetisches, Harmonisches Mittel \\
$(i j)$ & Symmetrisierung in den Indizes i und j \\
{$[i j]$} & Antisymmetrisierung in den Indizes i und j \\
$q, f$ & Zugehörigkeit zu $q, f$ \\
\hline
\end{tabular}

\section{A.1.2.2 Superskripte}

Superskripte sollen besonders als Matrixoperatoren eingesetzt werden. Bei einer Hintereinanderschaltung können die Klammern weggelassen worden sein, etwa

$$
\left(\left(\boldsymbol{A}^{-1}\right)^{T}\right)^{F}=\boldsymbol{A}^{-1 T F}
$$

Die am weitesten links stehende Operation wird demnach zuerst ausgeführt.

\begin{tabular}{l|l|l|l} 
Symbol & Name & Typ & Definition \\
\hline \hline$T$ & Transposition & Operator & $\left(\boldsymbol{A}^{T}\right)_{i j}:=(\boldsymbol{A})_{j i}$ \\
$S$ & Symmetrisierung & Matrixoperator & $\boldsymbol{A}^{S}:=\frac{1}{2}\left(\boldsymbol{A}+\boldsymbol{A}^{T}\right)$ \\
$A$ & Antisymmetrisierung & Matrixoperator & $\boldsymbol{A}^{A}:=\frac{1}{2}\left(\boldsymbol{A}-\boldsymbol{A}^{T}\right)$ \\
$D$ & Dualisierung & Vektoroperator & $\left(\boldsymbol{x}^{D}\right)_{i j}:=\epsilon_{i k j}(\boldsymbol{x})_{k} \equiv \boldsymbol{\Omega}_{x}$ \\
$D$ & Dualisierung & Matrixoperator & $\left(\boldsymbol{A}^{D}\right)_{i}:=-\frac{1}{2} \epsilon_{i j k}(\boldsymbol{A})_{j k}$ \\
$F$ & vgl. A.10 & Matrixoperator & $\left(\boldsymbol{A}^{F}\right)_{i m}:=\frac{1}{2}\left(A_{l l} \delta_{i m}-A_{i m}\right)$ \\
$G$ & Gauß-Transformation & Matrixoperator & $\boldsymbol{A}^{G}:=\boldsymbol{A}^{T} \boldsymbol{A}$ \\
0 & Normierung & Operator & $\boldsymbol{A}^{0}:=\boldsymbol{A} /\|\boldsymbol{A}\|$ \\
-1 & Inversion & Matrixoperator & $\boldsymbol{A}^{-1} \boldsymbol{A}=\boldsymbol{I}$ \\
- & Pseudoinversion & Matrixoperator & $\boldsymbol{A}^{-} \boldsymbol{A}=\boldsymbol{P}$ \\
\hline
\end{tabular}

Der Superskript 0 wird auch noch für eine Vorlast als $f^{0}$ bzw. $\boldsymbol{F}^{0}$ und $\boldsymbol{T}^{0}$ verwendet. 


\section{A.1.3 Kernbuchstaben}

\section{A.1.3.1 Normaldruck}

Mit normalen Buchstaben werden Indizes, Vektor-, Matrix- und Tensorkomponenten, skalare Parameter wie Winkel, Aufpunkte und sechskomponentige Vektoren gekennzeichnet. Dabei sollen die Komponenten einer vektoriellen/tensoriellen Größe zwar denselben Kernbuchstaben wie die Größe selbst bekommen, aber dann nicht im Fettdruck dargestellt werden.

\begin{tabular}{|c|c|c|}
\hline Symbol & Name & Тур \\
\hline $\bar{A} A, B, \ldots P$ & / allgemeine Punkte & \multirow{8}{*}{ Punkte im Ortsraum } \\
\hline$O$ & Ursprung & \\
\hline$L$ & Lastsystem & \\
\hline$M$ & Meßsystem & \\
\hline$D$ & Drehmomentszentrum & \\
\hline$T$ & Translationszentrum & \\
\hline$W$ & Widerstandszentrum & \\
\hline$Z$ & elastisches Zentrum & \\
\hline$F$ & Fläche & \multirow{6}{*}{ Geometrische Größen } \\
\hline$l, L, h, H, d, D$ & Länge, Höhe, Dicke & \\
\hline$R, r, \varrho$ & Radius & \\
\hline$\theta$ & Drehwinkel & \\
\hline$I, J$ & Flächenträgheitsmomente & \\
\hline$\vartheta, \phi$ & Winkel d. Kugelkoordinaten & \\
\hline$x_{i}:=(\boldsymbol{x})_{i}$ & & \multirow{5}{*}{$\begin{array}{l}\text { Komponenten von } \\
\text { Vektoren, Matrizen und } \\
\text { Tensoren }\end{array}$} \\
\hline$A_{i j}:=(\boldsymbol{A})_{i j}$ & & \\
\hline$\delta_{i j}, \delta_{i}^{j}$ & Kronecker-Delta & \\
\hline$\varepsilon_{i j k}$ & Epsilon-Tensor & \\
\hline$x, y, z$ & Kartesische Koordinaten & \\
\hline$F, T, d, \theta$. & Betrag von $\boldsymbol{F}, \boldsymbol{T}, \boldsymbol{d}, \boldsymbol{\theta} \ldots$ & \multirow[t]{2}{*}{ Betrag eines Vektors } \\
\hline G & Gewichtskraft & \\
\hline$\rho$ & Massendichte & Skalar \\
\hline$f:=\left(\begin{array}{l}\boldsymbol{F} \\
\boldsymbol{T}\end{array}\right)$ & Last & \multirow{2}{*}{ Sechskomponentige Vektoren } \\
\hline$q:=\left(\begin{array}{l}\boldsymbol{d} \\
\boldsymbol{\theta}\end{array}\right)$ & Lageänderung & \\
\hline$\lambda$ & & \multirow{4}{*}{ Eigenwerte, reelle Parameter } \\
\hline$w_{i}$ & Eigenwerte v. $\boldsymbol{W}$ & \\
\hline$w_{i j}$ & Differenz $w_{i}-w_{j}$ & \\
\hline$u_{i}$ & Flächen- Kongruenzparameter & \\
\hline$\Theta$ & Heaviside-Funktion & Stufenfunktion \\
\hline
\end{tabular}


Aufgrund ihrer Vielfalt bekommen die Symbole der Elemente der Elastizitätstheorie eine eigene Tabelle.

\begin{tabular}{l|l} 
Symbol & Name \\
\hline \hline$\Phi$ & Elastisches Potential \\
\hline$\sigma_{i j}, \epsilon_{i j}$ & Spannungs- Dehnungstensor \\
\hline$k_{i}, k^{\perp}, k^{\|}$ & Federkonstanten, Schichtgrößen \\
\hline$K_{i}=k_{i} d(\boldsymbol{x})$ & Materialkonstanten \\
\hline$E, G$ & Elastizitätsmoduln \\
\hline$\Lambda$ & Tensor der Elastizitätsmoduln \\
\hline$\lambda, \mu$ & Lamé-Moduln \\
\hline$\sigma$ & Querdehnzahl \\
\hline$k, \kappa, K$ & Scherkoeffizient, Beiwert, Korrekturfaktor \\
\hline$U, T, S$ & Thermodyn. Funktionen
\end{tabular}

\section{A.1.3.2 Fettdruck}

Fette lateinische Großbuchstaben werden für $3 \times 3$-Matrizen verwendet. Für allgemeine 3-er Vektoren werden, wie in der Mathematik üblich, möglichst kleine fette lateinische Buchstaben verwendet. Die Konventionen in der Physik erfordern hingegen oft Großbuchstaben, die ich wenn möglich natürlich beibehalte. Griechische Symbole werden bevorzugt für Drehungen oder durch Kreuzprodukte entstandene Objekte verwendet.

\section{$3 \times 3-$ Matrizen}

\begin{tabular}{l|l|l} 
Symbol & Name & Typ \\
\hline \hline $\mathbf{0}$ & Nullmatrix & \\
\hline $\boldsymbol{I}$ & Einheitsmatrix & \\
\hline $\boldsymbol{\Omega}_{x}=\boldsymbol{x}^{D}$ & & Antisymmetrische Matrix \\
\hline $\boldsymbol{D}, \boldsymbol{S}_{1}, \boldsymbol{S}_{2}, \boldsymbol{M}$ & & Bestandteile der Flexibilitätsmatrix $\mathcal{F}$ \\
\hline $\boldsymbol{A}, \boldsymbol{B}_{1}, \boldsymbol{B}_{2}, \boldsymbol{C}$ & & Bestandteile der Steifheitsmatrix $\mathcal{S}$ \\
\hline $\boldsymbol{W}$ & Widerstandsmatrix & \\
\hline $\boldsymbol{R}$ & & Drehmatrix \\
\hline $\boldsymbol{J}$ & Trägheitstensor & \\
\hline $\boldsymbol{P} \|_{x}:=\boldsymbol{x} \boldsymbol{x}^{T} / \boldsymbol{x}^{T} \boldsymbol{x}$ & Parallelprojektor & Projektor \\
\hline $\boldsymbol{P}_{x}^{\perp}:=\boldsymbol{I}-\boldsymbol{P}_{x}^{\|}$ & Orthogonalprojektor & Projektor \\
\hline $\boldsymbol{\Psi}$ & & Federdyade \\
\hline $\boldsymbol{K}$ & Federmatrix & \\
\hline $\boldsymbol{K}, \boldsymbol{G}, \boldsymbol{N}$ & & Bestandteile der $6 \times 6$-Federmatrix $\mathcal{K}$ \\
\hline
\end{tabular}

Abweichend davon werden in der Einleitung für die Bestandteile der $3 \times 3$-Flexibilitätsmatrix die Symbole $\boldsymbol{s}_{1}, \boldsymbol{s}_{2}$ und $m$ verwendet die ihren Dimensionen als $2 \times 1$ bzw. $1 \times 1$-Matrix näherkommen. 


\section{Nichtquadratische Matrizen}

Für statistische Überlegungen werden verschiedene Messungen zu Matrizen zusammengefaßt. Für linear von der Zeit abhängende Lasten $f(t)=f_{0}+t g_{0}$ und Lagen $q(t)=q_{0}+t p_{0}$ erhält man die folgenden $6 \times n$-Matrizen.

\begin{tabular}{l|l} 
Symbol & Name \\
\hline $\boldsymbol{L}=\left(f_{1}, f_{2}, \ldots, f_{n}\right)$ & Lastmatrix \\
$\boldsymbol{Q}=\left(q_{1}, q_{2}, \ldots, q_{n}\right)$ & Lagematrix \\
$\boldsymbol{G}=\left(g_{1}, g_{2}, \ldots, g_{n}\right)$ & \\
$\boldsymbol{P}=\left(p_{1}, p_{2}, \ldots, p_{n}\right)$ & \\
\hline
\end{tabular}

Ebenfalls für statistische Zwecke kann eine $6 \times 6$ Varianz-Kovarianzmatrix $\boldsymbol{\Sigma}$ bestimmt werden.

Der Translationsanteil der Transformationsmatrix für die Lageänderungen ist eine $3 \times 6$-Matrix und wird mit $\boldsymbol{X}$ bezeichnet.

\section{Dreikomponentige Vektoren}

\begin{tabular}{|c|c|c|}
\hline Symbol & Name & Тур \\
\hline 0 & Nullvektor & \multirow{7}{*}{ Phys. Vektoren } \\
\hline$F$ & Kraft & \\
\hline$T$ & Drehmoment & \\
\hline$d$ & Translation & \\
\hline$\theta$ & Drehvektor & \\
\hline$o, f, l$ & ortsfester, freier, linienflüchtiger V. & \\
\hline $\boldsymbol{\alpha}:=\boldsymbol{\theta} /|\theta|$ & Richtung des Drehvektors & \\
\hline $\boldsymbol{w}$ & Widerstandszentrum & \multirow{3}{*}{ spez. Orte } \\
\hline$z$ & elastisches Zentrum & \\
\hline$s$ & Schwerpunkt & \\
\hline$a$ & Anfangs- / Aufpunktvektor & \multirow{6}{*}{ Geom. Vektoren } \\
\hline $\boldsymbol{r}, \boldsymbol{l}$ & Richtungsvektor & \\
\hline$n, e$ & Normalen- / Einheitsvektor & \\
\hline$\xi$ & körperfester Vektor & \\
\hline $\boldsymbol{g}=\boldsymbol{a}+\lambda \boldsymbol{r}$ & Gerade & \\
\hline$t, n, b$ & Begleitendes Dreibein & \\
\hline $\boldsymbol{x}, \boldsymbol{y}, \boldsymbol{z}$ & & beliebige Vektoren \\
\hline$v, e$ & & Eigenvektor \\
\hline$\nabla$ & Gradient, Nabla & Differentialoperator \\
\hline
\end{tabular}

Ortsvektoren werden mit einem Pfeil über Anfangs- und Endpunkt gekennzeichnet, also etwa $\overrightarrow{A B}$ für einen Ortsvektor vom Punkt $A$ nach $B$. 


\section{A.1.3.3 Kalligraphische Symbole}

Kalligraphische Symbole werden bevorzugt für $6 \times 6$-Matrizen verwendet.

\begin{tabular}{|c|c|c|}
\hline Symbol & Name & Тур \\
\hline $\mathcal{F}:=\left(\begin{array}{cc}\boldsymbol{D} & \boldsymbol{S}_{2} \\
\boldsymbol{S}_{1} & \boldsymbol{M}\end{array}\right)$ & Flexibilitätsmatrix & \multirow{14}{*}{$6 \times 6$-Matrizen } \\
\hline $\mathcal{S}:=\left(\begin{array}{cc}\boldsymbol{A} & \boldsymbol{B}_{2} \\
\boldsymbol{B}_{1} & \boldsymbol{C}\end{array}\right)$ & Steifheitsmatrix & \\
\hline $\mathcal{E}$ & elementare Steifheitsmatrix & \\
\hline $\mathcal{I}:=\left(\begin{array}{ll}\boldsymbol{I} & \mathbf{0} \\
\mathbf{0} & \boldsymbol{I}\end{array}\right)$ & Einheitsmatrix & \\
\hline $\mathcal{I}_{p}:=\left(\begin{array}{cc}\boldsymbol{I} & \mathbf{0} \\
\mathbf{0} & |\boldsymbol{p}| \boldsymbol{I}\end{array}\right)$ & & \\
\hline $\mathcal{T}:=\left(\begin{array}{ll}\boldsymbol{I} & 0 \\
\Omega & \boldsymbol{I}\end{array}\right)$ & Transformationsmatrix & \\
\hline $\mathcal{N}:=\mathcal{T}-\mathcal{I}$ & & \\
\hline $\mathcal{J}:=\left(\begin{array}{ll}\mathbf{0} & \boldsymbol{I} \\
\boldsymbol{I} & \mathbf{0}\end{array}\right)$ & & \\
\hline $\mathcal{R}:=\left(\begin{array}{cc}\boldsymbol{R} & \mathbf{0} \\
\mathbf{0} & \boldsymbol{R}\end{array}\right)$ & Drehmatrix & \\
\hline \multicolumn{2}{|l|}{$\mathcal{U}:=\mathcal{R} \mathcal{T}$} & \\
\hline $\mathcal{M}:=\left(\begin{array}{cc}m \boldsymbol{I} & -m \boldsymbol{\Omega}_{s} \\
m \boldsymbol{\Omega}_{s} & \boldsymbol{J}\end{array}\right)$ & Massenmatrix & \\
\hline $\mathcal{D}$ & Dissipationsmatrix & \\
\hline $\mathcal{V}:=\left(\begin{array}{cc}\mathbf{0} & \boldsymbol{\Omega}_{F} \\
\Omega_{F} & \boldsymbol{\Omega}_{T}\end{array}\right)$ & Vorlastmatrix & \\
\hline $\mathcal{K}:=\left(\begin{array}{cc}K & 0 \\
0 & G\end{array}\right)$ & Federmatrix & \\
\hline $\mathcal{L}$ & & Linearer \\
\hline $\mathcal{P}(t, \tau)=\exp \left(-(t-\tau) \mathcal{D}^{-1} \mathcal{S}\right)$ & Propagator & Differentialoperator \\
\hline $\mathcal{O}$ & LANDAU-Symbol & \\
\hline
\end{tabular}

\section{A.1.3.4 Serifenfrei}

Serifenfreie Symbole sind Dimensionen pysikalischer Größen vorbehalten.

\begin{tabular}{l|l} 
Symbol & Größe \\
\hline \hline 1 & reine Zahl \\
$\mathrm{T}$ & Zeit \\
$\mathrm{L}$ & Länge \\
$\mathrm{M}$ & Masse \\
\hline
\end{tabular}




\section{A.2 Einfache Zusammenhänge}

\section{A.2.1 Projektoren}

Die Länge der Projektion eines beliebigen Vektors $\boldsymbol{v}$ auf einen Einheitsvektor $\boldsymbol{n}$, für den also $\boldsymbol{n}^{T} \boldsymbol{n}=1$ gilt, ist gegeben durch das Skalarprodukt $\boldsymbol{n} \cdot \boldsymbol{v}=n_{j} v_{j}$ und der projizierte Vektor $\boldsymbol{v}^{\|}$selber durch

$$
\left(\boldsymbol{v}^{\|}\right)_{i}=\underbrace{n_{i} n_{j}}_{=:\left(\boldsymbol{P}_{n}^{\|}\right)_{i j}} v_{j}
$$

mit dem Parallelprojekto $\imath^{1}$

$$
\boldsymbol{P}_{n}^{\|}=\boldsymbol{n} \boldsymbol{n}^{T}
$$

Für nichtnormierte Vektoren $\boldsymbol{x}$ verwendet man den zum normierten Vektor gehörenden Parallelprojektor $\boldsymbol{P}_{x}^{\|}=\frac{\boldsymbol{x} \boldsymbol{x}^{T}}{\|x\|^{2}}$. Den zu $\boldsymbol{n}$ senkrechten Anteil $\boldsymbol{v}^{\perp}$ von $\boldsymbol{v}$ erhält man durch Subtraktion des parallelen Anteils

$$
\left(\boldsymbol{v}^{\perp}\right)_{i}=v_{i}-\left(\boldsymbol{v}^{\|}\right)_{i}=v_{i}-n_{i} n_{j} v_{j}=\underbrace{\left(\delta_{i j}-n_{i} n_{j}\right)}_{=:\left(\boldsymbol{P}_{n}^{\perp}\right)_{i j}} v_{j}
$$

mit dem Orthogonalprojektor

$$
\boldsymbol{P}_{n}^{\|}=\boldsymbol{I}-\boldsymbol{n n ^ { T }} .
$$

Die Zerlegung $\boldsymbol{v}=\boldsymbol{v}^{\|}+\boldsymbol{v}^{\perp}$ lautet in Projektoren ausgedrückt

$$
\boldsymbol{P}^{\|}+\boldsymbol{P}^{\perp}=\boldsymbol{I}
$$

und für die Produkte zu gleichen Einheitsvektoren gilt $\boldsymbol{P}^{\perp} \cdot \boldsymbol{P}^{\perp}=\boldsymbol{P}^{\perp}$ und $\boldsymbol{P}^{\|} \cdot \boldsymbol{P}^{\|}=$ $\boldsymbol{P}^{\|}$, kurz

$$
\boldsymbol{P}^{2}=\boldsymbol{P}
$$

und

$$
\boldsymbol{P}^{\perp} \cdot \boldsymbol{P}^{\|}=\boldsymbol{P}^{\|} \cdot \boldsymbol{P}^{\perp}=\mathbf{0}
$$

In den entsprechenden Unterräumen wirken sie also wie die Einheitsmatrix. Das Produkt ungleicher Parallelprojektoren zu den Vektoren $\boldsymbol{x}$ und $\boldsymbol{y}$ ist gegeben durch die Dyade

$$
\boldsymbol{P}_{x}^{\|} \boldsymbol{P}_{y}^{\|}=\frac{\boldsymbol{x} \boldsymbol{x}^{T}}{\|\boldsymbol{x}\|^{2}} \frac{\boldsymbol{y} \boldsymbol{y}^{T}}{\|\boldsymbol{y}\|^{2}}=\frac{(\boldsymbol{x} \cdot \boldsymbol{y})}{\boldsymbol{x}^{2} \boldsymbol{y}^{2}} \boldsymbol{x} \boldsymbol{y}^{T}
$$

\footnotetext{
${ }^{1}$ Für das dyadische Produkt $\left(\boldsymbol{a} \boldsymbol{b}^{T}\right)_{i j}:=a_{i} b_{j}$ zweier Vektoren benutzen manche Autoren die Schreibweise $\boldsymbol{a} \otimes \boldsymbol{b}$ oder einfach $\boldsymbol{a} \boldsymbol{b}$. Das skalare Produkt wird dann zu $\boldsymbol{a} \odot \boldsymbol{b}$ oder einfach $\boldsymbol{a} \cdot \boldsymbol{b}$, wobei ein Punkt für eine Verjüngung $\boldsymbol{a} \cdot \boldsymbol{b}=\delta_{i j} a_{i} b_{j}$ und zwei Punkte für eine Kontraktion über zwei Indizes usw. steht. Die Spur einer Matrix $\boldsymbol{A}$ kann so geschrieben werden als als $\boldsymbol{I}: \boldsymbol{A}$. In Zweifelsfällen wie $\boldsymbol{a} \boldsymbol{b}$ werde ich, um meine Notation eindeutig zu halten, eine explizite Transposition oder für das Skalarprodukt einen Punkt bzw. eine runde Klammer oder besser gleich Indizes benutzen.
} 
wobei der Nenner für Einheitsvektoren wegfällt. Entsprechend können die symmetrischen bzw. antisymmetrischen Anteile auf die von $(\boldsymbol{x} \cdot \boldsymbol{y}) \boldsymbol{x} \boldsymbol{y}^{T}$ zurückgeführt werden.

Für gemischte Orthogonal- und Parallelprojektoren erhält man mit $\boldsymbol{P}^{\perp}=\boldsymbol{I}-\boldsymbol{P}^{\|}$, etwa

$$
\boldsymbol{P}_{x}^{\|} \boldsymbol{P}_{y}^{\perp}+\boldsymbol{P}_{y}^{\|} \boldsymbol{P}_{x}^{\perp}=\boldsymbol{P}_{x}^{\|}+\boldsymbol{P}_{y}^{\|}-\left(\boldsymbol{P}_{x}^{\|} \boldsymbol{P}_{y}^{\|}+\boldsymbol{P}_{y}^{\|} \boldsymbol{P}_{x}^{\|}\right)=\boldsymbol{P}_{x}^{\perp} \boldsymbol{P}_{y}^{\|}+\boldsymbol{P}_{y}^{\perp} \boldsymbol{P}_{x}^{\|}
$$

Gerade bei geometrischen Problemen ist dieser Formalismus nützlich, da sich viele Größen sofort hinschreiben lassen.

\section{A.2.2 Der $\varepsilon$-Tensor, Kreuzprodukte und antisymmetrische Matrizen}

Der $\varepsilon$-Tensor ist der vollständig antisymmetrische Tensor dritter Stufe. Er ist definiert durch

$$
\varepsilon_{i j k}:=\left\{\begin{array}{cl}
+1 & (i j k)=((123),(231),(312)) \\
-1 & (i j k)=((132),(321),(213)) \\
0 & \text { sonst }
\end{array}\right.
$$

d. h. der Wert von $\varepsilon_{i j k}$ ist gleich dem Signum der Permutation $\Pi(123) \mapsto(i j k)$. Bei Verwendung eines linkshändigen Koordinatensystems kehrt sich das Vorzeichen der Einträge um. Durch Multiplikation mit der (Inversen der) Wurzel der Determinante des metrischen Tensors entsteht ein kovariantes (kontravariantes) Pseudotensorfeld, der LEVI-CivitA-Pseudotensor. Die analoge Konstruktion ist somit in $n$ Dimensionen möglich.

Damit läßt sich das Kreuzprodukt schreiben als

$$
(\boldsymbol{x} \times \boldsymbol{y})_{i}=\varepsilon_{i j k} x_{j} y_{k},
$$

wobei sich in linkshändigen Koordinatensystemen das Vorzeichen wieder umkehrt. Da axiale Vektoren in den meisten Formeln zusammen mit einem Kreuzprodukt auftauchen, läßt sich dieser Vektor gleich besser als antisymmetrische Matrix beschreiben über

$$
(\boldsymbol{x} \times \boldsymbol{y})_{i}=\underbrace{\varepsilon_{i k j} x_{k}}_{=:\left(\boldsymbol{\Omega}_{x}\right)_{i j}} y_{j}=\left(\boldsymbol{\Omega}_{x} \boldsymbol{y}\right)_{i}
$$

Andere Autoren (z. B. [58]) benutzen für diese Isomorphie zwischen Vektoren und antisymmetrischen Matrizen die Schreibweise $\boldsymbol{x}^{\times}$. 


\section{A.2.2.1 Produkte antisymmetrischer Matrizen}

Über

$$
\varepsilon_{i j k} \varepsilon_{k l m}=\left|\begin{array}{cc}
\delta_{i}^{l} & \delta_{j}^{l} \\
\delta_{i}^{m} & \delta_{j}^{m}
\end{array}\right|=\delta_{i}^{l} \delta_{j}^{m}-\delta_{j}^{l} \delta_{i}^{m}
$$

erhält man für das Quadrat einer antisymmetrischen Matrix

$$
\begin{aligned}
\left(\boldsymbol{\Omega}_{x}^{2}\right)_{i j} & =\varepsilon_{i k l} x_{k} \varepsilon_{l m j} x_{m}=\left|\begin{array}{cc}
\delta_{i}^{m} & \delta_{k}^{m} \\
\delta_{i}^{j} & \delta_{k}^{j}
\end{array}\right| x_{k} x_{m}=x_{i} x_{j}-x_{k} x_{k} \delta_{i j} \\
& =-\boldsymbol{x}^{2}\left(\boldsymbol{P}_{x}^{\perp}\right)_{i j},
\end{aligned}
$$

also im wesentlichen einen Orthogonalprojektor.

Im zu den Vektoren isomorphen Raum der antisymmetrischen Matrizen wird das Kreuzprodukt durch den Kommutator der isomorphen Matrizen abgebildet. Aus dem Produkt zweier zu ungleichen Vektoren gehörenden antisymmetrischen Matrizen

$$
\left(\Omega_{x} \Omega_{y}\right)_{i j}=\varepsilon_{i k l} x_{k} \varepsilon_{l m j} y_{m}=\left|\begin{array}{cc}
\delta_{i}^{m} & \delta_{k}^{m} \\
\delta_{i}^{j} & \delta_{k}^{j}
\end{array}\right| x_{k} y_{m}=x_{j} y_{i}-x_{k} y_{k} \delta_{i j}
$$

bzw.

$$
\boldsymbol{\Omega}_{x} \boldsymbol{\Omega}_{y}=\boldsymbol{y} \boldsymbol{x}^{T}-(\boldsymbol{x} \cdot \boldsymbol{y}) \boldsymbol{I}
$$

und der zu ihrem Kreuzprodukt gehörenden antisymmetrischen Matrix

$$
\left(\boldsymbol{\Omega}_{x \times y}\right)_{i j}=\varepsilon_{i k j}(\boldsymbol{x} \times \boldsymbol{y})_{k}=\varepsilon_{i k j} \varepsilon_{k l m} x_{l} y_{m}=\left|\begin{array}{cc}
\delta_{j}^{l} & \delta_{i}^{l} \\
\delta_{j}^{m} & \delta_{i}^{m}
\end{array}\right| x_{l} y_{m}=x_{j} y_{i}-x_{i} y_{j}
$$

bzw.

$$
\boldsymbol{\Omega}_{x \times y}=\boldsymbol{y} \boldsymbol{x}^{T}-\boldsymbol{x} \boldsymbol{y}^{T}
$$

folgt nämlich

$$
\boldsymbol{\Omega}_{x \times y}=\boldsymbol{\Omega}_{x} \boldsymbol{\Omega}_{y}-\boldsymbol{\Omega}_{y} \boldsymbol{\Omega}_{x}=2\left(\boldsymbol{\Omega}_{x} \boldsymbol{\Omega}_{y}\right)^{A}=\left[\boldsymbol{\Omega}_{x}, \boldsymbol{\Omega}_{y}\right]
$$

wobei sich die Terme mit der Einheitsmatrix $\delta_{i j}$ gerade wegheben. Ebenso berechnet man den Antikommutator

$$
\left\{\boldsymbol{\Omega}_{x}, \boldsymbol{\Omega}_{y}\right\}=2\left(\boldsymbol{\Omega}_{x} \boldsymbol{\Omega}_{y}\right)^{S}=\boldsymbol{\Omega}_{x} \boldsymbol{\Omega}_{y}+\boldsymbol{\Omega}_{y} \boldsymbol{\Omega}_{x}=\boldsymbol{y} \boldsymbol{x}^{T}+\boldsymbol{x} \boldsymbol{y}^{T}-2(\boldsymbol{x} \cdot \boldsymbol{y}) \boldsymbol{I}
$$

mit der Eigenschaft, daß

$$
\left(\boldsymbol{\Omega}_{x} \boldsymbol{\Omega}_{y}\right)^{S} \boldsymbol{x}=\frac{1}{2} \boldsymbol{y}^{2} \boldsymbol{P}_{y}^{\perp} \boldsymbol{x} \quad \text { und } \quad\left(\boldsymbol{\Omega}_{x} \boldsymbol{\Omega}_{y}\right)^{S} \boldsymbol{y}=\frac{1}{2} \boldsymbol{x}^{2} \boldsymbol{P}_{x}^{\perp} \boldsymbol{y} .
$$




\section{A.2.2.2 Die Dualisierung}

Die Dualisierung ist eine nur im $\mathbb{R}^{3}$ mögliche eindeutige Zuordnung von Vektoren zu antisymmetrischen Matrizen. Sie ist so gemacht, daß aus

$$
\left(\boldsymbol{x}^{D}\right)_{i j}:=\varepsilon_{i k j}(\boldsymbol{x})_{k}
$$

und

$$
\left(\boldsymbol{A}^{D}\right)_{i}:=-\frac{1}{2} \varepsilon_{i j k}(\boldsymbol{A})_{j k}
$$

folgt, daß für die zweimalige Dualisierung

$$
\left(\boldsymbol{x}^{D}\right)_{i}^{D}=-\frac{1}{2} \varepsilon_{i j k} \varepsilon_{j l k} x_{l}=-\frac{1}{2}\left(\delta_{i j} \delta_{j l}-\delta_{j j} \delta_{i l}\right) x_{l}=(\boldsymbol{x})_{i}
$$

und

$$
\left(\boldsymbol{A}^{D}\right)^{D}=\boldsymbol{A}^{A}
$$

gilt, also daß zweimaliges Dualisieren einen Vektor unverändert läßt. Wegen $\boldsymbol{x}^{D} \equiv$ $\boldsymbol{\Omega}_{x}$ folgt für eine duale Dyade $(\boldsymbol{x y})^{D}=-\frac{1}{2} \boldsymbol{x}^{D} \boldsymbol{y}=-\frac{1}{2} \boldsymbol{x} \times \boldsymbol{y}$. Da nur der antisymmetrische Anteil einer Matrix in die Dualisierung eingeht, also $\boldsymbol{A}^{D}=\left(\boldsymbol{A}^{A}\right)^{D}$, folgt

$$
\left(\boldsymbol{A}^{T}\right)^{D}=\left(-\boldsymbol{A}^{A}\right)^{D}=-\boldsymbol{A}^{D}
$$

Die Dualisierung ist, bis auf ihr Vorzeichen, genau der HoDGEoperator des $\mathbb{R}^{3}$.

Oft sind Ausdrücke der Gestalt $(\boldsymbol{\Omega} \boldsymbol{A})^{D}=\left(\boldsymbol{x}^{D} \boldsymbol{A}\right)^{D}$ zu untersuchen. Es ergibt sich

$$
(\boldsymbol{\Omega} \boldsymbol{A})_{i}^{D}=-\frac{1}{2} \varepsilon_{i j k} \varepsilon_{j m l} x_{m} A_{l k}=\underbrace{\frac{1}{2}\left(A_{l l} \delta_{i m}-A_{i m}\right)}_{=:\left(\boldsymbol{A}^{F}\right)_{i m}} x_{i}
$$

mit der Abkürzung

$$
\boldsymbol{A}^{F}=\frac{1}{2}((\operatorname{Sp} \boldsymbol{A}) \boldsymbol{I}-\boldsymbol{A})
$$

Die wichtigsten Eigenschaften dieser neuen Matrixoperation lauten $\boldsymbol{I}^{F}=\boldsymbol{I}$ und $\left(\boldsymbol{A}^{F}\right)^{T}=\left(\boldsymbol{A}^{T}\right)^{F}$. Daraus folgt für $\boldsymbol{\Omega}=\boldsymbol{x}^{D}$ sofort

$$
(\boldsymbol{A} \boldsymbol{\Omega})^{D}=-\left((\boldsymbol{A} \boldsymbol{\Omega})^{T}\right)^{D}=-\left(\boldsymbol{\Omega}^{T} \boldsymbol{A}^{T}\right)^{D}=\left(\boldsymbol{\Omega} \boldsymbol{A}^{T}\right)^{D}=\left(\boldsymbol{A}^{T}\right)^{F} \boldsymbol{x}
$$

Auf einen Parallelprojektor angewandt ergibt sich

$$
\boldsymbol{P}^{\|^{F}}=\frac{1}{2}(\underbrace{\left(\operatorname{Sp}^{\|}\right)}_{=1} \boldsymbol{I}-\boldsymbol{P}^{\|})=\frac{1}{2} \boldsymbol{P}^{\perp},
$$


und die Transformierte eines Orthogonalprojektors ist

$$
\boldsymbol{P}^{\perp F}=\frac{1}{2}(\underbrace{\left(\mathrm{Sp} \boldsymbol{P}^{\perp}\right)}_{=2} \boldsymbol{I}-\boldsymbol{P}^{\perp})=\frac{1}{2}\left(\boldsymbol{I}+\boldsymbol{P}^{\|}\right)=\boldsymbol{I}-\frac{1}{2} \boldsymbol{P}^{\perp} .
$$

Aus der Spurfreiheit einer antisymmetrischen Matrix folgt $\Omega^{F}=-\Omega / 2$ und daher gilt für das Produkt zweier antisymmetrischer Matrizen

$$
\left(\boldsymbol{\Omega}_{x} \boldsymbol{\Omega}\right)^{D}=\boldsymbol{\Omega}^{F} \boldsymbol{x}=-\frac{1}{2} \boldsymbol{\Omega} \boldsymbol{x}
$$

Interessant ist noch eine Householdermatrix $\boldsymbol{I}-2 \boldsymbol{P}_{n}^{\|}$. Im von $\boldsymbol{n}$ aufgespannten Unterraum $\langle\boldsymbol{n}\rangle$ ist sie genau eine Punktspiegelung, im $\mathbb{R}^{3}$ also eine Spiegelung an der Ebene $\langle\boldsymbol{n}\rangle^{\perp}$. Ihre Transformierte lautet

$$
\left(\boldsymbol{I}-2 \boldsymbol{P}^{\|}\right)^{F}=\frac{1}{2}(\underbrace{\operatorname{Sp}\left(\boldsymbol{I}-2 \boldsymbol{P}^{\|}\right)}_{=1} \boldsymbol{I}-\boldsymbol{P}^{\perp})=\frac{1}{2} \boldsymbol{P}^{\|} .
$$

\section{A.2.2.3 Projektoren von Kreuzprodukten}

Die Berechnung des zu einem Kreuzprodukt gehörenden Parallelprojektors erfordert die Berechnung eines nichtkontrahierten Produktes zweier $\varepsilon$-Tensoren. Für einen Orthogonalprojektor hingegen vereinfacht sich die Rechnung etwas wegen

$$
\boldsymbol{P}_{x \times y}^{\perp}=-\frac{1}{(\boldsymbol{x} \times \boldsymbol{y})^{2}} \boldsymbol{\Omega}_{x \times y}^{2}
$$

Es ergibt sich mit $|\boldsymbol{x} \times \boldsymbol{y}|^{2}=\boldsymbol{x}^{2} \boldsymbol{y}^{2}-(\boldsymbol{x} \boldsymbol{y})^{2}$

$$
\begin{aligned}
\boldsymbol{P}_{x \times y}^{\perp} & =-\frac{1}{|\boldsymbol{x} \times \boldsymbol{y}|^{2}} \boldsymbol{\Omega}_{x \times y}^{2} \\
& =-\frac{1}{|\boldsymbol{x} \times \boldsymbol{y}|^{2}}\left(\boldsymbol{y} \boldsymbol{x}^{T}-\boldsymbol{x} \boldsymbol{y}^{T}\right)^{2} \\
& =\frac{1}{|\boldsymbol{x} \times \boldsymbol{y}|^{2}}\left(\boldsymbol{x}^{2} \boldsymbol{y} \boldsymbol{y}^{T}+\boldsymbol{y}^{2} \boldsymbol{x} \boldsymbol{x}^{T}-\boldsymbol{x} \boldsymbol{y}\left(\boldsymbol{x} \boldsymbol{y}^{T}+\boldsymbol{y} \boldsymbol{x}^{T}\right)\right) \\
& =\frac{1}{|\boldsymbol{x} \times \boldsymbol{y}|^{2}}\left(\boldsymbol{x}^{2} \boldsymbol{y}^{2}\left(\boldsymbol{P}_{x}^{\|}+\boldsymbol{P}_{y}^{\|}\right)-\boldsymbol{x}^{2} \boldsymbol{y}^{2}\left(\boldsymbol{P}_{x}^{\|} \boldsymbol{P}_{y}^{\|}+\boldsymbol{P}_{y}^{\|} \boldsymbol{P}_{x}^{\|}\right)\right) \\
& =\frac{\boldsymbol{x}^{2} \boldsymbol{y}^{2}}{|\boldsymbol{x} \times \boldsymbol{y}|^{2}}\left(\boldsymbol{P}_{x}^{\|}+\boldsymbol{P}_{y}^{\|}\right)-\left(\boldsymbol{P}_{x}^{\|} \boldsymbol{P}_{y}^{\|}+\boldsymbol{P}_{y}^{\|} \boldsymbol{P}_{x}^{\|}\right) \\
& =\frac{\boldsymbol{x}^{2} \boldsymbol{y}^{2}}{|\boldsymbol{x} \times \boldsymbol{y}|^{2}}\left(\boldsymbol{P}_{x}^{\|} \boldsymbol{P}_{y}^{\perp}+\boldsymbol{P}_{y}^{\|} \boldsymbol{P}_{x}^{\perp}\right),
\end{aligned}
$$

d. h. der Orthogonalprojektor des Kreuzproduktes zweier Vektoren läßt sich durch die Projektoren der Einzelvektoren ausdrücken. Daher lautet die Basis der Darstellungen von zwei Vektoren abhängenden symmetrischen Matrizen $\boldsymbol{I}, \boldsymbol{P}_{x}^{\|}, \boldsymbol{P}_{y}^{\|}$, und $\left(\boldsymbol{P}_{x}^{\|} \boldsymbol{P}_{y}^{\|}\right)^{S}$. 
Für geometrische Probleme ist eine andere Schreibweise günstiger. Aus

$$
\begin{aligned}
\boldsymbol{P}_{x \times y}^{\perp} & =\frac{\boldsymbol{x}^{2} \boldsymbol{y}^{2}}{|\boldsymbol{x} \times \boldsymbol{y}|^{2}}\left(\boldsymbol{P}_{x}^{\|}+\boldsymbol{P}_{y}^{\|}\right)-\frac{(\boldsymbol{x} \boldsymbol{y})}{|\boldsymbol{x} \times \boldsymbol{y}|^{2}}\left(\boldsymbol{x} \boldsymbol{y}^{T}+\boldsymbol{y} \boldsymbol{x}^{T}\right) \\
& =\frac{|\boldsymbol{x} \times \boldsymbol{y}|^{2}+(\boldsymbol{x y})^{2}}{|\boldsymbol{x} \times \boldsymbol{y}|^{2}}\left(\boldsymbol{P}_{x}^{\|}+\boldsymbol{P}_{y}^{\|}\right)-\frac{(\boldsymbol{x} \boldsymbol{y})}{|\boldsymbol{x} \times \boldsymbol{y}|^{2}}\left(\boldsymbol{x} \boldsymbol{y}^{T}+\boldsymbol{y} \boldsymbol{x}^{T}\right) \\
& =\boldsymbol{P}_{x}^{\|}+\boldsymbol{P}_{y}^{\|}+\frac{(\boldsymbol{x y})}{|\boldsymbol{x} \times \boldsymbol{y}|^{2}}\left((\boldsymbol{x} \boldsymbol{y})\left(\boldsymbol{P}_{x}^{\|}+\boldsymbol{P}_{y}^{\|}\right)-\left(\boldsymbol{x} \boldsymbol{y}^{T}+\boldsymbol{y} \boldsymbol{x}^{T}\right)\right)
\end{aligned}
$$

folgt insbesondere für aufeinander senkrechte Vektoren $\boldsymbol{x} \boldsymbol{y}=0$ und damit

$$
\boldsymbol{P}_{x \times y}^{\perp}=\boldsymbol{P}_{x}^{\|}+\boldsymbol{P}_{y}^{\|}
$$

Diese Formel ist dann genau die Zerlegung der Einheitsmatrix durch drei paarweise orthogonale Einheitsvektoren $\boldsymbol{x}, \boldsymbol{y}$ und $\boldsymbol{z}=\boldsymbol{x} \times \boldsymbol{y}$ in $\boldsymbol{I}=\boldsymbol{P}_{x}^{\|}+\boldsymbol{P}_{y}^{\|}+\boldsymbol{P}_{z}^{\|}$.

\section{A.2.2.4 Kreuzprodukte und Drehungen}

Es soll nun das Transformationsverhalten der antisymmetrischen Matrizen unter Drehungen studiert werden. Eine Drehmatrix $\boldsymbol{R}$ ist zusammengesetzt aus Spalten paarweise orthogonaler Eigenvektoren $\boldsymbol{e}_{i}$ und läßt sich daher schreiben als

$$
(\boldsymbol{R})_{i j}=\left(\boldsymbol{e}_{i}\right)_{j}
$$

insbesondere ist $(\boldsymbol{R} \boldsymbol{x})_{i}=\boldsymbol{e}_{i} \cdot \boldsymbol{x}$. Die Komponenten der gedrehten Matrix lauten

$$
\begin{aligned}
\left(\boldsymbol{R} \boldsymbol{\Omega}_{x} \boldsymbol{R}^{T}\right)_{i j} & =R_{i k} \varepsilon_{k l m} x_{l} R_{m j}^{T} \\
& =\left(\boldsymbol{e}_{i}\right)_{k} \varepsilon_{k l m} x_{l}\left(\boldsymbol{e}_{j}\right)_{m} \\
& =\left(\boldsymbol{e}_{j} \times \boldsymbol{e}_{i}\right)_{l} x_{l} \\
& =\left(\boldsymbol{e}_{j} \times \boldsymbol{e}_{i}\right) \cdot \boldsymbol{x} \\
& =\left(\begin{array}{ccc}
0 & -\boldsymbol{e}_{3} \boldsymbol{x} & \boldsymbol{e}_{2} \boldsymbol{x} \\
\boldsymbol{e}_{3} \boldsymbol{x} & 0 & -\boldsymbol{e}_{1} \boldsymbol{x} \\
-\boldsymbol{e}_{2} \boldsymbol{x} & \boldsymbol{e}_{1} \boldsymbol{x} & 0
\end{array}\right)_{i j} \\
& =\left(\boldsymbol{\Omega}_{R x}\right)_{i j},
\end{aligned}
$$

also kurz

$$
\boldsymbol{R} \boldsymbol{\Omega}_{x} \boldsymbol{R}^{T}=\boldsymbol{\Omega}_{R x}
$$

Daraus folgt sofort die Kovarianz des Kreuzproduktes unter Drehungen, denn es gilt

$$
\begin{aligned}
& (\boldsymbol{R} \boldsymbol{x}) \times(\boldsymbol{R} \boldsymbol{y})=\boldsymbol{\Omega}_{R x} \boldsymbol{R} \boldsymbol{y}=\boldsymbol{R} \boldsymbol{\Omega}_{x} \boldsymbol{R}^{T} \boldsymbol{R} \boldsymbol{y}=\boldsymbol{R} \boldsymbol{\Omega}_{x} \boldsymbol{y} \\
= & \boldsymbol{R}(\boldsymbol{x} \times \boldsymbol{y}) .
\end{aligned}
$$




\section{A.2.2.5 Determinante, Adjungierte und Inverse}

Die vollständige Antisymmetrie des $\varepsilon$-Tensors ermöglicht es weiterhin, die Determinante, Adjungierte und mit diesen sogar die Inverse einer $3 \times 3$-Matrix auszudrücken. Die Determinante lautet wegen der vollständigen Antisymmetrie und Multilinearität der Determinante in jedem Index

$$
\operatorname{det} \boldsymbol{A}=\frac{1}{6} \varepsilon_{i j k} \varepsilon_{l m n} A_{i l} A_{j m} A_{k n}
$$

nachzuprüfen mit der SARRUSschen Regel bzw. mit

$$
\varepsilon_{i j k} \varepsilon_{l m n}=\left|\begin{array}{ccc}
\delta_{i l} & \delta_{j l} & \delta_{k l} \\
\delta_{i m} & \delta_{j m} & \delta_{k m} \\
\delta_{i n} & \delta_{j n} & \delta_{k n}
\end{array}\right|
$$

Die Werte der algebraischen Komplemente ergeben sich aus den Werten der entsprechenden Unterdeterminante, in der die betreffende Zeile und Spalte weggestrichen wurden, versehen mit dem Vorzeichen nach der Schachbrettregel. Die adjungierte Matrix ist die Matrix dieser algebraischen Komplemente ${ }^{2}$. Für $3 \times 3$-Matrizen lautet sie

$$
\left(\boldsymbol{A}^{\mathrm{adj}}\right)_{i j}=\frac{1}{2} \varepsilon_{i k l} \varepsilon_{j m n} A_{k m} A_{l n}
$$

und die Inverse erhält man schließlich über

$$
\boldsymbol{A}^{-1}=\frac{1}{\operatorname{det} \boldsymbol{A}}\left(\boldsymbol{A}^{T}\right)^{\operatorname{adj}}
$$

\section{A.2.3 Die Güte einer Matrix}

Es ist oft notwendig zu beurteilen, wie symmetrisch eine Matrix ist, z. B. bei den Transformationsmatrizen. Die Güte einer Matrix soll ein solches Maß für ihre Symmetrie sein. Die von mir verwendete Funktion lautet

$$
G(\boldsymbol{M}):=\frac{2\left\|\boldsymbol{M}^{S}\right\|^{2}-3\left\|\boldsymbol{M}^{A}\right\|^{2}}{2\left\|\boldsymbol{M}^{S}\right\|^{2}+3\left\|\boldsymbol{M}^{A}\right\|^{2}} .
$$

Für rein antisymmetrische Matrizen ist $G=-1$; für rein symmetrische ist $G=$ +1 . Sind alle Einträge betragsmäßig gleich, so berücksichtigen die Vorfaktoren die unterschiedliche Anzahl der von Null verschiedenen Einträge: 9 für symmetrische und 6 für antisymmetrische Matrizen. Das Ergebnis ist dann $G=0$. Die Definition über die Normquadrate ist günstig für symbolische Rechnungen (also auch für Computeralgebra), da keine Wurzeln auftauchen.

\footnotetext{
${ }^{2}$ Einige Autoren, etwa ([7] $\left.\S 3\right)$, benutzen die Bezeichnung adjungierte Matrix für die transponierte Matrix der algebraischen Komplemente.
} 
In $n$ Dimensionen hat eine symmetrische Matrix $n^{2}$ Einträge und eine antisymmetrische, da sie auf der Hauptdiagonale keine Einträge hat, nur $n(n-1)$. Nach Kürzen von $n$ lautet die allgemeine Gütefunktion mit den obigen Eigenschaften demnach

$$
G(\boldsymbol{M}, n):=\frac{(n-1)\left\|\boldsymbol{M}^{S}\right\|^{2}-n\left\|\boldsymbol{M}^{A}\right\|^{2}}{(n-1)\left\|\boldsymbol{M}^{S}\right\|^{2}+n\left\|\boldsymbol{M}^{A}\right\|^{2}}
$$

\section{A.3 Rechnungen mit Mathematica}

Um mit dem Computeralgebra-System Mathematica die Berechnungen der Steifheitsmatrizen der Beispiele rechnen zu können, benötigt man Routinen und Prozeduren, die ich im Folgenden vorstellen möchte.

\section{A.3.1 Prozeduren zur Vektor- und Tensorrechnung und für Blockmatrizen}

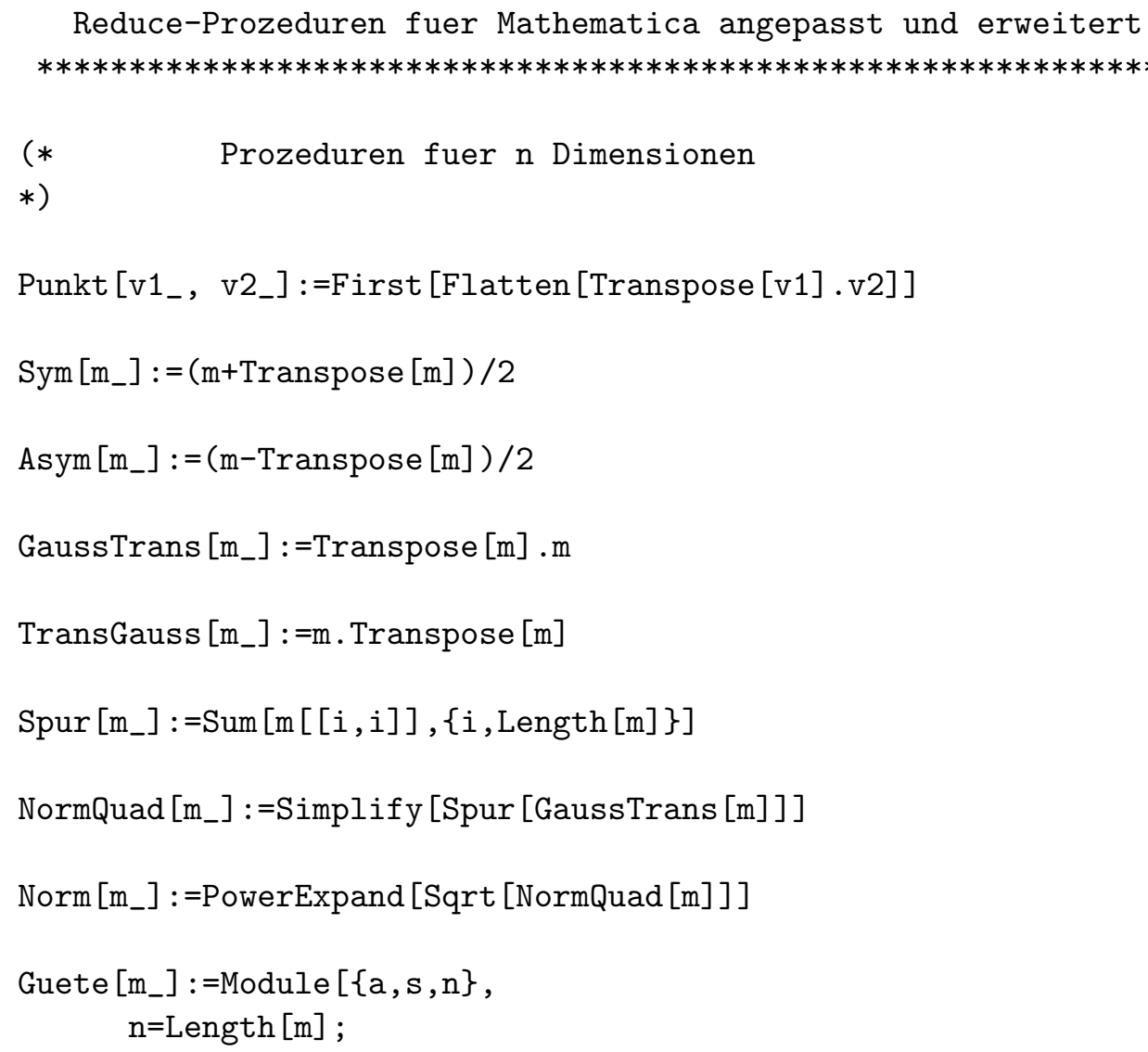




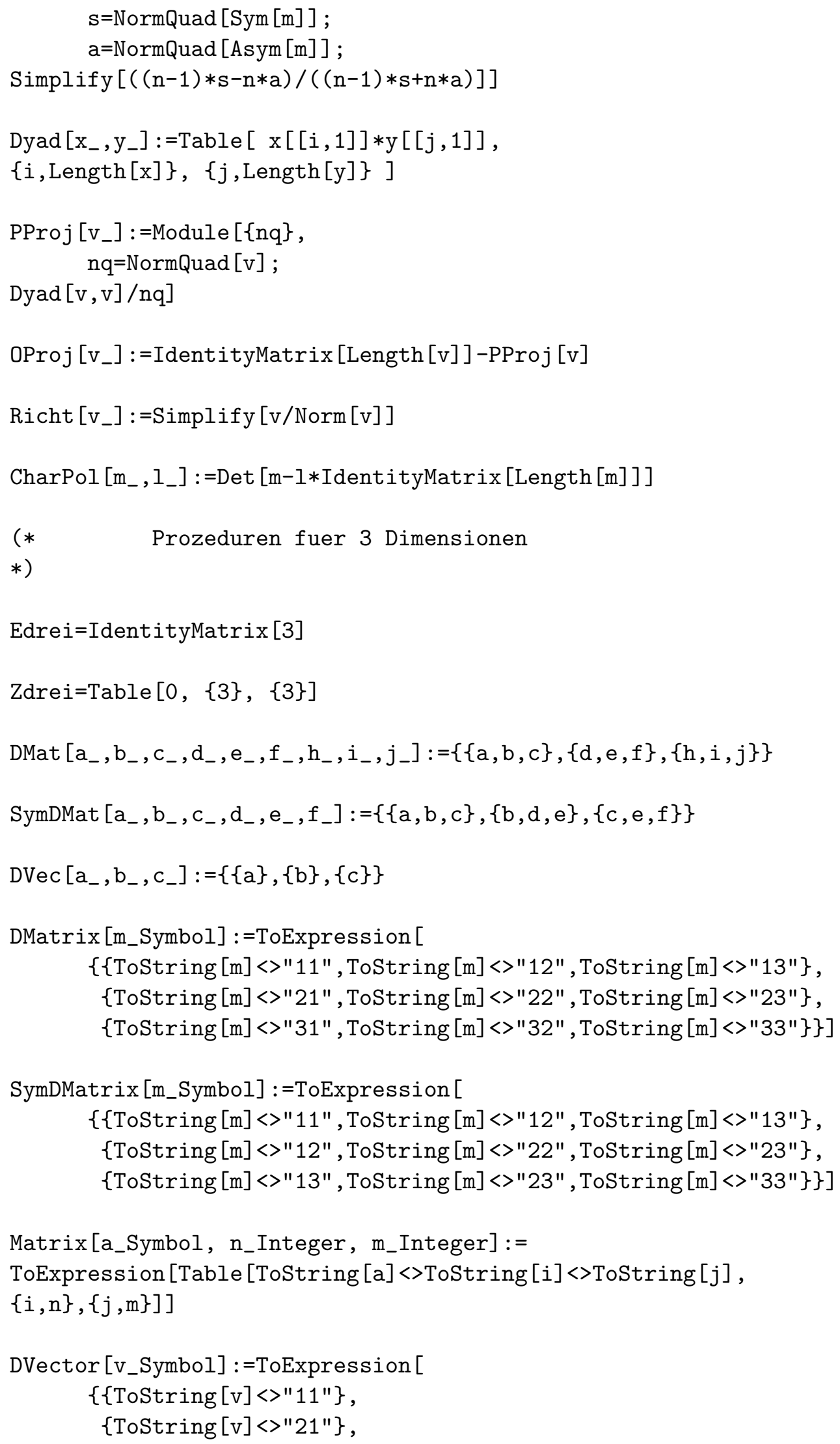




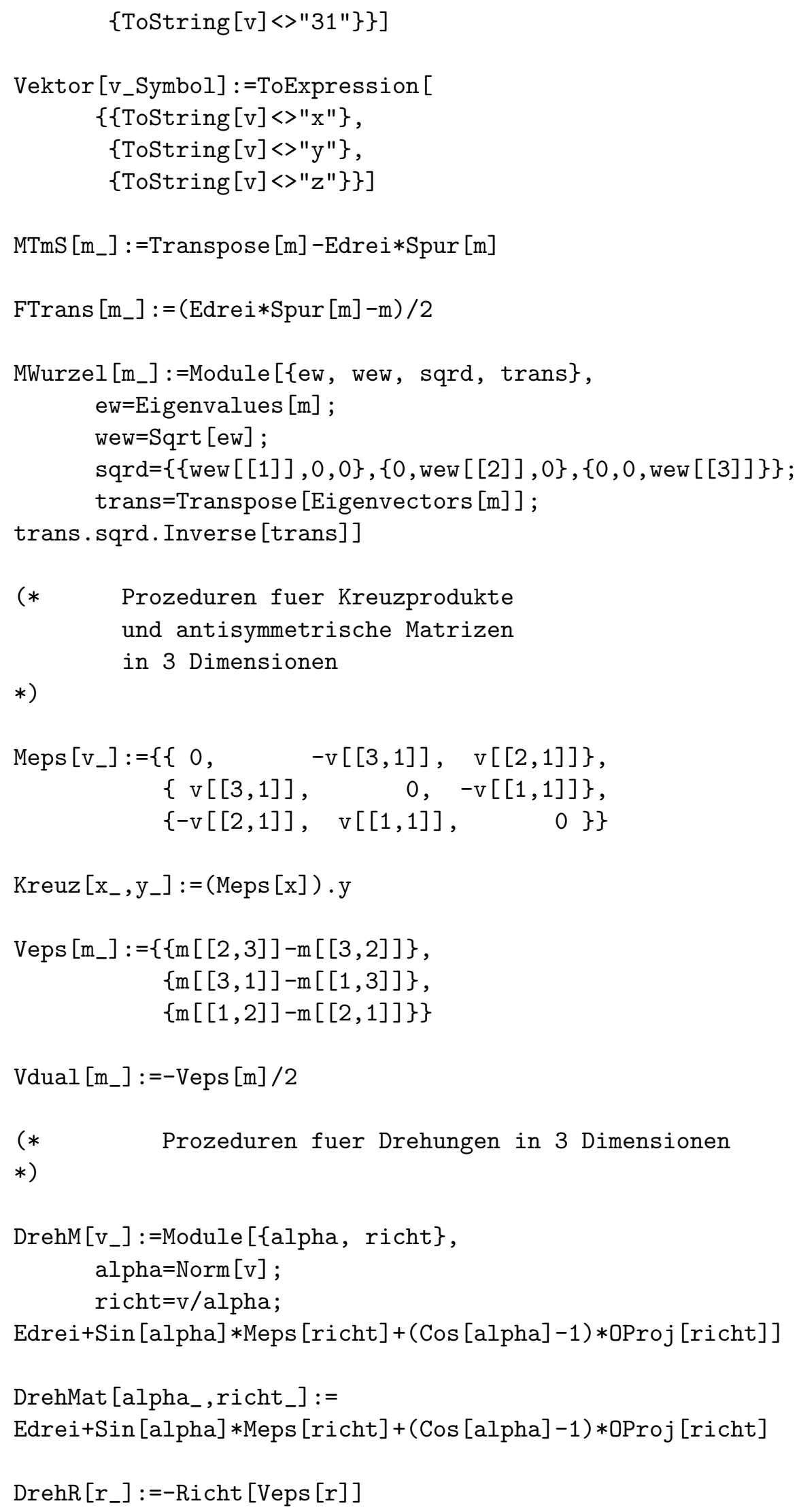




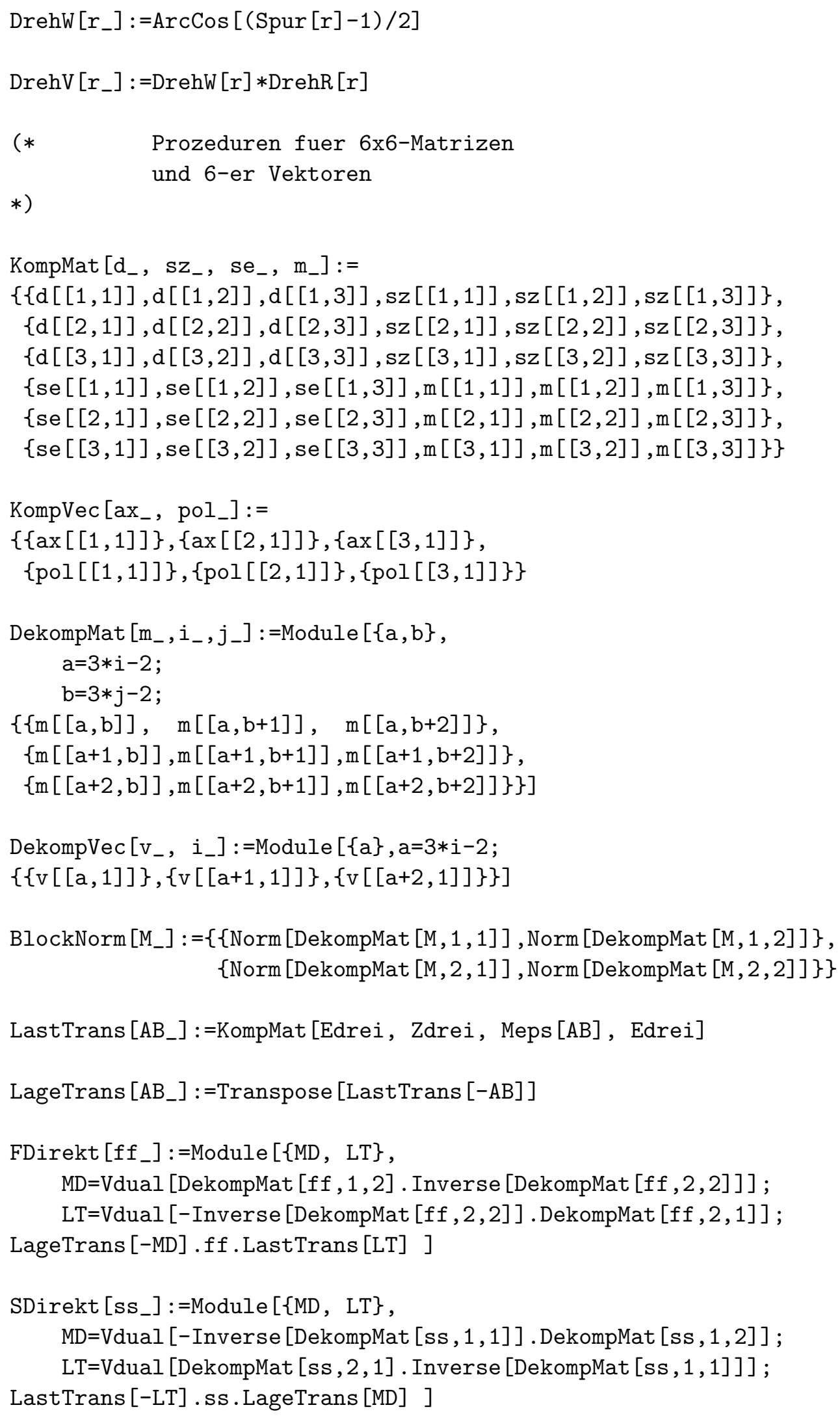


PermRot $\left[R_{-}\right]:=$Module $[\{\mathrm{DD}, \mathrm{DW}\}$,

$\mathrm{DD}=\{\mathrm{R}$, DiagonalMatrix $[1,-1,-1] \cdot \mathrm{R}$,

DiagonalMatrix $[-1,1,-1] . R$,

DiagonalMatrix $[-1,-1,1] . R\}$;

$\mathrm{DW}=\mathrm{Table}[\operatorname{DrehW}[\mathrm{DD}[\mathrm{i}]]],\{i, 4\}]$;

$\mathrm{DD}[[\mathrm{Position}[\mathrm{DW}, \operatorname{Min}[\mathrm{DW}][[1,1]]]]]$

FRealForm [f_] : =Module [\{m, SVD , RotMess, RotLast $\}$,

$\mathrm{m}=$ DekompMat $[\mathrm{f}, 2,2]$;

SVD=SingularValues [m];

RotMess=Part [SVD , 1] ;

RotMess $*=$ Det [RotMess];

RotMess $=$ PermRot [RotMess $]$;

RotLast $=$ Part [SVD , 3] ;

RotLast $*=$ Det [RotLast] ;

RotLast=Transpose [PermRot [RotLast] ] ;

Chop [KompMat [RotMess , Zdrei , Zdrei , RotMess] .

FDirekt [f] .KompMat [RotLast,Zdrei,Zdrei, RotLast]] ]

BlockSMult $\left[\mathrm{m}_{-}, \mathrm{d}_{-}\right]:=$

$\{\{\mathrm{m}[[1,1]] * \mathrm{~d}[[1,1]], \mathrm{m}[[1,2]] * \mathrm{~d}[[1,1]], \mathrm{m}[[1,3]] * \mathrm{~d}[[1,1]]$, $\mathrm{m}[[1,4]] * \mathrm{~d}[[1,2]], \mathrm{m}[[1,5]] * \mathrm{~d}[[1,2]], \mathrm{m}[[1,6]] * \mathrm{~d}[[1,2]]\}$,

$\{\mathrm{m}[[2,1]] * \mathrm{~d}[[1,1]], \mathrm{m}[[2,2]] * \mathrm{~d}[[1,1]], \mathrm{m}[[2,3]] * \mathrm{~d}[[1,1]]$, $\mathrm{m}[[2,4]] * \mathrm{~d}[[1,2]], \mathrm{m}[[2,5]] * \mathrm{~d}[[1,2]], \mathrm{m}[[2,6]] * \mathrm{~d}[[1,2]]\}$,

$\{\mathrm{m}[[3,1]] * \mathrm{~d}[[1,1]], \mathrm{m}[[3,2]] * \mathrm{~d}[[1,1]], \mathrm{m}[[3,3]] * \mathrm{~d}[[1,1]]$, $\mathrm{m}[[3,4]] * \mathrm{~d}[[1,2]], \mathrm{m}[[3,5]] * \mathrm{~d}[[1,2]], \mathrm{m}[[3,6]] * \mathrm{~d}[[1,2]]\}$,

$\{\mathrm{m}[[4,1]] * \mathrm{~d}[[2,1]], \mathrm{m}[[4,2]] * \mathrm{~d}[[2,1]], \mathrm{m}[[4,3]] * \mathrm{~d}[[2,1]]$, $\mathrm{m}[[4,4]] * \mathrm{~d}[[2,2]], \mathrm{m}[[4,5]] * \mathrm{~d}[[2,2]], \mathrm{m}[[4,6]] * \mathrm{~d}[[2,2]]\}$, $\{\mathrm{m}[[5,1]] * \mathrm{~d}[[2,1]], \mathrm{m}[[5,2]] * \mathrm{~d}[[2,1]], \mathrm{m}[[5,3]] * \mathrm{~d}[[2,1]]$, $\mathrm{m}[[5,4]] * \mathrm{~d}[[2,2]], \mathrm{m}[[5,5]] * \mathrm{~d}[[2,2]], \mathrm{m}[[5,6]] * \mathrm{~d}[[2,2]]\}$, $\{\mathrm{m}[[6,1]] * \mathrm{~d}[[2,1]], \mathrm{m}[[6,2]] * \mathrm{~d}[[2,1]], \mathrm{m}[[6,3]] * \mathrm{~d}[[2,1]]$, $\mathrm{m}[[6,4]] * \mathrm{~d}[[2,2]], \mathrm{m}[[6,5]] * \mathrm{~d}[[2,2]], \mathrm{m}[[6,6]] * \mathrm{~d}[[2,2]]\}\}$

(* Prozeduren fuer 9x9-Matrizen und 9-er Vektoren

$*)$

DreiKompMat $\left[a_{-}, b_{-}, c_{-}, d_{-}, e_{-}, f_{-}, g_{-}, h_{-}, i_{-}\right]:=$ $\{\{\mathrm{a}[[1,1]], \mathrm{a}[[1,2]], \mathrm{a}[[1,3]], \mathrm{b}[[1,1]], \mathrm{b}[[1,2]], \mathrm{b}[[1,3]]$, $c[[1,1]], c[[1,2]], c[[1,3]]\}$,

$\{a[[2,1]], a[[2,2]], a[[2,3]], b[[2,1]], b[[2,2]], b[[2,3]]$, $c[[2,1]], c[[2,2]], c[[2,3]]\}$,

$\{\mathrm{a}[[3,1]], \mathrm{a}[[3,2]], \mathrm{a}[[3,3]], \mathrm{b}[[3,1]], \mathrm{b}[[3,2]], \mathrm{b}[[3,3]]$, $c[[3,1]], c[[3,2]], c[[3,3]]\}$,

$\{d[[1,1]], d[[1,2]], d[[1,3]], e[[1,1]], e[[1,2]], e[[1,3]]$, $f[[1,1]], f[[1,2]], f[[1,3]]\}$,

$\{d[[2,1]], d[[2,2]], d[[2,3]], e[[2,1]], e[[2,2]], e[[2,3]]$, 


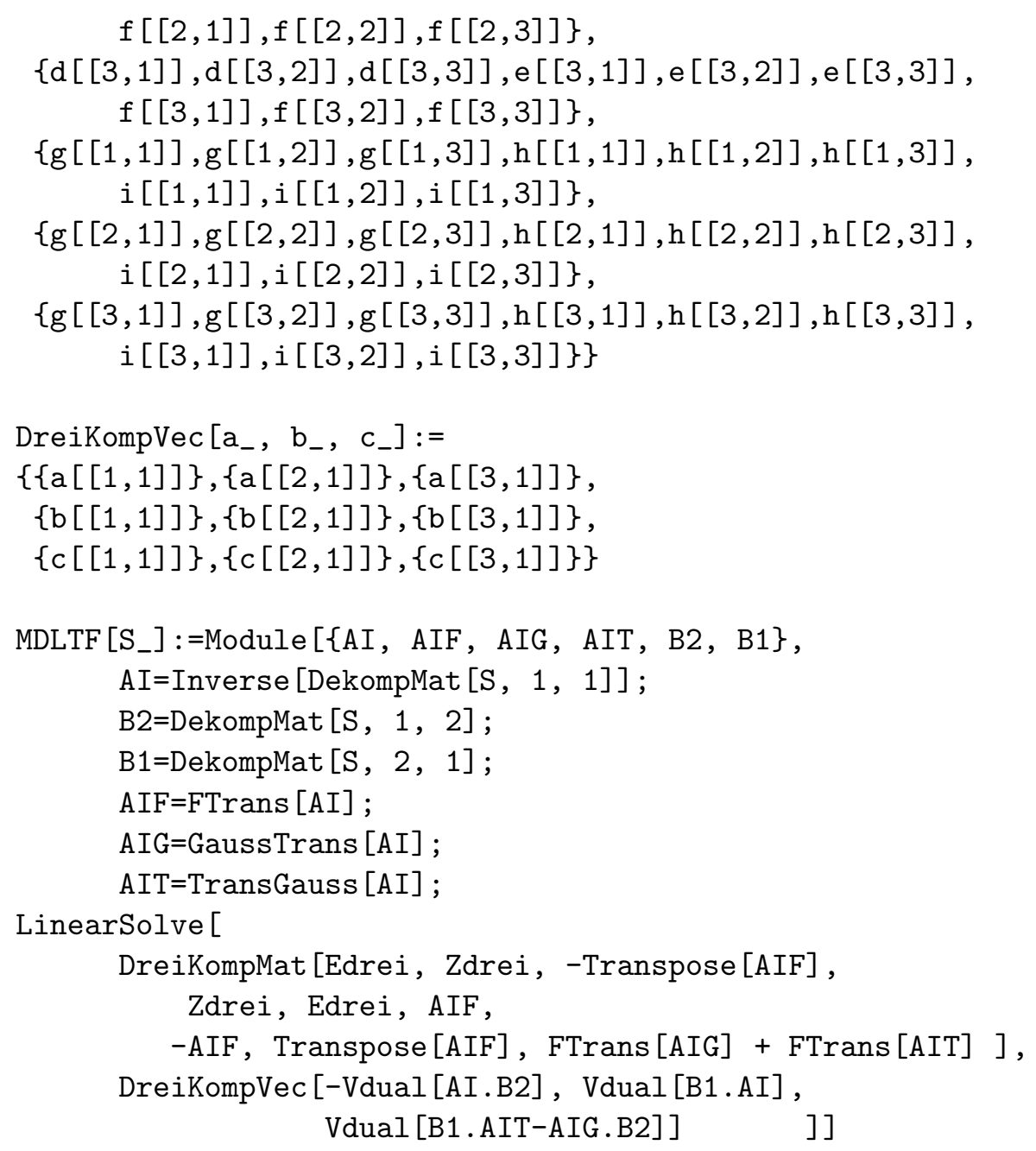

$\operatorname{MDF}\left[\mathrm{S}_{-}\right]:=$Module $[\{\mathrm{AI}, \mathrm{AIF}, \mathrm{AIG}, \mathrm{B} 2\}$,

$\mathrm{AI}=$ Inverse $[$ DekompMat $[\mathrm{S}, 1,1]]$;

$\mathrm{B} 2=$ DekompMat $[S, 1,2]$;

AIF $=$ FTrans $[\mathrm{AI}]$;

$\mathrm{AIG}=$ GaussTrans $[\mathrm{AI}]$;

LinearSolve [KompMat [Edrei, -Transpose[AIF], -AIF, FTrans[AIG] ], KompVec [-Vdual [AI.B2], Vdual[AIG.B2]] ]]

LTF $\left[S_{-}\right]:=$Module $[\{\mathrm{AI}, \mathrm{AIF}, \mathrm{AITG}, \mathrm{B} 1\}$,

$\mathrm{AI}=$ Inverse [DekompMat $[\mathrm{S}, 1,1]]$;

B1=DekompMat $[S, 2,1]$;

AIF=FTrans [AI] ;

AITG=TransGauss [AI] ;

LinearSolve [KompMat [Edrei, AIF, Transpose [AIF], FTrans [AITG] ], KompVec [Vdual [B1.AI], Vdual[B1.AITG] ] ] 


\section{A.3.2 Prozeduren zur Differentialgeometrie}

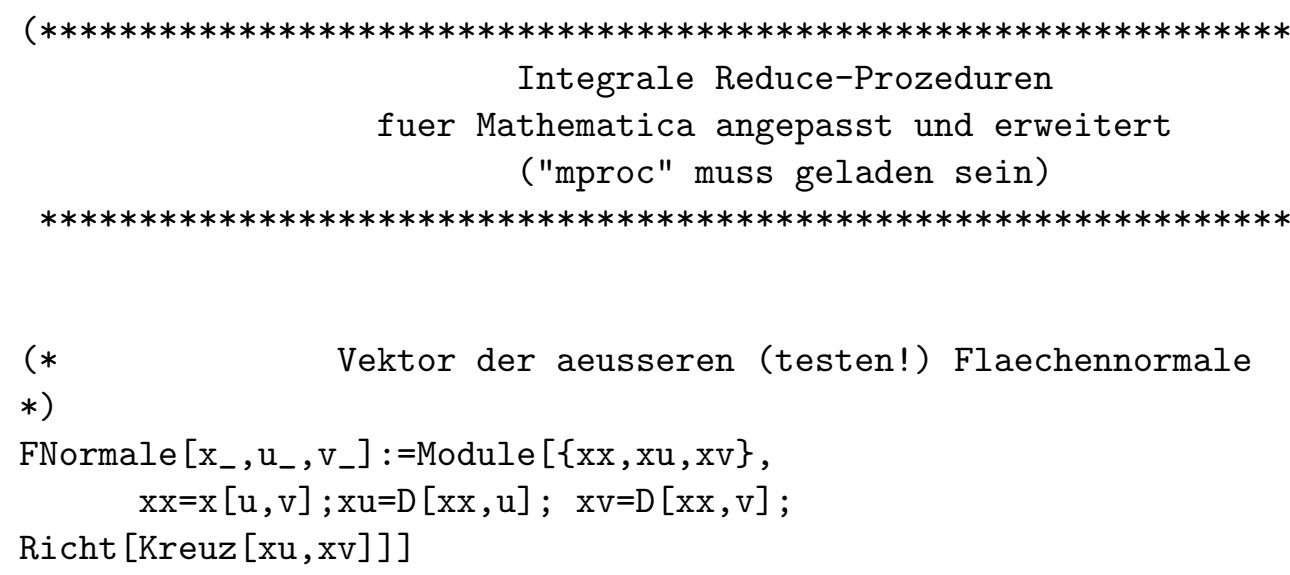




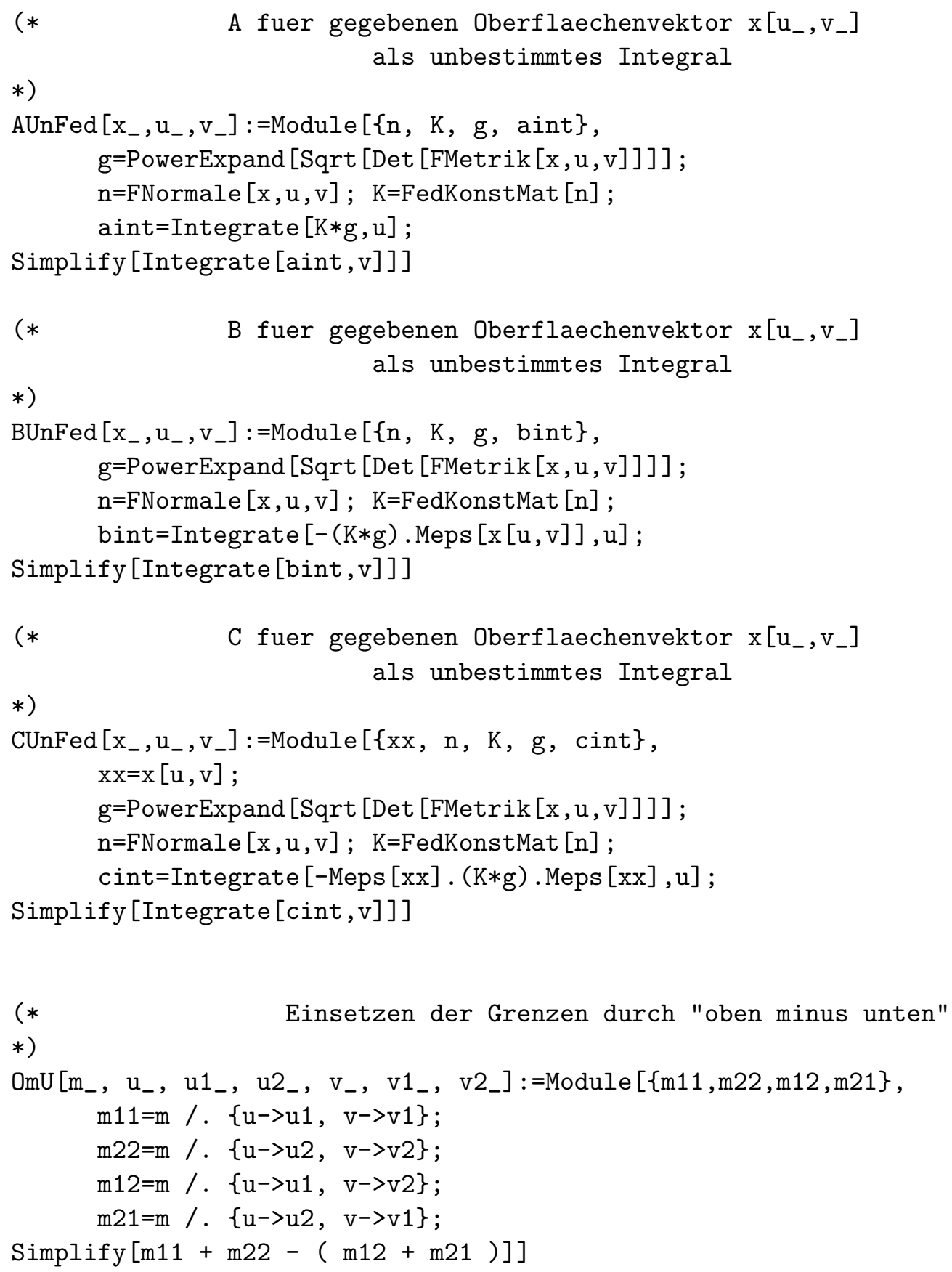

\section{A.3.3 Prozeduren zur direkten Auswertung}

Mit diesen Prozeduren ist eine schnelle Übersicht aller wichtigen Parameter zu einer gegebenen Flexibilitätsmatrix möglich. Die Matrix hat dazu in MAThematicASyntax in einem einzelnen File vorzuliegen. Steifheitsmatrizen sind vor der Bearbeitung zu invertieren und wieder herauszuschreiben. 


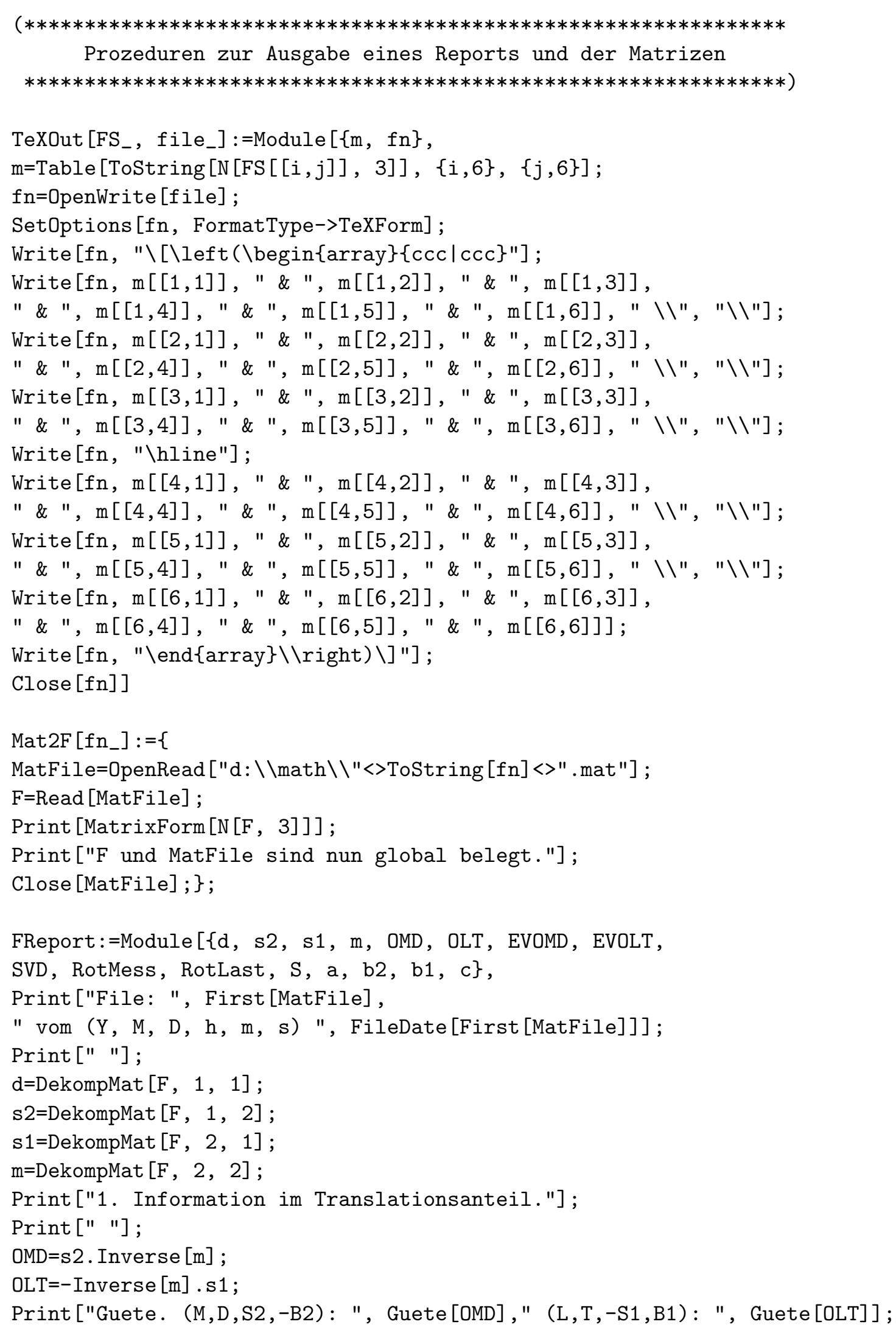

FReport:=Module $[\{\mathrm{d}, \mathrm{s} 2, \mathrm{~s} 1, \mathrm{~m}$, OMD, OLT, EVOMD, EVOLT, SVD, RotMess, RotLast, S, a, b2, b1, c\}, Print ["File: ", First [MatFile], 


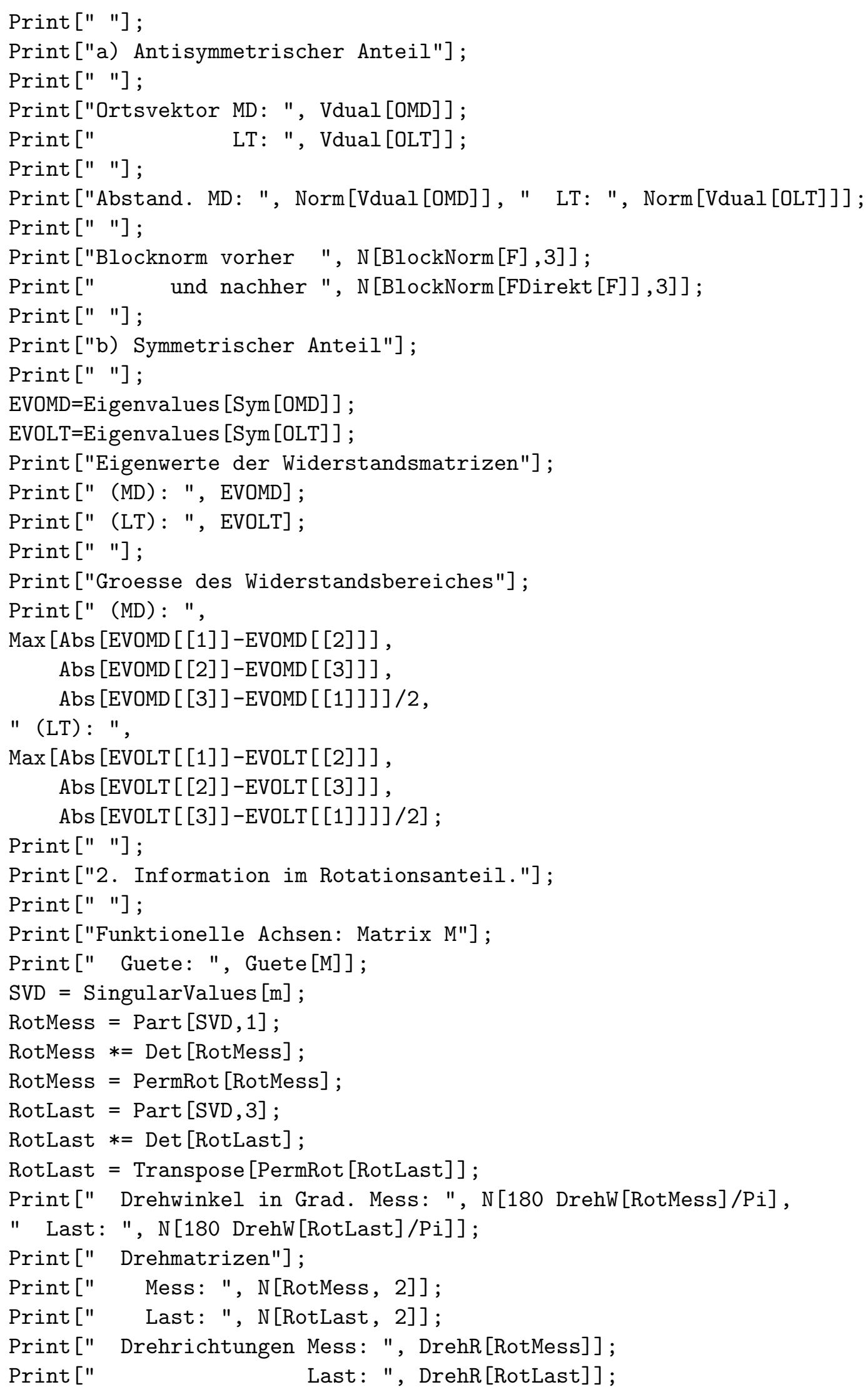




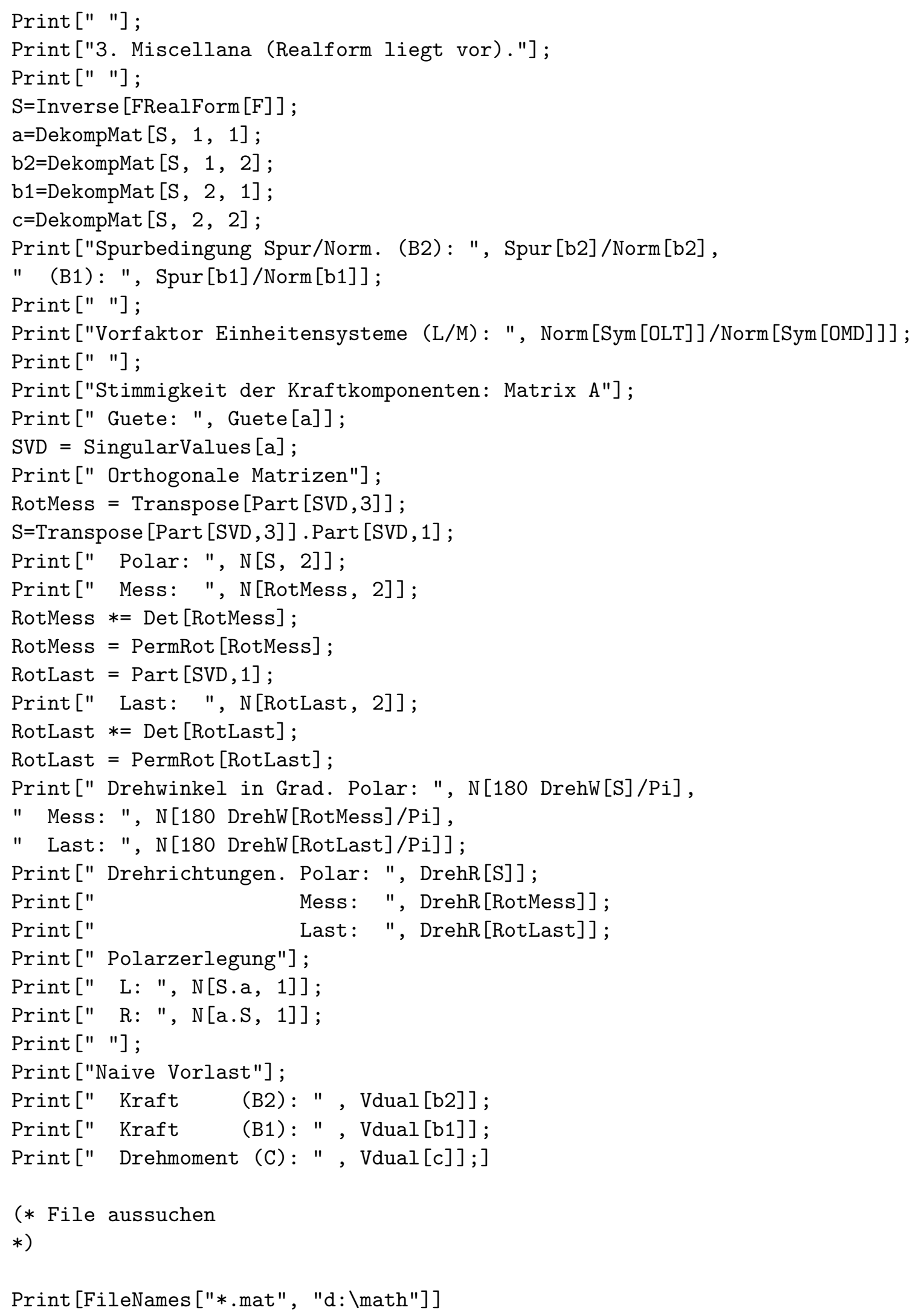




\section{Anhang B}

\section{Miscellana}

In diesem Abschnitt sollen einige Objekte untersucht werden, die möglicherweise später einmal von Bedeutung werden könnten.

\section{B.1 Widerstandsachsen}

Im Allgemeinen existiert kein einzelner Punkt mehr mit den Eigenschaften eines Widerstandszentrums. Die Abschwächung dieser Wunscheigenschaften zu Minimierungsproblemen führte in 2.2.5zur Definition des elastischen Zentrums. Ein anderer Definitionsversuch führte auf eine Punktmenge, die Aufpunktfläche.

Die Bedingungen an das Widerstandszentrum können statt formal auch mit einer geometrischen Motivation abgeschwächt werden: Statt Forderungen an einen einzelnen Punkt $W$ zu stellen

$$
\left\{\begin{array}{c}
\text { für alle Kräfte mit Wirkungslinien durch W... } \\
\text { für alle Drehungen mit Achsen durch W... }
\end{array}\right\}
$$

zeichnet man für Punkte $P$ mit

$\left\{\begin{array}{c}\text { es gibt Kraftwirkungslinien durch } P \\ \text { es gibt Drehachsen durch } P\end{array}\right\}$ für die gilt $\left\{\begin{array}{c}\boldsymbol{T}(P)=\mathbf{0} \Leftrightarrow \delta \boldsymbol{\theta}=\mathbf{0} \\ \delta \boldsymbol{d}(P)=\mathbf{0} \Leftrightarrow \boldsymbol{F}=\mathbf{0}\end{array}\right\}$

eine ganze Punktmenge aus, genauer Geraden im Raum. Diese Geraden, die Widerstandsachsen, berühren die Aufpunktfläche da sie spezielle Schraubachsen mit verschwindendem Schraubanteil sind. Widerstandsachsen sind also im Raum liegende Geraden, längs derer sich die Aufhängung benimmt, als habe sie ein Widerstandszentrum. Falls sich mehrere solcher Achsen finden lassen, die stetig ineinander übergehen und von nur einem Parameter abhängen, möchte ich diese zu einer Fläche zusammenfassen, der Achsfläche. 


\section{B.1.1 Die Achsfläche}

Die Achsfläche ist die Menge aller Widerstandsachsen. Als einparametrige Menge von Geraden ist sie eine Regelfläche; als zweiparametrige Schar wäre sie, wie die Menge aller Schraubachsen selbst, eine Geradenkongruenz. Existiert ein Widerstandszentrum, so entartet sie zum gesamten Raum.

Es wird sich in diesem Abschnitt zeigen, daß sie entweder ein einschaliges Hyperboloid oder eine Kombination aus Koordinatenebenen bzw. Koordinatenachsen ist. Falls die Widerstandsachsen in einer Doppelebene liegen, ist ihnen je Ebene ein Punkt gemeinsam.

Das Koordinatensystem liege im elastischen Zentrum $Z$. Es sollen die obigen Forderungen auf die Komponenten der hier als symmetrisch vorausgesetzten Flexibilitätsmatrix übertragen werden. Dann gilt in $Z$

$$
\left(\begin{array}{l}
\delta \boldsymbol{d} \\
\delta \boldsymbol{\theta}
\end{array}\right)_{Z}=\left(\begin{array}{cc}
\boldsymbol{D}_{Z} & \boldsymbol{S}_{Z} \\
\boldsymbol{S}_{Z}^{T} & \mathrm{M}
\end{array}\right)\left(\begin{array}{l}
\boldsymbol{F} \\
\boldsymbol{T}
\end{array}\right)_{Z}
$$

Sei $P$ ein Punkt einer Widerstandsachse. Auf einem solchen Punkt hat die Flexibilitätsmatrix die Gestalt

$$
\mathcal{F}_{P}=\mathcal{T}_{Z P}^{T} \mathcal{F}_{Z} \mathcal{T}_{Z P}=\left(\begin{array}{cc}
\boldsymbol{D}_{P} & \boldsymbol{S}_{Z}-\boldsymbol{\Omega}_{Z P} \boldsymbol{M} \\
\boldsymbol{S}_{Z}^{T}+\boldsymbol{M} \boldsymbol{\Omega}_{Z P} & \mathbf{M}
\end{array}\right)
$$

und das Entkoppeln von Translationen und Rotationen nach Definition bedeutet nun für eine reine Kraft

$$
\left(\begin{array}{c}
\delta \boldsymbol{d} \\
\mathbf{0}
\end{array}\right)_{P}=\left(\begin{array}{cc}
\boldsymbol{D}_{P} & \boldsymbol{S}_{Z}-\boldsymbol{\Omega}_{Z P} \boldsymbol{M} \\
\boldsymbol{S}_{Z}^{T}+\boldsymbol{M} \boldsymbol{\Omega}_{Z P} & \mathbf{M}
\end{array}\right)\left(\begin{array}{c}
\boldsymbol{F} \\
\mathbf{0}
\end{array}\right)_{P},
$$

bzw. für eine reine Drehung

$$
\left(\begin{array}{c}
\mathbf{0} \\
\delta \boldsymbol{\theta}
\end{array}\right)_{P}=\left(\begin{array}{cc}
\boldsymbol{D}_{P} & \boldsymbol{S}_{Z}-\boldsymbol{\Omega}_{Z P} \boldsymbol{M} \\
\boldsymbol{S}_{Z}^{T}+\boldsymbol{M} \boldsymbol{\Omega}_{Z P} & \mathbf{M}
\end{array}\right)\left(\begin{array}{c}
\mathbf{0} \\
\mathbf{T}
\end{array}\right)_{P} .
$$

Es ergibt sich also

$$
\mathbf{0}=\left(\boldsymbol{S}_{Z}^{T}+\boldsymbol{M} \Omega_{Z P}\right) \boldsymbol{F}=\boldsymbol{M}\left(\boldsymbol{M}^{-1} \boldsymbol{S}_{Z}^{T}+\Omega_{Z P}\right) \boldsymbol{F}
$$

für die Kräfte, und

$$
\mathbf{0}=\left(\boldsymbol{S}_{Z}-\boldsymbol{\Omega}_{Z P} \boldsymbol{M}\right) \boldsymbol{T}=\left(\boldsymbol{S}_{Z}-\boldsymbol{\Omega}_{Z P} \boldsymbol{M}\right) \boldsymbol{M}^{-1} \delta \boldsymbol{\theta}=\left(\boldsymbol{S}_{Z} \boldsymbol{M}^{-1}-\boldsymbol{\Omega}_{Z P}\right) \delta \boldsymbol{\theta}
$$

für die Drehungen. Mit der Matrix $\boldsymbol{S} \boldsymbol{M}^{-1}=\boldsymbol{W}$, die im elastischen Zentrum symmetrisch ist, lassen sich beide Gleichungen mit dem linienflüchtigen Richtungsvektor $\boldsymbol{r} \mathrm{zu}$

$$
\left(\boldsymbol{W} \pm \Omega_{Z P}\right) \boldsymbol{r}=\mathbf{0}
$$


zusammenfassen! Da $\boldsymbol{M}$ nichtsingulär ist, müssen jeweils die Matrizen in den Klammern singulär sein. Es folgt notwendig

$$
\operatorname{det}\left(\boldsymbol{W} \pm \Omega_{Z P}\right)=0
$$

Wegen (A.15) ist $\Omega_{Z P}$ im System der Eigenvektoren von $\boldsymbol{W}$ wieder antisymmetrisch. Seien $w_{i}$ die Eigenwerte von $\boldsymbol{W}$ und $\overrightarrow{Z P}=(x, y, z)^{T}$ die Komponenten des Aufpunktes der Widerstandsachse. Dann folgt im Eigensystem von $\boldsymbol{W}$

$$
\begin{aligned}
\operatorname{det}\left(\boldsymbol{W} \pm \Omega_{Z P}\right) & =\left|\left(\begin{array}{ccc}
w_{1} & \mp z & \pm y \\
\pm z & w_{2} & \mp x \\
\mp y & \pm x & w_{3}
\end{array}\right)\right| \\
& =w_{1} w_{2} w_{3} \mp x y z \pm x y z+w_{1} x^{2}+w_{2} y^{2}+w_{3} z^{2} \\
& =\operatorname{det} \boldsymbol{W}+\overrightarrow{Z P}^{T} \boldsymbol{W} \overrightarrow{Z P},
\end{aligned}
$$

und die Gleichung der Achsfläche

$$
\begin{aligned}
0 & =\operatorname{det}\left(\boldsymbol{W}-\boldsymbol{\Omega}_{Z P}\right) \\
& =\operatorname{det} \boldsymbol{W}+\overrightarrow{Z P}^{T} \boldsymbol{W} \overrightarrow{Z P} \\
& =w_{1} w_{2} w_{3}+w_{1} x^{2}+w_{2} y^{2}+w_{3} z^{2}
\end{aligned}
$$

beschreibt eine zum elastischen Zentrum symmetrische Fläche zweiter Ordnung.

Ist nun $\boldsymbol{x}$ ein Punkt dieser Fläche, so muß eine Lösung $\boldsymbol{p}:=\overrightarrow{Z P}$ zusammen mit dem linienflüchtigen Richtungsvektor $\boldsymbol{r}$, also wahlweise $\boldsymbol{F}$ oder $\delta \boldsymbol{\theta}$ eine Gerade im Raum beschreiben, also

$$
\boldsymbol{x}=\boldsymbol{p}+\lambda \boldsymbol{r}
$$

In der Tat gilt, falls ein Punkt $P$ und eine Richtung $\boldsymbol{r}$ mit den gewünschten Eigenschaften existieren, für alle Punkte der Geraden

$$
\left(\boldsymbol{W} \pm \boldsymbol{\Omega}_{x}\right) \boldsymbol{r}=\left(\boldsymbol{W} \pm \boldsymbol{\Omega}_{p+\lambda r}\right) \boldsymbol{r}=\left(\boldsymbol{W} \pm \boldsymbol{\Omega}_{p} \pm \lambda \boldsymbol{\Omega}_{r}\right) \boldsymbol{r}=\left(\boldsymbol{W} \pm \boldsymbol{\Omega}_{p}\right) \boldsymbol{r}=\mathbf{0}
$$

Aus dieser Gleichung folgt für den Aufpunkt

$$
\mathbf{0}=\boldsymbol{p}^{T}\left(\boldsymbol{W} \pm \boldsymbol{\Omega}_{p}\right) \boldsymbol{r}=\boldsymbol{p}^{T} \boldsymbol{W} \boldsymbol{r} \pm \boldsymbol{p}^{T} \boldsymbol{\Omega}_{p} \boldsymbol{r}=\boldsymbol{p}^{T} \boldsymbol{W} \boldsymbol{r}
$$

und für den Richtungsvektor selbst

$$
\mathbf{0}=\boldsymbol{r}^{T}\left(\boldsymbol{W} \pm \boldsymbol{\Omega}_{p}\right) \boldsymbol{r}=\boldsymbol{r}^{T} \boldsymbol{W} \boldsymbol{r} \pm \boldsymbol{r}^{T} \boldsymbol{\Omega}_{p} \boldsymbol{r}=\boldsymbol{r}^{T} \boldsymbol{W} \boldsymbol{r}
$$

\footnotetext{
${ }^{1}$ Hätte man diese Überlegungen mit einer nichtsymmetrischen Ausgangsmatrix angefangen, so bekäme man statt (B.2) die zwei Gleichungen

$$
\begin{aligned}
\left(\boldsymbol{M}^{-1} \boldsymbol{S}_{1}+\boldsymbol{\Omega}_{L P}\right) \boldsymbol{F}=\left(\boldsymbol{W}_{L}+\boldsymbol{\Omega}_{L P}\right) \boldsymbol{F} & =\mathbf{0} \text { und } \\
\left(\boldsymbol{\Omega}_{M P}-\boldsymbol{S}_{2} \boldsymbol{M}^{-1}\right) \delta \boldsymbol{\theta}\left(\boldsymbol{\Omega}_{M P}-\boldsymbol{W}_{M}\right) \delta \boldsymbol{\theta} & =\mathbf{0} .
\end{aligned}
$$

Die folgende Untersuchung wäre dann für Lastsystem und Meßsystem je einmal durchzuführen.
} 
Der Richtungsvektor ist demnach auch Element einer Fläche zweiter Ordnung.

Die Gleichungen (B.2) und (B.6) können dazu verwandt werden, die Art der Achsfläche näher zu spezifizieren. Die Unterscheidungskriterien sind hier die Vorzeichen der einzelnen Eigenwerte $w_{i}$. Da die Gleichungen mit -1 durchmultipliziert werden können, ist die erste Komponente oBdA. immer $\geq 0$.

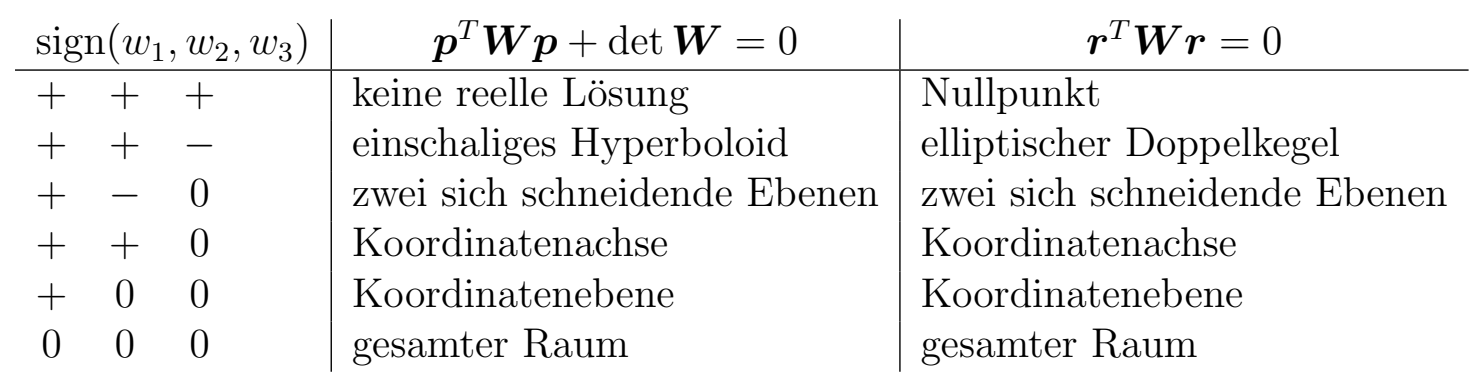

Im ersten Fall gibt es zu keiner Richtung einen passenden Aufpunkt und damit auch keine Widerstandsachse. Bis auf den letzten Fall, der bei einem Widerstandszentrum vorliegt, gibt es immer Richtungen im Raum zu denen kein passender Aufpunkt existiert. Daher ist es nicht möglich, eine der Aufpunktfläche ähnliche Menge solcher Punkte zu allen Richtungen zu gewinnen. Da keine absoluten Glieder in den Definitionsgleichungen auftauchen, kann die Achsfläche kein hyperbolisches Paraboloid sein, eine weitere nichttriviale Regelfläche, welche gleichzeitig Fläche zweiter Ordnung ist.

Als Nächstes sollen die Richtungen $\boldsymbol{r}$ näher untersucht werden. Die Gleichung (B.2) besagt, daß eine Zeile der Matrix $\boldsymbol{W} \pm \boldsymbol{\Omega}_{p}$, etwa die dritte, als Linearkombination durch die beiden anderen ausgedrückt werden kann. Eine Lösung $\boldsymbol{r}=\left(r_{1}, r_{2}, r_{3}\right)^{T}$ des homogenen Gleichungssystems

$$
\left(\begin{array}{ccc}
w_{1} & -z & y \\
z & w_{2} & -x \\
-y & x & w_{3}
\end{array}\right)\left(\begin{array}{l}
r_{1} \\
r_{2} \\
r_{3}
\end{array}\right)=0
$$

sollte das Kreuzprodukt der ersten beiden Zeilen der Koeffizientenmatrix sein, also

$$
\boldsymbol{r}=\left(\begin{array}{c}
w_{1} \\
-z \\
y
\end{array}\right) \times\left(\begin{array}{c}
z \\
w_{2} \\
-x
\end{array}\right)=\left(\begin{array}{c}
z x-w_{2} y \\
y z+w_{1} x \\
z^{2}+w_{1} w_{2}
\end{array}\right)
$$

In der Tat ergibt sich damit für die dritte Zeile

$$
\begin{aligned}
& \left(\begin{array}{lll}
-y, & x, \quad w_{3}
\end{array}\right) \cdot\left(\begin{array}{c}
z x-w_{2} y \\
y z+w_{1} x \\
z^{2}+w_{1} w_{2}
\end{array}\right) \\
& =-y\left(z x-w_{2} y\right)+x\left(y z+w_{1} x\right)+w_{3}\left(z^{2}+w_{1} w_{2}\right) \\
& =-y z x+w_{2} y^{2}+x y z+w_{1} x^{2}+w_{3} z^{2}+w_{1} w_{2} w_{3} \\
& =\operatorname{det} \boldsymbol{W}+\boldsymbol{p}^{T} \boldsymbol{W} \boldsymbol{p}=0
\end{aligned}
$$


Dadurch, daß die dritte Zeile eine Linearkombination der ersten beiden ist, liefert die analoge Überlegung für zwei andere Zeilen keine grundsätzlich neuen Lösungen. Die gewonnene Lösung (B.7) ist also prinzipiell bis auf Vorfaktoren eindeutig. Es kann aber vorkommen, daß eine Parametrisierung der Aufpunkte mit der Formel (B.7) nicht alle Richtungen liefert. Das ist der Fall, wenn die Matrix $\boldsymbol{W}$ selbst entartet ist. Dann ist es günstiger, das Kreuzprodukt zweier anderer Vektoren zu verwenden, etwa

$$
\boldsymbol{r}=\left(\begin{array}{c}
z \\
w_{2} \\
-x
\end{array}\right) \times\left(\begin{array}{c}
-y \\
x \\
w_{3}
\end{array}\right)=\left(\begin{array}{c}
x^{2}+w_{2} w_{3} \\
x y-w_{3} z \\
z x+w_{2} y
\end{array}\right) .
$$

Durch Nachrechnen kann man sich ferner unter Verwendung von (B.2) überzeugen, daß diese Lösung mit den Gleichungen (B.6) und (B.5) verträglich ist.

Sei im Folgenden $w_{i} \geq 0$, d. h. die Vorzeichen werden explizit angeschrieben. Das einschalige Hyperboloid

$$
x^{2} w_{1}+y^{2} w_{2}-z^{2} w_{3}=w_{1} w_{2} w_{3}
$$

hat den Kehlkreis

$$
\left(\begin{array}{l}
x \\
y \\
z
\end{array}\right)=\left(\begin{array}{c}
\sqrt{w_{1} w_{2}} \cos \phi \\
\sqrt{w_{1} w_{3}} \sin \phi \\
0
\end{array}\right)
$$

der hier eine Parameterdarstellung der Aufpunkte ist. Mit (B.7) ergibt sich eine Darstellung des Hyperboloiden als Regelfläche

$$
\left(\begin{array}{l}
x \\
y \\
z
\end{array}\right)=\left(\begin{array}{c}
\sqrt{w_{1} w_{2}} \cos \phi \\
\sqrt{w_{1} w_{3}} \sin \phi \\
0
\end{array}\right)+\lambda\left(\begin{array}{c}
w_{2} \sqrt{w_{1} w_{3}} \sin \phi \\
-w_{1} \sqrt{w_{2} w_{3}} \cos \phi \\
w_{1} w_{2}
\end{array}\right) .
$$

Dreht man nun den Umlaufsinn des Kehlkreises um, so erhält man die andere Schar von Erzeugenden, also insgesamt

$$
\left(\begin{array}{l}
x \\
y \\
z
\end{array}\right)=\left(\begin{array}{c}
\sqrt{w_{1} w_{2}} \cos \phi \\
\pm \sqrt{w_{1} w_{3}} \sin \phi \\
0
\end{array}\right)+\lambda\left(\begin{array}{c}
\mp w_{2} \sqrt{w_{1} w_{3}} \sin \phi \\
-w_{1} \sqrt{w_{2} w_{3}} \cos \phi \\
w_{1} w_{2}
\end{array}\right) .
$$

Analog erhält man für die beiden sich schneidenden Ebenen mit der Gleichung

$$
0=x^{2} w_{1}-y^{2} w_{2}=\left(x \sqrt{w_{1}}-y \sqrt{w_{2}}\right)\left(x \sqrt{w_{1}}+y \sqrt{w_{2}}\right)
$$

die Darstellung

$$
\begin{aligned}
\left(\begin{array}{l}
x \\
y \\
z
\end{array}\right) & =\left(\begin{array}{c}
\mu \sqrt{w_{2}} \\
\pm \mu \sqrt{w_{1}} \\
0
\end{array}\right)+\lambda\left(\begin{array}{c}
\mp \mu w_{2} \sqrt{w_{1}} \\
-\mu w_{1} \sqrt{w_{2}} \\
-w_{1} w_{2}
\end{array}\right) \\
& =\left(\begin{array}{c}
\mu \sqrt{w_{2}} \\
\pm \mu \sqrt{w_{1}} \\
0
\end{array}\right)+\lambda \sqrt{w_{1} w_{2}}\left(\begin{array}{c}
\mp \mu \sqrt{w_{2}} \\
-\mu \sqrt{w_{1}} \\
-\sqrt{w_{1} w_{2}}
\end{array}\right)
\end{aligned}
$$


Besonders interessant ist nun die Umparametrisierung

$$
\begin{aligned}
\left(\begin{array}{l}
x \\
y \\
z
\end{array}\right) & =\left(\begin{array}{c}
\mu \sqrt{w_{2}} \\
\pm \mu \sqrt{w_{1}} \\
0
\end{array}\right)+(\lambda \pm 1)\left(\begin{array}{c}
\mp \mu \sqrt{w_{2}} \\
-\mu \sqrt{w_{1}} \\
-\sqrt{w_{1} w_{2}}
\end{array}\right) \\
& =\left(\begin{array}{c}
\mu \sqrt{w_{2}} \\
\pm \mu \sqrt{w_{1}} \\
0
\end{array}\right) \pm\left(\begin{array}{c}
\mp \mu \sqrt{w_{2}} \\
-\mu \sqrt{w_{1}} \\
-\sqrt{w_{1} w_{2}}
\end{array}\right)+\lambda\left(\begin{array}{c}
\mp \mu \sqrt{w_{2}} \\
-\mu \sqrt{w_{1}} \\
-\sqrt{w_{1} w_{2}}
\end{array}\right) \\
& =\left(\begin{array}{c}
\mp \mu \sqrt{w_{2}} \\
0 \\
\mp \sqrt{w_{1} w_{2}}
\end{array}\right)+\lambda\left(\begin{array}{c}
\mp w_{1} \\
-\sqrt{w_{1} w_{2}}
\end{array}\right) .
\end{aligned}
$$

Allen Geraden einer Ebene ist daher der Punkt $\mp \sqrt{w_{1} w_{2}}$ der $z$-Achse gemein. Des weiteren steht der Vektor

$$
\boldsymbol{n}:=\left(\begin{array}{c}
\sqrt{w_{1}} \\
\mp \sqrt{w_{2}} \\
0
\end{array}\right)
$$

auf allen diesen Geraden senkrecht. Wegen des gemeinsamen Aufpunktes liegen diese Geraden in jeweils einer Ebene, die dadurch in der Punkt-Normalen-Form beschrieben ist. Beide Ebenen schneiden sich in der $z$-Achse.

Mit der Parameterdarstellung der Aufpunkte $(0,0, z)$ beginnend versagt hier übrigens die Formel (B.7). Erst mit (B.8) erhält man die andere Parameterdarstellung

$$
\left(\begin{array}{c}
0 \\
0 \\
\pm z
\end{array}\right)+\lambda\left(\begin{array}{c}
w_{2} w_{3} \\
\mp z w_{3} \\
0
\end{array}\right)
$$

der man die obigen Eigenschaften der beiden Ebenen nicht mehr so unmittelbar ansieht.

Die Gegenbeispielfläche aus Abschnitt 5.1.4 mit $\lambda=1$ hat bezüglich des Systems der Eigenvektoren die Widerstandsmatrix $\boldsymbol{W}=\operatorname{diag}(-1,1,0)$. Entsprechend ist die Achsfläche eine Doppelebene mit den Aufpunkten

$$
\boldsymbol{p}_{1,2}=\left(\begin{array}{c}
0 \\
0 \\
\mp 1
\end{array}\right)
$$

und den Normalenvektoren

$$
\boldsymbol{n}_{1,2}=\left(\begin{array}{c}
1 \\
\pm 1 \\
0
\end{array}\right)
$$

die Ebenen korrespondieren also mit dem oberen und unterem Grat der Aufpunktfläche, vgl. Abb. 2.6, Die Schraubachsen schneiden sich dabei im obersten bzw. untersten auf der $z$-Achse gelegenen Punkt der Fläche. 


\section{B.2 Das ebene Widerstandszentrum}

In diesem Abschnitt sollen die älteren Resultate bezüglich des Widerstandszentrums für ebene Aufhängungen im jetzigen Formalismus dargestellt und dabei überprüft werden. Gleichzeitig ist das ein Test für den von mir verwendeten Formalismus; es muß ja ein „Schwerpunkt der Federkräfte“ existieren. Da die von mir verwendete Definition des elastischen Zentrums angewandt auf ebene Aufhängungen nicht die gewünschten Resultate liefert, vgl. 2.2.4.2, muß für ebene Aufhängungen eine passendere Definition gefunden werden. Damit wird die Lücke zwischen der zweidimensionalen Rechnung der Einleitung 1.1 und dem später entwickelten Formalismus geschlossen.

\section{B.2.1 Definition des ebenen Widerstandszentrums}

Die Lage einer Ebene im Raum ist gegeben durch einen Aufpunkt und eine Ebenenormale $\boldsymbol{n}$. Ein Ebenes Widerstandszentrum $E$ als Aufpunkt einer solchen $W i$ derstandsebene liege genau dann vor, wenn

$$
\left\{\begin{array}{c}
\text { ein reines Drehmoment in Normalenrichtung } \\
\text { eine reine Kraft senkrecht zur Normale und durch das Zentrum }
\end{array}\right\}
$$

eine reine

$$
\left\{\begin{array}{c}
\text { Drehung in Normalenrichtung mit Achse durch das Zentrum } \\
\text { Translation senkrecht zur Normale }
\end{array}\right\}
$$

zur Folge hat. Mit den zu $\boldsymbol{n}$ gehörenden Projektoren ausgedrückt heißt das

$$
\left(\begin{array}{c}
\boldsymbol{F} \\
\boldsymbol{T}
\end{array}\right) \stackrel{E}{=}\left(\begin{array}{c}
\mathbf{0} \\
\boldsymbol{P}^{\|} \boldsymbol{T}
\end{array}\right) \Leftrightarrow\left(\begin{array}{c}
\delta \boldsymbol{d} \\
\delta \boldsymbol{\theta}
\end{array}\right) \stackrel{E}{=}\left(\begin{array}{c}
\mathbf{0} \\
\boldsymbol{P}^{\|} \delta \boldsymbol{\theta}
\end{array}\right)
$$

und

$$
\left(\begin{array}{c}
\boldsymbol{F} \\
\boldsymbol{T}
\end{array}\right) \underline{E}\left(\begin{array}{c}
\boldsymbol{P}^{\perp} \boldsymbol{F} \\
\mathbf{0}
\end{array}\right) \Leftrightarrow\left(\begin{array}{c}
\delta \boldsymbol{d} \\
\delta \boldsymbol{\theta}
\end{array}\right) \underline{E}\left(\begin{array}{c}
\boldsymbol{P}^{\perp} \boldsymbol{d} \\
\mathbf{0}
\end{array}\right) .
$$

Übertragen auf die Komponenten der Steifheitsmatrix mit

$$
\left(\begin{array}{c}
\delta \boldsymbol{d} \\
\delta \boldsymbol{\theta}
\end{array}\right)=\left(\begin{array}{cc}
\boldsymbol{A} & \boldsymbol{B}_{E} \\
\boldsymbol{B}_{E}^{T} & \boldsymbol{C}_{E}
\end{array}\right)\left(\begin{array}{c}
\boldsymbol{F} \\
\boldsymbol{T}
\end{array}\right)
$$

lautet das

$$
\begin{aligned}
& \mathbf{0}=\boldsymbol{B}_{E} \boldsymbol{P}^{\|} \\
& \mathbf{0}=\boldsymbol{P}^{\perp} \boldsymbol{C}_{E} \boldsymbol{P}^{\|} \\
& \mathbf{0}=\boldsymbol{P}^{\|} \boldsymbol{A}_{E} \boldsymbol{P}^{\perp} \\
& \mathbf{0}=\boldsymbol{B}_{E}^{T} \boldsymbol{P}^{\perp} .
\end{aligned}
$$


Diese Gleichungen können besser verstanden werden, wenn man die zu ihren Zahlenwerten gehörenden linearen Endomorphismen betrachtet. Die zweite Gleichung (B.11) etwa besagt, daß das Bild $\boldsymbol{C}_{E} \boldsymbol{n}$ von $\boldsymbol{n}$ wieder parallel zu $\boldsymbol{n}$ ist, d. h. daß $\boldsymbol{n}$ Eigenvektor von $\boldsymbol{C}_{E}$ ist. Dieses Eigenvektorproblem hängt über die Matrix $\boldsymbol{C}_{E}$ vom gewählten Koordinatensystemursprung ab, und stellt so eine starke Einschränkung an die Lösbarkeit des Problems dar. Die dritte Gleichung (B.12) lautet transponiert $\boldsymbol{P}^{\perp} \boldsymbol{A}^{T} \boldsymbol{P}^{\|}=\mathbf{0}$, und wegen $\boldsymbol{A}^{T}=\boldsymbol{A}$ muß $\boldsymbol{n}$ auch Eigenvektor von $\boldsymbol{A}$ sein, wobei dieses Eigenvektorproblem nicht vom Aufpunkt abhängt. Daher kann ein ebenes Widerstandszentrum nur dann existieren, wenn eine Achse konstanter Helizität, also ein Eigenvektor von $\boldsymbol{A} \boldsymbol{C}_{E}$, als Ebenennormale fungiert. Weiterhin liegen die Bilder von Vektoren aus $\langle\boldsymbol{n}\rangle^{\perp}$ wieder in $\langle\boldsymbol{n}\rangle^{\perp}$, so daß, wenn man $\boldsymbol{n}$ auf eine der Koordinatenrichtungen dreht, $\boldsymbol{A}$ und $\boldsymbol{C}$ eine Blockdiagonalstruktur besitzen, welche den Raum orthogonal in $\langle\boldsymbol{n}\rangle$ und $\langle\boldsymbol{n}\rangle^{\perp}$ zerlegt; etwa für $\boldsymbol{n}=\boldsymbol{e}_{z}$

$$
\left(\begin{array}{lll}
* & * & 0 \\
* & * & 0 \\
0 & 0 & *
\end{array}\right)
$$

Die Gleichungen (B.10) und (B.12) können ebenso als lineare Endomorphismen besser verstanden werden. Dann nämlich gelten die folgenden Aussagen (vgl. [36] $\S 21)$ :

$$
\begin{aligned}
& \langle\boldsymbol{n}\rangle \stackrel{\sqrt{B .10}=}{=} \operatorname{Kern}(\boldsymbol{B})=\left(\operatorname{Im}\left(\boldsymbol{B}^{T}\right)\right)^{\perp} \Rightarrow \operatorname{Im}\left(\boldsymbol{B}^{T}\right)=\langle\boldsymbol{n}\rangle^{\perp} \\
& \langle\boldsymbol{n}\rangle^{\perp} \stackrel{\sqrt{B .122}}{=} \operatorname{Kern}\left(\boldsymbol{B}^{T}\right)=(\operatorname{Im}(\boldsymbol{B}))^{\perp} \Rightarrow \operatorname{Im}(\boldsymbol{B})=\langle\boldsymbol{n}\rangle \quad,
\end{aligned}
$$

die starke Einschränkungen an die Matrix $\boldsymbol{B}_{E}$ stellen; wieder für $\boldsymbol{n}=\boldsymbol{e}_{z}$ hat $\boldsymbol{B}_{E}$ die Struktur

$$
\left(\begin{array}{lll}
0 & 0 & 0 \\
0 & 0 & 0 \\
* & * & 0
\end{array}\right)
$$

und $\boldsymbol{B}_{E}$ hat Rang 1. Existiert nun ein Widerstandszentrum, so gilt $\boldsymbol{B}_{E}=\boldsymbol{A} \boldsymbol{\Omega}_{Z E}$, und $\Omega_{Z E}=\boldsymbol{A}^{-1} \boldsymbol{B}_{E}$ hat dieselbe Struktur wie $\boldsymbol{B}_{E}$. Da das aber nur möglich ist für $Z=E$ wird klar, daß, falls ein Widerstandszentrum existiert, dieses mit dem Ebenen Widerstandszentrum übereinstimmt.

\section{B.2.2 Eine allgemeine ebene Aufhängung}

Die Bedingungen aus dem letzten Abschnitt sind nun für eine allgemeine ebene Aufhängung mit

$$
\boldsymbol{K}=\left(\begin{array}{ccc}
k_{1} & k_{4} & 0 \\
k_{4} & k_{2} & 0 \\
0 & 0 & k_{3}
\end{array}\right) \quad \text { und } \quad \boldsymbol{n}=\left(\begin{array}{c}
0 \\
0 \\
1
\end{array}\right)
$$


explizit nachzuprüfen. Es folgt

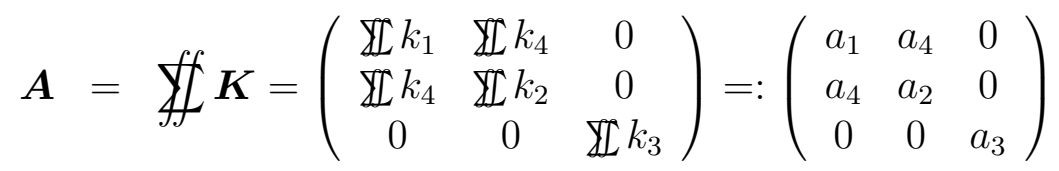

$$
\begin{aligned}
& \boldsymbol{B}=-\mathscr{f} \boldsymbol{K} \boldsymbol{\Omega}=\mathscr{f}\left(\begin{array}{ccc}
k_{1} & k_{4} & 0 \\
k_{4} & k_{2} & 0 \\
0 & 0 & k_{3}
\end{array}\right)\left(\begin{array}{ccc}
0 & 0 & -y \\
0 & 0 & x \\
y & -x & 0
\end{array}\right) \\
& =\mathscr{f}\left(\begin{array}{ccc}
0 & 0 & -k_{1} y+k_{4} x \\
0 & 0 & k_{2} x-k_{4} y \\
k_{3} y & -k_{3} x & 0
\end{array}\right) \\
& \boldsymbol{C}=-\int \boldsymbol{\Omega} \boldsymbol{K} \boldsymbol{\Omega}=\left(\begin{array}{ccc}
0 & 0 & -y \\
0 & 0 & x \\
y & -x & 0
\end{array}\right)\left(\begin{array}{ccc}
0 & 0 & -k_{1} y+k_{4} x \\
0 & 0 & k_{2} x-k_{4} y \\
k_{3} y & -k_{3} x & 0
\end{array}\right) \\
& =\mathscr{f}\left(\begin{array}{ccc}
k_{3} y^{2} & -k_{3} x y & 0 \\
-k_{3} x y & k_{3} x^{2} & 0 \\
0 & 0 & k_{1} y^{2}-2 k_{4} x y+k_{2} x^{2}
\end{array}\right) \text {. }
\end{aligned}
$$

An dem Aufbau von $\boldsymbol{A}$ und $\boldsymbol{C}$ lassen sich sofort die gewünschten Bedingungen (B.11) und (B.12) verifizieren. Die Bedingungen (B.10) und (B.12) führen beide auf die Gleichungen

$$
\begin{aligned}
0 & =-\oiiint k_{1} y+\oiiint k_{4} x \\
0 & =\oiiint k_{2} x-\oiiint k_{4} y
\end{aligned}
$$

welche die Bestimmungsgleichungen für die Koordinaten von $E$ sind. Mit der Translation $x=x_{E}+\xi$ und $y=y_{E}+\eta$ erhält man das folgende Gleichungssystem für das Ebene Widerstandszentrum $E$

$$
\begin{aligned}
a_{2} x_{E}-a_{4} y_{E} & =\oiiint k_{2} \xi-\oiiint k_{4} \eta \\
-a_{4} x_{E}+a_{1} y_{E} & =\oiiint k_{1} \eta-\oiiint k_{4} \xi
\end{aligned}
$$

mit der Lösung

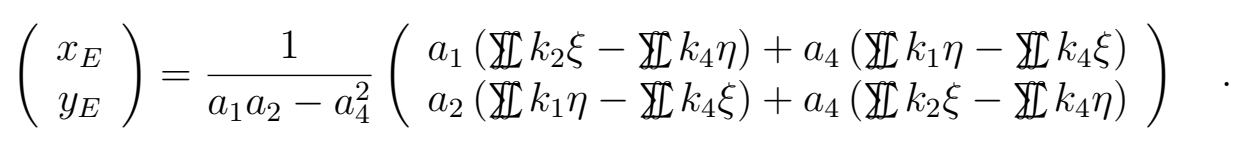

Das ist der gesuchte Mittelpunkt der Federkräfte einer allgemeinen ebenen Aufhängung. Selbst diese Formel entspricht noch nicht dem Schwerpunkt der Aufhängung; daher soll im Folgenden untersucht werden, wann das der Fall ist. 


\section{B.2.3 Vergleich mit dem Schwerpunkt der Aufhängung}

Für ein Medium ohne Scherelastizität gilt $k_{4}=0$ und $a_{4}=0$. Dann aber folgt

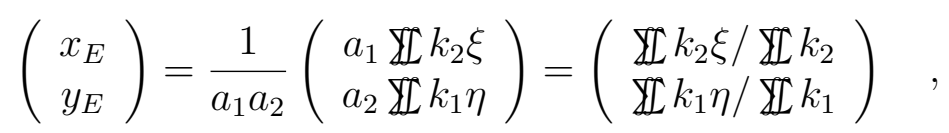

wobei die „Massen“ der Aufhängung gerade die sind, die zu den senkrechten Komponenten gehören. Bei isotropen Aufhängungen ist $k_{1}=k_{2}=: k$, und man darf durchaus von der „Stärke“ einer Aufhängung sprechen. In diesem Fall erst ist, wie auch aus dem räumlichen Fall bekannt, die Analogie zum Schwerpunkt perfekt, es gilt dann

$$
\left(\begin{array}{l}
x_{E} \\
y_{E}
\end{array}\right)=\frac{1}{\mathbb{Z} k}\left(\begin{array}{c}
\mathbb{I C} k \xi \\
\mathbb{Z L} k \eta
\end{array}\right)
$$

und die Federstärke $k$ hat die Rolle der Masse übernommen. Für eine konstante, aber nicht notwendigerweise isotrope Aufhängung kürzen sich die Stärken in der jeweiligen Richtung heraus, und es folgt

$$
\left(\begin{array}{l}
x_{E} \\
y_{E}
\end{array}\right)=\frac{1}{\mathbb{X} 1}\left(\begin{array}{l}
\mathbb{X L} \xi \\
\mathbb{Z L} \eta
\end{array}\right) \text {, }
$$

was diesmal als rein geometrischer Schwerpunkt der Aufhängung gedeutet werden kann.

\section{B.2.4 Im räumlichen Formalismus}

Die räumliche Formel für das elastische Zentrum liefert mit $a:=\left(a_{1} a_{2}-a_{4}^{2}\right)^{-1}$ zum Vergleich mit den obigen Ergebnissen

$$
\begin{aligned}
& \Omega_{Z}=-\left(\boldsymbol{A}^{-1} \boldsymbol{B}\right)^{A}
\end{aligned}
$$

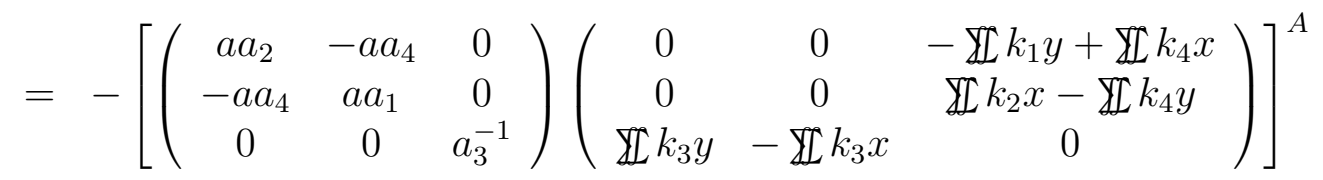

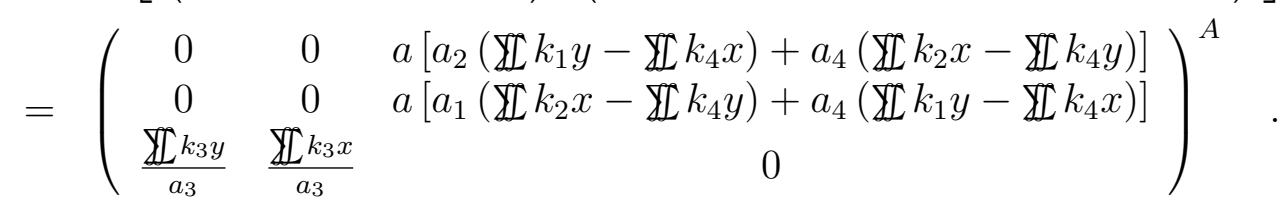

Die Übereinstimmung der Formel (B.13) in $E$ mit den Elementen $\boldsymbol{\Omega}_{13}$ und $\boldsymbol{\Omega}_{23}$ läßt hoffen, daß sich ein der räumlichen Formel ähnelnder Ausdruck finden läßt; auf den ersten Blick würde man statt $\boldsymbol{z}=-\left(\boldsymbol{A}^{-1} \boldsymbol{B}\right)^{D}$ als Abwandlung einfach $\boldsymbol{z}_{E}=-2\left(\boldsymbol{A}^{-1} \boldsymbol{B} \boldsymbol{P}_{n}^{\|}\right)^{D}$ erwarten, da nur noch die eine Hälfte der Matrixelemente 
in die Rechnung eingeht. Die nun folgende Rechnung liefert aber zusätzliche Terme, die in Richtung der Ebenennormale liegen.

Falls ein gemeinsamer Eigenvektor zu $\boldsymbol{A}$ und $\boldsymbol{C}$ an einem oder mehreren bestimmten Punkten existiert, wird $E$ wie im letzten Abschnitt bestimmt aus den Gleichungen

$$
\begin{aligned}
& \mathbf{0}=\boldsymbol{B}_{E} \boldsymbol{P}_{n}^{\|}=\left(\boldsymbol{B}+\boldsymbol{A} \boldsymbol{\Omega}_{O E}\right) \boldsymbol{P}_{n}^{\|} \\
& \mathbf{0}=\boldsymbol{B}_{E}^{T} \boldsymbol{P}_{n}^{\perp}=\left(\boldsymbol{B}+\boldsymbol{A} \boldsymbol{\Omega}_{O E}\right)^{T} \boldsymbol{P}_{n}^{\perp}=\left(\boldsymbol{B}^{T}-\boldsymbol{\Omega}_{O E} \boldsymbol{A}\right) \boldsymbol{P}_{n}^{\perp} .
\end{aligned}
$$

Für den Rest dieser Rechnung möchte ich die Subskripte weglassen. Formt man (B.15) in die Form $\boldsymbol{\Omega} \boldsymbol{P}^{\|}=-\boldsymbol{A}^{-1} \boldsymbol{B} \boldsymbol{P}^{\|}$um, so erhält man mit (A.10)

$$
-\left(\boldsymbol{A}^{-1} \boldsymbol{B} \boldsymbol{P}^{\|}\right)^{D}=\boldsymbol{P}^{\|^{F}} \overrightarrow{O E}=\frac{1}{2}\left(\operatorname{Sp}\left(\boldsymbol{P}^{\|}\right) \boldsymbol{I}-\boldsymbol{P}^{\|}\right) \overrightarrow{O E}=\frac{1}{2} \boldsymbol{P}^{\perp} \overrightarrow{O E}
$$

also für den senkrechten Anteil von $\overrightarrow{O E}$ wie erwartet

$$
\boldsymbol{P}^{\perp} \overrightarrow{O E}=-2\left(\boldsymbol{A}^{-1} \boldsymbol{B} \boldsymbol{P}^{\|}\right)^{D}
$$

wobei die rechte Seite dieser Gleichung wegen

$$
\boldsymbol{n}^{T}\left(\boldsymbol{A}^{-1} \boldsymbol{B} \boldsymbol{P}^{\|}\right)^{D}=n_{i} \varepsilon_{i j k}\left(\boldsymbol{A}^{-1} \boldsymbol{B}\right)_{j l} n_{l} n_{k}=0
$$

tatsächlich keinen Beitrag parallel zu $\boldsymbol{n}$ liefert. Aus (B.16) erhält man mit $\boldsymbol{x}:=\overrightarrow{O E}$ durch Dualisieren

$$
\left(\boldsymbol{B}^{T} \boldsymbol{P}^{\perp}\right)^{D}=\left(\boldsymbol{\Omega} \boldsymbol{A} \boldsymbol{P}^{\perp}\right)^{D} \stackrel{(A .10)}{=}\left(\boldsymbol{A} \boldsymbol{P}^{\perp}\right)^{F} \boldsymbol{x}=\frac{1}{2}\left(\operatorname{Sp}\left(\boldsymbol{A} \boldsymbol{P}^{\perp}\right) \boldsymbol{I}-\boldsymbol{A} \boldsymbol{P}^{\perp}\right) \boldsymbol{x} .
$$

Da die senkrechte Komponente von $\boldsymbol{x}$ bereits bekannt ist, kann man nach Multiplikation mit $\boldsymbol{P}^{\|}$wegen $\boldsymbol{P}^{\|} \boldsymbol{A} \boldsymbol{P}^{\perp}=\mathbf{0}$ folgern, daß

$$
\boldsymbol{P}^{\|} \overrightarrow{O E}=\boldsymbol{P}^{\|} \boldsymbol{x}=\frac{2}{\operatorname{Sp}\left(\boldsymbol{A} \boldsymbol{P}^{\perp}\right)} \boldsymbol{P}^{\|}\left(\boldsymbol{B}^{T} \boldsymbol{P}^{\perp}\right)^{D}
$$

Nun gilt aber

$$
\begin{aligned}
\left(\boldsymbol{B}^{T} \boldsymbol{P}^{\perp}\right)^{D} & =\left(-\boldsymbol{P}^{\perp^{T}} \boldsymbol{B}\right)^{D}=-\left(\boldsymbol{P}^{\perp} \boldsymbol{B}\right)^{D \stackrel{(A .2)}{=}}-\left(-\boldsymbol{\Omega}_{n}^{2} \boldsymbol{B}\right)^{D} \\
\stackrel{(A .100}{=} & \left(\boldsymbol{\Omega}_{n} \boldsymbol{B}\right)^{F} \boldsymbol{n}=\frac{1}{2}\left(\operatorname{Sp}\left(\boldsymbol{\Omega}_{n} \boldsymbol{B}\right) \boldsymbol{I}-\boldsymbol{\Omega}_{n} \boldsymbol{B}\right) \boldsymbol{n}
\end{aligned}
$$

und mit

$$
\operatorname{Sp}\left(\boldsymbol{\Omega}_{n} \boldsymbol{B}\right)=\varepsilon_{i j k} n_{j} B_{k i}=\varepsilon_{j k i} B_{k i} n_{j}=B_{j}^{D} n_{j}=\boldsymbol{n} \boldsymbol{B}^{D}
$$

erhält man insgesamt für das ebene Widerstandszentrum die Formel

$$
\begin{aligned}
\overrightarrow{O E} & =\boldsymbol{P}^{\|} \overrightarrow{O E}+\boldsymbol{P}^{\perp} \overrightarrow{O E} \\
& =-2\left(\boldsymbol{A}^{-1} \boldsymbol{B} \boldsymbol{P}^{\|}\right)^{D}+\frac{1}{\mathrm{Sp}\left(\boldsymbol{A} \boldsymbol{P}^{\perp}\right)} \boldsymbol{P}^{\|} \boldsymbol{B}^{D} .
\end{aligned}
$$


Bis auf den Zusatzterm entspricht diese der obigen Vermutung.

Diese Formel besagt, daß, falls eine Richtung (und ein Bezugssystem) mit den gewünschten Eigenschaften gefunden werden kann, immer ein ebenes Widerstandszentrum existiert. Das Hauptproblem liegt also bei der Bestimmung des Normalenvektors, insbesondere bei realen Daten. Einfacher ist die vorläufige Bestimmung von $\boldsymbol{z}_{E}$ aus (B.15) und (B.16) und die Überprüfung der Lösung an $\boldsymbol{C}_{E}$.

Der erste Teil der Formel (B.18) läß sich im elastischen Zentrum etwas verbindlicher schreiben. Für den senkrechten Anteil folgt mit der Definition der Dualisierung (A.9)

$$
\begin{aligned}
\left(\boldsymbol{P}^{\perp} \overrightarrow{Z E}\right)_{i} & =-2 \cdot-\frac{1}{2} \varepsilon_{i j k}\left(\boldsymbol{A}^{-1} \boldsymbol{B}\right)_{j m} n_{m} n_{k} \\
& =\varepsilon_{i j k}(-\boldsymbol{W} \boldsymbol{n})_{j} n_{k} \\
& =(\boldsymbol{n} \times(\boldsymbol{W} \boldsymbol{n}))_{i},
\end{aligned}
$$

also in Worten

Existiert das Ebene Widerstandszentrum, so liegt es auf einem Lotfußpunkt der Achsfläche bezüglich der Widerstandsebene.

\section{B.3 Der lineare Zwangskontakt}

In der Natur sind Synarthrosen und Diarthrosen oft mechanisch gekoppelt, etwa bei den Wirbelbogengelenken oder bei den Approximalkontakten der Zähne.

Für kleine Auslenkungen bekommt man in nicht allzu weit entfernten Punkten $P$ eine wiederum kleine Auslenkung. Ist nun $P$ der Kontaktpunkt eines Gelenkes, also der Berührpunkt der Gelenkflächen einer Synarthrose, so lassen sich beide Flächen in der Umgebung dieses Punktes durch die gemeinsame Schmiegeebene approximieren. Abgesehen also von der Wanderung des Kontaktpunktes während der Bewegung - welche nur Effekte in zweiter Ordnung hervorruft - findet die Bewegung des Kontaktpunktes für genügend kleine Auslenkungen in dieser Schmiegeebene statt.

Dann aber lassen sich die Zwangsbedingungen mathematisch durch lineare Gleichungen beschreiben, was das Problem lösbar macht. Andere Zwangsbedingungen, wie z. B. eine dimere $\operatorname{Kette}^{2}$ führen auf nichtlineare Gleichungen und stellen einen

\footnotetext{
${ }^{2}$ Zur Erklärung dieses Modells der Bewegung organischer Gelenke vgl. etwa [16] oder [49]. Eine dimere Kette ist ein Kontakt aus als Kreise genäherte Schnitten durch die Gelenkflächen in der Ebene der gedachten Bewegung. Da die beiden Mittelpunkte $M_{1}$ und $M_{2}$ der Kreise in allen Bewegungszuständen konstanten Abstand haben, ist die Anzahl der Freiheitsgrade um einen reduziert. Dieses Modell ist streng genommen, wie der lineare Zwangskontakt auch, auch
} 
dann vor mathematisch höchstens noch in sehr speziellen Fällen lösbare Aufgaben. Für die Anschauung sind sie dennoch brauchbar.

Daher halte ich lineare Zwangsbedingungen für angemessen, um die Bewegungseinschränkung z. B. durch Approximalkontakte oder Wirbelbogengelenke für kleine Bewegungen zu beschreiben.

Es soll der ungefesselte mit dem gefesselten Fall verglichen werden. Die gefesselten Größen werden im Folgenden mit einem Strich gekennzeichnet, etwa $*^{\prime}$.

\section{B.3.1 Angreifende und tatsächlich wirkende Lasten}

Im Punkt $P$ wirkt sich die Fesselung wie folgt aus:

1. Drehmomente werden nicht beeinflußt, d. h.

$$
\boldsymbol{T}_{P}{ }^{\prime}=\boldsymbol{T}_{P}
$$

2. Der zur Normalen $\boldsymbol{n}$ der Schmiegeebene paralle Anteil der im Kontaktpunkt $P$ angreifenden Kraft wird von der Gelenkfläche aufgefangen, d. h. die tatsächlich auf die elastische Aufhängung wirkende Kraft ist

$$
\boldsymbol{F}_{P}{ }^{\prime}=\boldsymbol{F}_{P}-\boldsymbol{P}_{n}^{\|} \boldsymbol{F}_{P}=\boldsymbol{P}_{n}^{\perp} \boldsymbol{F}_{P}
$$

3. Das wird nun als neues, in $\mathrm{P}$ wirkendes Lastsystem $f^{\prime}$ aufgefaßt, also im Matrixformalismus insgesamt

$$
f^{\prime}=\underbrace{\left(\begin{array}{cc}
\boldsymbol{P}_{n}^{\perp} & \mathbf{0} \\
\mathbf{0} & \boldsymbol{I}
\end{array}\right)}_{=: \mathcal{Z}_{P}} f .
$$

Als Nächstes wird die Wirkung der Fesselung im Punkt $A$ betrachtet. Dazu sind drei Schritte nötig:

nur lokal gültig, da die Gelenkflächen über den Bewegungsbereich eine veränderliche Krümmung haben. Für annähernd ebene Bewegungen und ungefähr konstante Krümmungen sollte dieses Modell aber eine gute Approximation der tatsächlichen Bewegung über einen großen Bereich liefern. Die Bewegung einer dimeren Kette kann im ebenen Fall auf die beiden Freiheitsgrade zweier Scharniergelenke mit konstantem Abstand reduziert werden, sie ist dann flächenläufig. Wegen dieser technischen Bauform trägt die dimere Kette auch den Namen Gelenkzweischlag. Die zugehörige auch im räumlichen Fall angemessene Nebenbedingung

$$
\left|\overrightarrow{M_{1} M_{2}}\right|^{2}=\text { const }
$$

ist quadratisch, und daher sind dimere Ketten nicht Gegenstand dieses Kapitels. Das räumliche Pendant einer dimeren Kette, etwa eines inkongruenten Eigelenkes, ist eine Anordnung aus vier um $90^{\circ}$ verdrehten Scharniergelenken. 
1. Transformation $f_{P}=\mathcal{T}_{P A} f_{A}$,

2. Fesselung $f_{P}{ }^{\prime}=\mathcal{Z}_{P} f_{P}$ und

3. Rücktransformation $f_{A}{ }^{\prime}=\mathcal{T}_{A P} f_{P}{ }^{\prime}$,

also insgesamt

$$
\mathcal{Z}_{A(P)}=\mathcal{T}_{A P} \mathcal{Z}_{P} \mathcal{T}_{P A}
$$

Die Komponenten der Fesselungsmatrix ergeben sich nun über

$$
\begin{aligned}
\mathcal{Z}_{A(P)} & =\left(\begin{array}{cc}
\boldsymbol{I} & \mathbf{0} \\
\boldsymbol{\Omega}_{A P} & \boldsymbol{I}
\end{array}\right)\left(\begin{array}{cc}
\boldsymbol{P}_{n}^{\perp} & \mathbf{0} \\
\mathbf{0} & \boldsymbol{I}
\end{array}\right)\left(\begin{array}{cc}
\boldsymbol{I} & \mathbf{0} \\
\boldsymbol{\Omega}_{P A} & \boldsymbol{I}
\end{array}\right) \\
& =\left(\begin{array}{cc}
\boldsymbol{I} & \mathbf{0} \\
\boldsymbol{\Omega}_{A P} & \boldsymbol{I}
\end{array}\right)\left(\begin{array}{cc}
\boldsymbol{P}_{n}^{\perp} & \mathbf{0} \\
\boldsymbol{\Omega}_{P A} & \boldsymbol{I}
\end{array}\right) \\
& =\left(\begin{array}{cc}
\boldsymbol{P}_{n}^{\perp} & \mathbf{0} \\
\boldsymbol{\Omega}_{A P} \boldsymbol{P}_{n}^{\perp}+\boldsymbol{\Omega}_{P A} & \boldsymbol{I}
\end{array}\right)
\end{aligned}
$$

Mit $\boldsymbol{\Omega}_{A P} \boldsymbol{P}_{n}^{\perp}+\boldsymbol{\Omega}_{P A}=\boldsymbol{\Omega}_{P A}-\boldsymbol{\Omega}_{P A} \boldsymbol{P}_{n}^{\perp}=\boldsymbol{\Omega}_{P A}\left(\boldsymbol{I}-\boldsymbol{P}_{n}^{\perp}\right)=\boldsymbol{\Omega}_{P A} \boldsymbol{P}_{n}^{\|}$folgt schließlich

$$
\mathcal{Z}_{A(P)}=\left(\begin{array}{cc}
\boldsymbol{P}_{n}^{\perp} & \mathbf{0} \\
\boldsymbol{\Omega}_{P A} \boldsymbol{P}_{n}^{\|} & \boldsymbol{I}
\end{array}\right) \quad .
$$

Aus der eben hergeleiteten Beziehung folgt aber sofort

$$
\begin{aligned}
\mathcal{T}_{B A} \mathcal{Z}_{A(P)} & =\mathcal{T}_{B A} \mathcal{T}_{A P} \mathcal{Z}_{P} \mathcal{T}_{P A}=\mathcal{T}_{B P} \mathcal{Z}_{P} \mathcal{T}_{P A}=\mathcal{T}_{B P} \mathcal{Z}_{P} \mathcal{T}_{P B} \mathcal{T}_{B A} \\
& =\mathcal{Z}_{B(P)} \mathcal{T}_{B A}
\end{aligned}
$$

also daß es gleich ist, ob man zunächst die Last transformiert und dann die Fesselung wirken läßt, oder umgekehrt. Daher kann tatsächlich von einem äquivalenten gefesselten Lastsystem gesprochen werden. Die Fesselung läßt sich offenbar in jedem Punkt durch eine lineare Abbildung der Lasten beschreiben, ein Grund, weshalb ich diese Art des Zwangskontaktes linear genannt habe. Der andere Grund ist die Form der Zwangsbedingung selber. Die Gleichung $\boldsymbol{n}^{T} \boldsymbol{F}=0$ lautet in verallgemeinerten Koordinaten

$$
z^{T} q=0
$$

mit $z^{T}:=\left(\boldsymbol{n}^{T}, \mathbf{0}^{\mathbf{T}}\right)$. Sie ist ebenfalls linear.

\section{B.3.2 Tatsächlich mögliche Lagen}

Eine Einschränkung der Bewegung im Ortsraum, wieder im Punkt $P$, wirkt sich nur auf die Translation $\delta \boldsymbol{d}_{P}$ aus. Die lineare Fesselung wirkt offenbar nur auf die polaren Vektoren, d. h. auch die Zwangsbedingung für die Lagen wird mit demselben Vektor 
$z$ beschrieben wie für die Lasten. Analog zu den Überlegungen für die Lasten gilt für die Lagen

$$
\delta \boldsymbol{\theta}_{P}{ }^{\prime}=\delta \boldsymbol{\theta}_{P} \quad \text { und } \quad \delta \boldsymbol{d}_{P}{ }^{\prime}=\boldsymbol{P}_{n}^{\perp} \delta \boldsymbol{d}_{P}
$$

also im Matrixformalismus wieder

$$
\delta q_{P}{ }^{\prime}=\mathcal{Z}_{P} \delta q_{P}
$$

Mit dem Transformationsverhalten der Lagen erhält man nun zum Punkt $A$ mit $\mathcal{Z}_{P}^{T}=\mathcal{Z}_{P}$ das Verhalten

$$
\delta q_{A}{ }^{\prime}=\mathcal{T}_{P A}^{T} \mathcal{Z}_{P} \mathcal{T}_{A P}^{T} \delta q_{A}=\left(\mathcal{T}_{A P}^{T} \mathcal{Z}_{P}^{T} \mathcal{T}_{P A}\right)^{T} \delta q_{A}=\left(\mathcal{T}_{A P}^{T} \mathcal{Z}_{P} \mathcal{T}_{P A}\right)^{T} \delta q_{A}=\mathcal{Z}_{A(P)}^{T} \delta q_{A}
$$

Durch Transposition der entsprechenden Gleichung für die Lasten folgt wieder die Vertauschbarkeit von Translation und Fesselung

$$
\mathcal{Z}_{B(P)}^{T} \mathcal{T}_{A B}^{T}=\mathcal{T}_{A B}^{T} \mathcal{Z}_{B(P)}^{T}
$$

und es kann, wieder analog zu den Lasten, von linear gefesselten Lagesystemen gesprochen werden. Damit ist auch eine lineare Abbildung zwischen den gefesselten Lasten und Lagen in allen Bezugssystemen linear.

\section{B.3.3 Die Flexibilitätsmatrix eines linear gefesselten Sys- tems}

Die Flexibilitätsmatrix soll wieder als lineares Input-Ouput-Modell für $q^{\prime}\left(f^{\prime}\right)$ betrachtet werden. Damit bekommt man die Flexibilitätsmatrix des gefesselten Systems über die drei Schritte

1. Die angreifende Last wird auf die tatsächlich wirkende Last zurechtgestutzt,

2. die Flexibilitätsmatrix liefert die zur tatsächlich wirkenden Last gehörenden Auslenkungen des ungefesselten Systems, und

3. auf diese von der elastischen Aufhängung allein angestrebten Lagen wirkt die Fesselung erneut.

Insgesamt erhält man die Flexibilitätsmatrix eines gefesselten Systems als Produkt der drei diese Operationen beschreibenden Matrizen, also

$$
\mathcal{F}_{A}^{\prime}=\mathcal{Z}_{A(P)}^{T} \mathcal{F}_{A} \mathcal{Z}_{A(P)}
$$

Die Gesamtmatrix ist offenbar wieder symmetrisch. Da die Fesselungsmatrix $\mathcal{Z}$ Projektoren enthält, ist die gefesselte Flexibilitätsmatrix $\mathcal{F}^{\prime}$ nicht mehr invertierbar, zumindest nicht im gesamten Raum. 
Ausgehend von der ursprünglichen Flexibilitätsmatrix ergeben sich nun die Komponenten der Flexibilitätsmatrix des gefesselten Systems. Da der Parallelprojektor $\boldsymbol{P}_{n}^{\|}$immer im Zusammenhang mit $\boldsymbol{\Omega}_{P A}$ auftaucht, erhält man mit $\boldsymbol{Z}:=\boldsymbol{\Omega}_{P A} \boldsymbol{P}_{n}^{\|}$

$$
\begin{aligned}
& \mathcal{F}_{A}{ }^{\prime}=\left(\begin{array}{cc}
\boldsymbol{P}_{n}^{\perp} & -\boldsymbol{P}_{n}^{\|} \boldsymbol{\Omega}_{P A} \\
\mathbf{0} & \boldsymbol{I}
\end{array}\right)\left(\begin{array}{cc}
\boldsymbol{D} & \boldsymbol{S} \\
\boldsymbol{S}^{T} & \boldsymbol{M}
\end{array}\right)\left(\begin{array}{cc}
\boldsymbol{P}_{n}^{\perp} & \mathbf{0} \\
\boldsymbol{\Omega}_{P A} \boldsymbol{P}_{n}^{\|} & \boldsymbol{I}
\end{array}\right) \\
& =\left(\begin{array}{cc}
\boldsymbol{P}_{n}^{\perp} & \boldsymbol{Z}^{T} \\
\mathbf{0} & \boldsymbol{I}
\end{array}\right)\left(\begin{array}{cc}
\boldsymbol{D} & \boldsymbol{S} \\
\boldsymbol{S}^{T} & \boldsymbol{M}
\end{array}\right)\left(\begin{array}{cc}
\boldsymbol{P}_{n}^{\perp} & \mathbf{0} \\
\boldsymbol{Z} & \boldsymbol{I}
\end{array}\right) \\
& =\left(\begin{array}{cc}
\boldsymbol{P}^{\perp} & \boldsymbol{Z}^{T} \\
\mathbf{0} & \boldsymbol{I}
\end{array}\right)\left(\begin{array}{cc}
\boldsymbol{D} \boldsymbol{P}^{\perp}+\boldsymbol{S} \boldsymbol{Z} & \boldsymbol{S} \\
\boldsymbol{S}^{T} \boldsymbol{P}^{\perp}+\boldsymbol{M} \boldsymbol{Z} & \boldsymbol{M}
\end{array}\right) \\
& =\left(\begin{array}{cc}
\boldsymbol{P}^{\perp}\left(\boldsymbol{D} \boldsymbol{P}^{\perp}+\boldsymbol{S} \boldsymbol{Z}\right)+\boldsymbol{Z}^{T}\left(\boldsymbol{S}^{T} \boldsymbol{P}^{\perp}+\boldsymbol{M} \boldsymbol{Z}\right) & \boldsymbol{P}^{\perp} \boldsymbol{S}+\boldsymbol{Z}^{T} \boldsymbol{M} \\
\boldsymbol{S}^{T} \boldsymbol{P}^{\perp}+\boldsymbol{M} \boldsymbol{Z} & \boldsymbol{M}
\end{array}\right) \\
& =\left(\begin{array}{cc}
\boldsymbol{P}^{\perp} \boldsymbol{D} \boldsymbol{P}^{\perp}+\boldsymbol{P}^{\perp} \boldsymbol{S} \boldsymbol{Z}+\boldsymbol{Z}^{T} \boldsymbol{S}^{T} \boldsymbol{P}^{\perp}+\boldsymbol{Z}^{T} \boldsymbol{M} \boldsymbol{Z} & \boldsymbol{P}^{\perp} \boldsymbol{S}+\boldsymbol{Z}^{T} \boldsymbol{M} \\
\boldsymbol{S}^{T} \boldsymbol{P}^{\perp}+\boldsymbol{M} \boldsymbol{Z} & \boldsymbol{M}
\end{array}\right) .
\end{aligned}
$$

Die Teilmatrix $\boldsymbol{M}$ hängt offenbar nicht von der Wirkung eines linearen Zwangskontaktes ab - ein weiterer Grund, sie für die Normal- und Realform einer elastischen Matrix heranzuziehen.

An den geänderten Nebendiagonalmatrizen erkennt man, daß bei linearen Zwangsbedingungen das elastische Zentrum nach der bisherigen Definition verschoben wird, denn es gilt

$$
\begin{aligned}
\overrightarrow{A Z^{\prime}} & =\left(\boldsymbol{S}^{\prime} \boldsymbol{M}^{\prime-1}\right)^{D}=\left(\left(\boldsymbol{P}^{\perp} \boldsymbol{S}+\boldsymbol{Z}^{T} \boldsymbol{M}\right) \boldsymbol{M}^{-1}\right)^{D} \\
& =\left(\boldsymbol{P}^{\perp}\left(\boldsymbol{S} \boldsymbol{M}^{-1}\right)+\boldsymbol{Z}^{T}\right)^{D}
\end{aligned}
$$

und mit $\boldsymbol{S} \boldsymbol{M}^{-1}=\boldsymbol{\Omega}_{A Z}+\boldsymbol{W}$ sowie $\boldsymbol{Z}^{T}=\left(\boldsymbol{\Omega}_{P A} \boldsymbol{P}^{\|}\right)^{T}=\boldsymbol{P}^{\|} \boldsymbol{\Omega}_{A P}$ folgt weiter

$$
\begin{aligned}
\overrightarrow{A Z^{\prime}} & =\left(\boldsymbol{P}^{\perp}\left(\boldsymbol{\Omega}_{A Z}+\boldsymbol{W}\right)\right)^{D}+\left(\boldsymbol{P}^{\|} \boldsymbol{\Omega}_{A P}\right)^{D} \\
\stackrel{\text { A.11 }}{=} & \left(\boldsymbol{P}^{\perp}\left(\boldsymbol{\Omega}_{A Z}+\boldsymbol{W}\right)\right)^{D}+\frac{1}{2} \boldsymbol{P}^{\perp} \overrightarrow{A P} \\
\stackrel{A .12}{=} & \overrightarrow{A Z}-\frac{1}{2} \boldsymbol{P}^{\perp} \overrightarrow{A Z}+\left(\boldsymbol{P}^{\perp} \boldsymbol{W}\right)^{D}+\frac{1}{2} \boldsymbol{P}^{\perp} \overrightarrow{A P} \\
& =\overrightarrow{A Z}+\frac{1}{2} \boldsymbol{P}^{\perp}(\overrightarrow{Z A}+\overrightarrow{A P})+\left(\boldsymbol{P}^{\perp} \boldsymbol{W}\right)^{D} \\
& =\overrightarrow{A Z}+\frac{1}{2} \boldsymbol{P}^{\perp} \overrightarrow{Z P}+\left(\boldsymbol{P}^{\perp} \boldsymbol{W}\right)^{D} .
\end{aligned}
$$

Existiert ein Widerstandszentrum, so ist $\boldsymbol{W}=\mathbf{0}$, und die dann gültige Formel

$$
\overrightarrow{A Z^{\prime}}=\overrightarrow{A Z}+\frac{1}{2} \boldsymbol{P}^{\perp} \overrightarrow{Z P}
$$

erlaubt eine einfache geometrische Interpretation: 
Der lineare Zwangskontakt verschiebt ein Widerstandszentrum um die Hälfte des zur Normalen senkrechten Anteils des Ortsvektors vom Widerstandszentrum zum Kontaktpunkt hin.

\section{B.3.4 Steifheitsmatrix und Potential eines linear gefessel- ten Systems}

Betrachtet man nun das lineare Input-Output-Modell für $f^{\prime}\left(q^{\prime}\right)$, also schon für die passend zurechtgestutzten Lasten und Lagen, so erhält man die Steifheitsmatrix des gefesselten Systems

$$
\mathcal{S}^{\prime}=\mathcal{Z} \mathcal{S} \mathcal{Z}^{T}
$$

welche wieder symmetrisch ist. Diese kann aus dem elastischen Potential

$$
\Phi=\frac{1}{2} q^{\prime T} \mathcal{S} q^{\prime}=\frac{1}{2}\left(\mathcal{Z}^{T} q\right)^{T} \mathcal{S}\left(\mathcal{Z}^{T} q\right)=\frac{1}{2} q^{T} \mathcal{Z} \mathcal{S} \mathcal{Z}^{T} q
$$

abgeleitet werden und ist nicht im gesamten Raum invertierbar. Der Grund dafür ist, daß bestimmte Koordinaten nicht mehr zur Beschreibung des Systems nötig sind, hier die zu $\boldsymbol{n}$ parallelen Anteile der Translation. Es wird, geometrisch gesprochen, nur noch in einem Unterraum der verallgemeinerten Koordinaten gearbeitet. Für die zugehörigen verallgemeinerten Kräfte folgt allein aus der Existenz eines Potentials

$$
\begin{aligned}
f^{\prime T} & =-\frac{\partial \Phi}{\partial q^{\prime}}=-\left(\frac{\partial q^{\prime}}{\partial \Phi}\right)^{-1}=-\left(\frac{\partial \mathcal{Z}^{T} q}{\partial \Phi}\right)^{-1}=-\left(\frac{\partial q}{\partial \Phi}\right)^{-1} \mathcal{Z}^{T-}=-\frac{\partial \Phi}{\partial q} \mathcal{Z}^{T-} \\
& =f^{T} \mathcal{Z}^{T-}
\end{aligned}
$$

Die Pseudoinverse $*^{-}$besagt hier nur, daß nach der einen Koordinate nicht differenziert wird, also daß auch keine zugehörige verallgemeinerte Kraft existiert. Diese Pseudoinverse eines Projektors auf einen Unterraum ist demnach der Projektor selber. Da er im entsprechenden Unterraum wie eine Einheitsmatrix wirkt, gilt also wie gewünscht

$$
f^{\prime}=\mathcal{Z} f
$$

Da Lasten und Lagen mit derselben Matrix gefesselt werden, ist der Raum der Lasten und Lagen vollständig separiert in Anteile parallel und senkrecht zu z. Die elastischen Matrizen sind daher von der Form $\langle z\rangle \otimes\langle z\rangle \oplus\langle z\rangle^{\perp} \otimes\langle z\rangle^{\perp}$. In $\langle z\rangle^{\perp} \otimes\langle z\rangle^{\perp}$ allein sind beide Matrizen invertierbar. Die im gesamten Raum davon unabhängigen Anteile in $\langle z\rangle \otimes\langle z\rangle$ äußern sich in der Flexibilitätsmatrix in Nullen, und in der Steifheitsmatrix entsprechend in unendlich großen Einträgen.

\section{B.3.5 Zwei lineare Fesselungen}

Die meisten über Diarthrosen gekoppelten Synarthrosen besitzen zwei Kontakte: Die Wirbelbogengelenken etwa liegen paarweise vor und Zähne haben zwei Appro- 
ximalkontakte. Selbst ein so kompliziertes Gelenk wie das Kniegelenk fällt in die Kategorie der zweikontaktigen Synarthrosen, und zwar wenn man den Bandapparat samt Knorpel als Synarthrose betrachtet und den Flexionswinkel als äußeren Parameter betrachtet.

Da die Anzahl der Freiheitsgrade nun um zwei vermindert ist, hat die Matrix der Fesselung nur noch Rang vier.

Bei den Lagen, also den verallgemeinerten Koordinaten, sind jetzt nur noch Translationen in der zu beiden Schmiegeebenen normalen Richtung möglich. Für eine reine Translation folgt also

$$
\delta \boldsymbol{d}^{\prime}=\boldsymbol{P}_{n_{1} \times n_{2}}^{\|} \delta \boldsymbol{d}
$$

wobei $\boldsymbol{n}_{1}$ und $\boldsymbol{n}_{2}$ die Normalenvektoren der Schmiegeebenen sind. Die Anteile längs des Kreuzproduktes der Normalen, $\boldsymbol{P}_{n_{1} \times n_{2}}^{\perp} \delta \boldsymbol{d}$ werden von den Gelenken behindert.

Drehungen rufen Translationen hervor und werden nur über deren Transformationsverhalten behindert. Diese Translationen müssen in den zwei Kontaktpunkten $P_{1}$ und $P_{2}$ immer senkrecht zu den Ebenennormalen sein. Die Verhältnisse werden besonders einfach im Punkt $S$, der in der Mitte zwischen $P_{1}$ und $P_{2}$ liegt, also in

$$
\overrightarrow{O S}:=\frac{1}{2}\left(\overrightarrow{O P}_{1}+\overrightarrow{O P}_{2}\right)
$$

Damit bei einer reinen Drehung die Punkte auf den Schmiegeebenen laufen, gilt dort

$$
\mathbf{0}=\boldsymbol{n}_{1}\left(\overrightarrow{S P}_{1} \times \delta \boldsymbol{\theta}\right) \quad \text { und } \quad \mathbf{0}=\boldsymbol{n}_{2}\left(\overrightarrow{S P}_{2} \times \delta \boldsymbol{\theta}\right),
$$

was sich mit $\boldsymbol{p}:=\frac{1}{2} \vec{P}_{1}$ kürzer schreiben läßt als

$$
\mathbf{0}=\boldsymbol{n}_{1}(\boldsymbol{p} \times \delta \boldsymbol{\theta})=\left(\boldsymbol{n}_{1} \times \boldsymbol{p}\right) \delta \boldsymbol{\theta} \quad \text { und } \quad \mathbf{0}=\boldsymbol{n}_{2}(\boldsymbol{p} \times \delta \boldsymbol{\theta})=\left(\boldsymbol{n}_{2} \times \boldsymbol{p}\right) \delta \boldsymbol{\theta} .
$$

Nur Drehungen um die gemeinsame Senkrechte

$$
\left(\boldsymbol{n}_{1} \times \boldsymbol{p}\right) \times\left(\boldsymbol{n}_{2} \times \boldsymbol{p}\right)=\boldsymbol{P}_{p}^{\|}\left(\boldsymbol{n}_{1} \times \boldsymbol{n}_{2}\right),
$$

welche einfach $\boldsymbol{p}$ selber ist, werden also von den Gelenken zugelassen, ohne Translationen hervorzurufen. Das ist anschaulich klar, denn die Drehachse ist dann die Verbindungslinie beider Kontaktpunkte.

Man vermutet also, daß die Matrix der Fesselung in $S$ die Form

$$
\mathcal{S}_{S}^{T}=\mathcal{Z}_{S}=\left(\begin{array}{cc}
\boldsymbol{P}_{n_{1} \times n_{2}}^{\|} & \mathbf{0} \\
\mathbf{0} & \boldsymbol{P}_{p}^{\|}
\end{array}\right)
$$

hat. In den beiden Kontaktpunkten erhält man mit diesem Projektor

$$
\mathcal{Z}_{P_{1,2}}^{T}=\left(\begin{array}{cc}
\boldsymbol{I} & \pm \boldsymbol{\Omega}_{p} \\
\mathbf{0} & \boldsymbol{I}
\end{array}\right)\left(\begin{array}{cc}
\boldsymbol{P}_{n_{1} \times n_{2}}^{\|} & \mathbf{0} \\
\mathbf{0} & \boldsymbol{P}_{p}^{\|}
\end{array}\right)\left(\begin{array}{cc}
\boldsymbol{I} & \mp \boldsymbol{\Omega}_{p} \\
\mathbf{0} & \boldsymbol{I}
\end{array}\right)
$$




$$
\begin{aligned}
& =\left(\begin{array}{cc}
\boldsymbol{I} & \pm \boldsymbol{\Omega}_{p} \\
\mathbf{0} & \boldsymbol{I}
\end{array}\right)\left(\begin{array}{cc}
\boldsymbol{P}_{n_{1} \times n_{2}}^{\|} & \mp \boldsymbol{P}_{n_{1} \times n_{2}}^{\|} \boldsymbol{\Omega}_{p} \\
\mathbf{0} & \boldsymbol{P}_{p}^{\|}
\end{array}\right) \\
& =\left(\begin{array}{cc}
\boldsymbol{P}_{n_{1} \times n_{2}}^{\|} & \mp \boldsymbol{P}_{n_{1} \times n_{2}}^{\|} \boldsymbol{\Omega}_{p} \\
\mathbf{0} & \boldsymbol{P}_{p}^{\|}
\end{array}\right),
\end{aligned}
$$

d. h. die Gleichung für die Fesselung hat in beiden Kontaktpunkten dieselbe Form, wie es sein muß. Diese Matrix kann aber den Fall zweier Kontaktpunkte nicht korrekt beschreiben, da sie nur den Rang zwei statt vier hat. Die Bewegungsanteile sind also immer durch die beiden Kontakte gekoppelt, und eine Betrachtung der Grenzfälle reiner Translation und Rotation wird dem nicht gerecht. Andererseits ist es klar, daß wieder eine elastische Matrix im Sinne eines Input-Output-Modells existieren sollte. Da die Anschauung hier offenbar nur begrenzt weiterhilft, wird im Folgenden abstrakt vorgegangen.

Die beiden Zwangsbedingungen in $P_{1}$ und $P_{2}$ lauten ausgedrückt in $S$

$$
\mathbf{0}=\boldsymbol{n}_{1} \delta \boldsymbol{d}-\left(\boldsymbol{n}_{1} \times \boldsymbol{p}\right) \delta \boldsymbol{\theta} \quad \text { und } \quad \mathbf{0}=\boldsymbol{n}_{2} \delta \boldsymbol{d}+\left(\boldsymbol{n}_{2} \times \boldsymbol{p}\right) \delta \boldsymbol{\theta} .
$$

In verallgemeinerten Koordinaten haben sie die Form

$$
z_{1}^{T} q=0 \quad \text { und } \quad z_{2}^{T} q=0
$$

wobei

$$
z_{1}^{T}=\left(\boldsymbol{n}_{1}^{T}, \quad-\left(\boldsymbol{n}_{1} \times \boldsymbol{p}\right)^{T}\right) \quad \text { und } \quad z_{2}^{T}=\left(\boldsymbol{n}_{2}^{T}, \quad\left(\boldsymbol{n}_{2} \times \boldsymbol{p}\right)^{T}\right),
$$

sie sind also beide linear. Um das Problem zu geometrisieren, müssen zunächst die unterschiedlichen Einheiten der Lagekomponenten berücksichtigt werden. Etwa mit

$$
\tilde{q}=\underbrace{\left(\begin{array}{cc}
\boldsymbol{I} & \mathbf{0} \\
\mathbf{0} & |\boldsymbol{p}| \boldsymbol{I}
\end{array}\right)}_{=: \mathcal{I}_{p}} q
$$

haben die verallgemeinerten Koordinaten die Einheit einer Länge, und die Zwangsbedingung hat mit

$$
\tilde{z}=\mathcal{I}_{p}^{-1} z
$$

weiterhin die Form

$$
\tilde{z}_{1}^{T} \tilde{q}_{1}=0 \quad \text { und } \quad \tilde{z}_{2}^{T} \tilde{q}_{2}=0,
$$

wobei die Vektoren $\tilde{z}_{1}$ und $\tilde{z}_{2}$ nun dimensionslos sind.

Gesucht ist nun ein Projektor, also eine symmetrische $6 \times 6$-Matrix $\tilde{\mathcal{Z}}_{S}^{T}$ mit

$$
\tilde{z}_{1}^{T} \tilde{\mathcal{Z}}_{S}^{T}=0 \quad \text { und } \quad \tilde{z}_{2}^{T} \tilde{\mathcal{Z}}_{S}^{T}=0 \quad \text { und } \quad\left(\tilde{\mathcal{Z}}_{S}^{T}\right)^{2}=\tilde{\mathcal{Z}}_{S}^{T} \quad \text { und } \quad \tilde{\mathcal{Z}}_{S}^{T}=\tilde{\mathcal{Z}}_{S}
$$

Den Projektor für die ursprünglichen Koordinaten $q$ erhält man durch Reskalierung auf die alten Einheiten, also aus $\tilde{q}^{\prime}=\tilde{\mathcal{Z}}_{S} \tilde{q}$ über

$$
q^{\prime}=\mathcal{I}_{p}^{-1} \tilde{q}^{\prime}=\mathcal{I}_{p}^{-1} \tilde{\mathcal{Z}}_{S} \tilde{q}=\underbrace{\mathcal{I}_{p}^{-1} \tilde{\mathcal{Z}}_{S} \mathcal{I}_{p}}_{=\mathcal{Z}_{S}} q
$$


Die allgemeine Form eines Projektors auf einen von den Vektoren $z_{1}, z_{2}, \ldots z_{n}$ aufgespannten Unterraum eines $\mathbb{R}^{m}$ ist wie folgt gegeben: ${ }^{3}$ Sei $\boldsymbol{X}$ die aus den Spaltenvektoren $z_{1}, z_{2}, \ldots z_{n}$ gebildete Matrix, also

$$
\boldsymbol{X}=\left(z_{1}, z_{2}, \ldots z_{n}\right) \quad .
$$

Dann ist der Projektor auf diesen Unterraum eindeutig gegeben durch

$$
\boldsymbol{P}^{\|}=\boldsymbol{X}\left(\boldsymbol{X}^{T} \boldsymbol{X}\right)^{-1} \boldsymbol{X}^{T}
$$

und hat den Rang $n$. Der Projektor senkrecht zu diesem Unterraum lautet

$$
\boldsymbol{P}^{\perp}=\boldsymbol{I}-\boldsymbol{P}^{\|}
$$

und hat Rang $m-n$. Da man statt mit den $n$ Vektoren $z_{1}, z_{2}, \ldots z_{n}$ mit $m-n$ Basisvektoren des orthogonalen Komplements hätte beginnen können, ist der Parallelprojektor des orthogonalen Komplements der Orthogonalprojektor des Unterraumes selber. In der Mathematik spricht man daher in beiden Fällen von einer orthogonalen Projektion.

Mit dieser Formel können also bis zu sechs lineare Zwangskontakte beschrieben werden. Berechnet wird dann zunächst der Parallelprojektor $\boldsymbol{P}^{\|}$der Zwangsbedingungen. $\boldsymbol{P}^{\|} q$ beschreibt die nicht möglichen Bewegungsanteile und $q-\boldsymbol{P}^{\|} q=\boldsymbol{P}^{\perp} q$ die unter Zwangsbedingungen möglichen, also gilt $\mathcal{Z}=\boldsymbol{P}_{z_{1}, z_{2}, \ldots z_{n}}^{\perp}$. Der Fall dreier Zwangsbedingungen könnte beispielsweise für das obere Sprunggelenk von Interesse sein. Zwanglauf, also der Fall einer einparametrigen Bewegung, liegt bei fünf punktförmigen Kontakten vor.

Im Fall zweier Zwangsbedingungen ist

$$
\begin{aligned}
\mathcal{Z}_{S} & =\mathcal{I}-\left(z_{1}, z_{2}\right)\left(\left(z_{1}, z_{2}\right)^{T}\left(z_{1}, z_{2}\right)\right)^{-1}\left(z_{1}, z_{2}\right)^{T} \\
& =\mathcal{I}-\left(z_{1}, z_{2}\right)\left(\left(\begin{array}{c}
z_{1}^{T} \\
z_{2}^{T}
\end{array}\right)\left(z_{1}, z_{2}\right)\right)^{-1}\left(\begin{array}{c}
z_{1}^{T} \\
z_{2}^{T}
\end{array}\right) \\
& =\mathcal{I}-\left(z_{1}, z_{2}\right)\left(\begin{array}{cc}
z_{1} \cdot z_{1} & z_{1} \cdot z_{2} \\
z_{2} \cdot z_{1} & z_{2} \cdot z_{2}
\end{array}\right)^{-1}\left(\begin{array}{c}
z_{1}^{T} \\
z_{2}^{T}
\end{array}\right) \\
& =\mathcal{I}-\frac{1}{z_{1}^{2} z_{2}^{2}-\left(z_{1} z_{2}\right)^{2}}\left(z_{1}, z_{2}\right)\left(\begin{array}{cc}
z_{2}^{2} & -z_{1} z_{2} \\
-z_{1} z_{2} & z_{1}^{2}
\end{array}\right)\left(\begin{array}{c}
z_{1}^{T} \\
z_{2}^{T}
\end{array}\right) \\
& =\mathcal{I}-\frac{1}{z_{1}^{2} z_{2}^{2}-\left(z_{1} z_{2}\right)^{2}}\left(z_{2}^{2} z_{1} z_{1}^{T}-\left(z_{1} z_{2}\right)\left(z_{1} z_{2}^{T}+z_{2} z_{1}^{T}\right)+z_{1}^{2} z_{2} z_{2}^{T}\right)
\end{aligned}
$$

die gesuchte Matrix für zwei lineare Fesselungen.

Es sollen nun die Komponenten des Parallelprojektors bestimmt werden. Mit den Abkürzungen

$$
\boldsymbol{r}:=\left(\boldsymbol{n}_{1} \times \boldsymbol{n}_{2}\right) /\left|\boldsymbol{n}_{1} \times \boldsymbol{n}_{2}\right|
$$

\footnotetext{
${ }^{3}$ Vgl. den Anhang zur Matrizenrechnung von 9 .
} 


$$
\begin{aligned}
c & :=\boldsymbol{n}_{1} \boldsymbol{n}_{2} \\
\boldsymbol{p}^{\circ} & :=\boldsymbol{p} /|\boldsymbol{p}| \\
p_{1} & :=\boldsymbol{n}_{1} \cdot \boldsymbol{p}^{\circ} \\
p_{2} & :=\boldsymbol{n}_{2} \cdot \boldsymbol{p}^{\circ}
\end{aligned}
$$

erhält man die Skalarprodukte

$$
\begin{aligned}
\tilde{z}_{1} \cdot \tilde{z}_{1} & =\boldsymbol{n}_{1}^{2}+\left(\boldsymbol{n}_{1} \times \boldsymbol{p}^{\circ}\right)^{2}=1+\left(\boldsymbol{n}_{1}^{2} \boldsymbol{p}^{\circ 2}-\left(\boldsymbol{p}^{\circ} \boldsymbol{n}_{1}\right)^{2}\right) \\
& =2-p_{1}^{2} \\
\tilde{z}_{2} \cdot \tilde{z}_{2} & =2-p_{2}^{2} \\
\tilde{z}_{1} \cdot \tilde{z}_{2} & =\boldsymbol{n}_{1} \boldsymbol{n}_{2}-\left(\boldsymbol{n}_{1} \times \boldsymbol{p}^{\circ}\right)\left(\boldsymbol{n}_{2} \times \boldsymbol{p}^{\circ}\right)=c-\left(\boldsymbol{n}_{1} \boldsymbol{n}_{2} \boldsymbol{p}^{\circ 2}-\left(\boldsymbol{p}^{\circ} \boldsymbol{n}_{1}\right)\left(\boldsymbol{p}^{\circ} \boldsymbol{n}_{1}\right)\right) \\
& =p_{1} p_{2},
\end{aligned}
$$

die Systemdeterminante

$$
\begin{aligned}
\tilde{z}_{1}^{2} \tilde{z}_{2}^{2}-\left(\tilde{z}_{1} \tilde{z}_{2}\right)^{2} & =\left(2-p_{1}^{2}\right)\left(2-p_{2}^{2}\right)-\left(p_{1} p_{2}\right)^{2} \\
& =4-2\left(p_{1}^{2}+p_{2}^{2}\right)
\end{aligned}
$$

und die Dyaden

$$
\begin{aligned}
\tilde{z}_{1} \tilde{z}_{1}^{T}= & \left(\begin{array}{cc}
\boldsymbol{n}_{1} \boldsymbol{n}_{1}^{T} & -\boldsymbol{n}_{1}\left(\boldsymbol{n}_{1} \times \boldsymbol{p}^{\circ}\right)^{T} \\
-\left(\boldsymbol{n}_{1} \times \boldsymbol{p}^{\circ}\right) \boldsymbol{n}_{1}^{T} & \left(\boldsymbol{n}_{1} \times \boldsymbol{p}^{\circ}\right)\left(\boldsymbol{n}_{1} \times \boldsymbol{p}^{\circ}\right)^{T}
\end{array}\right) \\
\tilde{z}_{2} \tilde{z}_{2}^{T}= & \left(\begin{array}{cc}
\boldsymbol{n}_{2} \boldsymbol{n}_{2}^{T} & \boldsymbol{n}_{2}\left(\boldsymbol{n}_{2} \times \boldsymbol{p}^{\circ}\right)^{T} \\
\left(\boldsymbol{n}_{2} \times \boldsymbol{p}^{\circ}\right) \boldsymbol{n}_{2}^{T} & \left(\boldsymbol{n}_{2} \times \boldsymbol{p}^{\circ}\right)\left(\boldsymbol{n}_{2} \times \boldsymbol{p}^{\circ}\right)^{T}
\end{array}\right) \\
\tilde{z}_{1} \tilde{z}_{2}^{T}= & \left(\begin{array}{cc}
\boldsymbol{n}_{1} \boldsymbol{n}_{2}^{T} & \boldsymbol{n}_{1}\left(\boldsymbol{n}_{2} \times \boldsymbol{p}^{\circ}\right)^{T} \\
-\left(\boldsymbol{n}_{1} \times \boldsymbol{p}^{\circ}\right) \boldsymbol{n}_{2}^{T} & -\left(\boldsymbol{n}_{1} \times \boldsymbol{p}^{\circ}\right)\left(\boldsymbol{n}_{2} \times \boldsymbol{p}^{\circ}\right)^{T}
\end{array}\right) \\
\tilde{z}_{1} \tilde{z}_{2}^{T}= & \left(\begin{array}{cc}
\boldsymbol{n}_{2} \boldsymbol{n}_{1}^{T} & -\boldsymbol{n}_{2}\left(\boldsymbol{n}_{1} \times \boldsymbol{p}^{\circ}\right)^{T} \\
\left(\boldsymbol{n}_{2} \times \boldsymbol{p}^{\circ}\right) \boldsymbol{n}_{1}^{T} & -\left(\boldsymbol{n}_{2} \times \boldsymbol{p}^{\circ}\right)\left(\boldsymbol{n}_{1} \times \boldsymbol{p}^{\circ}\right)^{T}
\end{array}\right)
\end{aligned}
$$

Die sich insgesamt mit (B.19) ergebenden Terme sind leider nicht einfach, so daß rückblickend klar wird, warum der Versuch einer anschaulichen Herleitung fehlschlug.

Eine letzte Bemerkung noch zu den Lasten. Da die Zwangskontakte zu der elastischen Aufhängung parallel geschaltet sind, lassen sich die Lasten in eine Summe aus den Zwangsbedingungen aufgenommenen und auf die Synarthrose wirkenden Anteile zerlegen, also

$$
f=f^{\prime}+f_{z}
$$

Die Zwangslasten stehen im Gleichgewicht mit den Normalkräften $\phi_{1} \boldsymbol{n}_{1}$ und $\phi_{2} \boldsymbol{n}_{2}$, genauer

$$
\begin{aligned}
& \mathbf{0}=\boldsymbol{F}_{z}+\boldsymbol{n}_{1} \phi_{1}+\boldsymbol{n}_{2} \phi_{2} \\
& \mathbf{0}=\boldsymbol{T}_{z}+\boldsymbol{p} \times\left(\boldsymbol{n}_{1} \phi_{1}-\boldsymbol{n}_{2} \phi_{2}\right) .
\end{aligned}
$$


Durch skalare Multiplikation mit $\boldsymbol{n}_{1}$ und $\boldsymbol{n}_{2}$ erhält man mit $F_{1,2}:=\boldsymbol{n}_{1,2} \boldsymbol{F}$ und $T_{1,2}:=\boldsymbol{n}_{1,2} \boldsymbol{T}$ daraus

$$
\left(\begin{array}{l}
F_{1} \\
F_{2}
\end{array}\right)=-\left(\begin{array}{ll}
1 & c \\
c & 1
\end{array}\right)\left(\begin{array}{l}
\phi_{1} \\
\phi_{2}
\end{array}\right)
$$

für die Kräfte, und

$$
\left(\begin{array}{l}
T_{1} \\
T_{2}
\end{array}\right)=\left(\begin{array}{cc}
0 & \left(\boldsymbol{n}_{1}, \boldsymbol{p}, \boldsymbol{n}_{2}\right) \\
-\left(\boldsymbol{n}_{2}, \boldsymbol{p}, \boldsymbol{n}_{1}\right) & 0
\end{array}\right)\left(\begin{array}{l}
\phi_{1} \\
\phi_{2}
\end{array}\right)=\left(\boldsymbol{p}, \boldsymbol{n}_{1}, \boldsymbol{n}_{2}\right)\left(\begin{array}{cc}
0 & -1 \\
-1 & 0
\end{array}\right)\left(\begin{array}{c}
\phi_{1} \\
\phi_{2}
\end{array}\right)
$$

für die Drehmomente. Insgesamt lassen sich daher die unbekannten Normalkräfte eliminieren, und es ergibt sich

$$
\begin{aligned}
\left(\begin{array}{l}
F_{1} \\
F_{2}
\end{array}\right) & =\frac{1}{\left(\boldsymbol{p}, \boldsymbol{n}_{1}, \boldsymbol{n}_{2}\right)}\left(\begin{array}{ll}
1 & c \\
c & 1
\end{array}\right)\left(\begin{array}{ll}
0 & 1 \\
1 & 0
\end{array}\right)\left(\begin{array}{l}
T_{1} \\
T_{2}
\end{array}\right) \\
& =\frac{1}{\left(\boldsymbol{p}, \boldsymbol{n}_{1}, \boldsymbol{n}_{2}\right)}\left(\begin{array}{ll}
c & 1 \\
1 & c
\end{array}\right)\left(\begin{array}{l}
T_{1} \\
T_{2}
\end{array}\right) .
\end{aligned}
$$

Es besteht also ein eindeutiger Zusammenhang zwischen auf den Zwangskontakt wirkenden Kräften und Drehmomenten. Ebenso muß ein eindeutiger Zusammenhang zwischen den in der Bewegung gehemmten Komponenten der Lage bestehen. Möglicherweise ist in einem geeigneten schiefwinkligen Koordinatensystem eine einfache Gestalt von $\mathcal{Z}_{S}$ ableitbar.

Eine alternative Methode zur Berücksichtigung von Zwangskräften beruht auf der Additivität von Steifheitsmatrizen bei parallel geschalteten Aufhängungen. Sie besteht darin, für die Zwangskontakte eine Steifheitsmatrix mit beliebig großen Einträgen anzusetzen und diese dann zu der der Aufhängung zu addieren ${ }^{4}$ Die potentielle Energie ist nach wie vor im Minimum, aber unter Nebenbedingungen. Mit der Methode der LAGRANGE-Multiplikatoren erhält man etwa für zwei Kontakte das Potential

$$
\Phi^{\prime}=\frac{1}{2} q^{T} \mathcal{S} q+\lambda_{1} z_{1}^{T} q+\lambda_{2} z_{2}^{T} q \stackrel{!}{=} \min
$$

mit den zugehörigen Lasten

$$
f=\mathcal{S} q+\lambda_{1} z_{1}+\lambda_{2} z_{2}
$$

Die Zwangsbedingungen sind hier also, bis auf Vorfaktoren, die unbekannten Vorlasten selber. Durch Quadrieren der Zwangsbedingungen ändert sich nur die Bedeutung der LAGRANGE-Multiplikatoren. Man erhält

$$
\Phi^{\prime}=\frac{1}{2} q^{T} \mathcal{S} q+\frac{1}{2} \lambda_{1} q^{T}\left(z_{1} z_{1}^{T}\right) q+\frac{1}{2} \lambda_{2} q^{T}\left(z_{2} z_{2}^{T}\right) q \stackrel{!}{=} \min
$$

und für die Lasten ergibt sich

$$
f=\left(\mathcal{S}+\lambda_{1}\left(z_{1} z_{1}^{T}\right)+\lambda_{2}\left(z_{2} z_{2}^{T}\right)\right) q
$$

Die $\lambda_{1,2}$ entsprechen nun den unbekannten und beliebig großen Einträgen in der Steifheitsmatrix. Sie modellieren also die willkürlichen Zwangskräfte für beliebige Lagen, die Aufhängung ist dann beliebig steif.

\footnotetext{
${ }^{4}$ Diese Methode nennt 43 treffend "Links and Joints as Virtual Springs".
} 


\section{Literaturverzeichnis}

[1] Jorge Angeles: Rational Kinematics; Springer Tracts in Natural Philosophy 34; Springer (1988)

[2] J. Angeles, A. Kecsméthy (EDs.): Kinematics and dynamics of multibody systems; International Centre for Mechanical Systems (CISM) courses and lectures No. 360; Springer-Verlag, Wien, New York (1995)

[3] Benedikt BeCKer: Physikalische Grundlagen orthodontischer Zahnbewegung; Diplomarbeit, IV. Physikalisches Institut, Universität Göttingen (1989)

[4] Benedikt Becker: Die Physik der Zahnbewegung; Schriftliche Mitteilung und unvollendete Dissertation, IV. Physikalisches Institut, Universität Göttingen

[5] B. K. B: Berkowitz, B. J. Moxham, H. N. Newman: The Periodontal Ligament in Health and Disease; Pergamon Press; Oxford, New York, Toronto, Sydney, Paris, Frankfurt (1982)

[6] C. Biezeno, R. Grammel: Technische Dynamik; Springer, Berlin (1939)

[7] Christoph Bourauel: Simulation of Orthodontic Tooth Movements - A Comparision of Numerical Models; Journal of Orofacial Orthopedics / Fortschritte der Kieferorthopädie 60, 136-51 Nr. 2, Urban \& Vogel München (1999)

[8] K. B. Broberg: On the Mechanical Behaviour of Intervertebral Discs; Spine, Vol. 8, Nr. 2; Harper \& Row Publishers Inc. (1983)

[9] Edgar Brunner: Angewandte Statistik II; Vorlesungsskript Universität Göttingen; Fassung vom 17.07.; (1994)

[10] R. A. Burn-Murdoch, D. W. Tyler: Physiological Evidence that Periodontal Collagen in the Rat Exists as Fibres Prior to Histological Fixation; Archs. Oral Biol. Vol. 26 pp. 995-999, (1981)

[11] Christiansen, R. L., Burstone, C. J.: Centers of rotation within the periodontal space; Am. J. Orthodontics, Vol. 55, Nr. 4 (1969) 
[12] Edgar D. Coolidge: The Thickness of the Human Periodontal Membrane; Journal of the American Dental Association, Vol. 24, pp. 1260-1270 (1937)

[13] E. J. Davidian: Use of a computer model to study the force distribution on the root of the maxillary central incisor; Am. J. Orthodont. Vol. 59 Nr. 6; St. Louis (1971)

[14] A. C. Eringen (Ed.): Continuum Physics; New York, London (Academic Press 1971)

[15] H. Feneis: Gefüge und Funktion des normalen Zahnfleischbindegewebes; Deutsche Zahnärztliche Zeitschrift 7, S. 467-76; Carl Hanser Zeitschriftenverlag; München (1952)

[16] Отто Fischer: Kinematik organischer Gelenke; Die Wissenschaft 18; Friedrich Vieweg und Sohn; Braunschweig (1907)

[17] Gilbert D. Fish: Some Engineering Principles of Possible Interest to Orthodontists; The Dental Cosmos, Vol. 59, pp. 881-889, Philadelphia, White (1917)

[18] S. FlügGe (Hrsg.): Handbuch der Physik; Berlin, Göttingen, Heidelberg (Springer 1960)

[19] W. FlüGGE: Viscoelasticity; 2. Ed., Berlin, Heidelberg, New York (Springer 1975)

[20] F. R. Gantmacher: Matrizenrechnung; Teil 1: Allgemeine Theorie, Teil 2 : Spezielle Fragen und Anwendungen; VEB Deutscher Verlag der Wissenschaften; Berlin (1958, 1959)

[21] Andrea GörTz: Meßverfahren zur Bestimmung der Bewegung von HüftEndo-Prothesen in Relation zur Knochenbewegung bei Anregung von Biegeschwingungen; Diplomarbeit, IV. Physikalisches Institut, Universität Göttingen (1996)

[22] Gray, A.: Differentialgeometrie; Spektrum Akademischer Verlag, Heidelberg, Berlin, Oxford (1994)

[23] A. Gysi: Resilency and the Like In Its Effect On the Facet Angulations of Artificial Teeth; Dental Digest 10, Vol. 36 (1930)

[24] R. Haller: Neopositivismus; Wiss. Buchgesellschaft, Darmstadt (1993)

[25] J. A. Haringx: On Highly Compressible Helical Springs and Rubber Rods and Their Application for Vibration-Free Mountings, parts 1 and 2; Philips Research Reports, Vol. 3 401-449, Vol. 4 49-80 (1949) 
[26] Hoffmann-Axthelm (Hrsg.): Lexikon der Zahnmedizin, 3.(8.) neubearbeitete Auflage; Quintessenz Verlags-GmbH, Berlin, Chicago, London, Rio de Janeiro, Tokio (1983)

[27] J. HoscheK: Liniengeometrie; Bibliographisches Institut, Zürich (1971)

[28] J. Hoschek, D. LASSER: Grundlagen der geometrischen Datenverarbeitung; B. G. Teubener, Stuttgart (1992)

[29] P. C. Hughes, G. B. Sincarsin: Dynamics of an elastic multibody chain: Part B-Global Dynamics; Dynamics and Stability of Systems Vol. 4 Nos. $3 \& 4$ (1989), pp. 227-244

[30] Manfred Husty, Adolf Karger, Hans Sachs, Waldemar SteinhilPER: Kinematik und Robotik; Springer-Verlag; Berlin, Heidelberg (1997)

[31] W. Kahle, H. Leonhardt, W. Platzer: Taschenatlas der Anatomie, Bd. 1: Bewegungsapparat; 5. überarbeitete Auflage; Georg Thieme Verlag Stuttgart, New York und Deutscher Taschenbuch Verlag München (1986)

[32] Thomas B. Kardos, Leslie O. Simpson: A theoretical consideration of the periodontal membrane as a collagenous thixotropic system and its relationship to tooth eruption; J. Periodontal Res. Vol. 14 pp. 444-451 (1979)

[33] M. KleiBer (Ed.): Handbook of Computational Solid Mechanics; SpringerVerlag; Berlin, Heidelberg, New York (1998)

[34] KARL KLOTTER: Technische Schwingungslehre II: Schwinger von mehreren Freiheitsgraden (Mehrläufige Schwinger); Reprint d. zweiten Auflage; Springer-Verlag; Berlin, Heidelberg, New York (1981)

[35] J. KorelC: Symbolic Approach in Computational Mechanics and its Application to the Enhanced Strain Method; Dissertation TH Darmstadt, FB Mechanik; Darmstadt (1996)

[36] Kowalsky, H. J.: Lineare Algebra; 9. Aufl., Walter de Gruyter, Berlin, New York (1979)

[37] E. Kreuzer (Ed.): Computerized Symbolic Manipulation in Mechanics; CISM Courses and Lectures No. 343, International Centre for Mechanical Sciences; Springer-Verlag; Wien-New York (1994)

[38] L. D. Landau, E. M. Lifschitz: Mechanik; 12. berichtigte Auflage, Berlin (Akademie-Verlag 1987)

[39] L. D. Landau, E. M. Lifschitz: Hydrodynamik; 5. überarbeitete Auflage, Berlin (Akademie-Verlag 1991)

[40] L. D. LAndau, E. M. Lifschitz: Elastizitätstheorie; 7. unveränderte Auflage, Berlin (Akademie-Verlag 1991) 
[41] L. LindKVIST: Three-Dimensional Load-Deformation Relationships of Arbitrarily Loaded Coiled Springs; Doktorsavhandlingar vid Chalmers Tekniska Högskola, Ny serie Nr. 1081 (Göteborg Sweden 1995)

[42] D. A. Loch, Z. Luo, J. L. Lewis, N. J. Stewart: A Theoretical Model of the Knee and the ACL: Theory and Experimental Verification; J. Biomechanics, Vol. 25, No. 1, pp. 81-95 (1992)

[43] MaRT MäGI: On the significance of the pre-load state in linearized analysis of vibration in lumped parameter mechanical systems; Report, Mechanical Engineering Department, Chalmers University of Technology; Göteborg, Sweden (1987)

[44] J. Middleton, M. Jones, A. Wilson: The role of the periodontal ligament in bone modeling: The initial development of a time-dependent finite element model; Am. J. Orthod. Dentofacial Orthop. 109(2) pp. 155-62 (1996)

[45] V. C. Mow, W. M: LAI: Recent developments in synovial joint biomechanics; SIAM Rev. 22, pp. 275-317 (1980)

[46] I. MüLLER: Thermodynamik; Bertelsmann Universitätsverlag, Düsseldorf (1973)

[47] R. J. Nikolai: Periodontal ligament reaction and displacements of a maxillary central incisor subjected to transverse crown loading; J. Biomechanics, Vol. 7 pp. 93-99; Pergamon Press (1974)

[48] H. NÄGerl, D. Kubein-Meesenburg, J. Fanghänel: Elements of a General Theory of Joints, 2. Introduction into a Theory of Synarthrosis; Anat. Anz., Jena 171 (1990) 323-333

[49] NÄGERL H: Biomechanische Prinzipien in Diarthrosen und Synarthrosen; Habilitationsschrift am Fachbereich Medizin, Göttingen (1990)

[50] H. Nägerl, D. Kubein-Meesenburg, Ch. J. Burstone, B. Becker: Theoretische und experimentelle Aspekte zur initialen Zahnbeweglichkeit; Inform. Orthodont. Kieferorthop. 23 43-61; Hüthig-Verlag, Heidelberg, München (1991)

[51] J. Palacios: Dimensional Analysis; MacMillan \& Co Ltd, London; St Martin's Press , New York (1964)

[52] M. M. PAnJabi: Three-Dimensional Mathematical Model of the Human Spine Structure; Journal of Biomechanics 6 (1973) pp. 671-680

[53] M. M. Panjabi, A. Brand JR., A. A. White III: Three-Dimensional Flexibility and Stiffness Properties of the Human Thoracic Spine; Journal of Biomechanics 9 (1976) pp. 185-192 
[54] U. Schramm, L. Kitis, W. Kang, W. D. Pilkey: On the shear deformation coefficient in beam theory; Finite Elements in Analysis and Design No. 16 pp. 141-162; Elsevier (1994)

[55] A. M. SchwarZ: Über die Bewegung belasteter Zähne; Zeitschrift für Stomatologie, Bd. 26 S. 40-83; Springer, Wien (1928)

[56] A. M. SCHWARZ: Mathematische Überprüfung des Verhaltens belasteter Zähne; Zeitschrift für Stomatologie, Bd. 26 S. 398-403; Springer, Wien (1928)

[57] J. C. Simo: The (symmetric) Hessian for geometrically nonlinear models in solid mechanics: Intrinsic definition and geometric interpretation; Computer Methods in Applied Mechanics and Engineering Vol. 96 pp. 189-200 (1992)

[58] G. B. Sincarsin, P. C. Hughes: Dynamics of an elastic multibody chain: Part A-Body motion equations; Dynamics and Stability of Systems Vol. 4 Nos. $3 \& 4$ (1989) pp. 209-226

[59] Stefan SPIERING: Kinematik der Lendenwirbelsäule unter Lastvariationen; Diplomarbeit IV. Phys. Inst., Universität Göttingen (1995)

[60] A. Sommerfeld: Mechanik, Vorlesungen über Theoretische Physik I; Nachdruck der 8. durchgesehenen Auflage, Verlag Harri Deutsch; Thun, Frankfurt/Main (1977)

[61] C. L. Steyn, W. S. Verwoerd, E. J. van der Merwe, O. L. Fourie: Calculation of the Position of the Axis of Rotation when Single-rooted teeth are Orthodontically tipped; Brit. J. Orthodont. Vol. 5 pp. 153-156 (1978)

[62] J. Stuelpnagel: On the Parametrization of the Three-Dimensional Rotation Group; SIAM Review Vol. 6 No. 4; (1964)

[63] W. J. Sutcliffe, J. D. Atherton: The Mechanics of Tooth Mobility; Brit. J. Orthodont.; Vol. 7 pp. 171-178, (1980)

[64] J. L. Synge: The Tightness of the Teeth considered as a Problem concerning the Equilibrium of a Thin Incompressible Elastic Membrane; Philosophical Transactions of the Royal Society Of London, Series A, 231, Pp. 435-477, London (Harrison and Sons 1933)

[65] J. L. Synge: The Equilibrium of a Tooth with a General Conical Root; The London, Edinburgh and Dublin Philosophical Magazine and Journal of Science 7. Ser. Vol. XV Nr. CI-May, Pp. 969-996, London (Taylor and Francis 1933)

[66] J. L. Synge: The Theory of an Incompressible Periodontal Membrane; The International Journal of Orthodontia and Dentistry for Children; Vol. XIX Nr. 6 June, Pp. 567-73; St. Louis (1933) 
[67] István Szabó: Geschichte der mechanischen Prinzipien; Birkhäuser Verlag; Basel und Stuttgart (1977)

[68] István Szabó: Höhere Technische Mechanik; Springer-Verlag; Berlin, Heidelberg, New York, Tokyo; Zweiter Nachdruck d. fünften verb. u. erw. Auflage (1985)

[69] H. Teichmann: Physikalische Anwendungen der Vektor- und Tensorrechnung; Bibliographisches Institut (1973)

[70] I. Todhunter: A history of the theory of elasticity and of the strength of materials from Galilei to the present time; Edited and compiled by Karl Pearson, Vol. 1: Galilei to Saint-Venant 1639 - 1850 Cambridge (1886), Vol. 2: Saint-Venant to Lord Kelvin, 2 Bde. Cambridge (1893)

[71] C. Truesdell, W. Noll: Representation Theorems in: [18] Bd.III/3 The Non-Linear Field Theories of Mechanics Ch. B.II

[72] Wolfgang SchäFer: Messung der räumlichen Mikrobewegung und der Verbiegung des Femurschaftes von Hüft-Endo-Prothesen in Abhängigkeit eines räumlichen Kraftsystems; Diplomarbeit, IV. Physikalisches Institut, Universität Göttingen (1995)

[73] R. Zurmühl, S. FALK : Matrizen und ihre Anwendungen; Fünfte, überarbeitete und erweiterte Auflage; Springer-Verlag, Berlin, Heidelberg, New York, Tokyo (1984) 


\section{Lebenslauf}

\section{Angaben zur Person}

$\begin{array}{ll}\text { Name } & \text { Dathe } \\ \text { Vorname } & \text { Henning } \\ \text { Wohnort } & \text { 37075 Göttingen, Arndtstr. 8 } \\ \text { Geburtstag } & \text { 16. März 1964 } \\ \text { Geburtsort } & \text { Braunschweig } \\ \text { Eltern } & \text { Wolfgang Friedrich Walter Dathe, } \\ & \text { Eva Elisabeth Dathe, geb. Görig. } \\ \text { Staatsangehörigkeit } & \text { deutsch } \\ \text { Familienstand } & \text { ledig }\end{array}$

\section{Schulausbildung}

1970 bis 1983

Schulbesuch in Braunschweig, zuletzt am Gymnasium Raabeschule.

Mai 1983 Abschluß: Allgemeine Hochschulreife

\section{Hochschulstudium Elektrotechnik}

Wintersemester 1983/84 Technische Universität Braunschweig

\section{Zivildienst}

Mai 1984

bis August 1985
Einberufung zum Zivildienst

Krankentransport/Rettungsdienst beim

Deutschen Roten Kreuz Wolfenbüttel

\section{Hochschulstudium Physik}

ab Wintersemester 1985/86

Oktober 1987

ab Wintersemester 1987/88

Oktober 1992
Technische Universität Braunschweig Vordiplom

Georg-August-Universität Göttingen

Diplom am Institut für Theoretische Physik

\section{Hochschulstudium Lehramt Gymnasien}

Sommersemester 1993

Wintersemester 1993

\section{Beschäftigungen}

1984

seit 1986

1988-1992

seit 1993
Georg-August-Universität Göttingen

Lehramt Gymnasien Mathematik/Physik

Zwischenprüfung in Mathematik

Praktikum bei Siemens/Braunschweig Lehrer an der Freien Musikschule Göttingen Wiss. Hilfskraft (Übungsgruppenleiter) Wissenschaftlicher Mitarbeiter am Klinikum der Universität Göttingen Abteilung Kieferorthopädie, Arbeitsgruppe Biomechanik 